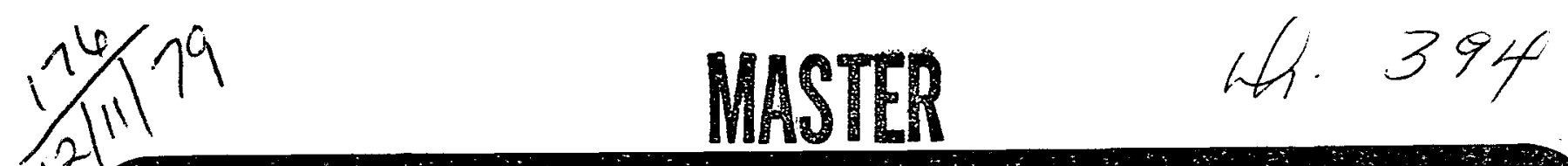

GA-A14726

UC-77

\title{
NUCLEAR AND THERMAL DESIGN VERIFICATION FOR THE PEACH BOTTOM HIGH-TEMPERATURE GAS-COOLED REACTOR
}

\author{
by \\ J. J. SAURWEIN and C. F. WALLROTH
}

\author{
Prepared under \\ Contract EY-76-C-03-0167 \\ Project Agreement No. 56 \\ for the San Francisco Operations Office \\ Department of Energy and the \\ Electric Power Research Institute
}

DATE PUBLISHED: SEPTEMBER 1979

\section{GENERAL ATOMIC COMPANY}




\section{DISCLAIMER}

This report was prepared as an account of work sponsored by an agency of the United States Government. Neither the United States Government nor any agency Thereof, nor any of their employees, makes any warranty, express or implied, or assumes any legal liability or responsibility for the accuracy, completeness, or usefulness of any information, apparatus, product, or process disclosed, or represents that its use would not infringe privately owned rights. Reference herein to any specific commercial product, process, or service by trade name, trademark, manufacturer, or otherwise does not necessarily constitute or imply its endorsement, recommendation, or favoring by the United States Government or any agency thereof. The views and opinions of authors expressed herein do not necessarily state or reflect those of the United States Government or any agency thereof. 


\section{DISCLAIMER}

Portions of this document may be illegible in electronic image products. Images are produced from the best available original document. 
This report was prepared as an account of work sponsored by the United States Government. Neither the United States nor the Department of Energy, nor any of their employees, nor any of their contractors, subcontractors, or their employees, makes any warranty, express or implied, or assumes any legal liability or responsibility for the accuracy, completeness or usefulness of any information, apparatus, product or process disclosed, or represents that its use would not infringe privately owned rights.

Printed in the United States of America Avallable from

National Technical Information Service

U.S. Department of Commerce 5285 Port Royal Road

Springfield, Virginta 22161

Price: Printed Copy $\$ 9.25$; Microfiche $\$ 3.00$ 
GA-A14726

UC-77

\title{
NUCLEAR AND THERMAL DESIGN VERIFICATION FOR THE PEACH BOTTOM HIGH-TEMPERATURE GAS-COOLED REACTOR
}

\author{
by \\ J. J. SAURWEIN and C. F. WALLROTH
}

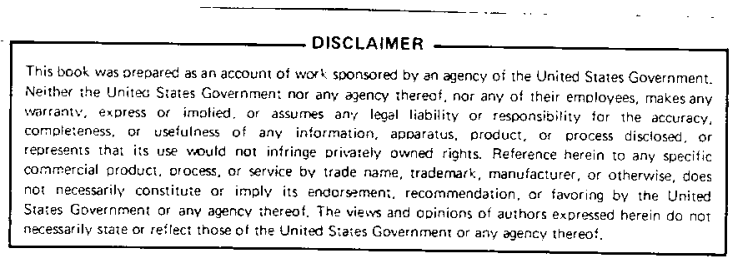

Prepared under

Contract EY-76-C-03-0167

Project Agreement No. 56

for the San Francisco Operations Office

Department of Energy and the

Electric Power Research Institute

\author{
GENERAL ATOMIC PROJECT 3238 \\ DATE PUBLISHED: SEPTEMBER 1979
}

\section{GENERAL ATOMIC COMPANY}


$\theta$

-

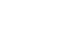

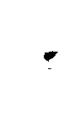

: 
ABSTRACT

This report describes the efforts by General Atomic Company to verify high-temperature gas-cooled reactor (HTGR) nuclear and thermal design methods through verification of the calculational methods for nuclear and thermal performance of Peach Bottom Core 2 standard driver fuel elements.

Time-averaged and end-of-life (EOL) axial power profiles for individual fuel elements were established from measured Cs-137, Zr-95, and La-140 distributions and used to determine the core average time and EOL unrodded axial power profiles for comparison with design code predictions. The compact-to-compact agreement between the measured and predicted time-averaged power distributions was found to be $\pm 7.4 \%$ ( $\left.1 \sigma^{*}\right)$, which is we11 within the $\pm 8 \%$ to $13 \%$ quoted for other nuclear reactors. Uncertainties in the predictive accuracy resulting from measurement uncertainties are $\pm 3.5 \%(1 \sigma)$ and $\pm 1.6 \%(1 \sigma)$ for the time-averaged and EOL power profiles, respectively. Comparisons of measured unrodded power distributions at 385, 701, and 897 effective full-power days (EFPD) verify the prediction that the power in elements whose axial power shapes were unperturbed by control rods shifted from the bottom of the core and smoothed out with fuel depletion although the shift of power to the top of the core was shown to be overpredicted.

The fuel burnup in each gamma-scanned driver element was determined from the total Cs-137 inventory and compared with the corresponding burnup prediction to determine the accuracy of element average (radial) power predictions. The core average power prediction was found to be within

$\star_{1} \sigma=1$ standard deviation 
$\pm 0.7 \%(1 \sigma)$, and the element-to-element agreement between predicted and measured power was $\pm 6.8 \%(1 \sigma)$, which is within the $\pm 3 \%$ to $8 \%$ quoted for other nuclear reactors. The uncertainty in the predictive accuracy for the element average power is $14.7 \%(1 \sigma)$.

The combined accuracy for radial and axial power predictions for the Peach Bottom HTGR is $\pm 10 \%(1 \sigma)$ compared to $\pm 8 \%$ to $15 \%$ for other reactor systems. The uncertainty for the combined accuracy is $\pm 6 \%$ ( $1 \sigma$ ).

A fuel compact irradiation strain correlation defining radial strain as a function of fast neutron fluence and temperature was developed from fuel compact metrology data. Use of this correlation in the thermal calculations permitted reasonable modeling of the radial gap between sleeve and fuel compact.

Temperature predictions were made and compared with measured peak fuel temperatures provided by in-pile thermocouple readings. The agreement between predicted and measured temperatures is within $\pm 87^{\circ} \mathrm{C}(1 \sigma)$ with an apparent bias of $+27^{\circ} \mathrm{C} \pm 7^{\circ} \mathrm{C}(1 \sigma)$ in the temperature predictions. The most probable causes for this general overprediction of temperatures are eccentricity of the sleeve-fuel compact gap and underestimation of the fuel compact thermal conductivity. The agreement between calculated and measured temperatures varied significantly in different regions of the core. Average biases (calculated minus measured) were determined to be $-18^{\circ} \mathrm{C} \pm 49^{\circ} \mathrm{C}(1 \sigma)$ in the inner six rings of fuel elements, $+85^{\circ} \mathrm{C} \pm 119^{\circ} \mathrm{C}$ $(1 \sigma)$ in rings 7 through 12 , and $+46^{\circ} \mathrm{C} \pm 54^{\circ} \mathrm{C}(1 \sigma)$ in rings 13 through 15. 
CONTENTS

ABSTRACT ................................ 11

1. INTRODUCTION . . . . . . . . . . . . . . . 1-1

2. NUCLEAR PERFORMANCE ................... 2- . . .

2.1. Physics Calculations ............. 2-1

2.2. Power Measurements . . . . . . . . . 2-3

2.3. Comparison of Measured and Predicted Power ...... 2-4

2.3.1. Axial Power Profiles........... 2-4

2.3.2. Radial Power Profiles ....... . . 2-7

2.3.3. Fue1 Burnup ............. . 2-7

3. SLEEVE-FUEL COMPACT GAP ............... . . . . . .

3.1. Correlation for Fuel Compact Radial Strain....... 3-1

3.2. Comparison of Predicted and Measured Sleeve-Fuel Compact Radial Gaps . . . . . . . . . . . 3-3

4. THERMAL PERFORMANCE ................. 4- . .

4.1. Temperature Calculations . . . . . . . . . . 4-1

4.2. Feedback of Power Measurements into Thermal Calculations 4-2

4.3. Temperature Measurements . . . . . . . . . 4-3

4.4. Thermocouple Recalibration ........... 4-5

4.5. Comparison of Calculated and Measured Temperatures . . 4-9

5. DISCUSSION AND CONCLUSIONS .............. . . 5-1

6. ACKNOWLEDGMENTS . . . . . . . . . . . . . 6-1

7. REFERENCES ........................ . . . . . .

APPENDIX: IRRADIATION CONDITIONS AND FUEL PERFORMANCE DATA FOR

PEACH BOTTOM FUEL ELEMENTS . . . . . . . . . . A-1

FIGURES

1-1. Driver fuel element with thermocouple, Peach Bottom Core 2 . 1-4

1-2. Driver element fuel compact, Peach Bottom Core 2 . . . . . 1-5 
FIGURES (continued)

1-3. Driver element solid spines, Peach Bottom Core 2 . . . . 1-6

1-4. Core locations of instrumented and examined driver fuel elements..................... . 1-7

2-1. Unrodded axial power profile predictions for Peach Bottom Core 2. . . . . . . . . . . . . . . . . . 2-9

2-2. Unrodded axial fast flux profile predictions for Peach Bottom Core 2. . . . . . . . . . . . . . 2-10

2-3. Partially rodded axial power profile predictions for Peach Bottom Core 2 . . . . . . . . . . . . . . . . 2-11

2-4. Partially rodded axial fast flux profile predictions for Peach Bottom Core 2 . . . . . . . . . . . . . 2-12

2-5. Normalized $\mathrm{Zr}-95$ and La-140 distributions for E14-01 . . . 2-13

2-6. Normalized $\mathrm{Zr}-95$ and La-140 distributions for F03-01 . . . 2-14

2-7. Comparison of measured and predicted time-averaged unrodded axial power profiles for Peach Bottom Core 2. . . . . . . 2-15

2-8. Comparison of measured and predicted EOL unrodded axial power profiles for Peach Bottom Core 2. . . . . . . . 2-16

2-9. Comparison of E06-01 normalized $\mathrm{Zr}-95$ distribution with predicted 300 and 600 EFPD unrodded axial power profiles . 2 2-17

2-10. Comparison of E11-07 normalized $\mathrm{Zr}-95$ distribution with predicted 600 and 900 EFPD unrodded axial power profiles; E11-07 was removed for postirradiation examination after 701 EFPD . . . . . . . . . . . . . . . . 2-18

2-11. Measured unrodded axial power profiles at 385,701 , and 897 EFPD ..................... 2-19

2-12. Comparison of measured and predicted radial power distributions for Peach Bottom Core 2............... 2-20

3-1. Fuel compact Irradiation-induced radial strain as a function of fast neutron fluence and temperature . . . . . . . 3-4

4-1. Peach Bottom core 2 thermal power . . . . . . . . . 4-13

4-2. Peach Bottom core 2 operating parameters . . . . . . . . 4-14

4-3. TREVER calculation locations for Peach Bottom driver fuel elements . . . . . . . . . . . . . . . 4-15

4-4. FTE-18, FPTE-3, and FTE-5 thermocouple EOL recalibration results .. . . . . . . . . . . . . . 4 4-16

4-5a. Peach Bottom thermocouple recalibration term $\mathrm{T}_{0}$. . . . . 4-17 4-5b. Peach Bottom thermocouple recalibration term K . . . . . 4-17 


\section{FIGURES (continued)}

4-6a. Height correlation for Type W Peach Bottom thermocouple recalibration term $\mathrm{T}_{\mathrm{o}}$.. . . . . . . . . . . . . . 4-18

4-6b. Height correlation for Type W Peach Bottom thermocouple recalibration term $\mathrm{K}$. . . . . . . . . . . . . 4-18

4-7. Comparison of predicted temperatures with E01-01 C/A thermocouple readings . . . . . . . . . . . . . 4-19

4-8. Comparison of predicted temperatures with E01-01 W/Re thermocouple readings . . . . . . . . . . . . . 4-19

4-9. Comparison of predicted temperatures with B02-02 C/A thermocouple readings . . . . . . . . . . . . . 4-20

4-10. Comparison of predicted temperatures with E02-01 C/A thermocouple readings . . . . . . . . . . . . 4-20

4-11. Comparison of predicted temperatures with E02-01 W/Re thermocouple readings .. . . . . . . . . . . . 4-21

4-12. Comparison of predicted temperatures with B03-03 C/A thermocouple readings . . . . . . . . . . . . 4-21

4-13. Comparison of predicted temperatures with E03-01 C/A thermocouple readings . . . . . . . . . . . . 4-22

4-14. Comparison of predicted temperatures with E03-01 W/Re thermocouple readings . . . . . . . . . . . . . 4-22

4-15. Comparison of predicted temperatures with E05-01 C/A thermocouple readings .. . . . . . . . . . . 4-23

4-16. Comparison of predicted temperatures with E05-01 W/Re thermocouple readings .. . . . . . . . . . 4-23

4-17. Comparison of predicted temperatures with E05-01 C/A thermocouple readings . . . . . . . . . . . . 4-24

4-18. Comparison of predicted temperatures with E07-01 W/Re thermocouple readings . . . . . . . . . . . . 4-24

4-19. Comparison of predicted temperatures with E09-01 W/Re thermocouple readings .. . . . . . . . . . . 4-25

4-20. Comparison of predicted temperatures with E11-01 W/Re thermocouple readings . . . . . . . . . . . 4-25

4-21. Comparison of predicted temperatures with E13-01 C/A thermocouple readings .. . . . . . . . . . 4-26

4-22. Comparison of predicted temperatures with E13-01 W/Re thermocouple readings . . . . . . . . . . . 4-26

4-23. Comparison of predicted temperatures with E14-01 C/A thermocouple readings . . . . . . . . . . . 4-27 
FIGURES (Continued)

4-24. Comparison of predicted temperatures with E15-01 W/Re thermocouple readings . . . . . . . . . . 4-27

4-25. Comparison of predicted temperatures with A14-14 C/A thermocouple readings . . . . . . . . . . . . . 4-28

TABLES

2-1. Peach Bottom core 2 GAUGE3 depletion . . . . . . . . . 2-21

2-2. Driver elements gamma-scanned by General Atomic . . . . 2-22

2-3. Comparison of measured and predicted time-averaged unrodded axial power profiles for Peach Bottom core 2 . . . . . . 2-23

2-4. Comparison of measured and predicted EOL unrodded axial power profiles for Peach Bottom core 2. . . . . . . . 2-24

3-1. Data base for fuel compact 1rradiation-Induced radial strain correlation . . . . . . . . . . . . 3-5

3-2. Comparison of measured and predicted sleeve-fuel compact radial gap... . . . . . . . . . . . . . . 3-6

4-1. TREVER time points . . . . . . . . . . . . . . 4-29

4-2. Summary of material property data used in TREVER analysis - 4-30

4-3. Thermocouple performance . . . . . . . . . . . . 4-31

4-4. Summary of Peach Bottom core 2 thermocouple performance . 4-32

4-5. E01-01 comparison of TREVER and C/A thermocouple temperatures.............. . 4-34

4-6. E01-01 comparison of TREVER and W/Re thermocouple temperatures............... 4-35

4-7. E02-02 comparison of TREVER and C/A thermocouple temperatures................. 4-36

4-8. E02-01 comparison of TREVER and C/A thermocouple temperatures.............. . . 4-37

4-9. E02-01 comparison of TREVER and W/Re thermocouple temperatures . . . . . . . . . . . . . . 4-38

4-10. B03-03 comparison of TREVER and C/A thermocouple temperatures . . . . . . . . . . . . . . . 4-39

4-11. E03-01 comparison of TREVER and C/A thermocouple temperatures... .. . . . . . . . . . . . 4 440

4-12. E03-01 comparison of TREVER and $W /$ Re thermocouple temperatures ... . . . . . . . . . . . 4 4-41 
TABLES (Continued)

4-13. E05-01 comparison of TREVER and C/A thermocouple temperatures . . . . . . . . . . . . . . . 4-42

4-14. E05-01 comparison of TREVER and W/Re thermocouple temperatures . . . . . . . . . . . . . . . . 4-43

4-15. E06-01 comparison of TREVER and C/A thermocouple temperatures . . . . . . . . . . . . . . . . . 4-44

4-16. E07-01 comparison of TREVER and W/Re thermocouple temperatures . . . . . . . . . . . . . . . 4 4-45

4-17. E09-01 comparison of TREVER and W/Re thermocouple temperatures... . . . . . . . . . . . . 4-46

4-18. E11-01 comparison of TREVER and W/Re thermocouple temperatures... . . . . . . . . . . . . . 4-47

4-19. E13-01 comparison of TREVER and C/A thermocouple temperatures . . . . . . . . . . . . . . . . . . 4-48

4-20. E13-01 comparison of TREVER and W/Re thermocouple temperatures . . . . . . . . . . . . . . . . . 4-49

4-21. E14-01 comparison of TREVER and C/A thermocouple temperatures . . . . . . . . . . . . . . 4-50

4-22. E15-01 comparison of TREVER and W/Re thermocouple data . . 4-51

4-23. A14-14 comparison of TREVER and C/A thermocouple temperatures.... . . . . . . . . . . 4-52

4-24. Summary of agreement between measured and predicted temperatures for Peach Bottom core 2 (fuel elements in rings 1 through 6) .............. . . 4-53

4-25. Summary of agreement between measured and predicted temperatures for Peach Bottom core 2 (fuel elements In rings 7 through 12 and rings 13 through 15) . . . . . . 4-54

4-26. Summary of agreement between measured and predicted temperatures for Peach Bottom core 2. . . . . . . . . 4-55

4-27. Measured and calculated temperatures.......... 4-56 


\section{INTRODUCTION}

The Peach Bottom Atomic Power Station Unit 1 was the first installation of a high-temperature gas-cooled reactor (HTGR) in the United States. Power operation began in January 1967 and the plant was operated successfully through October 31, 1974, when it was shut down for decommissioning. Subsequent to reactor shutdown, the Peach Bottom End-of-Life Program, cosponsored by the Department of Energy and the Electric Power Research Institute, was initiated. The primary objective of this program was to verify generic HTGR design methods through comparison of measured and predicted nuclear, thermal, fuel, and materials performance in Peach Bottom. This report presents the work done to verify the calculational methods for nuclear and thermal performance of the driver fuel elements irradiated in Core 2 of the Peach Bottom HTGR. A complete description of this reactor is given in Refs. 1 and 2; portions of these documents are summarized below.

The Peach Bottom HTGR core consisted of 804 batch-loaded fuel elements, 36 control rod guide tubes, and 19 emergency shutdown rod guide tubes vertically oriented in a close-packed array. The core was approximately cylindrical in shape with an effective diameter of $2743 \mathrm{~mm}$ ( $9 \mathrm{ft}$ ) and an active height of $2286 \mathrm{~mm}(7.5 \mathrm{ft})$. The design power of the reactor was $115 \mathrm{MW}(\mathrm{t})$, giving an average power density of $8.3 \mathrm{~kW} / 1 \mathrm{iter}$. Cold helium at $2.4 \mathrm{MPa}(350 \mathrm{psi})$ and $327^{\circ} \mathrm{C}\left(620^{\circ} \mathrm{F}\right)$ entered the core and was heated to $704^{\circ} \mathrm{C}\left(1300^{\circ} \mathrm{F}\right)$ during its upward passage through the tricuspid

channels formed by the elements. The reactor was controlled by 36 hydraulically driven control rods inserted from the bottom of the core. An additional 19 electrically driven emergency shutdown rods were under the control of the reactor operator. The guide tubes for the control rods and emergency shutdown rods were made of graphite and were similar in shape to the fuel elements. 
A standard Core 2 driver fuel element, shown in Fig. 1-1, consisted of an upper reflector section, a fuel-bearing middle section, and a bottom reflector section. Outwardly, the element had the appearance of a solid graphite cylinder $89 \mathrm{~mm}$ (3.5 in.) in diameter and $3658 \mathrm{~mm}$ (144 in.) in length, with a grappling knob at the top for handling. The primary components of the fuel element were a bottom connector, sleeve, filter screen, an internal fission product trap assembly, lower reflector piece, 30 fuel compacts, 3 spine pieces, burnable poison compacts (in selected elements), thermocouples (in selected elements), and an upper reflector assembly. Except for the fuel compacts, filter screen, and thermocouples, al1 components were made of graphite. The fuel compacts consisted of fuel particles in a graphite matrix. The fuel particles were between 200 and $600 \mu \mathrm{m}$ in diameter and consisted of uranium/thorium-carbide particles coated with pyrolytic carbon. This fuel was expected to experience temperatures as high as $1510^{\circ} \mathrm{C}\left(2750^{\circ} \mathrm{F}\right)$ in the Peach Bottom core.

The screen, internal trap assembly, lower reflector piece, and fuel compacts with spines were stacked in that order within the 2921-mm (115-in.) long sleeve, which extended from the bottom of the top reflector to the bottom connector of the fuel element. The annular fuel compacts were stacked on the cylindrical graphite spine sections, which were approximately $44.5 \mathrm{~mm}$ (1.75 in.) in diameter and $762 \mathrm{~mm}$ (30 in.) long. Details of the fuel compacts and spines are shown in Figs. 1-2 and 1-3. The top reflector assembly was cemented to the sleeve forming a single unit. Purge gas entered the element through a $6.3-\mathrm{mm}(0.25-\mathrm{in}$ ) channel down the centerline of the top reflector and flowed through the annular gaps between the fuel compacts and sleeve and between the fuel compacts and spine, around the bottom reflector, through the fission product trap and screen, and finally out the centerline hole in the bottom connector. In instrumented elements, the thermocouples were located in a notch cut in the spine, as shown in Fig. 1-3. 
The following work was performed to verify the nuclear and thermal calculations for the driver fuel elements.

1. Measured axial and radial power distributions were obtained and compared with design code predictions.

2. A fuel-compact irradiation-induced radial strain correlation based on fuel compact metrology data was developed.

3. Temperatures were calculated for each instrumented element and each element which underwent postirradiation examination (PIE).

4. Predicted and measured temperatures were compared.

Measured data characterizing the performance of driver fuel elements were obtained from in-pile thermocouple readings (17 elements), gamma scans ( 55 elements), and destructive PIE (7 elements). Six of the seven destructive PIEs were carried out at Oak Ridge National Laboratory (ORNL). The fuel elements for which measured data have been collected are shown in Fig. $1-4$.

Although many of the data presented in Sections 2.2 and 2.3 of this report have already been presented in Ref. 3, they are included in this document for convenience and continuity. In general, Ref. 3 presents the gamma scan data, a discussion of its usefulness, and some sample comparisons between nuclear predicitons and measurements. This report presents the detailed comparisons between nuclear predictions and measurements (Section 2.3) based on all available gamma scan data. These comparisons were performed for the specific purpose of verifying nuclear and thermal design calculations. 

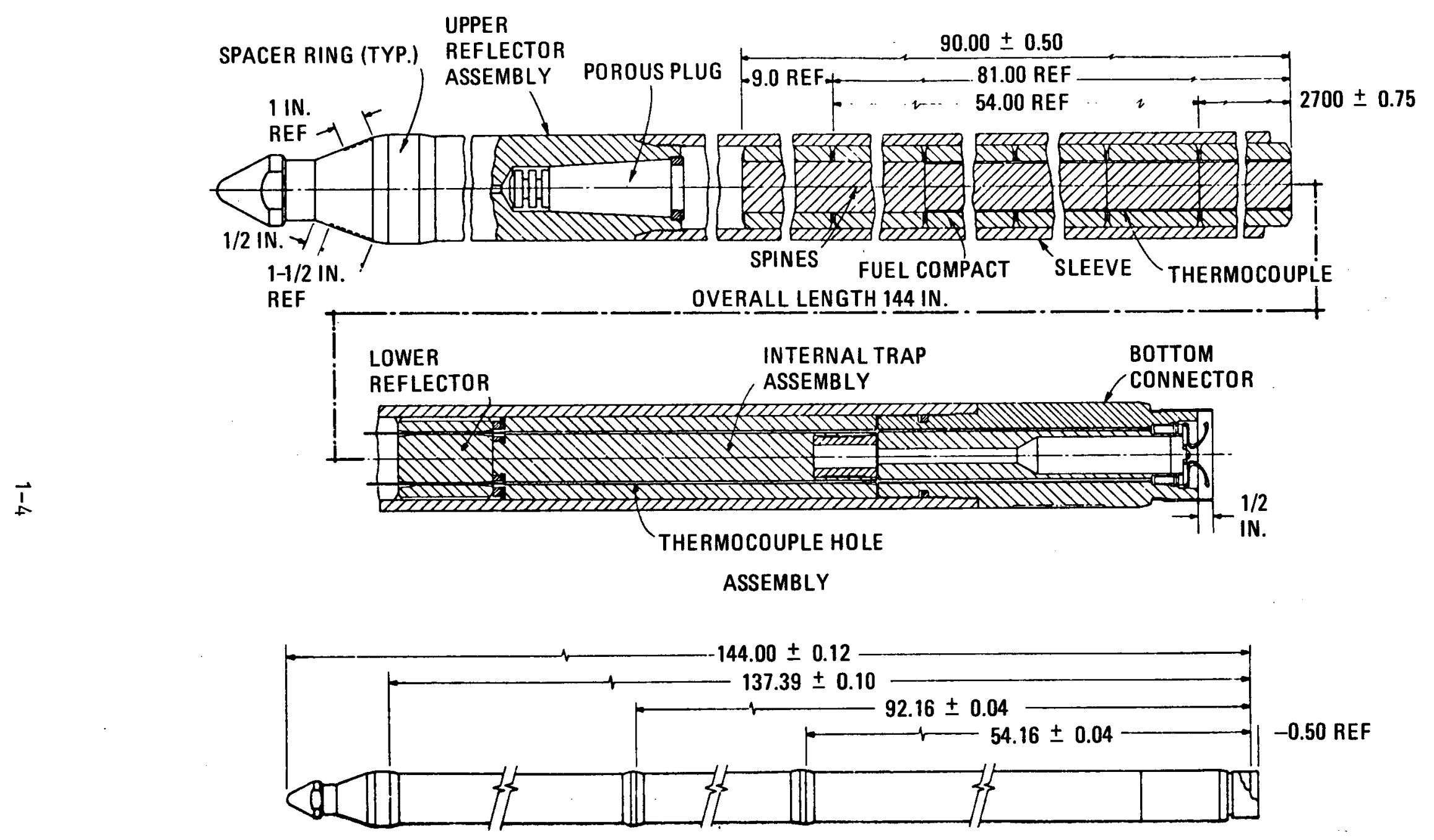

ALL DIMENSIONS IN INCHES

FUEL ELEMENT OUTLINE

Fig. 1-1. Driver fuel element with thermocouple, Peach Bottom Core 2 

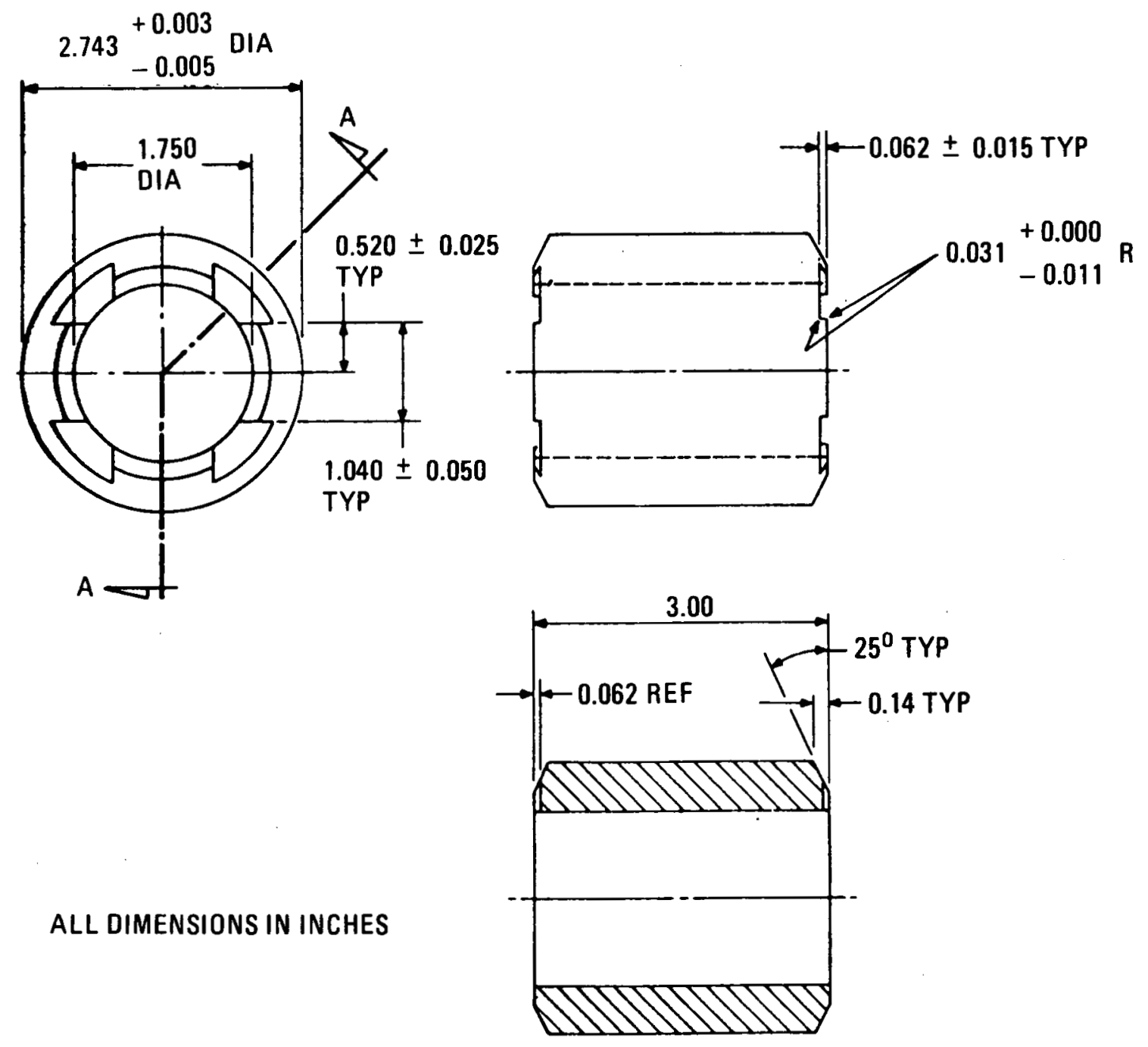

SECTION A-A

Fig. 1-2. Driver element fuel compact, Peach Bottom Core 2 


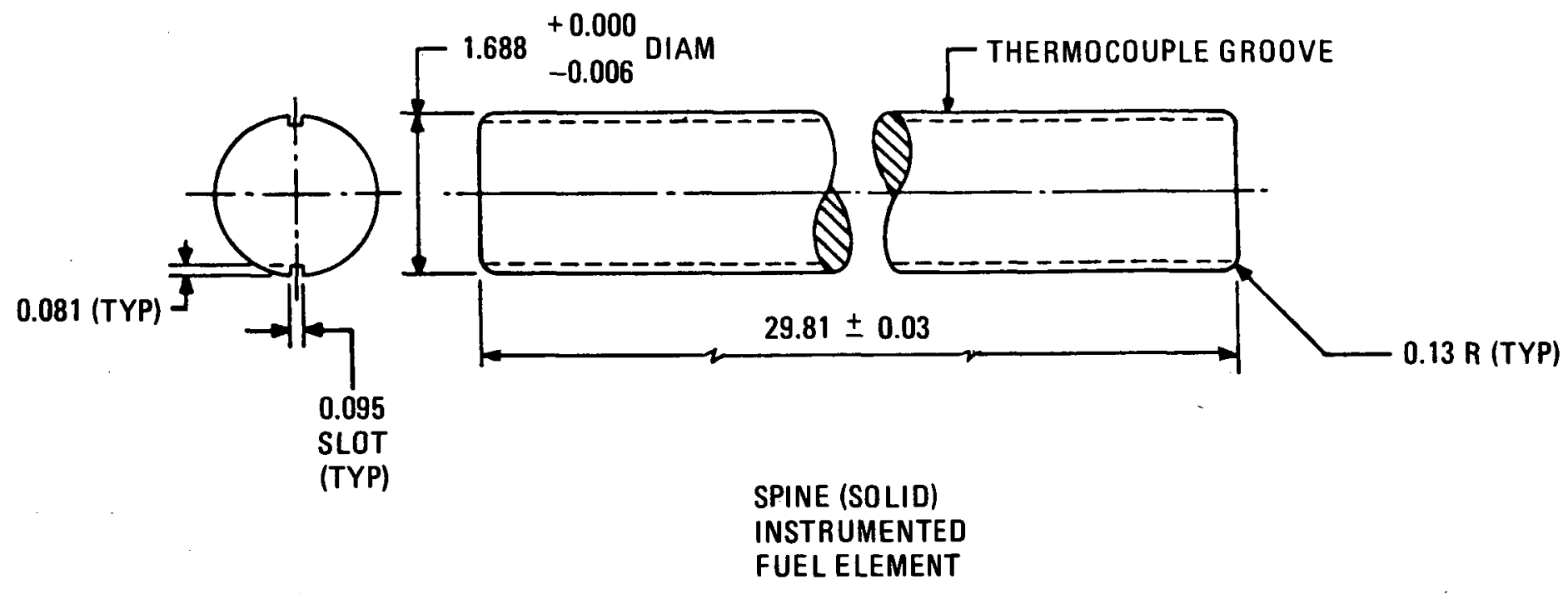

$\vec{a}$

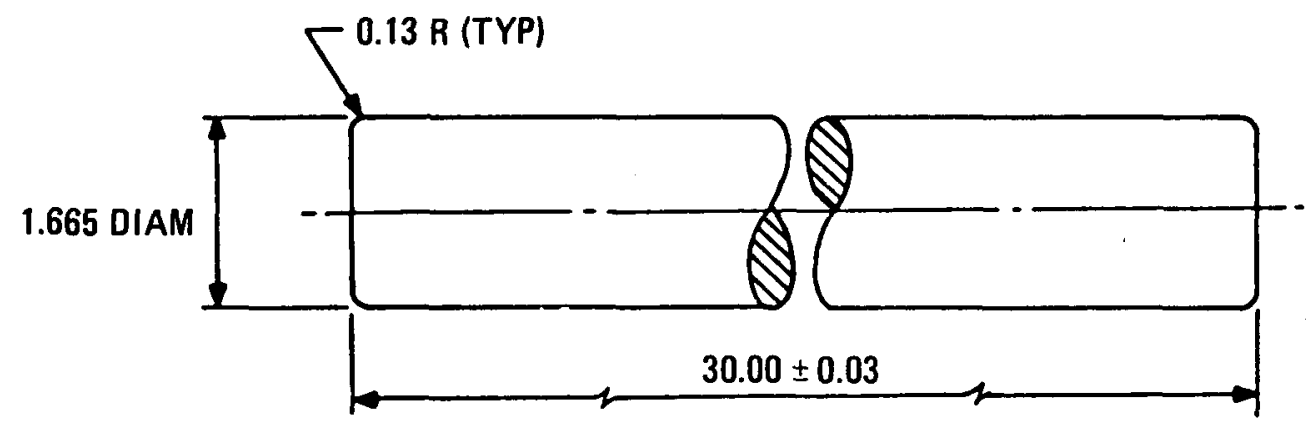

SPINE, SOLID

ALL DIMENSIONS IN INCHES

Fig. 1-3. Driver element solid spines, Peach Bottom Core 2 


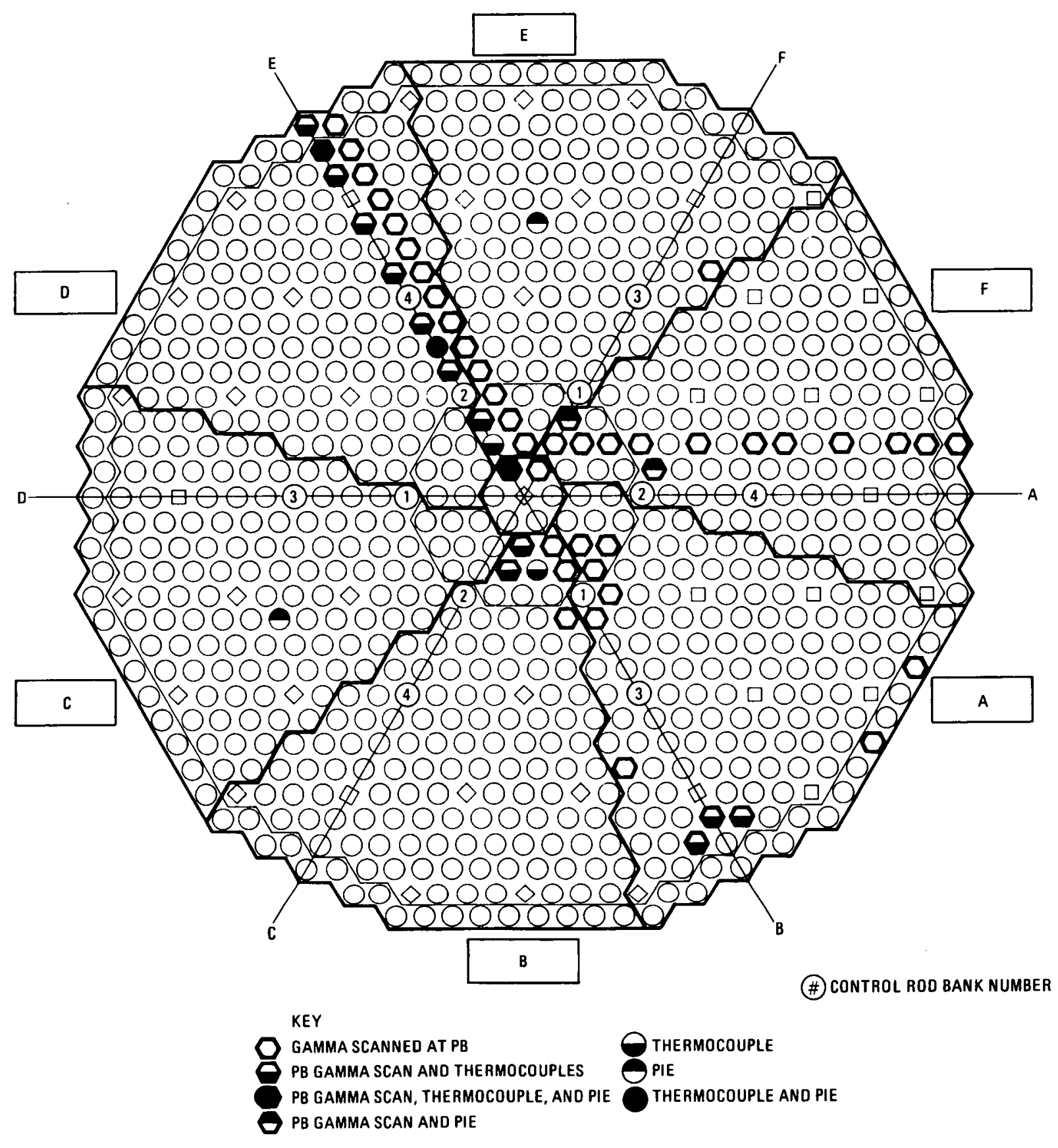

Fig. 1-4. Core locations of instrumented and examined driver fuel elements 


\section{NUCLEAR PERFORMANCE}

\subsection{PHYS ICS CALCULATIONS}

Meaningful verification of thermal design methods is dependent on accurate modeling of the material properties of the fuel elements and the nuclear conditions under which they were irradiated. The construction of a physics model which accurately describes the core operating history is therefore a prerequisite for such a verification procedure. In this context, the factors of primary importance are the radial and axial power and neutron flux distributions. These distributions were calculated using the GAUGE (Ref. 4) and BUG R-Z (Ref. 5) computer codes. These calculations are discussed briefly below.

A BUG R-Z control rod cell was constructed to model the Peach Bottom Core 2 axial power and flux distributions under various degrees of control rod insertion. This cell was then used to calculate control rod reactivity insertion curves. These curves are of primary importance since they provide the link between the axial and radial physics models. The reactivity insertion curve describes the relationship between negative reactivity and the extent of control rod insertion. The radial full-core physics model (GAUGE) associates the partially inserted control rod with an appropriate control rod atom density in the rodded region. The selection of the proper control rod atom density is keyed to the fraction of the total control rod reactivity worth required. Since the physical control rod insertion in the operating reactor is being modeled, it is imperative that the effect of control rod insertion on the reactivity be well modeled in the axial cell model in order to correctly calculate the radial power and flux distributions with the GAUGE code. After completion 
of the reactivity insertion curve calculations, the cell was depleted for 900 EFPD* and unrodded power and flux distributions were calculated at various stages of depletion.

At this point, results of an earlier unrodded axial FEVER (Ref. 6) depletion calculation and those of actual flux distribution measurements made in 1966 (Ref. 7) were taken into consideration for comparative evaluation. It became apparent from this comparison that accurate modeling of the reflectors was critical to the establishment of the overall unrodded power distribution. Unfortunately, the top and bottom reflectors, while having a large effect on the core power distribution, are poisoned in a manner that does not lend itself to easy modeling. The measurements taken in 1966 indicate that the effect of the reflector poisoning is somewhat underestimated in the physics modeling. This was overcome in the earlier FEVER study by arbitrarily increasing the macroscopic cross section of the control rod in the reflector by a factor of 2 to 3 until the calculated flux distribution matched the measurements more closely. A theoretical explanation for this adjustment was never found. A final set of unrodded normalized axial power and fast flux profiles was arrived at taking all three sources of information into account. This set includes profiles at 0, 300, 600, and 900 EFPD. These axial power and flux profiles are given in Figs. 2-1 and 2-2, respectively. As can be seen in Fig. 2-2, power shifts away from the bottom of the core and smooths out as the fuel depletes.

Normalized axial power and fast flux profiles were generated in a similar fashion for partially rodded configurations. These profiles are shown in Figs. 2-3 and 2-4 and are to be used for elements adjacent to partially inserted control rods. The fully rodded distributions were found to be well described by the corresponding unrodded distribution.

*EFPD is defined as 1 effective full-power [(115 MW( $t)$ ] day of reactor operation. Peach Bottom Core 2 operated for 897 EFPD. 
A detailed radial physics depletion calculation of the as-operated Peach Bottom Core 2 which carefully accounted for all shuffling of fuel and test elements was performed using the GAUGE computer code. During this depletion, four banks of three control rods each were used. The history of the core was divided into a series of time intervals, each of which covered a period of fairly uniform power generation. This depletion study is summarized in Table $2-1$.

\subsection{POWER MEASUREMENTS}

In a11, 55 standard driver fuel elements were gamma scanned at Peach Bottom after final shutdown of the reactor in October 1974. Twenty-four elements were scanned during November and December of 1974 (Phase I) and an additional 30 were scanned in May and June of 1975 (Phase II). These elements are listed in Table 2-2. Additional gamma-scan data from PIE of E01-01, E11-07, F03-01, E14-01, E06-01, and F05-05 at ORNL are available in Refs. 8 through 13 .

Since the concentration of a nonmobile isotope in a fuel compact is directly related by the fission yield to the number of fissions and therefore to power, isotopic profiles determined from gamma scanning can be used to establish axial power profiles for the fuel elements. Specifically, short- and long-lived isotopes can be used to establish end-oflife (EOL) and time-averaged axial power profiles, respectively. Three isotopes, $\mathrm{Cs}-137, \mathrm{Zr}-95$, and $\mathrm{La}-140$, are of interest in this respect. Since Cs-137 has a half life of 30.1 years, the Cs-137 distribution is representative of the time-averaged axial power profile in elements in which there was no cesium redistribution. The distributions of $\mathrm{Zr}-95$ (half life of 65.5 days) and La-140 (half life of 12.8 days) are representative of the EOL axial power profile in elements whose axial power distributions were unperturbed by control rod insertion over the 
last 150 days of irradiation.* The similarity in the distributions of these two isotopes in such an element (E14-01) is shown in Fig. 2-5. The dissimilarity of these two distributions in an element (F03-01) adjacent to a partially inserted control rod during the last 150 days of irradiation is shown in Fig. 2-6. In such cases, only the La-140 profile is representative of the EOL axial power distribution.

The Cs-137, $\mathrm{Zr}-95$, and La-140 concentrations can be used to measure radial as well as axial power distributions. Using the mean activities of these isotopes, time-averaged and EOL radial power profiles can be determined for Peach Bottom Core 2. In addition, in cases where there was no discernible Cs-137 loss from an element, the total Cs-137 inventory can be used to calculate an element average fuel burnup using the method described in Section 3.3 of Ref. 3. This burnup is directly proportional to the element average power.

\subsection{COMPARISON OF MEASURED AND PREDICTED POWER}

\subsubsection{Axial Power Profiles}

Time-averaged and EOL axial power distributions for individual fuel elements were established from Cs-137, $\mathrm{Zr}-95$, and La-140 distributions and used to determine core average time-averaged and EOL unrodded axial power distributions for comparison with design code predictions. The time-averaged profile was obtained by averaging the normalized Cs-137 distributions for A14-14, E13-01, E13-02, E14-01, E14-02, and F15-14, a11 of which had little or no cesium redistribution. The average EOL profile was obtained from the EOL profiles for 23 elements, all of which were three or more core locations away from a partially inserted control rod during the last 150 days of irradiation. The comparison between the measured and predicted time-averaged unrodded axial power profile is shown

*An element is assumed to have had an unperturbed axial power distribution if it was three or more core locations away from a partially inserted control rod. 
in Fig. 2-7 and Table 2-3. The comparison between the measured and predicted EOL unrodded power profiles is shown in Fig. 2-8 and Table 2-4.

The EOL 2r-95 distributions for E06-01 and E11-07, which were removed from the core at 385 and 701 EFPD, respectively, are available from gamma scans performed at ORNL. Since the axial power distributions in both of these elements were either unperturbed or only slightly perturbed by control rods during the last 150 days of irradiation, these $\mathrm{Zr}-95$ distributions are representative of unrodded axial power profiles at 385 and 701 EFPD and can be used along with the average EOL unrodded power profile determined from the Phase I and Phase II gamma scans to verify the predicted change in the unrodded axial power profile with fuel depletion. The E06-01 Zr-95 distribution is compared with the 300 and 600 EFPD power profile predictions in Fig. 2-9, the E11-07 $\mathrm{Zr}-95$ distribution is compared with the 600 and 900 EFPD power profile predictions in Fig. 2-10, and these two profiles along with the measured 897 EFPD (EOL) profile are shown in Fig. 2-11. THe following conclusions concerning axial power predictions can be drawn from the above comparisons.

1. The compact-to-compact agreement between the measured and predicted time-averaged unrodded axial power distributions is within $\pm 7.4 \%(1 \sigma)$, which is well within the $\pm 8 \%$ to $13 \%$ quoted for other nuclear reactors (Ref. 14). The uncertainty in the predictive accuracy resulting from measurement uncertainties is $\pm 3.5 \%(1 \sigma)$. For all compacts, the calculated and measured axial power factors are within $\pm 25 \%$ of each other. (If compact 1 is excluded, the agreement is $\pm 15 \%$ or better for all compacts.) The relation between the two profiles suggests there may have been some cesium redistribution from the hot compacts in the middle and upper middle portions of the element to the cooler elements near the bottom of the element. Without this redistribution, the agreement between the measured and predicted profiles would be better. 
2. The compact-to-compact agreement between the measured and predicted EOL unrodded axial power distributions is within $\pm 7.2 \%$ $(1 \sigma)$, which is again well within the $\pm 8 \%$ to $13 \%$ quoted as the predictive accuracy in axial power (pellet to average) calculations for other nuclear reactors. The uncertainty in the predictive accuracy resulting from measurement uncertainties is $\pm 1.6 \%(1 \sigma)$. In all compacts, the calculated and measured axial power factors are within $\pm 15 \%$ of each other. The relation between the two profiles indicates that the shift of power to the top of the core with fuel depletion has been somewhat overpredicted by the physics calculations.

3. The power in elements whose axial power shapes are not perturbed by control rods shifts from the bottom part of the element and smooths out with fuel depletion as predicted. The rate of change in the unrodded axial power distribution is well predicted since the measured 385 EFPD profile is bracketed by the predicted 300 and 600 EFPD profiles and the measured 701 EFPD profile is bracketed by the predicted 600 and 900 EFPD profiles.

4. The predicted reflector effect in the unrodded axial power profiles near the top reflector interface is confirmed by the E06-01 and E11-07 $\mathrm{Zr}-95$ distributions. The reflector effect is evident in E06-01 (in the data points but not the smooth curve since only a fourth-order fit was used) but has disappeared in E11-07. This is consistent with the predicted profiles which show the reflector effect in the 0,300, and 600 EFPD curves but not the 900 EFPD curve. A similar reflector interface is suggested by the E06-01 $\mathrm{Zr}-95$ distribution but was not predicted by the physics calculations. 


\subsubsection{Radial Power Profiles}

Normalized time-averaged and EOL radial power profiles determined from element average Cs-137, La-140, and $\mathrm{Zr}-95$ activities are compared with GAUGE-predicted beginning-of-life (BOL) time-averaged and EOL radial power profiles in Fig. 2-12. As can be seen, the normalized $\mathrm{Zr}-95$ and La-140 profiles are somewhat flatter than the predicted EOL profile, with the $\mathrm{Zr}-95$ profile being, as expected, the farther away. The Cs-137 profile confirms the flatness of the time-averaged profile. The BOL relative power distribution is a mirror image of the EOL distribution. A pivot point can be recognized approximately at core location 10 .

\subsubsection{Fuel Burnup}

An element-by-element comparison of measured and predicted burnup of all gamma-scanned fuel elements is presented in Ref. 3 and is therefore not offered here. However, the following conclusions from Ref. 3 comparisons are restated below.

1. There was no significant difference between the calculated and measured burnup on a core average basis since the established bias of $-0.4 \%$ is well within the measurement uncertainty of $\pm 0.7 \%$ and can therefore be ignored.

2. In all cases, the calculated and measured burnups were within $\pm 15 \%$ of each other.

3. The core average power was predicted within $\pm 0.7 \%(1 \sigma)$.

4. The element-to-element agreement between predicted and measured element average (radial) power was $\pm 6.8 \%(1 \sigma)$, which is within the $3 \%$ to $8 \%$ quoted for other nuclear reactors (Ref. 14). The 
uncertainty in the predictive accuracy resulting from measurement uncertainties is $4.7 \%(1 \sigma)$. The predictive accuracy for the element average power is simflar to the accuracy determined for the axial power predictions.

5. For 34 of the 48 driver elements for which measured burnup was determined, the difference between calculated and measured burnup was less than the $1 \sigma$ error on the measurements and therefore insignificant. 


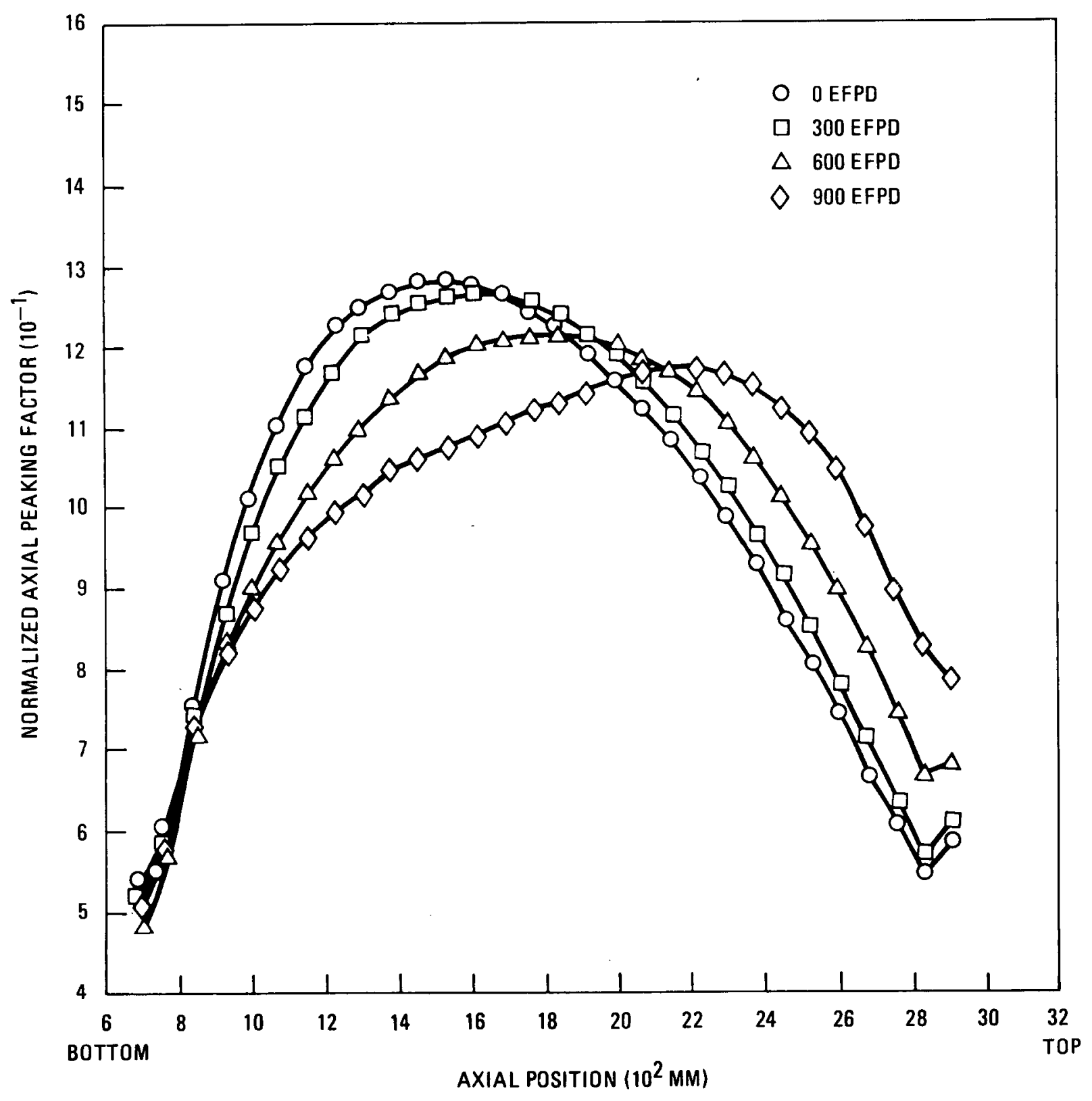

Fig. 2-1. Unrodded axial power profile predictions for Peach Bottom Core 2 


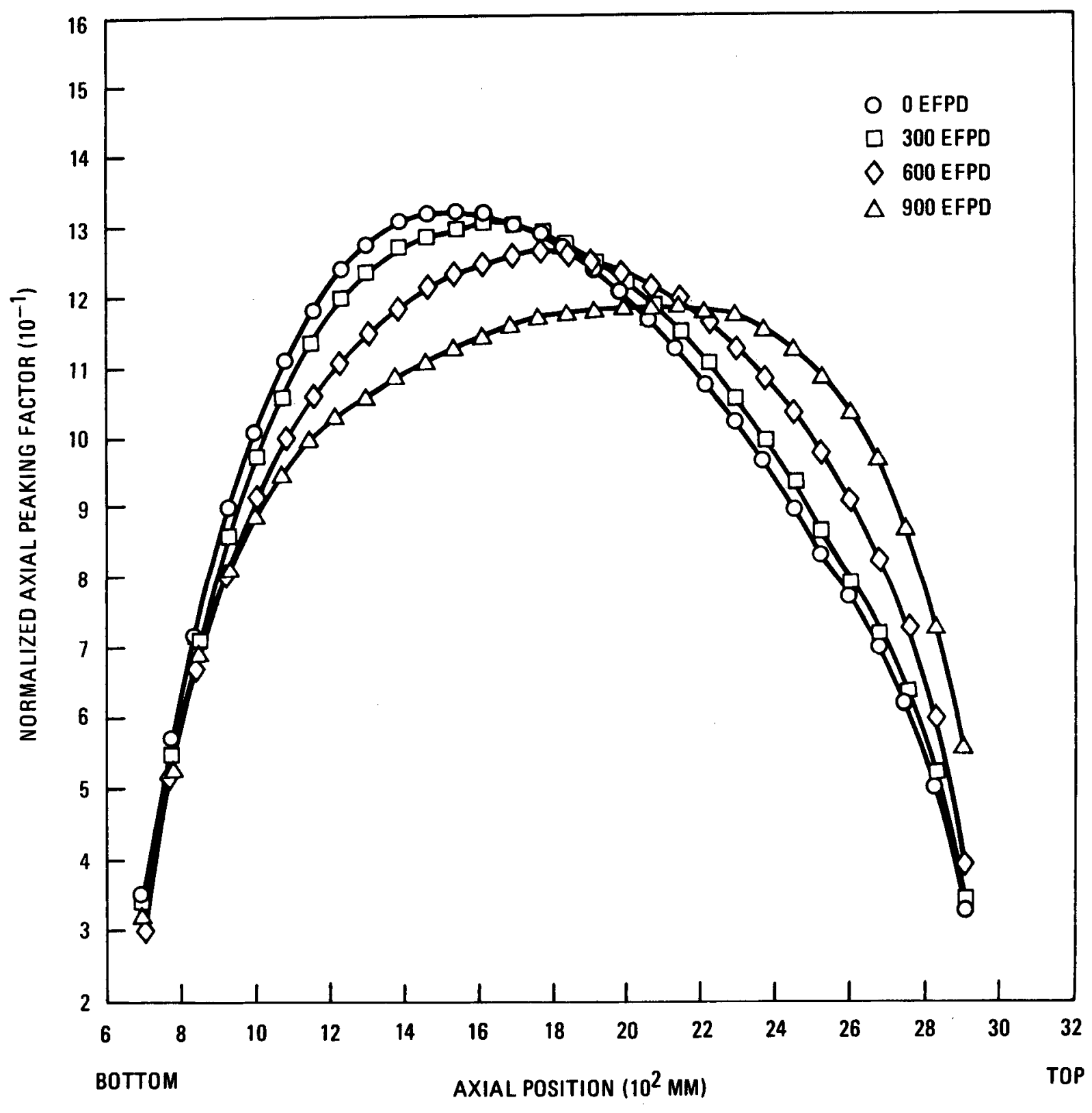

Fig. 2-2. Unrodded axial fast flux profile predictions for Peach Bottom Core 2 


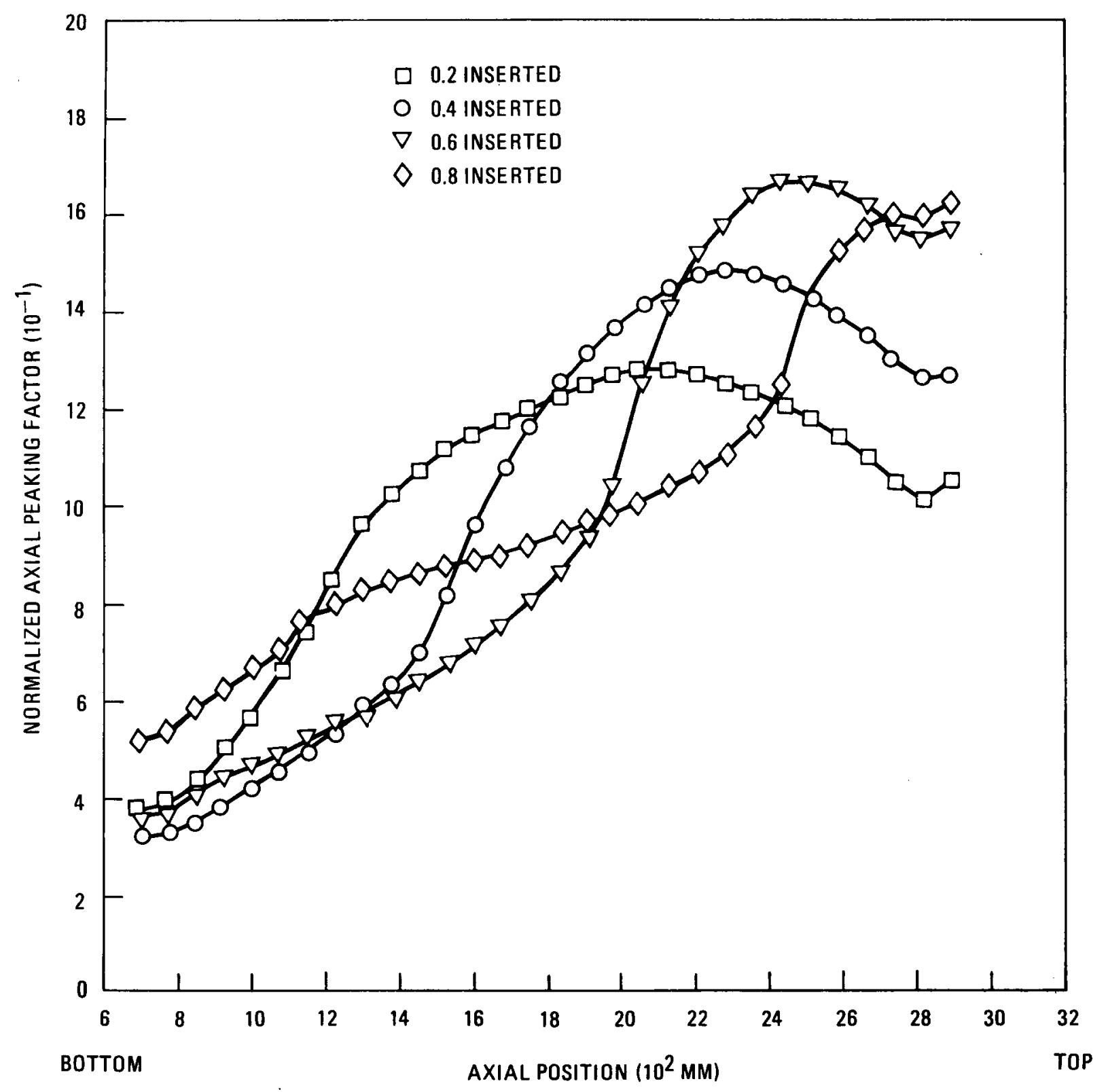

Fig. 2-3. Partially rodded axlal power profile predictions for Peach Bottom Core 2 


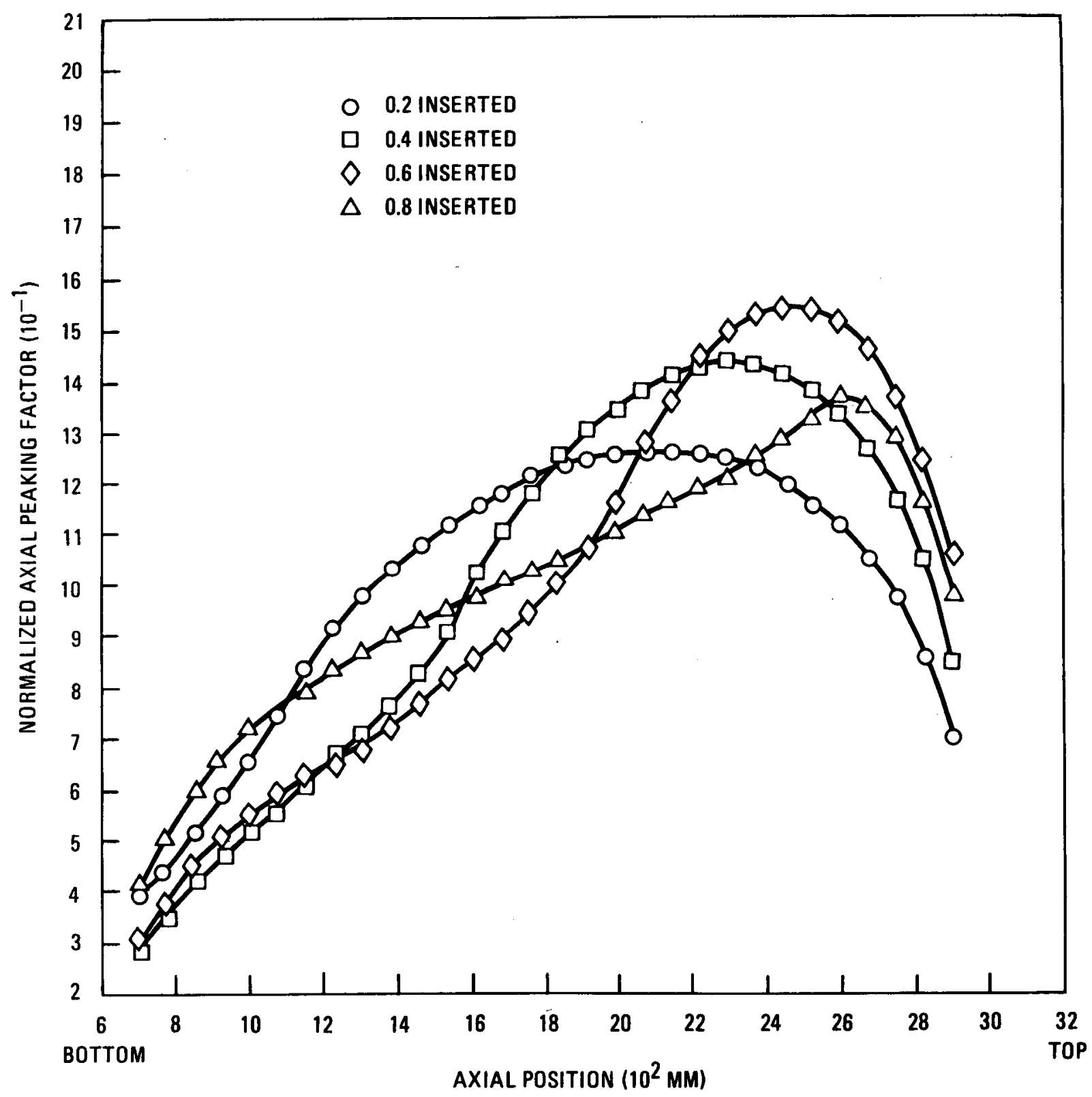

Fig. 2-4. Partially rodded axial fast flux profile predictions for Peach Bottom Core 2 


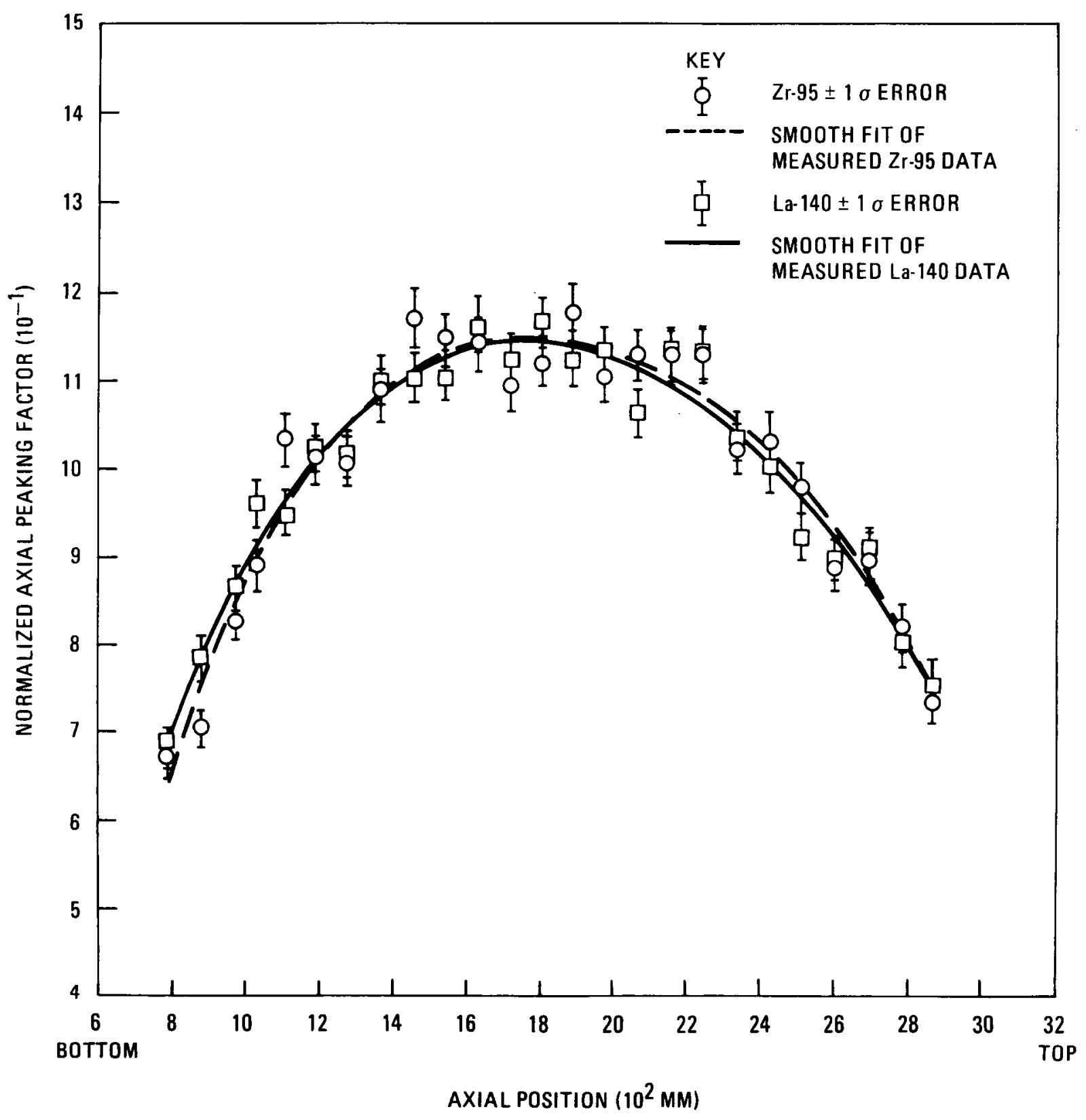

Fig. 2-5. Normalized $\mathrm{Zr}-95$ and La-140 distributions for E14-01 


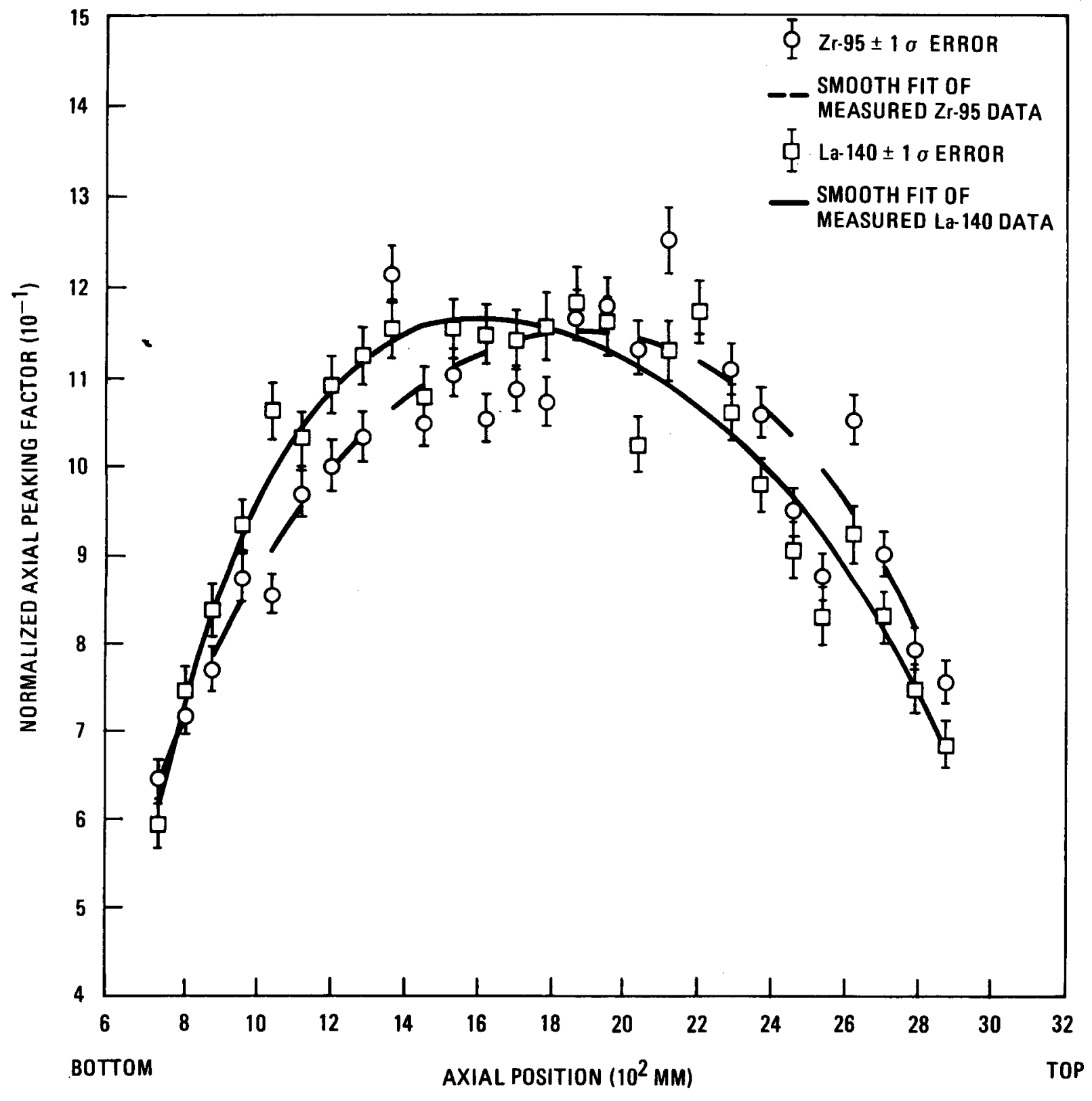

Fig. 2-6. Normalized $\mathrm{Zr}-95$ and La-140 distributions for F03-01 


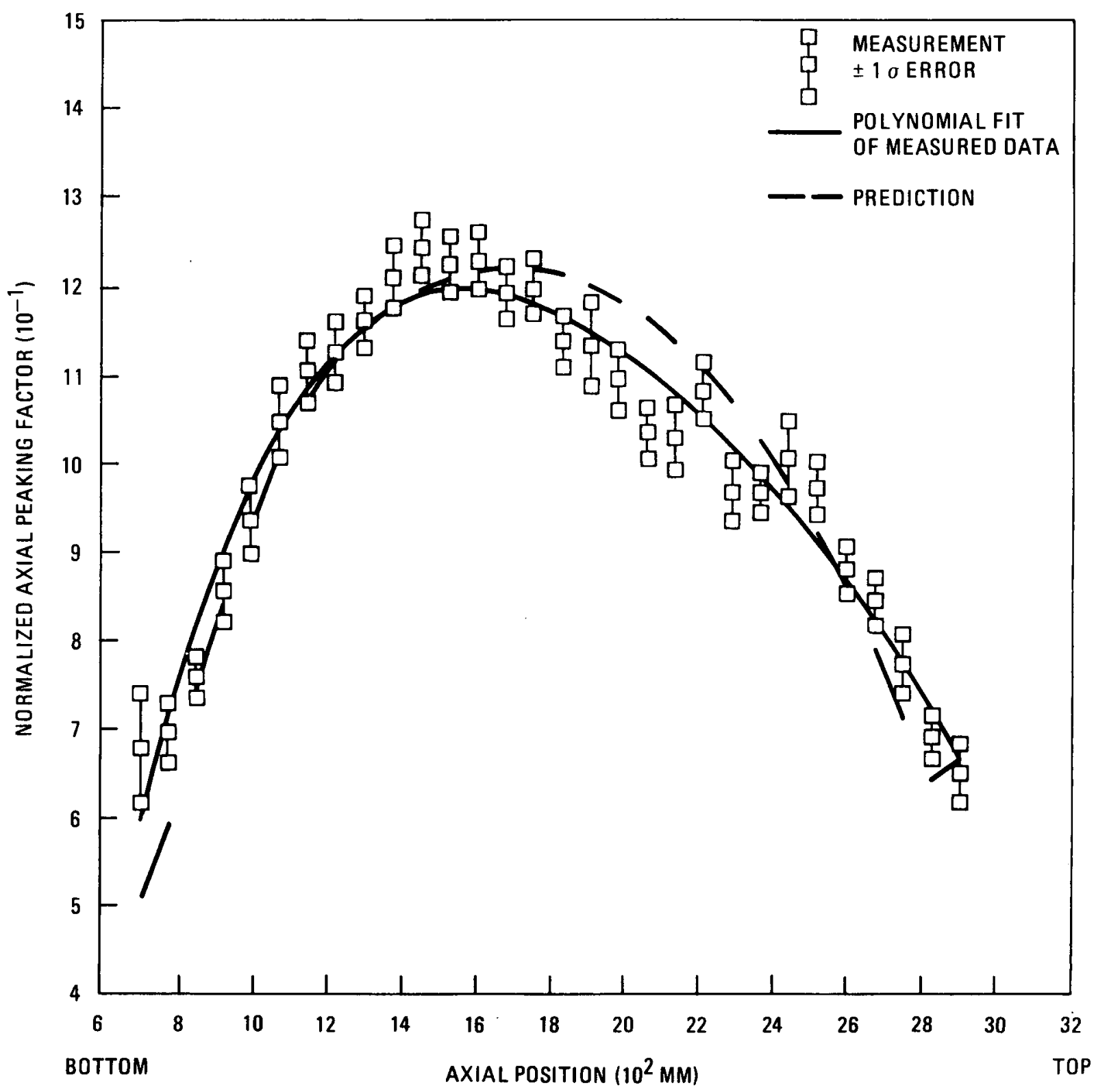

Fig. 2-7. Comparison of measured and predicted time-averaged unrodded axial power profiles for Peach Bottom Core 2 


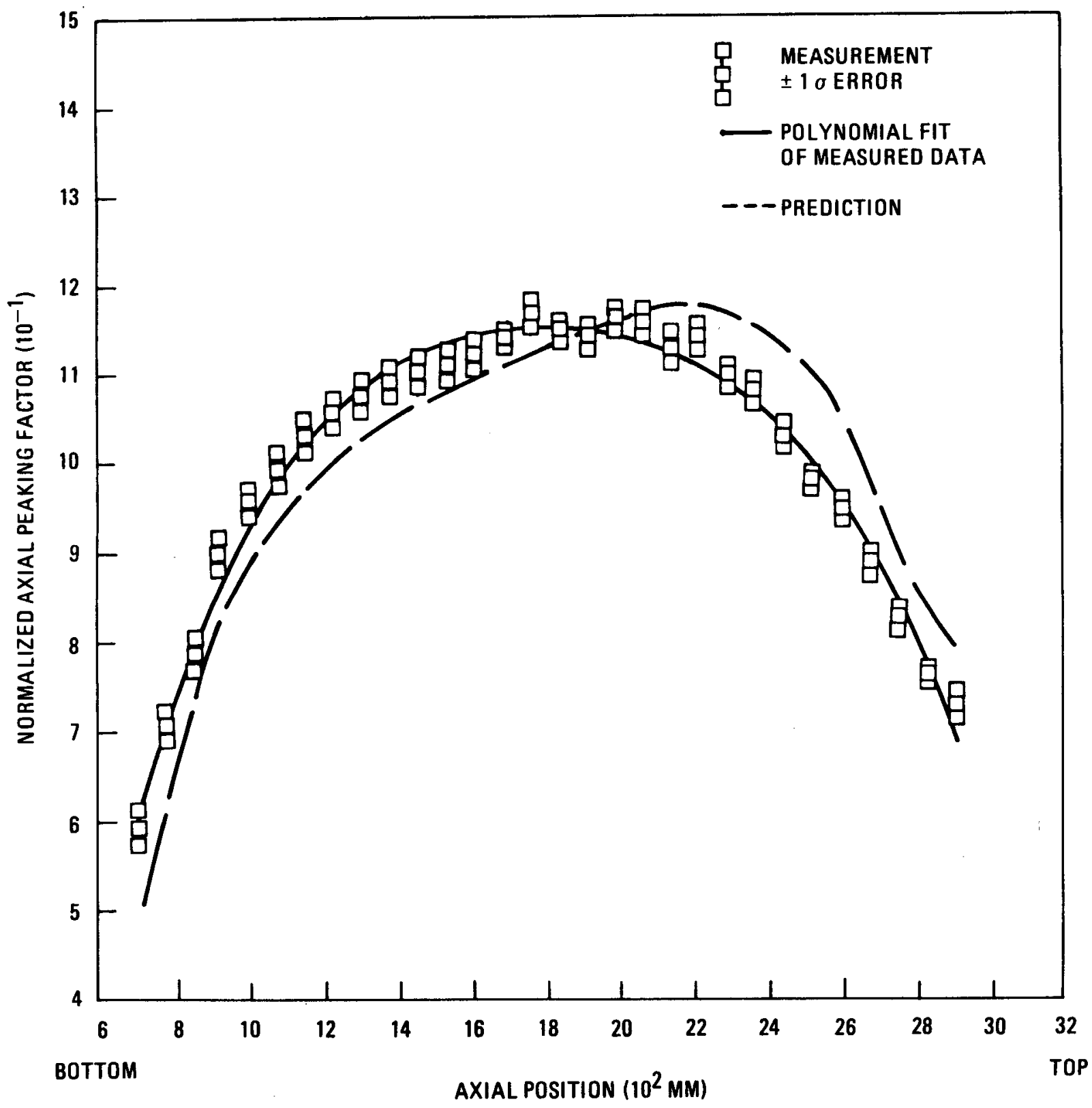

Fig. 2-8. Comparison of measured and predicted EOL unrodded axial power profiles for Peach Bottom Core 2 


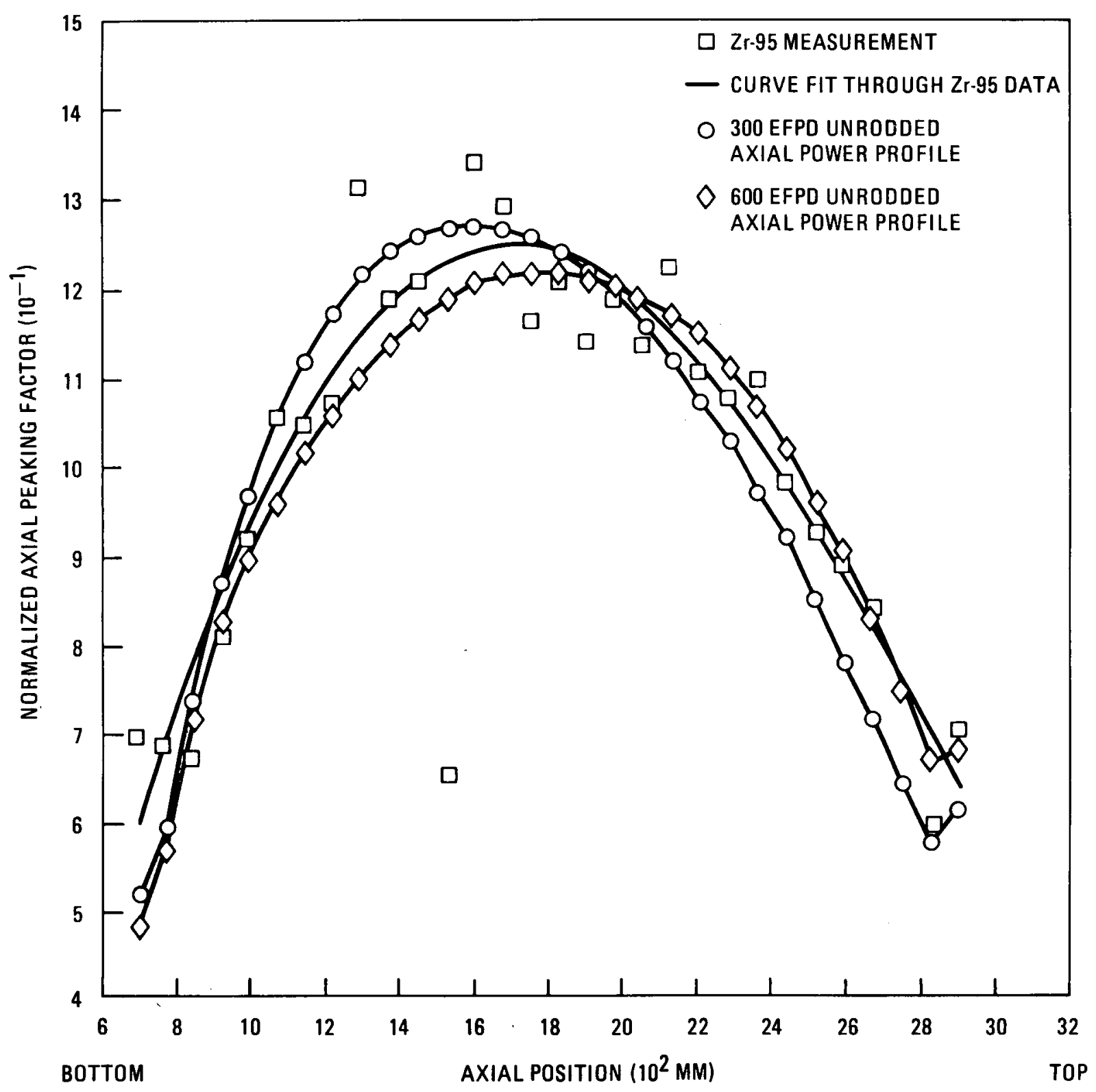

Fig. 2-9. Comparison of E06-01 normalized Zr-95 distribution with predicted 300 and 600 EFPD unrodded axial power profiles 


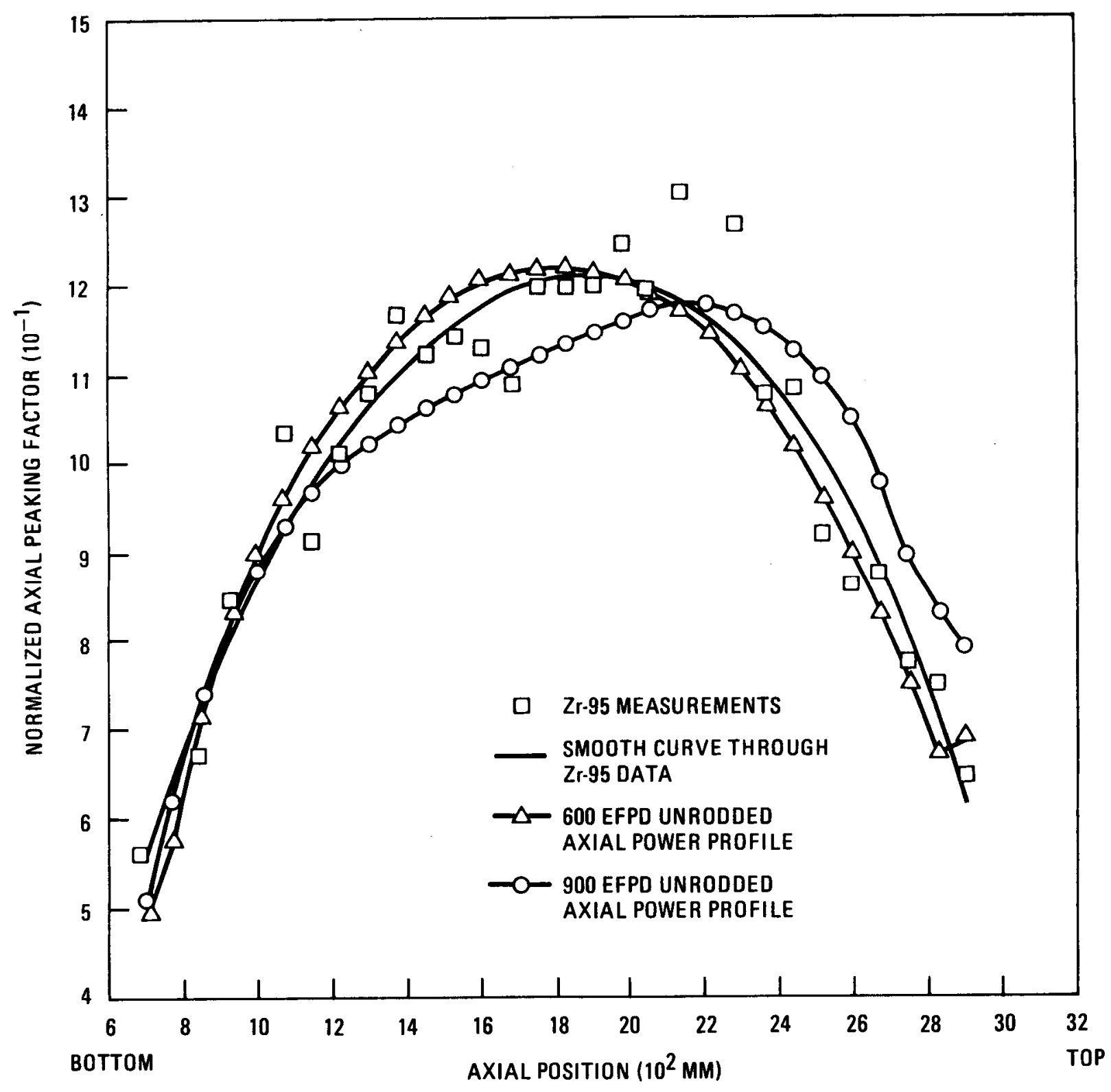

Fig. 2-10. Comparison of E11-07 normalized $\mathrm{Zr}-95$ distribution with predicted 600 and 900 EFPD unrodded axial power profiles; E11-07 was removed for postirradiation examination after 701 EFPD 


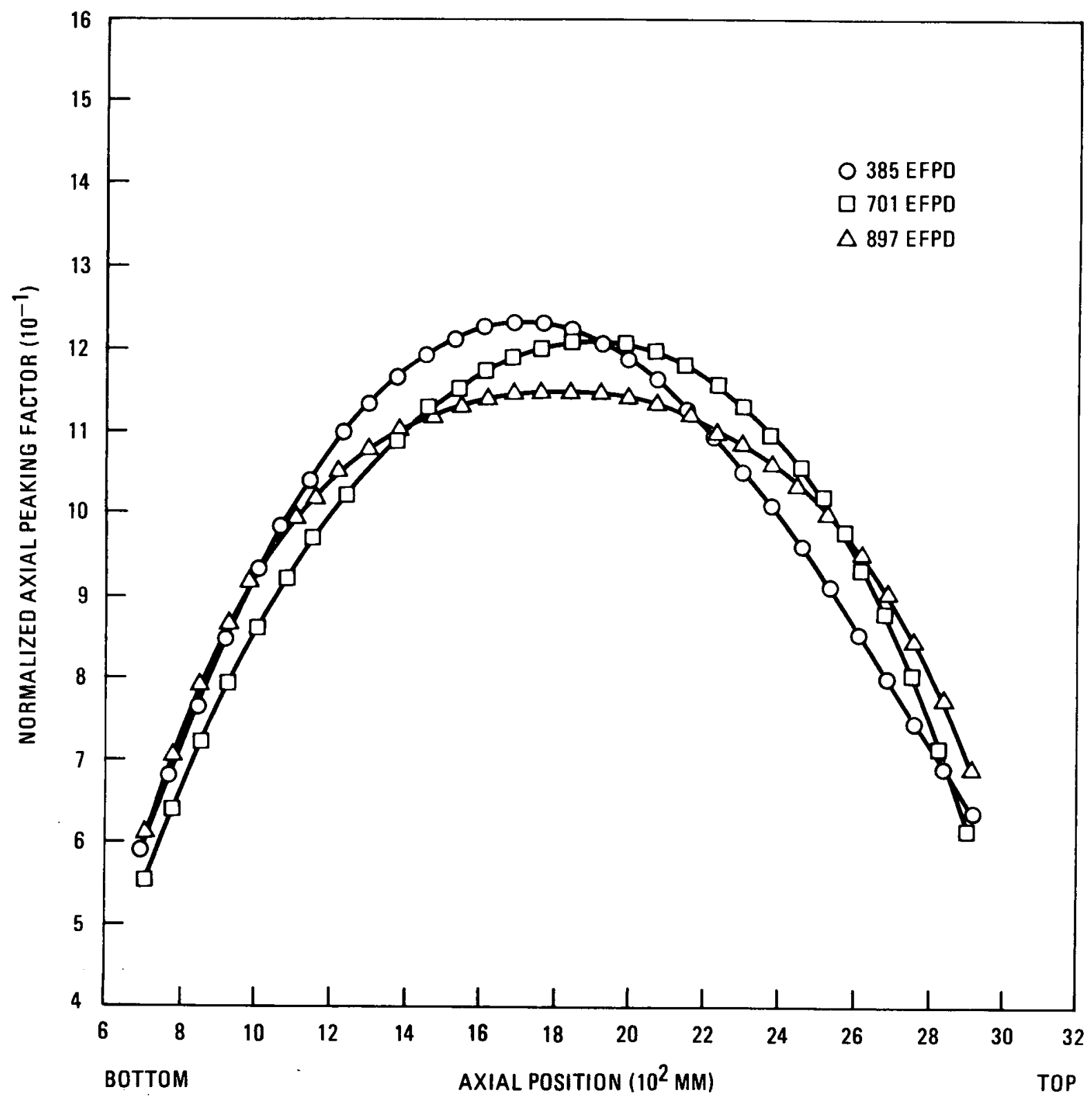

Fig. 2-11. Measured unrodded axial power profiles at 385, 701, and 897 EFPD 


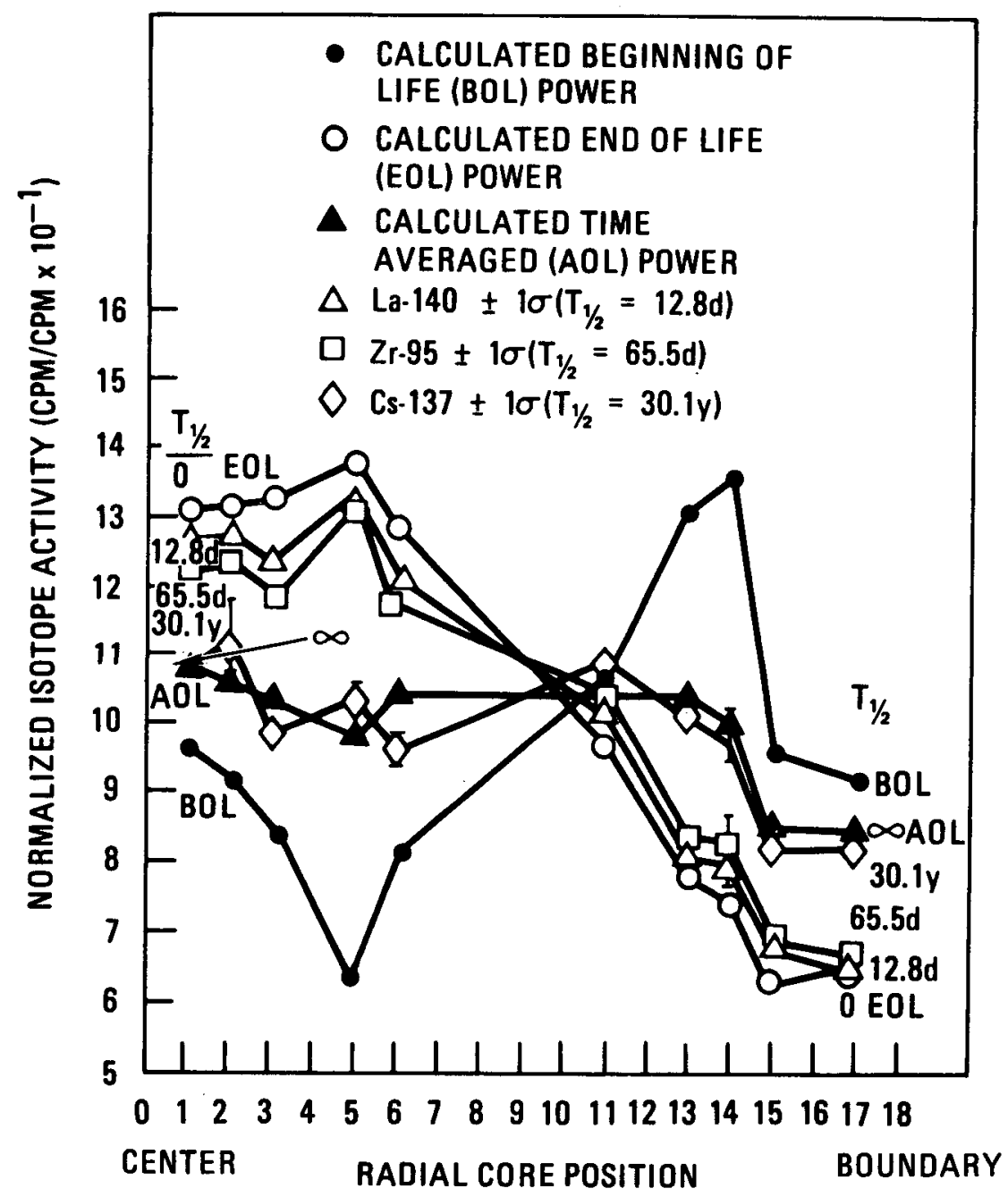

Fig. 2-12. Comparison of measured and predicted radial power distributions for Peach Bottom Core 2 
TABLE 2-1

PEACH BOTTOM CORE 2 GAUGE 3 DEPLETION

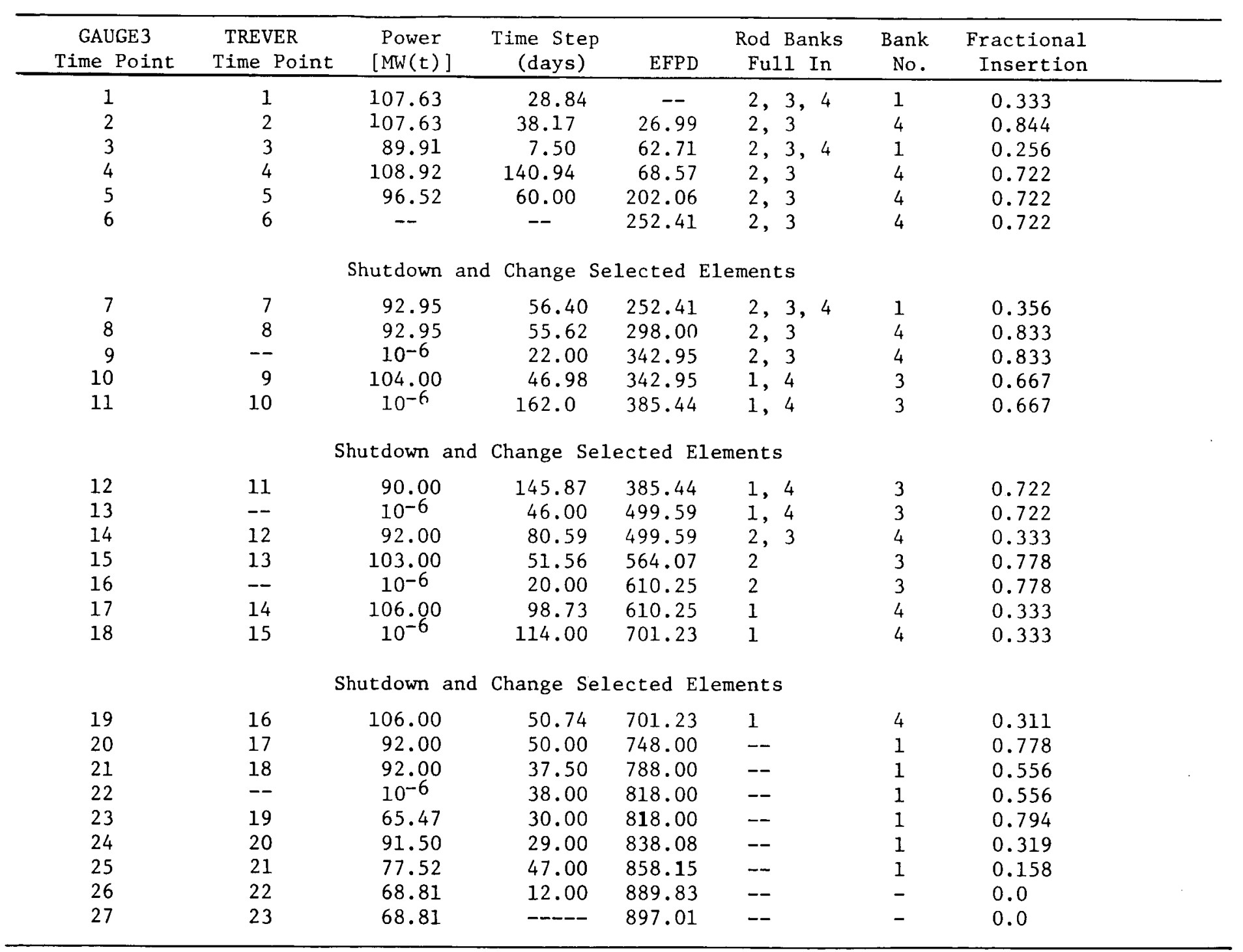


TABLE 2-2

DRIVER ELEMENTS GAMMA-SCANNED BY GENERAL ATOMIC

\begin{tabular}{ll|ll}
\hline \multicolumn{2}{c|}{ Phase I } & \multicolumn{2}{c}{ Phase II } \\
\hline & & F01-01 & E08-02 \\
E01-01 & E07-01 & B02-01 & E09-02 \\
B02-02 & E09-01 & F02-01 & F09-08 \\
E02-02 & E11-01 & E03-01 & F10-02 \\
A03-03 & E11-02 & E03-02 & F10-09 \\
B03-01 & F11-03 & F03-02 & B11-03 \\
B03-03 & B13-01 & A04-03 & E12-02 \\
F03-01 & B14-02 & A04-04 & F12-11 \\
A05-05 & E14-01 & E04-02 & E13-01 \\
B05-01 & E15-01 & F04-03 & E13-02 \\
E05-01 & E15-02 & B05-02 & A14-14 \\
F05-05 & A17-08 & E05-02 & E14-02 \\
E06-02 & A17-11 & F05-04 & F14-13 \\
& & E07-02 & F15-14 \\
& & F07-06 & F16-15 \\
\hline
\end{tabular}


TABLE 2-3

COMPARISON OF MEASURED AND PREDICTED TIME-AVERAGED

UNRODDED AXIAL POWER PROFILES FOR PEACH BOTTOM CORE 2

\begin{tabular}{|c|c|c|c|c|c|}
\hline \multirow[b]{2}{*}{$\begin{array}{l}\text { Fuel } \\
\text { Compact }\end{array}$} & \multirow[b]{2}{*}{ Predicted $\mathrm{APF}_{P}{ }^{(\mathrm{a})}$} & \multicolumn{2}{|c|}{ Measured $(b)$} & \multicolumn{2}{|c|}{ Relative Difference (\%) } \\
\hline & & $\mathrm{APF}_{\mathrm{M}}{ }^{(2)}$ & Error $\left(\begin{array}{ll}1 & \sigma\end{array}\right)$ & $\mathrm{Z}=\mathrm{APF} \mathrm{P}_{\mathrm{P}} / \mathrm{APF}_{\mathrm{M}}-1$ & Error $\left(\begin{array}{ll}1 & \sigma\end{array}\right)$ \\
\hline 1 & 0.510 & 0.678 & \pm 0.061 & -24.8 & \pm 6.7 \\
\hline 2 & 0.594 & 0.695 & \pm 0.033 & -14.5 & \pm 4.1 \\
\hline 3 & 0.737 & 0.759 & \pm 0.023 & -2.9 & \pm 2.9 \\
\hline 4 & 0.857 & 0.856 & \pm 0.031 & 0.1 & \pm 3.6 \\
\hline 5 & 0.941 & 0.937 & \pm 0.035 & 0.4 & \pm 3.8 \\
\hline 6 & 1.012 & 1.051 & \pm 0.040 & -3.7 & \pm 3.7 \\
\hline 7 & 1.072 & 1.107 & \pm 0.033 & -3.2 & \pm 2.9 \\
\hline 8 & 1.118 & 1.128 & \pm 0.032 & -0.9 & \pm 2.8 \\
\hline 9 & 1.152 & 1.163 & \pm 0.025 & -0.9 & \pm 2.1 \\
\hline 10 & 1.182 & 1.211 & \pm 0.036 & -2.4 & \pm 2.9 \\
\hline 11 & 1.202 & 1.246 & \pm 0.031 & -3.5 & \pm 2.4 \\
\hline 12 & 1.212 & 1.226 & \pm 0.030 & -1.1 & \pm 2.4 \\
\hline 13 & 1.222 & 1.230 & \pm 0.030 & -0.6 & \pm 2.4 \\
\hline 14 & 1.224 & 1.196 & \pm 0.029 & 2.3 & \pm 2.5 \\
\hline 15 & 1.222 & 1.201 & \pm 0.029 & 1.7 & \pm 2.5 \\
\hline 16 & 1.216 & 1.142 & \pm 0.028 & 6.5 & \pm 2.6 \\
\hline 17 & 1.202 & 1.137 & \pm 0.046 & 5.7 & \pm 4.3 \\
\hline 18 & 1.187 & 1.099 & \pm 0.033 & 8.0 & \pm 3.2 \\
\hline 19 & 1.167 & 1.039 & \pm 0.028 & 12.3 & \pm 3.0 \\
\hline 20 & 1.143 & 1.032 & \pm 0.039 & 10.8 & \pm 4.2 \\
\hline 21 & 1.110 & 1.087 & \pm 0.030 & 2.3 & \pm 2.8 \\
\hline 22 & 1.074 & 0.971 & \pm 0.033 & 10.6 & \pm 3.8 \\
\hline 23 & 1.028 & 0.970 & \pm 0.022 & 6.0 & \pm 2.4 \\
\hline 24 & 0.979 & 1.009 & \pm 0.043 & -3.0 & \pm 4.1 \\
\hline 25 & 0.923 & 0.974 & \pm 0.029 & -5.2 & \pm 2.8 \\
\hline 26 & 0.860 & 0.883 & \pm 0.025 & -2.6 & \pm 2.8 \\
\hline 27 & 0.791 & 0.846 & \pm 0.028 & -6.5 & \pm 3.1 \\
\hline 28 & 0.716 & 0.776 & \pm 0.033 & -7.7 & \pm 3.9 \\
\hline 29 & 0.645 & 0.694 & \pm 0.023 & -7.1 & \pm 3.1 \\
\hline 30 & 0.664 & 0.652 & \pm 0.035 & 1.8 & \pm 5.5 \\
\hline $\begin{array}{l}\text { Average } \\
\text { RMS }\end{array}$ & 1.000 & 1.000 & \pm 0.033 & $\begin{array}{l}-0.7 \\
\pm 7.4\end{array}$ & \pm 3.5 \\
\hline
\end{tabular}

(a) $\mathrm{APF}=$ axial peaking factor.

(b) As determined from the Cs-137 distributions in six driver fuel elements. 
TABLE 2-4

COMPARISON OF MEASURED AND PREDICTED EOL UNRODDED AXIAL POWER PROFILES FOR PEACH BOTTOM CORE 2

\begin{tabular}{|c|c|c|c|c|c|}
\hline \multirow[b]{2}{*}{$\begin{array}{l}\text { Fuel } \\
\text { Compact }\end{array}$} & \multirow[b]{2}{*}{ Predicted $\mathrm{APF}_{\mathrm{P}}(\mathrm{a})$} & \multicolumn{2}{|c|}{ Measured (b) } & \multicolumn{2}{|c|}{ Relative Difference (\%) } \\
\hline & & $\mathrm{APF}_{\mathrm{M}}^{(2)}$ & Error $(1 \quad \sigma)$ & $\mathrm{Z}=\mathrm{APF} \mathrm{P}_{\mathrm{P}} / \mathrm{APF}_{\mathrm{M}}-1$ & Error $\left(\begin{array}{ll}1 & \sigma\end{array}\right)$ \\
\hline 1 & 0.505 & 0.593 & \pm 0.018 & -14.8 & \pm 2.6 \\
\hline 2 & 0.620 & 0.699 & \pm 0.010 & $-11 \cdot 3$ & \pm 1.3 \\
\hline 3 & 0.740 & 0.783 & \pm 0.012 & -5.5 & \pm 1.4 \\
\hline 4 & 0.825 & 0.897 & \pm 0.011 & -8.0 & \pm 1.1 \\
\hline 5 & 0.885 & 0.950 & \pm 0.013 & -6.8 & \pm 1.3 \\
\hline 6 & 0.930 & 0.992 & \pm 0.017 & -6.2 & \pm 1.6 \\
\hline 7 & 0.970 & 1.024 & \pm 0.015 & -5.3 & \pm 1.4 \\
\hline 8 & 1.000 & 1.057 & \pm 0.018 & -5.4 & \pm 1.6 \\
\hline 9 & 1.020 & 1.077 & \pm 0.017 & -5.3 & \pm 1.5 \\
\hline 10 & 1.050 & 1.094 & \pm 0.016 & -4.0 & \pm 1.4 \\
\hline 11 & 1.065 & 1.099 & \pm 0.016 & -3.1 & \pm 1.4 \\
\hline 12 & 1.080 & 1.107 & \pm 0.018 & -2.4 & \pm 1.6 \\
\hline 13 & 1.095 & 1.119 & \pm 0.016 & -2.2 & \pm 1.4 \\
\hline 14 & 1.110 & 1.144 & \pm 0.012 & -3.0 & \pm 1.0 \\
\hline 15 & 1.125 & 1.166 & \pm 0.014 & -3.5 & \pm 1.2 \\
\hline 16 & 1.135 & 1.149 & \pm 0.014 & -1.2 & \pm 1.2 \\
\hline 17 & 1.150 & 1.141 & \pm 0.017 & 0.8 & \pm 1.5 \\
\hline 18 & 1.162 & 1.164 & \pm 0.017 & -0.2 & \pm 1.5 \\
\hline 19 & 1.175 & 1.157 & \pm 0.016 & 1.6 & \pm 1.4 \\
\hline 20 & 1.180 & 1.130 & \pm 0.020 & 4.4 & \pm 1.8 \\
\hline 21 & 1.180 & 1.138 & \pm 0.016 & 3.7 & \pm 1.5 \\
\hline 22 & 1.170 & 1.096 & \pm 0.013 & 6.7 & \pm 1.3 \\
\hline 23 & 1.155 & 1.080 & \pm 0.016 & 6.9 & \pm 1.6 \\
\hline 24 & 1.130 & 1.028 & \pm 0.014 & 9.9 & \pm 1.5 \\
\hline 25 & 1.100 & 0.978 & \pm 0.014 & 12.5 & \pm 1.6 \\
\hline 26 & 1.055 & 0.944 & \pm 0.012 & 11.8 & \pm 1.4 \\
\hline 27 & 0.980 & 0.884 & \pm 0.013 & 10.9 & \pm 1.6 \\
\hline 28 & 0.900 & 0.875 & \pm 0.015 & 9.1 & \pm 2.0 \\
\hline 29 & 0.830 & 0.760 & \pm 0.010 & 9.2 & \pm 1.4 \\
\hline 30 & 0.790 & 0.728 & \pm 0.019 & 8.5 & \pm 2.8 \\
\hline $\begin{array}{l}\text { Average } \\
\text { RMS }\end{array}$ & 1.000 & 1.000 & \pm 0.015 & $\begin{array}{l}+0.3 \\
\pm 7.2\end{array}$ & \pm 1.6 \\
\hline
\end{tabular}

(a) $\mathrm{APF}=$ axial peaking factor.

(b) As determined from the measured EOL power profiles for 23 driver fuel elements. 


\section{SLEEVE-FUEL COMPACT GAP}

\subsection{CORRELATION FOR FUEL COMPACT RADIAL STRAIN}

To accurately calculate temperatures in Peach Bottom fuel compacts, it is mandatory that the sleeve-fuel compact gap be reasonably well modeled. This gap is a function of the thermal and irradiation-induced radial strain in the sleeve and fuel compact and the preirradiation gap. Since the irradiation-induced strain in the fuel compact is the most important of these factors, it should be possible to model the sleeve-fuel compact gap reasonably well if a good radial strain correlation is available. The correlation given below was developed using the Peach Bottom fuel compact metrology data for E01-01 (Ref. 8), El1-07 (Ref. (9), F03-01 (Ref. 10), E14-01 (Ref.. 11), and C11-07 (Ref. 15):

$$
\begin{aligned}
\Delta D / D= & \left(C_{1}+C_{2} T+C_{3} T^{2}+C_{4} T^{3}\right) \phi \\
& +\left(C_{5}+C_{6} T+C_{7} T^{2}+C_{8} T^{3}\right) \phi^{2}+\left(C_{9}+C_{10} T\right) \phi^{3}
\end{aligned}
$$

where $\phi=$ fast neutron fluence $\left(x 10^{-25} \mathrm{n} / \mathrm{m}^{2}\right)$,

$$
\mathrm{T}=\text { temperature in }{ }^{\circ} \mathrm{F} \text {, }
$$

and the values for constants $C_{1}$ through $C_{10}$ are as given below:

$$
\begin{aligned}
& c_{1}=-0.9645824 \\
& c_{2}=0.9655615 \times 10^{-3} \\
& c_{3}=-0.5934919 \times 10^{-6}
\end{aligned}
$$




$$
\begin{aligned}
& c_{4}=0.7010922 \times 10^{-10} \\
& c_{5}=-0.2930805 \\
& c_{6}=0.1048583 \times 10^{-2} \\
& c_{7}=-0.6630441 \times 10^{-6} \\
& c_{8}=0.1251074 \times 10^{-9} \\
& c_{9}=-0.4022474 \times 10^{-1}
\end{aligned}
$$

Figure 3-1 shows the fuel compact irradiation strain as a function of fast neutron fluence and temperature.

The PIE data used to develop the above correlation are given in Table 3-1. Unfortunately, it was found that these data by themselves were not sufficient to give a good correlation. The absence of strain data for temperatures between $650^{\circ}$ and $800^{\circ} \mathrm{C}$, for temperatures above $1200^{\circ} \mathrm{C}$, and at low temperature and high fluence allowed the data fitting code to provide a correlation which gave obviously erroneous strains in these areas. However, it was observed that the slope of the family of strain-versus-fluence curves for the Peach Bottom fuel compacts resembled the irradiation-induced strain parallel to the axis of extrusion in $\mathrm{H}-327$ graphite. This was found to be reasonable since (1) the compacts consisted primarily of a needle coke graphite filler, and (2) the compacts were press-molded, which aligned the graphite particles preferentially in the radial direction (in $\mathrm{H}-327$ graphite, particles are aligned preferentially along the axis of extrusion). Therefore, it is not unreasonable that the radial compact strain resembles the axial strain in H-327 graphite. Since no PIE data for temperatures above $1200^{\circ} \mathrm{C}$ are available, additional strain information was obtained by observing the relationship between the $1200^{\circ} \mathrm{C}$ curve and higher temperature curves for $\mathrm{H}-327$ graphite and using these relations to extrapolate the Peach Bottom fuel compact $1200^{\circ} \mathrm{C}$ curve. Strain information at low temperature and high fluence was also obtained by extrapolating the Peach Bottom strain curves based on the shape of the H-327 graphite axial strain curves. 


\subsection{COMPARISON OF PREDICTED AND MEASURED SLEEVE-FUEL COMPACT RADIAL GAPS}

A comparison of predicted and measured sleeve-fuel compact gaps is presented in Table 3-2. The predicted gaps included in this table were calculated at room temperature to eliminate the effect of thermal strain to allow direct comparison with measured gaps. The agreement between measured and predicted gaps for E11-07, E01-01, E14-01, F03-01, and C11-07 is within $\pm 0.06 \mathrm{~mm}(1 \sigma)$ with no apparent bias. This confirms the hypothesis that the sleeve-fuel compact gaps could be well modeled if a correlation defining fuel compact irradiation-induced radial strain could be developed. Absence of a bias in the predicted gaps indicates that a good fit of the data has been obtained. In F05-05, the calculated gaps are underpredicted by $-0.13 \mathrm{~mm}(5 \mathrm{mils}) \pm 0.04 \mathrm{~mm}(1.4 \mathrm{mils})(1 \sigma)$. Comparison of the measured and calculated gaps in this element is of interest since its fuel compact strain data were not available at the time the strain correlation was developed and are therefore not included in the data base for the correlation. The $-0.13-\mathrm{mm}$ (5-mil) underprediction of the gap in F05-05 corresponds to an underprediction of approximately $-18^{\circ} \mathrm{C}$ in the average time-averaged peak fuel temperature for the five compacts considered. It can therefore be concluded that the gap is reasonably well modeled in F05-05 in spite of the average $-0.13-\mathrm{mm}$ (5-mil) bias. 


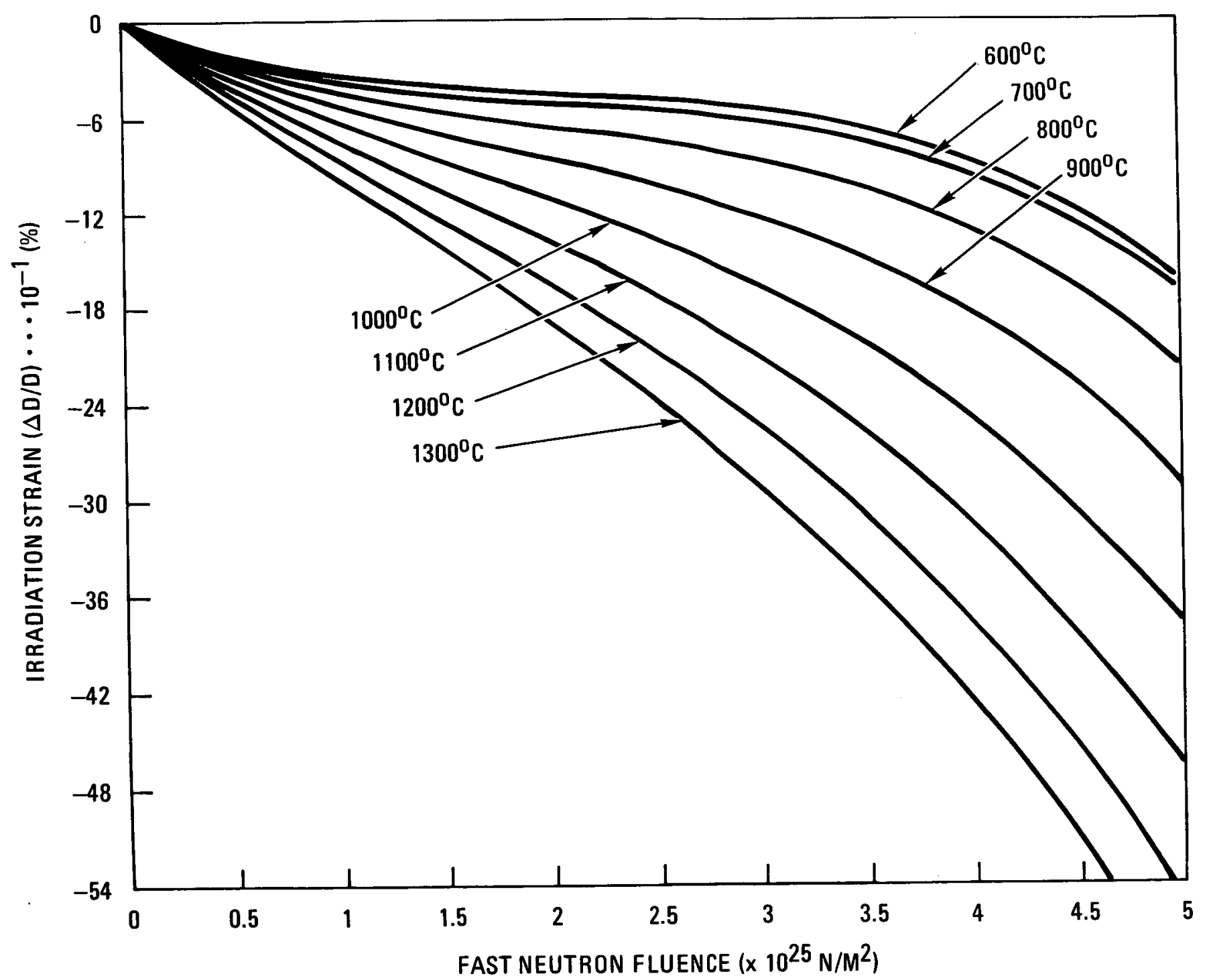

Fig. 3-1. Fuel compact irradiation-induced radial strain as a function of fast neutron fluence and temperature 
TABLE 3-1

DATA BASE FOR FUEL COMPACT IRRADIATION-INDUCED

RADIAL STRAIN CORRELATION

\begin{tabular}{|c|c|c|c|c|c|}
\hline $\begin{array}{c}\text { Fue1 } \\
\text { Element }\end{array}$ & $\begin{array}{l}\text { Fuel } \\
\text { Compact }\end{array}$ & $\begin{array}{c}\text { Strain } \\
(\%)\end{array}$ & $\begin{array}{c}\text { Error } \\
\%\end{array}$ & $\begin{array}{c}\text { Temperature } \\
\left({ }^{\circ} \mathrm{C}\right)\end{array}$ & $\begin{array}{c}\text { Fast F1uence } \\
\left(10^{25} \mathrm{n} / \mathrm{m}^{2}\right)\end{array}$ \\
\hline E14-01 & $\begin{array}{r}1 \\
9 \\
18 \\
24 \\
30 \\
\end{array}$ & $\begin{array}{l}-0.296 \\
-1.335 \\
-2.114 \\
-1.486 \\
-0.458 \\
\end{array}$ & $\begin{array}{l} \pm 0.052 \\
\pm 0.163 \\
\pm 0.130 \\
\pm 0.241 \\
\pm 0.043\end{array}$ & $\begin{array}{r}582 \\
974 \\
1129 \\
1069 \\
976 \\
\end{array}$ & $\begin{array}{l}0.82 \\
2.95 \\
3.05 \\
2.52 \\
1.01\end{array}$ \\
\hline E11-07 & $\begin{array}{r}2 \\
5 \\
9 \\
24 \\
27 \\
30 \\
\end{array}$ & $\begin{array}{l}-0.151 \\
-0.969 \\
-1.686 \\
-1.977 \\
-1.860 \\
-0.798 \\
\end{array}$ & $\begin{array}{l} \pm 0.122 \\
\pm 0.065 \\
\pm 0.063 \\
\pm 0.067 \\
\pm 0.188 \\
\pm 0.063 \\
\end{array}$ & $\begin{array}{r}660 \\
879 \\
1069 \\
1137 \\
1065 \\
1016 \\
\end{array}$ & $\begin{array}{l}1.35 \\
2.37 \\
2.98 \\
2.70 \\
2.15 \\
1.01 \\
\end{array}$ \\
\hline C11-07 & $\begin{array}{r}5 \\
9 \\
12 \\
15 \\
21 \\
24 \\
27 \\
30\end{array}$ & $\begin{array}{l}-0.306 \\
-0.791 \\
-0.615 \\
-0.848 \\
-0.947 \\
-0.589 \\
-0.468 \\
-0.210\end{array}$ & $\begin{array}{l} \pm 0.022 \\
\pm 0.052 \\
\pm 0.073 \\
\pm 0.131 \\
\pm 0.206 \\
\pm 0.102 \\
\pm 0.070 \\
\pm 0.045\end{array}$ & $\begin{array}{r}870 \\
1043 \\
1108 \\
1143 \\
1134 \\
1092 \\
1035 \\
1013\end{array}$ & $\begin{array}{l}0.93 \\
1.18 \\
1.24 \\
1.24 \\
1.06 \\
0.90 \\
0.70 \\
0.33\end{array}$ \\
\hline E01-01 & $\begin{array}{r}1 \\
9 \\
18 \\
24 \\
30\end{array}$ & $\begin{array}{l}-0.518 \\
-3.034 \\
-3.621 \\
-2.467 \\
-1.183\end{array}$ & $\begin{array}{l} \pm 0.082 \\
\pm 0.281 \\
\pm 0.217 \\
\pm 0.107 \\
+0.122\end{array}$ & $\begin{array}{r}610 \\
1054 \\
1165 \\
1103 \\
953\end{array}$ & $\begin{array}{l}1.07 \\
3.86 \\
3.99 \\
3.30 \\
1.32 \\
\end{array}$ \\
\hline F03-01 & $\begin{array}{r}1 \\
9 \\
18 \\
24 \\
30\end{array}$ & $\begin{array}{l}-0.440 \\
-2.320 \\
-3.213 \\
-2.505 \\
-0.921\end{array}$ & $\begin{array}{l} \pm 0.079 \\
\pm 0.157 \\
\pm 0.305 \\
\pm 0.279 \\
\pm 0.090\end{array}$ & $\begin{array}{r}568 \\
939 \\
1103 \\
1084 \\
999\end{array}$ & $\begin{array}{l}1.08 \\
3.60 \\
4.03 \\
3.53 \\
1.66\end{array}$ \\
\hline
\end{tabular}

(a) Temperatures were obtained from TREVER calculations. These are time-averaged temperatures.

(b) Fluences were calculated using element average fluxes from GAUGE, and axial flux profiles were generated by FEVER. 
TABLE 3-2

COMPARISON OF MEASURED AND PREDICTED SLEEVE-FUEL COMPACT RADIAL GAP

\begin{tabular}{|c|c|c|c|c|}
\hline \multirow{2}{*}{$\begin{array}{l}\text { Fuel } \\
\text { Element }\end{array}$} & \multirow{2}{*}{$\begin{array}{c}\text { Fuel } \\
\text { Compact }\end{array}$} & \multicolumn{2}{|c|}{ Sleeve-Fue1 Compact Gap (mm) } & Bias \\
\hline & & Measured & Predicted & Pred. - Meas. (mm) \\
\hline E11-07 & $\begin{array}{r}5 \\
9 \\
24 \\
27 \\
30 \\
\end{array}$ & $\begin{array}{l}0.41 \\
0.61 \\
0.68 \\
0.67 \\
0.36 \\
\end{array}$ & $\begin{array}{l}0.36 \\
0.67 \\
0.69 \\
0.53 \\
0.36 \\
\end{array}$ & $\begin{array}{r}-0.05 \\
0.06 \\
0.01 \\
-0.14 \\
0.00 \\
\end{array}$ \\
\hline E01-01 & $\begin{array}{r}1 \\
9 \\
18 \\
24 \\
30 \\
\end{array}$ & $\begin{array}{l}0.24 \\
1.00 \\
1.20 \\
0.79 \\
0.45\end{array}$ & $\begin{array}{l}0.23 \\
0.87 \\
1.18 \\
0.80 \\
0.38\end{array}$ & $\begin{array}{r}-0.01 \\
-0.13 \\
-0.08 \\
0.01 \\
-0.07\end{array}$ \\
\hline E14-01 & $\begin{array}{r}1 \\
9 \\
18 \\
24 \\
30 \\
\end{array}$ & $\begin{array}{l}0.28 \\
0.59 \\
0.75 \\
0.56 \\
0.24 \\
\end{array}$ & $\begin{array}{l}0.23 \\
0.55 \\
0.77 \\
0.58 \\
0.34 \\
\end{array}$ & $\begin{array}{r}-0.05 \\
-0.04 \\
0.02 \\
0.02 \\
0.10\end{array}$ \\
\hline F03-01 & $\begin{array}{r}1 \\
9 \\
18 \\
24 \\
30 \\
\end{array}$ & $\begin{array}{l}0.18 \\
0.73 \\
1.00 \\
0.78 \\
0.35 \\
\end{array}$ & $\begin{array}{l}0.23 \\
0.64 \\
1.02 \\
0.85 \\
0.46 \\
\end{array}$ & $\begin{array}{r}0.05 \\
-0.09 \\
0.02 \\
0.07 \\
0.11 \\
\end{array}$ \\
\hline $\mathrm{C} 11-07$ & $\begin{array}{r}1 \\
5 \\
9 \\
12 \\
15 \\
21 \\
24 \\
27 \\
30 \\
\end{array}$ & $\begin{array}{l}0.17 \\
0.19 \\
0.39 \\
0.38 \\
0.40 \\
0.39 \\
0.33 \\
0.22 \\
0.17 \\
\end{array}$ & $\begin{array}{l}0.19 \\
0.27 \\
0.36 \\
0.39 \\
0.41 \\
0.38 \\
0.34 \\
0.30 \\
0.24 \\
\end{array}$ & $\begin{array}{r}0.02 \\
0.08 \\
-0.03 \\
0.01 \\
0.01 \\
-0.01 \\
0.01 \\
0.08 \\
0.07 \\
\end{array}$ \\
\hline $\bar{Z} \mathrm{RMS}_{\mathrm{Z}}$ & & & & $\begin{array}{r}0.00 \\
\pm 0.06 \\
\end{array}$ \\
\hline$F 05-05^{(a)}$ & $\begin{array}{r}1 \\
9 \\
18 \\
24 \\
30\end{array}$ & $\begin{array}{l}0.26 \\
0.71 \\
1.16 \\
0.90 \\
0.46\end{array}$ & $\begin{array}{l}0.21 \\
0.65 \\
0.92 \\
0.70 \\
0.34\end{array}$ & $\begin{array}{l}-0.05 \\
-0.06 \\
-0.24 \\
-0.20 \\
-0.12\end{array}$ \\
\hline $\begin{array}{l}\text { Average } \\
\text { RMS }\end{array}$ & & & & $\begin{array}{l}-0.13 \\
\pm 0.08\end{array}$ \\
\hline
\end{tabular}

(a) F05-05 fuel compact strain data were not available at the time the strain correlation was developed and are therefore not included in the data base for the correlation. The data base is comprised of the strain data for the other five elements listed in this table. 


\section{THERMAL PERFORMANCE}

\subsection{TEMPERATURE CALCULATIONS}

A modified version of TREVER (Ref, 16), a one-dimensional heat transfer code, is used to calculate Peach Bottom fuel element temperatures. The version of TREVER employed is set up to perform thermal calculations at 30 axial nodes corresponding to the center of each fuel compact in a Peach Bottom fuel element. In the TREVER analysis, the history of Peach Bottom Core 2 is simulated by 23 time points which divide it into 22 intervals of steady-state operation. These time points are listed in Table 4-1. Each core operating parameter assigned to a given time point $i$ is the average value of that parameter for the interval from time point $i$ to time point $i+1$. The core power, flow rate, and inlet helium pressure and temperature for each of the TREVER time intervals are shown in Figs. 4-1 and 4-2. These data were obtained by averaging the hour-by-hour information recorded for Core 2. It may be observed that some of the core powers shown in Fig. 4-1 are higher than the corresponding powers given in Table 2-1. This difference occurs because some of the time intervals include short shutdowns or periods of very low power generation. These periods were included when the average powers were calculated for the time intervals of the GAUGE depletion study but were omitted for the TREVER analysis since their inclusion would have lowered the average power below normal operating power and resulted in unrealistically low temperatures.

The physics data required as input to the thermal analysis include element average power factors and fast neutron fluence and axial power and flux profiles. These data have already been discussed in Section 2.1. Other data utilized by TREVER are summarized in Table 4-2. 
Temperatures calculated by TREVER include the bulk coolant temperature and the maximum and minimum sleeve and fuel compact temperatures. The temperature in the spine is approximately equal to the peak fuel compact temperature. The locations at which temperatures are calculated are shown in Fig. 4-3. Temperature histories have been calculated for each instrumented driver element and for each of the elements which underwent PIE. The temperature histories for the latter group (E01-01, F03-01, F05-05, E06-01, C11-07, E11-07, and E14-02 are provided in the Appendix.

\subsection{FEEDBACK OF POWER MEASUREMENTS INTO THERMAL CALCULATIONS}

As dicussed in Section 2.2, measured Cs-137, Zr-95, and La-140 distributions can be used to establish time-averaged and EOL axial power profiles for the individual fuel elements. Since these profiles vary significantly from element to element and since the physics calculations do not exactly predict axial power distributions, measured power profiles should be fed back into thermal calculations to obtain more reliable temperatures. Theoretically, the thermal calculations could be further improved by correcting element average power using correction factors obtained through comparison of measured and predicted burnup (Section $2.3 .3)$.

Although it was originally planned to employ these techniques for the thermal analysis of Peach Bottom driver elements, it was decided not to do so because of the cesium redistribution in nearly all elements of the core and the statistical insignificance of the majority of element average power correction factors. These methods have been applied for the thermal analyses of fuel test elements FTE-14 and FTE-15 (Ref. 24) where cesium redistribution was not as widespread and the use of mass spectroscopy permitted a much more accurate determination of burnup. In FTE-14, for which an $8 \%$ underprediction in the element average power was established 
and corrected, the agreement between the predicted and measured temperatures for the $\mathrm{W} / \mathrm{Re}$ thermocuple was within $1^{\circ} \mathrm{C}$ on a time-averaged basis and within $\pm 14^{\circ} \mathrm{C}(1 \sigma)$ for all TREVER time intervals. This thermocouple was located within a slot along the inner surface of the graphite fuel body and effectively measured peak graphite temperature. Unfortunately, the other three thermocouples in FTE-14 and FTE-15 failed, preventing further comparison between predicted and measured temperatures. Nevertheless, it has been shown that the feedback of power measurements represents a significant improvement in thermal analysis and that efforts should be made to reduce the uncertainty associated with measured burnup so that these methods can be applied to the thermal analyses of all fuel elements.

\subsection{TEMPERATURE MEASUREMENTS}

Seventeen driver fuel elements in Peach Bottom Core 2 were instrumented with two thermocouples each. These elements are identified in Fig. 1-4. Of the 34 thermocouples, 11 are the $W /$ Re type and 23 are Chromel/Alumel (C/A) thermocouples. Each thermocouple is located in a slot in the spine (Fig. 1-3) and effectively measures the peak fuel compact temperature at the axial location of the hot junction. A summary of thermocouple performance is given in Table 4-3.

The lifetime of a thermocouple is defined as the period of time during which the thermocouple gave reliable readings. Reliable readings are defined as measurements from the axial location where the thermocouple hot junction was supposed to be located. Although in this discussion the thermocouple is said to have failed once it stopped giving reliable readings, many thermocouples did not fail entirely but apparently short circuited and continued to give measurements from an axial location lower in the core. It was not always clear when a thermocouple malfunctioned, and there was no way to know for certain whether the thermocouple hot 
junction was exactly at the axial location at which it was supposed to be. In evaluating the thermocouple data, it was assumed that all readings were reliable unless thermocouple failure was clearly indicated. Indications of obvious failure include sudden and unexpected large reductions in recorded temperature followed by continuous low readings, marked deviation from the temperature trend established by the other thermocouple in the element (if functional) and by thermocouples in other elements having a similar environment, and temperature readings that are so far below expected temperatures that they are unbelievable. Some likely reasons for thermocouple failure are:

1. Structural defectiveness or improper installation.

2. Thermal shock resulting from rapid and large changes in temperature such as those caused by startup and shutdown or by sudden changes on local or total core power.

3. Excessive temperature.

The temperature levels at which thermocouple reliability is jeopardized are indicated by the following observations:

1. The highest temperature recorded by a $\mathrm{C} / \mathrm{A}$ thermocouple in the Peach Bottom core was approximately $1180^{\circ} \mathrm{C}$ (in E05-01) and the thermocouple failed immediately afterwards.

2. Uncorrected $\mathrm{W} / \mathrm{Re}$ thermocouple readings between $1300^{\circ}$ and $1350^{\circ} \mathrm{C}$ were recorded for Peach Bottom fuel test elements FTE-13, FTE-14, and FTE-18 (Ref. 25). The actual temperatures were $50^{\circ} \mathrm{C}$ to $150^{\circ} \mathrm{C}$ higher as determined from recalibration (see Section 4.4). Barring failure for reasons 1 or 2 cited above, the C/A thermocouples and the $\mathrm{W} / \mathrm{Re}$ thermocouples should perform dependably up to temperatures of about $1200^{\circ} \mathrm{C}$ and $1400^{\circ} \mathrm{C}$, respectively. 
The performance of each thermocouple is summarized in Table 4-4.

Temperature readings were recorded on a weekly basis and had to be corrected for the following:

1. Changes in cold junction temperature (standoff pin temperature). This correction was done automatically at the reactor site.

2. Adjustment in calibration equipment for the $W /$ Re type, for which readings were $22^{\circ} \mathrm{C}$ too high because of change from the $W-5 \%$ $\mathrm{Re} / \mathrm{W}-26 \%$ type to the $\mathrm{W}-3 \% \operatorname{Re} / \mathrm{W}-25 \%$ Re type between Core 1 and Core 2.

3. Decalibration of the W/Re type due to effect on emf output of neutron bombardment and transmutation.

The derivation of the recalibration formula used on Peach Bottom W/Re thermocouples is discussed in Section 4.4 .

4.4. THERMOCOUPLE RECALIBRATION

Thermocouple recalibration has been carried out a group of six thermocouples recovered from Peach Bottom fuel test elements, including. two each from FTE-5, FTE-18, and FPTE-3. Five of these were $\mathrm{W}-3 \% \mathrm{Re} / \mathrm{W}-25 \%$ $\operatorname{Re}($ Type $W$ ) thermocouples and one was a $\mathrm{C} / \mathrm{A}$ (Type $\mathrm{K}$ ) thermocouple. The $\mathrm{C} / \mathrm{A}$ thermocouple, for which no decalibration was expected, was included as a control test for the recalibration experiment. Resistance measurements for the thermocouples were consistent with preirradiation values, indicating that all were intact after irradiation. Each thermocouple was inserted into a furnace at known temperatures ranging from $680^{\circ} \mathrm{C}$ to $980^{\circ} \mathrm{C}$ and the actual and indicated temperatures were recorded (Ref. 26). The recalibration results are shown in Fig. 4-4, which plots the actual 
temperature $T_{a}$ versus the indicated temperature $T_{1}$. The recalibration results for each thermocouple have been analyzed via linear regression by the method of least squares under the assumption of $0^{\circ} \mathrm{C}$ as the cold junction temperature:

$$
\mathrm{T}_{\mathrm{a}}=\mathrm{T}_{\mathrm{o}}+\mathrm{K} \cdot \mathrm{T}_{\mathrm{i}}
$$

with the regression constants being

$$
\mathrm{T}_{0}=1 / \mathrm{n} \sum \mathrm{T}_{\mathrm{a}}-\mathrm{K} / \mathrm{n} \sum \mathrm{T}_{\mathrm{i}}
$$

and

$$
\mathrm{K}=\frac{\sum_{\mathrm{T}_{i} \mathrm{~T}_{\mathrm{a}}}-\left(\sum_{\mathrm{T}_{i}} \sum_{\mathrm{T}_{\mathrm{a}}}\right) / \mathrm{n}}{{\sum \mathrm{T}_{\mathrm{i}}^{2}-\left(\sum_{\mathrm{T}}\right)^{2} / \mathrm{n}}^{2}}
$$

The correlation coefficients are in all cases very close or equal to 1.0 (see Fig. 4-4) indicating a strong linear correlation for the covered temperature range. This temperature range corresponds to irradiation temperatures from $1020^{\circ} \mathrm{C}$ to $1320^{\circ} \mathrm{C}$ based on a cold junction temperature of $340^{\circ} \mathrm{C}$ and covers the irradiation temperatures experienced by the majority of the thermocouples. An additional measurement at an actual furnace temperature of $420^{\circ} \mathrm{C}$ made with thermocouple FPTE-3B shows that the linear correlation also holds at lower temperatures. It is therefore concluded that the recalibration results can be extrapolated to higher temperatures as well. (These temperatures were not achievable during recalibration because of a furnace limitation.) The standard deviations along the regression lines range from $\pm 1^{\circ} \mathrm{C}$ to $\pm 8^{\circ} \mathrm{C}$ and average $\pm 4^{\circ} \mathrm{C}$, which is within the estimated $\pm 8^{\circ} \mathrm{C}$ error associated with the test equipment. The nearly 
identical decalibration experienced by the two identical thermocouples from FTE-18 shows decalibration to be a systematic effect for the Type $W$ thermocouples. A reproducibility of $\pm 8^{\circ} \mathrm{C}$ is concluded for the decalibration effect. This represents about $1 \%$ of the cold junction to hot junction temperature rise for the test element thermocouples. As expected, no decalibration was found for the $\mathrm{C} / \mathrm{A}$ thermocouple, which supports the validity of the recalibration exercise.

The regression constants $\mathrm{T}_{0}$ and $\mathrm{K}$ are correlated with the thermal fluence $\psi$ in Figs. 4-5a and 4-5b, respectively. A second-order fit was used for the intercept $T_{o}$ and a first-order fit was used for the slope $K$. As can be seen, the thermocouples from FTE-5 and FTE-18 follow a certain pattern but the FPTE-3 thermocouples are somewhat different. The dissimilar decalibration of the thermocouples can be attributed to the following. First, the hot junctions of the FPTE-3 thermocouples were about 7 $\mathrm{mm}$ inserted directly into fuel compacts while the FTE-5 and FTE-18 thermocouples were surrounded by graphite and about $7 \mathrm{~mm}$ away from the nearest fuel zone. The lower thermal neutron microflux in the fuel compacts resulted in less decalibration in the FPTE-3 thermocouples. Secondly, the active core lengths of the FPTE- 3 thermocouples were different $(648 \mathrm{~mm}$ and $1210 \mathrm{~mm}$ ) compared to FTE-5 and FTE-18 (1257 mm). Since the decalibration resulting from thermal neutron bombardment is accumulated over the thermocouple length, the thermocouple with the shortest active core length experienced the least decalibration.

The following recalibration formula has been developed for all Type $W$ Peach Bottom thermocouples:

$$
\mathrm{T}_{\mathbf{a}}=\mathrm{a}\left(\psi+\mathrm{b} \psi^{2}\right)+(1+\mathrm{c} \psi)\left(\mathrm{T}_{\mathrm{i}}-\mathrm{T}_{\mathrm{s}}\right)+\mathrm{T}_{\mathbf{s}},
$$

where

$$
\begin{aligned}
\mathrm{a}= & -1.303 \times 10^{-4} \mathrm{gh}^{1.667}(\text { see Fig. 4-6a), } \\
\mathrm{b}= & -0.0669, \\
\mathrm{c}= & 2.916 \times 10^{-3} \mathrm{gh}^{0.4544}(\text { see Fig. 4-6b), } \\
\mathrm{g}= & \text { geometry factor to account for location of thermocouple } \\
& \text { hot junction in fuel }(\mathrm{g}=1.0 \text { ) or in graphite }(g=1.64),
\end{aligned}
$$




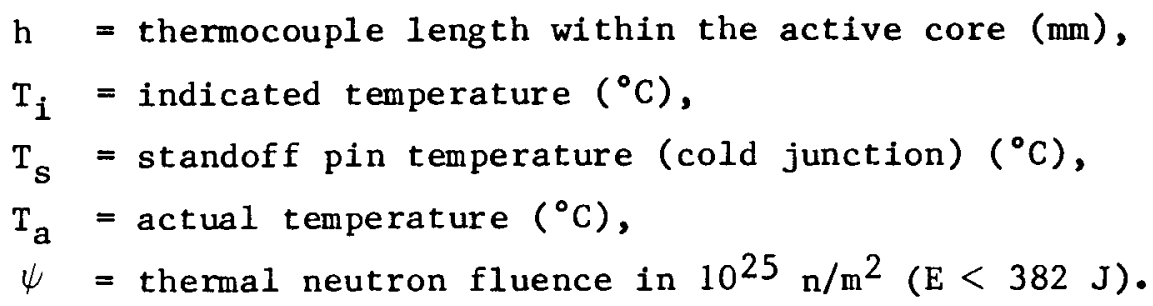

In developing the above equation, it was assumed that fluence and temperature gradients were similar for all Peach Bottom regular fuel and fuel test elements and would correlate with the thermocouple length within the active core. Under this assumption, the effect of these factors on thermocouple decalibration is accounted for by the $h$ term in the recalibration formula. In instrumented regular fuel elements, the thermocouples were situated within slots in the graphite spine directly adjacent to fuel compacts (Figs. 1-1 through 1-3). Because of this geometrical configuration, the geometry factor for regular fuel element thermocouples was taken to be 1.32 , the average of the geometry factors for thermocouples surrounded by graphite $(g=1.64)$ and those surrounded by fuel $(g=1.0)$.

The error $(1 \sigma)$ associated with the recalibration formula is given by the equation

$$
s=\sqrt{s_{1}^{2}+s_{2}^{2}+s_{3}^{2}+s_{4}^{2}},
$$

$$
\text { where } \begin{aligned}
\mathrm{S}_{1} & =\left[\mathrm{a}+2 \mathrm{~b} \psi+\mathrm{C}\left(\mathrm{T}_{\mathrm{i}}-\mathrm{T}_{\mathrm{s}}\right)\right] \mathrm{S} \psi \\
\mathrm{S}_{2} & \cong 0.8 \psi, \\
\mathrm{S}_{3} & =1.09 \times 10^{-3} \psi\left(\mathrm{T}_{\mathrm{i}}-\mathrm{T}_{\mathrm{s}}\right), \\
\mathrm{S}_{4} & \cong 0.4 \psi \\
\mathrm{S}_{\psi} & =0.096 \psi \text { or } 0.076 \psi
\end{aligned}
$$

The error terms $s_{1}, s_{2}, s_{3}$, and $s_{4}$ are the errors resulting from the uncertainties on the thermal neutron fluence, regression for terms a and $\mathrm{b}$, the term $\mathrm{K}=1+\mathrm{c} \psi$, and the geometry factor $\mathrm{g}$, respectively. $\mathrm{A} 6.8 \%$ $(1 \sigma)$ error on the thermal fluence was determined from calibrated gamma spectroscopy on 48 standard Peach Bottom fuel elements (Ref. 3). This 
error applies to FTE-18 and FPTE-3 as well. For elements like FTE-5 in which burnup and gamma spectroscopic measurements have been fed back into the nuclear calculations, the uncertainty on the thermal neutron fluence can be reduced to $3.4 \%(1 \sigma)$. Consequently the error of the fluence term $S \psi$ is $9.6 \%$ and $7.6 \%$ when applied to other than the recalibrated thermocouples from elements without and with burnup measurements, respectively.

\subsection{COMPARISON OF CALCULATED AND MEASURED TEMPERATURES}

Comparisons of measured and calculated temperatures have been made for 19 of the 34 driver element thermocouples. These comparisons are shown graphically in Figs. 4-7 through 4-25 and are tabulated in Tables 4-5 through 4-23. The measured temperatures appearing in the tables were obtained by time averaging the individual temperature measurements for each TREVER time interval. Of the 15 thermocouples for which no temperature comparisons were made, seven failed at BOL, four failed immediately after BOL, and the remaining four measured temperatures $76.2 \mathrm{~mm}$ ( $3 \mathrm{in}$.) from either the top or bottom of the active core. Since TREVER is a one-dimensional heat transfer code and cannot account for axial conduction at the fuel compact-reflector interfaces, the calculated temperatures at these locations are not expected to be representative of actual temperatures. Therefore, comparisons of measured and calculated temperatures at these locations were excluded from the thermal design verification.

A summary of the agreement between measured and calculated temperatures is given in Tables 4-24 through 4-26. The following observations and conclusions are based on the data presented in this table.

1. The agreement between all measured and calculated temperatures for the 19 thermocouple locations considered is within $\pm 87^{\circ} \mathrm{C}$ $(1 \sigma)$ with an apparent bias* of $\pm 7^{\circ} \mathrm{C}(1 \sigma)$. This is not necessarily indicative of the average uncertainty of all temperature predictions for Peach Bottom Core 2 driver elements

\footnotetext{
*Bias is defined as calculated minus measured property.
} 
since the thermocouple measurements do not constitute a representative distribution of temperatures with respect to core location or time. However, it is the best uncertainty estimate derivable from actual temperature measurements.

2. The disagreement between calculated and measured temperatures varies significantly in different regions of the core, as shown in Table 4-27.

3. Temperatures in elements within the inner six rings of the core are generally underpredicted prior to 342 EFPD, overpredicted from 342 to 500 EFPD, and underpredicted from 500 to 610 EFPD. Since rod bank 2 was fully inserted until 342 EFPD, withdrawn, and then fuly inserted again at 500 EFPD, overestimation of the effect of this rod bank on the radial power distribution is indicated.

4. Rod bank 1 was the last rod bank to be removed from the core, being gradually withdrawn from 610 EFPD until approximately 890 EFPD. Since temperatures in $\mathrm{B02-02}$, an element strongly influenced by this rod bank, were underpredicted until 858 EFPD and overpredicted after 858 EFPD, it appears that the effect of this rod bank on local power is also overestimated.

5. The agreement between measured and calculated temperatures in E07-01, E09-01, and E11-01, all of which are influenced by control rod bank 4 , is particularly poor. These temperatures are generally underpredicted prior to 500 EFPD, during which time rod bank 4 is either completely or almost completely inserted, and greatly overpredicted after 500 EFPD, when rod blank 4 is either completely or almost completely withdrawn. Once again, the influence of control rods on local power appears to be overestimated. 
6. Temperatures appear to be overpredicted in the outer elements for the entire 897 EFPD of Core 2.

There are too many possible reasons why calculated and measured temperatures could disgree to attempt to explain disagreements on a case-by-case basis. However, some of the most probable reasons for these differences are suggested below.

1. Incorrect recalibration of the $\mathrm{W} / \mathrm{Re}$ thermocouple.

2. The thermocouple hot junction physically or effectively (due to a short circuit) located at some other location than is assumed.

3. Incorrect element average power. Overestimation of the influence of control rods on the radial power distribution is indicated by the temperature comparisons. Unfortunately, the uncertainty in measured burnup was too large to allow accurate element average power correction factors to be determined.

4. Incorrect axial power profiles. Although the unrodded axial power profiles have been shown to be in good agreement with measurements, no means of verifying the partially rodded axial power profiles is available.

5. Eccentricity of the sleeve-fuel compact radial gap. The gaps used on the thermal calculations are average gaps. Since the $\Delta T$ per unit of gap width decreases with increasing gap size, eccentricity of the gap would generally cause temperatures within the fuel compact to be lower than they would be if the gap were concentric. Gap eccentricity was observed at ORNL during element PIE (Refs. 8 through 13). 
6. Thermal conductivity of the fuel compact. A constant value of $19 \mathrm{~W} / \mathrm{m} \cdot \mathrm{K}$ was used in the thermal calculations. Since the thermal conductivity is higher prior to irradiation and approaches this value with fast neutron exposure, use of a constant $19 \mathrm{~W} / \mathrm{m}^{*} \mathrm{~K}$ contributes somewhat to the overprediction of temperatures near BOL. Furthermore, there is uncertainty associated with the value of the irradiated thermal conductivity itself. This value was originally taken to be $27.6 \mathrm{~W} / \mathrm{m}^{\bullet} \mathrm{K}$ (Ref. 1) but was later changed to $19 \mathrm{~W} / \mathrm{m} \cdot \mathrm{K}$ (Ref. 18).

The most probable causes for the general $27^{\circ} \mathrm{C}$ overprediction of temperatures are eccentricity of the sleeve-fuel compact gap and underestimation of the fuel compact thermal conductivity. A change in fuel compact conductivity from $19 \mathrm{~W} / \mathrm{m}^{\bullet} \mathrm{K}$ to approximately $27.6 \mathrm{~W} / \mathrm{m}^{\bullet} \mathrm{K}$ would offset the observed bias.

Peak fuel temperatures in approximately $1 \%$ of the driver elements were calculated to be in excess of $1510^{\circ} \mathrm{C}$, the original prediction (Ref. 2) for the peak driver element fuel temperature in Peach Bottom Core 2. This increase in predicted temperatures is a result of the fuel compact-sleeve gap being larger than originally expected. The decision to reduce core power after 701 EFPD prevented fuel temperature predictions from becoming far out of line with design temperatures. Peak temperatures approaching $1600^{\circ} \mathrm{C}$ were calculated for driver elements but could not be verified due to thermocouple fallure in the high-temperature elements. Peak temperatures of approximately $1600^{\circ} \mathrm{C}$ were also calculated for fuel test elements and were indirectly verified in FTE-14 and FTE-18 by thermocouple measurements (cf Section 4.3; highest fuel temperatures did not necessarily coincide with the axial location of the thermocouple hot junction). 


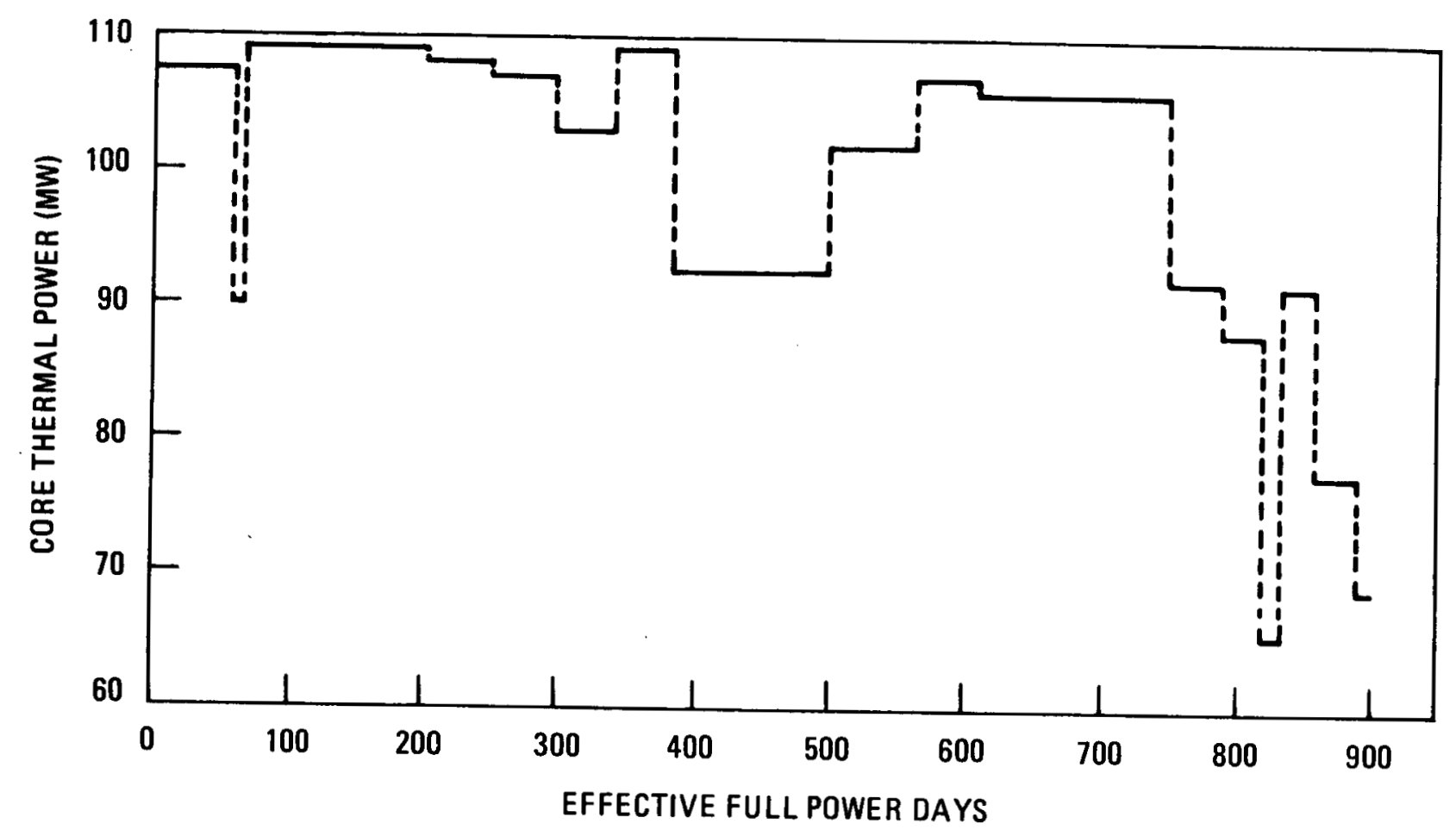

Fig. 4-1. Peach Bottom Core 2 thermal power 


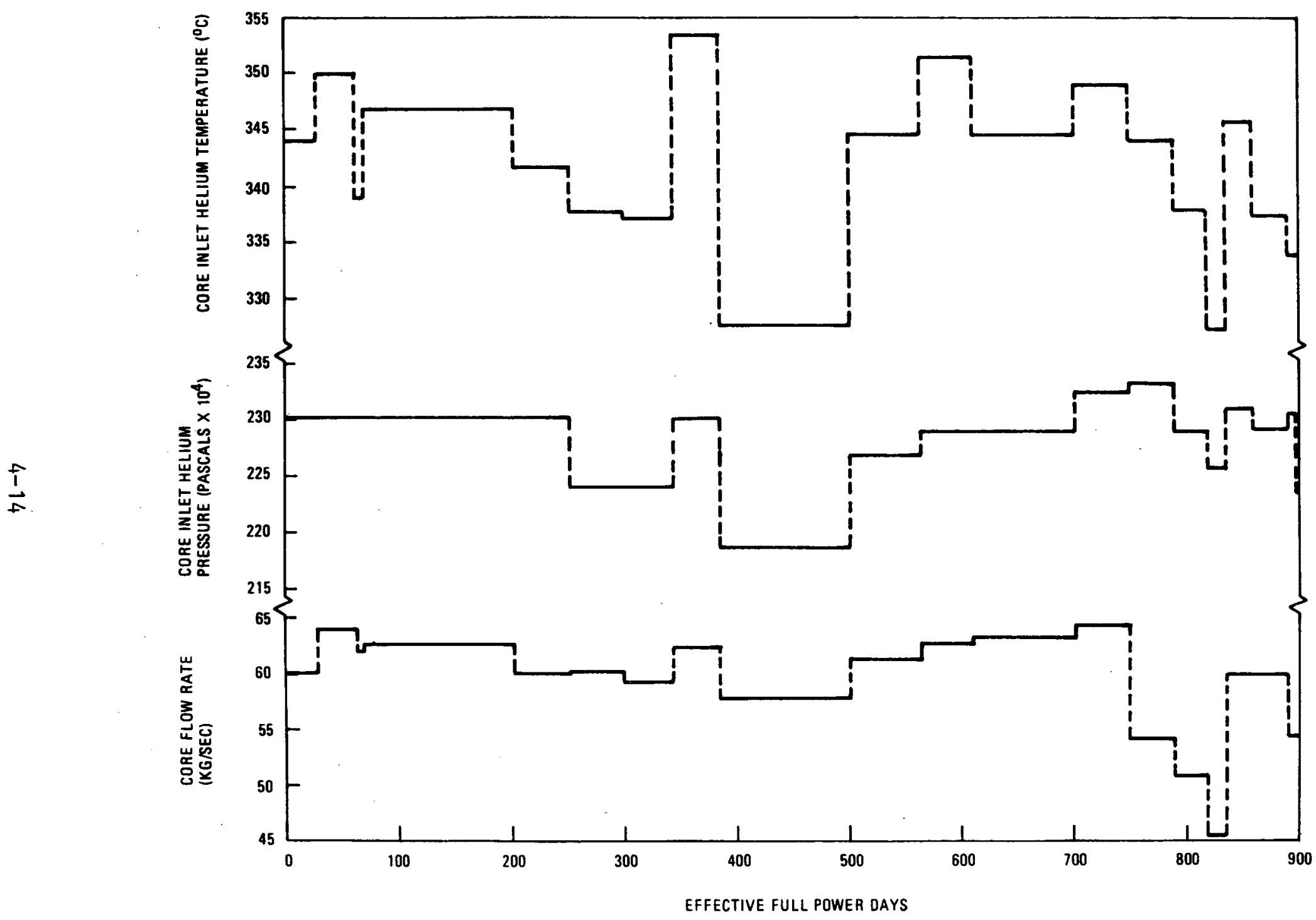

Fig. 4-2. Peach Bottom Core 2 operating parameters 


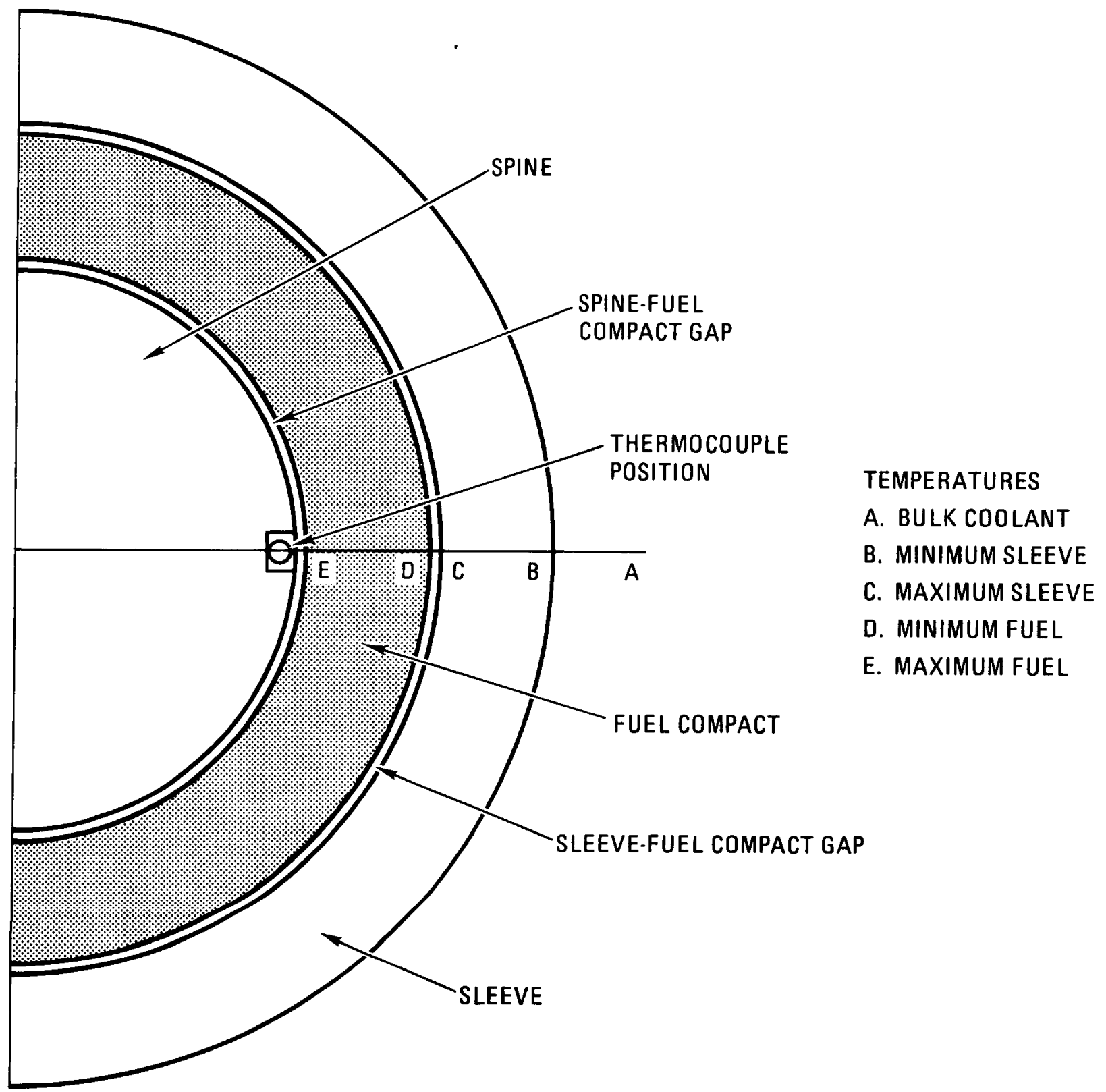

Fig. 4-3. TREVER calculation locations for Peach Bottom driver fuel elements 


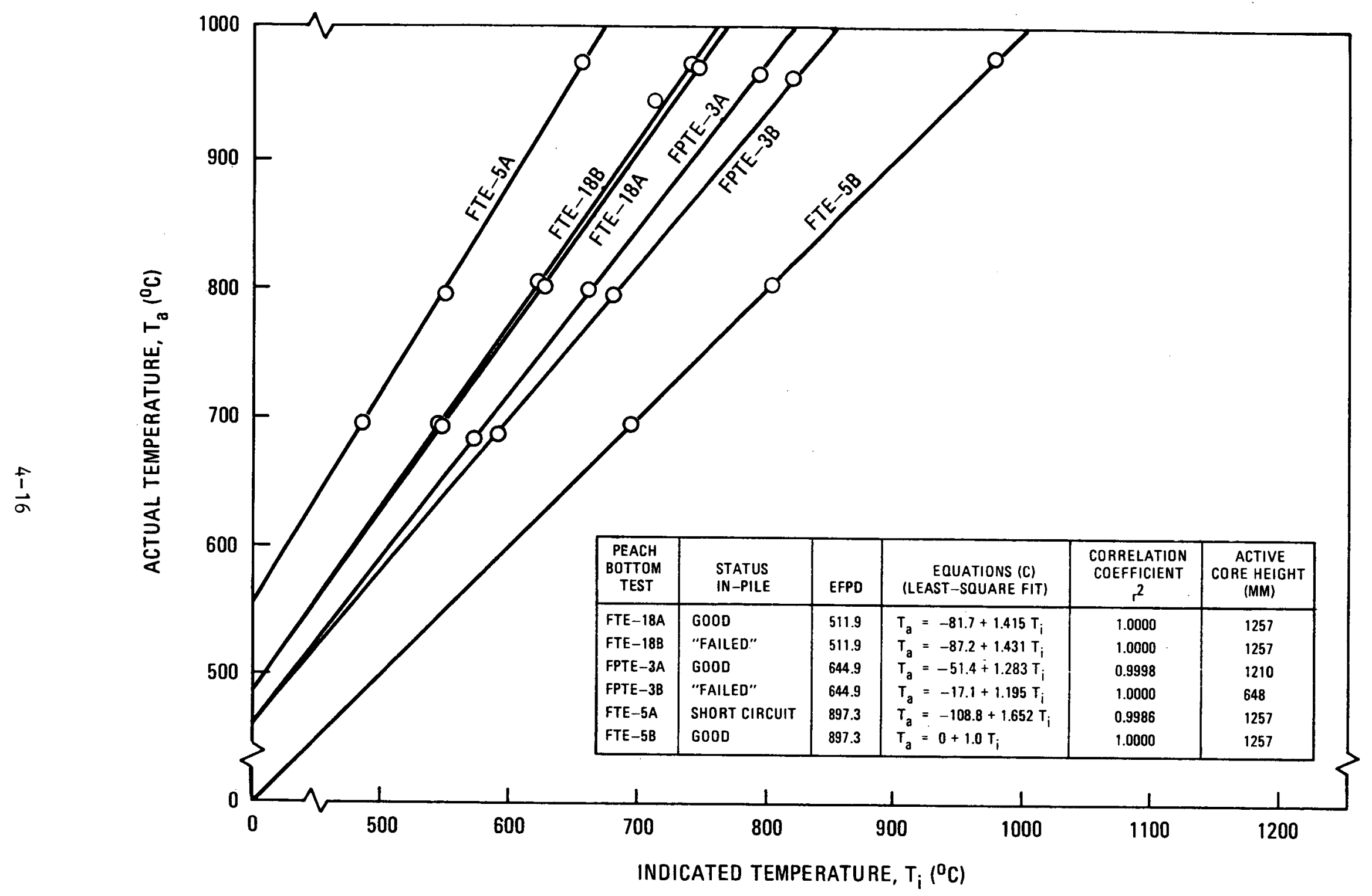

Fig. 4-4. FTE-18, FPTE-3, and FTE-5 thermocouple EOL recalibration results 


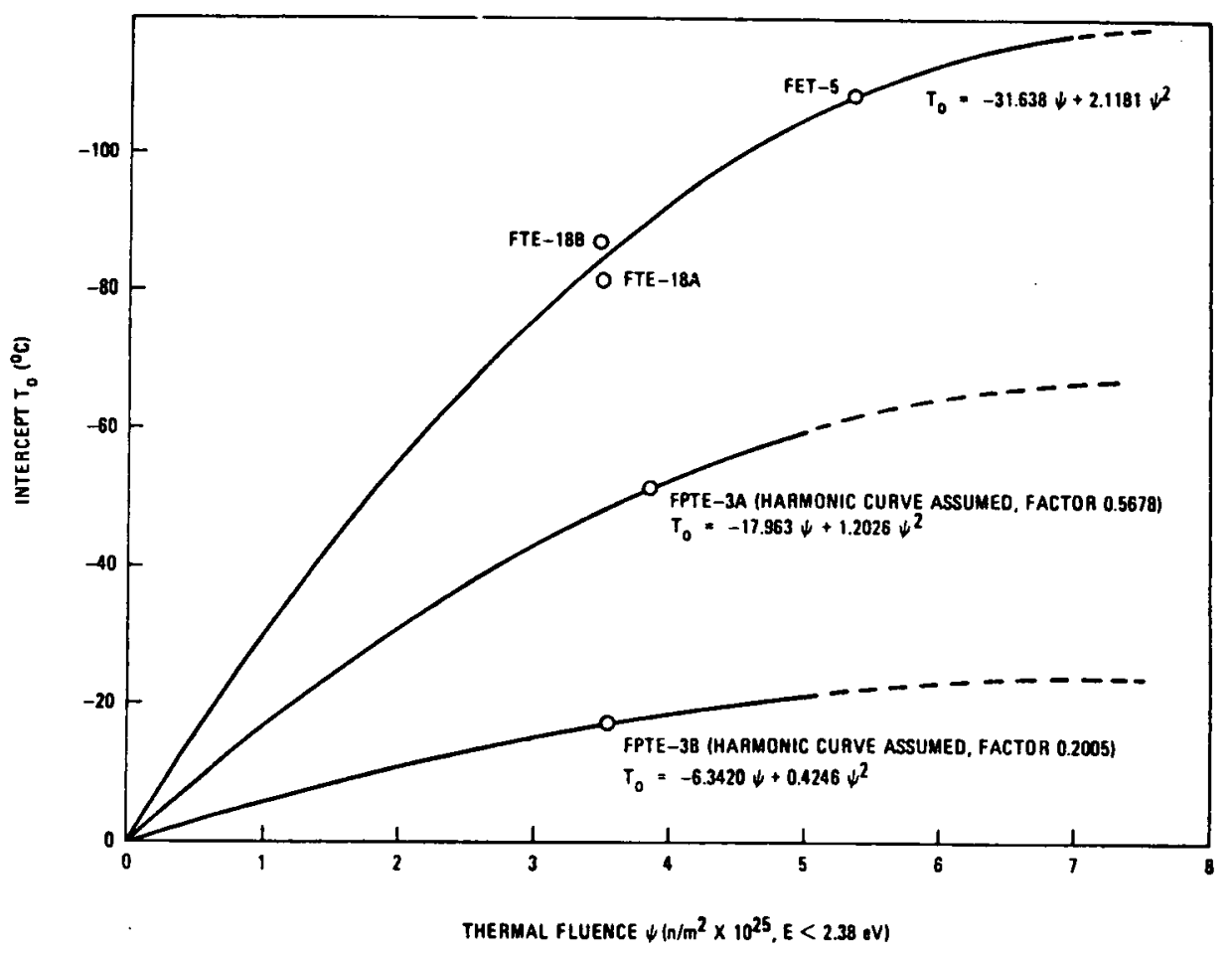

Fig. 4-5a. Peach Bottom thermocouple recalibration term $T_{0}$

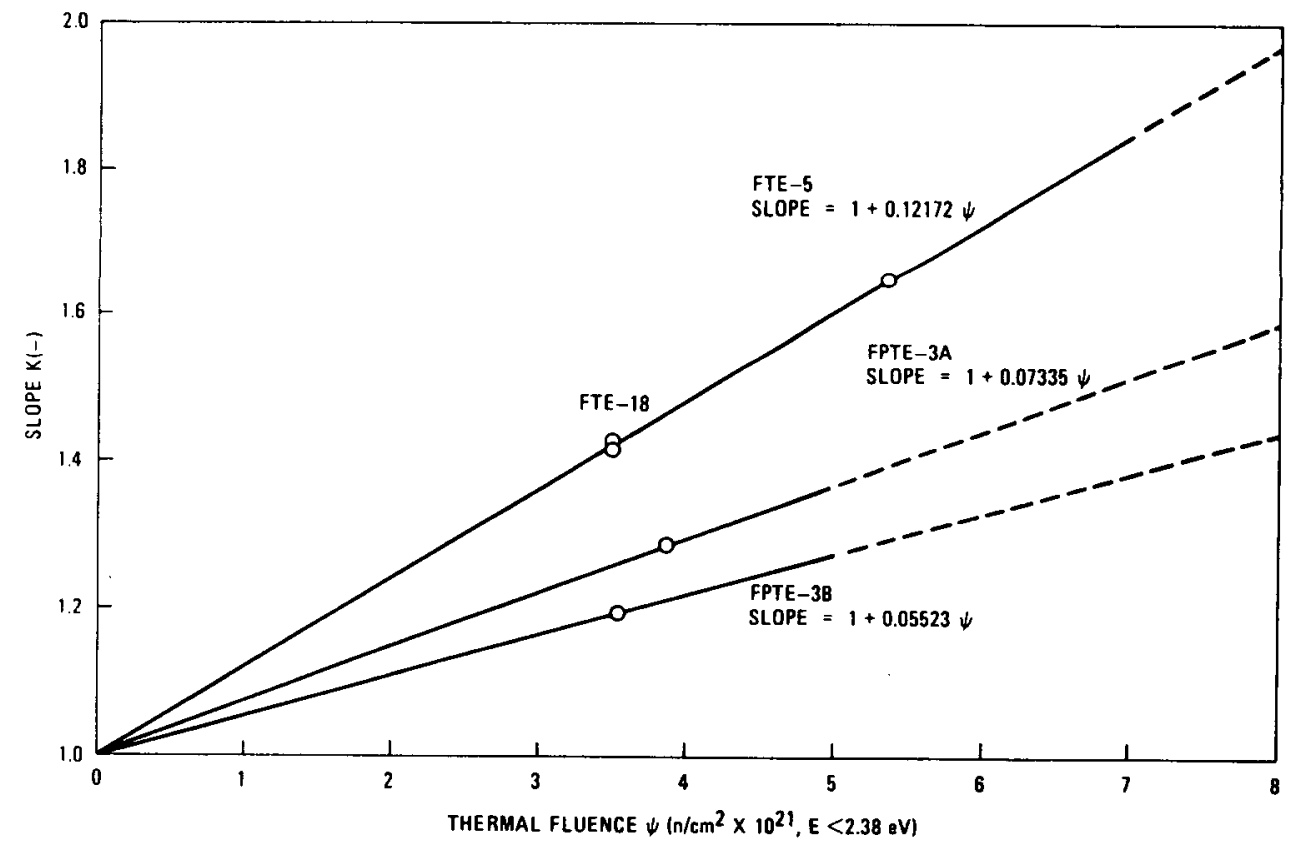

Fig. 4-5b. Peach Bottom thermocouple recalibration term $\mathrm{K}$ 


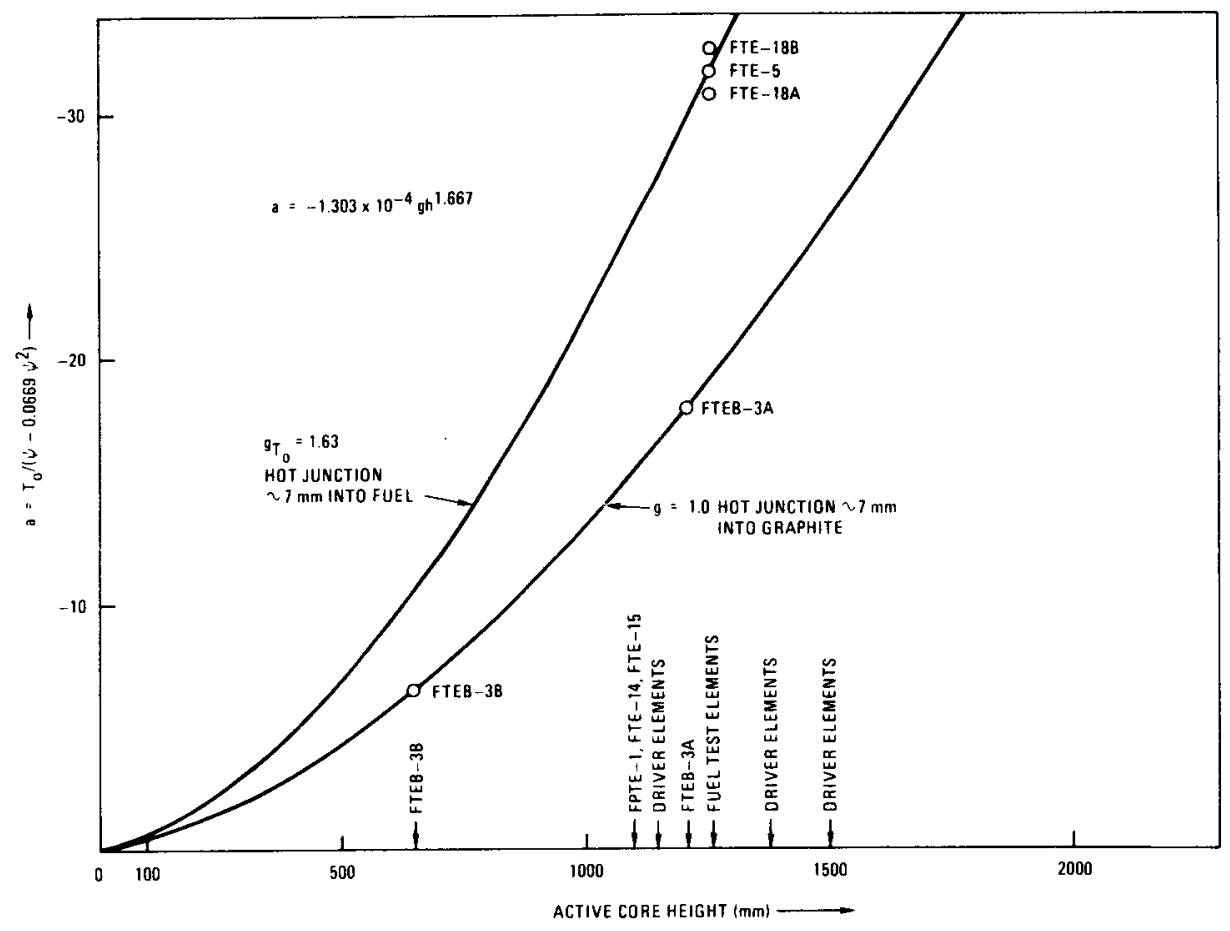

Fig. 4-6a. Height correlation for Type W Peach Bottom thermocouple recalibration term $\mathrm{T}_{\mathrm{o}}$

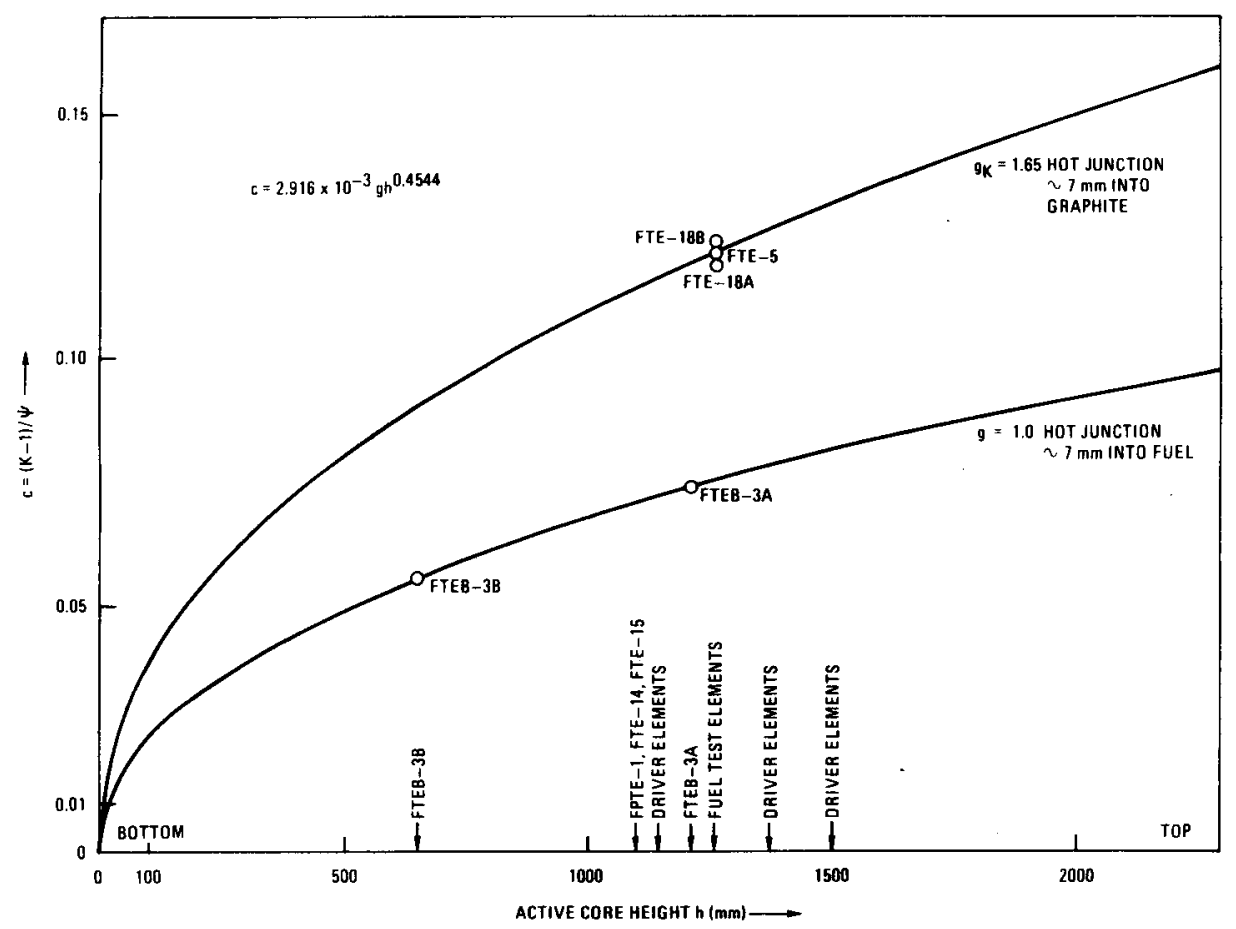

Fig. 4-6b. Height correlation for Type W Peach Bottom thermocouple recalibration term $\mathrm{K}$ 


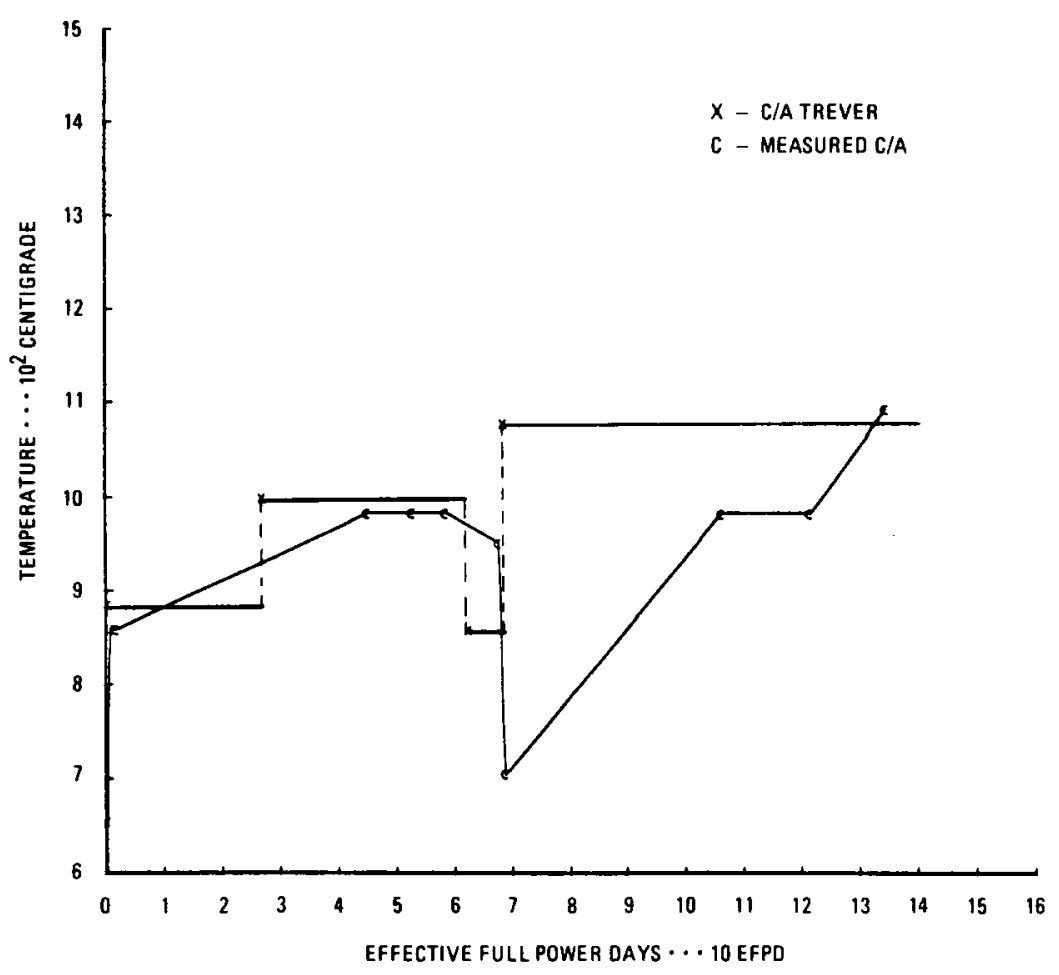

Fig. 4-7. Comparison of predicted temperatures with E01-01 C/A thermocouple readings

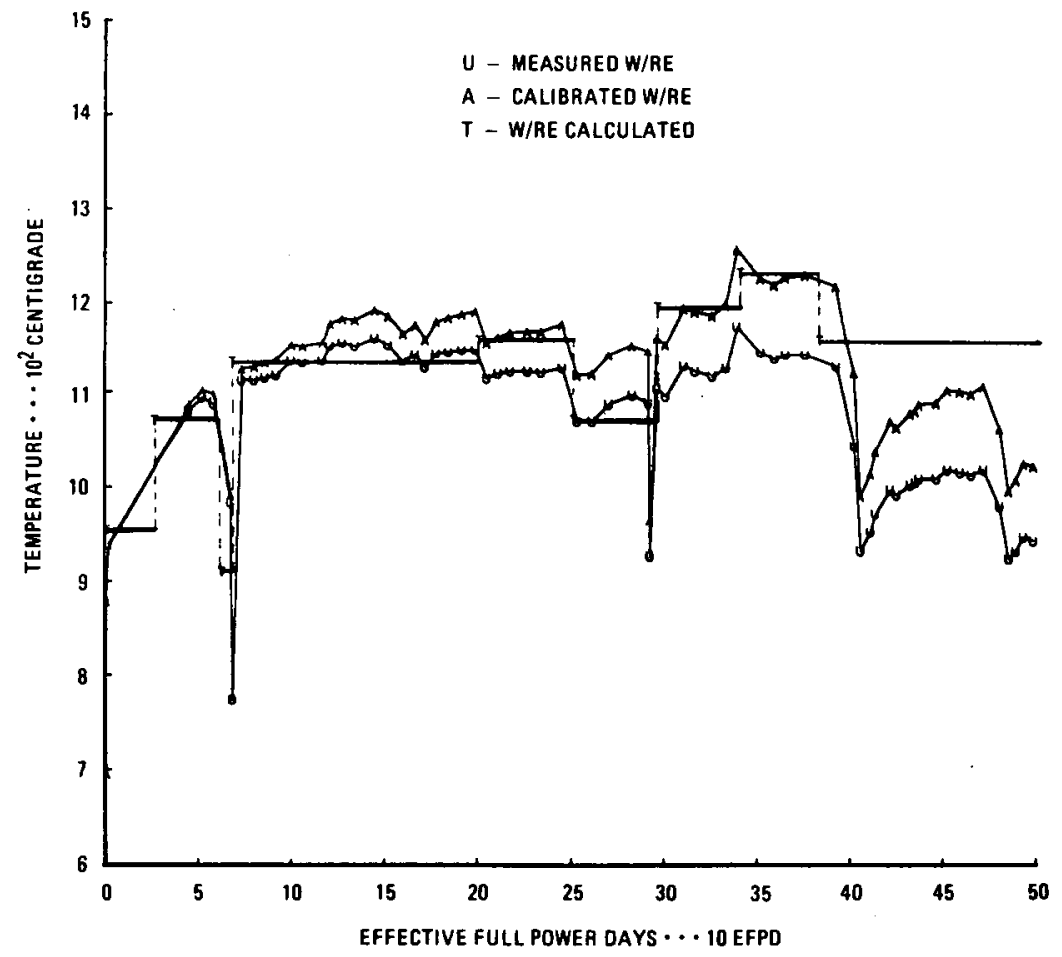

Fig. 4-8. Comparison of predicted temperatures with E01-01 W/Re thermocouple readings 


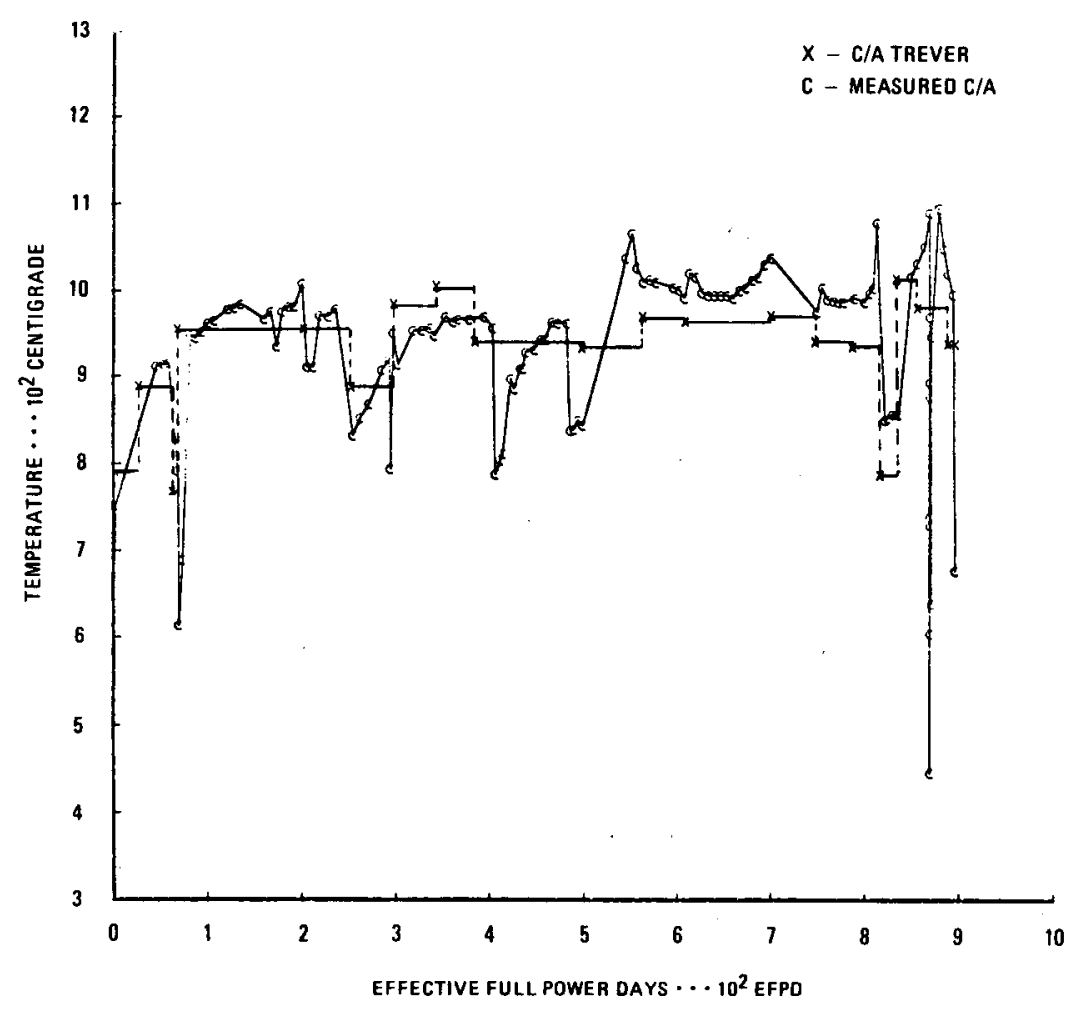

Fig. 4-9. Comparison of predicted temperatures with B02-02 C/A thermocouple readings

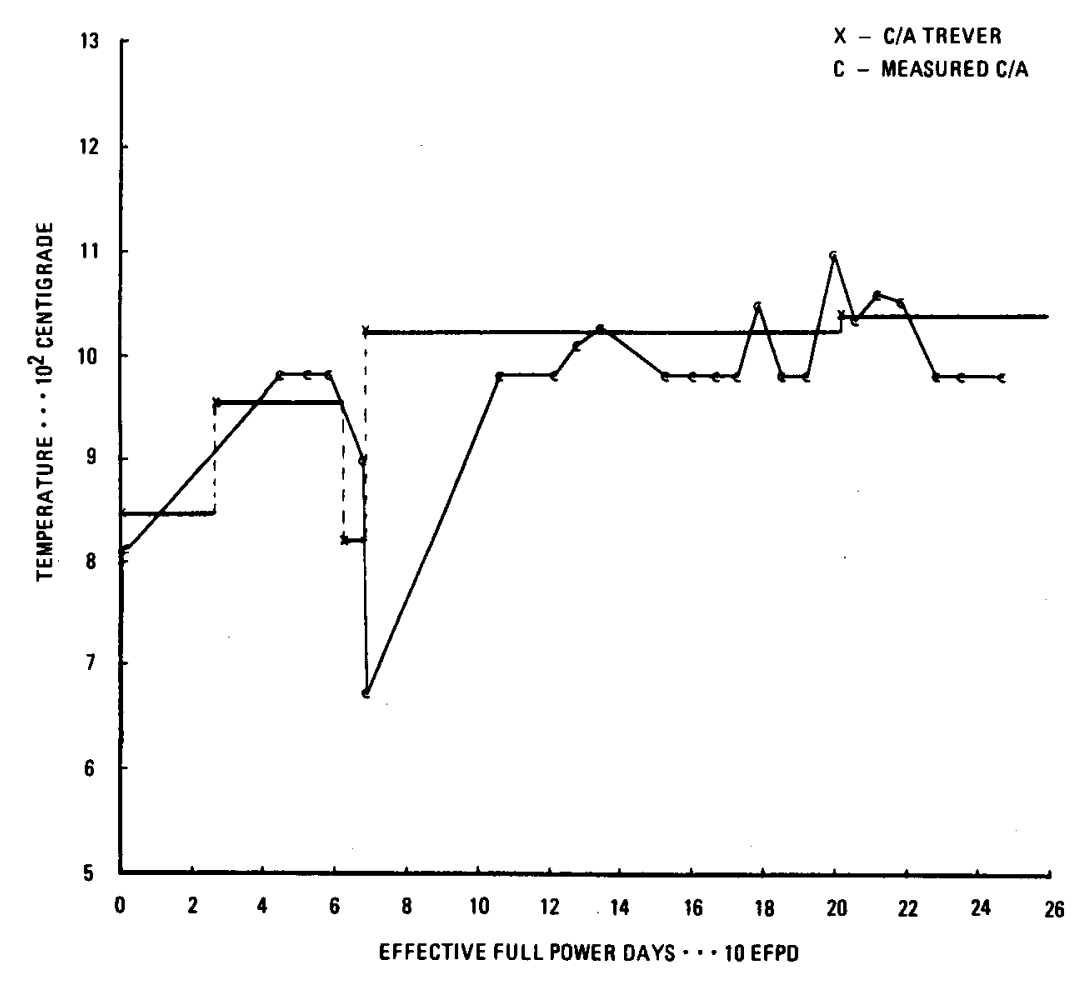

Fig. 4-10. Comparison of predicted temperatures with E02-01 C/A thermocouple readings 


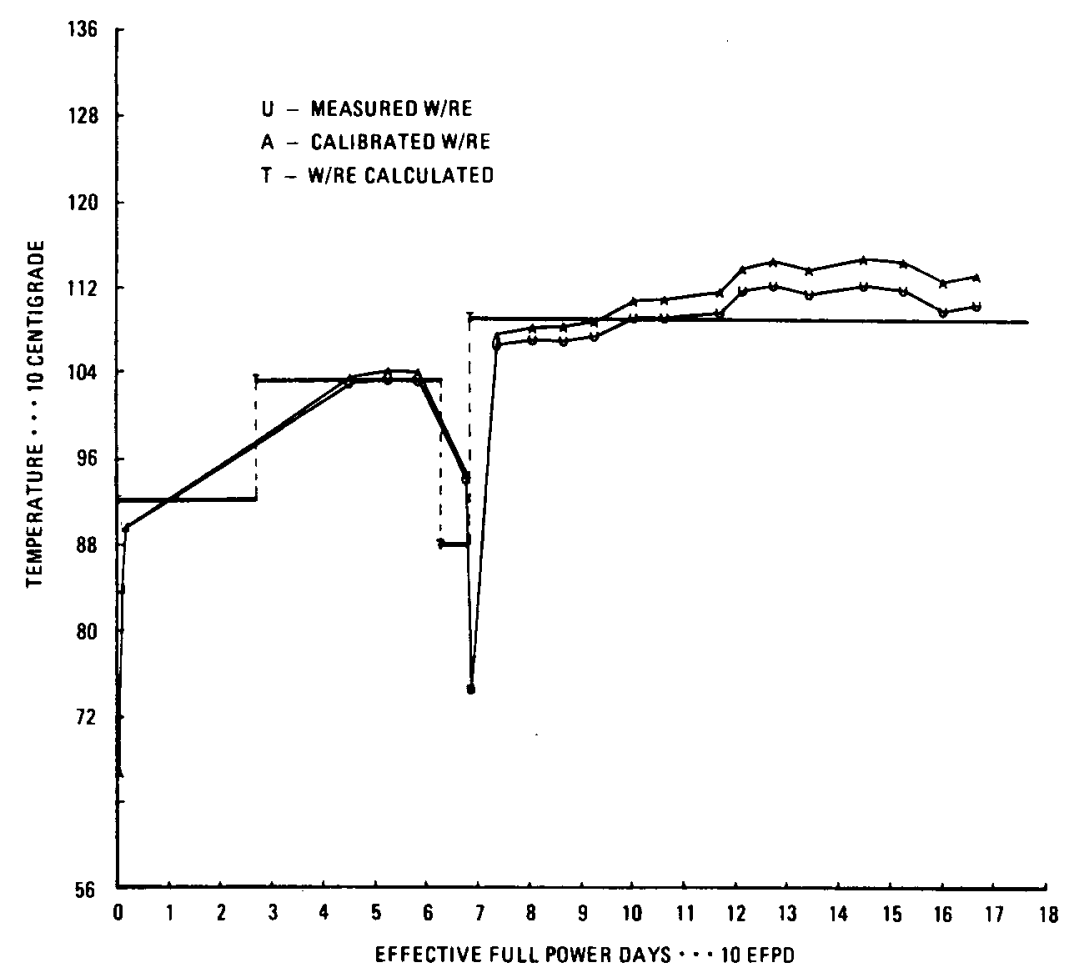

Fig. 4-11. Comparison of predicted temperatures with E02-01 W/Re thermocouple readings

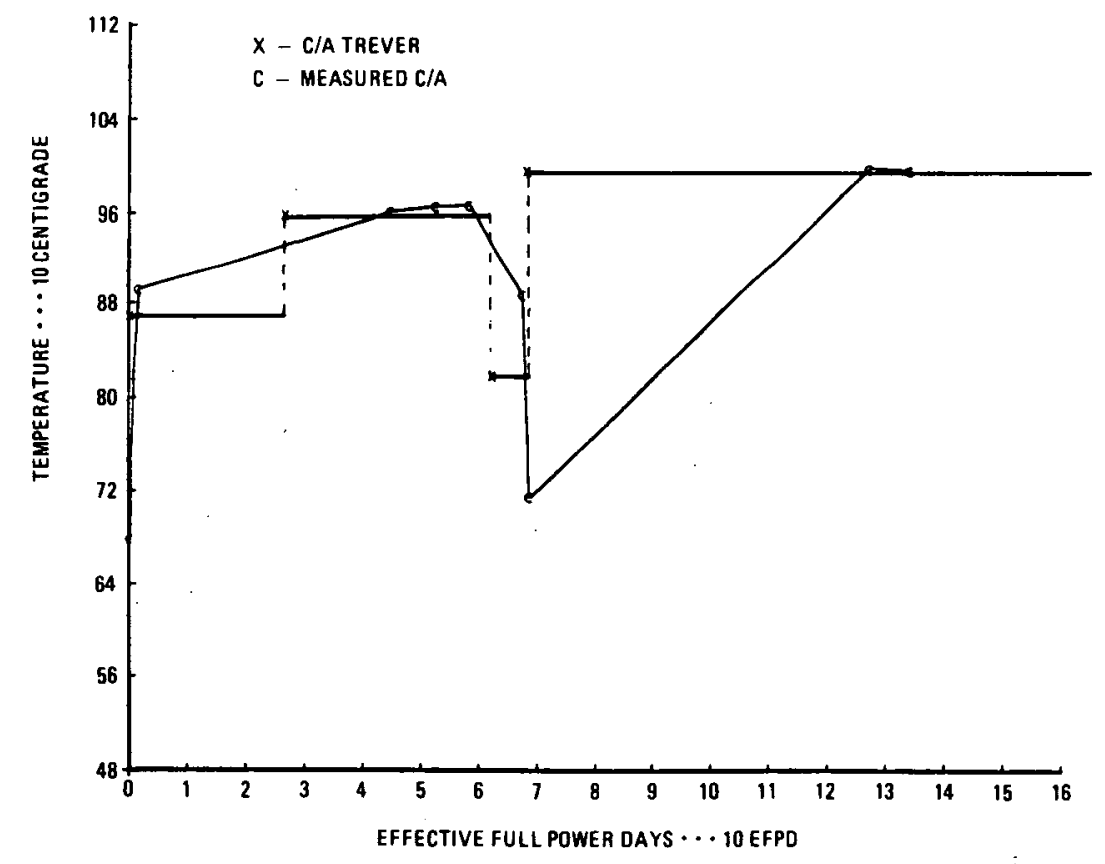

Fig. 4-12. Comparison of predicted temperatures with B03-03 C/A thermocouple readings 


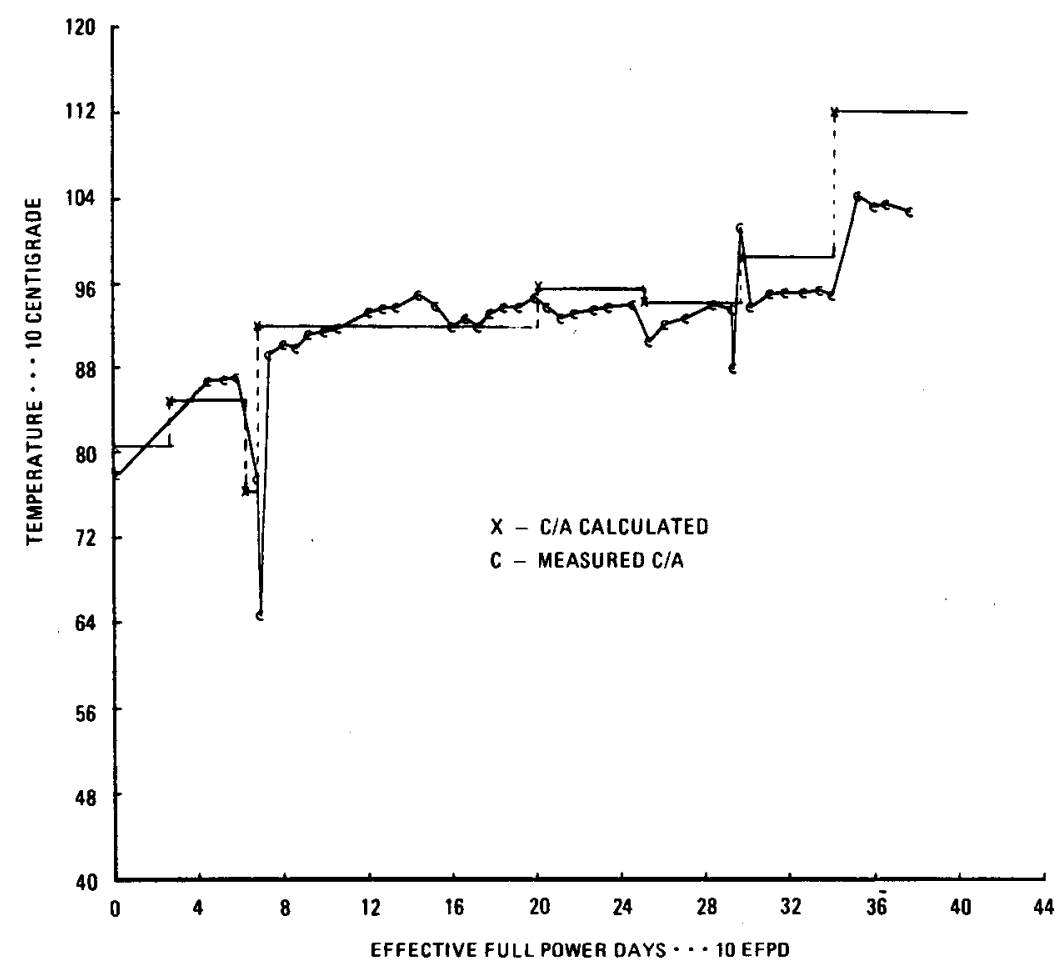

Fig. 4-13. Comparison of predicted temperatures with E03-01 C/A thermocouple readings

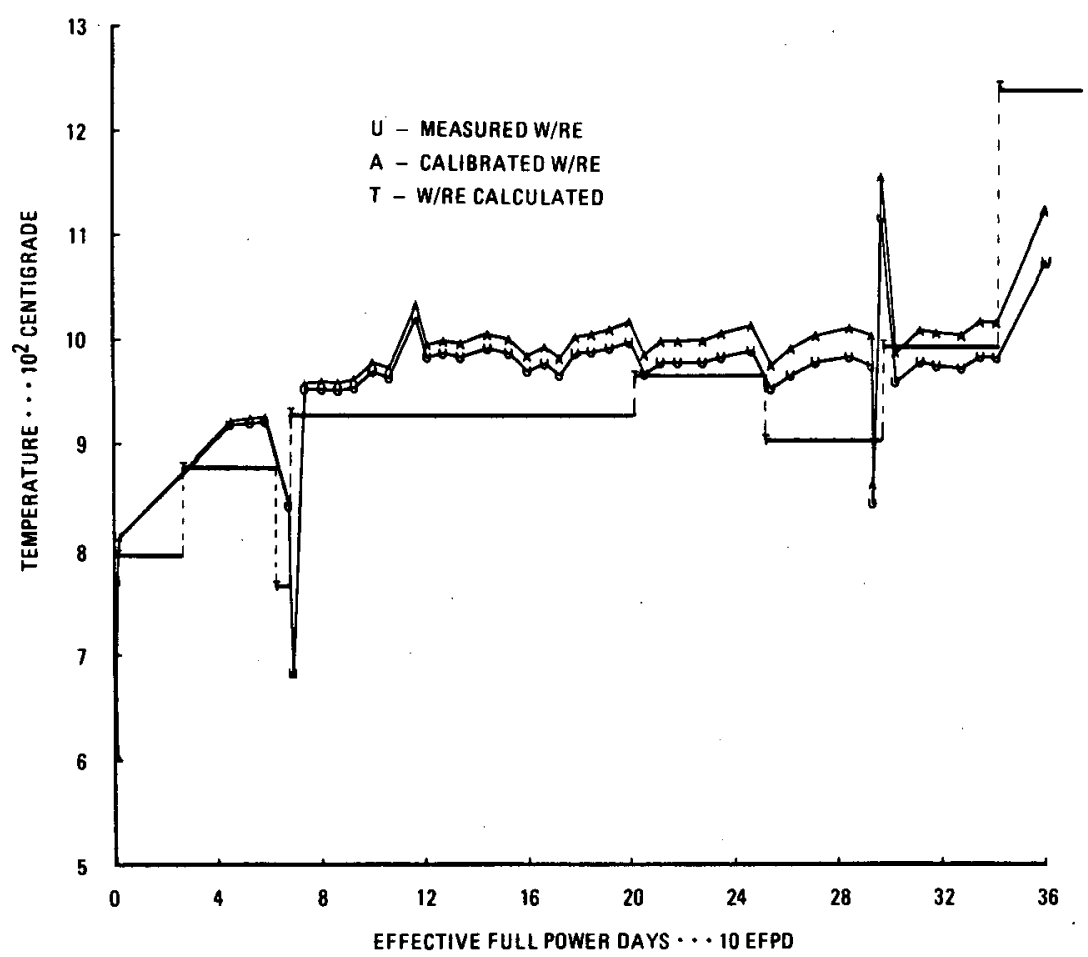

Fig. 4-14. Comparison of predicted temperatures with E03-01 W/Re thermocouple readings 


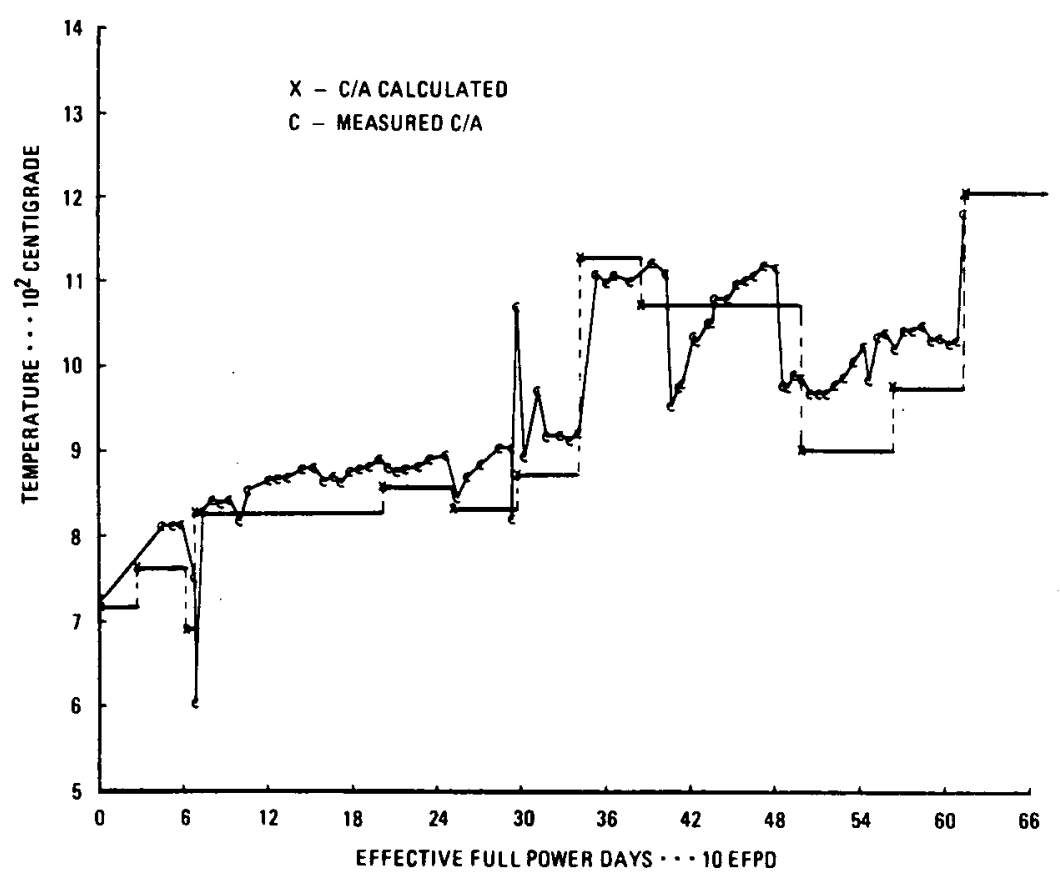

Fig. 4-15. Comparison of predicted temperatures with E05-01 C/A thermocouple readings

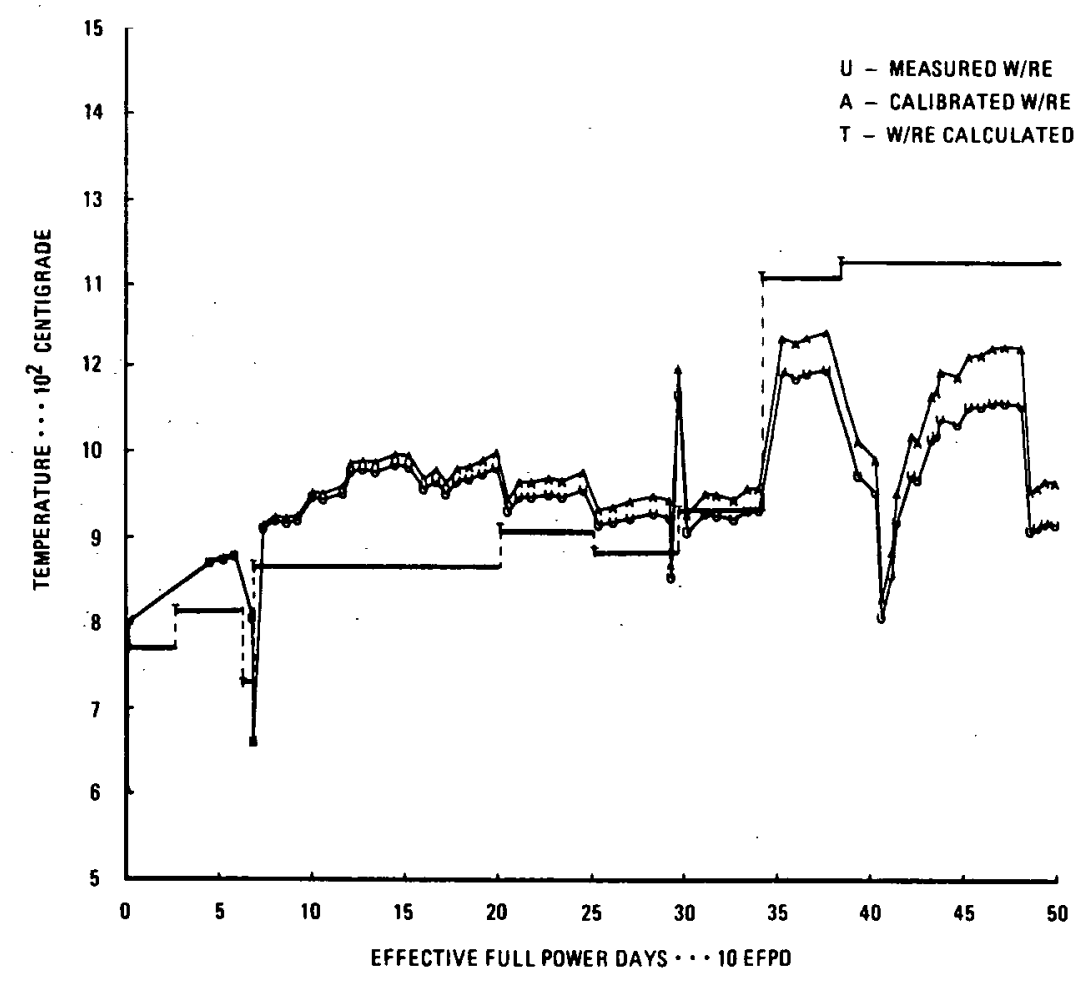

Fig. 4-16. Comparison of predicted temperatures with E05-01 W/Re thermocouple readings

$$
4-23
$$




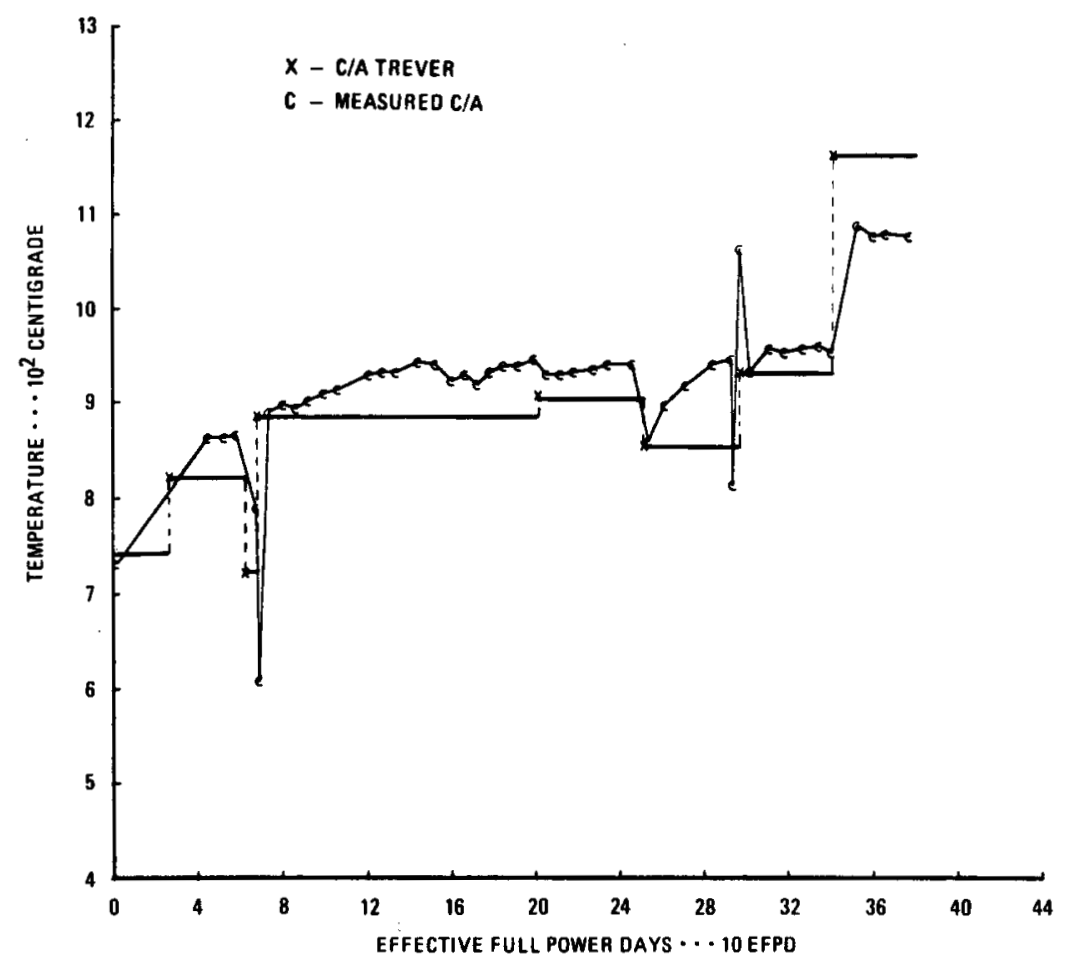

Fig. 4-17. Comparison of predicted temperatures with E05-01 C/A thermocouple readings

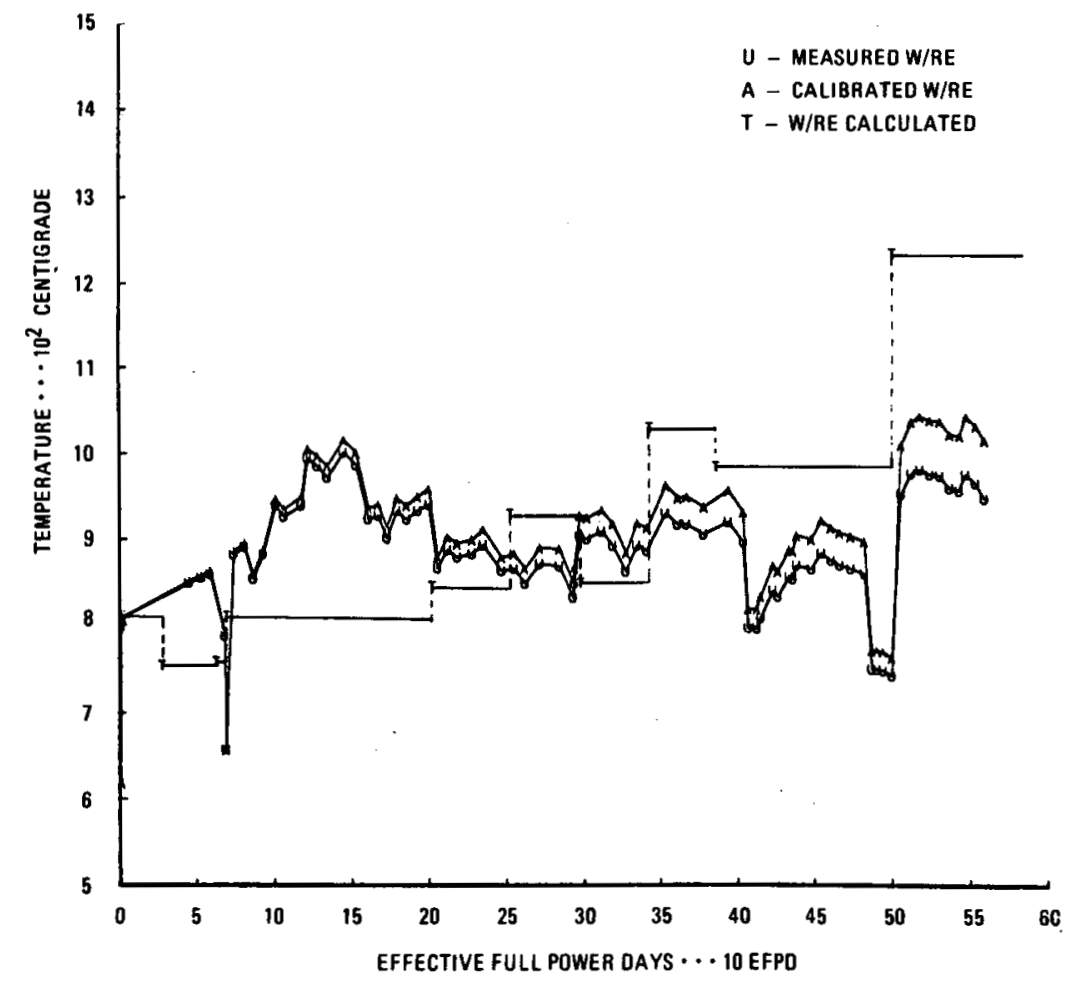

Fig. 4-18. Comparison of predicted temperatures with E07-01 W/Re thermocouple readings 4-24 


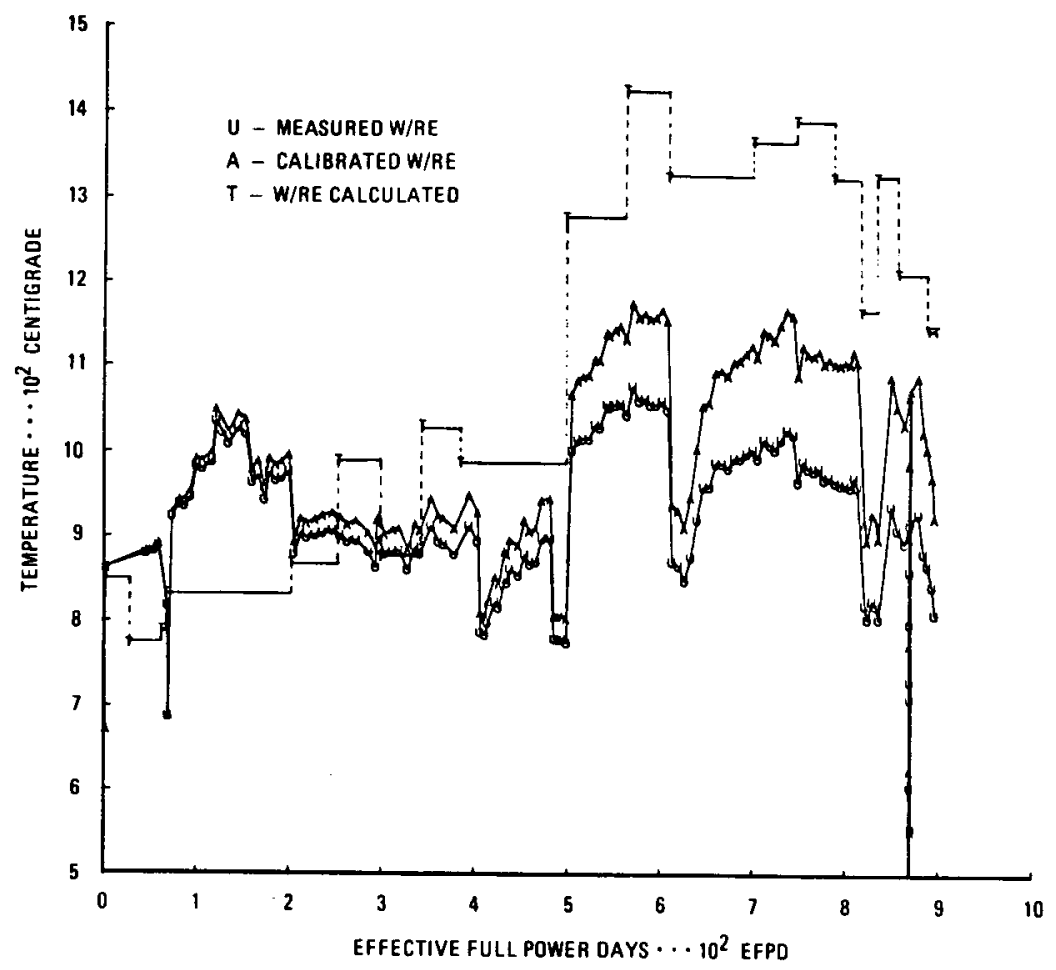

Fig. 4-19. Comparison of predicted temperatures with E09-01 W/Re thermocouple readings

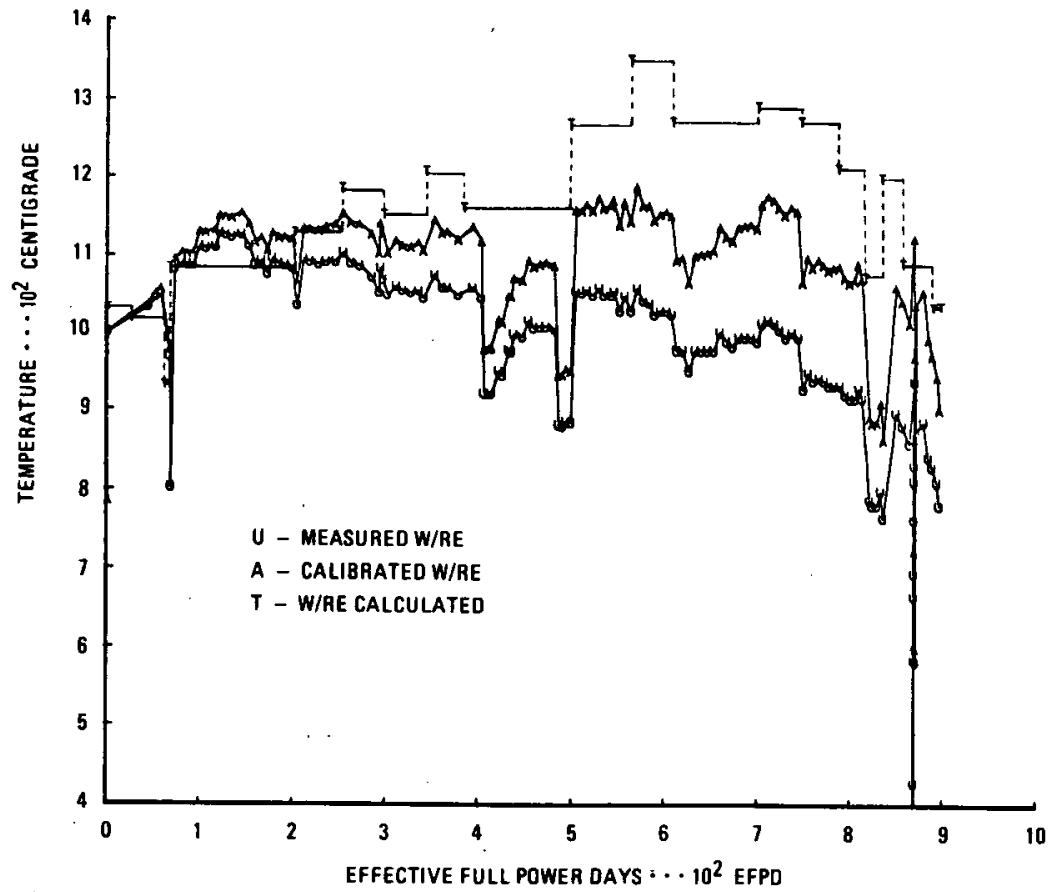

Fig. 4-20. Comparison of predicted temperatures with E11-01 W/Re thermocouple readings 


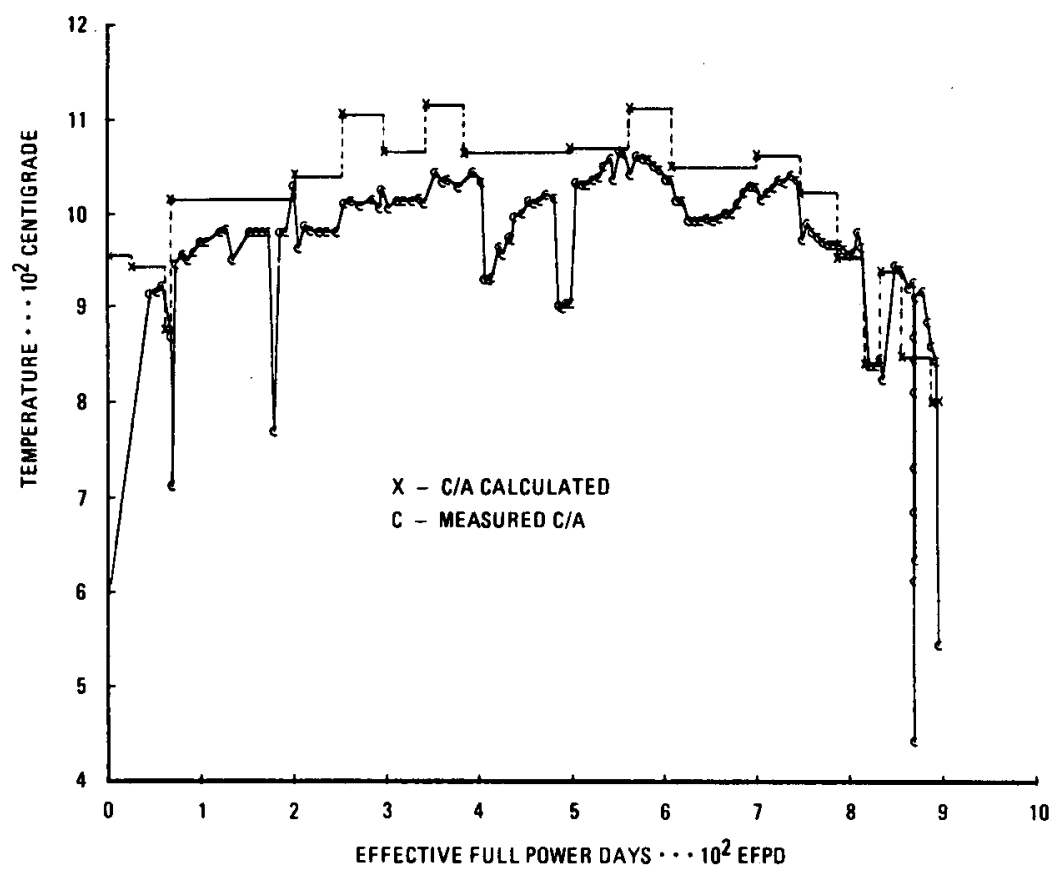

Fig. 4-21. Comparison of predicted temperatures with E13-01 C/A thermocouple readings

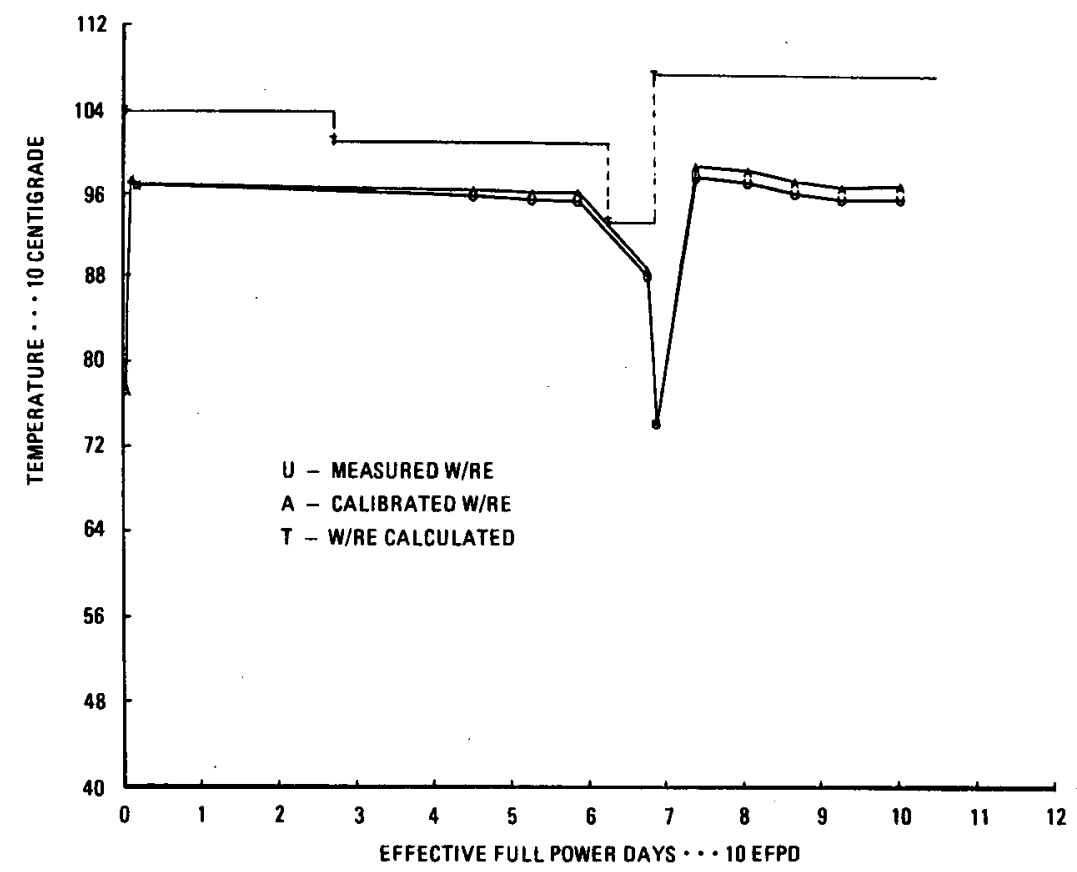

Fig. 4-22. Comparison of predicted temperatures with E13-01 W/Re thermocouple readings 


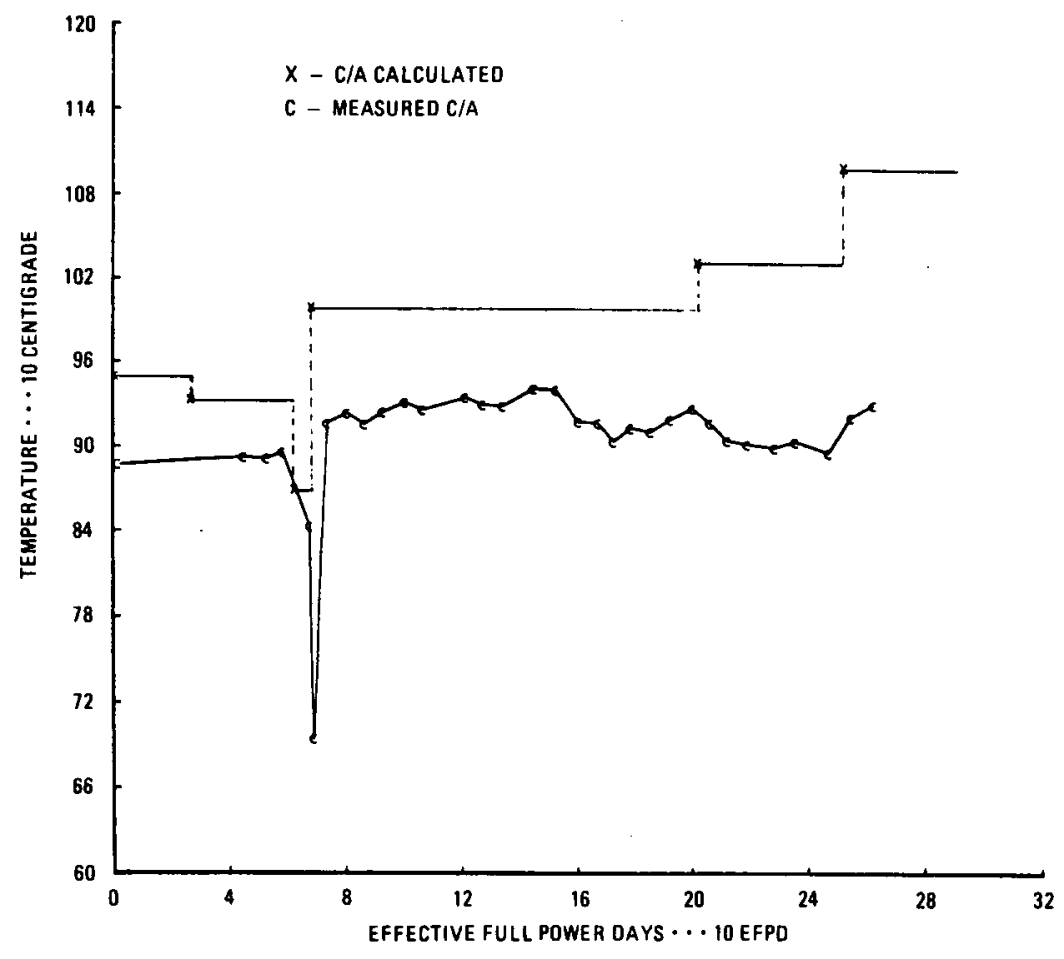

Fig. 4-23. Comparison of predicted temperatures with E14-01 C/A thermocouple readings

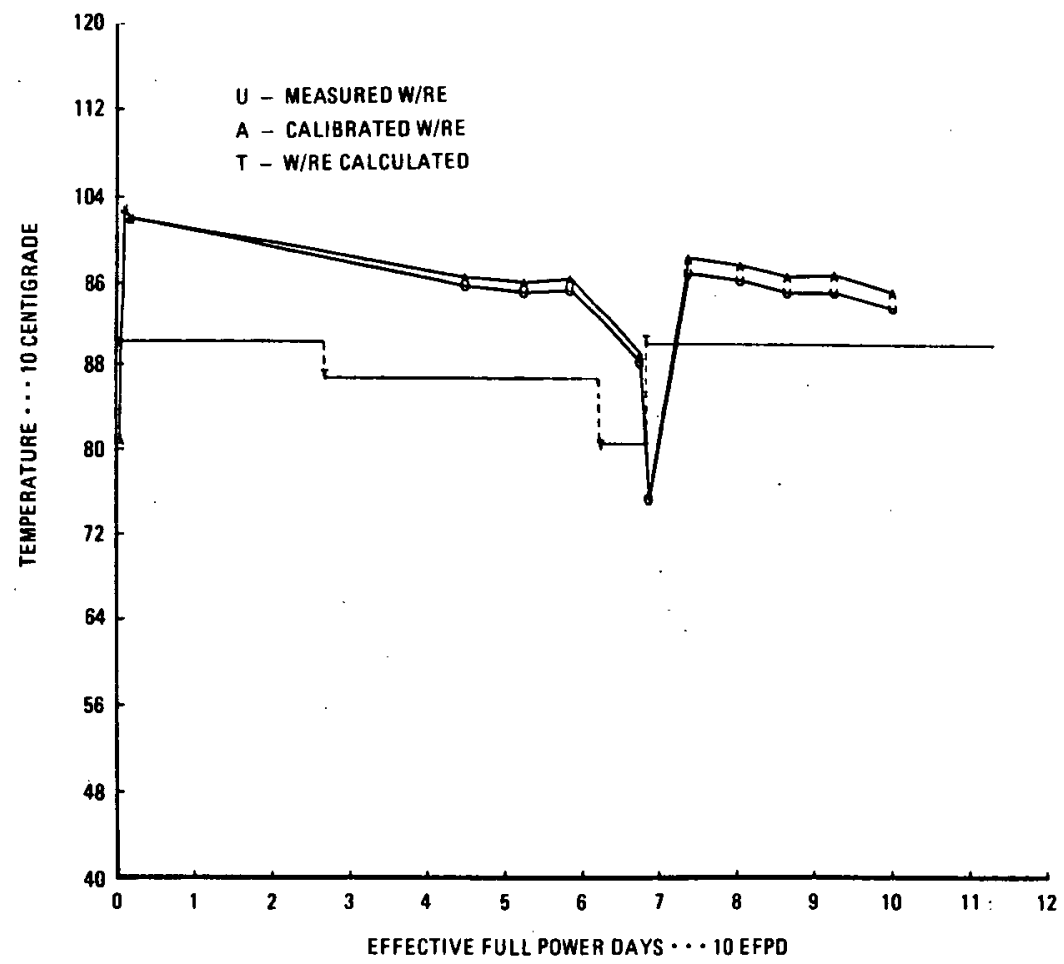

Fig. 4-24. Comparison of predicted temperatures with E15-01 W/Re thermocouple readings 4-27 


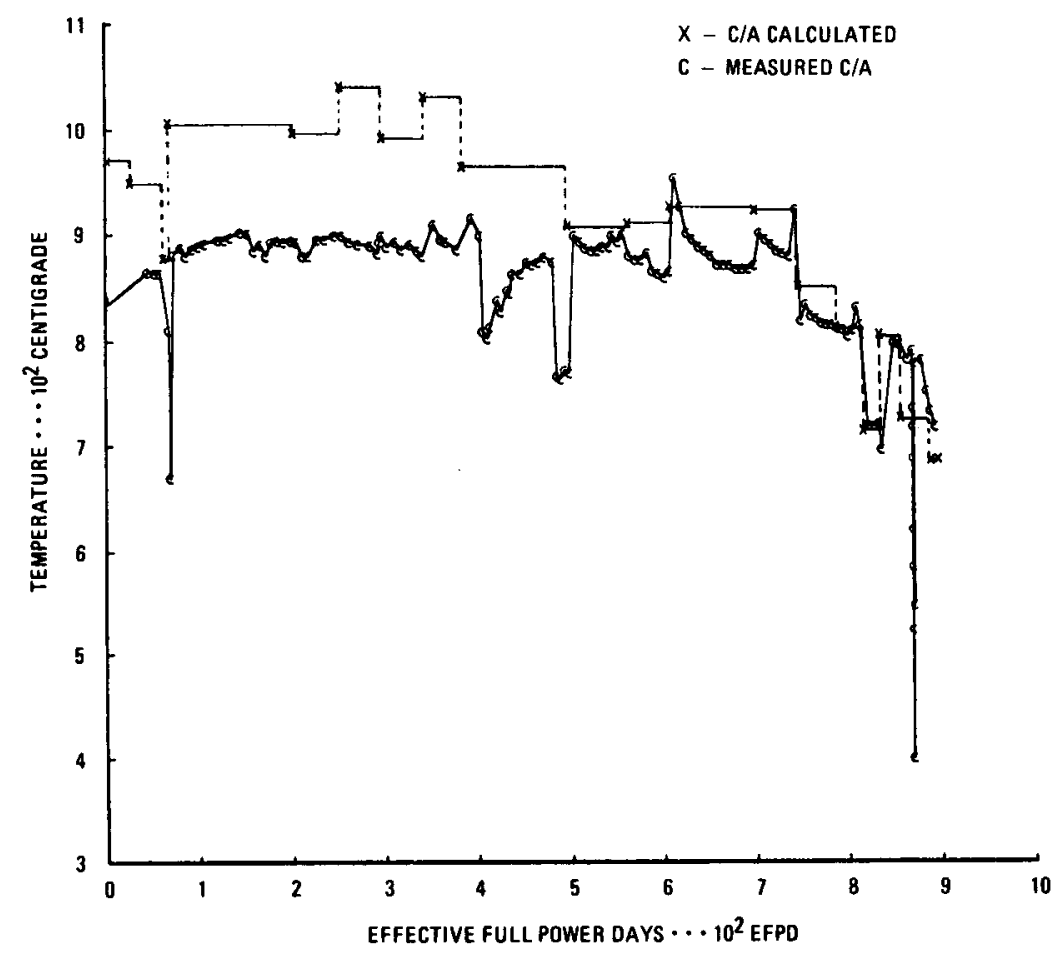

Fig. 4-25. Comparison of predicted temperatures with A14-14 C/A thermocouple readings 
TABLE $4-1$

TREVER TIME POINTS

\begin{tabular}{c|c|c}
\hline $\begin{array}{c}\text { Time } \\
\text { Point }\end{array}$ & $\begin{array}{c}\text { Effective Fu11 } \\
\text { Power Days(a) }\end{array}$ & $\begin{array}{c}\text { Operating } \\
\text { Days }(\mathrm{b})\end{array}$ \\
\hline 1 & 0.0 & 0.0 \\
2 & 26.99 & 28.84 \\
3 & 62.71 & 67.68 \\
4 & 68.57 & 75.18 \\
5 & 202.06 & 216.12 \\
6 & 252.41 & 269.74 \\
7 & 252.41 & 269.74 \\
8 & 298.00 & 318.73 \\
9 & 342.95 & 368.92 \\
10 & 385.44 & 413.75 \\
11 & 385.44 & 413.75 \\
12 & 499.59 & 555.68 \\
13 & 564.07 & 628.37 \\
14 & 610.25 & 678.00 \\
15 & 701.23 & 776.73 \\
16 & 701.23 & 776.73 \\
17 & 748.00 & 827.47 \\
18 & 788.00 & 877.47 \\
19 & 818.00 & 916.67 \\
20 & 835.00 & 946.67 \\
21 & 858.00 & 975.67 \\
22 & 889.70 & 1022.67 \\
23 & 897.00 & 1034.67 \\
\hline
\end{tabular}

(a) An effective full power day (EFPD) is defined as 1 equivalent day of reactor operation at $115 \mathrm{MW}(\mathrm{t})$.

(b) Operating days are defined as calendar days minus periods of reactor shutdown. 
TABLE 4-2

SUMMARY OF MATERIAL PROPERTY DATA USED IN TREVER ANALYSIS

\begin{tabular}{|c|c|c|}
\hline Material Property & Source of Data & Comments \\
\hline \multicolumn{3}{|l|}{ Conductivity } \\
\hline $\begin{array}{l}\text { Sleeve (H-381) } \\
\text { graphite) }\end{array}$ & $\begin{array}{l}\text { Method: Ref. } 17 \text {, Section } 8.5 .3 \\
\text { Constants: Ref. 18, Table } 5-3\end{array}$ & $\begin{array}{l}\text { Assumed to be } \\
\text { the same as } \\
\text { H-327 graphite }\end{array}$ \\
\hline Fuel compact & Ref. 19 & $\begin{array}{l}\text { Assumed to be } \\
\text { a constant } \\
19 \mathrm{~W} / \mathrm{m} \cdot \mathrm{K}\end{array}$ \\
\hline \multicolumn{3}{|l|}{ Irradiation strain } \\
\hline Sleeve & Ref. 20, Fig. 3 (1) & \\
\hline Fuel Compact & $\begin{array}{l}\text { Method: Ref. } 17 \text {, Section } 8.1 .1 \\
\text { Correlation: See Section } 3.1\end{array}$ & $\begin{array}{l}\text { Correlation based } \\
\text { on PIE metrology } \\
\text { data }\end{array}$ \\
\hline \multicolumn{3}{|l|}{ Thermal strain } \\
\hline Sleeve & Ref. 21 & \\
\hline Fuel compact & Ref. 22 & \\
\hline Fuel performance & Ref. 23 & \\
\hline
\end{tabular}


TABLE 4-3

THERMOCOUPLE PERFORMANCE

\begin{tabular}{l|c|c}
\hline & $\begin{array}{c}\text { Number of Thermocouples } \\
\text { with Specified } \\
\text { Lifetime }\end{array}$ \\
\cline { 2 - 3 } Lifetime of Thermocouple & W/Re & C/A \\
\hline O EFPD (BOL failures) & 0 & 7 \\
<2 EFPD & 2 & 2 \\
<150 EFPD & 3 & 3 \\
150 <t> 397 EFPD (EOL) & 4 & 4 \\
BOL - EOL & 2 & 7 (a) \\
\hline $\begin{array}{l}\text { Average 1ifetime (\% of } \\
\text { full irradiation exposure) }\end{array}$ & $47 \%$ & $53 \%$ \\
\hline
\end{tabular}

(a) Includes the $\mathrm{C} / \mathrm{A}$ thermocouple in E02-01 that was irradiated for 252 EFPD and the C/A thermocouple in E06-01 that was irradiated for 385 EFPD.

(b) Based on the 27 thermocouples which gave at least one reliable reading. The seven thermocouples that were unreliable from BOL are assumed to have failed due to faulty assembly and are therefore not included in the thermocouple performance statement. 
TABLE 4-4

SUMMARY OF PEACH BOTTOM CORE 2 THERMOCOUPLE PERFORMANCE

\begin{tabular}{|c|c|c|c|c|}
\hline $\begin{array}{l}\text { Driver } \\
\text { Element }\end{array}$ & $\begin{array}{c}\text { Thermocouple } \\
\text { Type }\end{array}$ & $\begin{array}{l}\text { Location of Hot Junction } \\
\text { (Total Core Height) (mm) }\end{array}$ & $\begin{array}{l}\text { Duration of Reliable } \\
\text { Readings (EFPD) }\end{array}$ & Remarks \\
\hline E01-01 & $\begin{array}{l}\mathrm{C} / \mathrm{A} \\
\mathrm{W} / \mathrm{Re}\end{array}$ & $\begin{array}{l}1346.2 \\
2032.0\end{array}$ & $\begin{array}{l}0-134 \\
0-500\end{array}$ & $\begin{array}{l}\text { Complete failure after } 134 \text { EFPD. } \\
\text { Steady decline in recorded temperature } \\
\text { after } 500 \text { EFPD. }\end{array}$ \\
\hline E02-01 & $\begin{array}{l}\mathrm{C} / \mathrm{A} \\
\mathrm{W} / \mathrm{Re}\end{array}$ & $\begin{array}{l}1346.2 \\
2032.0\end{array}$ & $\begin{array}{l}0-252 \\
0-167\end{array}$ & $\begin{array}{l}\text { OK throughout life. EOL at } 252 \text { EFPD. } \\
\text { Sudden drop at } 167 \text { EFPD suggests short } \\
\text { circuit. }\end{array}$ \\
\hline E03-01 & $\begin{array}{l}\mathrm{C} / \mathrm{A} \\
\mathrm{W} / \mathrm{Re}\end{array}$ & $\begin{array}{l}1346.2 \\
2032.0\end{array}$ & $\begin{array}{l}0-377 \\
0-341\end{array}$ & $\begin{array}{l}\text { Complete failure after } 377 \text { EFPD. } \\
\text { Erratic after } 341 \text { EFPD and complete } \\
\text { failure at } 450 \text { EFPD. }\end{array}$ \\
\hline E05-01 & $W / \operatorname{Re}$ & 2032.0 & $0-613$ & $\begin{array}{l}\text { Complete failure after } 613 \text { EFPD. Last } \\
\text { recorded temperature of } 1180^{\circ} \mathrm{C} \text { sug- } \\
\text { gests failure due to excessive tem- } \\
\text { perature. } \\
\text { Complete failure after } 499 \mathrm{EFPD} \text {. }\end{array}$ \\
\hline Ł06-01 & $\begin{array}{l}\mathrm{C} / \mathrm{A} \\
\mathrm{W} / \mathrm{Re}\end{array}$ & $\begin{array}{l}1346.2 \\
2032.0\end{array}$ & $\begin{array}{l}0-385 \\
0\end{array}$ & $\begin{array}{l}\text { OK throughout life. EOL at } 385 \text { EFPD. } \\
\text { Functional but reliability is } \\
\text { questionable. Appears to have short } \\
\text { circuited at BOL. }\end{array}$ \\
\hline E07-01 & $\mathrm{C} / \mathrm{A}$ & $\begin{array}{l}1346.2 \\
2032.0\end{array}$ & 0 & $\begin{array}{l}\text { Functional but readings are clearly } \\
\text { too low. BOL failure. } \\
\text { Functional but erratic after } 558 \text { EFPD. }\end{array}$ \\
\hline E09-01 & $\mathrm{C} / \mathrm{A}$ & $\begin{array}{l}1346.2 \\
2032.0\end{array}$ & $\begin{array}{l}0 \\
0-879\end{array}$ & $\begin{array}{l}\text { Functional but readings clearly too } \\
\text { low BOL failure. } \\
\text { OK throughout life. }\end{array}$ \\
\hline $\mathrm{E} 11-01$ & $\begin{array}{l}\mathrm{C} / \mathrm{A} \\
\mathrm{W} / \mathrm{Re}\end{array}$ & $\begin{array}{l}1346.2 \\
2032.0\end{array}$ & $\begin{array}{l}0 \\
0-897\end{array}$ & $\begin{array}{l}\text { Apparent short circuit shortly after BOL. } \\
\text { OK throughout life. }\end{array}$ \\
\hline E13-01 & $\begin{array}{l}\mathrm{C} / \mathrm{A} \\
\mathrm{W} / \mathrm{Re}\end{array}$ & $\begin{array}{l}1346.2 \\
2032.0\end{array}$ & $\begin{array}{l}0-897 \\
0-100\end{array}$ & $\begin{array}{l}\text { OK throughout life. } \\
\text { Functional but appears to have short } \\
\text { circuited at } 100 \text { EFPD. }\end{array}$ \\
\hline
\end{tabular}


TABLE 4-4 (continued)

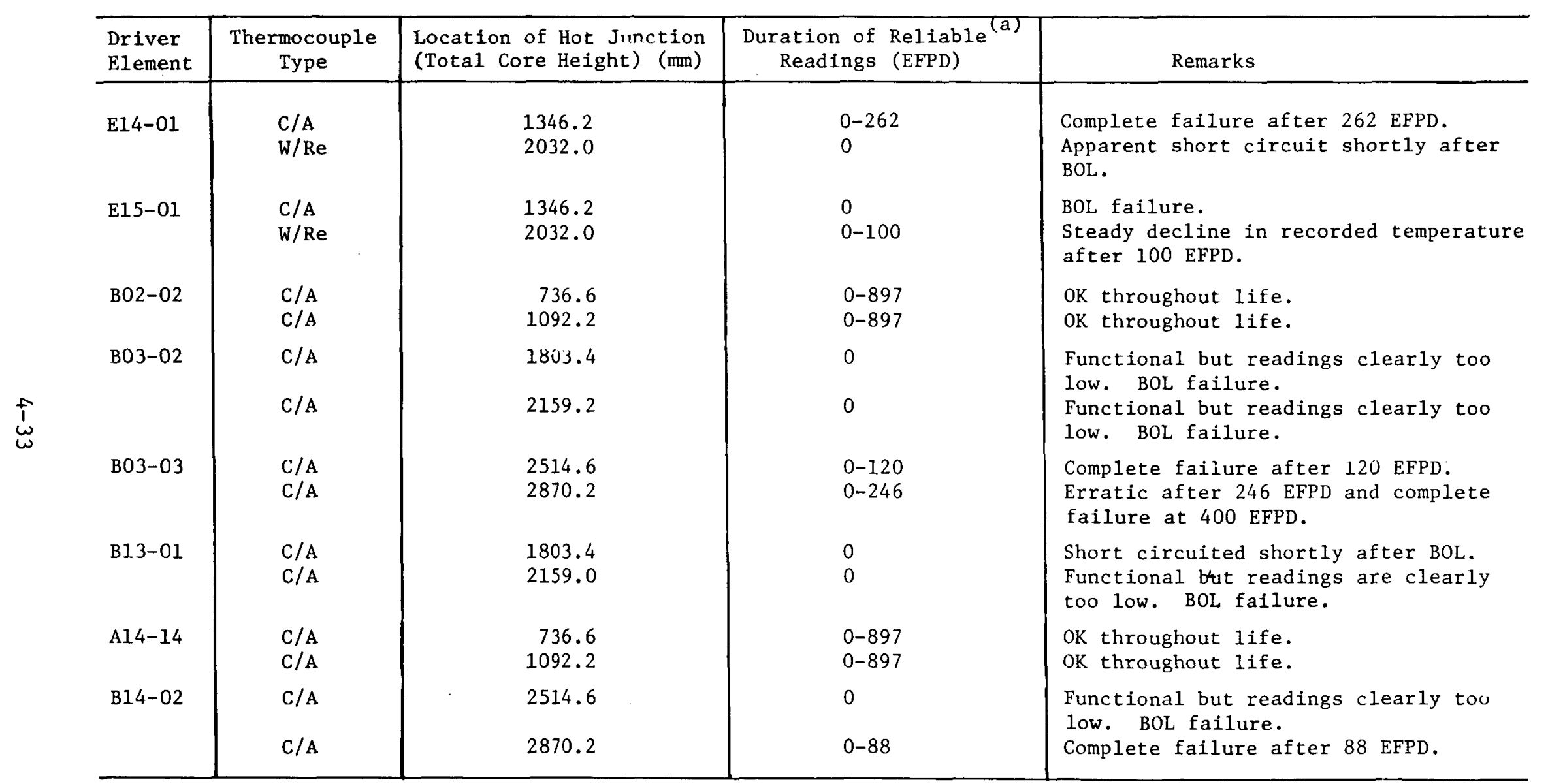

(a) Reliable readings are defined as readings made at the axial location where the thermocouple hot junction was supposed to be located. 
TABLE 4-5

E01-01 COMPARISON OF TREVER AND C/A THERMOCOUPLE TEMPERATURES

TREVER SIPIILATION

THEAMOCOUPLE DATA

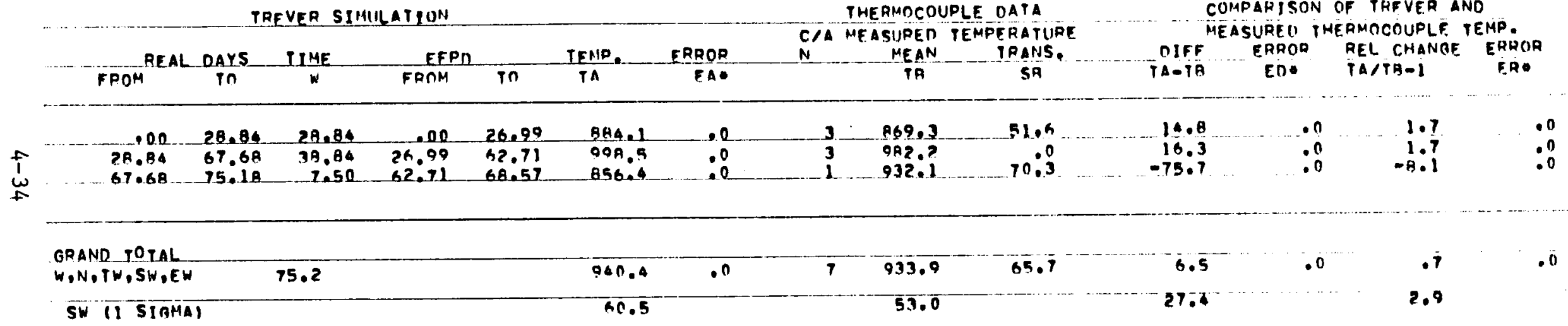


TABLE 4-6

E01-01 COMPARISON OF TREVER AND W/Re THERMOCOUPLE TEMPERATURES

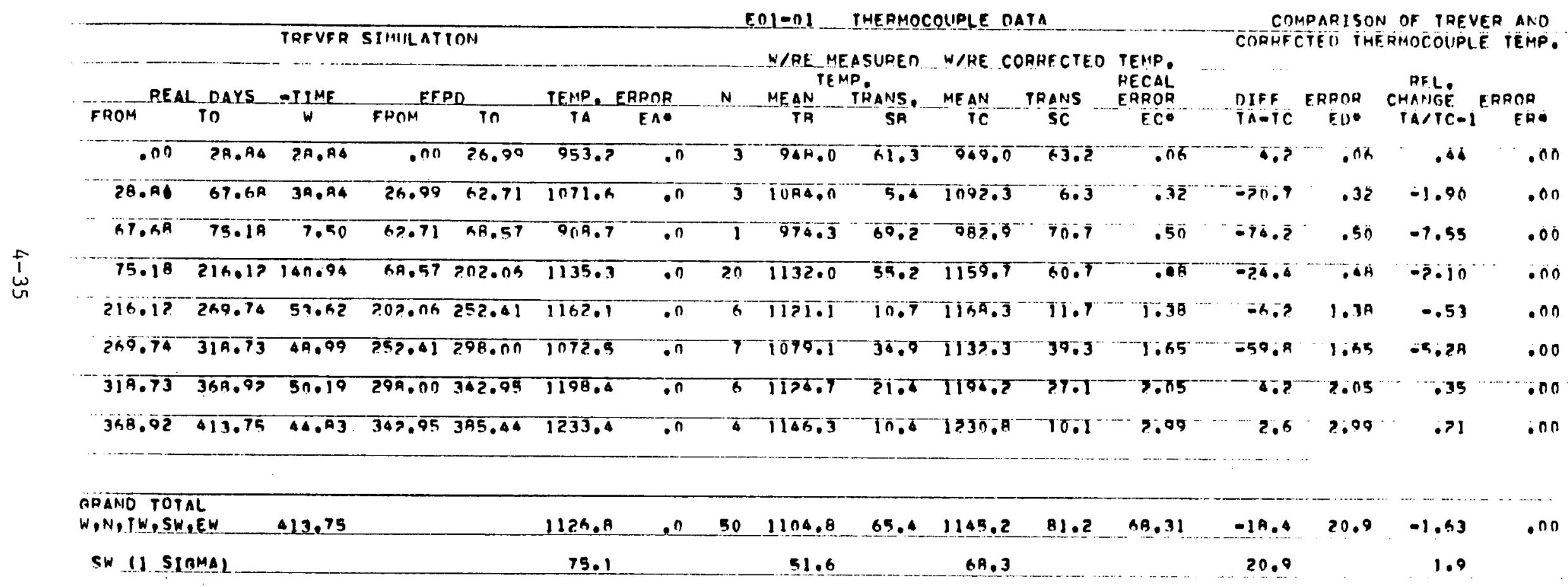


TABLE $4-7$

E02-02 COMPARISON OF TREVER AND C/A THERMOCOUPLE TEMPERATURES

TRFVER SIMULATION

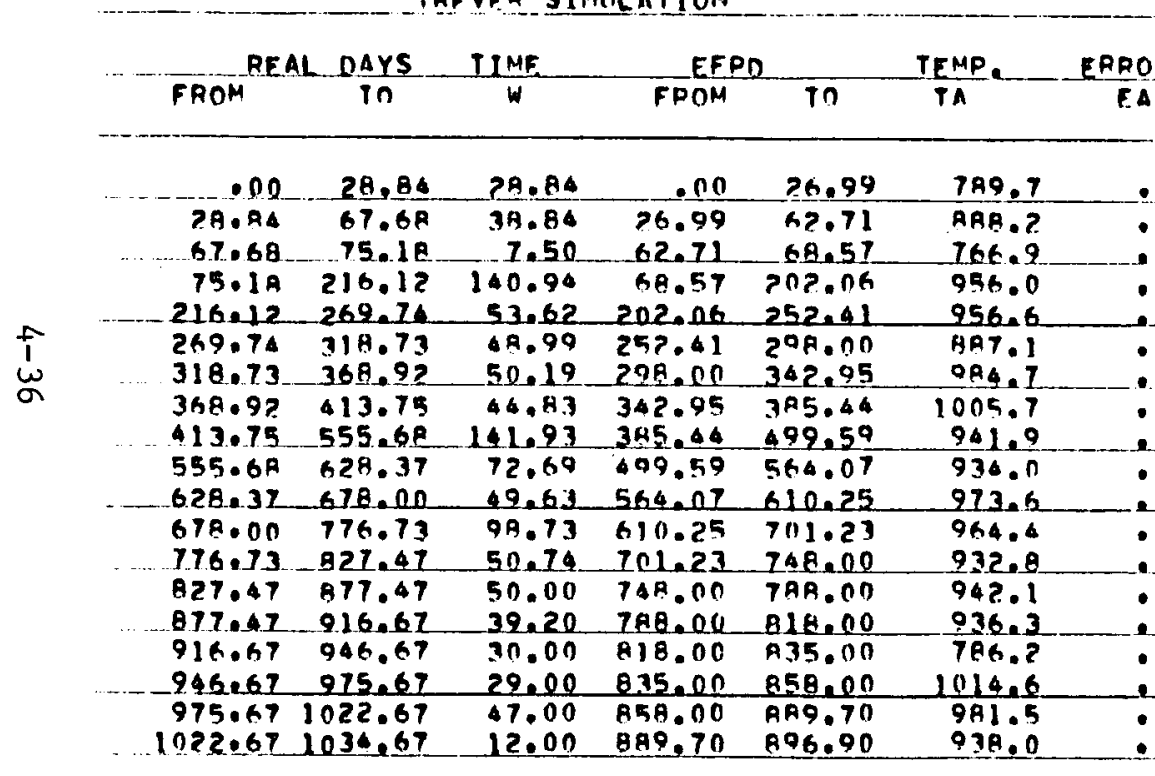

THERMOCOUPLE DATA

C/A MEASURE $n$ TEMPERATURE

EAO N TH MEAN TRANIS

COMPARISON OF TREVER AND

MEASUAF D THERMOCOUPLE TEMP OIFF EPNOR REL CHANGE ERROR
TA-TA
EOD

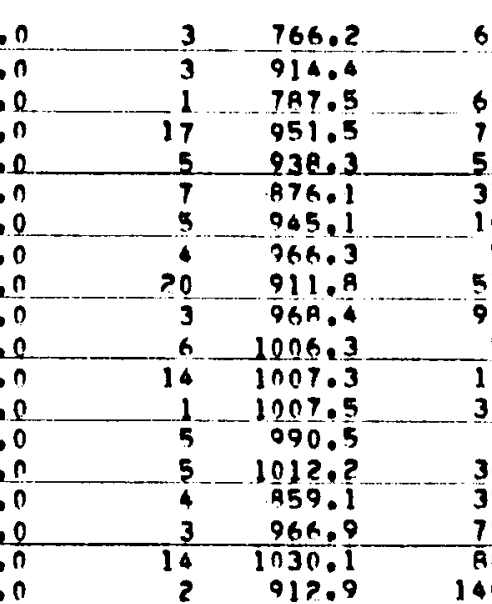

62.5

$23 \cdot 5$
-26.2
-20.6

-26.2
-20.6

1.4 $7.0-0.5$

$2.3+19.3$

(16.

5.9

17.3

11.0

39.6

30.4 $.1+3 \frac{34.6}{4}$ $5.4 \quad-32.7$ $3.0-42.9$ $30.5 \quad-74.7$ $7.1-4 \overline{8} \cdot \frac{7}{4}$ $34.9--75.9$ -12.9
-12.7 $=\frac{47 \cdot \frac{7}{4}}{2}$ $46.0 \quad 25.1$ ED TA/IRAI ER.

GRANO TOTAL

W.N.TW.SW,EW

1034.7

941.0

.0

$12 . \overline{2}$

951.3

$7 \overline{9} 3$

$-10.3$

36.5

$-1.0$ 
TABLE 4-8

E02-01 COMPARISON OF TREVER AND C/A THERMOCOUPLE TEMPERATURES

TREVFR SIMUILATION

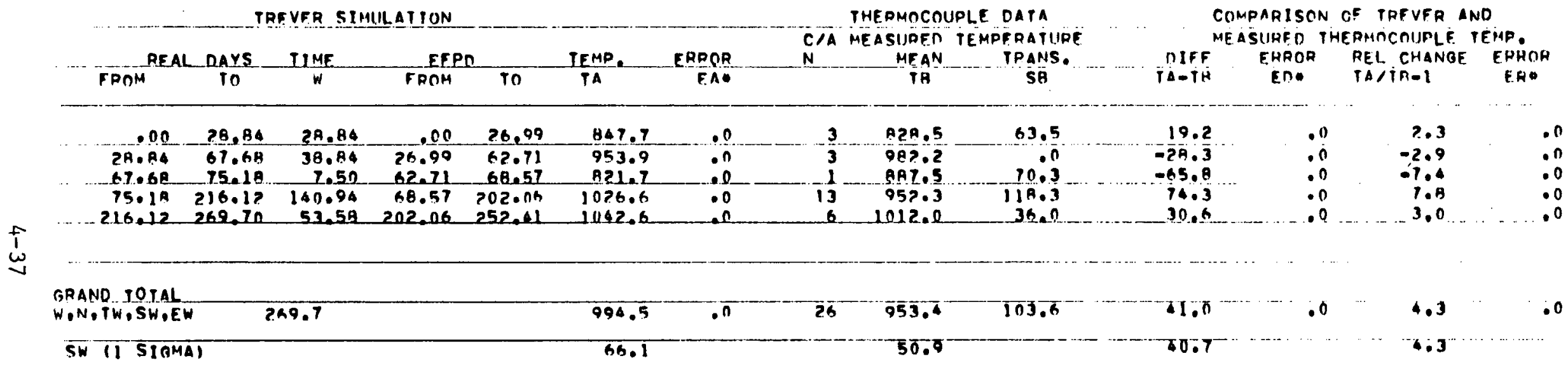


TABLE 4-9

E02-01 COMPARISON OF TREVER AND W/Re THERMOCOUPLE TEMPERATURES

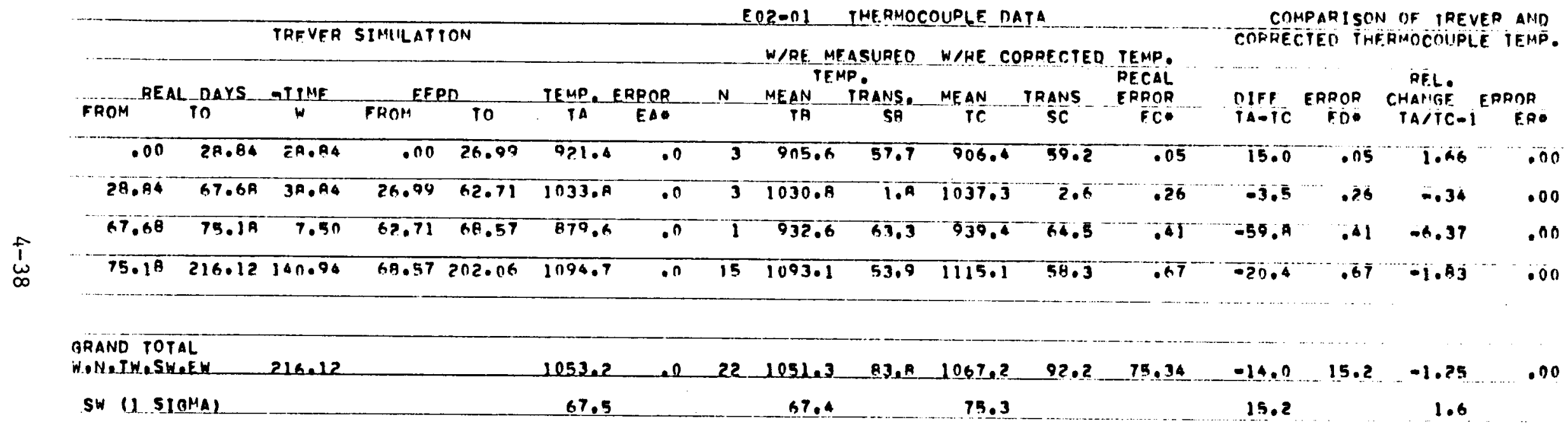


TABLE $\quad 4-10$

B03-03 COMPARISON OF TREVER AND C/A THERMOCOUPLE TEMPERATURES

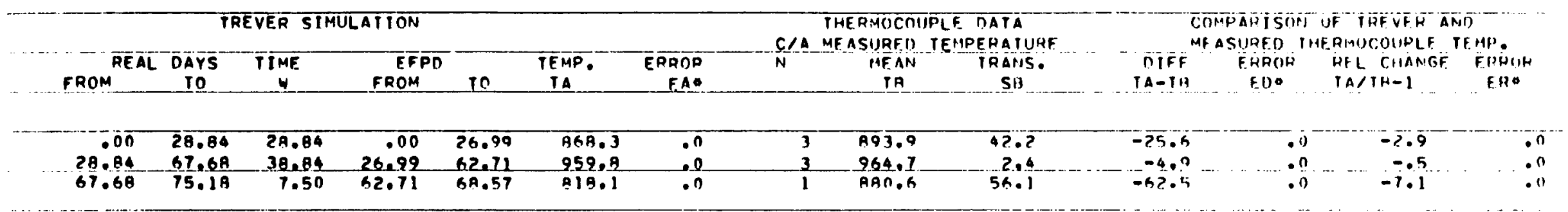

GĀAND TOTAL

W,N,TW, SWEEW

75.2

910.6

1929.2

4.

$-18.6$

$-2 \cdot 1$

(1)

SW (1 SIOMA)

$5 ? .8$

36.9

_ 17.6

$2 . ?$ 
TABLE $4-11$

E03-01 COMPARISON OF TREVER AND C/A THERMOCOUPLE TEMPERATURES

TREYFA SIMULAIION

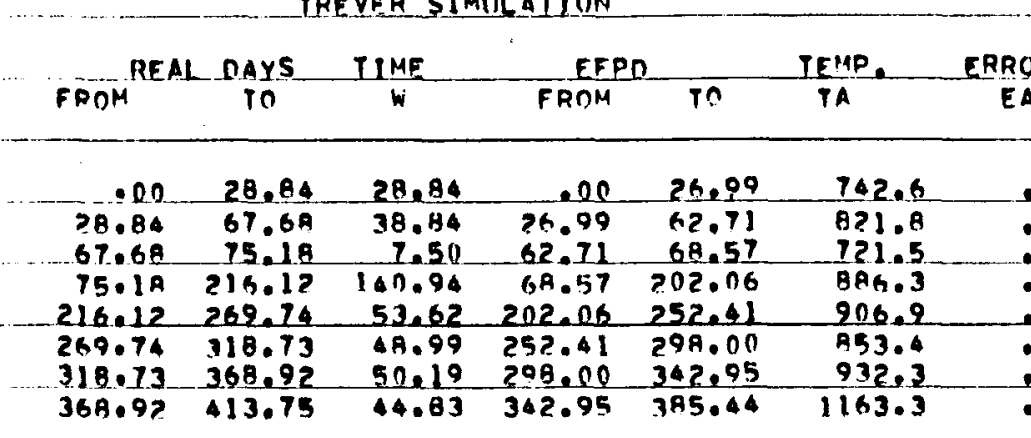

THEPMOCOUPLE DATA C/A MEASURED TEMPERATURE N N MEAN TRANS.

S̄

$\begin{array}{ll}0 & 3 \\ 0 & 3 \\ 0 & 1 \\ 0 & 10 \\ 0 & 6 \\ 0 & ? \\ .0 & 6\end{array}$
863.4 7 790. 917.2 932,6 917.6 $957 \cdot 0$ $1067 \cdot 6$

-

$50 \cdot 3$

57.6
57.6 4.5
15.0

4 ค. 3

$2 \frac{3}{3} \cdot \frac{3}{0}$

TIFF
TAOTAB
COMFARISON OF TREVER AND MEASUREO THERMOCOUPLE TEMP.
ERROR REL CHANEF ERROR TA-TA FIO TA/THAI

BRAND TOTAL

W.N.TW,SW.EW

413.7

901.6

49

920.8

A3.?

$-41.6$

-59.3
-30.9

$-25.7$

SW (1 SIOMA)

104.1

73.2

42.7

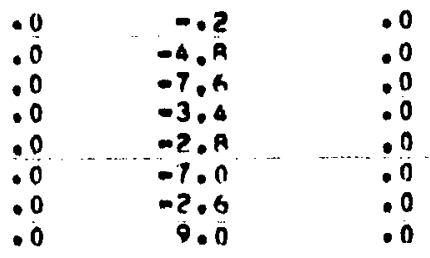


TABLE $4-12$

E03-01 COMPARISON OF TREVER AND W/Re THERMOCOUPLE TEMPERATURES

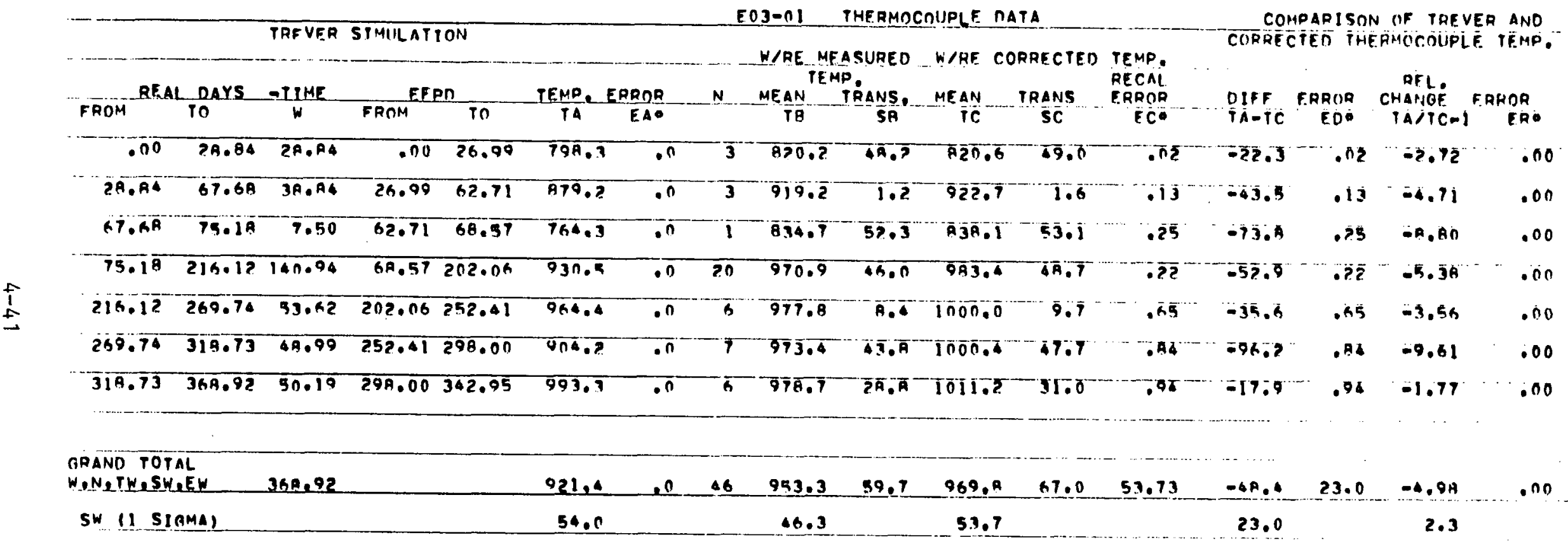


TABLE $4-13$

E05-01 COMPARISON OF TREVER AND C/A THERMOCOUPLE TEMPERATURES

TRFVER SIMULATION

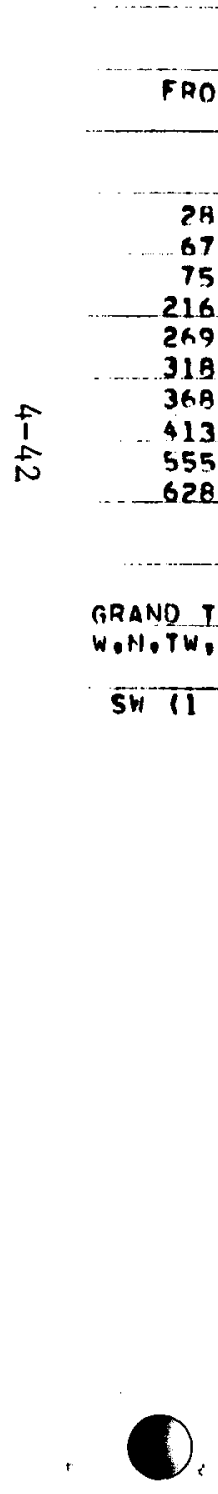
PFAL DAYS IIME

$\begin{array}{rrr}28.84 & 28.84 & 28.84 \\ 67.69 & 39.84\end{array}$
THERMOCOUPLE DATA C/A MEASURED TEMPERATLIAF.

IO $W$ FROM TO TA
SB

39.7

$\begin{array}{llllll}39.84 & 26.99 & 62.71 & 763.6\end{array}$

$68.57 \quad 68.57 \quad 698.9$

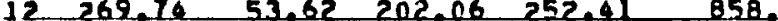

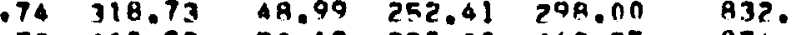

$369.92 \quad 50.19208 .0034205$

$\begin{array}{lllll} & 13.75 & 44.83 & 342.95 & 345.44\end{array}$

$\begin{array}{rrrr}141.93 & 395.44 & 499.59 & 1072.0\end{array}$

976.8

0
0
0
0
0
0
0
0
0
0
0

3
3
1
19
6
7
6
20
10

731.7
$91 \geq .5$
744.9
957.9

39.7
.7
.1 .0

A57.1

A8.4. 1

990.6

029.0

1025.7

$106 ? \cdot 5$

997.0
1036.8

4.0
41.0

41.9

18.2

37.6

$35 \cdot 6$

57.1

26.4

$9 \overline{14.1} .0$

ค 7

944.1

111.0

.0

3.7
$-30.1$

$-3.8$

COMPARISON OF TAEVER ANO MFASIJPFD THERMOCOUPLE TEMT. DIFF FARUP PEI CHANGE ERROR TAETA FDE TA/IR-I ER.

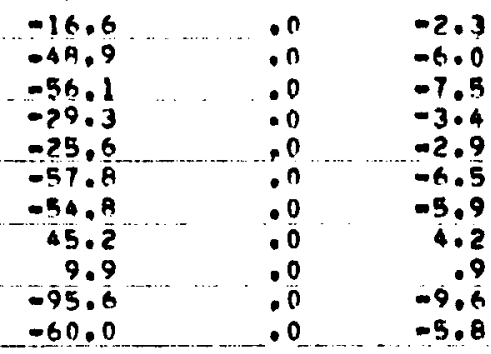


TABLE 4-14

E05-01 COMPARISON OF TREVER AND W/Re THERMOCOUPLE TEMPERATURES

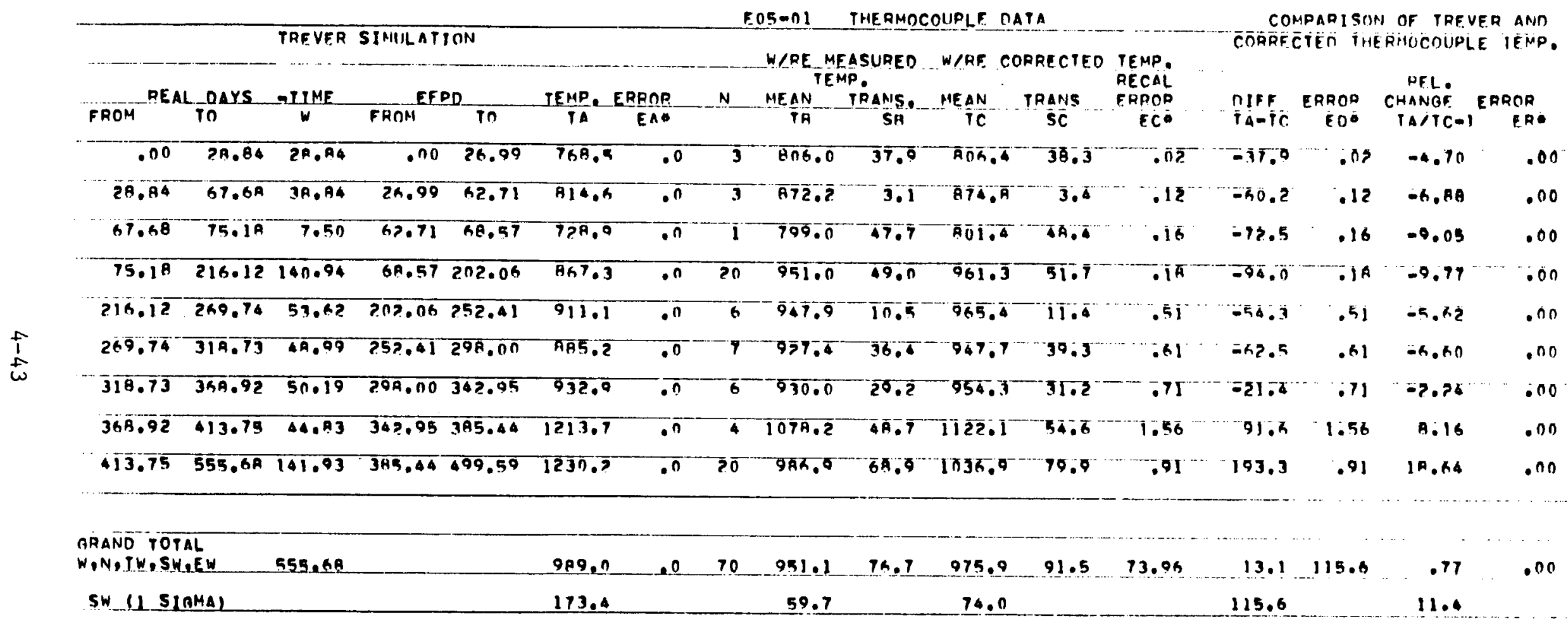


TABLE 4-15

E06-01 COMPARISON OF TREVER AND C/A THERMOCOUPLE TEMPERATURES

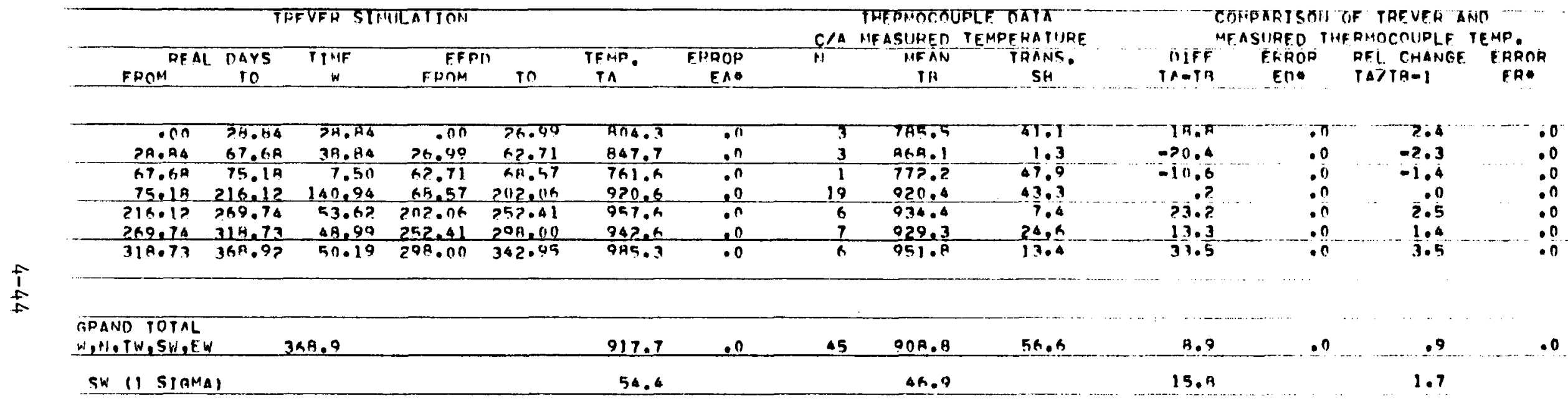


TABLE 4-16

E07-01 COMPARISON OF TREVER AND W/Re THERMOCOUPLE TEMPERATURES

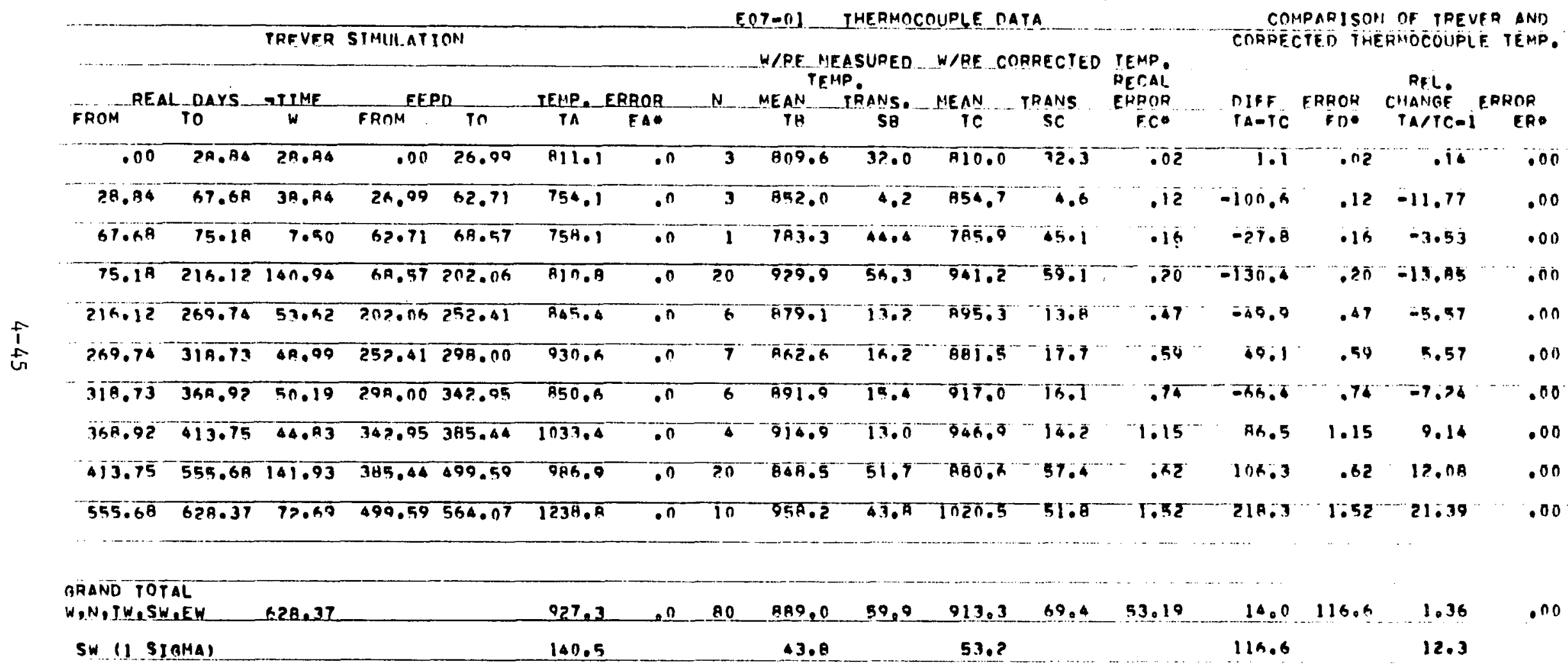


TABLE $4-17$

E09-01 COMPARISON OF TREVER AND W/Re THERMOCOUPLE TEMPERATURES

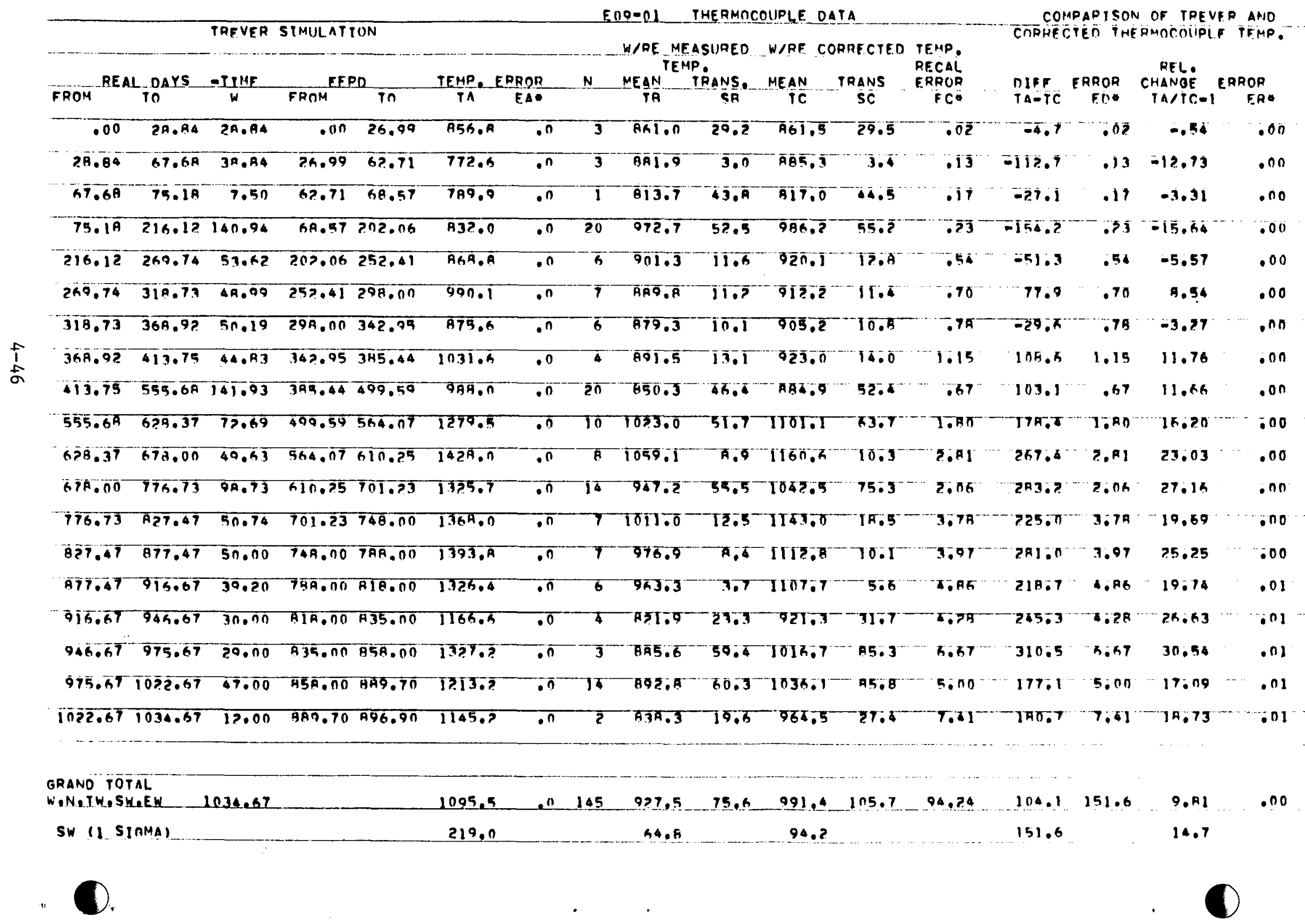


TABLE 4-18

E11-01 COMPARISON OF TREVER AND $W /$ Re THERMOCOUPLE TEMPERATURES

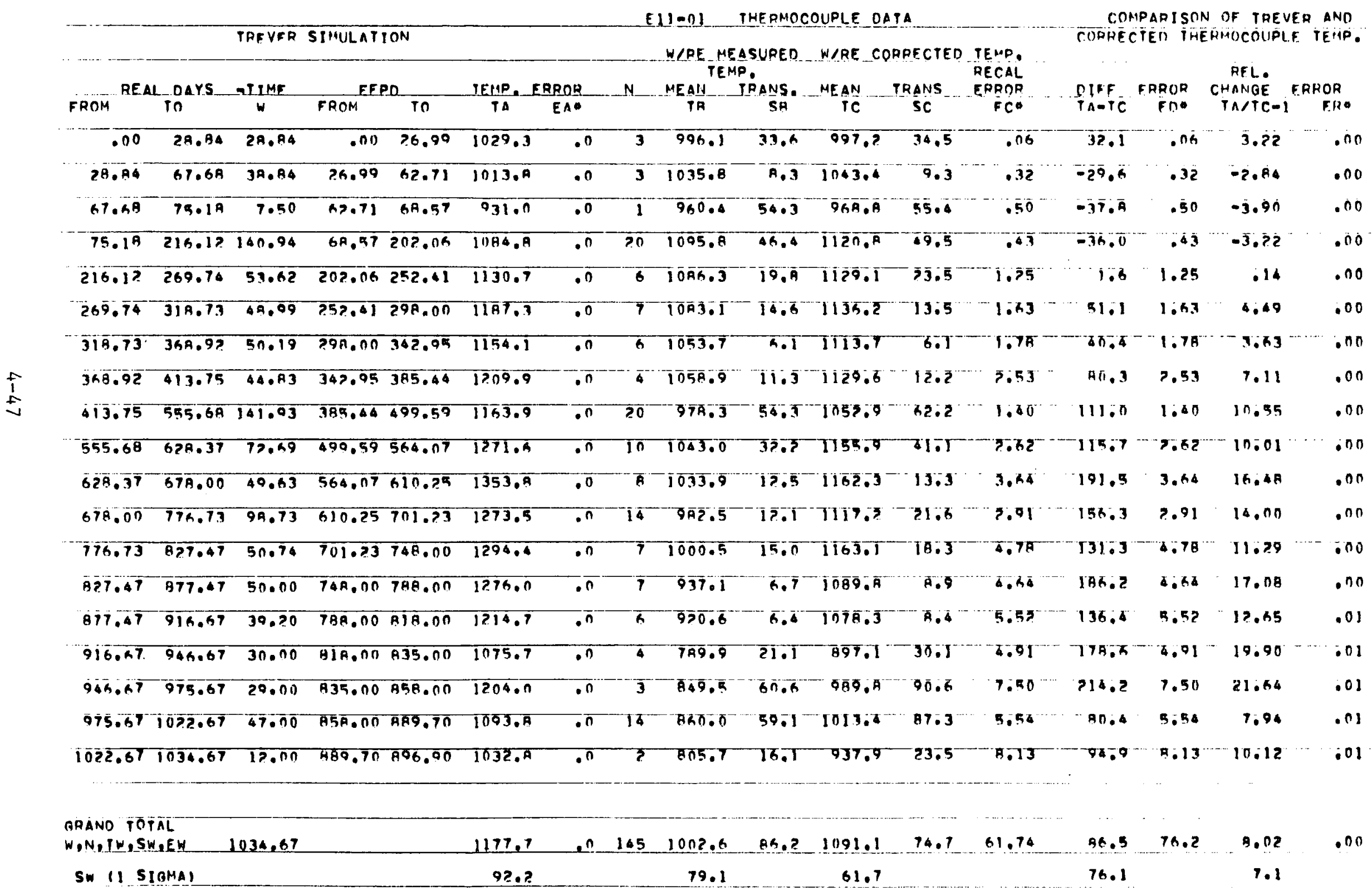


TABLE 4-19

E13-01 COMPARISON OF TREVER AND C/A THERMOCOUPLE TEMPERATURES

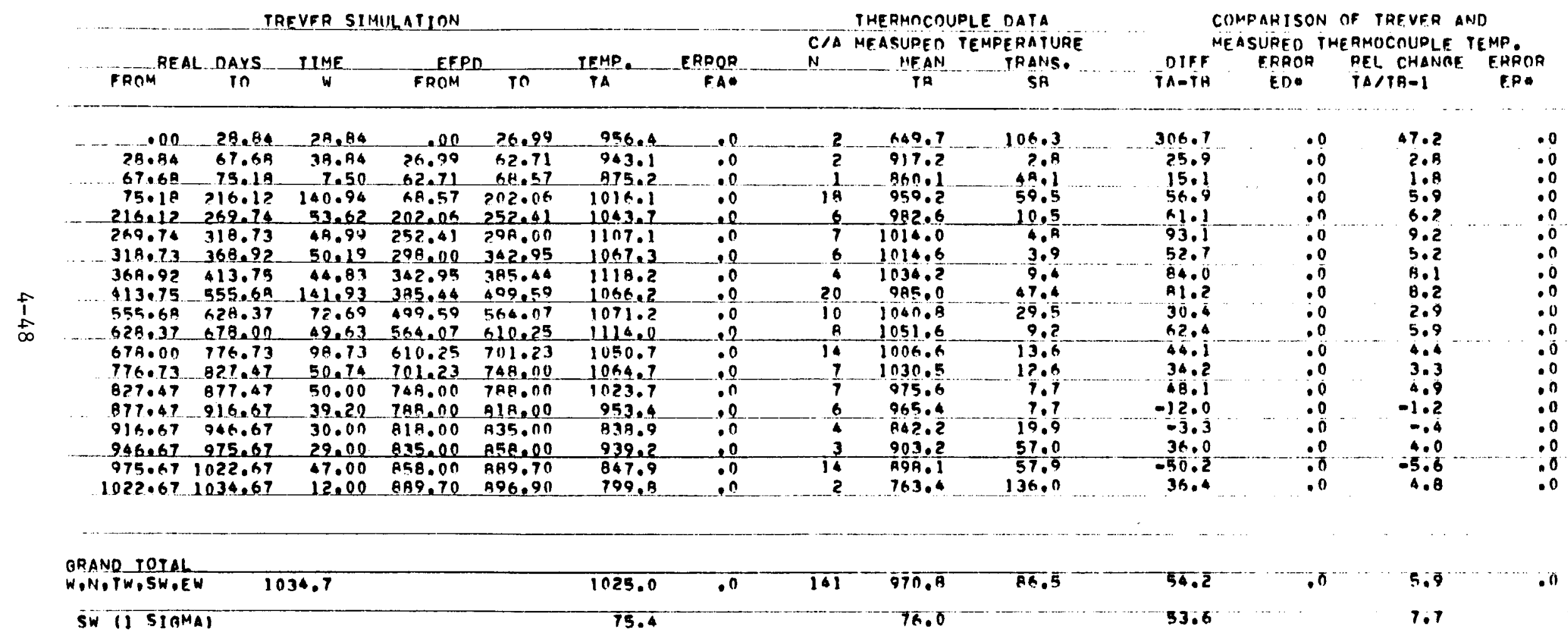


TABLE $4-20$

E13-01 COMPARISON OF TREVER AND W/Re THERMOCOUPLE TEMPERATURES

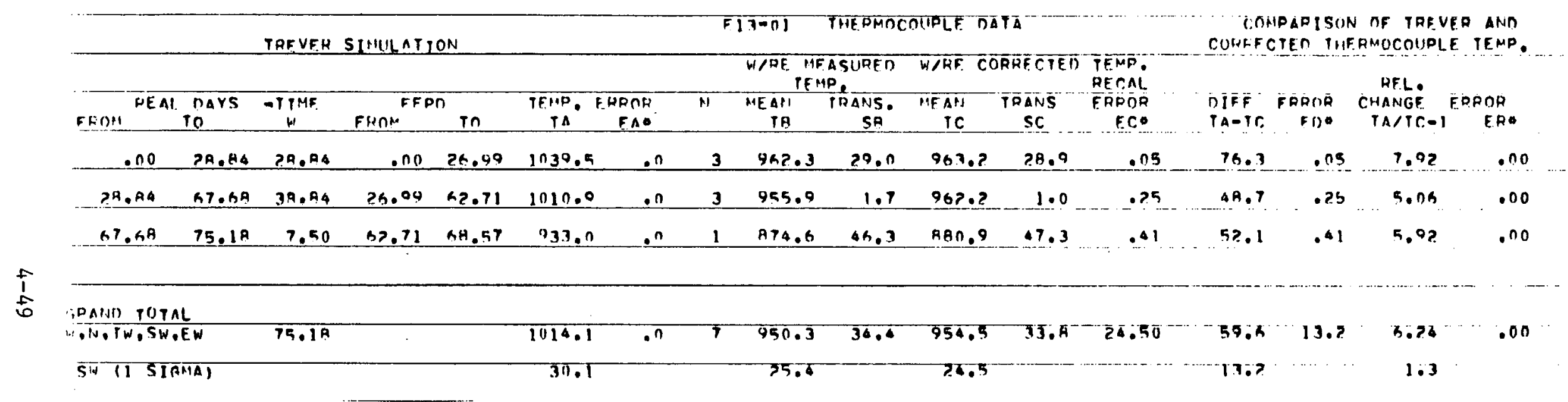


TABLE 4-21

E14-01 COMPARISON OF TREVER AND C/A THERMOCOUPLE TEMPERATURES

\begin{tabular}{|c|c|c|c|c|c|c|c|c|c|c|c|c|}
\hline \multicolumn{6}{|c|}{ 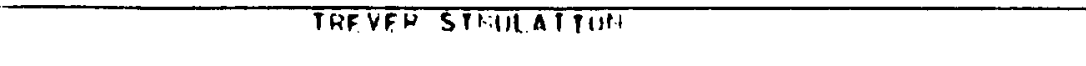 } & \multicolumn{3}{|c|}{ 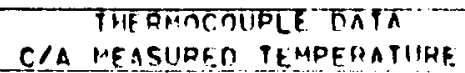 } & \multicolumn{4}{|c|}{ 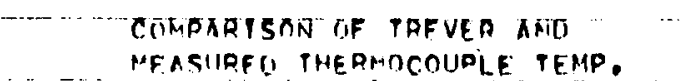 } \\
\hline $\begin{array}{l}\text { RFAL DAYS } \\
\text { FROII II } \\
\end{array}$ & $\begin{array}{c}T T: F \\
w \\
\end{array}$ & $\begin{array}{l}\text { FFFE } \\
\text { FPOM }\end{array}$ & in & TFMP. & $\begin{aligned} \text { FRPOP } \\
\text { FAQ }\end{aligned}$ & N & $\begin{array}{l}\text { ME } \\
\text { IA }\end{array}$ & $\begin{array}{l}\text { Thans. } \\
\text { Sh }\end{array}$ & $\begin{array}{l}\text { NIFH } \\
\text { TA-TH }\end{array}$ & $\begin{array}{l}\text { FHHOD } \\
\text { EN" }\end{array}$ & $\begin{array}{l}\text { HE THANAGF } \\
\text { TATTH=1 }\end{array}$ & $\begin{array}{l}\text { ERPOP } \\
\text { ERP }\end{array}$ \\
\hline $\begin{array}{rr}.01 & 79.44 \\
29.84 & 67.69 \\
97.60 & 75.19 \\
75.19 & 215.17 \\
216.17 & 269.74\end{array}$ & $\begin{array}{r}79.40 \\
38.84 \\
7.50 \\
140.44 \\
53.67\end{array}$ & $\begin{array}{r}.04 \\
28.99 \\
2.71 \\
28.57 \\
2112.164\end{array}$ & $\begin{array}{l}26.49 \\
62.71 \\
64.57 \\
208.06 \\
752.41\end{array}$ & $\begin{array}{l}969.9 \\
933,3 \\
84 . .5 \\
909.5 \\
1031.0\end{array}$ & $\begin{array}{l}.0 \\
.0 \\
.0 \\
.0 \\
.0\end{array}$ & $\begin{array}{r}2 \\
2 \\
11 \\
19 \\
4\end{array}$ & 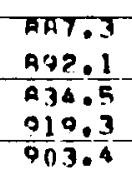 & $\begin{array}{r}1.5 \\
1.5 \\
45.9 \\
34.0 \\
7.5\end{array}$ & $\begin{array}{r}23.4 \\
41.2 \\
36.0 \\
80.3 \\
179.6\end{array}$ & $\begin{array}{l}.0 \\
: n \\
: 0 \\
: 0\end{array}$ & $\begin{array}{r}7.0 \\
4.5 \\
4.1 \\
4.7 \\
14.1\end{array}$ & $\begin{array}{l}: 0 \\
.0 \\
.0 \\
.0 \\
.0\end{array}$ \\
\hline 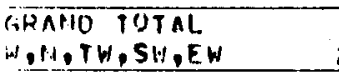 & 209.7 & & & 997.3 & .0 & 30 & 906.4 & 31.7 & Ro. 9 & .0 & R.9 & .0 \\
\hline SW (1 SIGNA) & & & & 37.7 & & & 17.3 & & 27.5 & & 3.0 & \\
\hline
\end{tabular}


TABLE $4-22$

E15-01 COMPARISON OF TREVER AND W/Re THERMOCOUPLE DATA

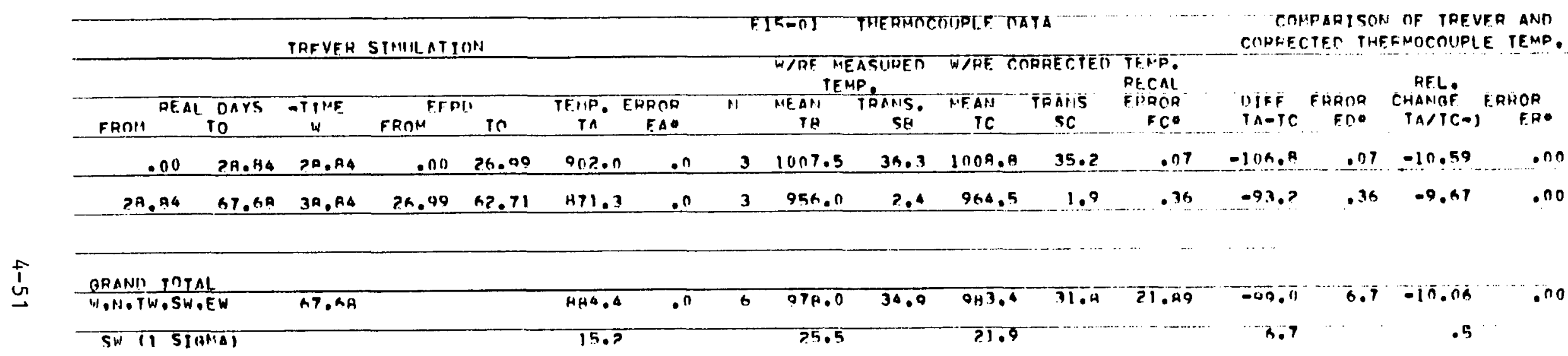


TABLE 4-23

A14-14 COMPARISON OF TREVER AND C/A THERMOCOUPLE TEMPERATURES

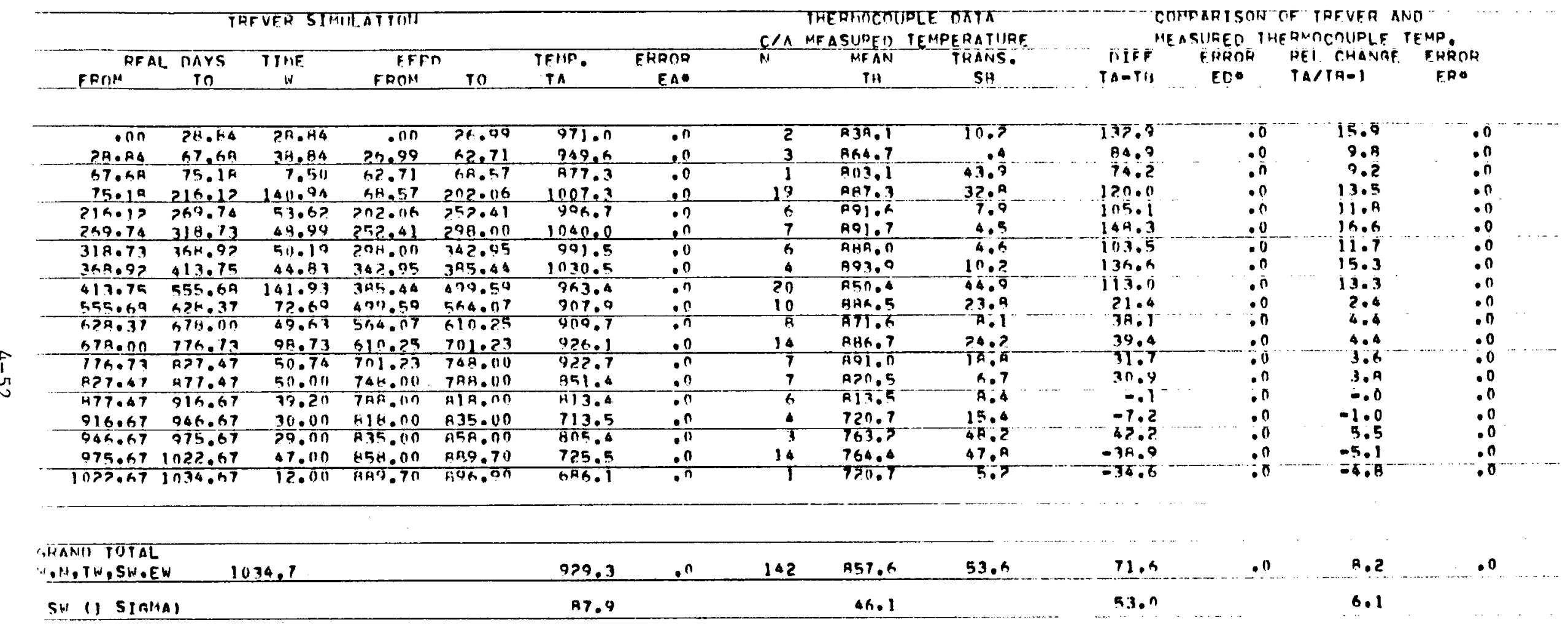


TABLE 4-24

SUMMARY OF AGREEMENT BETWEEN MEASURED AND PREDICTED TEMPERATURES FOR PEACH BOTTOM CORE 2 (FUEL ELEMENTS IN RINGS 1 THROUGH 6)

\begin{tabular}{|c|c|c|c|c|c|c|c|c|c|c|c|c|c|c|}
\hline \multirow{3}{*}{$\begin{array}{c}\text { Time } \\
\text { Interval } \\
\text { (EFPD) }\end{array}$} & \multicolumn{14}{|c|}{ Predicted Temperature - Measured Temperature $\left({ }^{\circ} \mathrm{C}\right)$} \\
\hline & \multicolumn{2}{|c|}{ E01-01 } & \multirow{2}{*}{$\frac{\mathrm{BO} 2-02}{\mathrm{C} / \mathrm{A} 2}$} & \multicolumn{2}{|c|}{$\mathrm{E} 02-01$} & \multirow{2}{*}{$\frac{\mathrm{B} 03-03}{\mathrm{C} / \mathrm{Al}}$} & \multicolumn{2}{|c|}{ E03-01 } & \multicolumn{2}{|c|}{$\mathrm{E} 05-01$} & \multirow{2}{*}{\begin{tabular}{|c|} 
E06-01 \\
C/A \\
\end{tabular}} & \multicolumn{3}{|c|}{ Average } \\
\hline & $\mathrm{W} / \mathrm{Re}$ & $\mathrm{C} / \mathrm{A}$ & & $\mathrm{W} / \mathrm{Re}$ & $\mathrm{C} / \mathrm{A}$ & & $\mathrm{W} / \operatorname{Re}$ & $\mathrm{C} / \mathrm{A}$ & $\mathrm{W} / \mathrm{Re}$ & $\mathrm{C} / \mathrm{A}$ & & $\begin{array}{l}\text { No. of } \\
\text { Meas. }\end{array}$ & Mean & RMS \\
\hline $\begin{array}{c}0-26.99 \\
26.99-62.71 \\
62.71-68.57 \\
68.57-202.06 \\
202.06-252.41\end{array}$ & $\begin{array}{r}4 \\
-21 \\
-74 \\
-24 \\
-6\end{array}$ & $\begin{array}{r}15 \\
16 \\
-76\end{array}$ & $\begin{array}{r}23 \\
-26 \\
-21 \\
4 \\
18\end{array}$ & $\begin{array}{r}15 \\
-3 \\
-60 \\
-20\end{array}$ & $\begin{array}{r}19 \\
-28 \\
-66 \\
74 \\
31\end{array}$ & $\begin{array}{r}-26 \\
-5 \\
-62\end{array}$ & $\begin{array}{l}-22 \\
-43 \\
-74 \\
-53 \\
-36\end{array}$ & $\begin{array}{l}-2 \\
-42 \\
-59 \\
-31 \\
-26\end{array}$ & $\begin{array}{l}-38 \\
-60 \\
-72 \\
-94 \\
-54\end{array}$ & $\begin{array}{l}-17 \\
-49 \\
-56 \\
-29 \\
-26\end{array}$ & $\begin{array}{r}19 \\
-20 \\
-11 \\
0 \\
23\end{array}$ & $\begin{array}{r}11 \\
11 \\
11 \\
9 \\
8\end{array}$ & $\begin{array}{r}-1 \\
-26 \\
-57 \\
-19 \\
-9\end{array}$ & $\begin{array}{l} \pm 20 \\
\pm 21 \\
\pm 21 \\
\pm 43 \\
\pm 29\end{array}$ \\
\hline $\begin{array}{l}252.41-298.00 \\
298.00-342.95 \\
342.95-385.44 \\
385.44-499.59 \\
499.59-564.07\end{array}$ & $\begin{array}{r}-60 \\
4 \\
3 \\
76\end{array}$ & & $\begin{array}{r}11 \\
40 \\
39 \\
30 \\
-34\end{array}$ & & & & $\begin{array}{l}-96 \\
-18\end{array}$ & $\begin{array}{r}-64 \\
-25 \\
96\end{array}$ & $\begin{array}{r}-62 \\
-21 \\
92 \\
193\end{array}$ & $\begin{array}{r}-58 \\
-55 \\
45 \\
10 \\
-96\end{array}$ & $\begin{array}{l}13 \\
33\end{array}$ & $\begin{array}{l}7 \\
7 \\
5 \\
4 \\
2\end{array}$ & $\begin{array}{r}-45 \\
-6 \\
55 \\
77 \\
-65\end{array}$ & $\begin{array}{l} \pm 38 \\
\pm 31 \\
\pm 35 \\
\pm 71 \\
\pm 31\end{array}$ \\
\hline $\begin{array}{l}564.07-610.25 \\
610.25-701.23 \\
701.23-748.00 \\
748.00-788.00 \\
788.00-818.00\end{array}$ & & & $\begin{array}{l}-33 \\
-43 \\
-75 \\
-48 \\
-76\end{array}$ & & & & & & & -60 & & $\begin{array}{l}2 \\
1 \\
1 \\
1 \\
1\end{array}$ & $\begin{array}{l}-46 \\
-43 \\
-75 \\
-48 \\
-76\end{array}$ & $\begin{array}{l} \pm 13 \\
\pm 35 \text { (a) } \\
\pm 35 \text { (a) } \\
\pm 35 \text { (a) } \\
\pm 35 \text { (a) }\end{array}$ \\
\hline $\begin{array}{l}818.00-835.00 \\
835.00-858.00 \\
858.00-899.70 \\
889.70-896.90 \\
\end{array}$ & & & $\begin{array}{r}-73 \\
48 \\
-49 \\
25 \\
\end{array}$ & & & & & & & & & $\begin{array}{l}1 \\
1 \\
1 \\
1\end{array}$ & $\begin{array}{r}-73 \\
48 \\
-49 \\
25 \\
\end{array}$ & $\begin{array}{l} \pm 35(a) \\
\pm 35(a) \\
\pm 35(a) \\
\pm 35(a)\end{array}$ \\
\hline $.0-896.90$ & & & & & & & & & & & & 83 & -18 & \pm 49 \\
\hline
\end{tabular}

(a) $\mathrm{RMS}=\left[\sum_{t=1}^{11} \mathrm{RMS}_{\mathrm{t}}^{2} / 11\right]^{1 / 2}=$ average root mean square deviation of the first eleven time intervals. 
TABLE 4-25

SUMMARY OF AGREEMENT BETWEEN MEASURED AND PREDICTED TEMPERATURES FOR PEACH BOTTOM CORE 2 (FUEL ELEMENTS IN RINGS 7 THROUGH 12 AND RINGS 13 THROUGH 15)

\begin{tabular}{|c|c|c|c|c|c|c|c|c|c|c|c|c|c|c|}
\hline \multirow{3}{*}{$\begin{array}{c}\text { Time } \\
\text { Interval } \\
\text { (EFPD) }\end{array}$} & \multicolumn{14}{|c|}{ Predicted Temperature.- Measured Temperature. $\left({ }^{\circ} \mathrm{C}\right)$} \\
\hline & $\mathrm{E} 07-01$ & E09-01 & E11-01 & \multicolumn{3}{|c|}{ Average } & \multicolumn{2}{|c|}{$\mathrm{E} 13-01$} & \multirow{2}{*}{$\begin{array}{l}\mathrm{A} 14-14 \\
\mathrm{C} / \mathrm{A} 2\end{array}$} & \multirow{2}{*}{$\begin{array}{c}E 14-01 \\
C / A\end{array}$} & \multirow{2}{*}{$\begin{array}{c}\mathrm{E} 15-01 \\
\mathrm{~W} / \mathrm{Re}\end{array}$} & \multicolumn{3}{|c|}{ Average } \\
\hline & W/Re & $\mathrm{W} / \mathrm{Re}$ & $W / \operatorname{Re}$ & $\begin{array}{l}\text { No. of } \\
\text { Meas. }\end{array}$ & Mean & RMS & W/Re & $\mathrm{C} / \mathrm{A}$ & & & & $\begin{array}{l}\text { No. of } \\
\text { Meas. }\end{array}$ & Mean & RMS \\
\hline $\begin{array}{c}0-26.99 \\
26.99-62.71 \\
62.71-68.57 \\
68.57-202.06 \\
202.06-252.41\end{array}$ & $\begin{array}{r}1 \\
-101 \\
-28 \\
-130 \\
50\end{array}$ & $\begin{array}{r}-5 \\
-113 \\
-27 \\
-154 \\
-51\end{array}$ & $\begin{array}{r}32 \\
-30 \\
-38 \\
-36 \\
2\end{array}$ & $\begin{array}{l}3 \\
3 \\
3 \\
3 \\
3\end{array}$ & $\begin{array}{r}9 \\
-81 \\
-31 \\
-107 \\
-33\end{array}$ & $\begin{array}{r} \pm 16 \\
\pm 37 \\
\pm 5 \\
\pm 51 \\
\pm 25\end{array}$ & $\begin{array}{l}76 \\
49 \\
52\end{array}$ & $\begin{array}{l}-- \\
26 \\
15 \\
57 \\
61\end{array}$ & $\begin{array}{r}133 \\
85 \\
74 \\
120 \\
105\end{array}$ & $\begin{array}{r}62 \\
41 \\
34 \\
80 \\
129\end{array}$ & $\begin{array}{r}-107 \\
-93\end{array}$ & $\begin{array}{l}4 \\
5 \\
4 \\
3 \\
3\end{array}$ & $\begin{array}{l}41 \\
22 \\
44 \\
86 \\
98\end{array}$ & $\begin{array}{l} \pm 89 \\
\pm 60 \\
\pm 22 \\
\pm 26 \\
\pm 28\end{array}$ \\
\hline $\begin{array}{l}252.41-298.00 \\
298.00-342.95 \\
342.95-385.44 \\
385.44-499.59 \\
499.59-564.07\end{array}$ & $\begin{array}{r}49 \\
-66 \\
86 \\
106 \\
218\end{array}$ & $\begin{array}{r}78 \\
-30 \\
109 \\
103 \\
178\end{array}$ & $\begin{array}{r}51 \\
40 \\
80 \\
111 \\
116\end{array}$ & $\begin{array}{l}3 \\
3 \\
3 \\
3 \\
3\end{array}$ & $\begin{array}{r}59 \\
-19 \\
92 \\
107 \\
171\end{array}$ & $\begin{array}{r} \pm 13 \\
\pm 44 \\
\pm 12 \\
\pm 3 \\
\pm 42\end{array}$ & & $\begin{array}{l}93 \\
53 \\
84 \\
81 \\
30\end{array}$ & $\begin{array}{r}148 \\
103 \\
137 \\
113 \\
21\end{array}$ & & & $\begin{array}{l}2 \\
2 \\
2 \\
2 \\
2\end{array}$ & $\begin{array}{r}120 \\
78 \\
111 \\
97 \\
25\end{array}$ & $\begin{array}{r} \pm 28 \\
\pm 25 \\
\pm 26 \\
\pm 16 \\
\pm 4\end{array}$ \\
\hline $\begin{array}{l}564.07-610.25 \\
610.25-701.23 \\
701.23-748.00 \\
748.00-788.00 \\
788.00-818.00\end{array}$ & & $\begin{array}{l}267 \\
283 \\
225 \\
281 \\
219\end{array}$ & $\begin{array}{l}192 \\
156 \\
131 \\
186 \\
136\end{array}$ & $\begin{array}{l}2 \\
2 \\
2 \\
2 \\
2\end{array}$ & $\begin{array}{l}229 \\
219 \\
178 \\
233 \\
178\end{array}$ & $\begin{array}{l} \pm 37 \\
\pm 63 \\
\pm 47 \\
\pm 47 \\
\pm 42\end{array}$ & & $\begin{array}{r}62 \\
44 \\
34 \\
48 \\
-12\end{array}$ & $\begin{array}{r}38 \\
39 \\
32 \\
31 \\
0\end{array}$ & & & $\begin{array}{l}2 \\
2 \\
2 \\
2 \\
2\end{array}$ & $\begin{array}{l}50 \\
42 \\
33 \\
39 \\
-6\end{array}$ & $\begin{array}{r} \pm 12 \\
\pm 3 \\
\pm 1 \\
\pm 8 \\
\pm 6\end{array}$ \\
\hline $\begin{array}{l}818.00-835.00 \\
835.00-858.00 \\
858.00-889.70 \\
889.70-896.90\end{array}$ & & $\begin{array}{l}245 \\
311 \\
177 \\
181\end{array}$ & $\begin{array}{r}179 \\
214 \\
80 \\
95\end{array}$ & $\begin{array}{l}2 \\
2 \\
2 \\
2\end{array}$ & $\begin{array}{l}212 \\
263 \\
128 \\
138\end{array}$ & $\begin{array}{l} \pm 33 \\
\pm 49 \\
\pm 48 \\
\pm 43\end{array}$ & & $\begin{array}{r}-3 \\
36 \\
-50 \\
36\end{array}$ & $\begin{array}{r}-7 \\
42 \\
-39 \\
-35\end{array}$ & & & $\begin{array}{l}2 \\
2 \\
2 \\
2\end{array}$ & $\begin{array}{r}-5 \\
39 \\
-44 \\
0\end{array}$ & $\begin{array}{r} \pm 2 \\
\pm 3 \\
\pm 5 \\
\pm 35\end{array}$ \\
\hline $0-896.90$ & & & & 48 & 85 & \pm 119 & & & & & & 47 & 46 & \pm 54 \\
\hline
\end{tabular}


TABLE 4-26

SUMMARY OF AGREEMENT BETWEEN MEASURED AND PREDICTED TEMPERATURES FOR PEACH BOTTOM CORE 2

\begin{tabular}{|c|c|c|c|c|c|c|c|c|c|c|c|c|}
\hline \multirow{3}{*}{$\begin{array}{l}\text { Time } \\
\text { Interval } \\
\text { EFPD }\end{array}$} & \multicolumn{12}{|c|}{ Predicted Temperature - Measured Temperature $\left({ }^{\circ} \mathrm{C}\right)$} \\
\hline & \multicolumn{3}{|c|}{ Inner 6 Rings } & \multicolumn{3}{|c|}{ Rings 7-12 } & \multicolumn{3}{|c|}{ Rings $13-15$} & \multicolumn{3}{|c|}{ Entire Core } \\
\hline & $\begin{array}{l}\text { No. of } \\
\text { Meas. }\end{array}$ & Mean & RMS & $\begin{array}{l}\text { No. of } \\
\text { Meas. }\end{array}$ & Mean & RMS & $\begin{array}{l}\text { No. of } \\
\text { Meas. }\end{array}$ & Mean & RMS & $\begin{array}{l}\text { No. of } \\
\text { Meas. }\end{array}$ & Mean & RMS \\
\hline $\begin{array}{c}0-26.99 \\
26.99-62.71 \\
62.71-68.57 \\
68.57-202.06 \\
202.06-252.41\end{array}$ & $\begin{array}{r}11 \\
11 \\
11 \\
9 \\
8\end{array}$ & $\begin{array}{r}-1 \\
-26 \\
-57 \\
-19 \\
-9\end{array}$ & $\begin{array}{l} \pm 20 \\
\pm 21 \\
\pm 21 \\
\pm 43 \\
\pm 29\end{array}$ & $\begin{array}{l}3 \\
3 \\
3 \\
3 \\
3\end{array}$ & $\begin{array}{r}9 \\
-81 \\
-31 \\
-107 \\
-33\end{array}$ & $\begin{array}{r} \pm 16 \\
\pm 37 \\
\pm 5 \\
\pm 51 \\
\pm 25\end{array}$ & $\begin{array}{l}4 \\
5 \\
4 \\
3 \\
3\end{array}$ & $\begin{array}{l}41 \\
22 \\
44 \\
86 \\
98\end{array}$ & $\begin{array}{l} \pm 89 \\
\pm 60 \\
\pm 22 \\
\pm 26 \\
\pm 28\end{array}$ & $\begin{array}{l}18 \\
19 \\
18 \\
15 \\
14\end{array}$ & $\begin{array}{r}10 \\
-22 \\
-30 \\
-16 \\
9\end{array}$ & $\begin{array}{l} \pm 48 \\
\pm 50 \\
\pm 45 \\
\pm 74 \\
\pm 55\end{array}$ \\
\hline $\begin{array}{l}252.41-298.00 \\
298.00-342.95 \\
342.95-385.44 \\
385.44-499.59 \\
499.59-564.07\end{array}$ & $\begin{array}{l}7 \\
7 \\
5 \\
4 \\
2\end{array}$ & $\begin{array}{r}-45 \\
-6 \\
55 \\
77 \\
-65\end{array}$ & $\begin{array}{l} \pm 38 \\
\pm 31 \\
\pm 35 \\
\pm 71 \\
\pm 31\end{array}$ & $\begin{array}{l}3 \\
3 \\
3 \\
3 \\
3\end{array}$ & $\begin{array}{r}59 \\
-19 \\
92 \\
107 \\
171\end{array}$ & $\begin{array}{r} \pm 13 \\
\pm 44 \\
\pm 12 \\
\pm 3 \\
\pm 42\end{array}$ & $\begin{array}{l}2 \\
2 \\
2 \\
2 \\
2\end{array}$ & $\begin{array}{r}120 \\
78 \\
111 \\
97 \\
25\end{array}$ & $\begin{array}{r} \pm 28 \\
\pm 25 \\
\pm 26 \\
\pm 16 \\
\pm 4\end{array}$ & $\begin{array}{r}12 \\
12 \\
10 \\
9 \\
7\end{array}$ & $\begin{array}{r}8 \\
5 \\
77 \\
91 \\
62\end{array}$ & $\begin{array}{r} \pm 73 \\
\pm 47 \\
\pm 35 \\
\pm 50 \\
\pm 105\end{array}$ \\
\hline $\begin{array}{l}564.07-610.25 \\
610.25-701.23 \\
701.23-748.00 \\
748.00-788.00 \\
788.00-818.00\end{array}$ & $\begin{array}{l}2 \\
1 \\
1 \\
1 \\
1\end{array}$ & $\begin{array}{l}-46 \\
-43 \\
-75 \\
-48 \\
-76\end{array}$ & $\begin{array}{l} \pm 13 \\
\pm 35 \\
\pm 35 \\
\pm 35 \\
\pm 35\end{array}$ & $\begin{array}{l}2 \\
2 \\
2 \\
2 \\
2\end{array}$ & $\begin{array}{l}229 \\
219 \\
178 \\
233 \\
178\end{array}$ & $\begin{array}{l} \pm 37 \\
\pm 63 \\
\pm 47 \\
\pm 47 \\
\pm 42\end{array}$ & $\begin{array}{l}2 \\
2 \\
2 \\
2 \\
2\end{array}$ & $\begin{array}{l}50 \\
42 \\
33 \\
39 \\
-6\end{array}$ & $\begin{array}{r} \pm 12 \\
\pm 3 \\
\pm 1 \\
\pm 8 \\
\pm 6\end{array}$ & $\begin{array}{l}6 \\
5 \\
5 \\
5 \\
5\end{array}$ & $\begin{array}{l}78 \\
96 \\
69 \\
99 \\
54\end{array}$ & $\begin{array}{l} \pm 116 \\
\pm 114 \\
\pm 103 \\
\pm 119 \\
\pm 109\end{array}$ \\
\hline $\begin{array}{l}818.00-835.00 \\
835.00-858.00 \\
858.00-889.70 \\
889.70-896.90\end{array}$ & $\begin{array}{l}1 \\
1 \\
1 \\
1\end{array}$ & $\begin{array}{r}-73 \\
48 \\
-49 \\
25\end{array}$ & $\begin{array}{l} \pm 35 \\
\pm 35 \\
\pm 35 \\
\pm 35\end{array}$ & $\begin{array}{l}2 \\
2 \\
2 \\
2\end{array}$ & $\begin{array}{l}212 \\
263 \\
128 \\
138\end{array}$ & $\begin{array}{l} \pm 33 \\
\pm 49 \\
\pm 48 \\
\pm 43\end{array}$ & $\begin{array}{l}2 \\
2 \\
2 \\
2\end{array}$ & $\begin{array}{r}-5 \\
39 \\
-44 \\
0\end{array}$ & $\begin{array}{r} \pm 2 \\
\pm 3 \\
\pm 5 \\
\pm 35\end{array}$ & $\begin{array}{l}5 \\
5 \\
5 \\
5\end{array}$ & $\begin{array}{r}68 \\
130 \\
24 \\
60\end{array}$ & $\begin{array}{r} \pm 123 \\
\pm 114 \\
\pm 92 \\
\pm 75\end{array}$ \\
\hline $0-896.90$ & 85 & -18 & \pm 49 & 48 & 85 & \pm 119 & 47 & 46 & \pm 54 & 180 & 27 & \pm 87 \\
\hline
\end{tabular}


TABLE 4-27

MEASURED AND CALCULATED TEMPERATURES

\begin{tabular}{|c|c|c|c|c|c|c|}
\hline $\begin{array}{c}\text { Core } \\
\text { Region }\end{array}$ & $\begin{array}{l}\text { No. of } \\
\text { Thermo- } \\
\text { couples }\end{array}$ & $\begin{array}{c}\text { No. of } \\
\text { Comparisons }\end{array}$ & $\begin{array}{l}\text { Average } \\
\text { Lifetime of } \\
\text { Thermocouple } \\
\text { (EFPD) }\end{array}$ & $\begin{array}{c}\text { Average } \\
\text { Bias } \\
(\text { Calc-Meas.) } \\
\left({ }^{\circ} \mathrm{C}\right)\end{array}$ & $\begin{array}{c}\text { Uncertainty } \\
\text { on Bias }(1 . \sigma) \\
(\mathrm{RMS} / \sqrt{\mathrm{n}}) \\
\left({ }^{\circ} \mathrm{C}\right)\end{array}$ & $\begin{array}{c}\text { RMS of } \\
\text { Bias }\left(\begin{array}{ll}1 & \sigma\end{array}\right) \\
\left({ }^{\circ} \mathrm{C}\right)\end{array}$ \\
\hline $\begin{array}{l}\text { Inner } \\
6 \text { rings }\end{array}$ & 11 & 85 & 372 & -18 & \pm 5 & \pm 49 \\
\hline $\begin{array}{l}\text { Rings } \\
7-12\end{array}$ & 3 & 48 & 786 & +85 & \pm 17 & \pm 119 \\
\hline $\begin{array}{l}\text { Rings } \\
13-15\end{array}$ & 5 & 47 & 437 & +46 & \pm 8 & \pm 54 \\
\hline $\begin{array}{l}\text { Al1 } \\
\text { rings }\end{array}$ & 19 & 180 & 454 & +27 & \pm 7 & \pm 87 \\
\hline
\end{tabular}




\section{DISCUSSION AND CONCLUSION}

The conclusions of the nuclear design verification using Peach Bottom EOL data are as follows:

1. The compact-to-compact agreement between the measured and predicted time-averaged power distributions and the measured and predicted EOL axial power distributions is within $\pm 7.4 \%$ ( $1 \sigma)$, which is well within the $\pm 8 \%$ to $13 \%$ quoted for other nuclear reactors (Ref. 14). The uncertainty in predictive accuracy resulting from measurement uncertainties is $\pm 3.5 \%(1 \sigma)$ and $\pm 1.6 \%(1 \sigma)$ for the time-averaged and EOL axial power distributions, respectively.

2. The power in elements whose axial power shapes were unperturbed by control rods shifted from the bottom of the core and smoothed out with depletion as predicted. The rate of change of the unrodded power profile with depletion was well modeled, although the shift of power toward the top of the core was slightly overpredicted.

3. The core average power was predicted within $\pm 0.7 \%(1 \sigma)$ and the element-to-element agreement between predicted and measured element average power is within $\pm 6.8 \%$ ( $1 \sigma)$, which is within the $3 \%$ to $8 \%$ quoted for other nuclear reactors (Ref. 14). The uncertainty in the predictive accuracy resulting from measurement uncertainties is $\pm 4.7 \%(1 \sigma)$. 
4. The combined accuracy for radial and axial power predictions for the Peach Bottom HTGR is $\pm 10 \%$ ( $1 \sigma$ ) compared to $\pm 8 \%$ to $15 \%$ for other nuclear reactors (Ref, 14). The uncertainty in the predictive accuracy resulting from measurement uncertainties is $\pm 6 \%(1 \sigma)$.

5. The core power distribution appears to have been less perturbed by control rod insertion than anticipated.

6. The validity of gamma spectroscopic fuel examination for nuclear design verification purposes has been demonstrated.

Although it was originally planned to feed power measurements back into the thermal calculations to eliminate some of the uncertainties associated with the nuclear predictions from thermal design verification, it was decided not to do so because of the cesium redistribution in almost all driver elements and the large uncertainties associated with the element average power corrections determined from burnup measurements. The thermal calculations were improved, however, through use of a radial strain correlation developed from fuel compact metrology data which permitted accurate modeling of radial sleeve compact gaps. Thermal calculations were performed with the TREVER code and predicted temperatures were obtained for comparison with thermocouple measurements. In all, 180 comparisons between predicted and measured temperatures in 14 different driver fuel elements were made.

The average lifetimes of the $16 \mathrm{C} / \mathrm{A}$ and $11 \mathrm{~W} / \mathrm{Re}$ driver element thermocouples which survived beyond BOL were $53 \%$ and $47 \%$ of full irradiation exposure, respectively. Seven C/A thermocouples failed at BOL, presumably due to faulty assembly. Failure temperatures for the C/A and W/Re thermocouples were observed to be approximately $1200^{\circ} \mathrm{C}$ and $\geq 1400^{\circ} \mathrm{C}$, respectively. 
The conclusions of thermal design verification are:

1. The agreement between measured and calculated temperatures for the 19 thermocouple locations considered is within $\pm 87^{\circ} \mathrm{C}(1 \sigma)$ with an apparent bias of $\pm 27^{\circ} \mathrm{C} \pm 7{ }^{\circ} \mathrm{C}(1 \sigma)$ in the predicted temperatures. The most probable causes of this general overprediction of temperature are eccentricity of the sleeve-fuel compact gap and underestimation of the fuel compact thermal conductivity.

2. The agreement between calculated and measured temperatures varies significantly in different regions of the core. Average disagreements (calculated minus measured temperatures) were determined to be $-18^{\circ} \mathrm{C} \pm 5^{\circ} \mathrm{C}(1 \sigma)$ for the inner six rings of fuel elements, $+85^{\circ} \mathrm{C} \pm 17^{\circ} \mathrm{C}(1 \sigma)$ for rings 7 through 12 , and $+46^{\circ} \mathrm{C} \pm 8^{\circ} \mathrm{C}(1 \sigma)$ for rings 13 through 15 . The root mean square deviations in the disagreement are $\pm 49^{\circ} \mathrm{C}(1 \sigma), \pm 119^{\circ} \mathrm{C}(1 \sigma)$, and $\pm 54^{\circ} \mathrm{C}(1 \sigma)$ for rings 1 through 6,7 through 12 , and 13 through 15, respectively

3. Peak fuel temperatures in approximately $1 \%$ of the driver elements were calculated to be in excess of $1510^{\circ} \mathrm{C}$, the original prediction (Ref. 2) for the peak driver element fuel temperature in Peach Bottom Core 2. This increase in predicted temperatures is a result of the fuel compact-sleeve gap being larger than originally expected. The decision to reduce core power after 701 EFPD prevented fuel temperature predictions from becoming far out of line with design temperatures. Peak temperatures approaching $1600^{\circ} \mathrm{C}$ were calculated for driver elements but could not be verified due to thermocouple failure in the high-temperature elements. Peak temperatures of approximately 
$1600^{\circ} \mathrm{C}$ were also calculated for fuel test elements and were indirectly verified in FTE-14 and FTE-18 by thermocouple measur ements.

4. The usefulness of high-temperature thermocouple instrumentation for verification of temperature predictions has been demonstrated. 


\section{ACKNOWLEDGMENTS}

The authors wish to acknowledge the following individuals for their contributions to the nuclear and thermal design verification efforts.

\section{Nuclear Predictions}

M. Schwartz, D. Hoppes, W. Lefler, K. E. Asmussen

Thermocouple Data

W. Lefler, F. McCord, A. Mehner, L. Mayweather, M. Scott

Gamma Scan Data

J. F. Holzgraf

Thermocouple Recalibration

J. M. McNair

Materials Property Data

L. A. Beavan and R. J. Price

Error Analysis Consultant

R. H. Leary 
Radial Strain Correlation

K. E. Asmussen

Editing

Isabel Buechler and Editorial Staff 


\section{REFERENCES}

1. "Final Hazards Summary Report, Peach Bottom Atomic Power Station," Volume II.

2. "Peach Bottom Atomic Power Station Unit 1 Core 2 Design and Operational Evaluation, Proposed Facility Change and Technical Specification Change (No. 13)," Philadelphia Electric Company, January 1970.

3. Holzgraf. J. F., "Gamma Spectroscopic Examination of the Peach Bottom HTGR Reactor Core Components," DOE Report GA-A13453, General Atomic Company, April 1978.

4. Wagner, M. R., "GAUGE, A Two-Dimensional Few Group Neutron Diffusion-Depletion Program for a Uniform Triangular Mesh," Gulf General Atomic Report GA-8307, March 15, 1968.

5. Dorsey, J. P., R. Froelich, and F. Todt, "BUG-2/BUGTRI, Two-Dimensional Multigroup Burnup Codes for Rectangular and Hexagonal Geometry," Gulf General Atomic Report GA-8272, August 22, 1969.

6. Todt, F. W., and L. J. Todt, "FEVER/Ml, A One-Dimensional Depletion Program for Reactor Fuel Cycle Analysis," Gulf General Atomic Report. GA-9780, October 22, 1969.

7. Lane, R. K., and A. S. Weiman, "Flux Distribution Measurements in Peach Bottom - Results of Post Construction R\&D Test Procedures B. P. 3, 10, 12," USAEC Report GAMD-7353, General Atomic Company, October $1,1966$. 
8. Wichner, R. P., et al., "Distribution of Fission Products in Peach Bottom HTGR Fuel Element F01-01," DOE Report ORNL/TM-6353, Oak Ridge National Laboratory, October 1978.

9. Wichner, R. P., et al., "Distribution of Fission Products in Peach Bottom HTGR Fuel Element E11-07," ERDA Report ORNL-5214, Oak Ridge National Laboratory, April 1977.

10. Wichner, R. P., et al., "Distribution of Fission Products in Peach Bottom HTGR Fuel Element F03-01," DOE Report ORNL/TM-5996 Oak Ridge National Laboratory, May 1978.

11. Wichner, R. P., et al., "Distribution of Fission Products in Peach Bottom HTGR Fuel Element E14-01," ERDA Report ORNL/TM-5730, Oak Ridge National Laboratory, August 1977.

12. Dyer, F. F., et al., "Postirradiation Examination of Peach Bottom HTGR Driver Fuel Element E06-01," ERDA Report OKNL-5126, Oak Ridge National Laboratory, April 1976.

13. Wichner, R. P., et al., "Distribution of Fission Products in Peach Bottom HTGR Fuel Element F05-05," DOE Report ORNL/TM-6455, Oak Ridge National Laboratory, January 1979.

14. "Reactor Burnup Physics," in Proceedings of a Panel on Reactor Burnup Physics, IEAE, Vienna, 1973.

15. Harmon, D. P., C. B. Scott, and W. J. Scheffel, "Postirradiation Examination of Peach BOttom Core 2 Fuel Element C11-07," General Atomic Report GA-A12460, August 6, 1974.

16. Shenoy, A. S., and D. W. McEachern, "HTGR Core Thermal Design Methods and Analysis," General Atomic Report GA-A12985, December 31, 1974. 
17. "Fuel Design Data Manual," Issue B, General Atomic unpublished data.

18. Beavan, L. A., "H-327 Graphite-Design Data Package," General Atomic, unpublished data, December 19, 1975.

19. Beavan, L. A., "Thermal Conductivity of Peach Bottom Fuel Compacts," General Atomic unpublished data, August 20, 1976.

20. Price, R. J., and L. A. Beavan, "Graphite Data Package for Analysis of Peach Bottom Test Elements," General Atomic unpublished data, November $26,1974$.

21. Koyama, K., "Thermal Expansion of Peach Bottom II Sleeve No. 2466," General Atomic unpublished data, May 27, 1970.

22. Koyama, K., "Thermal Expansion of Peach Bottom II Compacts," General Atomic unpublished data, September 24, 1969.

23. Smith, C. L., "Design Memo - Peak Bottom Fuel Performance Mode1s," General Atomic unpublished data, December 16, 1975.

24. Holzgraf, J. F., "Postirradiation Examination and Evaluation of Peach Bottom Fuel Test Elements FTE-14 and FTE-15," General Atomic Company Report GA-A13944, February 1979.

25. Wallroth, C. F., et al., "Postirradiation Examination and Evaluation of Peach Bottom Molded Fuel Test Element FTE-18," General Atomic Company Report GA-A13699, June 1, 1976.

26. McNair, J. M., "Calibration of Peach Bottom Test Element Thermocouples," General Atomic unpublished data, August 27, 1976. 
$\theta$

$\ddot{\theta}$

(2) 


$$
\begin{gathered}
\text { APPENDIX } \\
\text { IRRADIATION CONDITIONS AND FUEL PERFORMANCE DATA } \\
\text { FOR PEACH BOTTOM FUEL ELEMENTS } \\
\text { E01-01, F03-01, F05-05, E06-01, C11-07, E11-07, AND E14-01 }
\end{gathered}
$$

Temperature, fast neutron fluence, and fuel performance data for Peach Bottom fuel elements E01-01, F03-01, F05-05, E06-01, C11-07, E11-07, and E14-01 are presented in the following tables. These results were obtained using the Peach Bottom driver element version of the TREVER (Ref. 16) code. The TREVER analyses included:

1. No feedback of gamma scan data.

2. A fuel compact radial strain correlation developed from PIE strain data.

3. A thermal conductivity of $27.6 \mathrm{~W} / \mathrm{m} \cdot \mathrm{K}\left(13 \mathrm{Btu} / \mathrm{hr}-\mathrm{ft}-{ }^{\circ} \mathrm{F}\right)$ for the fuel compacts.

Except for the fuel compact thermal conductivity, these calculations were identical to those performed in support of this report. The thermal conductivity was changed from $19 \mathrm{~W} / \mathrm{m}^{\circ} \mathrm{K}\left(11 \mathrm{Btu} / \mathrm{hr}-\mathrm{ft}-{ }^{\circ} \mathrm{F}\right)$ to $27.6 \mathrm{~W} / \mathrm{m} \cdot \mathrm{K}\left(15 \mathrm{Btu} / \mathrm{hr}-\mathrm{ft}-{ }^{\circ} \mathrm{F}\right)$, in accordance with the conclusions of the report. Kernel migration distances and failure fractions were calculated using the $50 \%$ confidence level performance models given in Ref. 23. 
EOL-OL COMNACT 1

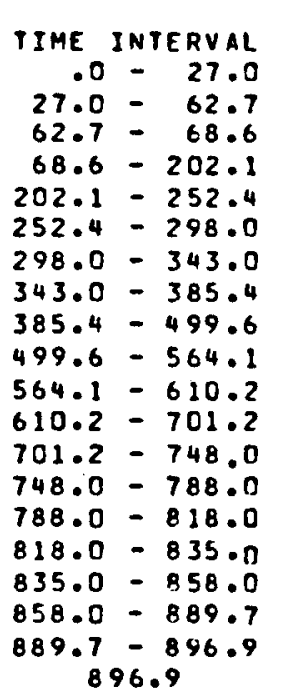

896.9

TIME AVERAGED RMS

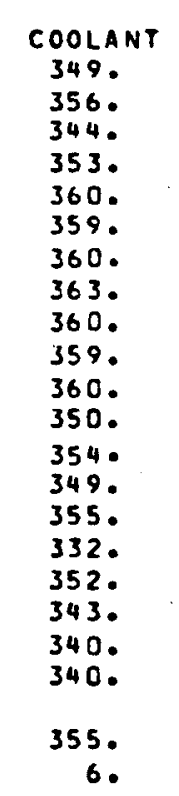

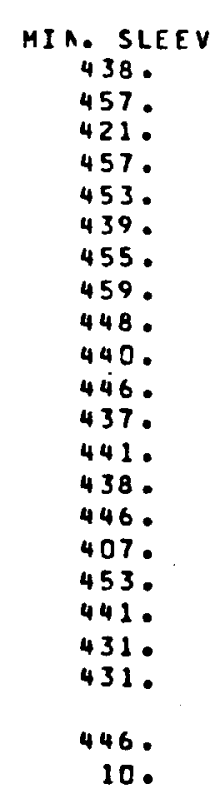

TEMPERATURES (C)

MAX. SLEEVE
$450^{\circ}$
474.
$4355^{\circ}$
484.

MIN. FUEL

$\begin{array}{ll}508 . & 540 . \\ 544 . & 582 .\end{array}$

484.

489.

475 .

501.

512.

497.

495.
505.

505.

503.

494.

594.

404.

522.

505 .

489.

488 .

492.

15.

492 .

560 .

566.

544.

582 .

596.

572.

573.

588.

581 .

587.

570.

584.

508 .

611 .

589.

566.

566.

570.

21.

540.

582.

599.

602 .

575 .

617 .

633.

604.

621.

620 .

$590^{\circ}$

0.

614.

529.

647 .

622.

596.

FASI FLUENCE

120**25 N/M**21

.00

.03
.07

.08

.24

.34
.39

.39
.44

.44
.57

.63

.68

.78

.83
.88

.92

.94

.97

1.02

1.03

EO1-01 COMPACT 2

TIME INTERVAL
$.0-27.0$
$27.0-62.7$
$62.7-68.6$
$68.6-202.1$
$202.1-252.4$
$252.4-298.0$
$298.0-343.0$
$343.0-385.4$
$385.4-499.6$
$499.6-564.1$
$564.1-610.2$
$610.2-701.2$
$701.2-748.0$
$743.0-788.0$
$788.0-818.0$
$818.0-935.0$
$835.0-858.0$
$858.0-889.7$
$889.7-896.9$
896.9

\begin{tabular}{|c|c|}
\hline COOLANT & MI A. SLEEVE \\
\hline $\begin{array}{l}3630 \\
349 \\
360\end{array}$ & $\begin{array}{l}476 . \\
436 . \\
476 .\end{array}$ \\
\hline $\begin{array}{l}367 . \\
365 . \\
367\end{array}$ & $\begin{array}{l}473 . \\
456 . \\
475 .\end{array}$ \\
\hline $\begin{array}{l}370 . \\
366 . \\
365 .\end{array}$ & $\begin{array}{l}480^{\circ} \\
4670^{\circ} \\
461 .\end{array}$ \\
\hline $\begin{array}{l}366^{\circ} \\
356^{\circ} \\
360^{\circ}\end{array}$ & $\begin{array}{l}468 . \\
4600^{\circ} \\
463 .\end{array}$ \\
\hline $\begin{array}{l}3560^{\circ} \\
362^{\circ} \\
338^{\circ}\end{array}$ & $\begin{array}{l}461 . \\
474 . \\
430 .\end{array}$ \\
\hline $\begin{array}{l}360 . \\
351 . \\
347 .\end{array}$ & $\begin{array}{l}483 . \\
471 . \\
459 .\end{array}$ \\
\hline 347. & 459. \\
\hline 362. & 467. \\
\hline 6. & 10. \\
\hline
\end{tabular}

IEMPERATURES (C)

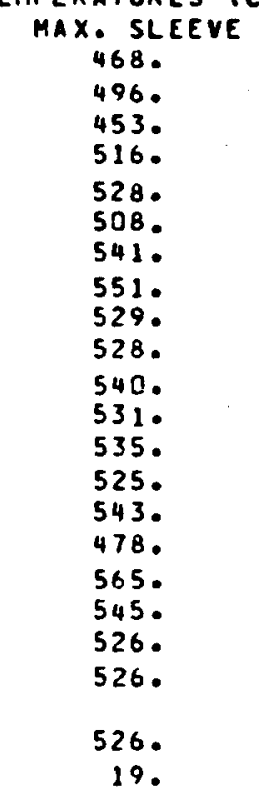

MIN. FUEL

531.

568 .

FAST FLUENCE

$(10 * 25 N / M * 2)$

$574 . .617$.

$517.55 \mathrm{C}$.

605.648 .

620 . 661.

592.627.

637.627.

651.693.

618.654.

623.661.

639.678.

629.669.

633.672.

614.0648.

$637.674^{\circ}$

549.5575.

669.7213.

643.663.

616.653.

616.652.

.00

.04

.12

.39
.49

.56

.65

.93

1.04

1.13

1.29

1.37

1.45

1.51

1.55

1.60

1.68

1.70

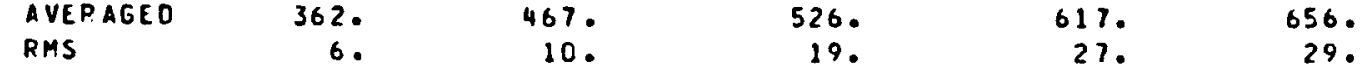




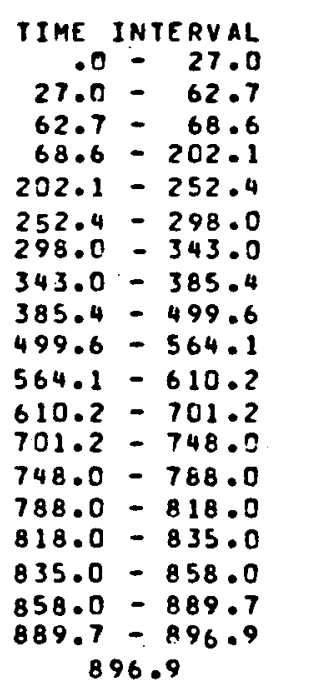

TIHE AVERAGEO RMS

\begin{tabular}{|c|c|}
\hline $\begin{array}{l}\text { COOLANT } \\
363 . \\
372 . \\
356 . \\
370 .\end{array}$ & $\begin{array}{c}\text { MIA. SLEEVE } \\
486 . \\
513 . \\
464 . \\
514 .\end{array}$ \\
\hline 375. & 506. \\
\hline $\begin{array}{l}372 . \\
375 .\end{array}$ & $\begin{array}{l}486 . \\
509 .\end{array}$ \\
\hline $\begin{array}{l}378^{\circ} \\
374^{\circ} \\
372^{\circ}\end{array}$ & $\begin{array}{l}515^{\circ} \\
499^{\circ} \\
493^{\circ}\end{array}$ \\
\hline $\begin{array}{l}374 . \\
364 . \\
368 .\end{array}$ & $\begin{array}{l}502^{\circ} \\
494^{\circ} \\
497^{\circ}\end{array}$ \\
\hline $\begin{array}{l}364 . \\
370^{\circ} \\
345^{\circ}\end{array}$ & $\begin{array}{l}496 . \\
504 . \\
454 .\end{array}$ \\
\hline $\begin{array}{l}369^{\circ} \\
360^{\circ} \\
355^{\circ}\end{array}$ & $\begin{array}{l}516^{\circ} \\
503{ }^{\circ} \\
489^{\circ}\end{array}$ \\
\hline 355. & 489. \\
\hline $\begin{array}{r}370 . \\
6 .\end{array}$ & $\begin{array}{r}501 . \\
12 .\end{array}$ \\
\hline
\end{tabular}

TEMPERATURES I $C$,

MAX. SLEEVE

504 .

539.

486.

566.

575.

549.
590.

600.

572

572 .

586.

577 .

581.

571 .

582.

609

587 .

565.

564 .

571.

21.

HIN. FUEL

581.

634.

566.

676.

691 .

655.

710 .

725.

685.

710.

701.

701.

682.

694.

595 .

733.

704.

674.

673.

684.
32.

MAX. FUEL

626.

606.

730.

742.

742.

699.

760.
777.

777.

738 .

759 .

749.

752 .

725.

739.

626.

786.

753.

718

717.

733.

33.

EOI-OL COMPACT

TIHE INTERVAL

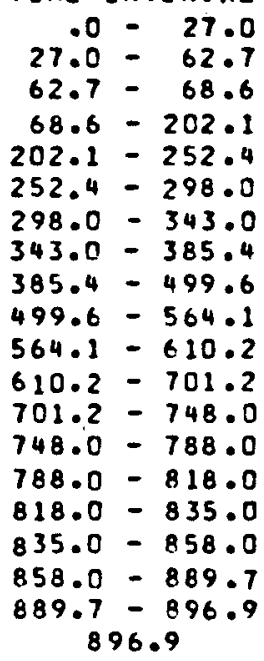

TIME AVERAGED RMS
TEMPERATURES (C)

\begin{tabular}{|c|c|}
\hline OOLANT & MIA. SLEEVE \\
\hline $\begin{array}{l}373 . \\
383 . \\
364 .\end{array}$ & $\begin{array}{l}520^{\circ} \\
552^{\circ} \\
495 .\end{array}$ \\
\hline $\begin{array}{l}380^{\circ} \\
385 \\
380\end{array}$ & $\begin{array}{l}554 . \\
539^{\circ} \\
5130^{\circ}\end{array}$ \\
\hline $\begin{array}{l}385 \\
388 .\end{array}$ & $\begin{array}{l}542 . \\
548^{\circ} .\end{array}$ \\
\hline $\begin{array}{l}383 \\
381\end{array}$ & $\begin{array}{l}530 . \\
519 .\end{array}$ \\
\hline & \\
\hline $\begin{array}{l}377^{\circ} \\
374^{\circ}\end{array}$ & $\begin{array}{l}525 . \\
525 .\end{array}$ \\
\hline $\begin{array}{l}380 \\
353 \\
379\end{array}$ & $\begin{array}{l}529^{\circ} \\
474{ }^{\circ} \\
5430^{\circ}\end{array}$ \\
\hline $\begin{array}{l}370 \\
365^{\circ} \\
365^{\circ}\end{array}$ & $\begin{array}{l}529 . \\
514^{\circ} \\
514^{\circ}\end{array}$ \\
\hline $\begin{array}{r}379 . \\
6 .\end{array}$ & $\begin{array}{r}532 \\
15 .\end{array}$ \\
\hline
\end{tabular}

MAX. SLEEVE 542 .

584.

616.

585.

633.

312 .

606

623.

614.

618.

608 .

611.

532.

643.

619.

594.

610.
MIN. FUEL 631.

695 .

616.

747.

755.
713.

713.

795.

749.

750.

772 .

764.

768 .

745.

748.

639.

795 .

765.

732.

732 .

747
35
FAST FLUENCE

$(10 * 25 N / M * 2)$

.00

.06

.15

.51

.64

.83

.95

1.22

1.37

1.48

1.69

1.80

1.90

1.98

2.02

2.09

2.22
FAST FLUENCE

MAX. FUEL

685 .

665 .

812.

764.

836

856

801 .

804 .

829 .

820 .

825 .

795 .

799 .

674.

854 .

819 .

780 .

780 .

804.
$(10 * 25 N / M * 2)$$$
.00
$$$$
.07
$$

.19

.20

.62

.78
.89

.89
1.03

1.16

1.48

1.66

1.79

2.04

2.17

2.29

2.43

2.51

2.63
2.66 
EOL-DI CUMPACT 5

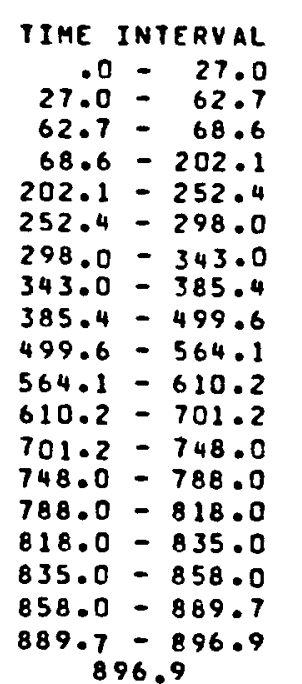

IIME AVERAGED RMS

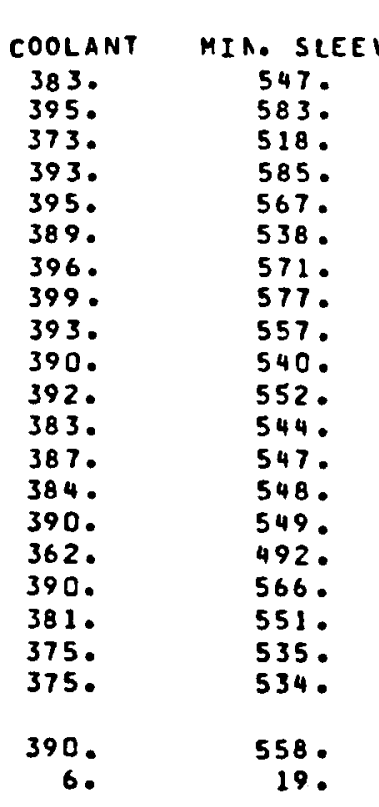

TEMPERATURES (C)

MAX. SLEEVE
573.
619.
548.
654.
651.
6150
669.
680.
644.
630.
649.
641.
644.
634.
635.
551.
669.
644.
618.
618.
641.
24.

MIN FUEL 668 . 740. 653. 800. 808 .

761.

833.

853.

803.

796.

16.

823.

823.

800 .

682.

854.

824.

789.

789.

799. 39.
MAX. FUEL

729.

812.

873.

875 .

818.

899.

21.

861.

884.

877.

854.

854.

854.

720 .

917.

882 .

841.

841.

861.

EO1-01 COMPACT 6

TIME INTERVAL $.0=27.0$
$27.0=62.7$ $27.0-62.7$ $62.7=68.6$
$68.6-202.1$

$202.1-252.4$

$252.4-298.0$

$298.0-343.0$

$343.0-385.4$

$385.4=499.6$

$499.6-564.1$

$564.1=610.2$

$701.2-748.0$

$748.0-788.0$

$788.0-818.0$

$818.0-835.0$

$835.0-858.0$

$858.0-889.7$

$889.7-896.9$

896.9

TIME AVERAGED RMS

\begin{tabular}{|c|c|}
\hline $\begin{array}{l}\text { COOLANT } \\
394 .\end{array}$ & $\begin{array}{c}\text { MIA. SLEEV } \\
573 .\end{array}$ \\
\hline $\begin{array}{l}408 . \\
383 . \\
406 .\end{array}$ & $\begin{array}{l}612 . \\
541 . \\
615 .\end{array}$ \\
\hline $\begin{array}{l}407 . \\
399 . \\
407 .\end{array}$ & $\begin{array}{l}593 . \\
560 . \\
598 .\end{array}$ \\
\hline $\begin{array}{l}411 . \\
405 . \\
400 .\end{array}$ & $\begin{array}{l}604 . \\
582 . \\
559 .\end{array}$ \\
\hline $\begin{array}{l}403 . \\
394 . \\
398 .\end{array}$ & $\begin{array}{l}572 . \\
565 . \\
568 .\end{array}$ \\
\hline $\begin{array}{l}395 . \\
400 . \\
370 .\end{array}$ & $\begin{array}{l}569 . \\
568 . \\
507 .\end{array}$ \\
\hline $\begin{array}{l}402 . \\
392 .\end{array}$ & $\begin{array}{l}586 . \\
571 .\end{array}$ \\
\hline $\begin{array}{l}386 . \\
386 .\end{array}$ & $\begin{array}{l}553 . \\
553 .\end{array}$ \\
\hline 401. & 581. \\
\hline 7 & 22 \\
\hline
\end{tabular}

TEMPERATURES (C)

$\begin{array}{ccc}\text { MAX. SLEEVE } & \text { MIN. FUEL } & \text { MAX. FUEL } \\ 601 . & 702 . & 768 . \\ 652 . & 781 . & 860 . \\ 573 . & 687 . & 746 . \\ 689 . & 848 . & 927 . \\ 682 . & 855 . & 928 . \\ 641 . & 804 . & 86 . \\ 700 . & 883 . & 956 . \\ 712 . & 906 . & 981 . \\ 673 . & 855 . & 918 . \\ 652 . & 841 . & 903 . \\ 673 . & 872 . & 937 . \\ 665 . & 869 . & 935 . \\ 668 . & 879 . & 945 . \\ 659 . & 856 . & 915 . \\ 655 . & 852 . & 908 . \\ 567 . & 726 . & 766 . \\ 692 . & 913 . & 979 . \\ 666 . & 883 . & 943 . \\ 638 . & 846 . & 901 . \\ 638 . & 846 . & 901 . \\ 668 . & 849 . & 916 . \\ 27 . & 43 . & 46 .\end{array}$

FAST FLUENCE

(10*25 N/M**2)

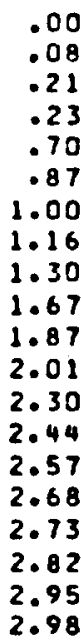

08

.21

70

.87

1.00

1.30

1.67

2.01

2.30

2.44

2.57

2.73

2.98

FAST FLUENCE

$110 * 25 N / M * 21$

.00

.09

.23
.25

.77

.77
.96

1.10

1.27

1.42

1.82

2.04

2.20

2.51

2.67

2.81

2.92

2.97

3.07

3.21

3.24 
EOL-01 COMPACT,

TIHE INTERVAL $.0-27.0$ $27.0=27.0$ $62.7-68.6$ $68.6-202.1$ $202.1-252.4$ $252.4-298.0$ $298.0-343.0$ $343.0-385.4$ $385.4-499.6$

$499.6-564.1$

$564.1-610.2$ $610.2-701.2$ $701.2=748.0$

$748.0-788.0$ $788.0-818.0$ $818.0-835.0$

$835.0-858.0$ $858.0=889.7$ $889.7-896.9$ 896.9

TIME AVERAGED RHS

COOLANT
406.
421.
394.
420.
420.
409.
420.
424.
416.
410.
414.
405.
409.
407.
412.
380.
414.
404.
397.
397.
413.
8.

MI A. SLEEVE MAX. SLEEYE

$$
596 .
$$

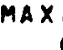

$$
626 .
$$

638 .

561.

642 .

616.

580 .

621 .

628.

604 .

579.

594.

587 .

589.

592.

586.

522 .

605.

571.

571.

\section{1.}

595.

719

708.

664.

698 .

675 .

698 .

690.

693.

684.

$583^{\circ}$.

713.

687.

658.

657 .

MIN

T31.

MAX FUEL

817.

716.

889.

895.

841.

927.

952.

900 .

888 .

922.

924.

938.

915.

770.

970.

940.

902 .

903.

693.

25 .

29.

895.

801.

900.

779.

972.

973.

1003.

1031.

967.

9540

992.

1007.

977.

962.

812.

1039.

1004.

960.

960 .

967.

EO1-01 COMPACT 8

TIME INTERVAL

$.0-27.0$

$27.0-62.7$

$62.7-68.6$

$68.6-202.1$

$202.1-252.4$

$252.4-298.0$

$298.0-343.0$

$343.0-385.4$

$385.4-499.6$

$499.6-564.1$

$564.1-610.2$

$610.2=701.2$

$701.2-748.0$

$748.0-788.0$

$788.0-8.18 .0$

$818.0-835.0$

$835.0-858.0$

$858.0-889.7$

$889.7-896.9$

896.9

TIME AVERAGED

\begin{tabular}{|c|c|}
\hline $\begin{array}{l}\text { COOLANT } \\
419 . \\
436 .\end{array}$ & $\begin{array}{c}\text { MIA. SLEEV } \\
616 . \\
661 .\end{array}$ \\
\hline $\begin{array}{l}4050^{\circ} \\
4350^{\circ}\end{array}$ & $\begin{array}{l}579 . \\
665^{\circ}\end{array}$ \\
\hline $\begin{array}{l}433 \\
420 \\
433\end{array}$ & $\begin{array}{l}639 . \\
599^{\circ} \\
644 .\end{array}$ \\
\hline $\begin{array}{l}437 . \\
429 . \\
421 .\end{array}$ & $\begin{array}{l}651 . \\
625 . \\
597 .\end{array}$ \\
\hline $\begin{array}{l}425 \\
417 \\
420\end{array}$ & $\begin{array}{l}613 . \\
606 . \\
608 .\end{array}$ \\
\hline $\begin{array}{l}420 . \\
423 . \\
389 .\end{array}$ & $\begin{array}{l}612 . \\
602 . \\
535\end{array}$ \\
\hline $\begin{array}{l}426 . \\
416 . \\
409 .\end{array}$ & $\begin{array}{l}623 . \\
608 . \\
588^{\circ}\end{array}$ \\
\hline 409. & 587. \\
\hline $\begin{array}{r}425 . \\
9 .\end{array}$ & $\begin{array}{r}624^{\circ} \\
27\end{array}$ \\
\hline
\end{tabular}
RHS

IEMPERATURES ( $C$ )

648.
707.
615.

846.

615.740.

745.

694.

752.

765.

721.

694.

718.

711.

714.

706 .

691.

597.

731.

705.

675.

715.

715.
31.
829.

933.

805.

1013.

944.
1046.

1076 .

1011.

997.

1039.

1043.

1060 .

1029.

1008.

852

1090 .

1054.

1009.

1010 .

1010.
FAST FLUENCE

(20*25 N/M**2)

.00

.09

.25
.27

.82

1.02

1.17

1.36
1.52

1.95

2.18

2.68

2.85

2.99

3.11

$3 \cdot 17$

3.27

3.42

3.45

FAST FLUENCE

$(10 * 25$ N/M**2)

.00

.10

.26

.86

1.08

1.23

1.43

1.60

2.05

2.29

2.47

2.81

2.99

3.14

3.26

3.32

3.42

3.58

3.61 
COI-OL COMPACY

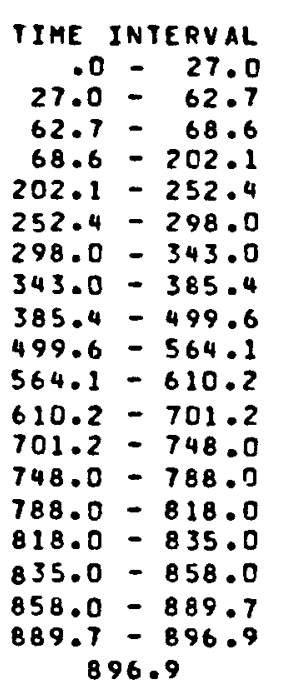

896.9

TIME AVERAGED RMS
COOLANT MIR. SLEEVE MAX. SLEEVE 432 . 450 .

416.

450.

446.

432 .

447.

451 .

442 .

432 .

437.

429.

432.

432.

435 .

439.

429.

420.

420 .

438.

10.
632 .

679.

593.

684 .

660 .

617.

665.

645

613 .

630 .

624.

626

630.

617.
548.

639 .

623 .

603 .

602 .

642.
28.
665 .

726.

630.

764.

754.

703.

788.

742 .

711.

737.

730.

725.

705 .

609 .

747.

720.

690 .

689.

734.
32.
TIME INTERYAL $.0-27.0$ $27.0=62.7$

$68.6-202.1$

$202.1=252.4$

$252.4-298.0$

$298.0=343.0$

$343.0-385.4$

$385.4-499.6$

$499.6-564.1$

$564.1-610.2$

$610.2-701.2$

$701.2=748.0$

$788.0-818.0$

$818.0-835.0$

$835.0-858.0$

$858.0-889.7$

$89.7-896.9$

896.9

IIME AVERAGEO RMS
MS

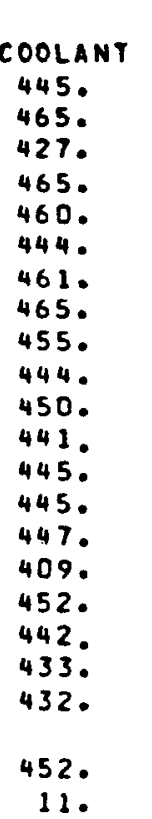

TEMPERATURES (C)

HIN. SLEEYE
647.
696.
606.
701.
677.
632.
683.
691.
662.
631.
649.
643.
645.
650.
634.
562.
658.
641.
620.
619.
660.
28.

EO1-01 COMPACT 10
MIN. FUEL 774.

868.

945.

963.

903.

998.

1029.

976.

1003.

1010 .

1028.

1006 .

982.

1027.

987

968.

59. 849 .

857

824.

1034.

1047.

975

1083.

1115 .

1048

1033 .

1078.

1085.

1103.

1073.

1044.

884

1129.

1093.

1047 .

1048 .

1045.

60.
MAX. FUEL

FAST FLUENCE

$(10 * 25 \quad N / M * 2)$

$$
\begin{aligned}
& .00 \\
& .10 \\
& .27 \\
& .29 \\
& .89 \\
& 1.11 \\
& 1.27 \\
& 1.47 \\
& 1.65 \\
& 2.11 \\
& 2.36 \\
& 2.55 \\
& 2.91 \\
& 3.09 \\
& 3.25 \\
& 3.37 \\
& 3.43 \\
& 3.53 \\
& 3.69 \\
& 3.73
\end{aligned}
$$

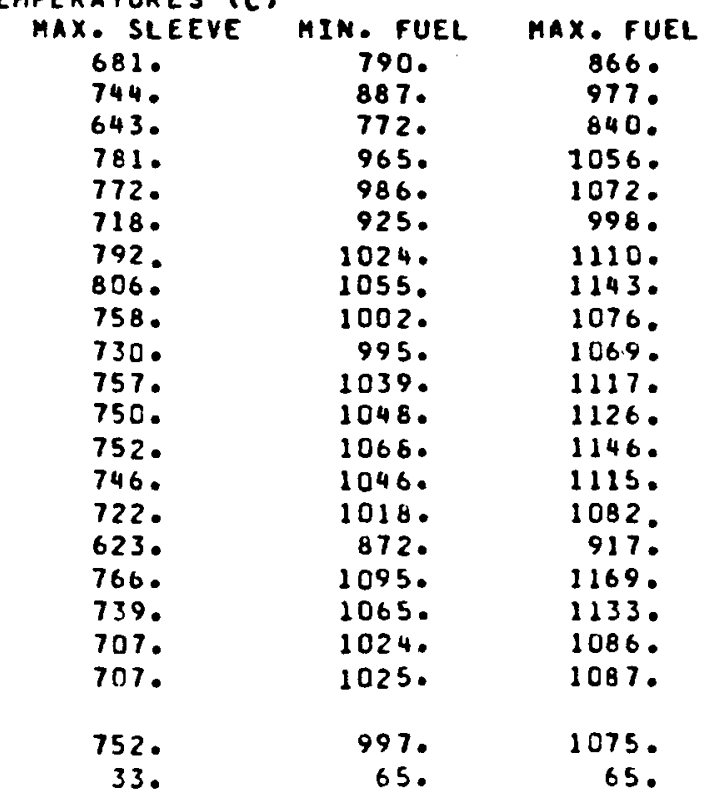

FAST FLUENCE

$110 * 25 N / M * 2$

.00
.10
.27

.27

.30

.91
1.14

1.30

1.51

1.70

2.17

2.43

2.62

2.99

3.18

3.34

3.46

3.53

3.63

3.63

3.80
3.83 
EO1-01 COMPACT 11

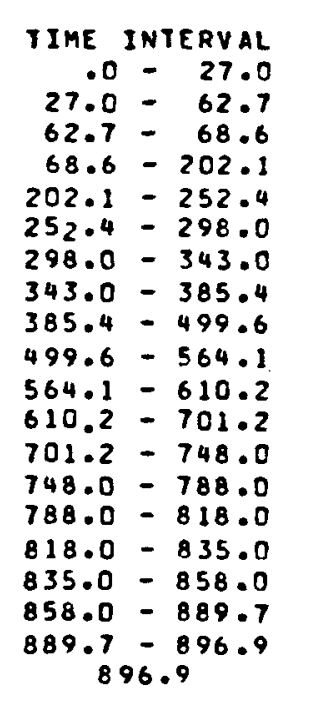

TIME AVERAGED RMS
COOLANT

458 .

458 .

480.

480.

474.

455.

475.

479.

468.

456.

462 .

454.

457 .

459.

459.

420.

465.

445.

445 .

465.

12.

TEMPERATURES (C)

AIA. SLEEVE
662.
713.
619.
719.
693.
646.
699.
707.
677.
647.
667.
661.
662.
668.
648.
574.
674.
657.
634.
634.
676.
29.

MAX. SLEEVE

MIN. FUEL 806.
906.
787.

762.

798.

787.

730.

821.

773.

747.

747.

769.

770.

765.

736.

635.
781.

781.

721.

721.

787.

984.

1004 .

1044 .

1075 .

1022 .

1022 .

1068 .

1076 .

1099 .

1077.

1042 .

894.

1121.

1091 .

1049.
1050.

996

1015.

1130 .

1165.

1097 .

1098.

1158.

1178.

1148.

940.

$11960^{\circ}$

1160 .

1112 .

768.

1020.

1100 .

68.68 .

EO1-01 COMPACT 12

TIME INTERVAL

$$
.0-27.0
$$

$27.0-62.7$

$62.7-68.6$

$68.6-202.1$

$202.1-252.4$

$252.4-298.0$

$298.0-343.0$

$343.0-385.4$

$385.4-499.6$

$499.6-564.1$

$564.1-610.2$

$610.2-701.2$

$701.2-748.0$

$748.0-788.0$

$788.0-818.0$

$818.0-835.0$

$835.0-858.0$

$858.0-889.7$

$889.7-896.9$

896.9

IIAE AVERAGED RMS

COOLANT MIA. SLEEVE MAX. SLEEVE

471.

495 .

450.

496.

488 .

467.

489.

493.

481.

475.

467.
470.

471 .

430.

479.

468

457.

457.

478.

13.
710.

777.

668.

800 .

741.

820 .

834.

785 .

762 .

791.

785.

781.

750 .

647.

797.

769.

735.

735.

691.

29.
MIN. FUEL

819.

920.

798.
998.

1018 .

954.

1059 .

1091 .

1037.

1043.

1090.

1101 .

1122.

1100.

1063.

914.

1143.

1113.

1071 .

1071.

1038.

71.
883.

855.

1076.

1091 .

1148 .

1107.

FAST FLUENCE

(10*25 $N / M * 2)$

.00

.10
.27

.30

.92
.15

1.32

1.53

1.53

1.71
2.20

2.46

.66

3.03

3.23

3.39

3.52

3.58

3.69

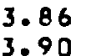

FAST FLUENCE

$120 * 25$ N/M**21

MAX. FUEL

895 .

1011.

866.

1089 .

1106.

$1145^{\circ}$

1112 .

1120 .

1172 .

1182 .

1203 .

1172.

1129.

960 .

1220 .

1183.

1134 .

1135.

.00

.10

.27
.30

.92

1.15

1.53

.72

2.21

2.48

2.68

3.06

3.26

3.43

3.56

3.62

3.74

3.90

3.94

1118 . 
EO1-01 COAPACT 13

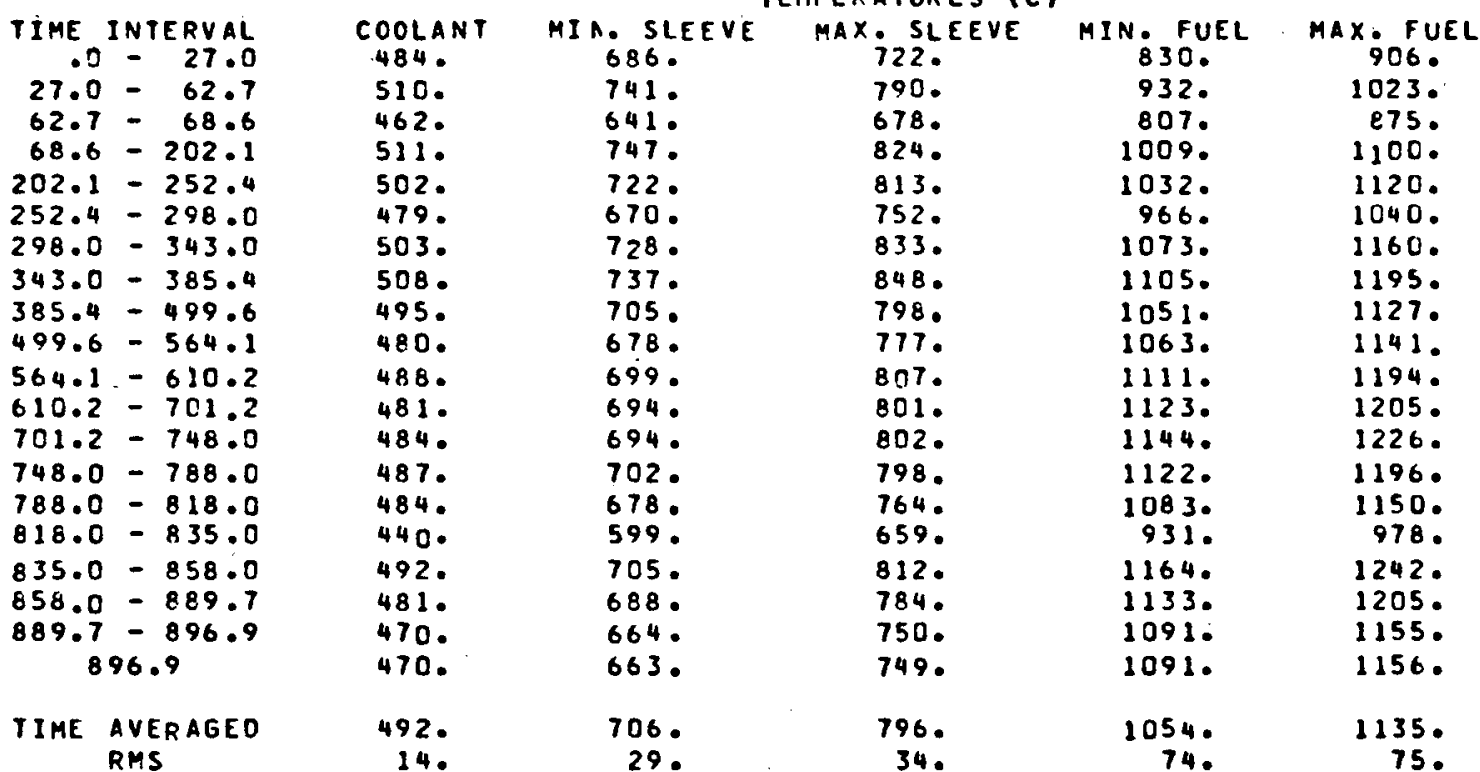

FAST FLUENCE

$(20 * 25 N / M * 2)$ .00
.10
.27
.30
.92
1.15
1.33
1.54
1.73
2.22
2.50
2.70
3.09
3.29
3.45
3.59
3.65
3.77
3.94
3.98

\section{E01-01 COMPACT I4} TIRE INTERVAL $27.0=27.0$ $62.7-68.6$ $68.6-202.1$ $202.1-252.4$ $252.4-298.0$ $298.0=343.0$ $343.0-385.4$

$385.4=499.6$ $499.6-564.1$ $564.1-610.2$ $610.2-701.2$ $701.2-748.0$ $748.0-788.0$ $788.0-818.0$ $818.0=835.0$ $835.0-858.0$ $858.0-889.7$ $889.7-896.9$ 896.9

IIME AVERAGED RMS

TEMPERATURES (C)

\begin{tabular}{|c|c|c|c|c|}
\hline \multirow[b]{2}{*}{$\begin{array}{l}\text { COOLANT } \\
497 . \\
525 .\end{array}$} & & \multirow[b]{2}{*}{$\begin{array}{c}\text { MIN. FUEL } \\
840 . \\
943\end{array}$} & \multirow[b]{2}{*}{$\begin{array}{c}\text { MAX. FUEL } \\
915 \\
1032\end{array}$} \\
\hline & $\begin{array}{c}\text { MIA. SLEEVE } \\
697 . \\
753 .\end{array}$ & $\begin{array}{c}\text { MAX. SLEEVE } \\
733 . \\
803 .\end{array}$ & & \\
\hline $\begin{array}{l}473 . \\
526 \\
516\end{array}$ & $\begin{array}{l}650 . \\
760 . \\
735\end{array}$ & $\begin{array}{l}687 . \\
835 . \\
824 .\end{array}$ & $\begin{array}{l}815 . \\
1018 . \\
1042 .\end{array}$ & $\begin{array}{r}882 . \\
1108 . \\
1129 .\end{array}$ \\
\hline $\begin{array}{l}491 . \\
517 . \\
522 .\end{array}$ & $\begin{array}{l}681 . \\
741 . \\
750 .\end{array}$ & $\begin{array}{l}761 . \\
844 . \\
859 .\end{array}$ & $\begin{array}{l}974 . \\
1083 . \\
1115 .\end{array}$ & $\begin{array}{l}1049 . \\
1170 \\
1205\end{array}$ \\
\hline $\begin{array}{l}508 . \\
492 . \\
502 .\end{array}$ & $\begin{array}{l}717 \\
690 \\
712\end{array}$ & $\begin{array}{l}809 . \\
789 \\
820\end{array}$ & $\begin{array}{l}10410 \\
1075 \\
1124 .\end{array}$ & $\begin{array}{l}1137 \\
1154 \\
1207\end{array}$ \\
\hline $\begin{array}{l}494 . \\
497^{\circ}\end{array}$ & $\begin{array}{l}707 \\
708 \text {. }\end{array}$ & $\begin{array}{l}814 . \\
815 .\end{array}$ & $\begin{array}{l}1136 . \\
1156^{\circ}\end{array}$ & $\begin{array}{l}1219^{\circ} \\
1239^{\circ}\end{array}$ \\
\hline 501. & 716. & 812. & 1136. & 1209. \\
\hline $\begin{array}{l}497 . \\
451 .\end{array}$ & $\begin{array}{l}693 . \\
611 .\end{array}$ & $\begin{array}{l}779 . \\
671 .\end{array}$ & $\begin{array}{r}1100 . \\
946 .\end{array}$ & $\begin{array}{r}1167 . \\
993 .\end{array}$ \\
\hline $\begin{array}{l}506 . \\
495 \\
483\end{array}$ & $\begin{array}{l}722 . \\
704 \\
679\end{array}$ & $\begin{array}{l}829 . \\
800 . \\
764 .\end{array}$ & $\begin{array}{l}1181 . \\
1150 . \\
1107\end{array}$ & $\begin{array}{l}1260 \\
1223 \\
1173\end{array}$ \\
\hline 483. & 678. & 764. & 1108. & 1173 \\
\hline $\begin{array}{r}506 . \\
15 .\end{array}$ & $\begin{array}{r}719 . \\
29 .\end{array}$ & $\begin{array}{r}808 . \\
34 .\end{array}$ & $\begin{array}{r}1066 . \\
76 .\end{array}$ & $\begin{array}{r}1147^{\circ} \\
77^{\circ}\end{array}$ \\
\hline
\end{tabular}

FAST FLUENCE (10*25 $N / M * 21$

.00
.10
.27
.29
.91
1.14
1.32
1.53
1.72
2.21
2.49
2.69
3.08
3.28
3.45
3.59
3.65
3.77
3.94
3.98

.29

.91
1.14

1.32

1.53

1.72

2.49

2.69

3.08

3.28

3.59

3.65

3.94

3.98 
EO1-01 COMPACT 15

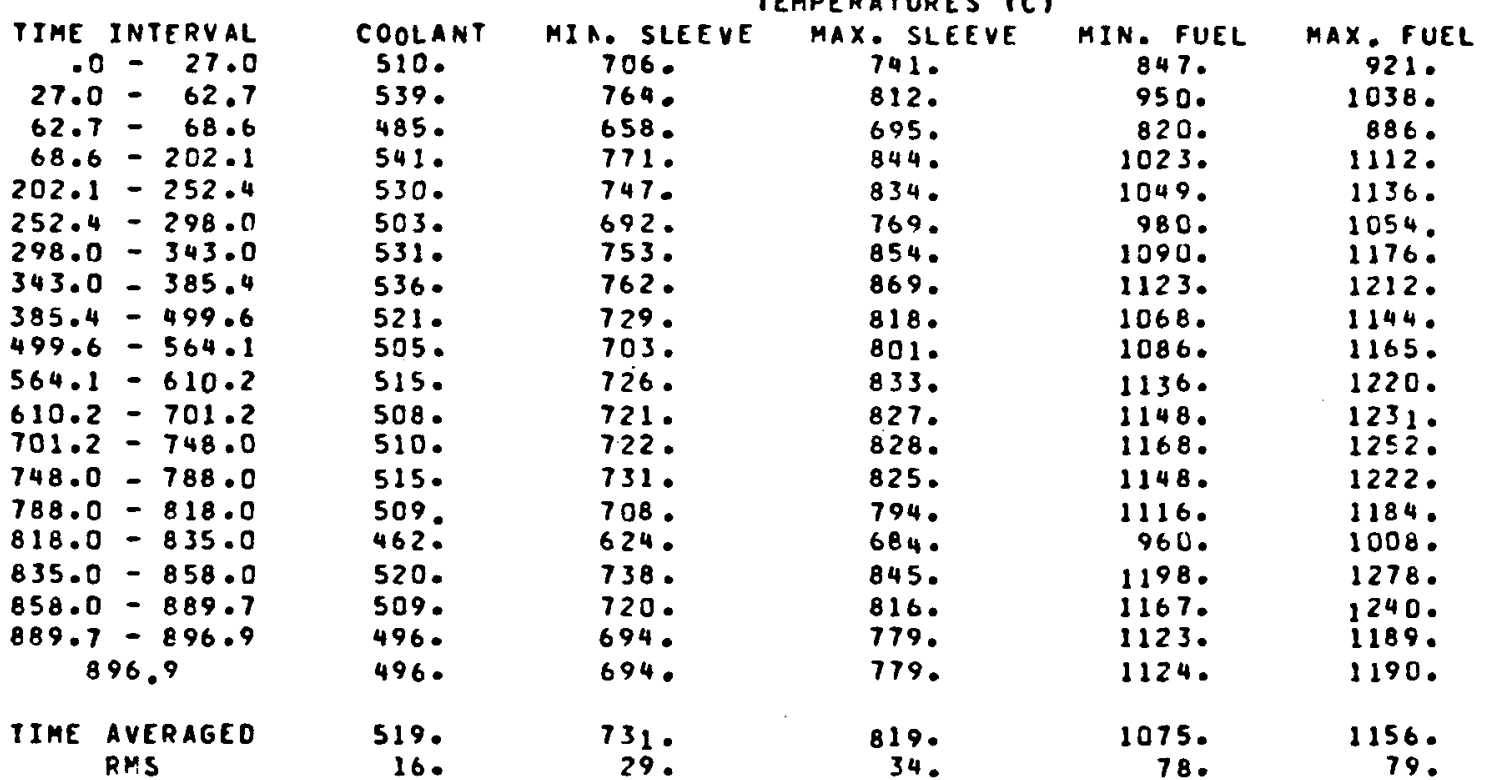

FAST FLUENCE

(10*23 N/M**2)

$$
.00
$$

.10
.27

.29

.90

1.13

1.52

1.71

2.20

2.48

2.69

3.08

3.28

3.45

3.66

3.77

3.95

EO1-01 COMPACT 16

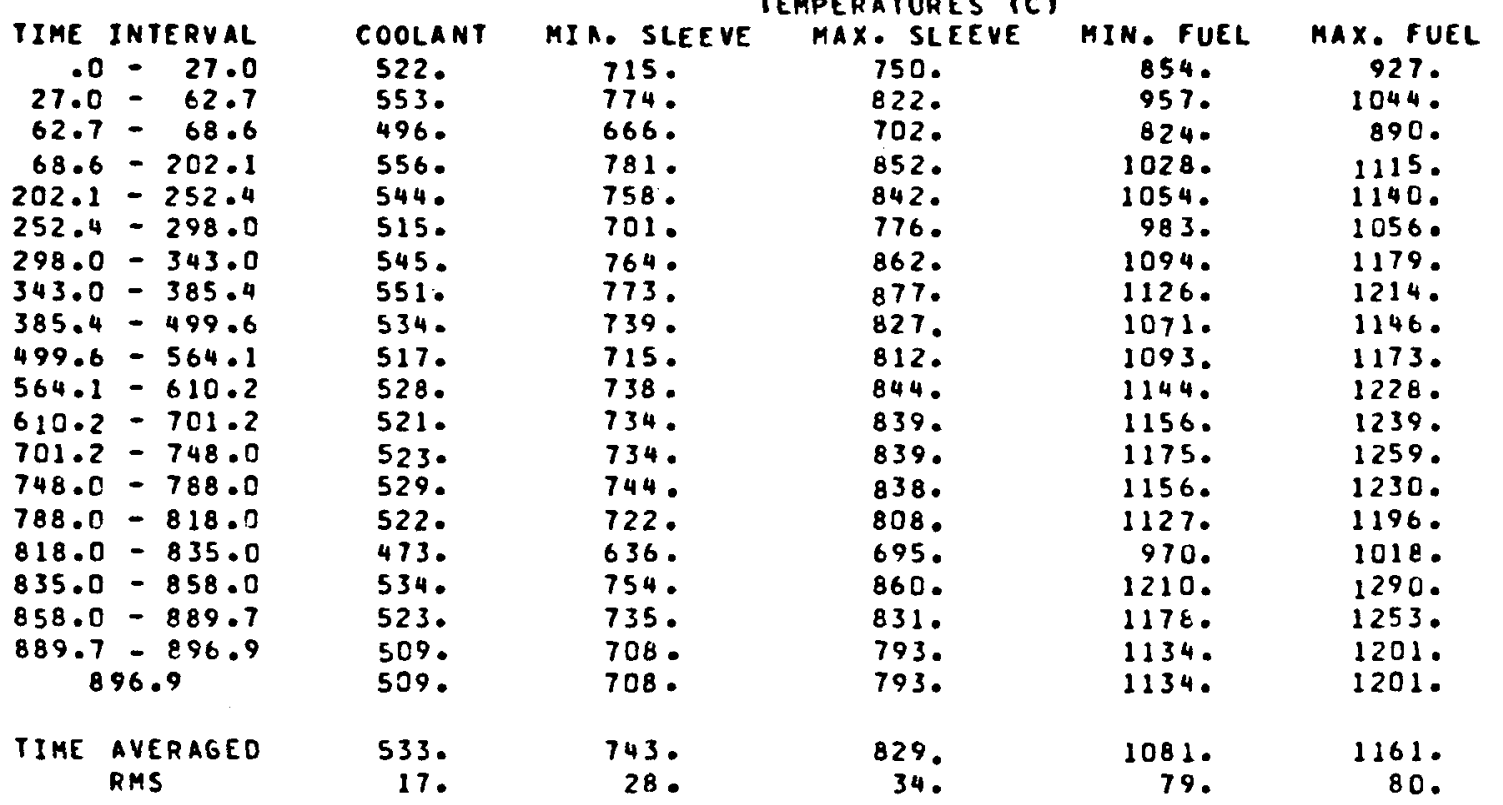

FAST FLUENCE

$(10 * 25 N / M * 2)$

.00
.10
.26
.29
.89
1.12
1.29
1.49
1.68
2.17
2.45
2.65
3.04
3.25
3.42
3.55
3.62
3.74
3.92
3.96


COI-DL COMPACY 17

TIME INTERYAL COOLANT MIA. SLEEVE TEMPERATURES (C)

$27.0=27.0$

$68.6-202.1$

$202.1-252.4$

$252.4-298.0$

$298.0-343.0$

$343.0-385.4$

$385.4-499.6$

$499.6-564.1$

$564.1-610.2$

$610.2-701.2$

$701.2-748.0$

$746.0-788.0$

$788.0=818.0$

$818.0-835.0$

$835.0-858.0$

$858.0-889.7$

$889.7-896.9$

896.9

TIME AVERAGED

RMS
$62.7-68.6$

\section{4. \\ 722 .}

567.

570 .

570.

526 .

559 .

564.

547.

529.

541.

534.

537 .

543.

536.

484.

549.

537 .

522.

522 .

546.
17.

781.

672 .

789.

708 .

773 .

782.

747 .

726.

750 .

746.

746.

737 .

649.

770.
752.
724.

723 .

753.

28.

MAX. SLE
756.

756.
828.
706.

706.

848.

780.

883.

832.

822 .

855.

849.

850 .

824.

708 .

877 .

847 .

809 .

808 .

838.

34.
MIN. FUEL

857.
959.

959.

825.
1026.

1026.

982 .

1092 .

1124.

1069.

1097.

1148.

1159.

1179.

1160.

1138.

979.

1222.

1190.

1145 .

1145 .

1083.

81.

E01-01 COMPACT 18

$\frac{p}{0}$

IIME INTERVAL

$$
.0-27.0
$$

$27.0-62.7$

$62.7-68.6$

$68.6-202.1$

$202.1-252.4$

$252.4-298.0$

$298.0-343.0$

$343.0-385.4$

$385.4-499.6$

$499.6-564.1$

$564.1-610.2$

$610.2-701.2$

$701.2-748.0$

$748.0-788.0$

$788.0-818.0$

$818.0-835.0$

$835.0-858.0$

$858.0-889.7$

$889.7-896.9$

896.9

TIME AVERAGEO RMS

$\begin{array}{cc}\text { COOLANT } & \text { HIA. SLEEVE } \\ 546 . & 728 . \\ 581 . & 788 . \\ 517 . & 677 . \\ 584 . & 796 . \\ 571 . & 775 . \\ 537 . & 715 . \\ 572 . & 781 . \\ 578 . & 791 . \\ 560 . & 755 . \\ 541 . & 736 . \\ 554 . & 761 . \\ 548 . & 757 . \\ 550 . & 757 . \\ 557 . & 769 . \\ 549 . & 752 . \\ 495 . & 661 . \\ 563 . & 787 . \\ 551 . & 768 . \\ 536 . & 739 . \\ 535 . & 738 . \\ & \\ 559 . & 762 . \\ 18 . & 27 .\end{array}$

TEMPERATURES (C)
MAX. SLEEVE
761 .

834.

710.
861.

861.

785 .

872 .

888 .

838 .

830 .

864.

659.

859.

839.

720.

893.

863.

824

845.

34.
MIN. FUEL

MAX. FUEL

859.928 .

960.11042.

$\begin{array}{rr}825 . & 887 . \\ 1025 . & 1107 .\end{array}$

$1053 . \quad 1135$.

$980 . \quad 1050$.

1091.1172.

1122.1206.

1067.1139 .

$1090^{\circ} \quad 1177$.

$\begin{array}{ll}1150 . & 1232 . \\ 1161 . & 1243 .\end{array}$

1179.21262 .

$1162 . \quad 1235$.

$1148 . \quad 1219$.

987.1037.

1233.1315 .

1200.1276 .

$\begin{array}{ll}11540^{\circ} & 12233^{\circ} \\ 11555^{\circ} & 12230^{\circ}\end{array}$

1084.

82.

1162.
FASI FLUENCE

$(10 * 25 \quad N / M * * 2)$

.00

.10
.26

.28

.87

1.09

1.26
1.46

1.46

2.13

2.40

2.60

2.99

3.19

3.36

3.50

3.57

3.69

3.87

3.91

FAST FLUENCE

(10*25 $N / R * 2$,

.00

.09

.25
.27

.85
1.07

1.43

1.60

2. 08

2.35
2.55

2.94

3.13

3.31

3.45

3.52

3.64

3.81

3.85 
EOI-01 COMPACT 19

TIME INTERYAL $.0-27.0$ $27.0-62.7$ $62.7-68.6$ $68.6-202.1$ $202.1-252.4$ $252.4-298.0$ $298.0^{\circ}-343.0$ $343.0-385.4$ $385.4-499.6$ $499.6-564.1$ $564.1-610.2$ $610.2-701.2$ $701.2-748.0$ $748.0-788.0$ $788.0-818.0$

$818.0-835.0$ $835.0-858.0$ $858.0-889.7$ $889.7-896.9$ 896.9

TIME AVERAGEO RMS

\begin{tabular}{|c|c|}
\hline $\begin{array}{l}\text { COOLANT } \\
558 .\end{array}$ & $\begin{array}{c}\text { MI } \mathrm{A}, \mathrm{SLEEV} \\
734 .\end{array}$ \\
\hline $\begin{array}{l}594 . \\
527^{\circ} \\
598^{\circ}\end{array}$ & $\begin{array}{l}795 . \\
683 . \\
803 .\end{array}$ \\
\hline $\begin{array}{l}584^{\circ} \\
548 .\end{array}$ & $\begin{array}{l}782 . \\
721 .\end{array}$ \\
\hline $\begin{array}{l}585 . \\
591 . \\
572^{\circ}\end{array}$ & $\begin{array}{l}780^{\circ} \\
797 . \\
761\end{array}$ \\
\hline 554 . & 745. \\
\hline $\begin{array}{l}567 . \\
561 .\end{array}$ & $\begin{array}{l}771 . \\
767 .\end{array}$ \\
\hline $\begin{array}{l}563 . \\
570 . \\
562 .\end{array}$ & $\begin{array}{l}7670^{\circ} \\
7790^{\circ} \\
768^{\circ}\end{array}$ \\
\hline $\begin{array}{l}506 . \\
578^{\circ} \\
565 .\end{array}$ & $\begin{array}{l}6744^{\circ} \\
8030^{\circ} \\
784 .\end{array}$ \\
\hline $\begin{array}{l}549 . \\
549 .\end{array}$ & $\begin{array}{l}754 . \\
754 .\end{array}$ \\
\hline $\begin{array}{l}572 . \\
18 .\end{array}$ & $\begin{array}{r}771 . \\
27 .\end{array}$ \\
\hline
\end{tabular}

TEMPERATURES (C)

MAX. SLEEYE

767.
840.
715.
865.

862. 962. 963.

826.
1024.

856

787.

874.

890 .

840.

838.
872.

867 .

867.

868.

855 .

733.

910.

839 .

839.

852.
34.

34 .

1048

1084 .

1115.

1061.

1098 .

1149.

1177 .

1260.

1157.

995 .

1242 .

1210 .

1163.

1164.

1083.

83.

MAX FUEL 930.

1043.

886.

1104.

1043.

1164.

1197.

$1130^{\circ}$

1175.

1240 .

1258.

1233 .

1229.

1045

1325

1233
1233.

1159.

86.
FAST FLUENCE

$(10 * 25 N / M * 2)$

.00

.09

.24
.26

.82

1.03

1.19

1.39

1.56

2.03
2.29

2.49

2.87

3.06

3.24

3.38

3.45

3.57

3.74
3.78

E01-01 COMPACT 20

TIME INTERVAL

$$
\begin{aligned}
& .0-27.0 \\
& .0-62.7
\end{aligned}
$$

$$
\begin{array}{r}
.0=27.0 \\
27.0=62.7
\end{array}
$$

$62.7-68.6$

$68.6-202.1$

$202.1-252.4$

$252.4-298.0$

$298.0-343.0$

$343.0-385.4$

$385.4-499.6$

$499.6-564.1$

$564.1-610.2$

$610.2-701.2$

$701.2-748.0$

$748.0-788.0$

$788.0-818.0$

$818.0-835.0$

$835.0-858.0$

$858.0-889.7$

$89.7-896.9$

896.9

IIME AVERAGED RMS
TEMPERATURES (C)

$\begin{array}{cc}\text { COOLANT MIA. SLEEV } \\ 569 . & 739 . \\ 607 . & 800 . \\ 537 . & 687 . \\ 611 . & 809 . \\ 596 . & 787 . \\ 559 . & 725 . \\ 597 . & 793 . \\ 604 . & 802 . \\ 584 . & 766 . \\ 565 . & 755 . \\ 580 . & 781 . \\ 574 . & 777 . \\ 575 . & 777 . \\ 584 . & 790 . \\ 576 . & 781 . \\ 518 . & 686 . \\ 592 . & 818 . \\ 580 . & 799 . \\ 563 . & 768 . \\ 562 . & 768 . \\ 584 . & 779 . \\ 19 . & 27 .\end{array}$

MAX. SLEEVE

\begin{abstract}
770.
\end{abstract}
843.
718.

718.
868.

858 .

787 .

875.

890 .

842 .

845 .

880.

875 .

877.

869.

745.
925.

925.

853.

852 .

857.

35 .
MIN. FUEL MAX. FUEL

863.

962.

825.
1020.

1041 .

966.

1075.

1105 .

1051.

1096.

1146.

1174 .

1158 .

1162 .

999.

1248 .

1215 .

1168 .

1169.

1079.

85.

928.

1039.

883.
1097.

1118.

1032.

1152 .

1184 。

1118 .

1172 .

1227 .

1237 .

1254.

1230 .

1234.

1049.

1331 .

1292 .

1238 .

1238 .

1154.

88.
FAST FLUENCE

(10*25 N/M**2)

$$
.00
$$

.09

.23
.25

.79

1.00

1.15

1.34

1.50

1.96
2.22

2.42

2.79

2.98

3.15

3.36

3.48

3.66

3.70 
COI-01 COMPACT 21

TIME INTERVAL $.0-27.0$ $27.0-62.7$ $62.7=68.6$
$68.6-202.1$ $68.6-202.1$
$202.1-252.4$ $202.1-252.4$ $252.4-298.0$ $298.0-343.0$ $343.0-385.4$ $385.4-499.6$ $499.6-564.1$ $564.1-610.2$ $610.2=701.2$ $701.2-748.0$ $748.0=788.0$ $788.0-818.0$ $818.0-835.0$ $835.0-858.0$ $858.0-889.7$ $889.7-896.9$ 896.9

TIME AVERAGEO RMS

TIME INTERVAL $.0=27.0$
$27.0=62.7$ $62.7-68.6$ $68.6-202.1$ $202.1-252.4$ $252.4-298.0$ $298.0-343.0$ $343.0-385.4$ $385.4-499.6$ $499.6-564.1$ $564.1-610.2$ $610.2-701.2$ $701.2-748.0$ $748.0-788.0$ $788.0-818.0$ $818.0-835.0$ $835.0-858.0$ $858.0-889.7$ $889.7-896.9$ 896.9

IIME AVERAGEO RMS

COOLANT
580.
619.
546.
623.
608.
569.
609.
616.
595.
577.
592.
587.
588.
597.
589.
529.
607.
594.
576.
576.
597.
19.

MIA. SLEEVE

IEMPERATURES (C)

741.
803.
689.
812.
790.
727.
796.
805.
769.
762.
789.
785.
785.
798.
795.
697.
833.
813.
781.
781.
785.
27.

MAX. SLE IC

MIN. FUEL

841.

844.

867.

856.

785.

873 .

888.

841.

850 .

885 .

880.
880 .

883 .

781.

938 .

907.

865.

859.
37.

860.

958 .

820 .

1011 .

1029.

1062 .

1090 .

1037.

1089.

1139.

1149.

1165.

1150.

1164.

1249 .

1217.

1169.

1170 .

1071.

86.

MAX F FUEL

922 .

1031.

875.
1085.

1017.

1135 .

1166.

1101.

1164.

1216.

1227 .

1243 .

$1230^{\circ}$

1333.

1294.

1239 .

1143.

\section{E01-01 COMPACT 22}

TEMPERATURES (C)

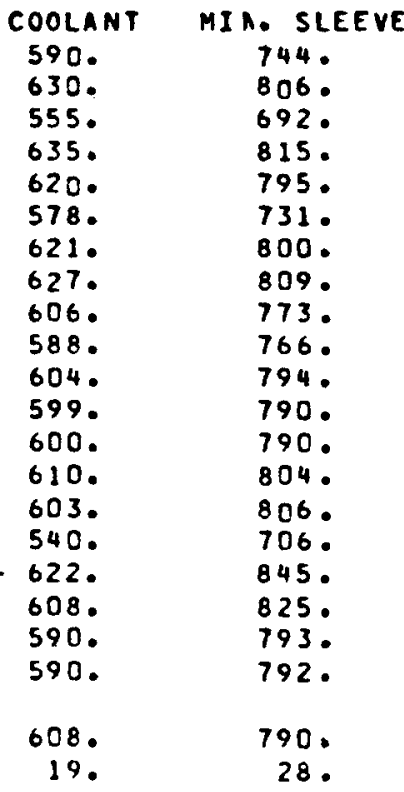

MAX. SLEEVE

773.

846.

867.

856.

785 .

872 .

888 .

841 .

885 .

881 .

881 .

885 .

892 .

764.

949.

918.

875.

875.

861.
38.
HIN. FUEL 859.

954.

1004.

1021.

945.

1051.

1026.

1125 .

1134.

1149.

1136.

1161.

996.

1246.

1214.

1166.

1166.

1061.
85.
1103 .

1220 .

FAST FLUENCE

$(10 * 25 N / M * 2)$

.00
.08
.22
.24
.76
.95
1.10
1.28
1.44
1.88
2.14
2.33
2.69
2.87
3.05
3.18
3.26
3.37
3.55
3.59

90 .
FAST FLUENCE

$(10 * 25 N / M * * 2)$

MAX, FUE

918.

869 .

1074 .

1092 .

1006 .

1122 .

1152.

1088 .

1149 .

1202 .

$121 \mathrm{C}$.

1225 .

1204.

1232 .

1046 .

1329 .

1290.

1235 .

1235 .

1131.
.00

.21

.23

.72

.91

1.05

1.22
1.37

1.79

2.04

2.22

2.57

2.75

2.93

3.06

3.14

3.25

3.43 
E01-01 COMPACT 23

\begin{tabular}{|c|c|c|c|c|c|c|c|}
\hline TIME & INTERVAL & COOLANT & MI . SLEEVE $^{\text {SLEV }}$ & $\begin{array}{l}\text { MPERATURES (C) } \\
\text { MAX. SLEEVE }\end{array}$ & MIN. FUEL & MAX. FUEL & $\begin{array}{l}\text { FAS T FLUENCE } \\
(10 * 25 \quad N / H * 2)\end{array}$ \\
\hline 27.0 & $\begin{array}{l}-\quad 27.0 \\
-\quad 62.7\end{array}$ & $\begin{array}{l}599 . \\
641 .\end{array}$ & $\begin{array}{l}744 . \\
806\end{array}$ & $\begin{array}{l}771 . \\
843 .\end{array}$ & $\begin{array}{l}852 . \\
945 .\end{array}$ & $\begin{array}{r}908 . \\
1011 .\end{array}$ & $\begin{array}{l}.00 \\
.08\end{array}$ \\
\hline 62.7 & $-\quad 68.6$ & 563. & 691. & 717. & 808 . & 858. & .20 \\
\hline $\begin{array}{r}68.6 \\
202.1 \\
252.4\end{array}$ & $\begin{array}{l}-202.1 \\
-252.4 \\
-298.0\end{array}$ & $\begin{array}{l}646 . \\
630 . \\
587 .\end{array}$ & $\begin{array}{l}8150^{\circ} \\
7950^{\circ} \\
731 .\end{array}$ & $\begin{array}{l}863 . \\
852 . \\
781 .\end{array}$ & $\begin{array}{r}991 . \\
1005 . \\
929 .\end{array}$ & $\begin{array}{l}1057 . \\
10720^{\circ} \\
986 .\end{array}$ & $\begin{array}{l}.22 \\
.68 \\
.86\end{array}$ \\
\hline $\begin{array}{l}298.0 \\
343.0\end{array}$ & $\begin{array}{l}-343.0 \\
-385.4\end{array}$ & $\begin{array}{l}632 \\
638\end{array}$ & $\begin{array}{l}800 \\
810\end{array}$ & $\begin{array}{l}866 . \\
882 .\end{array}$ & $\begin{array}{l}1033 . \\
1059 .\end{array}$ & $\begin{array}{l}1099 . \\
1128\end{array}$ & $\begin{array}{r}.99 \\
1.15\end{array}$ \\
\hline $\begin{array}{l}610.2 \\
701.2 \\
748.0\end{array}$ & $\begin{array}{l}-701.2 \\
-748.0 \\
-768.0\end{array}$ & $\begin{array}{l}611 . \\
612 . \\
622 .\end{array}$ & $\begin{array}{l}795 . \\
704 . \\
809 .\end{array}$ & $\begin{array}{l}882 . \\
882 . \\
887 .\end{array}$ & $\begin{array}{l}1119.0 \\
11320 \\
1121 .\end{array}$ & $\begin{array}{l}1192 . \\
12050^{\circ} \\
11860^{\circ}\end{array}$ & $\begin{array}{l}2.12 \\
2.45 \\
2.63\end{array}$ \\
\hline $\begin{array}{l}788.0 \\
818.0\end{array}$ & $\begin{array}{l}-818.0 \\
-835.0\end{array}$ & $\begin{array}{l}616 . \\
551 .\end{array}$ & $\begin{array}{l}816 . \\
715 .\end{array}$ & 901. & $\begin{array}{r}1155 . \\
990 .\end{array}$ & $\begin{array}{l}1226 . \\
1040 .\end{array}$ & $\begin{array}{l}2.80 \\
2.93\end{array}$ \\
\hline 835.0 & -858.0 & 636. & 856. & 958. & 1240. & 1322 & 3.01 \\
\hline
\end{tabular}

E01-01 COMPACT 24

TIME INTERVAL $00-27$ $27.0-27.0$ $62.7-62.7$ $68.6-202.1$ $202.1-252.4$ $252.4-298.0$ $298.0-343.0$

$343.0-385.4$

$385.4-499.6$

$499.6-564.1$

$564.1-610.2$

$610.2-701.2$

$701.2-748.0$

$748.0-788.0$

$788.0-818.0$

$818.0-835.0$

$835.0-858.0$

$858.0-889.7$

$889.7-896.9$

896.9

TIME AVERAGED RMS

TEMPERATURES (C)

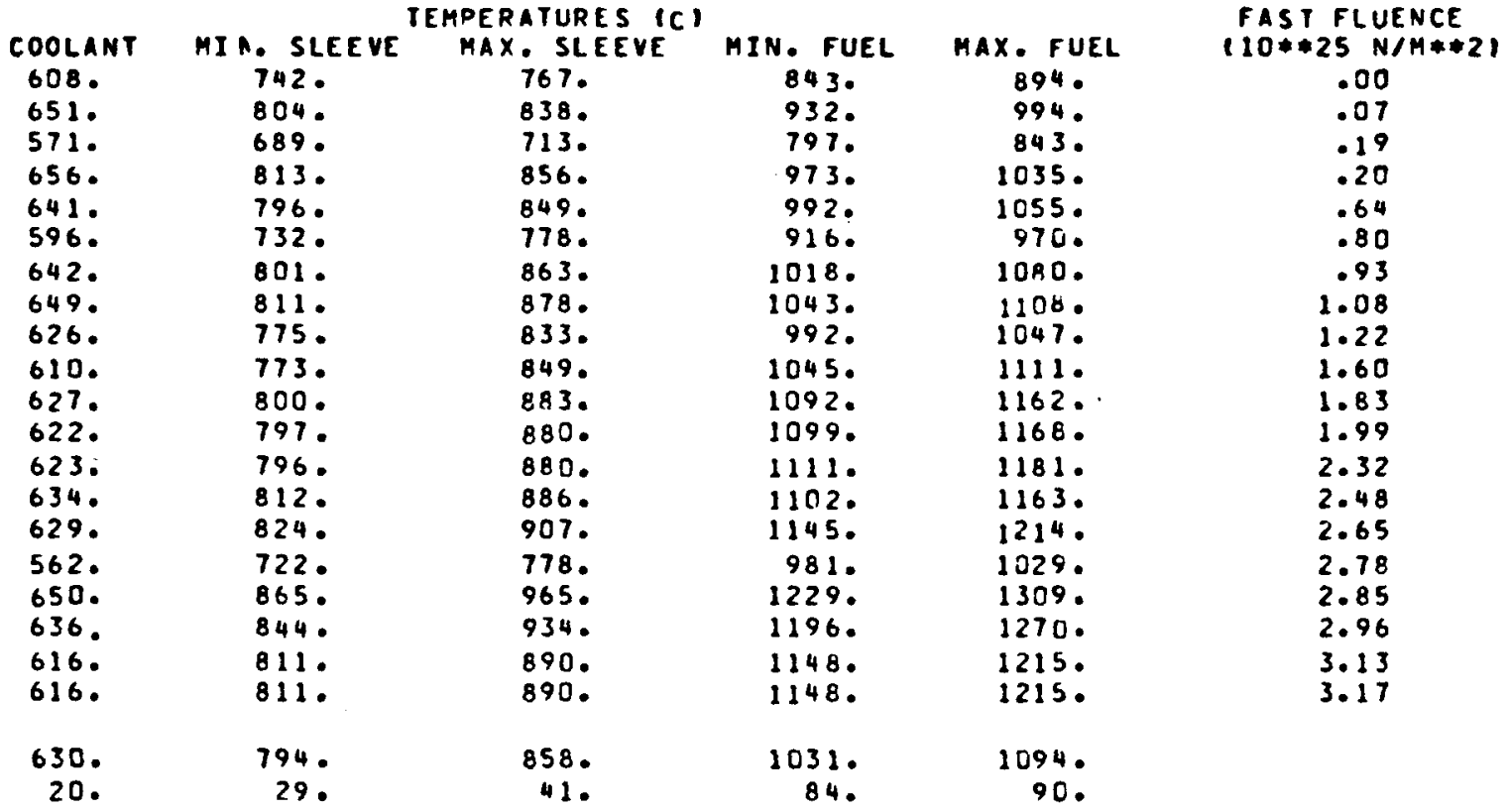


EOL-OI COMPACT 25

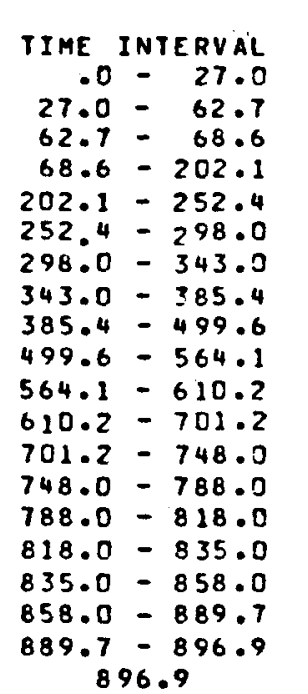

TIME AVERAGED RMS

COOLANT
616.
661.
578.
666.
650.
604.
651.
659.
635.
619.
637.
632.
633.
645.
641.
573.
664.
650.
629.
629.

640.

20.

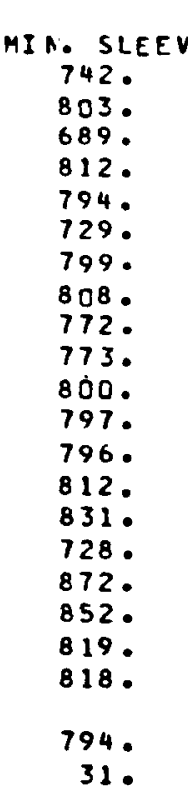

TEMPERATURES (C)

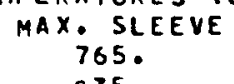

$$
\begin{aligned}
& 765 . \\
& 835 .
\end{aligned}
$$

$$
835 .
$$

$$
852 \text {. }
$$$$
841 .
$$

$$
771 .
$$

$$
853 .
$$

$$
843 .
$$

$$
878 \text {. }
$$

875 .$$
982 .
$$

$$
912 .
$$

$$
970 .
$$

$$
\begin{aligned}
& 939 . \\
& 895 . \\
& 895 .
\end{aligned}
$$

MIN

MIN. FUEL

836.
924.
789.

924.

960.

971.

994.

1018.

968 .

1022 .

1074 .

1084 .

1077.

1132.

968 .

1214.

1182.

1133.

853.

1012.

82 .

MAX. FUEL

885 .

1018 .

1029.

1052 .

1078.

1019.

1134 .

1139.

$115 \mathrm{C}$.

1135 .

1199.

1015 .

1253 .

$1198^{\circ}$.

1071.

EO1-01 COMPACT 26

TIME INTERVAL

$$
\begin{array}{r}
\text { IME INTERVAL } \\
.0-27.0
\end{array}
$$

$.0=27.0$
$27.0-62.7$

$62.7-68.6$

$68.6-202.1$

$202.1-252.4$

$252.4-298.0$

$343.0=385.4$

$343.0=385.4$
$385.4=499.6$

$499.6=564.1$

$564.1-610.2$

$610.2=701.2$

\begin{tabular}{|c|c|c|c|c|}
\hline $\begin{array}{l}\text { COOLANT } \\
624 . \\
669 . \\
585 \\
675 .\end{array}$ & $\begin{array}{c}\text { MIA. SLEEVE } \\
739 . \\
800 . \\
687 . \\
809 .\end{array}$ & $\begin{array}{l}\text { MAX. SLEEVE } \\
760 . \\
829 . \\
707 . \\
845 .\end{array}$ & $\begin{array}{l}\text { MIN. FUEL } \\
827 . \\
911 . \\
778 . \\
944 .\end{array}$ & $\begin{array}{c}\text { MAX. FUEL } \\
871 . \\
964 . \\
818 . \\
997 .\end{array}$ \\
\hline $\begin{array}{l}659 . \\
611 . \\
660 .\end{array}$ & $\begin{array}{l}790 . \\
726 . \\
795 .\end{array}$ & $\begin{array}{l}832 . \\
7630^{\circ} \\
843 .\end{array}$ & $\begin{array}{l}950 . \\
876 . \\
971 .\end{array}$ & $\begin{array}{r}1004 \\
921 . \\
1024 .\end{array}$ \\
\hline $\begin{array}{l}667 . \\
643 . \\
628\end{array}$ & $\begin{array}{l}804 . \\
769 . \\
772 .\end{array}$ & $\begin{array}{l}858 . \\
816 . \\
837\end{array}$ & $\begin{array}{r}993 . \\
944 . \\
1000 .\end{array}$ & $\begin{array}{r}1048 . \\
991 . \\
1059 .\end{array}$ \\
\hline $\begin{array}{l}647 . \\
642 . \\
643 .\end{array}$ & $\begin{array}{l}800 . \\
797 . \\
796 .\end{array}$ & $\begin{array}{l}871 . \\
869 . \\
869 .\end{array}$ & $\begin{array}{l}1044 . \\
1049 . \\
1058 .\end{array}$ & $\begin{array}{l}11060^{\circ} \\
1110^{\circ} \\
1119 .\end{array}$ \\
\hline $\begin{array}{l}656 . \\
654 \\
583\end{array}$ & $\begin{array}{l}812^{\circ} \\
8355^{\circ} \\
732^{\circ}\end{array}$ & $\begin{array}{l}877 . \\
913 . \\
784 .\end{array}$ & $\begin{array}{l}1052 . \\
1114 . \\
952 .\end{array}$ & $\begin{array}{r}1107 . \\
1178 . \\
997 .\end{array}$ \\
\hline $\begin{array}{l}677 . \\
663 .\end{array}$ & $\begin{array}{l}876 . \\
856 .\end{array}$ & $\begin{array}{l}970 . \\
940 .\end{array}$ & $\begin{array}{l}1193 . \\
1161 .\end{array}$ & $\begin{array}{l}1268 . \\
1230^{\circ}\end{array}$ \\
\hline 641. & 823 . & 896. & 1112. & 1175. \\
\hline 641. & $822^{\circ}$ & 896. & 1112. & 1175. \\
\hline $\begin{array}{r}650 \\
20\end{array}$ & $\begin{array}{r}792^{\circ} \\
32^{\circ}\end{array}$ & $\begin{array}{r}847^{\circ} \\
44^{\circ}\end{array}$ & $\begin{array}{r}991 . \\
80 .\end{array}$ & $\begin{array}{r}1046 . \\
86 .\end{array}$ \\
\hline
\end{tabular}

$701.2-748.0$

$748.0-788.0$

$786.0-818.0$

$818.0-835.0$

$835.0-858.0$

$858.0-889.7$

$889.7-896.9$

$$
896.9
$$

IIME AVEPAgEO

$$
\text { RMS }
$$

TEMPERATURES (C)
832 .

946.

1292 .

FAST FLUENCE

$110 * 25$ N/M* 21$$
.00
$$$$
\begin{array}{r}
.00 \\
.07 \\
.17
\end{array}
$$

.07
.17
.19

019

.74

.86

1.00
1.12

1.12
1.48

1.70

1.85

2.16

2.31

2.607

2.78

2.94
2.98

$8 B$.

FAST FLUENCE

$(10 * 25 N / H * 2)$

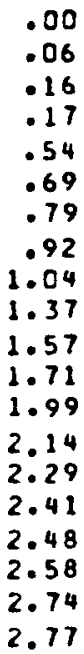

.06

.16

.54

.69
.79

.79
.92

1.04
1.37

1.57

1.71

2.14
2.29

2.41

2.48
2.58

2.74

2.77 
TOI-01 COMPACT 27

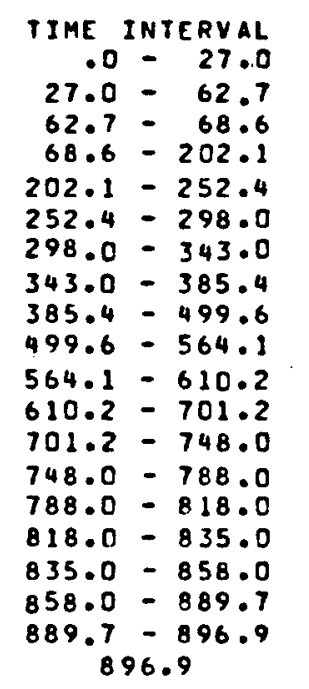

TIME AVERAGED RMS
TEMPERATURES (C)

$\begin{array}{cc}\text { COOLANT MIN. SLEEVE } \\ 631 . & 7350^{\circ} \\ 670^{\circ} & 796 . \\ 591 . & 683 . \\ 683 . & 804 . \\ 667 . & 787 . \\ 618 . & 723 . \\ 668 . & 792 . \\ 675 . & 801 . \\ 651 . & 766 . \\ 637 . & 769 . \\ 656 . & 797 . \\ 651 . & 794 . \\ 652 . & 793 . \\ 665 . & 809 . \\ 665 . & 833 . \\ 592 . & 730 . \\ 689 . & 874 . \\ 675 . & 854 . \\ 652 . & 821 . \\ 652 . & 820 . \\ 658 . & 789 . \\ 21 . & 32 .\end{array}$

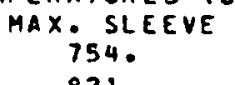

MIN. FUEL

$8150^{\circ}$

821.

836 .

824.

756.

834.

848.

827 .

861.

859.

869.

869.

778 .

961.

932.

889.

889 .

$766^{\circ}$

925.

930.

857.

949.

969.

974.

1017.

1020.

1028.

1024.

1084.
927.

1160 .

1128.

1080 .

838.

1080.

967.
75.

MAX FUEL

855 .

944.

802.

973.

$98 \mathrm{C}$.

899.

1020 .

965.
1028.

1028.

1077.

1077.

1074.

1143 .

968 .

1229.

11380

1017.

EO1-01 COMPACT 28

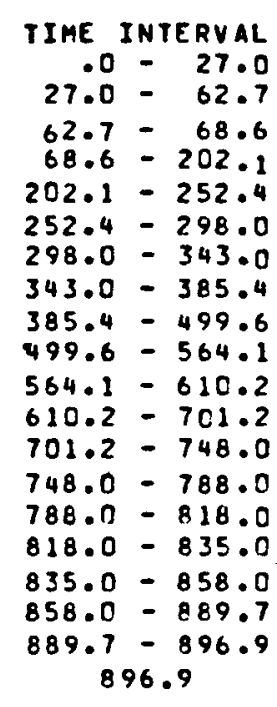

TIME AVERAGEO RMS

$\begin{array}{lc}\text { COOLANT } & \text { MIA. SLEEVE } \\ 637 . & 7320^{\circ} \\ 684 . & 792 . \\ 596 . & 680 . \\ 690^{\circ} & 801 . \\ 674 . & 782 . \\ 624 . & 718 . \\ 675 . & 786 . \\ 683 . & 795 . \\ 657 . & 761 . \\ 644^{\circ} & 764 . \\ 664 . & 791 . \\ 660 . & 788 . \\ 660 . & 787 . \\ 674 . & 804 . \\ 675 . & 830 . \\ 601 . & 728 . \\ 700 . & 870 . \\ 686 . & 850 . \\ 663 . & 817 . \\ 662 . & 817 . \\ 660^{\circ} & 784 . \\ 21 . & 32 .\end{array}$

TEMPERATURES (C)

MAX SLEEVE
749.
815.
697.
829.
813.
746.
822.
835.
796.
814.
848.
846.
847.
857.
895.
772.
949.
921.
880.
880.
827.
43.

IN. FUEL

805.

884.

755.

909.

907.

834 .

922.

941.

896.

943.

983.

986 .

992 .

991.

1051 .

899.

1123.

1092 .

1045 .

1045 .

MAX. FUE

842 .

$788^{\circ}$

951.

872 .

872.

966.

986.

$934^{\circ}$

1035 .

1037.

1043.

1036.

1106.

940.

69

1151.

1098 .

986.

FAST FLUENCE

(10*25 $N / M * 2)$

FAST FLUENCE

$(10 * 25 N / M+\infty 2)$
.00
.05
.14
.16
.49
.62
.72
.84
.95
1.25
1.43
1.56
1.82
1.95
2.09
2.21
2.27
2.37
2.51
2.54

.00

.05

.13

.14

.44
.55

.64

.74

.83
1.10

1.26

1.26

1.37

1.60
1.72

1.72
1.84

1.95

2.00

2.09

2.22
2.25 
EO1-01 COMPACT 29

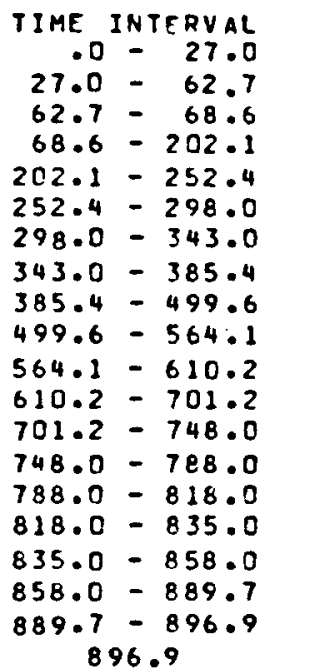

TIME AVERAGED RMS

COOLANT
643.
691.
601.
697.
680.
629.
682.
689.
663.
651.
672.
667.
668.
682.
685.
609.
711.
696.
672.
672.
673.
21.

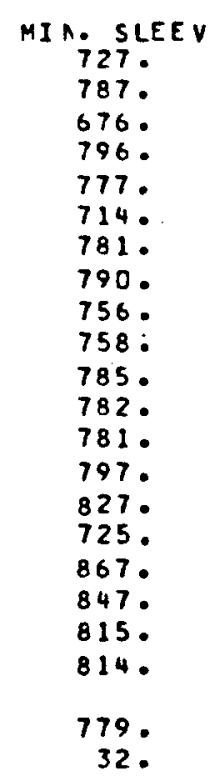

TEMPERATURES (C)

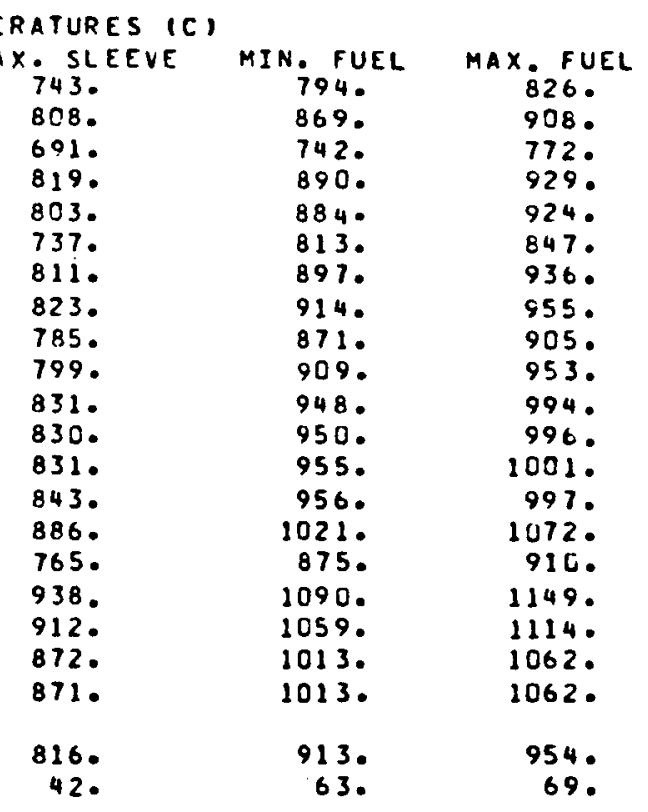

EO1-01 COMPACI 30

TIME INTERVAL $.0-27.0$ $27.0-62.7$ $62.7-68.6$ $68.6-202.1$ $202.1-252.4$ $252.4-298.0$ $298.0-343.0$ $343.0=385.4$ $385.4=499.6$ $499.6-564.1$ $564.1-610.2$ $610.2 .-701.2$ $701.2-748.0$ $748.0-788.0$ $788.0-818.0$ $818.0-835.0$ $835.0-858.0$ $858.0-889.7$ $889.7-896.9$ 896.9

TIME AVERAGED RMS

COOLANT
649.
698.
607.
704.
687.
$635 \circ$
689.
696.
670.
658.
679
675.
675
690.
694.
616.
721.
706.
682.
681.
680.
22.

MIA. SLEEVE
740.
802.
688.
811.
790.
725.
794
804.
769.
767.
794.
792.
791.
808.
829
727.
869.
850.
817.
816.
790.
31.

TEMPERATURES (C)

$\begin{array}{ccc}\text { MAX. SLEEVE } & \text { MIN. FUEL } & \text { MAX. FUEL } \\ 757^{\circ} & 812 . & 847 . \\ 824 . & 888 . & 930 . \\ 703 . & 757 . & 788 . \\ 835 . & 906 . & 948 . \\ 816 . & 895 . & 937 . \\ 747 . & 820 . & 857 . \\ 822 . & 906 . & 948 . \\ 833 . & 922 . & 965 . \\ 795 . & 876 . & 913 . \\ 801 . & 903 . & 948 . \\ 830^{\circ} & 941 . & 988 . \\ 832 . & 943 . & 989 . \\ 833 . & 948 . & 994 . \\ 847 . & 951 . & 993 . \\ 876 . & 995 . & 1044 . \\ 759 . & 855 . & 88.9 \\ 927 . & 1062 . & 1118 . \\ 903 . & 1034 . & 1085 . \\ 865 . & 989 . & 1036 . \\ 865 . & 989 . & 1036 . \\ & & \\ 822 . & 914 . & 957 . \\ 38 . & 53 . & 57 .\end{array}$

FAST FLUENCE

(10*25 N/M*2)

.00
.03
.07
.07
.23
.29
.34
.40
.45
.59
.67
.74
.86
.92
1.00
1.07
1.11
1.16
1.24
1.26

FAST FLUENCE

(10**25 N/M**21 - 00

.04

.11

.36

.52

.60

.68
.90

1.03

1.13

. 31

1.41

1.60

1.65

1.83

1.85 


\section{EO1-01 FUEL PERFORMANCE}

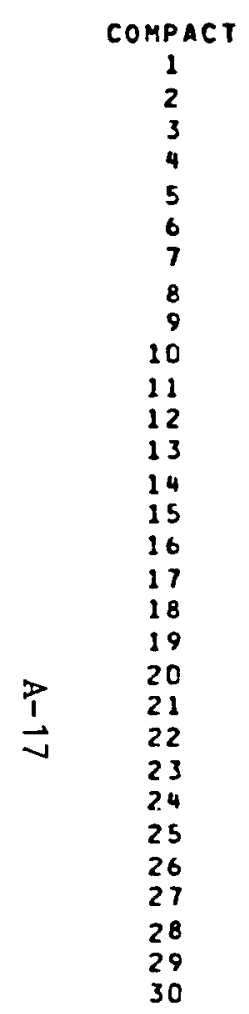

$\begin{array}{ccc}\text { KERNEL MIGRATION IRICRONSI } & \\ \text { AVERAGE } & \text { AMOEBAEQ. TEMP. } & \text { PEAK } \\ .00 & 599 . & .00 \\ .00 & 652 . & .00 \\ .00 & 127 . & .00 \\ .00 & 794 . & .00 \\ .00 & 850 . & .01 \\ .01 & 904 . & .02 \\ .04 & 957 . & .06 \\ .10 & 1001 . & .13 \\ .19 & 1030 . & .25 \\ .34 & 1065 . & .44 \\ .52 & 1091 . & .66 \\ .70 & 1111 . & .90 \\ .94 & 1133 . & 1.20 \\ 1.13 & 1144 . & 1.44 \\ 1.32 & 1156 . & 1.69 \\ 1.46 & 1164 . & 1.86 \\ 1.53 & 1168 . & 1.96 \\ 1.58 & 1171 . & 2.02 \\ 1.58 & 1171 . & 2.02 \\ 1.54 & 1171 . & 1.97 \\ 1.40 & 1166 . & 1.79 \\ 1.20 & 1157 . & 1.54 \\ .99 & 1148 . & 1.27 \\ .77 & 1131 . & .99 \\ .56 & 1116 . & .72 \\ .38 & 1094 . & .49 \\ .22 & 1063 . & .28 \\ .12 & 1029 . & .15 \\ .06 & 998 . & .08 \\ .04 & 980 . & .06\end{array}$

DEFECTIVE COATINGS
.08
.14
.18
.21
.24
.26
.28
.29
.30
.31
.31
.32
.32
.32
.32
.32
.31
.31
.30
.30
.29
.28
.27
.25
.24
.22
.20
.18
.15
.10

FUEL FAILURE (\$)

PRESSURE VESSEL KERNEL MIGRATION TOTAL

$\begin{array}{ccc}.23 & \text { KERNEL MIGRATION } & \text { TOTAL } \\ .37 & .00 & .31 \\ .49 & .00 & .51 \\ .59 & .00 & .67 \\ .66 & .00 & .80 \\ .71 & .00 & .89 \\ .76 & .00 & .97 \\ .79 & .00 & 1.04 \\ .82 & .00 & 1.08 \\ .84 & .00 & 1.12 \\ .86 & .00 & 1.15 \\ .87 & .00 & 1.17 \\ .87 & .00 & 1.18 \\ .88 & .00 & 1.19 \\ .88 & .00 & 1.19 \\ .87 & .00 & 1.20 \\ .86 & .00 & 1.19 \\ .85 & .00 & 1.17 \\ .83 & .00 & 1.16 \\ .81 & .00 & 1.13 \\ .79 & .00 & 1.11 \\ .76 & .00 & 1.08 \\ .73 & .00 & 1.04 \\ .70 & .00 & 1.00 \\ .66 & .00 & .95 \\ .61 & .00 & .89 \\ .56 & .00 & .83 \\ .49 & .00 & .76 \\ .41 & .00 & .67 \\ .28 & .00 & .56 \\ & .00 & .38\end{array}$

AMOEBA EQUIVALENT TEMPERATURES (C) CORRE SPOND TO AVERAGE KERNEL MIGRATIONS

KERNEL MIGRATION DISTANCES CALCULATED USING SOI CONFIOENCE LEVEL KMC

FUEL FAILURE CORRESPONDS TO 5 DI CONFIDENCE LEVEL 
F03-01 JMPACT 1

\begin{tabular}{|c|c|c|c|c|c|c|c|}
\hline TIME & $\begin{array}{r}\text { INTERVAL } \\
-\quad 27.0\end{array}$ & $\begin{array}{c}\text { COOLANT } \\
347 .\end{array}$ & $\begin{array}{c}\text { MIA. SLEEVE } \\
392 .\end{array}$ & $\begin{array}{l}\text { MPERATURES }(C) \\
\text { MAX. SLEEVE } \\
398 \text {. }\end{array}$ & MIN FUEL & $\begin{array}{c}\text { MAX. FUEL } \\
448 .\end{array}$ & $\begin{array}{c}\text { FAST FLUENCE } \\
(10 * 25 N / H * 2) \\
.00\end{array}$ \\
\hline $\begin{array}{l}27.0 \\
62.7 \\
68.6\end{array}$ & $\begin{array}{l}=\quad 62.7 \\
=\quad 68.6 \\
-202.1\end{array}$ & $\begin{array}{l}356 . \\
342 . \\
353 .\end{array}$ & $\begin{array}{l}456 . \\
391 . \\
456 .\end{array}$ & $\begin{array}{l}474 . \\
400 . \\
491 .\end{array}$ & $\begin{array}{l}543 . \\
439 . \\
567\end{array}$ & $\begin{array}{l}581 . \\
457 . \\
605\end{array}$ & $\begin{array}{l}.02 \\
.07 \\
.08\end{array}$ \\
\hline $\begin{array}{l}202.1 \\
252.4 \\
298.0\end{array}$ & $\begin{array}{l}-252.4 \\
-298.0 \\
-343.0\end{array}$ & $\begin{array}{l}360 . \\
357 \\
360\end{array}$ & $\begin{array}{l}453 . \\
400 \\
454 .\end{array}$ & $\begin{array}{l}498 \\
422 \\
510\end{array}$ & $\begin{array}{l}575 \\
462 \\
589\end{array}$ & $\begin{array}{l}611 . \\
479 . \\
624 .\end{array}$ & $\begin{array}{l}.24 \\
.30 \\
.33\end{array}$ \\
\hline $\begin{array}{l}343.0 \\
385.4 \\
499.6\end{array}$ & $\begin{array}{l}-385.4 \\
-499.6 \\
-564.1\end{array}$ & $\begin{array}{l}362 . \\
359^{\circ} \\
360 .\end{array}$ & $\begin{array}{l}428 \\
420 \\
444 .\end{array}$ & $\begin{array}{l}471 . \\
459 . \\
506 .\end{array}$ & $\begin{array}{l}534 . \\
515 . \\
586 .\end{array}$ & $\begin{array}{l}560 . \\
537 . \\
619 .\end{array}$ & $\begin{array}{l}.38 \\
.43 \\
.54\end{array}$ \\
\hline $\begin{array}{l}564.1 \\
610.2\end{array}$ & $\begin{array}{l}-610.2 \\
-701.2\end{array}$ & 360. & $\begin{array}{l}450 . \\
412 .\end{array}$ & $\begin{array}{l}516 . \\
459 .\end{array}$ & $\begin{array}{l}601 . \\
523 .\end{array}$ & $\begin{array}{l}635 . \\
546 .\end{array}$ & .61 \\
\hline $\begin{array}{l}701.2 \\
748.0\end{array}$ & $\begin{array}{l}-748.0 \\
-788.0 \\
-818.0\end{array}$ & $\begin{array}{l}353 . \\
349 . \\
353\end{array}$ & $\begin{array}{l}417 \\
421 . \\
407\end{array}$ & 466. & 532. & 557. & $\begin{array}{l}.75 \\
.80\end{array}$ \\
\hline $\begin{array}{l}788.0 \\
818.0\end{array}$ & $\begin{array}{l}-818.0 \\
-835.0\end{array}$ & $\begin{array}{l}353 . \\
332 .\end{array}$ & $\begin{array}{l}407 . \\
391 .\end{array}$ & $\begin{array}{l}443 . \\
426^{\circ}\end{array}$ & $\begin{array}{l}494 . \\
476 .\end{array}$ & $\begin{array}{l}513 . \\
493 .\end{array}$ & $\begin{array}{l}.86 \\
.89\end{array}$ \\
\hline $\begin{array}{l}835.0 \\
858.0\end{array}$ & $\begin{array}{l}-858.0 \\
-\quad 889.7\end{array}$ & $\begin{array}{l}350 . \\
342 .\end{array}$ & $\begin{array}{l}410 . \\
418 .\end{array}$ & $\begin{array}{l}454 . \\
470 .\end{array}$ & $\begin{array}{l}512 . \\
537\end{array}$ & $\begin{array}{l}534 . \\
563 .\end{array}$ & $\begin{array}{l}.92 \\
.95\end{array}$ \\
\hline $\begin{array}{r}889.7 \\
8\end{array}$ & $\begin{array}{l}-896.9 \\
96.9\end{array}$ & $\begin{array}{l}340 . \\
340 .\end{array}$ & $\begin{array}{l}438 . \\
438 .\end{array}$ & $\begin{array}{l}503 . \\
502 \text {. }\end{array}$ & $\begin{array}{l}584 . \\
583 .\end{array}$ & $\begin{array}{l}615 . \\
615 .\end{array}$ & $\begin{array}{l}1.00 \\
1.02\end{array}$ \\
\hline TIME & $\begin{array}{l}\text { A VERAGED } \\
\text { RMS }\end{array}$ & $\begin{array}{r}354 . \\
6 .\end{array}$ & $\begin{array}{r}430 . \\
21 .\end{array}$ & $\begin{array}{r}472 . \\
28 .\end{array}$ & $\begin{array}{l}538 . \\
41 .\end{array}$ & $\begin{array}{r}566 . \\
47 .\end{array}$ & \\
\hline
\end{tabular}

FO3-01 COMPACT 2

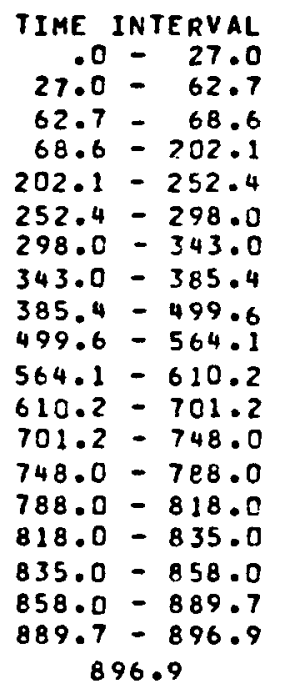

$$
896.9
$$

TIME AVERAGED RMS

\begin{tabular}{|c|c|}
\hline $\begin{array}{l}\text { COOLANT } \\
350 . \\
363 .\end{array}$ & $\begin{array}{c}\text { MIA. SLEEVE } \\
3977^{\circ} \\
475 .\end{array}$ \\
\hline $\begin{array}{l}345 \\
360 \\
367\end{array}$ & $\begin{array}{l}396 \\
475 \\
473\end{array}$ \\
\hline $\begin{array}{l}360 . \\
367 .\end{array}$ & $\begin{array}{l}404 . \\
474 .\end{array}$ \\
\hline 367. & 443. \\
\hline $\begin{array}{l}364 . \\
366 .\end{array}$ & $\begin{array}{l}434 . \\
466 .\end{array}$ \\
\hline $\begin{array}{l}367 \\
354 \\
358\end{array}$ & $\begin{array}{l}474 . \\
429 . \\
434 .\end{array}$ \\
\hline $\begin{array}{l}354 . \\
356 . \\
336\end{array}$ & $\begin{array}{l}429 . \\
412 . \\
397 .\end{array}$ \\
\hline $\begin{array}{l}354 . \\
347 . \\
348 .\end{array}$ & $\begin{array}{l}416 . \\
425 . \\
468 .\end{array}$ \\
\hline 348. & 468. \\
\hline $\begin{array}{r}360 . \\
7 .\end{array}$ & $\begin{array}{r}445 . \\
26\end{array}$ \\
\hline
\end{tabular}

EMPERATURES (C)

MAX. SLEEVE
403.
497.
407.
532.
539.
432.
549.
497.
481.
541.
551.
482.
491.
477.
449.
433.
460.
477.
546.
546.
498.
39.

MIN FUEL

437.

MAX FUEL

454.

574.

447.

618.

631 .

477.

642.

572 .

548.
637.

652.

560.

569.

544.

501.

483.

520.

546.

639.

638 .

616.

466.

661.

672.

$494^{\circ}$

683.

602 .

573 .

675 .

693.

589.

$598^{\circ}$

598 .

570.
520.

520.
501.

542.

572.

678 .

677 .

FAST FLUENCE

(10**25 N/M**2)

.00

.03

.11

.38

.47

.60

.60
.67

.67
.86

.98

1.07

1.22

1.30

1.30

1.37

1.41

1.44
1.48

1.54

1.56

$\begin{array}{rr}573 . & 605 . \\ 55 . & 64 .\end{array}$ 
rO3-08 COMPACT 3

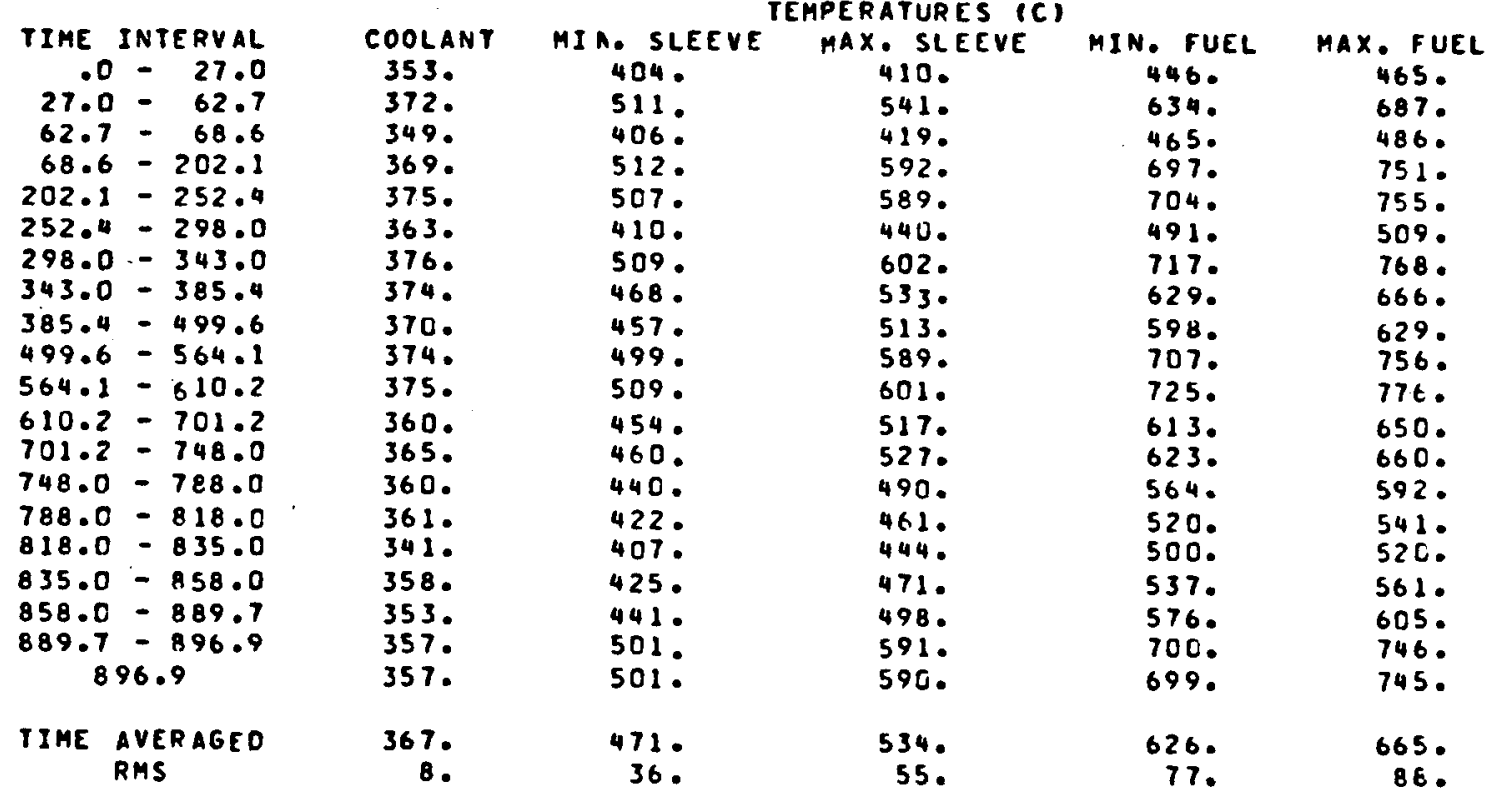

$\frac{p}{1}$

FO3-01 COMPACT 4

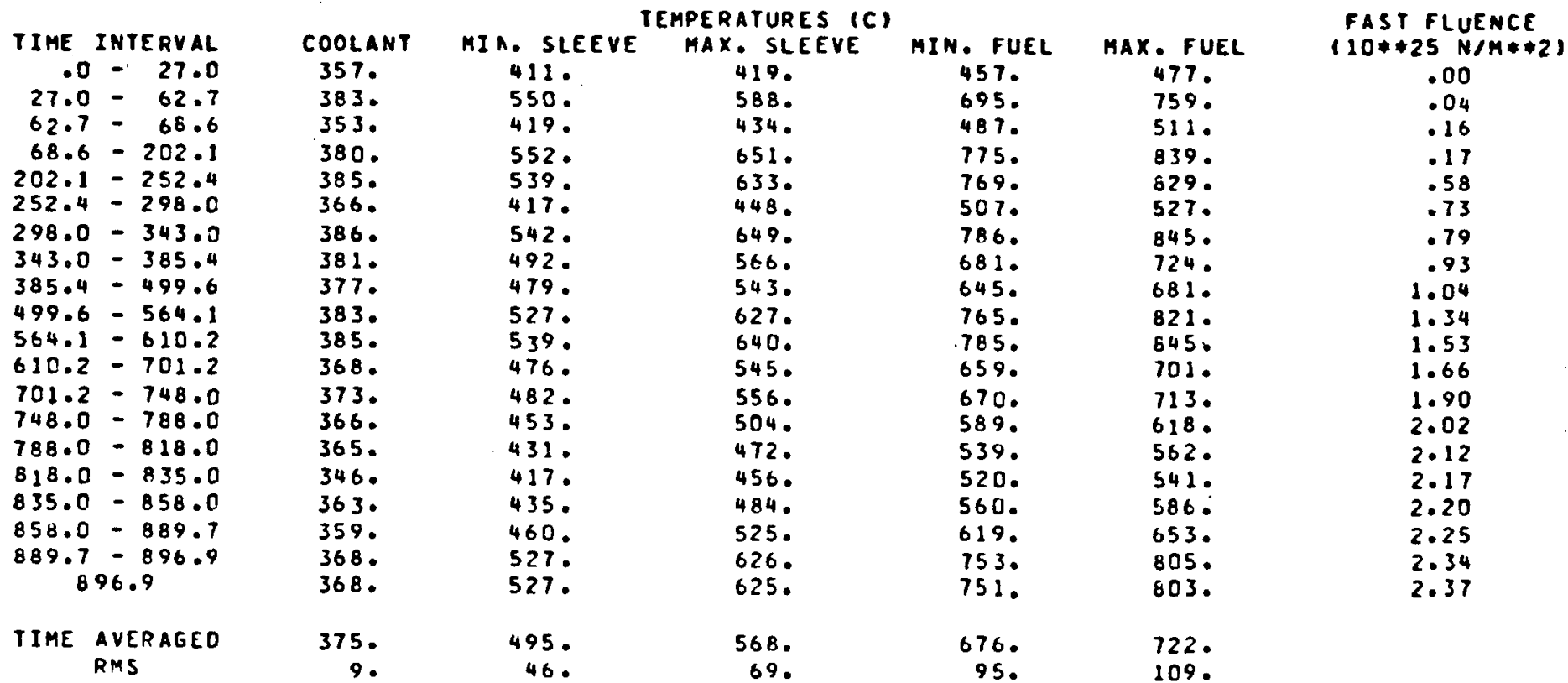


FO3-01 LUMPACT 5

TIME INTERVAL

.0 - 27.0

$27.0=62.7$

$62.7-68.6$

$68.6-202.1$

$202.1-252.4$

$252.4-298.0$

$298.0-343.0$

$343.0-385.4$

$385.4-499.6$

$499.6-564.1$

$564.1-610.2$

$610.2-701.2$

$701.2-748.0$

$748.0=788.0$

$788.0-818.0$

$818.0-835.0$

$835.0-858.0$

$858.0-889.7$

$889.7-896.9$

$$
896.9
$$

TIME AVERAGED RHS

$p$
1
0

TIME INTERVAL

$.0-27.0$

$27.0-62.7$

$62.7-68.6$

$68.6-202.1$

$202.1-252.4$

$252.4-298.0$

$298.0-343.0$

$343.0-385.4$

$385.4-499.6$

$499.6-564.1$

$564.1-610.2$

$610.2-701.2$

$701.2-748.0$

$748.0-788.0$

$788.0-818.0$

$818.0-835.0$

$835.0-858.0$

$858.0-889.7$

$889.7-896.9$

896.9

TIME AVERAGED FMS
361 .

394.

358 .

393.

396.

370.

397.

385.

393.

395.

376.

381.

372 .

351.

368.

379.

383.

11.

MIA. SLEEVE

421

$580^{\circ}$

584.

568 .

426.

570.

513

513.

498.

562 .

493 .

499 .

464.

439.

427 .

448 .

481 .

550.

516.

53.

MAX. SLEE IC

X. SLEEVE
429.
624.
450.

MIN. FUEL

$47 \%$

450 .

692 .

669.

459.

688 .

593.

657.

668 .

565.

577.

516 .

481.

467.

554.

653.

652 .

594.

78.

740 .

510 .

829.

822 .

526.

842.

724.
684.

808 .

832.

696.

709.

613.

559.

541.

692.

800 .

799.

717.

105.

MAX . FUEL

F03-01 COMPACT 6

\begin{tabular}{|c|c|}
\hline $\begin{array}{l}\text { COOLANT } \\
365 . \\
407 . \\
363 . \\
406 .\end{array}$ & $\begin{array}{l}\text { MI A. SLEEVE } \\
430 . \\
609 . \\
450 . \\
613 .\end{array}$ \\
\hline $\begin{array}{l}408 . \\
374 . \\
409 .\end{array}$ & $\begin{array}{l}594 . \\
435 . \\
597 .\end{array}$ \\
\hline $\begin{array}{l}399 . \\
394 . \\
403 .\end{array}$ & $\begin{array}{l}533^{\circ} \\
517^{\circ} \\
569^{\circ}\end{array}$ \\
\hline $\begin{array}{l}407 . \\
3850^{\circ} \\
389 .\end{array}$ & $\begin{array}{l}584 . \\
509 . \\
515 .\end{array}$ \\
\hline $\begin{array}{l}379 . \\
375 .\end{array}$ & $\begin{array}{l}476 . \\
448 .\end{array}$ \\
\hline 357. & 437. \\
\hline $\begin{array}{l}374 . \\
375 . \\
391 . \\
391 .\end{array}$ & $\begin{array}{l}460 . \\
508 . \\
570 . \\
569 .\end{array}$ \\
\hline $\begin{array}{r}393 . \\
24 .\end{array}$ & $\begin{array}{r}536^{\circ} \\
59^{\circ}\end{array}$ \\
\hline
\end{tabular}

TEMPERATURES (C)

MAX. SLEEVE
439.
657.

MIN. FUEL

484.

470 .

728 .

700 .

468 .

722 .

617 .

589.

678 .

694.

596.

530 .

478.

516 .

590.

676.

674 .

617.

86.
493.

511.

900 .

889.

548 .

908 .

772.

725.

869 .

696.

755

755.

644.

563.

621.

707 .

856.

854 .

767.

122 .

FAST FLUENCE

$110 * 25 N / M * 21$

.00

.17

.18

.65

.82
.88

1.04

1.17

1.51

1.71

1.87

2.14

2.27

2.47

2.53

2.63

2.66

FAST FLUENCE
$(10 * 25$ N/M**2)

AX. FUEL

508 .

859 .

572 .

954.

942.

568 .

964.

816 .

766.

914.

946.

782 .

798 .

676 .

607.

589.

656 :

778 .

904

904.

.00

.04

- 19

.21

.71
.90
.96

.96
1.14

1.14
1.28

1.65

1.87

2.33

2.48

2.59

2.65

2.69

2.75

2.86

2.90

810 .

132 . 
PO3-01 COMPACT

TIME INTERVAL $.0-27.0$ $27.0=62.7$ $62.7-68.6$ $68.6-202.1$ $202.1-252.4$ $252.4-298.0$ $298.0-343.0$ $343.0-385.4$ $385.4-499.6$ $499.6-564.1$

$564.1-610.2$

$610.2-701.2$ $701.2-748.0$ $748.0-788.0$ $788.0-818.0$ $818.0-835.0$ $835.0-858.0$ $858.0-889.7$ $889.7-896.9$ 896.9

TIME AVERAGED RMS

\section{IME INTERVAL} $.0-27.0$ $27.0=62.7$ $62.7-68.6$ $68.6-202.1$ $202.1-252.4$ $252.4-298.0$ $298.0-343.0$ $343.0-385.4$ $385.4=490.6$ $490.6=564.1$ $564.1-610.2$ $610.2-701.2$ $701.2-748.0$ $748.0-788.0$ $788.0-818.0$ $818.0-835.0$

$835.0-858.0$ $858.0-889.7$ $889.7-896.9$ 896.9

TIME AVERAGED RMS 370 . 421. 370 .

420 .

420.

378 .

421.

409 .

403 .

414.

394.

387 .

380 .

364.

380.

385 .

403.

403.

403.

17.

HIA. SLEEVE 440 .

635 .

467.

467.
640.

640.

618.

621 .

550 .

534.

590.

606.

526.

532 .

493.

458 .

451 .

474 .

535 .

589.

589 .

556.

64 .

MAX. SLEEVE

450.

686.

491.

756.

725 .

479.
750.

750.
637.

608.

703.

720.

602 .

615.

551.

495.

533.

627.

696.

695 .

640.

91.

MIN. FUEL

498.

816.

568.

913.

907.

565.

798.

753.

892.

924.

773.

790.
686.
609.

609.

663.

801.

891.

793.

119.

524 .

$890^{\circ}$

605 .

906.

985 .

591.

1011.

854.

801.

961.

824.

843.

636 .

627 .

697 .

852 .

952.

951.

851.
137 .

FO3-01 COMPACT 8

\begin{tabular}{|c|c|}
\hline $\begin{array}{l}\text { COOLANT } \\
375\end{array}$ & MI A. SLEEVE \\
\hline 377. & 487 \\
\hline $\begin{array}{l}435 . \\
4330\end{array}$ & $\begin{array}{l}663 . \\
640 .\end{array}$ \\
\hline $\begin{array}{l}383 . \\
435 . \\
419 .\end{array}$ & $\begin{array}{l}454 . \\
644 . \\
567 .\end{array}$ \\
\hline $\begin{array}{l}413 . \\
426 .\end{array}$ & $\begin{array}{l}549 . \\
609 .\end{array}$ \\
\hline 431. & 626. \\
\hline $\begin{array}{l}404 . \\
408 .\end{array}$ & $\begin{array}{l}541^{\circ} \\
547 .\end{array}$ \\
\hline 394. & 503. \\
\hline $\begin{array}{l}386 . \\
370 .\end{array}$ & $\begin{array}{l}468 . \\
460 .\end{array}$ \\
\hline 386. & 488 \\
\hline $\begin{array}{l}396 . \\
415 .\end{array}$ & $\begin{array}{l}566 . \\
607 .\end{array}$ \\
\hline 415. & 607 . \\
\hline $\begin{array}{r}413 . \\
19 .\end{array}$ & $\begin{array}{r}573 . \\
68 .\end{array}$ \\
\hline
\end{tabular}

TEMPERATURES (C)

MAX. SLEEVE

HIN. FUEL

513.

462

514.

777.

749.

490.

775

656

625.

724 .

743

618 .

632

561

513.

504.

550.

668 .

15

713.

659.

95.

5 .

845.

601.

942.

942 .

586.

829.

783.

930 .

965 .

808 .

828 .

712 .

637.

624.

703.

875.

933.

MAX. FUE

541.

642 .

1029.

1023.

614.
1052.

886.

833.

1002 .

1041 .

862 .

883

749.

665 .

651.

739.

933.

996 .

995 .

827.

123.

142 .
FAST FLUENCE

$110 * 25 \quad N / M * 21$

FAST FLUENCE

(10*25 N/M**2)

.00

.20

.22

.76

.97
1.04

1.22

1.37

2.01

2.49

2.66

2.76

2.83

2.94

3.07

3.10

.00

.05

.22

.23

.80

1.02

1.09

1.29

1.45

1.86

2.11

2.30

2.62

2.79

2.91

2.98

3.02

3.09

3.23

3.26 
FO3-01 LUPPACT

TIME INTERVAL

$$
\begin{array}{r}
.0-27.0 \\
07.0-62.7
\end{array}
$$

$.0-27.0$
$27.0-62.7$

$27.0-62.7$
$62.7-68.6$

$68.6-202.1$

$202.1-252.4$

$252.4-299.0$

$298.0-343.0$

$343.0-385.4$

$385.4-499.6$

$499.6-564.1$

$564.1-610.2$

$610.2-701.2$

$701.2-748.0$

$748.0-788.0$

$788.0-818.0$

$818.0-835.0$

$835.0-858.0$

$858.0-889.7$

$889.7-896.9$

$$
896.9
$$

TIME AVERAGED RMS

$\stackrel{P}{N}$

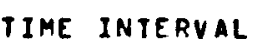

$.0-27.0$

$27.0=62.7$

$62.7-68.6$

$68.6-202.1$

$202.1-252.4$

$252.4-298.0$

$298.0-343.0$

$343.0-385.4$

$385.4-499.6$

$499.6-564.1$

$564.1-610.2$

$610.2-701.2$

$701.2-748.0$

$748.0-788.0$

$788.0-818.0$

$818.0-835.0$

$835.0-858.0$

$858.0-889.7$

$889.7-896.9$

$$
896.9
$$

380 .

450 .

385 .

447.

388 .

449.

430.

423.

444.

413.

418.

402 .

392 .

377.

394.

409.

428.

428 .

424.

22.

MiA. SLeEVe

MAXTTURES IC$$
462
$$

676 .

510 .

682.

661 .

464.

665.

584.
564.

626 .

645 .

555.

562 .

515 .

478 .

470 .

502 .

600 .

623.

623 .

473.

539 .

791 .

770.

S01.

798.

673.

641.

763 .

633.

647 .

574.

524.

515.

568.

730 .

728 .

676.

590.
72.

97.

MIN. FUEL

528 .

866.

635.

972.

606.

1004 .

856.

910.

1000 .

839.

861 .

741.

663.
648.

648 .

952 .

968 .

968.

MAX. FUEL

S58.

953.

1049 .

1056.

636 .

$1087^{\circ}$

917.

861 .

1036 .

1079.

694.

917.

780.

692 .

676.

780 .

1017.

1032 .

1032.

855.
126.

\begin{tabular}{|c|c|}
\hline COOLANT & MI h. SLEEVE \\
\hline 386. & 474 \\
\hline $\begin{array}{l}464 . \\
394 . \\
465 .\end{array}$ & $\begin{array}{l}693 . \\
526 . \\
699 .\end{array}$ \\
\hline $\begin{array}{l}461 . \\
394 .\end{array}$ & $\begin{array}{l}679 . \\
476 .\end{array}$ \\
\hline 463. & 683. \\
\hline $\begin{array}{l}441 . \\
433 .\end{array}$ & $\begin{array}{l}597 . \\
577^{\circ}\end{array}$ \\
\hline $\begin{array}{l}450 . \\
457 .\end{array}$ & $\begin{array}{l}645 . \\
665 .\end{array}$ \\
\hline 424. & 571. \\
\hline 428. & 577. \\
\hline $\begin{array}{l}411 . \\
398 .\end{array}$ & $\begin{array}{l}526 . \\
489 .\end{array}$ \\
\hline 384. & 479. \\
\hline 401. & 519. \\
\hline 422. & 625. \\
\hline 442. & 642 . \\
\hline 441. & 641. \\
\hline 436. & 606. \\
\hline 25 & 74. \\
\hline
\end{tabular}

918.

F03-01 COMPACT 10

TEMPERATURES (C)

TIME AVERAGED

25.

74 .
MIN. FUEL

545.

MAX. FUEL

578.

972 .

707 .

1067.

$994 . \quad 1080$.

$\begin{array}{rr}994 . & 1080 . \\ 630 . & 662 . \\ 1026 . & 1113 .\end{array}$

876.0939.

830.

1072

1118

894.0953

766.805.

$693 . \quad 724$.

$670 . \quad 698$.

$785 . \quad 627$.

$10055^{\circ} \quad 1074$.

$1006 . \quad 1072$

1006.1072 .

$881 . \quad 946$
FAST FLUENCE

$110 * 25 \quad N / M * 21$

.00

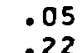

.22

.83

1.05

1.13

1.33

1.50

1.93

2.18

2.38

2.71

2.89

3.01

3.08

3.13

3.20

3.35

3.38

FAST FLUENCE

$110 * 25 \quad N / H * 2$

.00

.06

.23

.85

1.08

1.17

1.38

1.55

1.99

2.25

2.45

2.80

2.98

3.10

3.18

3.23

3.46

3.50 
103-01 JMPACT II

\begin{tabular}{|c|c|c|c|c|c|c|}
\hline \multirow{2}{*}{\multicolumn{2}{|c|}{ TIME INIERVAL }} & & & & & \\
\hline & & $\begin{array}{l}\text { COOLANT } \\
392 .\end{array}$ & $\begin{array}{c}\text { MIA. SLEEVE } \\
4,91 .\end{array}$ & $\begin{array}{l}\text { MAX. SLEEVE } \\
\text { SOS. }\end{array}$ & $\begin{array}{c}\text { MIN, FUEL } \\
\text { S6E. }\end{array}$ & $\begin{array}{c}\text { MAX, FUEL } \\
605 .\end{array}$ \\
\hline $\begin{array}{l}27.0 \\
62.7 \\
68.6\end{array}$ & $\begin{array}{l}=62.7 \\
=\quad 68.6 \\
=202.1\end{array}$ & $\begin{array}{l}479 . \\
403 . \\
480\end{array}$ & $\begin{array}{l}710 . \\
541 . \\
717 .\end{array}$ & $\begin{array}{l}764 . \\
572 . \\
819\end{array}$ & $\begin{array}{l}902 . \\
677 . \\
994 .\end{array}$ & $\begin{array}{r}991 . \\
730 . \\
1084 .\end{array}$ \\
\hline $\begin{array}{l}202.1 \\
252.4 \\
298.0\end{array}$ & $\begin{array}{l}-252.4 \\
-298.0 \\
-343.0\end{array}$ & $\begin{array}{l}475 . \\
400 . \\
477 .\end{array}$ & $\begin{array}{l}695 . \\
491 . \\
700 .\end{array}$ & $\begin{array}{l}801 . \\
533 . \\
829 .\end{array}$ & $\begin{array}{l}1012 . \\
660 \\
1047\end{array}$ & $\begin{array}{r}1099 . \\
6960 \\
1132 .\end{array}$ \\
\hline $\begin{array}{l}343.0 \\
385.4 \\
499.6\end{array}$ & $\begin{array}{l}=385.4 \\
=499.6 \\
-564.1\end{array}$ & $\begin{array}{l}452 . \\
444 . \\
463 .\end{array}$ & $\begin{array}{l}610 . \\
589 . \\
662 .\end{array}$ & $\begin{array}{l}697 . \\
666 . \\
782 .\end{array}$ & $\begin{array}{r}892 . \\
846 . \\
1022 .\end{array}$ & $\begin{array}{l}956: \\
900 . \\
1101 .\end{array}$ \\
\hline $\begin{array}{l}564.1 \\
610.2\end{array}$ & $\begin{array}{l}-610.2 \\
-701.2\end{array}$ & $\begin{array}{l}471 . \\
434 .\end{array}$ & $\begin{array}{l}684 . \\
585 .\end{array}$ & $\begin{array}{l}805 . \\
664 .\end{array}$ & $\begin{array}{r}1067 . \\
897\end{array}$ & $\begin{array}{l}1150 . \\
956 .\end{array}$ \\
\hline 701.2 & -748.0 & 439. & 591. & 678. & 922 . & 982. \\
\hline $\begin{array}{l}748.0 \\
788.0 \\
818.0\end{array}$ & $\begin{array}{l}-788.0 \\
-818.0 \\
-835.0\end{array}$ & $\begin{array}{l}419 . \\
405 . \\
391 .\end{array}$ & $\begin{array}{l}537 . \\
501 . \\
488 .\end{array}$ & $\begin{array}{l}596 \\
550 \\
533\end{array}$ & $\begin{array}{l}788 \\
721 \\
689\end{array}$ & $\begin{array}{l}829^{\circ} \\
754 . \\
718^{\circ}\end{array}$ \\
\hline $\begin{array}{l}835.0 \\
858.0\end{array}$ & $\begin{array}{l}-\quad 58.0 \\
-\quad 889.7\end{array}$ & $\begin{array}{l}410 . \\
436 .\end{array}$ & $\begin{array}{l}540 . \\
648 .\end{array}$ & $\begin{array}{l}618 . \\
769 .\end{array}$ & $\begin{array}{r}837 \\
1049\end{array}$ & $\begin{array}{r}884 \\
1122^{\circ}\end{array}$ \\
\hline $\begin{array}{r}889.7 \\
8\end{array}$ & $\begin{array}{l}-896.9 \\
96.9\end{array}$ & $\begin{array}{l}455 . \\
455 .\end{array}$ & $\begin{array}{l}657 . \\
657 .\end{array}$ & $\begin{array}{l}763 . \\
762 .\end{array}$ & $\begin{array}{l}1034 \\
1034 .\end{array}$ & $\begin{array}{l}1101 . \\
1101 .\end{array}$ \\
\hline TIME & $\begin{array}{l}\text { AVERAGED } \\
\text { RMS }\end{array}$ & $\begin{array}{r}447 \\
28\end{array}$ & $\begin{array}{r}621 . \\
75 .\end{array}$ & $\begin{array}{l}707 . \\
99 .\end{array}$ & $\begin{array}{l}905 . \\
127 .\end{array}$ & $\begin{array}{l}971 . \\
144 .\end{array}$ \\
\hline
\end{tabular}

FAST FLUENCE

$110 * 25 N / H * 21$

.00

.06

.23

.86

1.10

1.19

1.40
1.58

2.02

2.30
2.50

2.85

3.04

3.17

3.25

3.30
3.38

3.54
3.58

144.

\section{F03-01 COMPACT 12}

TIME INTERVAL

$.0-27.0$

$27.0=62.7$

$62.7=68.6$

$68.6-202.1$
$202.1-252.4$

$202.1-252.4$

$252.4-298.0$

$298.0-343.0$

$343.0-385.4$

$385.4=499.6$

$499.6-564.1$

$564.1-610.2$

$610.2-701.2$

$701.2=748.0$

$748.0-788.0$

$788.0-818.0$

$818.0-835.0$

$835.0-858.0$

$858.0-889.7$

$889.7-896.9$

896.9

IIME AVERAGEO RMS

\begin{tabular}{|c|c|}
\hline $\begin{array}{l}\text { COOLANT } \\
400 . \\
494 .\end{array}$ & $\begin{array}{c}\text { MI A. SLE E VE } \\
515 . \\
724 .\end{array}$ \\
\hline $\begin{array}{l}412 . \\
495 .\end{array}$ & $\begin{array}{l}557 . \\
731 .\end{array}$ \\
\hline 489. & 709. \\
\hline $\begin{array}{l}407 . \\
492 .\end{array}$ & $\begin{array}{l}514 . \\
714 .\end{array}$ \\
\hline $\begin{array}{l}463 . \\
454 . \\
475 .\end{array}$ & $\begin{array}{l}621 \\
600 \\
678\end{array}$ \\
\hline $\begin{array}{l}484 . \\
4450^{\circ} \\
450\end{array}$ & $\begin{array}{l}701 . \\
598 . \\
604 .\end{array}$ \\
\hline $\begin{array}{l}428 . \\
412 . \\
398 .\end{array}$ & $\begin{array}{l}547 . \\
513 . \\
497 .\end{array}$ \\
\hline $\begin{array}{l}420 . \\
451: \\
469 .\end{array}$ & $\begin{array}{l}573 . \\
672 . \\
673 .\end{array}$ \\
\hline 468. & 673. \\
\hline $\begin{array}{r}459 . \\
30\end{array}$ & $\begin{array}{r}636 . \\
75 .\end{array}$ \\
\hline
\end{tabular}

TEMPERATURES (C)

MAX. SLEE
$5320^{\circ}$
777.
589.
828.
812.
562.
839.
706.
675.
798.
822.
677.
691.
606.
564.
542.
662.
795.
778.
776.

MIN. FUEL

605 .

MAX. FUEL

915.1005

697.

1004 .

1025.

706.
1060.

905.

859.

1045.

1091 .

918.

945.
807.

748.

706.

912.

1091.

1060 .

752.

1146.

978.
1006.

$1060^{\circ} \quad 1128^{\circ}$

721.

926.
648 .

1112 .

748 .

968.

$913 \circ$

1176

1176.

849.

783 .

735 .

968 .

1167.

993.

FAST FLUENCE $110 * 25 \quad N / M * 21$

$$
\begin{aligned}
& .00 \\
& .07 \\
& .24 \\
& .26 \\
& .88 \\
& 1.11 \\
& 1.22 \\
& 1.43 \\
& 1.60 \\
& 2.05 \\
& 2.33 \\
& 2.54 \\
& 2.90 \\
& 3.09 \\
& 3.22 \\
& 3.31 \\
& 3.36 \\
& 3.45 \\
& 3.62 \\
& 3.66
\end{aligned}
$$

139. 
FO3-01 WOMPACT 13

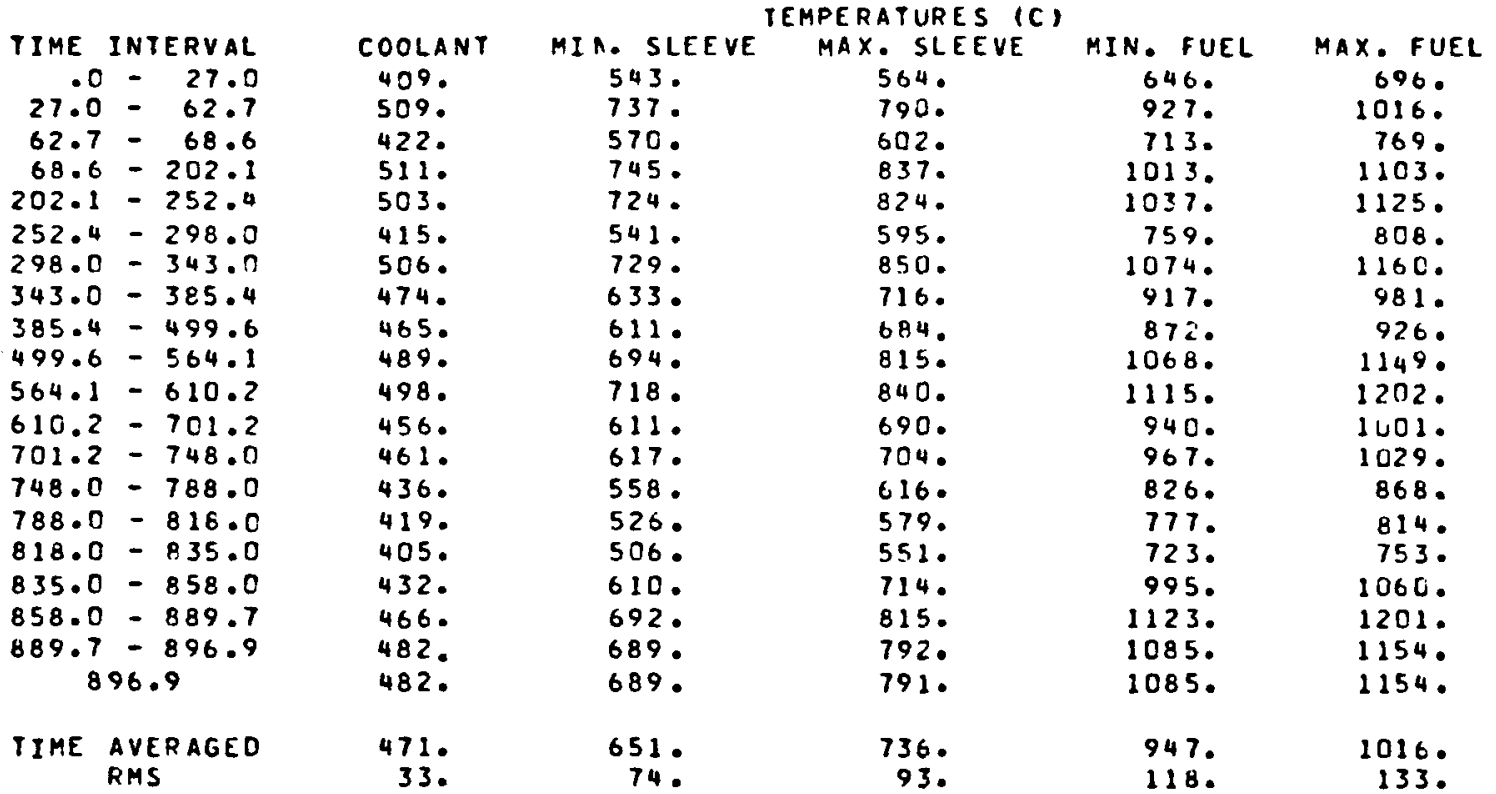

FAST FLUENCE

$(10 * 25$ N/M*2)

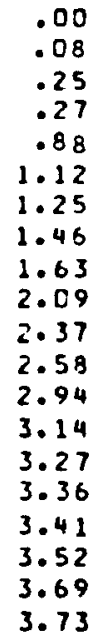

FO3-01 COMPACT IA

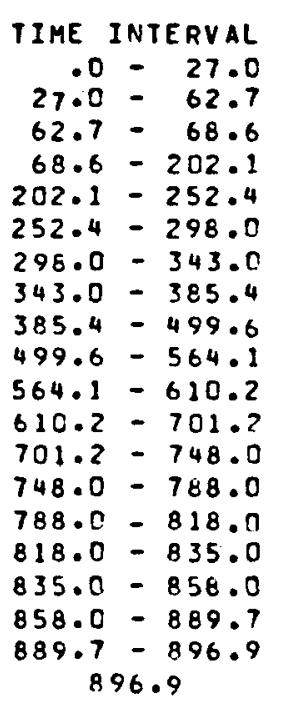

COOLANT
419.
524.
432.
526.
517.
424.
521.
486.
475.
502.
513.
467.
472.
445.
427.
413.
445.
481.
496.
496.
484.
35.

MIA. SLEEVE
570.
750.
583.
758.
737.
565.
743.
644.
621.
708.
733.
622.
629.
568.
539.
514.
645.
712.
706.
705.
665.
73.

TEMPERATURES (C)

$\begin{array}{ccc}\text { MAX. SLEEVE } & \text { MIN FUEL } & \text { MAX.FUEL } \\ 594 . & 683 . & 739 . \\ 801 . & 937 . & 1026 . \\ 615 . & 727 . & 785 . \\ 845 . & 1019 . & 1109 . \\ 834 . & 1047 . & 1135 . \\ 625 . & 803 . & 858 . \\ 859 . & 1084 . & 1170 . \\ 724 . & 926 . & 990 . \\ 693 . & 881 . & 935 . \\ 827 . & 1082 . & 1164 . \\ 853 . & 1131 . & 1218 . \\ 700 . & 953 . & 1014 . \\ 714 . & 980 . & 1043 . \\ 626 . & 841 . & 883 . \\ 595 . & 804 . & 843 . \\ 559 . & 735 . & 766 . \\ 761 . & 1064 . & 1136 . \\ 835 . & 1150 . & 1230 . \\ 898 . & 1107 . & 1176 . \\ 806 . & 1107 . & 1176 . \\ & & \\ 749 . & 963 . & 1033 . \\ 91 . & 113 . & 129 .\end{array}$

129.
(10*25 $N / H * 21$
FAST FLUENCE

IIME AVERAGEO RMS

73. 
FO3-01 LOMPACT 15

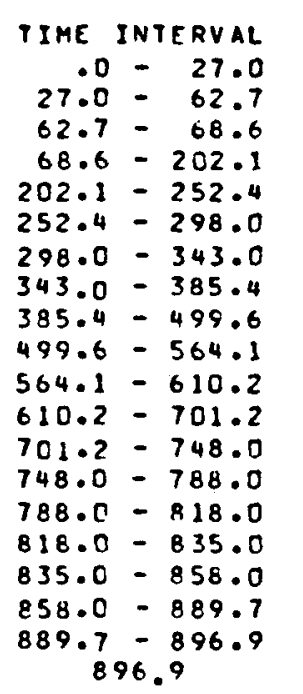

IIME AVERAGED RMS

TEMPERATURES (C)

MAX. SLEEVE MIN. FUEL MA

FUEL MAX. FUEL

FAST FLUENCE

$(10 * 25$ N/A * 2 )

TIME INTERVAL

$$
. C-27.0
$$

$27.0-62.7$

$62.7-68.6$

$68.6-202.1$

$202.1-252.4$

$252.4-298.0$

$298.0-343.0$

$343.0-385.4$

$385.4-499.6$

$499.6-564.1$

$564.1-610.2$

$610.2-701.2$

$701.2-748.0$

$748.0-788.0$

$788.0-818.0$

$818.0-835.0$

$835.0-358.0$

$858.0-889.7$

$89.7-896.9$

896.9

TIME AVERAGED PMS

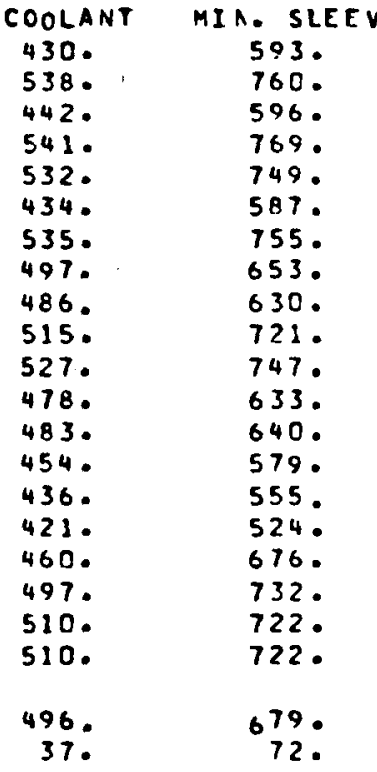

. FUEL
775.
1032.
801.
112.
1141.
898.
1177.
996.
942.
1179.
1234.
1027.
1056.
901.
875.
782.
1195.
1257.
1198.
1196.

FO3-01 COMPACT 16

619.
810.

810.
628.

851 .

843.

867 .

731 .

700 .

840.

710.

725.

613.

569.

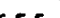

824 .

822 .

761.

713.

944.

1024.

1054 .

838 .

1091.

889.

1096 .

1146.

966.

850 .

834.

751 .

1116.

1175.

1127.

977.

126.

TEMPERATURES (C)

\begin{tabular}{|c|c|}
\hline $\begin{array}{l}\text { COOLANT } \\
441 .\end{array}$ & $\begin{array}{c}\text { MIA. SLEEVE } \\
615 .\end{array}$ \\
\hline $\begin{array}{l}552^{\circ} \\
452^{\circ} \\
550^{\circ}\end{array}$ & $\begin{array}{l}770 . \\
609 . \\
779 .\end{array}$ \\
\hline $\begin{array}{l}545 . \\
445 .\end{array}$ & $\begin{array}{l}760 . \\
608 .\end{array}$ \\
\hline 549. & 766. \\
\hline $\begin{array}{l}508 . \\
496 .\end{array}$ & $\begin{array}{l}662 . \\
638 .\end{array}$ \\
\hline $\begin{array}{l}528 . \\
541 . \\
489 .\end{array}$ & $\begin{array}{l}734 . \\
7610^{\circ} \\
644 .\end{array}$ \\
\hline $\begin{array}{l}494 . \\
463{ }^{\circ} \\
444 .\end{array}$ & $\begin{array}{l}651 . \\
591 . \\
571 .\end{array}$ \\
\hline $\begin{array}{l}428^{\circ} \\
4750^{\circ} \\
513 .\end{array}$ & $\begin{array}{l}534^{\circ} \\
705 . \\
752^{\circ}\end{array}$ \\
\hline $\begin{array}{l}525 . \\
524:\end{array}$ & $\begin{array}{l}738 . \\
737 .\end{array}$ \\
\hline 508. & 691. \\
\hline 38. & 72. \\
\hline
\end{tabular}

MAX. SLEEVE

MIN. FUEL

743.

MAX. FUEL

808 .

644.

952.

1028 .

642.

858 .

851 .

873.

737.

737.

851 .

851 .

720.

734.

650.

632 .

579.

836.

838 .

837 .

1058

870 .

1096.

938.

893.

1107.
1158.

975.

1002.

873.

862.

764.

1162 .

1198.

1143.

1038 .

817.
1114.
1145.

1145.

1180.

1000 .

946.

1189.

1245 .

1037 .

1065.

917.

926.

795.

1246 :

1281 .

1214.

$1214^{\circ}$

.00
.09

.09
.25

.25
.27

.88

1.11

1.26

1.65

2.10

2.10

2.39
2.60

2.97

3.17

3.31

3.41

3.47

3.58

3.76

3.81

989.

111 .

1059.

124.

773.
FAST FLUENCE

$110 * 25 N / M * 21$$$
.00
$$

.10

.25
.28

.87

1.10

1.26

1.47

1.64

2.09

2.38

2.59

2.96

3.15

3.30

3.41

3.47

3.59

3.77 
FO3-01 LOMPACT 17

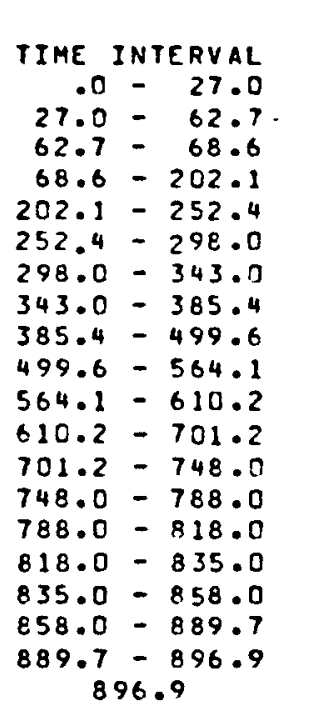

IIME AVERAGED RMS

$p$
1
$N$

COOLANT
453.
566.
462.
570.
559.
456.
563.
519.
506.
541.
555.
500.
505.
473.
454.
436.
491.
529.
539.
539.
520.
40.

TEMPERATURES (C)

MIA. SLEEVE
$635 \circ$
$777 \circ$
$622:$
786.
769.
627.
$775 \circ$
670.
645.
746.
774.
654.
661.
603.
591.
544.
732.
773.
755.
754.
702.
71.

660.
$8250^{\circ}$
656.

656.

860 .

855 .

695.

877 .

741 .

862 .

891 .

729 .

743.

663.

656.

589 .

868.

896.

855.

782.

86.

FO3-01 COMPACT 18

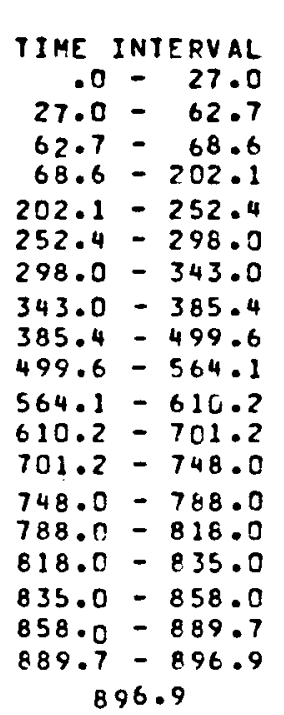

TIME AVERAGEO

\begin{tabular}{|c|c|}
\hline $\begin{array}{l}\text { COOLANT } \\
466 .\end{array}$ & MIA. SLEEVE \\
\hline $\begin{array}{l}579 . \\
4730^{\circ} \\
583 .\end{array}$ & $\begin{array}{l}784 \\
635{ }^{\circ} \\
793 .\end{array}$ \\
\hline $\begin{array}{l}573 . \\
468 . \\
577 .\end{array}$ & $\begin{array}{l}778 \\
645 \\
784\end{array}$ \\
\hline $\begin{array}{l}529^{\circ} \\
516^{\circ} \\
554^{\circ}\end{array}$ & $\begin{array}{l}677^{\circ} \\
652^{\circ} \\
757^{\circ}\end{array}$ \\
\hline $\begin{array}{l}569 . \\
511 . \\
516 .\end{array}$ & $\begin{array}{l}785 . \\
663 . \\
670 .\end{array}$ \\
\hline $\begin{array}{l}482^{\circ} \\
465{ }^{\circ} \\
444 .\end{array}$ & $\begin{array}{l}615^{\circ} \\
6190^{\circ} \\
554^{\circ}\end{array}$ \\
\hline $\begin{array}{l}508 . \\
546 . \\
554 . \\
554 .\end{array}$ & $\begin{array}{l}758^{\circ} \\
793^{\circ} \\
771^{\circ} \\
770^{\circ}\end{array}$ \\
\hline $\begin{array}{r}533 . \\
41 .\end{array}$ & $\begin{array}{r}713 . \\
69 .\end{array}$ \\
\hline
\end{tabular}

TEMPERATURES (C)

$\begin{array}{cr}\text { MAX. SLEEVE } & \text { MIN FUEL } \\ 688 . & 792 . \\ 830 . & 956 . \\ 669 . & 786 . \\ 863 . & 1024 . \\ 860 . & 1057 . \\ 715 . & 918 . \\ 881 . & 1095 . \\ 745 . & 937 . \\ 715 . & 893 . \\ 871 . & 1118 . \\ 901 . & 1170 . \\ 737 . & 984 . \\ 750 . & 1010 . \\ 675 . & 899 . \\ 692 . & 946 . \\ 599 . & 787 . \\ 898 . & 1228 . \\ 916 . & 1237 . \\ 870 . & 1173 . \\ 869 . & 1173 . \\ 792 . & 1003 . \\ 84 . & 109 .\end{array}$

768 .

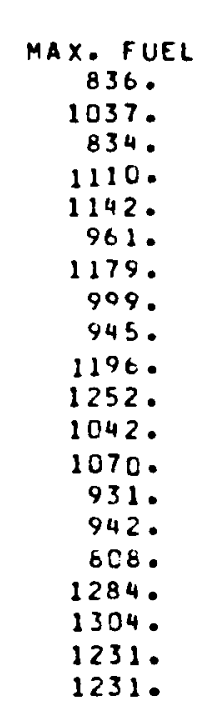

$\begin{array}{ll}996 . & 1066 . \\ 110 . & 122 .\end{array}$

FAST FLUENCE

110*25 N/M**2)

.00

.10

.25
.28

.28

1.09

1.26

1.46

1.63

2.07

2.35

2.56

2.93

3.12

3.27

3.39

3.45

3.57

3.76

3.80
FAST FLUENCE

MAX. FUEL 864.

1037.

1106.

1106.

1140.
987.

987.
1176.

997.

944.

1200 .

1256 .

045 .

1072 .

945.
1000.

820 .

$1320^{\circ}$

1323.

1246 .

1246 .

1072.
$(10 * 25 N / H * 2)$

.00

.10

.25

.85
1.07

1.25

1.45

1.61

2.04

2.32

2.53

2.89

3.08
3.23

3.37

3.43

3.56

3.75

3.79 
FO3-01 -OMPACT 19

TIME INTERVAL $.0-27.0$ $27.0-62.7$ $62.7-68.6$ $68.6-202.1$ $202.1-252.4$ $252.4-298.0$ $298.0-343.0$ $343.0-385.4$ $385.4-499.6$ $499.6-564.1$

$564.1-610.2$ $610.2-701.2$ $701.2-748.0$

$748.0-788.0$ $788.0-818.0$ $818.0-835.0$ $835.0-858.0$ $858.0-889.7$ $889.7-896.9$ 896.9

IIME AVERAGED RMS

TIME INTERVAL $.0-27.0$ $27.0-62.7$ $62.7-68.6$ $68.6-202.1$ $202.1-252.4$ $252.4-298.0$ $298.0-343.0$ $343.0-385.4$ $385.4=499.6$ $499.6=564.1$ $564.1-610.2$ $610.2-701.2$ $701.2-748.0$ $748.0=788.0$ $788.0=318.0$ $818.0-835.0$ $835.0-8.58 .0$ $858.0-889.7$ $9.7-896.9$

$$
896.9
$$

IIME AVEDAGEO RMS

\begin{tabular}{|c|c|}
\hline $\begin{array}{l}\text { COOLANT } \\
479 .\end{array}$ & $\begin{array}{c}\text { MIA. SLEE VE } \\
674 .\end{array}$ \\
\hline $\begin{array}{l}592 . \\
494 . \\
597 .\end{array}$ & $\begin{array}{l}791 . \\
647 . \\
801 .\end{array}$ \\
\hline $\begin{array}{l}585^{\circ} \\
480^{\circ} \\
590^{\circ}\end{array}$ & $\begin{array}{l}784 . \\
663 . \\
791 .\end{array}$ \\
\hline $\begin{array}{l}539 . \\
526 . \\
567 .\end{array}$ & $\begin{array}{l}682 . \\
658 . \\
767 .\end{array}$ \\
\hline $\begin{array}{l}583 . \\
521 . \\
527 .\end{array}$ & $\begin{array}{l}796 . \\
672 . \\
679 .\end{array}$ \\
\hline $\begin{array}{l}492 . \\
478 . \\
453 .\end{array}$ & $\begin{array}{l}629^{\circ} \\
662 . \\
565 .\end{array}$ \\
\hline $\begin{array}{l}526 . \\
562 . \\
569 .\end{array}$ & $\begin{array}{l}783 . \\
811 . \\
788 .\end{array}$ \\
\hline 568. & 787 . \\
\hline $\begin{array}{r}545 . \\
42 .\end{array}$ & $\begin{array}{r}724 . \\
68 .\end{array}$ \\
\hline
\end{tabular}

EMPERATURES IC

MAX. SLEEVE
708.
836.
681.
866.
862.
733.
883.
747.
718.
878.
909.
744.
757.
690.
750.
611.
925.
932.
886.
885.
801.
82.
814. 959. 798. 1023. 1053.

$$
937 .
$$

1090.

933 .

889.
1120.

1171 .

984 .

1005.

913.

1027.

800 .

1255 .

1250.

1186.

1186.

1006.

108.

888 .

1038 .

861.

1133.

1009 .

$1169^{\circ}$

169.

939.

1200.

1256 .

1044.

1071.

961.

091.

834

1349.

1337 .

1260.

1260 .

1077

120.

FO3-01 COMPACT 20

$\begin{array}{cc}\text { COOLANT } & \text { MIN. SLEEVE } \\ 492^{\circ} & 691 . \\ 605 . & 797 . \\ 495 . & 657 . \\ 610 . & 808 . \\ 598 . & 790 . \\ 493 . & 679 . \\ 603 . & 796 . \\ 549 . & 687 . \\ 535 . & 662 . \\ 580 . & 777 . \\ 590^{\circ} & 807 . \\ 532 . & 680 . \\ 537 . & 687 . \\ 502 . & 643 . \\ 492 . & 698 . \\ 461 . & 577 . \\ 543 . & 805 . \\ 579 . & 827 . \\ 584 . & 803 . \\ 583 . & 802 . \\ & \\ 557 . & 734 . \\ 43 . & 67 .\end{array}$

TEMPERATURES (C)

$X$. SLEEVE
726.
840.
691.
868.
862.
749.
883.
749.

MIN DUEL

MAX. FUEL

834.

958.
807.

1018.

1045.

952 .

1082 .

926.

719.

886.

918 .

751.

764 .

705 .

796.

623.

948.

946.

899.

883.
1120.

1120.

1172.

984.

927.

1083.

812.

1274.

1258.

1195.

1196.

909.
1034.

870 .

1095 .

1123.

1026.

1156.

$988^{\circ}$

931 .

1199.

1250.

1043.

1069 .

970.

1155 .

847.

1371 .

1344.

1270 .

1270.

1011.

808 .
81 .

109.

1079
FAST FLUENCE

110*25 N/M* 21

.00

.11

.25

1.04

1.04

1.42

1.58

2.01

2.28

2.49

2.84

3.03

3.33

3.33
3.39

3.53

3.72

3.76

FAST FLUENCE

(10**25 N/A**2)

.00

.11

.25
.27

.80

1.01

1.20

1.39

1.54

1.96

2.23

2.43

2.96

3.12

3.28

3.35

3.67

3.71 
F03-01 LUMPACT 21

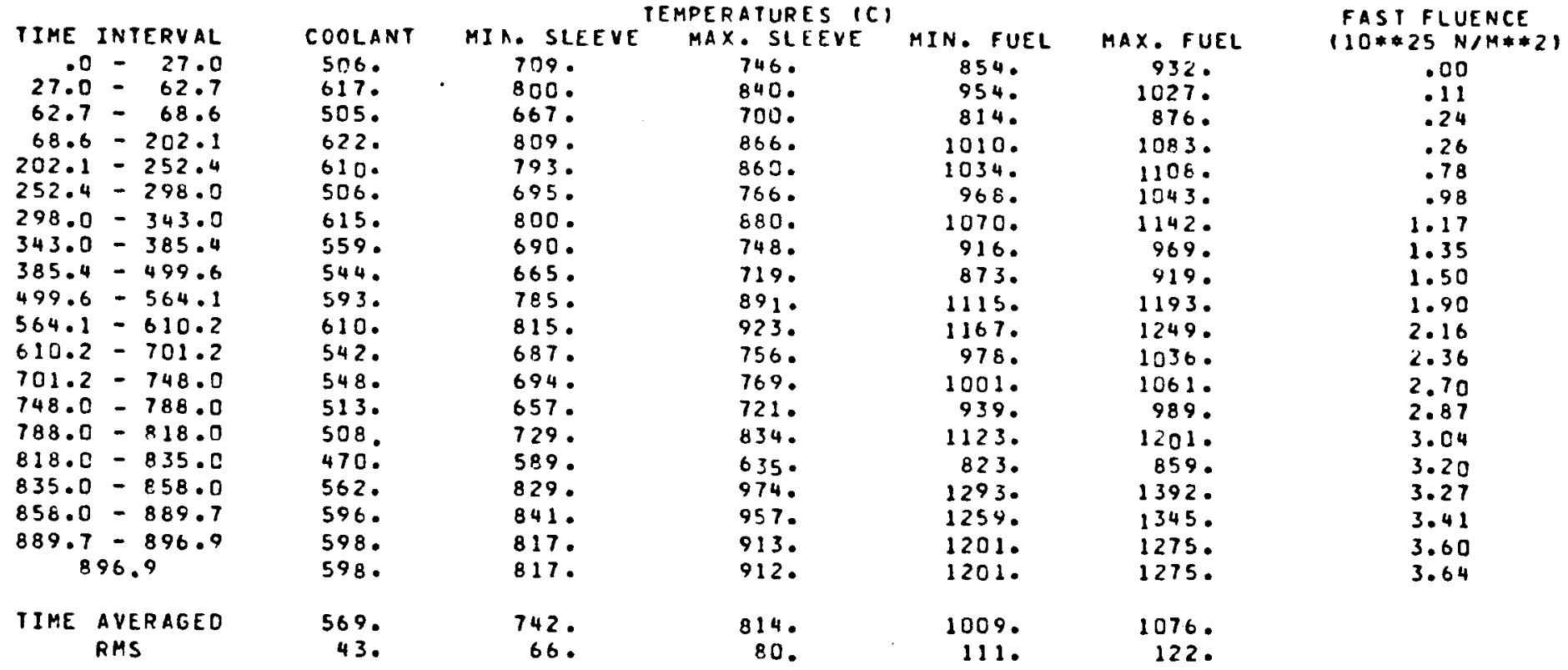

FO3-01 COMPACI 22
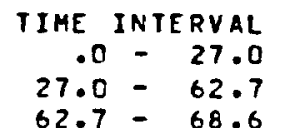

$62.7-68.6$

$68.6-202.1$

$202.1=252.4$

$298.0-343.0$

$343.0-385.4$

$385.4-499.6$

$499.6-564.1$

$564.1=610.2$

$701.2-748.0$

$748.0-788.0$

$788.0-818.0$

$818.0-835.0$

$835.0=858.0$
$858.0-889.7$

$689.7-896.9$

896.9

TIME AVERAGEO RMS

COOLANT
520.
629.
516.
634.
622.
518.
627.
568.
552.
605.
623.
552.
558.
524.
524.
479.
580.
612.
613.
613.
580
44.

MIA. SLEEV
723.
803.
674.
813.
797.
708.
804.
694.
669.
$790^{\circ}$
$820^{\circ}$
$692^{\circ}$
699.
673.
754.
603.
847.
852.
830.
829.
749.
65.

TEMPERATURES (C)

$\begin{array}{ccc}\text { MAX. SLEEVE } & \text { MIN.FUEL } & \text { MAX.FUEL } \\ 760 . & 869 . & 946 . \\ 842 . & 951 . & 1020 . \\ 707 . & 818 . & 880 . \\ 866 . & 1003 . & 1073 . \\ 861 . & 1025 . & 1097 . \\ 778 . & 975 . & 1050 . \\ 879 . & 1060 . & 1230 . \\ 748 . & 908 . & 959 . \\ 720 . & 866 . & 910 . \\ 891 . & 1105 . & 1180 . \\ 924 . & 1155 . & 1235 . \\ 758 . & 968 . & 1024 . \\ 770 . & 989 . & 1047 . \\ 739 . & 953 . & 1006 . \\ 863 . & 1146 . & 1227 . \\ 649 . & 835 . & 873 . \\ 990 . & 1300 . & 1400 . \\ 966 . & 1256 . & 1341 . \\ 924 . & 1201 . & 1274 . \\ 922 . & 1201 . & 1274 . \\ 819 . & 1005 . & 1070 . \\ 79 . & 110 . & 122 .\end{array}$

FAST FLUENCE

(10*25 N/M**2)

.00

.11
.23
.026

.23

.75

1.13

1.30

1.44

1.83

2.08

2.60

2.94

3.11

3.19

3.33

3.51

3.55 
F03-01 LOMPACT 23

TIME INTERVAL COOLANT MIA. SLEEVE TEMPERATURES (C)

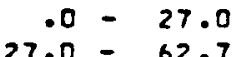

$27.0-62.7$

$62.7-68.6$

$68.6-202.1$

$252.4-298.0$

$298.0-343.0$

$343.0-385.4$

$385.4-499.6$

$499.6-564.1$

$564.1-610.2$

$610.2=701.2$
$701.2-748.0$

$748.0-788.0$

$788.0-818.0$

$818.0-835.0$

$835.0-858.0$

$858.0-889.7$

$889.7=896.9$

896.9

TIME AVERAGED RHS
$202.1-252.4$

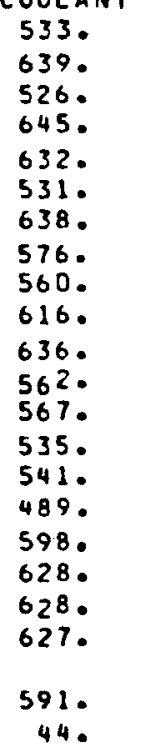

IN. SLEEVI

735.

683

813 .

797.

720 :

805 .

695.

670 .

794 .

825 .

697 .

703.

691.

779.

617.

864.

841 .

840 .

754.
65.

MAX. SLE

773.
839.

713.

861.

855.
788.

873.

745.

717.

925.

925.

772.

760.

606.

1004.

1004.
974.

932 .

931.

821.
79.
MIN. FUEL

881.

942.

822.
990.

1010.
979.

1043.

893.

852.

1093.
1143.
956.

976.

970.

1168.

1303 .

1252 .

1198 .

1198.

997.

111.

MAX. FUEL

958 .

1008 .

883.

1056 .

1077 .

1054 .

1108 .

942.

1165

1165.

1219.

1032 .

1025 .

1252.

889.

1402 .

1335.

1271 .

1271.

1060.

123 .
FAST FLUENCE

$(10 * 25 \quad N / M * * 2)$

.00

.11
.23

.25

.71

1.09

1.25

1.38

1.75

1.99
2.18

2.50

2.66

2.66

3.01

3.09

3.23

3.41

F03-01 COMPACT 24

TIME INTERVAL

$.0-27.0$

$27.0=62.7$

$62.7-68.6$

$68.6-202.1$

$202.1-252.4$

$252.4-298.0$

$298.0-343.0$

$343.0-385.4$

$385.4-499.6$

$499.6-564.1$

$564.1-610.2$

$610.2=701.2$

$701.2-748.0$

$748.0-788.0$

$788.0-818.0$

$818.0-835.0$

$835.0-858.0$

$858.0-889.7$

$89.7-896.9$

896.9

TIME AVERAGED RMS $\therefore$

$\begin{array}{lc}\text { COOLANT } & \text { MIA. SLEEV } \\ 547 . & 746 . \\ 649 . & 800 . \\ 536 . & 689 . \\ 656 . & 811 . \\ 643 . & 799 . \\ 544 . & 730 . \\ 648 . & 806 . \\ 584 . & 697 . \\ 568 . & 672 . \\ 627 . & 797 . \\ 647 . & 828 . \\ 571 . & 699 . \\ 577 . & 706 . \\ 547 . & 715 . \\ 558 . & 799 . \\ 499 . & 638 . \\ 616 . & 878 . \\ 644 . & 876 . \\ 642 . & 850 . \\ 641 . & 649 . \\ & \\ 602 . & 759 . \\ 44 . & 64 .\end{array}$

TEMPERATURES (C)

MAX. SLE
783.
$8340^{\circ}$
720.
$854^{\circ}$
852.
$7950^{\circ}$
$870^{\circ}$
743.
715.
887.
922.
759.
771.
790.
913.
689.
1013.
982.
939.
937.

MIN. FUEL

889 .

1299.

1247.

1191 .

1191.

822.
79.
930.

626.

972.

996.

978.

1028 .

881 .

841.

1076.

1126.

941 .

960 .

999.

1181 .

874 .

966.

885.

1033.

1060 .

1053 .

1090.

927.

680

1145

1199.

992.
1012.

1058 .

1266

416.

1396.

1329 .

1262 .

1262 .

988.11049 .
FAST FLUENCE

$110 * 25 \quad N / M * * 21$$$
.00
$$

.11

.22

.067

.84

1.04

1.19

1.32

1.66

1.89

2.07
2.37

2.53

2.71

2.89

2.97

3.11

3.28

3.32
124. 
FO3-01 WOMPACT 25

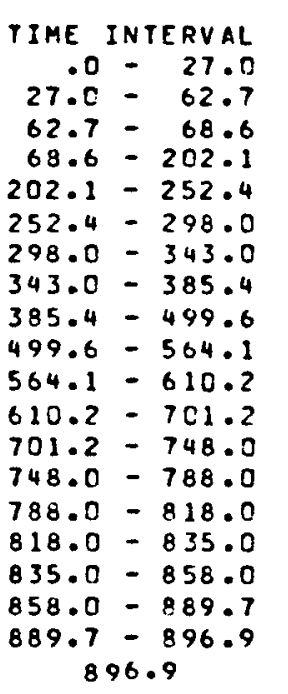

TIME AVERAGED RMS

$\stackrel{p}{1}$

COOLANT
560.
659.
546.
665.
652.
556.
658.
592.
575.
638.
659.
580
585.
561.
576.
511.
634.
659.
656.
655.
613.
44.
6

MIA. TEMPERATURES (C)

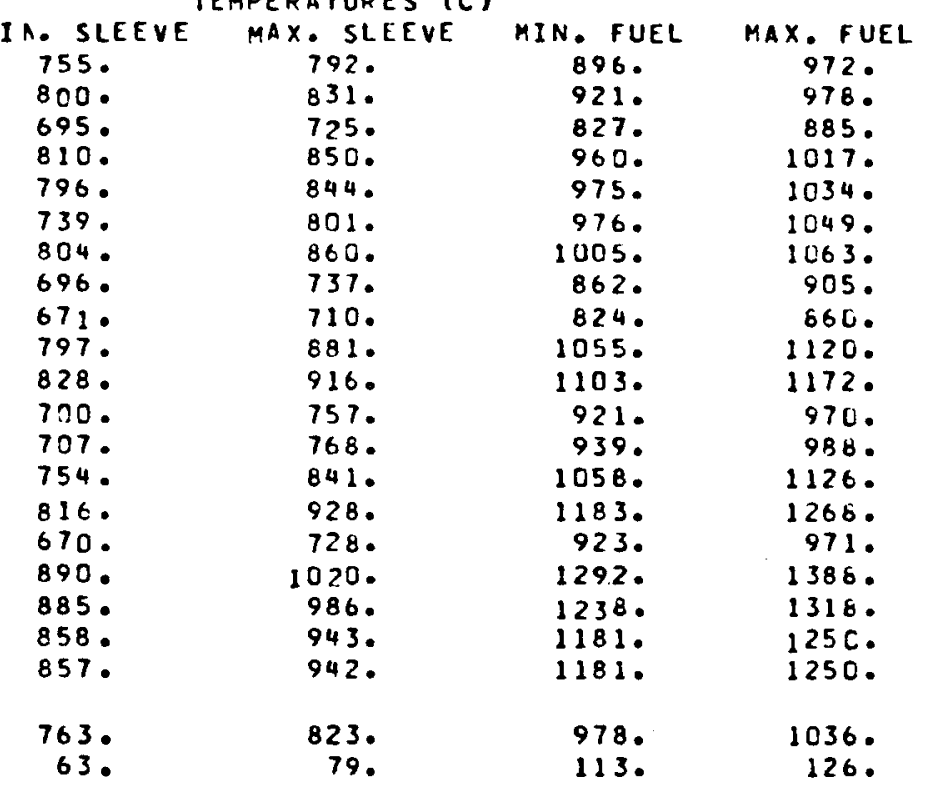

F03-01 COMPACT 26

TIME INTERVAL .0 - 27.0 $27.0=27.0$ $27.0-62.7$
$62.7-68.6$ $68.6-202.1$ $202.1-252.4$ $252.4-298.0$ $298.0-343.0$ $343.0-385.4$ $385.4=499.6$ $499.6-564.1$ $564.1-610.2$ $610.2-701.2$ $701.2-748.0$ $748.0=788.0$ $788.0-818.0$ $818.0-835.0$ $835.0-858.0$ $858.0-889.7$ $889.7-8.96 .9$ 896.9

TIME AVERAGED RMS

$\begin{array}{lc}\text { COOLANT } & \text { MIA. SLEEV } \\ 573 \circ & 7620^{\circ} \\ 667 . & 797 . \\ 556 . & 699 . \\ 674 . & 807 . \\ 661 . & 793 . \\ 568 . & 745 . \\ 667 . & 800 . \\ 599 . & 694 . \\ 581 . & 669 . \\ 647 . & 796 . \\ 669 . & 828 . \\ 588 . & 701 . \\ 593 . & 707 . \\ 576 . & 779 . \\ 593 . & 830 . \\ 524 . & 691 . \\ 651 . & 899 . \\ 674 . & 891 . \\ 669 . & 863 . \\ 669 . & 862 . \\ & \\ 622 . & 765 . \\ 44 . & 64 .\end{array}$

IEMPERATURES (C)

$\begin{array}{ccc}\text { TEMPERATURES } \\ \text { MAX. SLEEVE } & \text { MIN. FUEL } & \text { MAX. FUEL } \\ 798 . & 900 . & 973 . \\ 825 . & 909 . & 961 . \\ 728 . & 827 . & 883 . \\ 843 . & 944 . & 996 . \\ 835 . & 950^{\circ} & 1009 . \\ 804 . & 970 . & 1041 . \\ 851 . & 982 . & 1035 . \\ 731 . & 843 . & 882 . \\ 704 . & 806 . & 839 . \\ 873 . & 1033 . & 1094 . \\ 909 . & 1081 . & 1145 . \\ 753 . & 902 . & 948 . \\ 764 . & 918 . & 965 . \\ 872 . & 1083 . & 1155 . \\ 939 . & 1180 . & 1264 . \\ 752 . & 943 . & 994 . \\ 1024 . & 1280 . & 1374 . \\ 989 . & 1224 . & 1302 . \\ 943 . & 1165 . & 1231 . \\ 942 . & 1165 . & 1231 . \\ 821 . & & \\ 80 . & 964 . & 1019 . \\ & 115 . & 129 .\end{array}$

FAST FLUENCE

$110 * 25 N / M * 2)$

.00
.10
.20
.22
.59
.73
.92
1.05
1.16
1.45
1.66
1.81
2.08
2.21
2.41
2.58
2.68
2.80
2.97
3.01

FAST FLUENCE

$(10 * 25 N / M * 2)$

.00
.11
.21
.23
.63
.78
.98
1.12
1.23
1.56
1.78
1.94
2.23
2.38
2.57
2.74
2.83
2.96
3.14
3.17

3.17

3.01 
TO3-OI -OMFACI 27

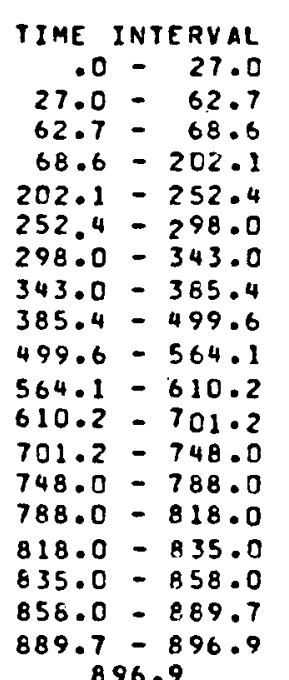

896.9

TIME AVEPAGED RMS

COOLANT
586.
$675 \circ$
565.
682.
669.
580.
675.
605.
587.
656.
679
596.
601.
591.
609.
537
668.
689.
681.
681.
632.
44.

MIA. SLEEVE
769.
793.
703.
803.
790.
751.
797.
692.
668.
794.
825.
699.
706.
800.
841.
709.
908.
898.
861.
860.
766.
65.

TEMPERATURES $(C)$

$\begin{array}{ccc}\text { MAX. SLEEVE } & \text { MIN FUEL } & \text { MAX. FUEL } \\ 804 . & 903 . & 974 . \\ 818 . & 894 . & 941 . \\ 731 . & 825 . & 879 . \\ 834 . & 925 . & 972 . \\ 827 . & 935 . & 985 . \\ 807 . & 963 . & 1032 . \\ 841 . & 960 . & 1009 . \\ 725 . & 826 . & 861 . \\ 699 . & 790 . & 820 . \\ 862 . & 1008 . & 1064 . \\ 899 . & 1054 . & 1113 . \\ 748 . & 880 . & 922 . \\ 758 . & 895 . & 937 . \\ 897 . & 1106 . & 1174 . \\ 948 . & 1174 . & 1256 . \\ 772 . & 956 . & 1008 . \\ 1028 . & 1267 . & 1358 . \\ 991 . & 1204 . & 1284 . \\ 935 . & 1135 . & 1197 . \\ 934 . & 1135 . & 1197 . \\ 817 . & 948 . & 1000 . \\ 82 . & 117 . & 131 .\end{array}$

FAST FLUENCE
$10 * 25$ N/M*21

.00

.00

.10
.18

.18
.20

.54

.67

.85

.97
1.07

1.34

1.52

1.66

1.91

2.03

2.23

2.39

2.49

2.61

2.77

2.80

\section{FO3-01 COMPACT 28}

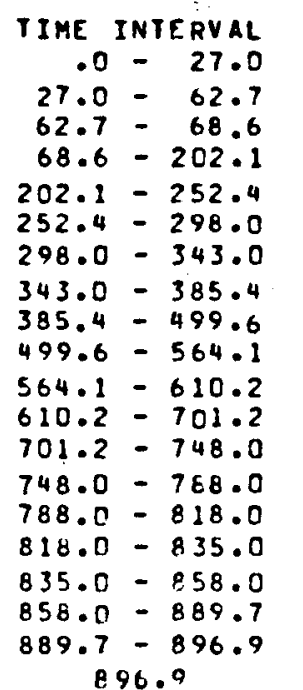

$\begin{array}{cc}\text { COOLANT } & \text { MIA. SLEEVE } \\ 598 . & 774 . \\ 682 . & 789 . \\ 574 . & 706 . \\ 690 . & 799 . \\ 676 . & 784 . \\ 591 . & 756 . \\ 682 . & 792 . \\ 611 . & 689 . \\ 593 . & 665 . \\ 665 . & 788 . \\ 688 . & 820 . \\ 602 . & 696 . \\ 608 . & 702 . \\ 607 . & 819 . \\ 625 . & 850 . \\ 550 . & 725 . \\ 684 . & 915 . \\ 702 . & 902 . \\ 693 . & 857 . \\ 692 . & 857 . \\ 640 . & 765 . \\ 44 . & 67 .\end{array}$

TEMPERATURES (C)

$\begin{array}{ccc}\text { MAX. SLEEVE } & \text { MIN. FUEL } & \text { MAX. FUEL } \\ 808 . & 904 . & 972 . \\ 812 . & 881 . & 924 . \\ 732 . & 821 . & 873 . \\ 827 . & 909 . & 952 . \\ 810 . & 911 . & 956 . \\ 807 . & 953 . & 1020 . \\ 829 . & 933 . & 977 . \\ 796 . & 804 . & 836 . \\ 691 . & 770 . & 797 . \\ 848 . & 976 . & 1027 . \\ 884 . & 1021 . & 1074 . \\ 739 . & 854 . & 892 . \\ 749 . & 867 . & 906 . \\ 918 . & 1110 . & 1185 . \\ 952 . & 1160 . & 1241 . \\ 789 . & 963 . & 1016 . \\ 1028 . & 1249 . & 1337 . \\ 990 . & 1189 . & 1260 . \\ 925 . & 1102 . & 1158 . \\ 924 . & 1101 . & 1158 . \\ 812 . & & \\ 84 . & 929 . & 978 . \\ 9 & 119 . & 133 .\end{array}$

FAST FLUENCE

110*25 N/M*21

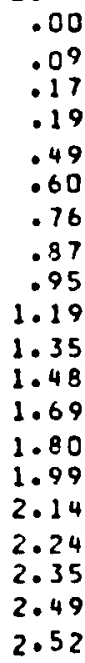

IIME AVERAGED RMS

67

84.

133. 
FO3-01 LOMPACT 29

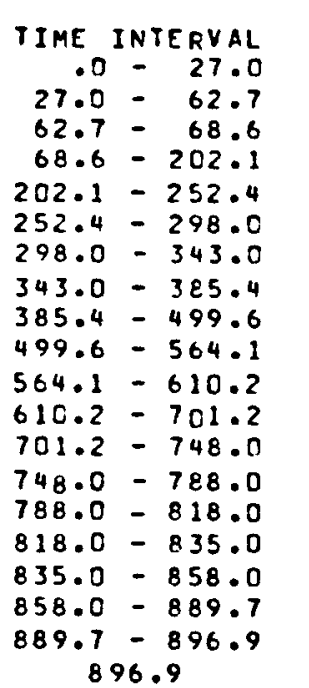

IIME AVERAGED RMS

\begin{tabular}{|c|c|}
\hline $\begin{array}{l}\text { COOLANT } \\
609 .\end{array}$ & $\begin{array}{c}\text { MIA. SLEEVE } \\
780 \text {. }\end{array}$ \\
\hline $\begin{array}{l}689 . \\
582 . \\
696 .\end{array}$ & $\begin{array}{l}784 . \\
700^{\circ} \\
794^{\circ}\end{array}$ \\
\hline $\begin{array}{l}682 . \\
602 . \\
689 .\end{array}$ & $\begin{array}{l}780 . \\
761 . \\
787 .\end{array}$ \\
\hline $\begin{array}{l}616 . \\
597^{\circ} \\
672^{\circ}\end{array}$ & $\begin{array}{l}686^{\circ} \\
662^{\circ} \\
782^{\circ}\end{array}$ \\
\hline $\begin{array}{l}695 . \\
608 . \\
614 .\end{array}$ & $\begin{array}{l}813 . \\
692 . \\
698 .\end{array}$ \\
\hline $\begin{array}{l}622 . \\
641 . \\
563 .\end{array}$ & $\begin{array}{l}833 . \\
862 . \\
737 .\end{array}$ \\
\hline $\begin{array}{l}699 . \\
715 . \\
703 . \\
703 .\end{array}$ & $\begin{array}{l}923 . \\
907 . \\
855 . \\
854 .\end{array}$ \\
\hline $\begin{array}{r}648 \\
44 .\end{array}$ & $\begin{array}{r}763 . \\
69 .\end{array}$ \\
\hline
\end{tabular}

TEMPERATURES (C)

\begin{tabular}{|c|c|}
\hline $\begin{array}{l}\text { IN. FUEL } \\
\text { 906. }\end{array}$ & $\begin{array}{c}\text { MAX. FUEL } \\
972 .\end{array}$ \\
\hline $\begin{array}{l}866 . \\
818 . \\
889 .\end{array}$ & $\begin{array}{l}905 . \\
868 . \\
928 .\end{array}$ \\
\hline $\begin{array}{l}889 . \\
943 . \\
909 .\end{array}$ & $\begin{array}{r}929 . \\
1007 . \\
948 .\end{array}$ \\
\hline $\begin{array}{l}784 . \\
752 . \\
942 .\end{array}$ & $\begin{array}{l}813 . \\
776 . \\
988 .\end{array}$ \\
\hline $\begin{array}{l}985 . \\
827 . \\
839 .\end{array}$ & $\begin{array}{l}1033 . \\
861 . \\
874\end{array}$ \\
\hline $\begin{array}{l}1107 \\
1155 \\
960\end{array}$ & $\begin{array}{l}1182 . \\
1234^{\circ} \\
1013^{\circ}\end{array}$ \\
\hline $\begin{array}{l}1234 . \\
1172 . \\
1071 . \\
1071 .\end{array}$ & $\begin{array}{l}1319 . \\
1240 . \\
11230^{\circ} \\
1123 .\end{array}$ \\
\hline $\begin{array}{l}910 . \\
121 .\end{array}$ & $\begin{array}{l}954 . \\
136 .\end{array}$ \\
\hline
\end{tabular}

FO3-01 COMPACT 30

TIME INTERYAL

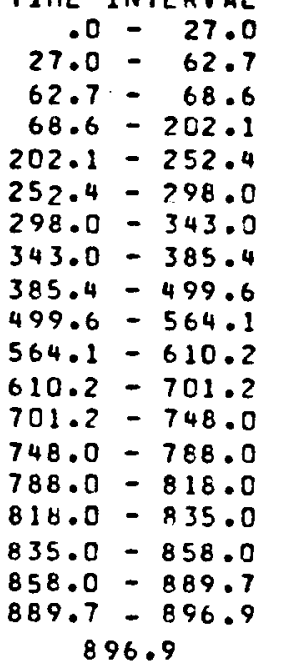

COOLANT
621.
696.
591.
703.
689.
613.
696.
621.
602.
679.
703.
615.
620.
638.
658.
576.
715.
729.
713.
713.
657.
44.

MIA. SLEEVE
793.
799.
723.
809.
793.
773.
801.
696.
672.
792.
824.
700.
706.
852.
881.
753.
940.
928.
857.
856.
776.
72.

TEMPERATURES (C)

$\begin{array}{ccc}\text { MAX. SLEEVE } & \text { MIN. FUEL } & \text { MAX. FUEL } \\ 827 . & 920 . & 986 . \\ 821 . & 886 . & 927 . \\ 748 . & 833 . & 884 . \\ 833 . & 906 . & 948 . \\ 819 . & 900 . & 943 . \\ 815 . & 940 . & 1005 . \\ 830 . & 918 . & 960 . \\ 717 . & 790 . & 821 . \\ 690 . & 757 . & 783 . \\ 832 . & 937 . & 984 . \\ 868 . & 980 . & 1029 . \\ 730 . & 823 . & 857 . \\ 739 . & 835 . & 870 . \\ 935 . & 1104 . & 1181 . \\ 970 . & 1152 . & 1232 . \\ 810 . & 961 . & 1016 . \\ 1039 . & 1230 . & 1315 . \\ 1009 . & 1182 . & 1253 . \\ 912 . & 1050 . & 1100 . \\ 911 . & 1050 . & 1099 . \\ 813 . & 914 . & \\ 89 . & 119 . & 960 .\end{array}$

FAST FLUENCE

(10*25 N/M*2)

.00
.06
.11
.12
.28
.35
.47
.52
.57
.69
.78
.85
.96
1.02
1.16
1.28
1.36
1.43
1.54
1.56


FO3-01 FUEL PERFORMANCE

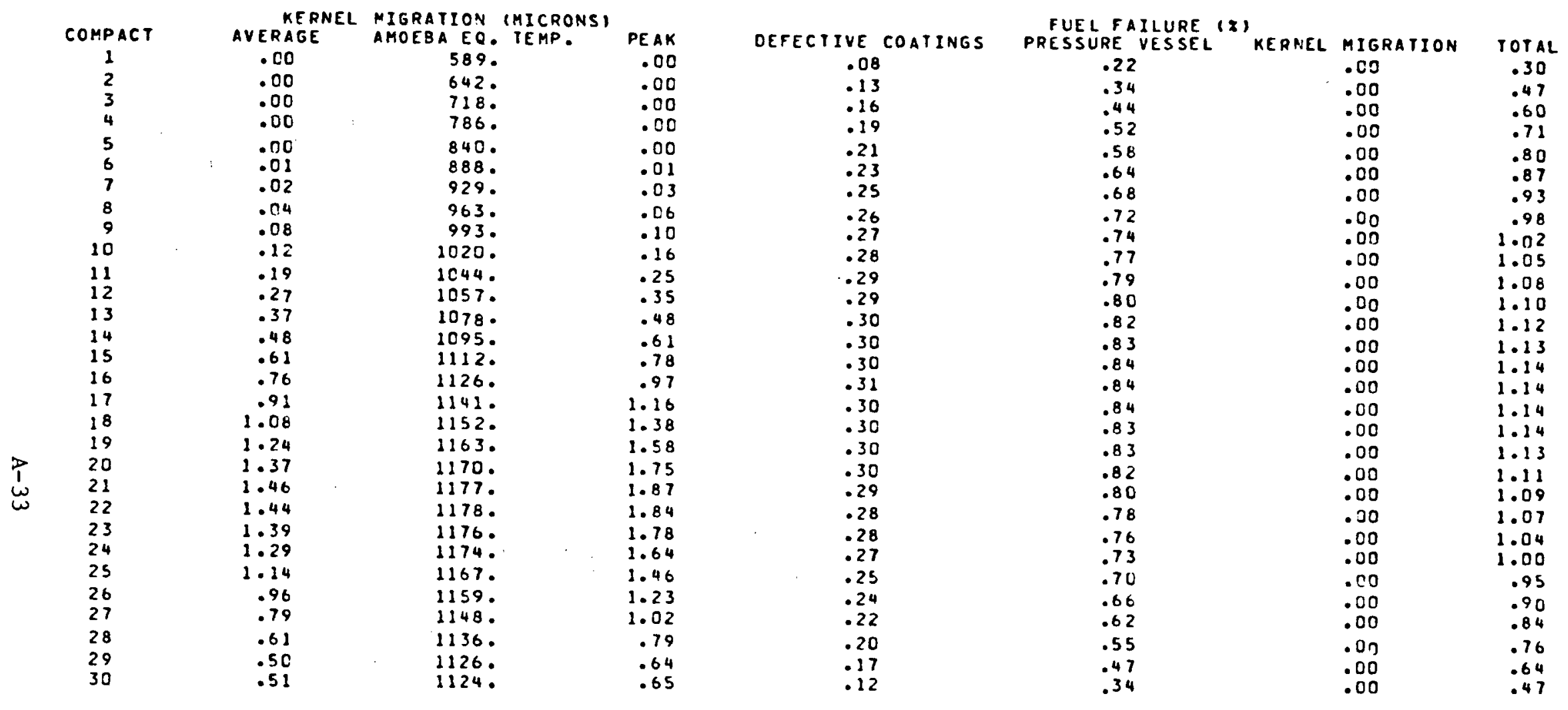

AMOEBA EOUIVALENT TEMPERATURES (C) CORRESPONO TO AVERAGE KERNEL MIGRATIONS KERNEL MIGRATION DISTANCES CALCULATED USING 5OZ CONFIOENCE LEVEL KMC FUEL FAILURE CORRESPONDS TO SOZ CONF IDENCE LEVEL 
F05-05 LOMPACT 1

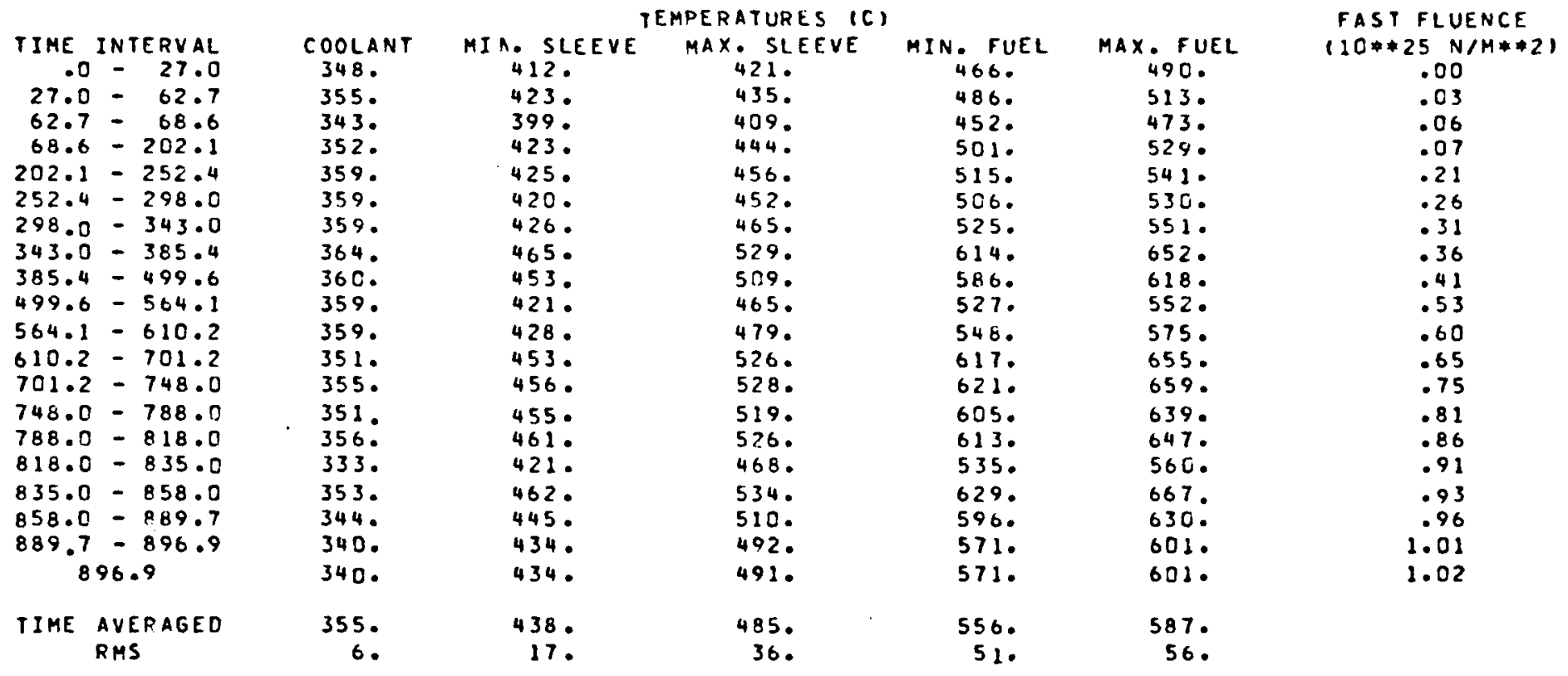

\begin{tabular}{|c|c|c|c|c|c|c|}
\hline TIME & INTERVAL & COOLANT & MIA. SLEEYE & $\begin{array}{l}\text { MPERATURES }(C) \\
\text { MAX. SLEEYE }\end{array}$ & MIN, FUEL & MAX, FUEL \\
\hline 27.0 & $\begin{array}{l}27.0 \\
-\quad 62.7\end{array}$ & $\begin{array}{l}353 \\
360\end{array}$ & $\begin{array}{l}4250 \\
436\end{array}$ & $\begin{array}{l}435 . \\
451\end{array}$ & $\begin{array}{l}484 . \\
508 .\end{array}$ & $\begin{array}{l}511 . \\
538 .\end{array}$ \\
\hline $\begin{array}{r}62.7 \\
68.6 \\
202.1\end{array}$ & $\begin{array}{l}=\quad 68.6 \\
=\quad 202.1 \\
-\quad 252.4\end{array}$ & $\begin{array}{l}347 . \\
357 \\
364 .\end{array}$ & $\begin{array}{l}409 . \\
437 . \\
440 .\end{array}$ & $\begin{array}{l}422 . \\
469 . \\
487 .\end{array}$ & $\begin{array}{l}471 . \\
535 . \\
557 .\end{array}$ & $\begin{array}{l}495 . \\
566 . \\
587 .\end{array}$ \\
\hline $\begin{array}{l}252.4 \\
298.0 \\
343.0\end{array}$ & $\begin{array}{l}-298.0 \\
=343.0 \\
-\quad 385.4\end{array}$ & $\begin{array}{l}363 . \\
364 . \\
371 .\end{array}$ & $\begin{array}{l}433 . \\
441 . \\
487 .\end{array}$ & $\begin{array}{l}479 . \\
494 . \\
569 .\end{array}$ & $\begin{array}{l}545 . \\
560 . \\
669 .\end{array}$ & $\begin{array}{l}572 . \\
595 . \\
713 .\end{array}$ \\
\hline $\begin{array}{l}385.4 \\
499.6 \\
564.1\end{array}$ & $\begin{array}{l}=499.6 \\
-564.1 \\
-610.2\end{array}$ & $\begin{array}{l}367 \\
364^{\circ} \\
365 .\end{array}$ & $\begin{array}{l}473 . \\
437 . \\
447 .\end{array}$ & $\begin{array}{l}540 . \\
490 . \\
507 .\end{array}$ & $\begin{array}{l}631 . \\
566 . \\
590 .\end{array}$ & $\begin{array}{l}666 . \\
595 . \\
622 .\end{array}$ \\
\hline $\begin{array}{l}610.2 \\
701.2\end{array}$ & $\begin{array}{l}-701.2 \\
-748.0\end{array}$ & $\begin{array}{l}358 . \\
363 .\end{array}$ & $\begin{array}{l}480 . \\
482 .\end{array}$ & $\begin{array}{l}564 . \\
564 .\end{array}$ & $\begin{array}{l}671 . \\
671 .\end{array}$ & $\begin{array}{l}716 . \\
717 .\end{array}$ \\
\hline 748.0 & -788.0 & 359. & 483. & 555. & 653. & 694. \\
\hline $\begin{array}{l}788.0 \\
818.0\end{array}$ & $\begin{array}{l}-818.0 \\
-835.0\end{array}$ & $\begin{array}{l}364 . \\
340 .\end{array}$ & $\begin{array}{l}493 . \\
448 .\end{array}$ & $\begin{array}{l}569 . \\
503 .\end{array}$ & $\begin{array}{l}670 . \\
582 .\end{array}$ & $\begin{array}{l}713 . \\
613 .\end{array}$ \\
\hline 835.0 & -858.0 & 361. & 495. & 580. & 689. & $7360^{\circ}$ \\
\hline $\begin{array}{l}858.0 \\
889.7\end{array}$ & $\begin{array}{l}-889.7 \\
-\quad 896.9\end{array}$ & $\begin{array}{l}352 . \\
347 .\end{array}$ & $\begin{array}{l}476 . \\
463 .\end{array}$ & $\begin{array}{l}551 . \\
530 .\end{array}$ & $\begin{array}{l}650 . \\
621 .\end{array}$ & $\begin{array}{l}692 . \\
658 .\end{array}$ \\
\hline B & 96.9 & 347. & 463. & 529. & 620. & 658. \\
\hline IIME & $\begin{array}{l}\text { AVERAGEO } \\
\text { FHS }\end{array}$ & $\begin{array}{l}361 . \\
6 .\end{array}$ & $\begin{array}{r}458 . \\
22 .\end{array}$ & $\begin{array}{l}516 . \\
43 .\end{array}$ & $\begin{array}{l}599 . \\
60 .\end{array}$ & $\begin{array}{r}635 . \\
66 .\end{array}$ \\
\hline
\end{tabular}

FAST FLUENCE

(10*25 $N / M * 2)$ .00

.04

.11

.12

.35

.44

. 51

.58

.67

.88

1.07

1.25

1.34

1.43
1.50

1.54

1.59

1.59

1.67
1.69
PHS

22.

43.

60 .

66 . 
r05-05 _.JMPACT 3

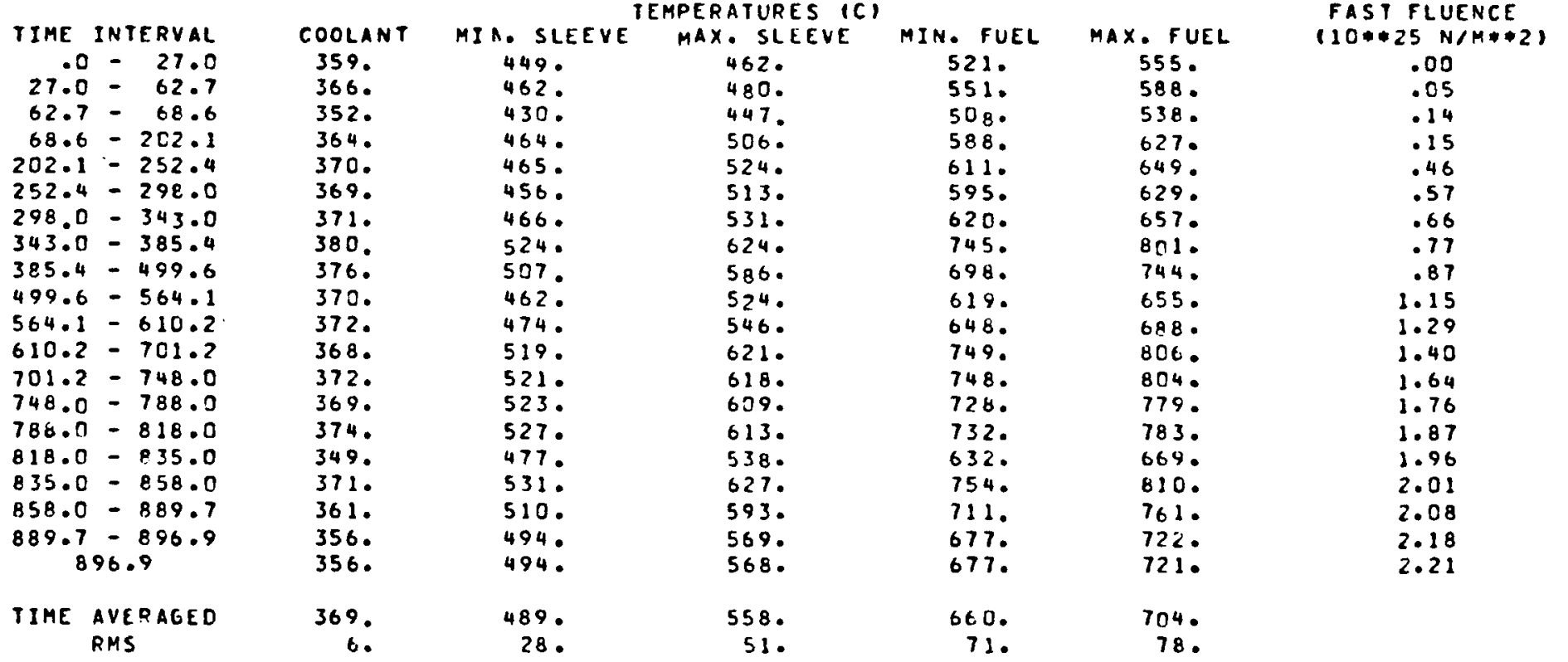

TIHE INTERVAL

$.0-27.0$
$27.0=62.7$
$62.7=68.6$
$68.6=202.1$
$202.1=252.4$
$252.4=298.0$
$298.0=343.0$
$343.0=385.4$
$385.4=499.6$
$499.6=564.1$
$564.1=610.2$
$610.2-701.2$
$791.2=748.0$
$748.0=788.0$
$788.0-818.0$
$818.0=835.0$
$835.0-858.0$
$858.0=889.7$
$889.7=896.9$

$\begin{array}{cc}\text { COOLANT } & \text { MIR. SLEEVE } \\ 367 . & 474 . \\ 374 . & 489 . \\ 359 . & 453 . \\ 372 . & 492 . \\ 378 . & 489 . \\ 376 . & 478 . \\ 378 . & 490 . \\ 390^{\circ} & 560 . \\ 385 . & 540 . \\ 377 . & 483 . \\ 380 . & 498 . \\ 379 . & 553 . \\ 383 . & 555 . \\ 380 . & 558 . \\ 385 . & 555 . \\ 358 . & 501 . \\ 383 . & 560 . \\ 372 . & 537 . \\ 366 . & 519 . \\ 366 . & 519 . \\ 378 . & 517 . \\ 6 . & 32 .\end{array}$

TEMPERATURES (C)

MIN. FUEL

MAX. FUEL

FASI FLUENCE

(10*25 N/M**2)

$\begin{array}{ll}\text { FUEL } & M A X . F U E L \\ 559 . & 600 . \\ 595 . & 640 . \\ 545 . & 581 . \\ 641 . & 687 . \\ 659 . & 703 . \\ 639 . & 679 . \\ 669 . & 712 . \\ 814 . & 879 . \\ 760 . & 815 . \\ 662 . & 704 . \\ 695 . & 742 . \\ 815 . & 880 . \\ 814 . & 879 . \\ 794 . & 853 . \\ 786 . & 843 . \\ 678 . & 719 . \\ 814 . & 877 . \\ 768 . & 824 . \\ 731 . & 781 . \\ 731 . & 781 . \\ & \\ 715 . & 766 . \\ 79 . & 87 .\end{array}$

.00
.07
.17
.18
.55
.69
.80
.93
1.06
1.40
1.57
1.70
1.98
2.12
2.26
2.36
2.42
2.50
2.62
2.65

IIME AVERAGEO RMS

590.

473.

543.

556.

564.

673 .

628.
550.

577 .

665 .

661.

652.

566.

662.

$599^{\circ}$

599 .

594.
56. 
FO5-05 COMPACT 5

TIME INTERVAL
$.0-27.0$

$27.0-62.7$

$62.7-68.6$

$68.6-202.1$

$202.1-252.4$

$298.0=343.0$

$343.0=385.4$

$385.4-499.6$

$499.6=564.1$

$564.1-610.2$

$610.2=701.2$

$701.2=748.0$
$748.0=788.0$

$788.0-818.0$

$818.0-835.0$

$835.0-858.0$

$858.0-889.7$

$889.7-896.9$

$$
896.9
$$

IIME AVERAGED RMS

$\underset{⿱ 乛}{w}$

COOLANT
375 .
383.
366.
381.
386.
384.
387.
402.
396.
385.
389.
391.
395.
393.
397.
369.
395.
383.
377.
377.
388.
7.

MIA. SLEEVE TEMPERATURES ( $C$ ) 495 .

511.

515 .

510 .

497.

511.

569 :

499.

516.

579.

580.

585 .

522 .

584 .

560 .

541 .

540 .

541.

35 .

Aax. SLE

513.

536.
493.

571.

582.

582.

591.

713.

663.

$$
\begin{aligned}
& 569 . \\
& 599 .
\end{aligned}
$$

698.

692 .

683.

588 .

691.

623.

623.

623.
60.

MIN. FUEL 589.

627.

573.

679.
697.

676.

708 .

870.
812.

695 .

732 .

866 .

867.

849.

837.

870 .

823.

784.

784.

759.

87.

MAX. FUEL

634.

678.

613.

731.

747.

757.

757.
943.

873.

741.

783.

936.

938.

913.

898 .

763 .

937.

882

837.

816.

96.

\section{F05-05 COMPACT 6}

IIME INTERVAL $.0-27.0$ $27.0-62.7$ $62.7-68.6$ $63.6-202.1$ $202.1-252.4$ $252.4-298.0$ $298.0-343.0$ $343.0-385.4$ $385.4-499.6$ $499.6=564.1$ $564.1-610.2$ $610.2-701.2$ $701.2-748.0$ $748.0-788.0$ $788.0-818.0$ $818.0-835.0$ $835.0=858.0$ $858.0-889.7$ $889.7=896.9$ B46.9

TIME AVERAGED RMS

COOLANT
384.
393.
374.
391.
396.
392.
396.
415.
408.
393.
398.
403.
407
406.
409.
380.
408.
$395 \circ$
388.
388.
399.
9.

MIR. SLEEVE
514.
532.
487.
536.
529.
515.
531.
620.
595.
515.
534.
604.
605.
611.
691.
540.
606.
581.
560.
559.
563.
38.

TEMPERATURES (C)

$\begin{array}{ccc}\text { MAX. SLEEVE } & \text { MIN. FUEL } & \text { MAX. FUEL } \\ 534 . & 615 . & 664 . \\ 559 . & 657 . & 712 . \\ 512 . & 599 . & 64 . \\ 596 . & 714 . & 770 . \\ 606 . & 732 . & 785 . \\ 589 . & 708 . & 756 . \\ 615 . & 744 . & 796 . \\ 749 . & 921 . & 999 . \\ 694 . & 860 . & 926 . \\ 586 . & 727 . & 776 . \\ 619 . & 769 . & 822 . \\ 727 . & 916 . & 991 . \\ 720 . & 921 . & 997 . \\ 712 . & 905 . & 972 . \\ 695 . & 887 . & 951 . \\ 607 . & 765 . & 811 . \\ 714 . & 925 . & 990 . \\ 675 . & 877 . & 940 . \\ 644 . & 836 . & 893 . \\ 644 . & 837 . & 893 . \\ 648 . & 801 . & 862 . \\ 63 . & 90 . & 105 .\end{array}$

FAST FLUENCE

$110 * 25 N / M * 21$

.00
.08
.21
.23
.68
.85
.99
1.14
1.30
1.72
1.93
2.09
2.43
2.61
2.77
2.89
2.95
3.05
3.19
3.22

FAST FLUENCE

.00
.07
.19
.21
.62
.78
.90
1.05
1.19
1.57
1.76
1.91
2.23
2.39
2.54
2.65
2.71
2.80
2.93
2.96

68

.99

.140

.72

.93

.09

.61

2.95

.05

3.22

55 . 
FOS-05 LOMPACT 7

\begin{tabular}{|c|c|c|c|c|c|c|c|}
\hline & \multicolumn{5}{|c|}{ IEMPERATURES $(\mathrm{C})$} & FAST FLUENCE \\
\hline $\begin{array}{l}\text { TIME } \\
.00 \\
27.0\end{array}$ & $\begin{array}{l}\text { INTERVAL } \\
-\quad 27.0 \\
-\quad 62.7\end{array}$ & $\begin{array}{l}\text { COOLANT } \\
394 . \\
403\end{array}$ & $\begin{array}{l}\text { MIA. SLEEVE } \\
532 . \\
550 .\end{array}$ & $\begin{array}{l}\text { MAX. SLEEVE } \\
553 . \\
580 .\end{array}$ & $\begin{array}{c}\text { MIN. FUEL } \\
637 . \\
683 .\end{array}$ & $\begin{array}{c}\text { MAX. FUCL } \\
689 . \\
741 .\end{array}$ & $\begin{array}{c}110 * 25 N / M * 21 \\
.00 \\
.09\end{array}$ \\
\hline $\begin{array}{r}62.7 \\
63.6 \\
202.1\end{array}$ & $\begin{array}{l}=68.6 \\
=202.1 \\
-252.4\end{array}$ & $\begin{array}{l}382 \\
402 \\
405\end{array}$ & $\begin{array}{l}502 \\
555 \\
547\end{array}$ & $\begin{array}{l}529 . \\
618 . \\
626 .\end{array}$ & $\begin{array}{l}621 \\
743 \\
761\end{array}$ & $\begin{array}{l}667 . \\
802 . \\
816\end{array}$ & $\begin{array}{l}.22 \\
.24 \\
.73\end{array}$ \\
\hline $\begin{array}{l}252.4 \\
298.0\end{array}$ & $\begin{array}{l}=298.0 \\
=343.0\end{array}$ & $\begin{array}{l}401 . \\
406 .\end{array}$ & $\begin{array}{l}532 . \\
549 .\end{array}$ & $\begin{array}{l}607 . \\
636 .\end{array}$ & $\begin{array}{l}736 . \\
774 .\end{array}$ & $\begin{array}{l}787 . \\
430 .\end{array}$ & $\begin{array}{r}.91 \\
1.06\end{array}$ \\
\hline 343.0 & -385.4 & 429. & 645. & 779. & 963. & 1047. & 1.22 \\
\hline $\begin{array}{l}385.4 \\
499.6\end{array}$ & $\begin{array}{l}-499.6 \\
-564.1\end{array}$ & $\begin{array}{l}421 . \\
402 .\end{array}$ & $\begin{array}{l}619 . \\
532 .\end{array}$ & $\begin{array}{l}721 . \\
605 .\end{array}$ & $\begin{array}{l}901 . \\
761 .\end{array}$ & $\begin{array}{l}971 . \\
813 .\end{array}$ & $\begin{array}{l}1.40 \\
1.84\end{array}$ \\
\hline 564.1 & -610.2 & 408 & 552. & $64 C$. & 807 & 864. & 2.06 \\
\hline $\begin{array}{l}610.2 \\
701.2\end{array}$ & $\begin{array}{l}-701.2 \\
-\quad 748.0\end{array}$ & $\begin{array}{l}417 . \\
420 .\end{array}$ & $\begin{array}{l}629 . \\
630 .\end{array}$ & $\begin{array}{l}757 . \\
748 .\end{array}$ & $\begin{array}{l}968 . \\
978 .\end{array}$ & $\begin{array}{l}1048 . \\
1058\end{array}$ & $\begin{array}{l}2.23 \\
2.60\end{array}$ \\
\hline 748.0 & -788.0 & 421. & 637. & 742. & 963. & 1035. & 2.79 \\
\hline $\begin{array}{l}788.0 \\
818.0\end{array}$ & $\begin{array}{l}-818.0 \\
-835.0\end{array}$ & $\begin{array}{l}422 . \\
391 .\end{array}$ & $\begin{array}{l}621 . \\
558 .\end{array}$ & $\begin{array}{l}717 . \\
626 .\end{array}$ & $\begin{array}{l}937 . \\
809 .\end{array}$ & $\begin{array}{r}1004 . \\
857\end{array}$ & $\begin{array}{l}2.95 \\
3.08\end{array}$ \\
\hline $\begin{array}{r}835.0 \\
858.0 \\
839.7 \\
8\end{array}$ & $\begin{array}{l}-858.0 \\
-889.7 \\
-896.9 \\
96.9\end{array}$ & $\begin{array}{l}421 . \\
408 . \\
400 . \\
400 .\end{array}$ & $\begin{array}{l}628 . \\
601 . \\
578 . \\
578 .\end{array}$ & $\begin{array}{l}737 . \\
696 . \\
663 . \\
663 .\end{array}$ & $\begin{array}{l}980 . \\
932 . \\
890 . \\
890 .\end{array}$ & $\begin{array}{l}1054 . \\
997 . \\
948^{\circ} \\
949 .\end{array}$ & $\begin{array}{l}3.14 \\
3.25 \\
3.40 \\
3.43\end{array}$ \\
\hline TI ME & $\begin{array}{l}\text { A VER RGED } \\
\text { RMS }\end{array}$ & $\begin{array}{l}410 . \\
10 .\end{array}$ & $\begin{array}{l}584 . \\
41 .\end{array}$ & $\begin{array}{r}672 . \\
67 .\end{array}$ & $\begin{array}{l}841 . \\
106 .\end{array}$ & $\begin{array}{l}905 . \\
116 .\end{array}$ & \\
\hline
\end{tabular}

FO5-05 COMPACT 8

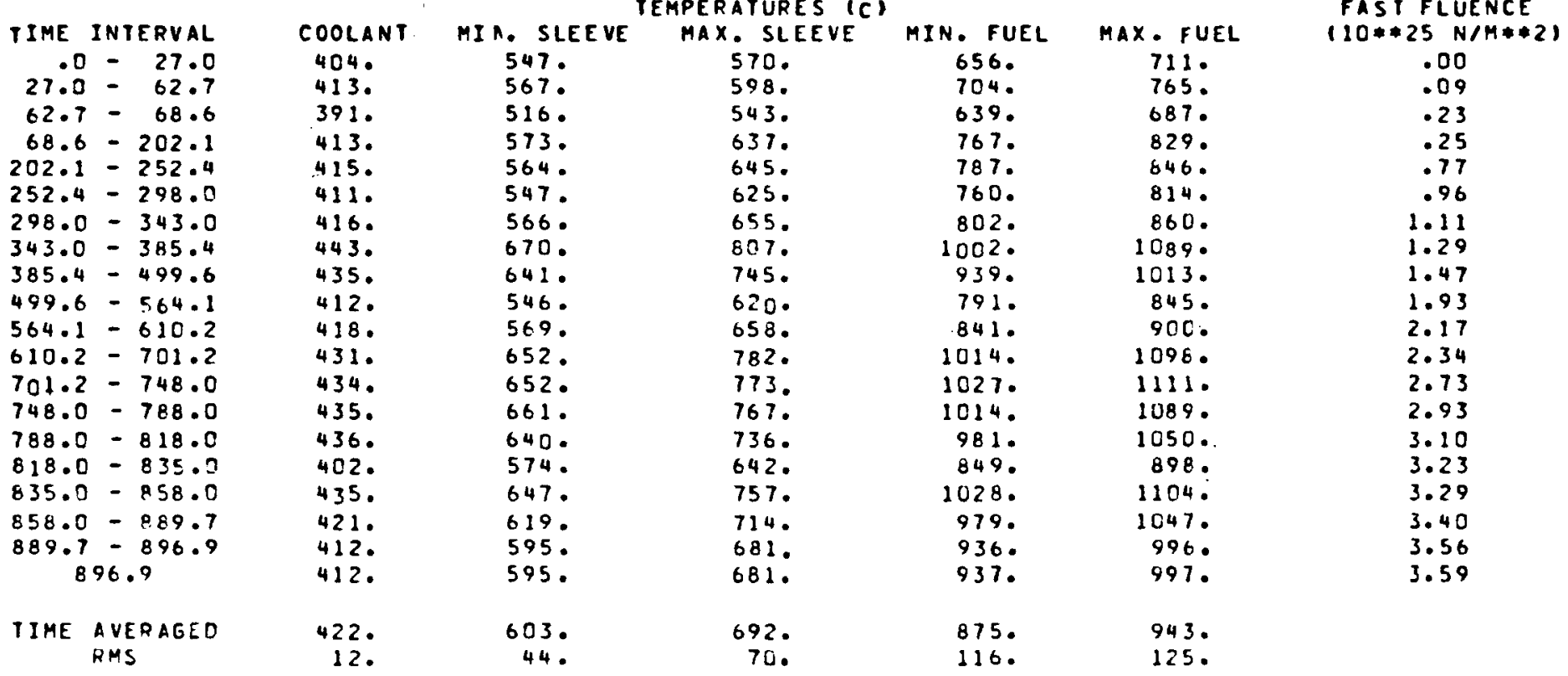


FOS-05 COMPACT 9

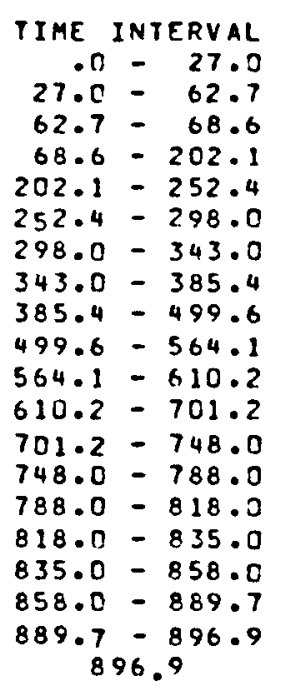

IIME AVERAGEO RMS

COOLANT
414.
424.
399.
424.
426.
421.
427.
458.
448.
421.
429.
445.
448.
451.
450.
414.
449.
434.
424.
424.
435.
13.

MIA. SLEEVE
560.
581.
527.
587.
580.
562.
582.
693.
663.
560.
584.
673.
673.
683.
658.
589.
665.
636.
611.
611.
620.
48.

EMPERATURES (C)

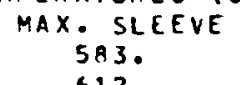

583.

555 .

651.

662 .

640.

673.

833.
768.

634 .

674.

805 .

705 .

705.
790.

790.

753.
656.

774.

731.

696.

696.

$M I$

MIN. FUE 671. 720.
652.

784.

810.

826.

1036 .

973.

871 .

1054 .

1070 .

1057.

1017.

882.

1067 .

1017.

973.

MAX FUEL

726.

782.

847.

872 .

838 .

887 .

1127

1049 .

873.

1141.

1156

$1156^{\circ}$

1135.

1088 .

1144.

1086.

1035 .

1035 .

905 .

974.

711.
74.

125.

135.

\section{F05-05 COMPACT 10}

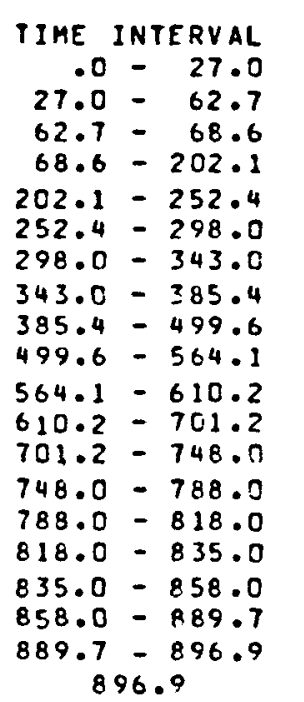

$\begin{array}{ll}\text { COOLANT } & \text { MIA. SLEEVE } \\ 424 . & 572 . \\ 435 . & 593 . \\ 408 . & 537 . \\ 436 . & 600 . \\ 437 . & 593 . \\ 430 . & 575 . \\ 438 . & 596 . \\ 473 . & 712 . \\ 463 . & 681 . \\ 431 . & 575 . \\ 440 . & 600 . \\ 460 . & 696 . \\ 463 . & 696 . \\ 467 . & 707 . \\ 464 . & 677 . \\ 426 . & 606 . \\ 463 . & 685 . \\ 448 . & 654 . \\ 437 . & 629 . \\ 437 . & 628 . \\ 447 . & 637 . \\ 15 . & 51 .\end{array}$

TEMPERATURES (C)

MAX. SLEEVE
$5955^{\circ}$
$6250^{\circ}$
$565^{\circ}$
$664^{\circ}$
676.
653.
687.
853.
786.
650.
692.
$830^{\circ}$
819.
816.
$7730^{\circ}$
673.
$795 \circ$
750.
714.
714.
728.
78.

MIN. FUEL

683.

739.

734.796.

664.713.

798. 862 .

827.890.

799.856.

845 .

1062 .

998.

845.

901.

1095 .

1112.

1101.

917.

1108.

1057

1012.

1013.

956.

1155.

1076.

902.

965.

1184.

1201.

1181.

1128 .

969.

1128.

1075.

1076.

FAST FLUENCE

I1O*25 N/M*21

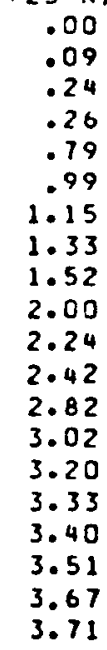

.09
.24

28

.79

.15

1.33

1.52
2.00

2.24

2.42

2.82

3.20

3.33

3.51

3.67

3.71

931.1003.

145.

FAST FLUENCE

$(10 * 25 N / M * 2)$

.00

.10

.24

.81

1.01

1.18

1.36

1.56

2.05

2.30

2.90

3.11

3.29

3.43

3.50

3.61

3.77

AVERAGED

$$
\text { RMS }
$$

15.

51. 
FOS-05 LUMACT 12

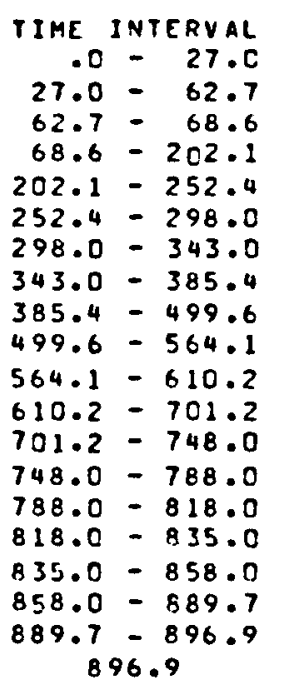

TIME AVEPAGED RMS

COOLANT
$4350^{\circ}$
446.
417.
447.
448.
441.
449.
489.
477.
441.
451.
476.
478.
483.
478.
439.
478.
461.
450.
450.
460.
17.

MIA. SLEEVE
584.
606.
548.
613.
606.
596.
609.
729.
697.
588.
615.
717.
716.
729.
694.
620.
702.
671.
644.
643.
653.
54.

TEMPERATURES (C)

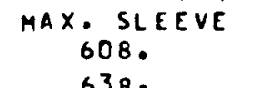

608 .

578.

676.

687 .

663.

698 .

870 .

802 .

664.

852.

$852 \circ$

838 .

789.

697.

812.

728.

728.

744.

81.
MIN. FUEL

696.

747.

812.

841 .

812.

812.

1083.

1019.

867.

927.

1128.

1146.

1135.

1083.
942.

1136.

1085 .

1038 .

1039.

953.

141.
MAX. FUEL

753.

811.

725 .

905 .

870 .

922.

1177.

1496.

927.

992.

1216

1216.

1156.

994.
1217.

1156.

1103.

1104 .

1026.

152 .

F05-05 COMPACT 12

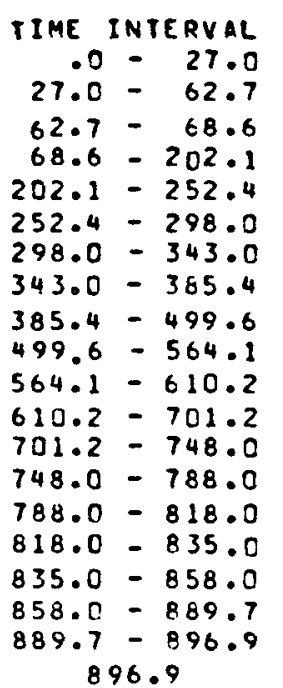

896.9

TIME AVERAGED RMS

$\begin{array}{cc}\text { COOLANT } & \text { MIR. SLEEVE } \\ 445 . & 594 . \\ 457 . & 616 . \\ 426 . & 556 . \\ 459 . & 624 . \\ 459 . & 617 . \\ 451 . & 597 . \\ 460 . & 620 . \\ 504 . & 745 . \\ 491 . & 712 . \\ 452 . & 601 . \\ 463 . & 629 . \\ 492 . & 736 . \\ 494 . & 735 . \\ 500 . & 749 . \\ 492 . & 711 . \\ 451 . & 635 . \\ 493 . & 720 . \\ 475 . & 687 . \\ 463 . & 659 . \\ 463 . & 659 . \\ 473 . & 667 . \\ 19 . & 57 .\end{array}$

TEMPERATURES (C)

$\begin{array}{cc}\text { MAX. SLEEVE } & \text { MIN FUEL } \\ 618 . & 706 . \\ 649 . & 758 . \\ 584 . & 683 . \\ 685 . & 821 . \\ 697 . & 852 . \\ 673 . & 823 . \\ 708 . & 871 . \\ 885 . & 1099 . \\ 815 . & 1035 . \\ 677 . & 885 . \\ 721 . & 947 . \\ 872 . & 1155 . \\ 860 . & 1173 . \\ 859 . & 1163 . \\ 805 . & 1107 . \\ 701 . & 963 . \\ 829 . & 1161 . \\ 781 . & 1109 . \\ 743 . & 1062 . \\ 743 . & 1062 . \\ 757 . & 971 . \\ 84 . & 147 .\end{array}$

MAX. FUEL

763.

FAST FLUENCE

110**25 N/H**2)

.00

.10

.25

.27
.82

1.03

1.19

1.38

1.58
2.09

2.35

2.55
2.97

3.18

3.38

3.52

3.60

3.71

3.92 
F05-05 LOMPACT 13

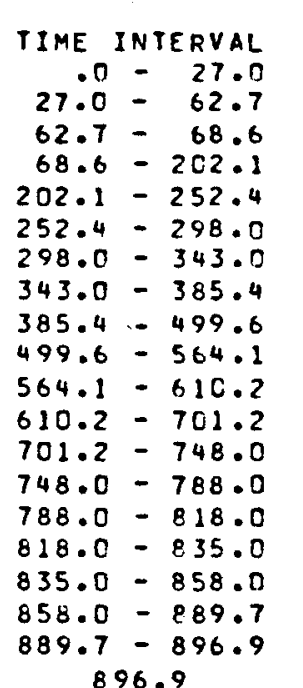

896.9

TIME AVERAGEO RMS

$\begin{array}{cc}\text { COOLANT } & \text { MIA. SLEEVE } \\ 456 . & 603 . \\ 468 . & 626 . \\ 435 . & 565 . \\ 470 . & 635 . \\ 470 . & 628 . \\ 461 . & 607 . \\ 471 . & 632 . \\ 520 . & 761 . \\ 506 . & 726 . \\ 462 . & 614 . \\ 475 . & 644 . \\ 508 . & 755 . \\ 510 . & 754 . \\ 517 . & 769 . \\ 507 . & 728 . \\ 464 . & 650 . \\ 508 . & 738 . \\ 489 . & 704 . \\ 476 . & 674 . \\ 476 . & 674 . \\ 486 . & 681 . \\ 21 . & 60 .\end{array}$

MA

MAX. SLEEVE

628 .

659.

692 .

706.

682.

719.

829.

689.

735.

892.

879.

879.

716.

846.

797.

758.

758.

771.
88. MIN. FUEL 715. 767.

690.

862 .

862.

883 .

1115.

1051.

967.

1181.

1199.

1189.

1130.

1184.

1131 .

1083.

1084 .

987.

153.

772 .

830.

740.

894.

926.

891.

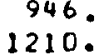

1130.

1034.

1275.

1294 .

1274.

1205.

1039 .

1267.

1205 .

1149.

$115 \mathrm{C}$.

1061.
165.

F05-05 COMPACT 14

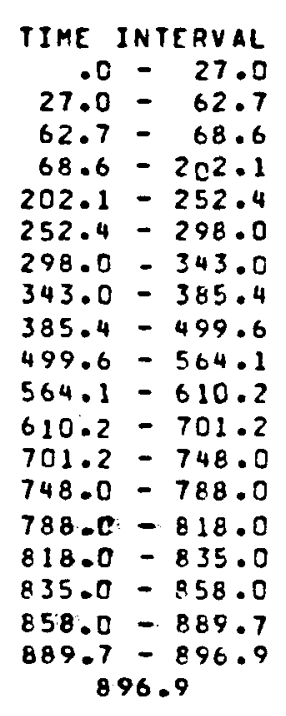

TIME AVERAGED

COOLANT
466.
479.
444.
482.
481.
471.
483.
535
520
473.
487
524
525.
533
522
477.
523.
504.
489.
489.
499.
24.

MIA. SLEEVE
612.
636.
572.
645.
639.
617.
642.
775.
740.
625.
656.
772.
770.
787.
746.
665.
755.
720.
690.
690.
694.
63.

TEMPERATURES (C)
MAX. SLEEVE

MAX. SLEEVE
637.
668.
599.
702.
715.
690.
727.
932.
841.
700.
747.
907.
895.
896.
839.
730.
864.
814.
773.
773.
782.
90.

MIN. FUEL

723.
775.

696.

836.

870.

840 .

891.

1063 .

915.

980.

1197.

1215.

1205 .

1149.

1002 .

1204 .

1150 .

1101.

MAX. FUE

779.

837 .

746.

900 .

934.

698 .

954 .

1221.

1142.

977.

1048 .

1292 .

1310 .

1291 .

1226 .

$1057 \circ$

1289.

1225 .

$1102 . \quad 1168$.

1169.

999.

1073.

FAST FLUENCE

(10*25 $N / M * 2$ )

.00
.10
.25
.27
.82
1.03
1.20
1.39
1.59
2.10
2.36
2.56
2.99
3.21
3.40
3.55
3.62
3.74
3.91
3.95

157.

FAST FLUENCE

$110 * 25 N / M * 21$ .00

.10

.24

.27

.81
1.02

1.02
1.19

1.38

1.57

2.09

2.35

2.55

2.99

3.21

3.40

3.55

3.63

3.75

3.92

3.96 
FO5-05 WUMPACT IS

TIME INTERVAL COOLANT MIA. SLEEVE TEMPERATURES (C)

$62.7-68.6$

$202.1-252.4$

$252.4-298.0$

$298.0=343.0$

$343.0=385.4$

$385.4-499.6$

$499.6-564.1$

$564.1-610.2$

$610.2=701.2$

$701.2-748.0$

$786.0-818.0$

$816.0-835.0$

$835.0-858.0$

$858.0-889.7$

$889.7-896.9$

896.9

TIME A VERAGEO RMS

$$
620 \text {. }
$$

644.
579.

579.
653.

648 .

626 .

652 .

789.

752.

636.

668 .

788 .

787 .

$7630^{\circ}$

763.

680.

737.

737.

705.

706.
66.

MAX SLEE

644.

675.
605.

708.

722.

697.

922 .

852 .

710 .

758.

923.

912.

857.

745.

882 .

831.

788 .

793.$$
\text { D - } 27.0
$$

$27.0=27.0$

$68.6-202.1$

$748.0-786.0$

COOLANT
476.
490.
453.
493.
491.
481.
494.
550.
534.
483.
498.
540.
541.
551.
537.
490.
538.
518.
503.
503.

512.

26.
93.

MIN. FUEL

729.

780.

701.

840.

846.

898.

1136.

1072 .

926.

992 .

1213.

1230 .

1221.

1169.

1019

1224.

1119.

1120.

1009.

161.

MAX F FEL

784.

842.
750.

903 .

940 .

904.

961.

$123 \mathrm{C}$.

1151.

1060.

1308 .

1326.

1307 .

1246.

1075 .

1309.

1245.

1288.

1083.

F05-05 COMPACT 16

$\stackrel{P}{1}$

TIME INTERVAL

$.0-27.0$
$27.0=62.7$

$27.0=62.7$
$62.7-68.6$

$68.6-262.1$

$202.1-252.4$

$252.4-298.0$

$298.0-343.0$

$343.0-385.4$

$385.4-499.6$

$499.6-564.1$

$564.1-610.2$

$610.2-701.2$

$701.2-748.0$

$748.0-788.0$

$788.0-818.0$

$B 18.0-835.0$

$835.0-858.0$

$858.0-889.7$

$889.7-896.9$

$$
896.9
$$

TIME AVERAGED RMS
COOLANT 486.

501.

462.

504.

502.

491.

505 .

566.

549.

494.

557.

568 .

552 .

503.

554.

533.

517.

516.

525
28
MI A. SLEEVE 627 .

652 .

585 .

661.

657 .

634 .

661.

800 .

763.

646.

680 .

804 .

802 .

820 .

780 .

694.

790.

753 .

721 .

720 .

718.
68.
TEMPERATURES (C)

MAX. SLEEYE MIN FUEL

651 .

683.

611

714.

728 .

702 .

741.

931.

861.

719.

768.

937.

924.

927.

874 .

759 .

898 .

846.

803.

803.

603.
96.

96.
MIN FUEL

735.
786.

705 .

844 .

880 .

850.

902 .

1141.

1077.

934.

1001 .

1224.

1241.

1232 .

1183.

1032.

1238.

1182.

1132.

1017.

164.

MAX. FUEL

789.

846

753.

906.

943.

907.

964.

1234.

1155 .

996.

1319.

1336.

1318.

1261 .

1088 .

1324 .

1259 .

1200.

$12 \pi 1$.

1090.

176.

FAST FLUENCE

$110 * 25$ N/M**21

.00

.09

.24

.26
.80

\begin{aligned} .80 \\ \hline 1018\end{aligned}

1.18

1.37

1.56
2.08

2.35

2.55

2.98

3.21

3.40

3.55

3.63

3.75

3.93
3.97

FAST FLUENCE

$110 * 25 N / M * 21$

.00

.09

.24

.26

.79
1.00

1.00

1.16

1. 35

1.54

2.05 
F05-05 LOMPACT 17

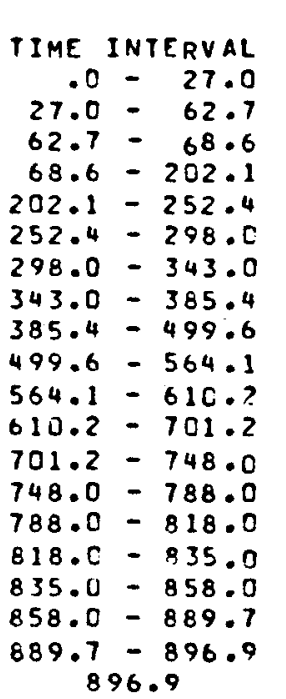

IIME AVERAGED RMS

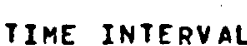

IME INTERVAL

$$
.0-27.0
$$$$
27.0-62.7
$$

$62.7-68.6$

$68.6-202.1$

$202.1-252.4$

$252.4-298.0$

$298.0-343.0$

$343.0-385.4$

$385.4=499.6$

$499.6-564.1$

$564.1-610.2$

$610.2-701.2$

$701.2-748.0$

$748.0-788.0$

$788.0-818.0$

$818.0=835.0$

$835.0-858.0$

$858.0-889.7$

$889.7-896.9$

$$
896.9
$$

TIME AVERAGEO RMS

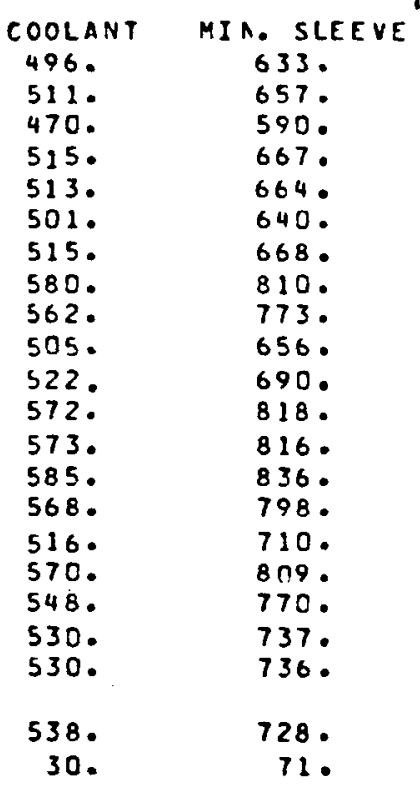

EMPERATURES IC

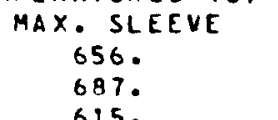

615.

717.

732.

746.

936 .

868.

778.

949.

936.

941.

892.

917.

834.

819.

819 .

812.

99.

MIN FUEL

737 .

787.

706.

843.

880 .

850 .

1142.
1076.

940.

1007.

1231.

1247 .

1240 .

1197.

1044.

1252.

1145 .

1021.

167.

790.

846.

752 .

903.

942.

906.

963.

1233.

1154.

1075 .

1326.

1342 .

1325 .

1277 .

1101.

1339.

1214.

1093.

180 .

FO5-05 COMPACT 18

\begin{tabular}{|c|c|c|c|c|}
\hline $\begin{array}{l}\text { COOLANT } \\
505 . \\
521 . \\
478 . \\
525\end{array}$ & $\begin{array}{l}\text { MIA. SLEEVE } \\
638 . \\
663 . \\
595 . \\
673 .\end{array}$ & $\begin{array}{c}\text { MAX. SLEEVE } \\
661 . \\
692 . \\
619 . \\
721 .\end{array}$ & $\begin{array}{c}\text { MIN. FUEL } \\
740 . \\
789 . \\
706 . \\
842 .\end{array}$ & $\begin{array}{c}\text { MAX FUEL } \\
791 . \\
846 . \\
752 . \\
901 .\end{array}$ \\
\hline $\begin{array}{l}523 . \\
510^{\circ} \\
526 .\end{array}$ & $\begin{array}{l}6711^{\circ} \\
647 \circ^{\circ} \\
6750^{\circ}\end{array}$ & $\begin{array}{l}736 . \\
710^{\circ} \\
750 .\end{array}$ & $\begin{array}{l}880 . \\
850 . \\
903 .\end{array}$ & $\begin{array}{l}941 . \\
9050^{\circ} \\
962 .\end{array}$ \\
\hline $\begin{array}{l}595 . \\
576 . \\
515 .\end{array}$ & $\begin{array}{l}819 . \\
781 . \\
665 .\end{array}$ & $\begin{array}{l}941 . \\
874 . \\
736 .\end{array}$ & $\begin{array}{r}1142 . \\
1078^{\circ} \\
943 .\end{array}$ & $\begin{array}{l}1231^{\circ} \\
1153^{\circ} \\
1004^{\circ}\end{array}$ \\
\hline $\begin{array}{l}534 . \\
588 . \\
589 .\end{array}$ & $\begin{array}{l}700 . \\
831 . \\
829 .\end{array}$ & $\begin{array}{l}786 . \\
960 . \\
947 .\end{array}$ & $\begin{array}{l}1010 . \\
1236 . \\
1251 .\end{array}$ & $\begin{array}{l}1078^{\circ} \\
1330^{\circ} \\
1340^{\circ}\end{array}$ \\
\hline $\begin{array}{l}601 . \\
583 . \\
530 .\end{array}$ & $\begin{array}{l}850^{\circ} \\
815^{\circ} \\
725 .\end{array}$ & $\begin{array}{l}954 . \\
910 . \\
790 .\end{array}$ & $\begin{array}{l}1245 . \\
1211 . \\
1050^{\circ}\end{array}$ & $\begin{array}{l}1330 . \\
1291 . \\
1114 .\end{array}$ \\
\hline $\begin{array}{l}586 . \\
563 . \\
544 .\end{array}$ & $\begin{array}{l}827 . \\
787 . \\
753 .\end{array}$ & $\begin{array}{l}934 . \\
880 . \\
835 .\end{array}$ & $\begin{array}{l}1265 . \\
1208 . \\
1156 .\end{array}$ & $\begin{array}{l}1354 . \\
1286 . \\
1226 .\end{array}$ \\
\hline 544. & 752 . & 834. & 1156. & 1227. \\
\hline $\begin{array}{r}551 . \\
33 .\end{array}$ & $\begin{array}{r}738 . \\
74 .\end{array}$ & $\begin{array}{l}819 . \\
102 .\end{array}$ & $\begin{array}{r}1024 . \\
169 .\end{array}$ & $\begin{array}{r}1095 . \\
183 .\end{array}$ \\
\hline
\end{tabular}

TEMPERATURES (C)
FAST FLUENCE

$(10 * 25$ N/M*2)

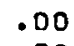

.09

.23

.25

.77

.97

1.13
1.32

1.51

2.01

2.27

2.90
3.12

3.12
3.32

3.47

3.55

3.67

3.84

3.88

FAST FLUENCE

(10*25 N/M**2)

.00

.09

.22

.25

.75
.95

.95
1.11

1.29

1.47

1.96

2.22

2.42

2.84

3.06

3.26

3.41

3.49

3.61

3.79

3.83 
FOS-OS DMPACT 19

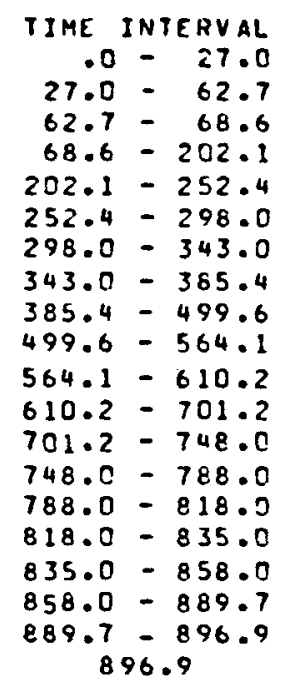

TIME AVERAGED

3
1
$\omega$

TIME INTERVAL

$$
\begin{array}{r}
\text { IHE INTERVAL } \\
.0-27.0
\end{array}
$$

$.0=27.0$
$27.0=62.7$

$62.7-68.6$

$68.6-202.1$

$202.1-252.4$

$252.4-298.0$

$343.0=385.4$

$385.4=499.6$

$385.4=499.6$
$499.6=564.1$

$564.1-610.2$

$610.2=701.2$

$701.2=748.0$

$748.0-788.0$

$788.0-818.0$

$818.0-835.0$

$835.0-858.0$

$858.0-889.7$

$889.7-896.9$

896.9

IIME AVEPAGED RMS

$\begin{array}{cc}\text { COOLANT } & \text { MIA. SLEEVE } \\ 514 . & 643 . \\ 530 . & 669 . \\ 486 . & 599 . \\ 5355^{\circ} & 679 . \\ 533 . & 677 . \\ 520 . & 652 . \\ 536 . & 681 . \\ 609 . & 827 . \\ 589 . & 78.0^{\circ} \\ 525 . & 673 . \\ 545 . & 710 . \\ 603 . & 843 . \\ 604 . & 841 . \\ 618 . & 863 . \\ 599 . & 833 . \\ 544 . & 740 . \\ 602 . & 845 . \\ 578 . & 804 . \\ 559 . & 769 . \\ 558 . & 768 . \\ 563 . & 747 . \\ 35 . & 77 .\end{array}$

TEMPERATURES (C)

MAX. SLEEVE MIN TUEL

666.

697.

623.
724.

724.

711.

752.

943.

877.
743.

794.

970.

965.

928.

806.

953.

897.
851.

850 .

826.
105.

791.

708.

842 .

877 .

847 .

900 .

1137 .

1074.

944.
1012.

1012.

1252 .

1247.

1223 .

1067.

1278 .

1219 .

1166 .

1167.

1025 .

171 .

793.

847.

752 .

899 .

936 .

900.

957 .

1223

1147

1005

1078 .

1331.

1345.

1331.

1304

1125 .

1367 .

1237 .

1238 .

1095 .

185.

FO5-05 COMPACT 20

TEMPERATURES (C)

$\begin{array}{cc}\text { COOLANT } & \text { RIA. SLEEVE } \\ 523 . & 648^{\circ} \\ 540^{\circ} & 673 . \\ 494 . & 603 . \\ 5450^{\circ} & 683 . \\ 543 . & 681 . \\ 529 . & 656 . \\ 546 . & 685 . \\ 623 . & 832 . \\ 602 . & 794 . \\ 536 . & 681 . \\ 557 . & 719 . \\ 619 . & 855 . \\ 619 . & 853 . \\ 634 . & 875 . \\ 615 . & 849 . \\ 557 . & 754 . \\ 618 . & 862 . \\ 593 . & 820 . \\ 573 . & 783 . \\ 572 . & 783 . \\ 576 . & 755 . \\ 38 . & 80 .\end{array}$

MAX. SLEEVE

MIN. FUEL

MAX. FUEL

669.

700.
625.
726.

739 .

712.

752.

943.

879.

750.
801.

979.

967.

976.

944.

920.

969.

913.

865 .

743.

791.

707.
839.

873.

843.

894.

1129.

1067 .

945.
1.012.

1239

1252 .

1248 .

1232 .

1074 .

1286 .

1227 .

1173

1174.

792.

845.
749.

749.

930.

894.

950.

1213.

1137.

1078 .

1331 .

1344.

1331.

1313.

1133 .

1376.

1306.

1245.

FAST FLUENCE

$110 * 25$ N/M**21

.00

.09

.22

.24

.73
.92

1.08
1.08

1.25

1.25
1.43

1.91

2.17

2.36
2.78

2.99

3.19

3.34

3.42

3.54

3.72
3.76

FAST FLUENCE

$(10 * 25$ N/M**2)

.00

.21

.23

.70

.89
1.04

1.21

1.38

.85

2.10

2.29

2.70

2.91

3.11

3.26

3.34

3.46

3.64

832.

108.

1023.

1092.

187. 
FOS-C5 LOMPACT 21

\begin{tabular}{|c|c|c|c|c|c|c|c|}
\hline & & & & MPPERATURES IC & & & $\begin{array}{l}\text { FASI FLUENCE } \\
110 * 25 N / 4 * * 21\end{array}$ \\
\hline $\begin{array}{r}\text { IIME } \\
27.0\end{array}$ & $\begin{array}{r}\text { INTERVAL } \\
-\quad 27.0 \\
-\quad 62.7\end{array}$ & $\begin{array}{l}\text { COOLANT } \\
532 . \\
549 .\end{array}$ & $\begin{array}{l}\text { MIN. SLEEVE } \\
650 . \\
676 .\end{array}$ & $\begin{array}{l}\text { MAX. SLEEVE } \\
\text { 671. } \\
701 .\end{array}$ & $\begin{array}{c}\text { MIN. FUEL } \\
\text { 742. } \\
788 .\end{array}$ & $\begin{array}{c}\text { MAX. FUEL } \\
788^{\circ} \\
839 .\end{array}$ & $\begin{array}{c}110 * 25 N / M * * 21 \\
.00 \\
.08\end{array}$ \\
\hline $\begin{array}{l}62.7 \\
68.6\end{array}$ & $\begin{array}{r}\quad 68.6 \\
-202.1\end{array}$ & $\begin{array}{l}501 . \\
554 .\end{array}$ & $\begin{array}{l}605 . \\
686 .\end{array}$ & $\begin{array}{l}826 . \\
726 .\end{array}$ & $\begin{array}{l}704 . \\
833 .\end{array}$ & $\begin{array}{l}745 . \\
886\end{array}$ & $\begin{array}{l}.20 \\
.22\end{array}$ \\
\hline 202.1 & -252.4 & 552. & 684. & 737. & 865. & 919. & .67 \\
\hline $\begin{array}{l}252.4 \\
298.0\end{array}$ & $\begin{array}{l}-298.0 \\
-343.0\end{array}$ & $\begin{array}{l}537 \\
555\end{array}$ & $\begin{array}{l}659 . \\
688 .\end{array}$ & $\begin{array}{l}711 . \\
751\end{array}$ & $\begin{array}{l}835 \\
886\end{array}$ & $\begin{array}{l}883 . \\
939 .\end{array}$ & $\begin{array}{l}.85 \\
.99\end{array}$ \\
\hline 343.0 & -385.4 & 636. & 836. & 940. & 1117 & 1197. & 1.16 \\
\hline $\begin{array}{l}385.4 \\
499.6 \\
564.1\end{array}$ & $\begin{array}{l}-499.6 \\
-564.1 \\
-610.2\end{array}$ & $\begin{array}{l}614 . \\
546 . \\
568 .\end{array}$ & $\begin{array}{l}797 . \\
688 . \\
726 .\end{array}$ & $\begin{array}{l}878 . \\
755 . \\
876 .\end{array}$ & $\begin{array}{r}1056 . \\
941 . \\
1008 .\end{array}$ & $\begin{array}{l}1123 . \\
1000 . \\
1072 .\end{array}$ & $\begin{array}{l}1.32 \\
1.78 \\
2.02\end{array}$ \\
\hline $\begin{array}{l}610.2 \\
701.2 \\
748.0\end{array}$ & $\begin{array}{r}-701.2 \\
-748.0 \\
-768.0\end{array}$ & $\begin{array}{l}634 . \\
634 . \\
650 .\end{array}$ & $\begin{array}{l}865^{\circ} \\
862^{\circ} \\
886 .\end{array}$ & $\begin{array}{l}986 . \\
973 . \\
983 .\end{array}$ & $\begin{array}{l}1234 \\
12460 \\
12430\end{array}$ & $\begin{array}{l}1324^{\circ} \\
1336^{\circ} \\
1324^{\circ}\end{array}$ & $\begin{array}{l}2.21 \\
2.60 \\
2.81\end{array}$ \\
\hline $\begin{array}{l}788.0 \\
818.0\end{array}$ & $\begin{array}{l}-818.0 \\
-835.0\end{array}$ & $\begin{array}{l}631 . \\
571 .\end{array}$ & $\begin{array}{l}864^{\circ} \\
767 .\end{array}$ & $\begin{array}{l}959 . \\
833 .\end{array}$ & $\begin{array}{l}1237 . \\
1078 \text {. }\end{array}$ & $\begin{array}{l}1316 . \\
1137\end{array}$ & $\begin{array}{l}3.00 \\
3.15\end{array}$ \\
\hline 835.0 & -858.0 & 634. & 877. & 984. & 1290. & 1380 & 3.23 \\
\hline $\begin{array}{l}858.0 \\
889.7\end{array}$ & $\begin{array}{l}-8.89 .7 \\
=896.9\end{array}$ & $\begin{array}{l}608 \\
587\end{array}$ & 835. & $\begin{array}{l}927 . \\
878 .\end{array}$ & $\begin{array}{l}1230 \\
1176\end{array}$ & 1310. & $\begin{array}{l}3.35 \\
3.53\end{array}$ \\
\hline & 196.9 & 587. & 796. & 877. & 1177. & 1248. & 3.57 \\
\hline TIME & $\begin{array}{l}\text { AVERAGED } \\
\text { RMS }\end{array}$ & $\begin{aligned} 587 \\
40 .\end{aligned}$ & $\begin{array}{r}761 . \\
83 .\end{array}$ & $\begin{array}{l}835 . \\
110 .\end{array}$ & $\begin{array}{r}1018 . \\
174\end{array}$ & $\begin{array}{l}1084 \\
188\end{array}$ & \\
\hline
\end{tabular}

F05-05 COMPACT 22

TIME INTERVAL $.0-27.0$ $27.0-62.7$ $27.0-62.7$
$62.7-68.6$ $68.6-202.1$ $202.1-252.4$ $252.4-298.0$ $298.0-343.0$ $343.0-385.4$ $385.4-499.6$ $499.6-564.1$ $564.1=610.2$ $610.2-701.2$ $701.2-748.0$ $748.0-788.0$ $788.0-818.0$ $818.0-835.0$ $835.0-858.0$ $858.0-889.7$ $889.7-896.9$ 896.9

TIME AVERAGEO RMS

$\begin{array}{cc}\text { COOLANT } & \text { MIN. SLEEVE } \\ 540 . & 653 . \\ 557 . & 678 . \\ 508 . & 607 . \\ 563 . & 689 . \\ 561 . & 688 . \\ 545 . & 662 . \\ 564 . & 692 . \\ 648 . & 840 . \\ 626 . & 801 . \\ 555 . & 692 . \\ 579 . & 731 . \\ 649 . & 871 . \\ 649 . & 868 . \\ 666 . & 893 . \\ 647 . & 878 . \\ 585 . & 779 . \\ 650 . & 891 . \\ 624 . & 847 . \\ 601 . & 809 . \\ 601 . & 808 . \\ 599 . & 766 . \\ 43 . & 85 .\end{array}$

TEMPERATURES (C) MAX. SLEEVE MIN. FUEL

$$
\begin{aligned}
& 673 . \\
& 703 . \\
& 627 . \\
& 726 . \\
& 738 . \\
& 711 . \\
& 751 . \\
& 938 . \\
& 879 . \\
& 757 . \\
& 808 . \\
& 987 . \\
& 975 . \\
& 987 . \\
& 972 . \\
& 843 . \\
& 996 . \\
& 938 . \\
& 819 . \\
& 888 .
\end{aligned}
$$

837.

837. 741.

786.

786.

828.

859 .

829.

879.

1108.

1048 .

933 .

1222.

1233

1232

1238 .

1078 .

1290.

1230 .

1175 .

1175 .

1011.
MAX. FUEL

785 .

835.

870.

879.
911.

876 .

931.

1184.

1112 .

990.

1061 .

1309.

1320.

1310

1316.

1136.

1374.

1309.

1245 .

1246 .

1076.
FAST FLUENCE

$(10 * 25 N / H * 2)$

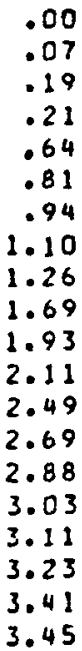

.00

.21

.64

.94

1.26

1.93

2.11

2.49

3.03

11

3.41

3.45 
FOS-OS LOMPACT 23

TIME INTERVAL $0-27.0$ $27.0-62.7$ $62.7-68.6$ $66.6-202.1$ $68.6-202.1$ $252.4=298.0$ $298.0-343.0$ $343.0-385.4$ $385.4-499.6$ $499.6-564.1$

$564.1-610.2$

$610.2-701.2$

$701.2-748.0$

$748.0-788.0$

$788.0-818.0$

$818.0-835.0$

$835.0-858.0$

$858.0-889.7$

$889.7-896.9$

896.9

TIHE AVERAGED

RMS

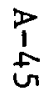

TIME INTERVAL

.0 -27.0

$27 . c-62.7$

$62.7-68.6$

$68.6-202.1$

$202.1=252.4$

$252.4-298.0$

$298.0-343.0$

$343.0-385.4$

$385.4-499.6$

$499.6-564.1$

$564.1-610.2$

$610.2=701.2$

$701.2-748.0$

$748.0=788.0$

$788.0-818.0$

$818.0-835.0$

$835.0-858.0$

$858.0-889.7$

$889.7-896.9$

896.9

IIME AVERAGED RMS

COOLANT
547.
565.
515.
572.
569.
553.
573.
660.
637.
565.
589.
663.
663.
681.
662.
598.
666.
638.
615.
615.

610.

45.

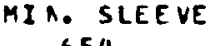
654.

679 .

608 .

689.

663 .

693.

841.

802 .

696.

736.

877 .

874 .

899 .

890 .

789.

903 .

859 .

820 .

819.

770.

87 .

MAX. SLEEVE)

MAX. SLEEVE

MIN. FUEL

737.

702.

626.

734.

707.

947.

874.

758.

810.
988.

977 .

989.

983.

853.

1007.

898 .

897 .

837.

114.

780.

696.

819.

847.

867 .

1090 .

1031.

924.

989.

1210 .

1219.

$1235^{\circ}$

1076.

1076.

$1287^{\circ}$

1226.

1171.

1001.

172.

MAX. FUEL

$826^{\circ}$.

1162.

1092.

979.

1049 .

1293 .

1303.

1315 .

1133.

1304.

1240.
1240.

1062.

FO5-05 COMPACT 24

COOLANT
554.
573.
5210
580.
577.
560.
581.
671.
647.
573.
599.
676.
676.
695.
677.
611.
682.
653.
629.
628.
620.
48.

TEMPERATURES (C)

\begin{tabular}{|c|c|c|c|}
\hline $\begin{array}{c}\text { MIA. SLEEVE } \\
653 \text {. }\end{array}$ & $\begin{array}{l}\text { MPERATURES (C) } \\
\text { MAX. SLEEVE } \\
\text { 670. }\end{array}$ & $\begin{array}{c}\text { MIN. FUEL } \\
730 .\end{array}$ & $\begin{array}{c}\text { MAX. FUEL } \\
768 .\end{array}$ \\
\hline $\begin{array}{l}678 . \\
607 . \\
689 .\end{array}$ & $\begin{array}{l}699 . \\
624 . \\
719 .\end{array}$ & $\begin{array}{l}771 . \\
688 . \\
807\end{array}$ & $\begin{array}{l}814 . \\
722 . \\
851 .\end{array}$ \\
\hline $\begin{array}{l}690 . \\
665 .\end{array}$ & $\begin{array}{l}732 . \\
705 .\end{array}$ & $\begin{array}{l}838 . \\
808 .\end{array}$ & 885. \\
\hline $\begin{array}{l}695 . \\
842 .\end{array}$ & $\begin{array}{l}744 . \\
926\end{array}$ & $\begin{array}{l}857 . \\
1075 .\end{array}$ & $\begin{array}{r}902 . \\
1244 .\end{array}$ \\
\hline & & $\begin{array}{r}1018 . \\
912 .\end{array}$ & $\begin{array}{l}1075 \text {. } \\
964 .\end{array}$ \\
\hline $\begin{array}{l}739 . \\
880 .\end{array}$ & $\begin{array}{l}809 . \\
985 .\end{array}$ & $\begin{array}{l}976 . \\
1192 .\end{array}$ & $\begin{array}{l}1033 . \\
1272 .\end{array}$ \\
\hline $\begin{array}{l}877 . \\
902 . \\
899 .\end{array}$ & $\begin{array}{l}975 . \\
989 . \\
991 .\end{array}$ & $\begin{array}{l}1201 . \\
1203 \\
1229\end{array}$ & $\begin{array}{l}1281 . \\
1275 . \\
1307 .\end{array}$ \\
\hline $\begin{array}{l}798 . \\
913 \\
868\end{array}$ & $\begin{array}{l}860 \\
1015 \\
955\end{array}$ & $\begin{array}{l}1069 \\
1279 \\
1217\end{array}$ & $\begin{array}{l}1125 \\
13650 \\
1293\end{array}$ \\
\hline $\begin{array}{l}828 . \\
828 .\end{array}$ & $\begin{array}{l}905 . \\
904 .\end{array}$ & $\begin{array}{l}11610 \\
11610\end{array}$ & $\begin{array}{l}1229^{\circ} \\
1230^{\circ}\end{array}$ \\
\hline 772 & 836. & 986. & 1047. \\
\hline 89. & 115. & 170. & 185. \\
\hline
\end{tabular}

778.

867 .

897.

662 .

915.

FAST FLUENCE

$(10 * 25 N / M * 21$

.00

.07
.18

.20

.60

.89

1.04

1.19

1.61

1.84
2.01

2.38

2.57

2.76

2.90

3.10

3.27
3.31

186.

FAST FLUENCE

$(10 * 25 N / M * 2)$

.00

.07

.17
.18

.57
.72

.84

1.12

1.51
1.73

1.73
1.89

2.24

2.43

2.61

2.75

2.83

2.95

3.11 
FO5-05 LOMPACT 25

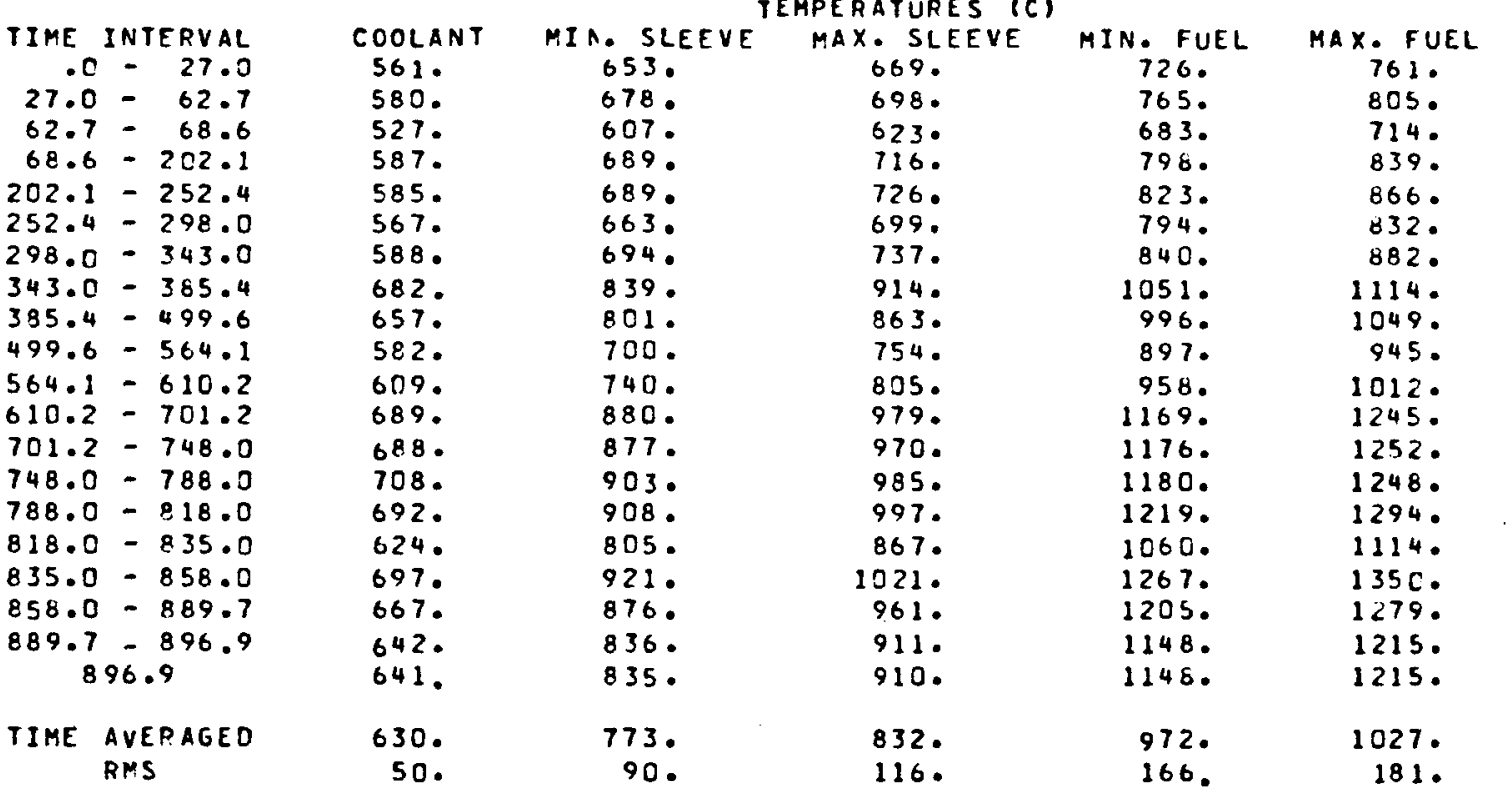

FAST FLUENCE (10*25 N/ $H * 2$ ) .00 .06 .15 .17 .52 .66
.77 .77
.90 1.03 1.40 1.60 1.76 2.09 2.26 2.44 2.57 2.65 2.76 2.92 2.92
2.96

F05-05 COMPACT 26

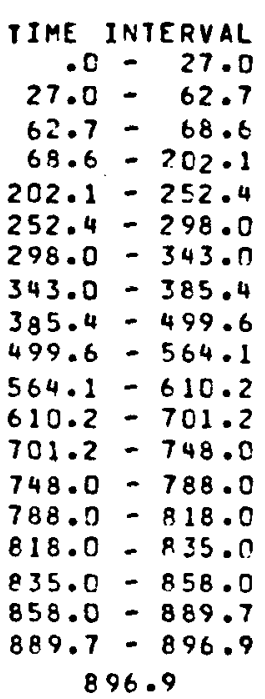

COOLANT
567.
586.
532.
594.
591.
573.
595.
691.
666.
590.
617.
701.
700
721.
706.
636.
712.
681.
655.
654.
639.
52.

MIA. SLEEVE
651.
676.
606.
687.
687.
662.
692.
836.
798.
700.
740.
880.
877.
903.
913.
810.
926.
881.
840.
840.
772.
92.

TEMPERATURES (C)

\begin{tabular}{|c|c|}
\hline $\begin{array}{c}\text { MAX. SLEEVE } \\
\text { GS6. }\end{array}$ & $\begin{array}{c}\text { MIN F FUEL } \\
\text { i19. }\end{array}$ \\
\hline $\begin{array}{l}694 . \\
620 . \\
712 .\end{array}$ & $\begin{array}{l}757^{\circ} \\
675{ }^{\circ} \\
787 .\end{array}$ \\
\hline $\begin{array}{l}719 . \\
693 . \\
730 .\end{array}$ & $\begin{array}{l}808 . \\
779 . \\
824 .\end{array}$ \\
\hline $\begin{array}{l}902 . \\
853 . \\
751 .\end{array}$ & $\begin{array}{r}1027^{\circ} \\
974^{\circ} \\
881 .\end{array}$ \\
\hline $\begin{array}{l}801 . \\
972 . \\
964 .\end{array}$ & $\begin{array}{l}941 . \\
11460 \\
11520\end{array}$ \\
\hline $\begin{array}{l}980 . \\
999 . \\
869 .\end{array}$ & $\begin{array}{l}1157 . \\
1203 . \\
1045 .\end{array}$ \\
\hline $\begin{array}{r}1022 . \\
963 .\end{array}$ & $\begin{array}{l}1248 . \\
1186 .\end{array}$ \\
\hline 912. & 1130. \\
\hline 911. & 1130. \\
\hline
\end{tabular}

MAX. FUEL 752. 794. 704.

824.

824.

848.

814.

1085 .

1023.

926.

991.

1217.

1222.

1275 .

1096 .

1329 .

1257.

1193 .

1193.

FAST FLUENCE

(10*25 $N / M * 2)$ .00

.06

.14

.16

.16
.48

.61

.71

.83

.95
1.29

1.48

1.48

1.63
1.93

2.09

2.26

2.39

2.46

2.57

2.72

2.76

TIME AVERAGED FMS

116.

955.
163. 
FOS-05 - JMFACT 27

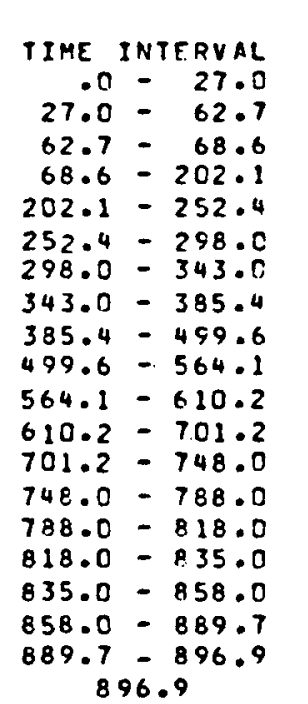

TIME AVERAGEO RMS

$\begin{array}{cc}\text { COOLANT } & \text { MIA. SLEEVE } \\ 572 . & 649 . \\ 592 . & 674 . \\ 537 . & 604 . \\ 600 . & 685 . \\ 598 . & 685 . \\ 579 . & 660 . \\ 601 . & 690 . \\ 700 . & 832 . \\ 674 . & 795 . \\ 597 . & 699 . \\ 625 . & 738 . \\ 712 . & 877 . \\ 711 . & 873 . \\ 733 . & 901 . \\ 719 . & 911 . \\ 647 . & 809 . \\ 725 . & 924 . \\ 694 . & 879 . \\ 666 . & 839 . \\ 666 . & 838 . \\ 647 . & 770 . \\ 55 . & 91 .\end{array}$

MAX OSLES (C)

662.
690 .

690 .

616.

714.

$614^{\circ}$.

688.

890 .

844.

$$
793 .
$$

960.

953.

971.

992.
864.

1013 .

955 .

905.

819.

114.

710.

740.

$747 . \quad 780$.

666.692.

$794 . \quad 830$.

765.798.

$\begin{array}{rr}808 . & 844 . \\ 1004 . & 1057 .\end{array}$

953.0998.

$920.0960^{\circ}$

$1118^{\circ} \quad 1183$.

$1123.1188^{\circ}$

$1130.1188^{\circ}$.

1174.1242 .

$1020 . \quad 1069$.

$12160^{\circ} \quad 1291$.

$1155^{\circ} \quad 12211^{\circ}$

1099.1158.

935.

156.

169.

$\frac{7}{1}$

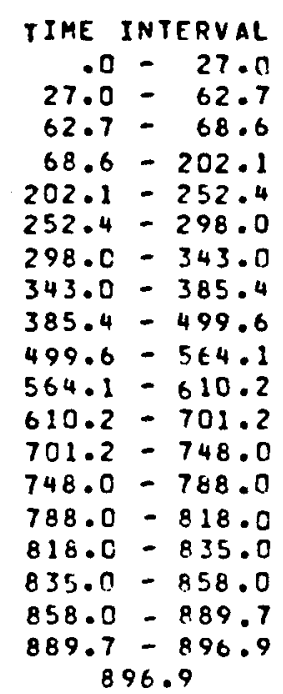

896.9

TIME AVEPAGED RMS

$\begin{array}{cc}\text { COOLAN } & \text { KIN. SLEEVE } \\ 577 . & 647 . \\ 597 . & 672 . \\ 541 . & 602 . \\ 605 . & 682 . \\ 603 . & 681 . \\ 584 . & 656 . \\ 607 . & 686 . \\ 708 . & 826 . \\ 681 . & 789 . \\ 603 . & 695 . \\ 633 . & 735 . \\ 722 . & 870 . \\ 721 . & 867 . \\ 743 . & 895 . \\ 731 . & 907 . \\ 658 . & 806 . \\ 737 . & 920 . \\ 705 . & 875 . \\ 677 . & 835 . \\ 677 . & 835 . \\ & \\ 655 . & 765 . \\ 57 . & 90 .\end{array}$

MAX. SLEEVE

MAX. FUEL

$774 . \quad 808$.

FO5-05 COMPACT 28

TEMPERATURES (C)

$\begin{array}{cc}\text { MAX. SLEEVE } & \text { MIN. FUEL } \\ 659 . & 703 . \\ 686 . & 738 . \\ 613 . & 658 . \\ 701 . & 762 . \\ 705 . & 776 . \\ 680 . & 748 . \\ 714 . & 789 . \\ 875 . & 976 . \\ 831 . & 927 . \\ 735 . & 838 . \\ 783 . & 894 . \\ 945 . & 1084 . \\ 939 . & 1088 . \\ 959 . & 1096 . \\ 981 . & 1142 . \\ 857 . & 993 . \\ 1002 . & 1181 . \\ 946 . & 1120 . \\ 897 . & 1065 . \\ 896 . & 1064 . \\ & \\ 809 . & 911 . \\ 111 . & 147 .\end{array}$

731 .

768 .

682.

793 .

809.

820 .

1023 .

967 .

876.

$930^{\circ}$

1143.

1146.

1149.

1038 .

1249 :

1180 .

1119.

1119.

FAST FLUENCE
$(10 * 25 N / M * 2)$
.00
.05
.13
.14
.44
.56
.65
.76
.87
1.18
1.35
1.48
1.76
1.91
2.06
2.18
2.26
2.35
2.49
2.53

FAST FLUENCE

$(10 * 25 N / M * 2)$

.00
.05
.12
.13
.39
.49
.57
.67
.76
1.04
1.19
1.30
1.55
1.68
1.82
1.93
1.99
2.08
2.20
2.23 
FO5-05 COMPACT 29

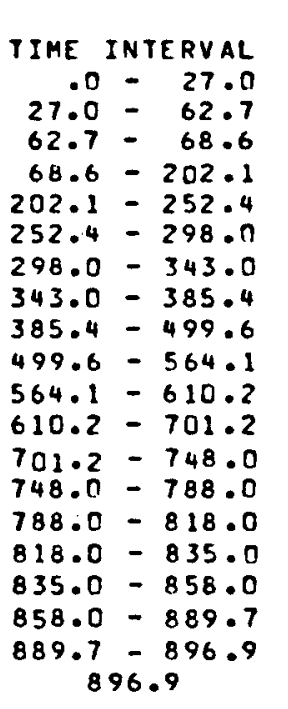

TIME AVERAGED RMS

$\underset{\infty}{1}$
COOLANT MIA. SLEEVE MEMPERATURES IC;

582.

602 .

545 .

610 .

608 .

$589^{\circ}$

589.
612.

715 .

688 ;

609.

639.

731.

729.

752.

667.

749.

716.

687.

687 .

662.

59.$$
644 .
$$

669 .

599 .

679.

678.

654.

683.

721.

691.

730 .

863 .

860 .

888 .

904 .

804 .

917.

873.

833.

832 .

761.

89.

655 .

681.

695 .

698 .

673 .

706.

860.

819.

724.

926.

922.

944.

972.

850.

937 .

890.

889.

798.

108 .

MIN. FUEL

695.

728.
649.

649.

$760^{\circ}$.

732.

732.

971.

949.

813.

866.

1047 .

1050.

1062.

1113.

968.

1148.

1034.

1033.

887.

139.

MAX. FUEL

719.

755 .

671.

776.

758 .

758.

$799^{\circ}$

$9920^{\circ}$

847.

904 .

1100 .

1103

$1100^{\circ}$

$11090^{\circ}$

1170.

1009.

1211.

1144.

1084.

925.

150 .

FOS-05 COMPACT 30

TEMPERATURES (C)

TIME INTERVAL

$.0-27.0$
$27.0=62.7$
$62.7-68.6$
$68.6-202.1$
$202.1-252.4$
$252.4=298.0$
$298.0=343.0$
$343.0=385.4$
$385.4=499.6$
$499.6-564.1$
$564.1=610.2$
$610.2=701.2$
$701.2-748.0$
$748.0=788.0$
$788.0=818.0$
$818.0-835.0$
$835.0=858.0$
$858.0=889.7$
$889.7=896.9$
896.9

TIME AVERAGEO RMS

COOLANT
587.
607.
549.
616.
613.
594.
618.
722.
695
615.
646.
740
738.
762.
753.
677.
760.
726.
697.
696.
669.
60.

MIA. SLEEVE
654.
679.
608.
690.
689.
663.
693.
836.
799.
699.
739.
$875 \circ$
872.
900.
907.
806.
919.
875.
835.
834.
771.
89.

MAX. SLE
$6660^{\circ}$
693.
618.
706.
707.
680.
713.
870.
828.
726.
771.
927.
925.
949.
963.
845.
983.
931.
884.
884.
803.
104.

MIN. FUEL

709.

MAX. FUEL

735 .

771.

683.

790.

799.

$766^{\circ}$

807.

768.

738 .

777.

957.

808.

861 .

1041.

1044.

1059.

1086 .

948 .

1121.

1063.

1010 .

1010.

1003 .

947.

843.

899

1095 .

1098 .

1098.

1142 .

987.

1181 :

1116.

1058 .

1058 .

927.

130.

140 .

FASI FLUENCE

$(10 * 25 \quad N / M * 2)$

$$
\begin{array}{r}
.00 \\
.04 \\
.09
\end{array}
$$

.10
.32
.40

.40

.47

.54
.62

.62
.85

.97

1.07

1.27

1.38

1.49
1.58

1.64

1.71

1.82

1.84

FAST FLUENCE

(10**25 $N / M * 2)$

.00

.02

.06

.07

.21

.26

.31

.36

.56

.64

.70

.83

.90
.99

1.06

1.10

1.15

1.24

1.26 


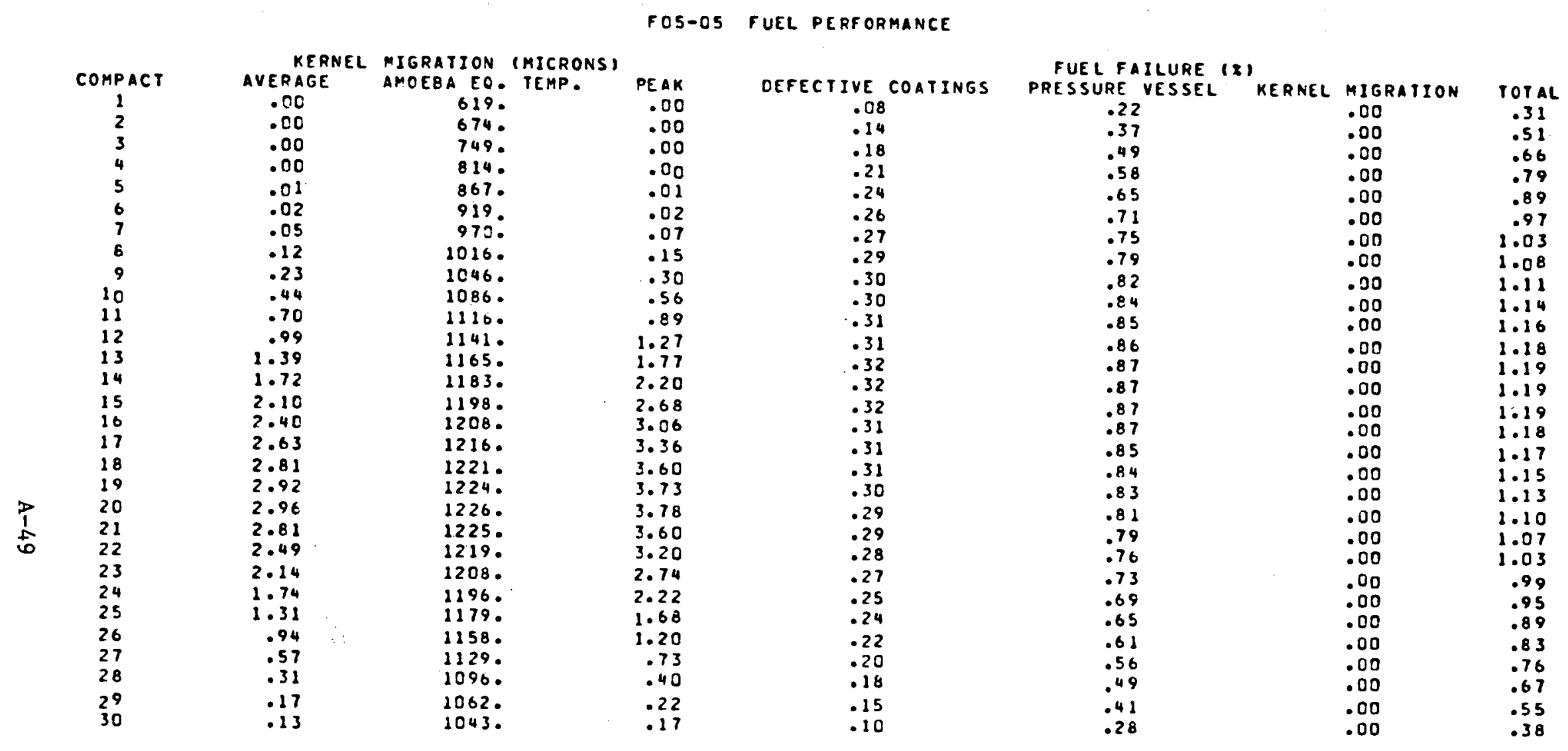

AMOEBA EOUIVALENT TEMPERATURES (C) CORRESPOND TO AVERAGE KERNEL MIGRAIIONS

KERNEL MIGRATION DISTANCES CALCULATED USING $50:$ CONFIDENCE LEVEL KMC

FUEL FAILURE CORRESPONOS TO 508 CONF IDENCE LEVEL 


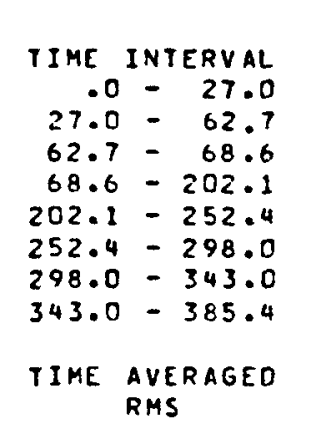

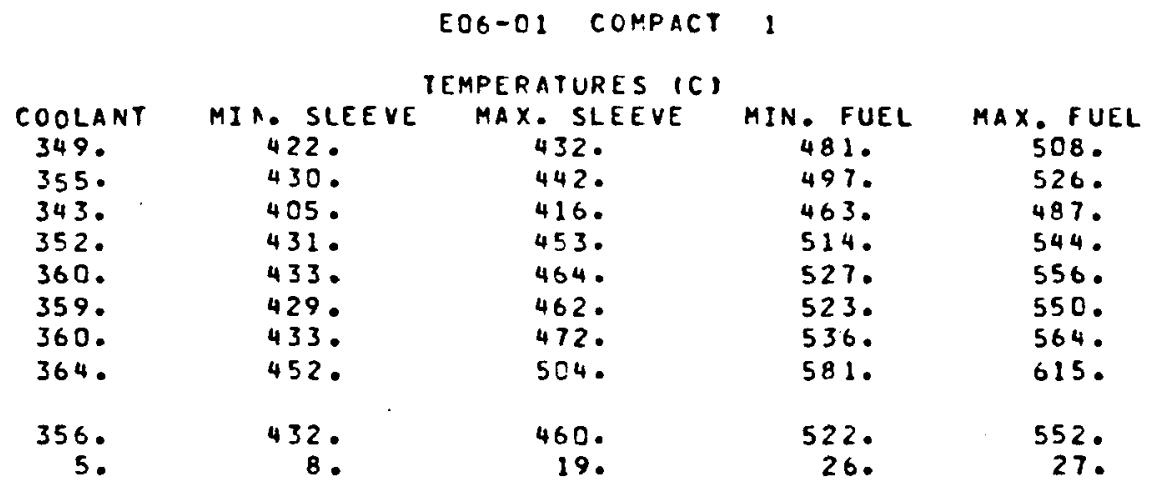

FAST FLUENCE
$(10 * 25 N / M * 21$
.00
.03
.06
.07
.21
.26
.31
.35

\section{EO6-01 COMPACT 2}

TIHE INTERVAL $.0=27.0$
$27.0=62.7$
$62.7=68.6$ $68.6-202.1$ $202.1-252.4$ $252.4-298.0$ $298.0-343.0$ $343.0-385.4$

IIME AVERAGED RMS

COOLANT
$3555^{\circ}$
361.
348.
358.
$3650^{\circ}$
365.
365.
371.
362.
5

MI $\wedge$. SLEEVE

TEMPERATURES (C)
MAX. SLEEVE

436.
444.
417.
446.
449.
444.
$4490^{\circ}$
472.
448.
9.

MIN. FUEL

MAX. FUEL

FAST FLUENCE

$110 * 25 N / M * 21$

$\begin{array}{cc}\text { NFUEL } & \text { MAX.FUEL } \\ 5020^{\circ} & 5320^{\circ} \\ 5211^{\circ} & 553 . \\ 4850^{\circ} & 511 . \\ 550 . & 5830^{\circ} \\ 5720^{\circ} & 6050^{\circ} \\ 566 . & 597 . \\ 5810^{\circ} & 613 . \\ 632 . & 6711^{\circ} \\ 560 . & 593 . \\ 34 . & 36 .\end{array}$

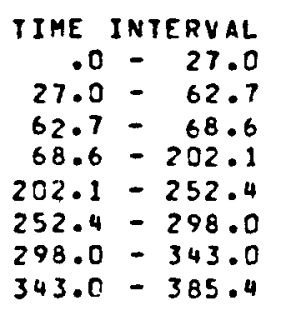

$\begin{array}{cc}\text { COOLANT } & \text { MIA. SLEEVE } \\ 362 . & 463 . \\ 368 . & 473 . \\ 354 . & 441 . \\ 366 . & 476 . \\ 373 . & 477 . \\ 372 . & 471 . \\ 373 . & 477 . \\ 380 . & 5050^{\circ} \\ 370 . & 477 . \\ 5 . & 11 .\end{array}$

TEMPERATURES (C)

.00
.04
.11
.12
.35
.43
.50
.58

FAST FLUENCE

$\begin{array}{ccc}\text { MAX. SLEEVE } & \text { MIN. FUEL } & \text { MAX. FUEL } \\ 478 . & 543 . & 581 . \\ 492 . & 567 . & 608 . \\ 459 . & 525 . & 557 . \\ 519 . & 607 . & 649 . \\ 537 . & 631 . & 672 . \\ 531 . & 622 . & 660 . \\ 543 . & 639 . & 679 . \\ 588 . & 701 . & 749 . \\ & & \\ 527 . & 617 . & 658 . \\ 29 . & 40 . & 43 .\end{array}$

(10*25 N/M*2)

.00
.06
.14
.15
.45
.57
.66
.76

IIME AVEPAGED RMS

E06-01 COMPACT 3

$$
\begin{aligned}
& 447 . \\
& 4590^{\circ} \\
& 4310^{\circ} \\
& 4790^{\circ} \\
& 4970^{\circ} \\
& 4930^{\circ} \\
& 5040^{\circ} \\
& 5410 \\
& 488 . \\
& 25
\end{aligned}
$$$$
\text { 4 }
$$

43. 


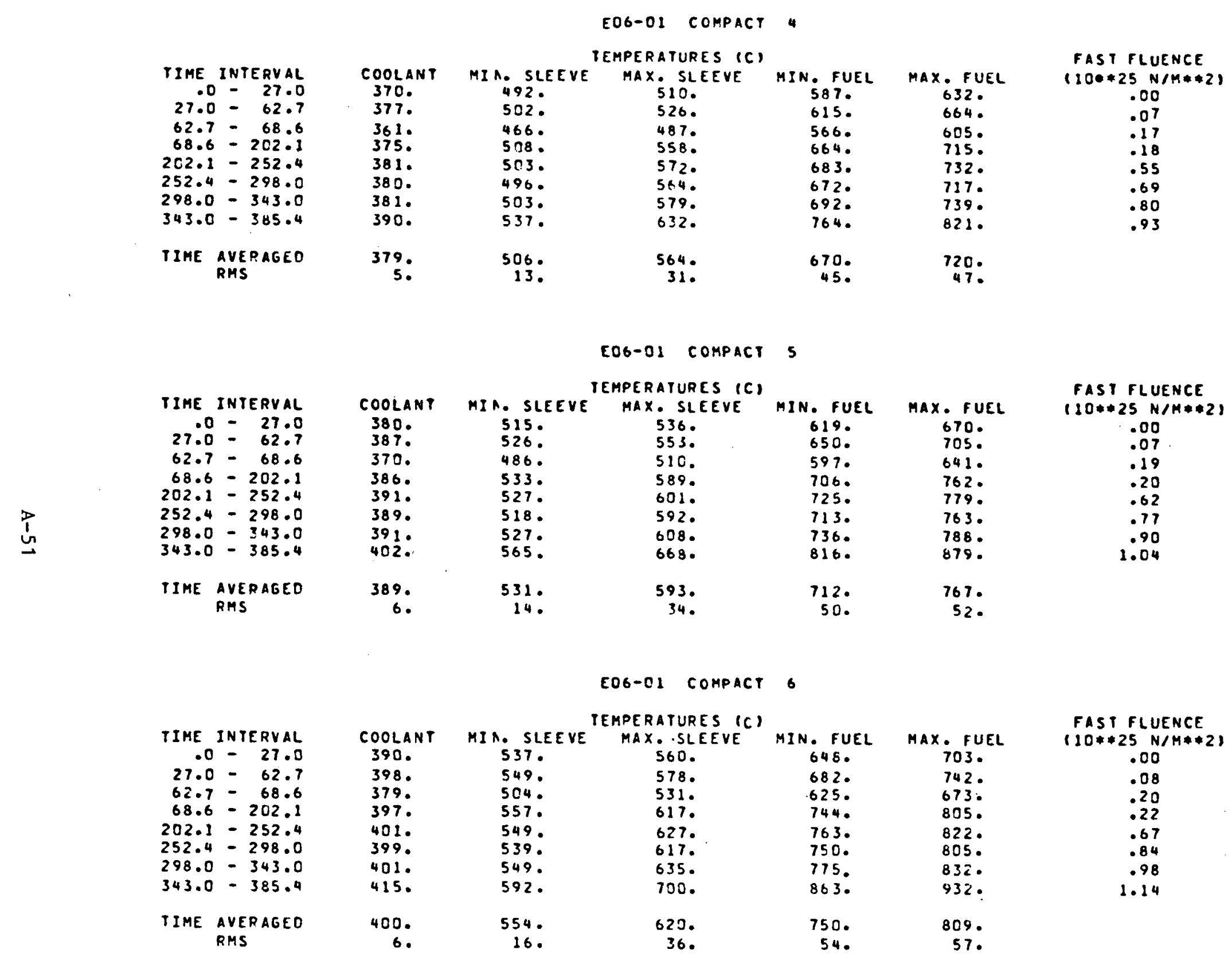


E06-01 COMPACT 7

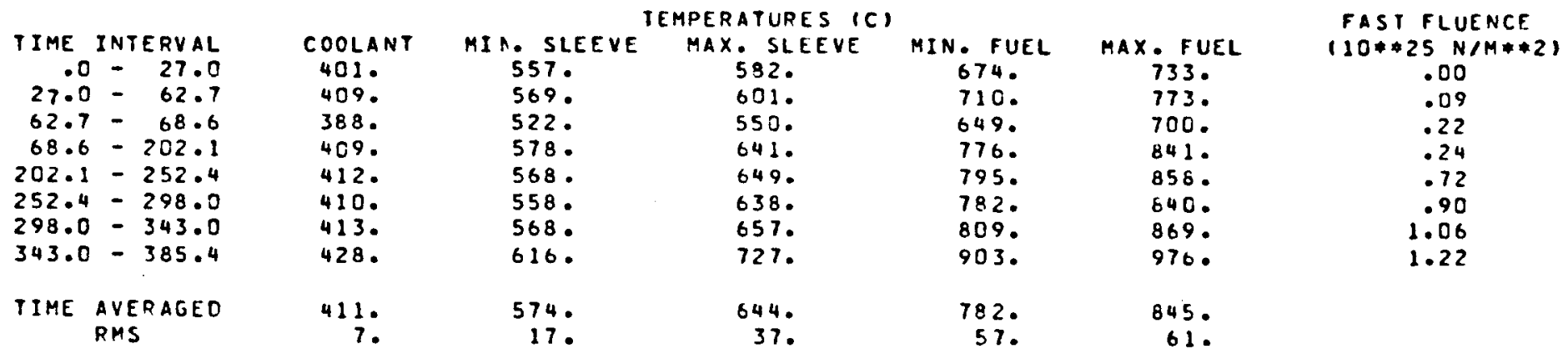

EO6-01 COMPACT

TIME INTERVAL

$$
.0-27.0
$$

$27.0-62.7$

$62.7-68.6$

$68.6-202.1$

$202.1-252.4$

$252.4-298.0$

$298.0-243.0$

$343.0-385.4$

TIME AVERAGED RHS

$\begin{array}{cc}\text { COOLANT } & \text { MIA. SLEE } \\ 413 . & 5750^{\circ} \\ 421 . & 580^{\circ} \\ 398^{\circ} & 537 . \\ 4220^{\circ} & 59.8^{\circ} \\ 424^{\circ} & 587 . \\ 421 . & 576 . \\ 424 . & 587 . \\ 442 . & 6390^{\circ} \\ 423 . & 594 . \\ 8 . & 19 .\end{array}$

TEMPERATURES (C)
MAX. SLEEVE

MIN. FUEL

MAX. FUEL

FAST FLUENCE

(10*25 N/M**21

696.

601.

621.
566.

662.

670.

658.

678 .

734.

669 .

802 .

824.

$81 \mathrm{C}$.

839.

$$
757 .
$$

799.

723.

871.

890.

871 .

903.

940.

665.

810.

876.

E06-01 COMPACI?

TIME INTERVAL
$.0-27.0$
$27.0-62.7$
$62.7-68.6$
$68.6-202.1$
$202.1-252.4$
$252.4-298.0$
$298.0-343.0$
$343.0-385.4$
TIME AVERAGED
RMS

$\begin{array}{ccc} & \\ & & \\ \text { CEMPERATURES (C) } \\ \text { COOLANT MIR. SLEEVE MAX. SLEEVE } \\ 425 . & 590 . & 617 . \\ 433 . & 603 . & 637 . \\ 408 . & 549 . & 579 . \\ 434 . & 614 . & 678 . \\ 436 . & 605 . & 689 . \\ 432 . & 593 . & 676 . \\ 436 . & 605 . & 697 . \\ 457 . & 660 . & 776 . \\ 436 . & 611 . & 682 . \\ 9 . & 20 . & 41 .\end{array}$

MIN. FUEL

712.

684.

821 .

850 .

836.

866.

973.

MAX. FUE

FAST FLUENCE

(10*25 N/M**2)

832.

774 .

.00

618.

738 .

.10

891.

918.

898.

932.

1052 .

.00
.09
.23
.25
.76
.95
1.11
1.28

00

23

.25

.28 


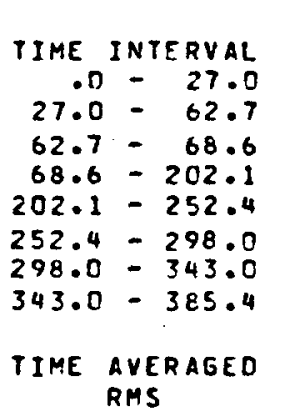

COOLANT
437.
445.
418.
447.
448.
444.
449.
472.
448.
9.

\begin{tabular}{cccc}
\multicolumn{4}{c}{ TEMPERATURES $(C)$} \\
MIA. SLEEVE MAX. SLEEVE \\
603. & 631. & MIN.FUEL & MAX.FUEL \\
617. & 651. & 727. & 790. \\
561. & 591. & 69. & 834. \\
628. & 692. & 838. & 752. \\
620. & 704. & 869. & 908. \\
607. & 690. & 855. & 939. \\
621. & 712. & 887. & 919. \\
679. & 794. & 998. & 954. \\
626. & 697. & 851. & 1074. \\
22. & 42. & 69. & 920. \\
& & & 73.
\end{tabular}

FAST FLUENCE

(10*25 N/M**2)

.00

.24

.80

1.00

1.17

1.36

E06-01 COMPACT 11

TIME INTERVAL

$.0=27.0$
$27.0=62.7$

$62.7-68.6$

$68.6-202.1$

$202.1-252.4$

$252.4-298.0$

$298.0=343.0$

$343.0-385.4$

TIME AVERAGED RMS

COOLAN
449.
457.
428.
460
460.
455.
461.
487.
461.
10.

MIA. SLEEVE
617.
631.
573.
643.
635.
621.
635.
696.
640.
23.

TEMPERATURES (C)

$\begin{array}{ccc}\text { MAX. SLEEVE } & \text { MIN. FUEL } & \text { MAX. FUEL } \\ 645 . & 741 . & 805 . \\ 666 . & 781 . & 850 . \\ 603 . & 710 . & 765 . \\ 706 . & 853 . & 924 . \\ 716 . & 884 . & 955 . \\ 702 . & 870 . & 935 . \\ 725 . & 903 . & 971 . \\ 811 . & 1018 . & 1100 . \\ 711 . & & \\ 43 . & 867 . & 937 . \\ & 70 . & 74 .\end{array}$

FAST FLUENCE

(10*25 N/M**2)

.00
.10
.24
.27
.81
1.01
1.18
1.37

EO6-01 COMPACT 12

TIME INTERVAL $.0=27.0$
$27.0=62.7$ $62.7=68.6$ $68.6-202.1$ $202.1-252.4$ $252.4-298.0$ $298.0-343.0$ $343.0-385.4$

TIME AVERAGED RMS

$\begin{array}{cc}\text { COOLANT MIA. SLEEVE } \\ 461 . & 629 . \\ 469 . & 642 . \\ 439 . & 583 . \\ 473 . & 656 . \\ 473 . & 647 . \\ 467 . & 633 . \\ 474 . & 647 . \\ 503 . & 722 . \\ 474 . & 653 . \\ 11 . & 24 .\end{array}$

TEMPERATURES (C)

$\begin{array}{ccc}\text { MAX.SLEEVE } & \text { MIN FUEL } & \text { MAX. FUEL } \\ 657 . & 753 . & 816 . \\ 678 . & 793 . & 862 . \\ 613 . & 719 . & 775 . \\ 717 . & 864 . & 935 . \\ 727 . & 897 . & 967 . \\ 713 . & 882 . & 947 . \\ 737 . & 916 . & 984 . \\ 825 . & 1034 . & 1116 . \\ 723 . & 879 . & 949 . \\ 43 . & 72 . & 76 .\end{array}$

FAST FLUENCE

(10*25 N/M**2)

$$
\begin{array}{r}
.00 \\
.10 \\
.24 \\
.27 \\
.81 \\
1.02 \\
1.19 \\
1.38
\end{array}
$$


E06-01 COMPACT 13

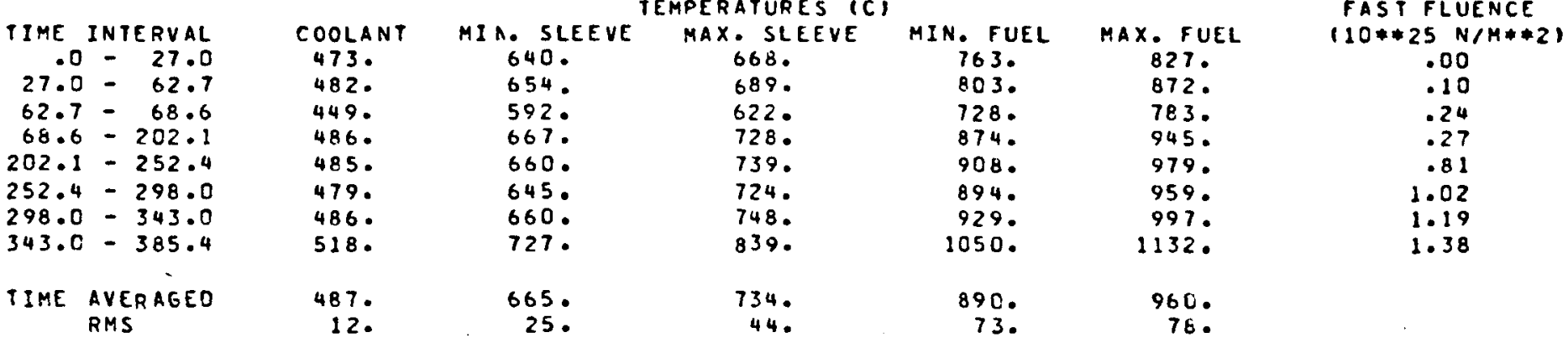

E06-01 COMPACT 14

TIME INTERYAL

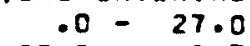

$27.0-62.7$

$62.7-68.6$

$68.6-202.1$

$202.1-252.4$

$252.4-298.0$

$298.0-343.0$

$343.0-385.4$

IIME AVERAGED RMS

COOLANT
485.
494.
459.
499.
498.
491.
499.
533.
500.
13.

MIA. SLEEVE
650.
664.
601.
679.
672.
656.
672.
742.
677
26

IEMPERATURES (C)

$\begin{array}{ccc}\text { MAX. SLEEVE } & \text { MIN. FUEL } & \text { MAX. FUEL } \\ 678 . & 772 . & 835 . \\ 699 . & 812 . & 881 . \\ 630 . & 735 . & 790 . \\ 737 . & 882 . & 952 . \\ 748 . & 918 . & 988 . \\ 733 . & 903 . & 968 . \\ 758 . & 939 . & 1007 . \\ 852 . & 1062 . & 1144 . \\ 744 . & 899 . & 969 . \\ 45 . & 74 . & 79 .\end{array}$

EO6-01 COMPACT 15

TIME INTERVAL

TIME -27.0
$27.0-62.7$
$62.7-68.6$
$68.6-202.1$
$202.1=252.4$
$252.4=298.0$
$298.0-343.0$
$343.0=385.4$
TIME AVERAGEO
RMS

COOLANT
497.
506.
469.
512.
510.
503.
511.
548.
512.
14.

MIA. SLEEVE
659.
$6739^{\circ}$
608.
688.
683.
667.
683.
$755^{\circ}$
687.
27

TEMPERATURES (C)

$\begin{array}{ccc} & \\ \text { MAX.SLEEVE } & \text { MIN. FUEL } & \text { MAX. FUEL } \\ 687 . & 779 . & 841 . \\ 707 . & 818 . & 885 . \\ 637 . & 740 . & 794 . \\ 745 . & 887 . & 956 . \\ 757 . & 925 . & 995 . \\ 741 . & 910 . & 975 . \\ 767 . & 946 . & 1014 . \\ 363 . & 1071 . & 1153 . \\ 752 . & & \\ 46 . & 906 . & 975 . \\ & 75 . & 80 .\end{array}$

FAST FLUENCE

(10*25 N/M**2)

$$
\begin{aligned}
& .00 \\
& .10 \\
& .24 \\
& .26 \\
& .80 \\
& 1.01 \\
& 1.18 \\
& 1.37
\end{aligned}
$$

FAST FLUENCE

(10*25 N/M**2)

.00

.10

.10
.24
.26

.26

.79
1.00

1.18

1.36 
E06-01 COMPACT 16

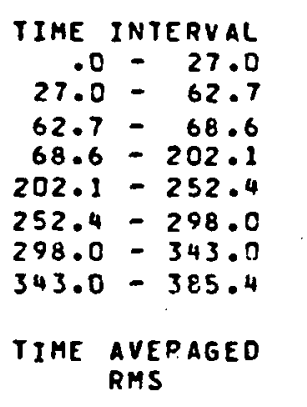

COOLANT
508.
518.
479.
525.
522.
515
523.
563.
525.
15.

MIN. SLEEVE
667 .
682.
616.
697.
693.
676.
693.
767.
697.
28.

TEMPERATURES (C)

$\begin{array}{ccc}\text { MAX. SLEEVE } & \text { MIN.FUEL } & \text { MAX. FUEL } \\ 605 . & 786 . & 847 . \\ 716 . & 825 . & 890 . \\ 644 . & 745 . & 798 . \\ 752 . & 891 . & 959 . \\ 764 . & 929 . & 999 . \\ 748 . & 914 . & 978 . \\ 774 . & 951 . & 1618 . \\ 872 . & 1077 . & 1158 . \\ 760 . & 911 . & 979 . \\ 46 . & 75 . & 80 .\end{array}$

FAST FLUENCE

$110 * 25 \quad N / M * 2$,

.00
.09
.23
.26
.78
.99
1.16
1.34

E06-01 COMPACT 17

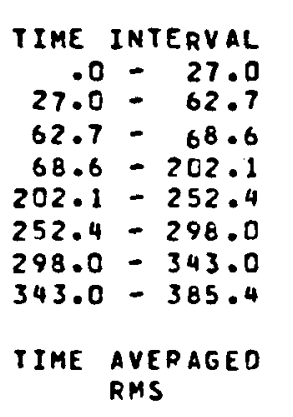

COOLANT
519.
529.
488.
537.
534.
526.
536.
578.
537.
16.

MIA. SLEEVE
674.
688.
621.
704.
701.
684.
701.
777.
704.
29.

IEMPERATURES (C)

$\begin{array}{cc}\text { MAX. SLEEVE } & \text { MIN. FUEL } \\ 701 . & 789 . \\ 721 . & 827 . \\ 648 . & 746 . \\ 756 . & 891 . \\ 770 . & 930 . \\ 753 . & 915 . \\ 779 . & 952 . \\ 879 . & 1078 . \\ 765 . & 911 . \\ 47 . & 75 . \\ & \end{array}$

MAX. FUEL
848.
891.
798.
957.
998.
977.
1017.
1158.
978.
80.

FAST FLUENCE

$(10 * 25 N / M * 2)$

$$
\begin{array}{r}
.00 \\
.09 \\
.23 \\
.25 \\
.76 \\
.97 \\
1.13 \\
1.31
\end{array}
$$

80.

\section{E06-01 COMPACT 18}

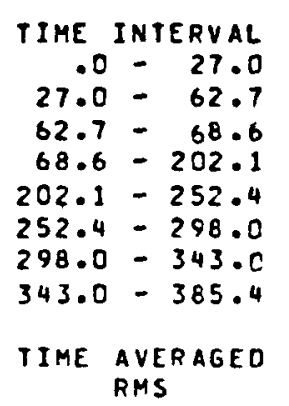

COOLANT
530.
540.
498.
549.
546.
537.
547.
593.
549.
17.

MIA. SLEEV
680.
695.
626.
711.
709.
692.
710.
787
712
30

TEMPERATURES (C)

$\begin{array}{ccc}\text { MAX.SLEEVE } & \text { MIN. FUEL } & \text { MAX. FUEL } \\ 706 . & 792 . & 850 . \\ 726 . & 829 . & 891 . \\ 653 . & 747 . & 797 . \\ 760 . & 891 . & 955 . \\ 7750^{\circ} & 931 . & 998 . \\ 758 . & 915 . & 977 . \\ 784 . & 952 . & 1016 . \\ 885 . & 1079 . & 1157 . \\ 770 . & 912 . & 977 . \\ 47 . & 74 . & 79 .\end{array}$

FAST FLUENCE $(10 * 25 \quad N / M * 2)$

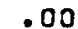

.00

.22

.24

.75

.94

1.28 
E06-01 COMPACT 19

\begin{tabular}{|c|c|c|c|c|c|c|c|}
\hline $\begin{array}{l}\text { TIME } \\
27.0 \\
27.0\end{array}$ & $\begin{array}{r}\text { INTERVAL } \\
-\quad 27.0 \\
-\quad 62.7\end{array}$ & $\begin{array}{l}\text { COOLANT } \\
541 . \\
551 .\end{array}$ & $\begin{array}{c}\text { MIR. SLEEVE } \\
686 . \\
701 .\end{array}$ & $\begin{array}{l}\text { MPERATURES IC } \\
\text { MAX SLEEVE } \\
712 . \\
732 .\end{array}$ & $\begin{array}{c}\text { MIN FUEL } \\
796 . \\
832 .\end{array}$ & $\begin{array}{c}\text { MAX. FUEL } \\
852^{\circ} \\
892\end{array}$ & $\begin{array}{c}\text { FAST FLUENCE } \\
(10 * 25 N / M * 21 \\
.00 \\
.09\end{array}$ \\
\hline $\begin{array}{r}62.7 \\
68.6 \\
202.1\end{array}$ & $\begin{array}{l}6=68.6 \\
=202.1 \\
-\quad 252.4\end{array}$ & $\begin{array}{l}507 . \\
560 . \\
558 .\end{array}$ & $\begin{array}{l}632 . \\
718 . \\
715 .\end{array}$ & $\begin{array}{l}657 . \\
765 . \\
777 .\end{array}$ & $\begin{array}{l}749 . \\
892 . \\
929 .\end{array}$ & $\begin{array}{l}798 . \\
954 . \\
993 .\end{array}$ & $\begin{array}{r}.22 \\
.24 \\
.72\end{array}$ \\
\hline $\begin{array}{l}252.4 \\
298.0 \\
343.0\end{array}$ & $\begin{array}{r}-298.0 \\
-343.0 \\
-385.4\end{array}$ & $\begin{array}{l}548 . \\
559 . \\
607 .\end{array}$ & $\begin{array}{l}698 . \\
716 . \\
795 .\end{array}$ & $\begin{array}{l}760 . \\
787 . \\
888 .\end{array}$ & $\begin{array}{r}912 . \\
949 . \\
1075 .\end{array}$ & $\begin{array}{l}972 . \\
1011 . \\
1151 .\end{array}$ & $\begin{array}{l}.91 \\
1.07 \\
1.25\end{array}$ \\
\hline TIME & $\begin{array}{l}\text { AVERAGED } \\
\text { RMS }\end{array}$ & $\begin{array}{r}560 . \\
18 .\end{array}$ & $\begin{array}{r}718 . \\
30 .\end{array}$ & $\begin{array}{r}774 . \\
46 .\end{array}$ & $\begin{array}{r}911 . \\
72 .\end{array}$ & $\begin{array}{r}974 . \\
77 .\end{array}$ & \\
\hline
\end{tabular}

EO6-01 COMPACT 20

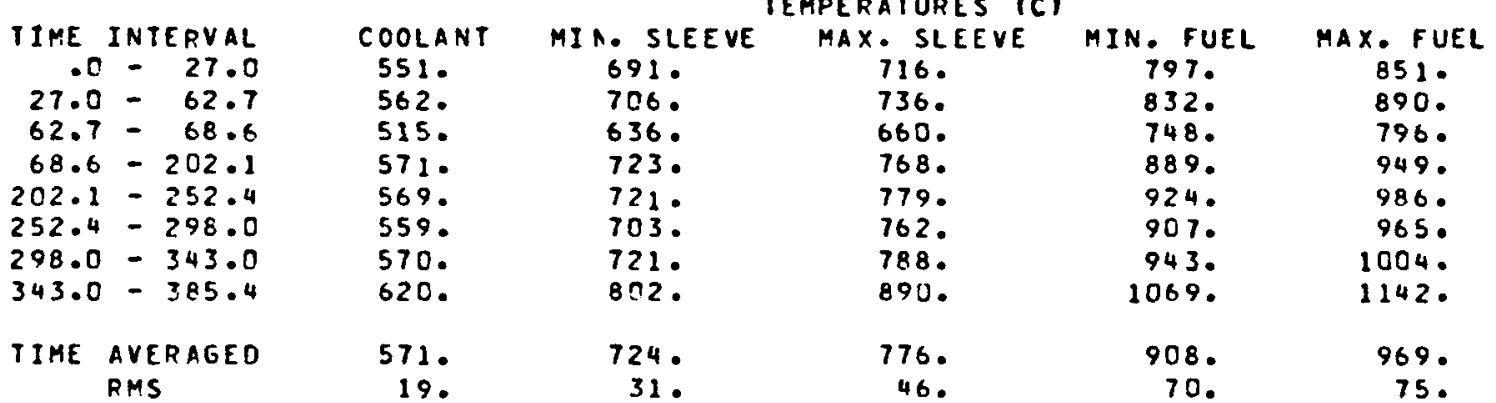

FAST FLUENCE

$(10 * 25 N / M * 2)$

.00

.08

.21

.23

.70

.88

1.03

1.20

EO6-01 COMPACT 21

TIME INTERVAL

$.0-27.0$

$27.0=62.7$

$62.7-68.6$

$68.6-202.1$

$202.1-252.4$

$252.4-298.3$

$298.0-343.0$

$343.0-385.4$

TEMPERATURES (C)
MAX. SLEEYE

COOLANT
561.
572.
524.
582.
579.
569.
581.
633.

MIA. SLEEVE

MIN. FUEL

694 .

738.

638 .

638 .
726.

724 .

706

725 .

662 .

768 .

778.

761.

806 .

889 .

796.

829 .

.745 .

883.

916.

899.

934.

75.

IIME AVERAGED

582.
20.

727

776.

901.

MAX. FUEL

847 .

885 .

785.

941 .

976.

954.

992.

1129 .

960.
FAST FLUENCE

$(20 * 25 \quad N / M * * 2)$
.00
.08
.20
.22
.67
.84
.99
1.15

31 .

45.

601.

73. 
E06-01 COMPACT 22

TIME INTERVAL COQLANT MEMPERATURES (C)

\begin{tabular}{|c|c|c|c|c|c|c|}
\hline 27.0 & $\begin{array}{r}27.0 \\
-\quad 62.7\end{array}$ & $\begin{array}{l}570 \text {. } \\
581 \text {. }\end{array}$ & $\begin{array}{c}\text { MIA. SLEEVE } \\
698 . \\
713 .\end{array}$ & $\begin{array}{c}\text { MAX. SLEEVE } \\
721 . \\
740 .\end{array}$ & $\begin{array}{c}\text { MIN. FUEL } \\
795 . \\
827 .\end{array}$ & $\begin{array}{c}\text { MAX. FUEL } \\
845 . \\
880\end{array}$ \\
\hline 62.7 & -68.6 & 532. & 641. & 663. & 743. & 786. \\
\hline $\begin{array}{r}68.6 \\
202.1\end{array}$ & $\begin{array}{l}-202.1 \\
-\quad 252.4\end{array}$ & $\begin{array}{l}592 . \\
589 .\end{array}$ & $\begin{array}{l}730 . \\
728 .\end{array}$ & $\begin{array}{l}769 . \\
779 .\end{array}$ & $\begin{array}{l}878 . \\
910 .\end{array}$ & $\begin{array}{l}933 . \\
968 .\end{array}$ \\
\hline 252.4 & -298.0 & 578. & 711. & 762. & 893. & 946. \\
\hline $\begin{array}{l}298.0 \\
343.0\end{array}$ & $\begin{array}{l}-343.0 \\
-385.4\end{array}$ & $\begin{array}{l}591 . \\
645 .\end{array}$ & $\begin{array}{l}730 . \\
812 .\end{array}$ & $\begin{array}{l}788 . \\
890 .\end{array}$ & $\begin{array}{r}928 . \\
1051 .\end{array}$ & $\begin{array}{r}983 . \\
1118 .\end{array}$ \\
\hline TIHE & $\begin{array}{l}\text { AVERAGEO } \\
\text { RMS }\end{array}$ & $\begin{array}{r}592 . \\
21 .\end{array}$ & $\begin{array}{r}731 . \\
32 .\end{array}$ & $\begin{array}{r}777 . \\
45 .\end{array}$ & $\begin{array}{r}896 . \\
66 .\end{array}$ & $\begin{array}{r}952 . \\
71 .\end{array}$ \\
\hline
\end{tabular}

FAST FLUENCE

$110 * 25 \quad N / M * * 21$

.00

.19

.21

.63

.94

1.09

E06-01 COMPACT 23

TIME INTERVAL $.0-27.0$
$27.0-62.7$ $62.7-68.6$ $68.6-202.1$ $202.1-252.4$ $252.4-298.0$ $298.0-343.0$ $343.0-385.4$ TIME AVERAGED
RMS

COOLANT
579.
590.
539.
601.
599.
587.
600.
657.
602.
22.

MIN. SLEEVE
$6999^{\circ}$
$7130^{\circ}$
$6420^{\circ}$
$7310^{\circ}$
$7300^{\circ}$
712.
731.
814.
732
32

IEMPERATURES (C)

$\begin{array}{ccc}\text { TEMPERATURES (C) } & \\ \text { MAX. SLEEVE } & \text { MIN. FUEL MAX.FUEL } \\ 720 . & 791 . & 837 . \\ 739 . & 821 . & 871 . \\ 663 . & 737 . & 778 . \\ 767 . & 869 . & 921 . \\ 776 . & 898 . & 952 . \\ 759 . & 881 . & 931 . \\ 784 . & 915 . & 967 . \\ 885 . & 1036 . & 1099 . \\ 775 . & & \\ 44 . & 886 . & 938 .\end{array}$

FAST FLUENCE

$(10 * 25 N / M * 2)$

.00

.07

.18

.19

.60

.76

.89
1.04

EO6-01 COMPACT 24

IIME INTERVAL
$.0-27.0$
$27.0-62.7$
$62.7-68.6$
$68.6=202.1$
$202.1-252.4$
$252.4-298.0$
$298.0-343.0$
$343.0=385.4$
TIME AVERAGED
RMS

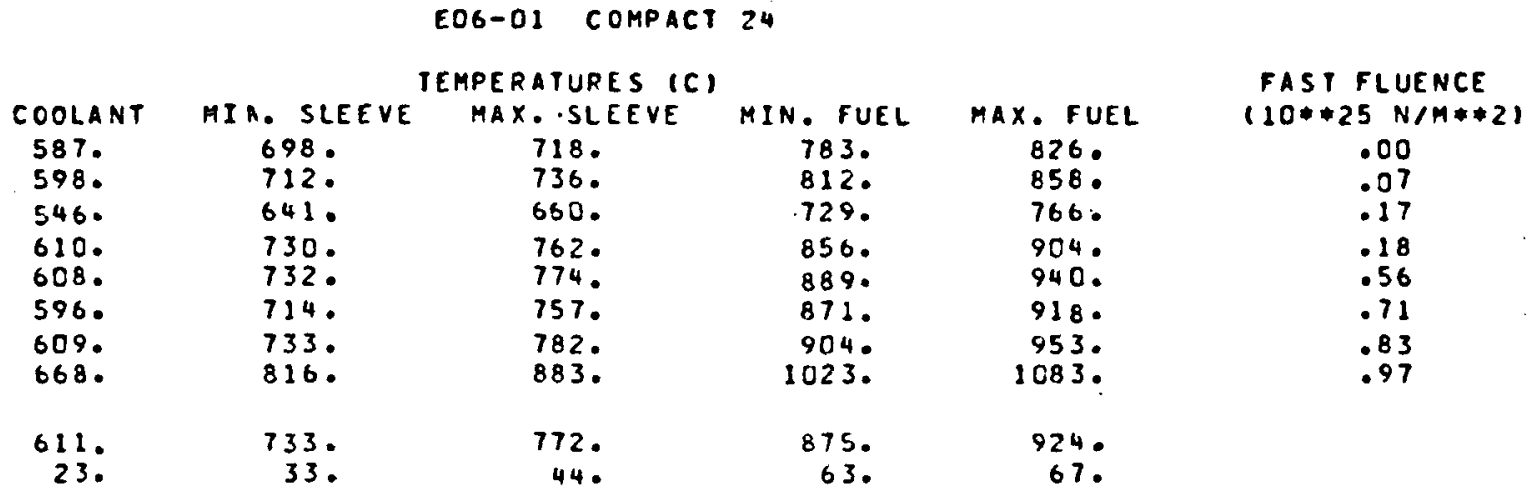


E06-01 COMPACT 25

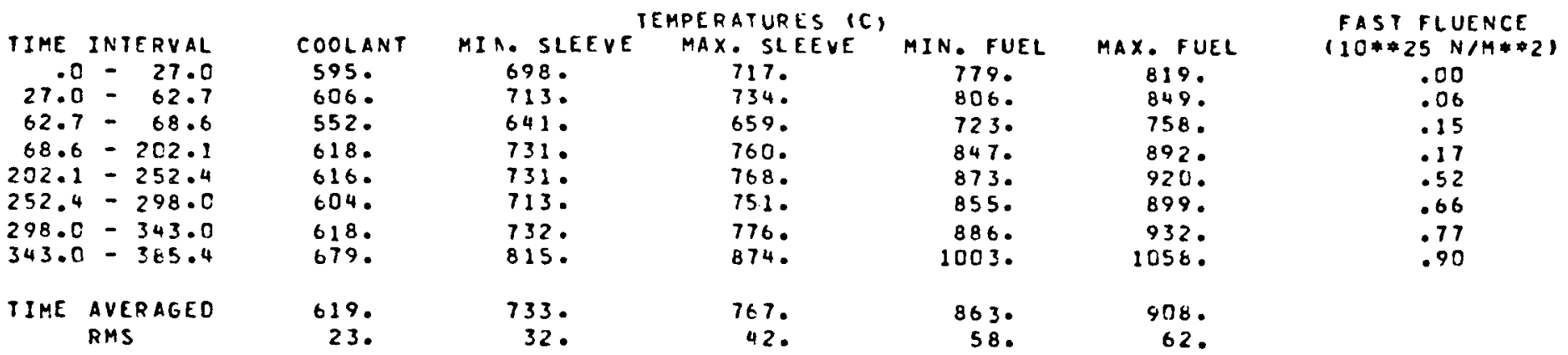

EO6-CL COMPACT 26

TIME INTERVAL

$$
.0-27.0
$$

$.0=27.0$
$27.0-62.7$

$62.7-68.6$

$68.6-202.1$

$202.1-252.4$

$252.4-298.0$

$298.0-343.0$

$343.0-385.4$

TIME AVERAGED RMS

COOLANT
602.
613.
558.
626.
624.
611.
626.
688.
627
24.

IEMPERATURES $(C)$

MIR. SLEEV

$$
697 \text {. }
$$

640 .

MAX. SLEEVE

TI1. FUE

729 .

729 .

711 .

730 .

714.

731.

656.
755.

762 .

745.

813.

$7690^{\circ}$

797.

715 .

835 .

857 .

839.

869.

982 .

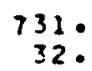

762.

849.

55 .

MAX. FUEL

FAST FLUENCE

(10**25 N/M**21

808 .

837 .

748.

$$
901 .
$$

879.

1033.

.00
.06
.14
.16
.48
.61
.71
.83

E06-01 COMPACT 27

TIME INTERVAL

$.0-27.0$
$27.0-62.7$

$62.7-68.6$

$68.6-202.1$

$252.4-298.0$

$252.4=298.0$

$343.0-385.4$

TIME AVERAGED

RMS

$\begin{array}{lc}\text { COOLANT MIA. SLEEVE } \\ 608 . & 694 . \\ 620 . & 708 . \\ 564 . & 638 . \\ 633 . & 726 . \\ 631 . & 727 . \\ 610^{\circ} & 709 . \\ 633 . & 728 . \\ 697 . & 811 . \\ & \\ 634 . & 729 . \\ 25 . & 32 .\end{array}$

TEMPERATURES (C)

$\begin{array}{ccc}\text { MAX.SLEEVE } & \text { MIN. FUEL } & \text { MAX.FUEL } \\ 709 . & 762 . & 796 . \\ 726 . & 786 . & 822 . \\ 652 . & 706 . & 735 . \\ 749 . & 821 . & 859 . \\ 756 . & 842 . & 882 . \\ 739 . & 824 . & 861 . \\ 762 . & 853 . & 89 . \\ 857 . & 963 . & 1009 . \\ 756 . & & \\ 40 . & 834 . & 873 . \\ & 53 . & 56 .\end{array}$

FAST FLUENCE

$(10 * 25 N / M * 2)$

.00
.05

.13

.12

.43

.05

.55
.65

.65
.75 


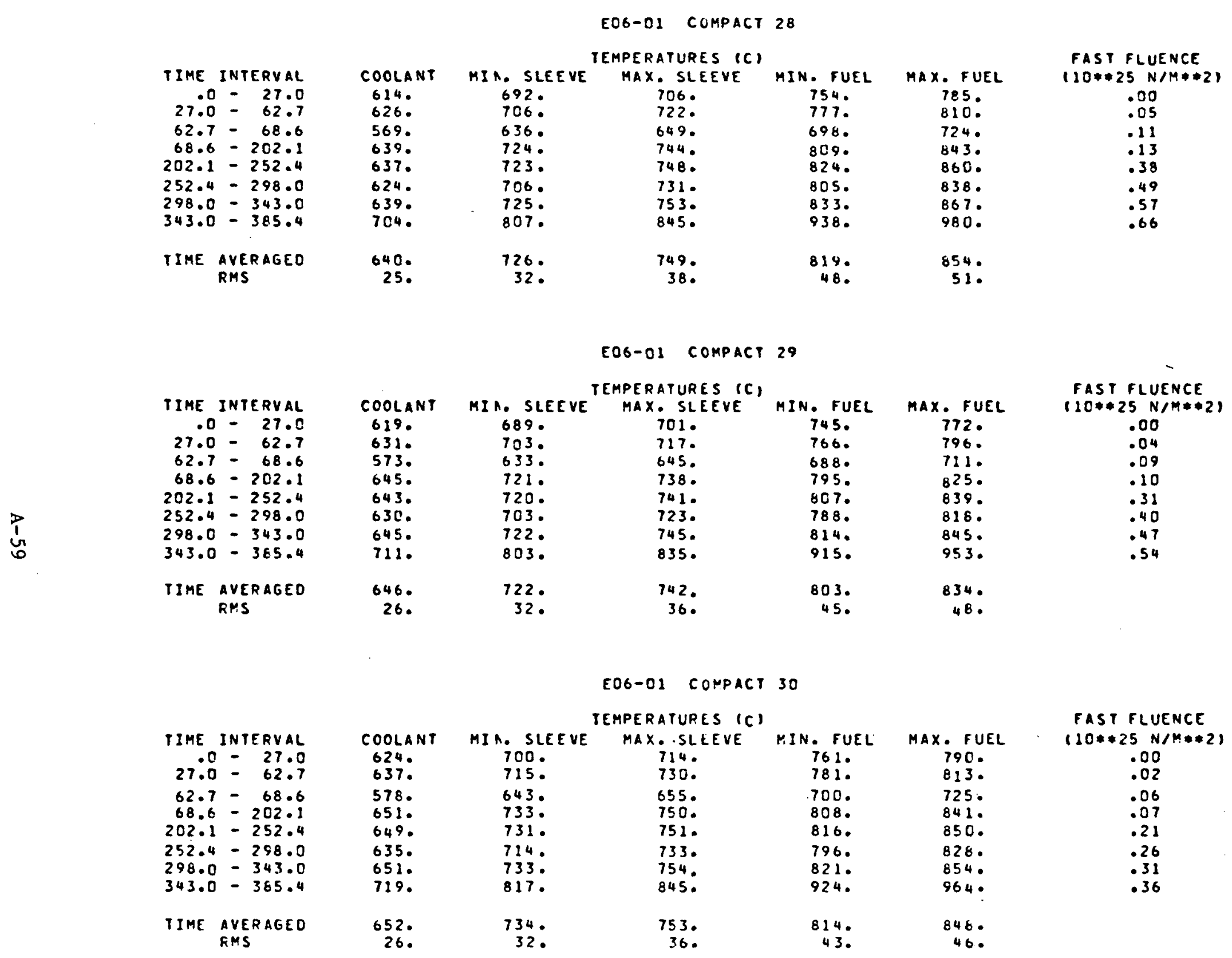




\section{EO6-01 FUEL PERFORMANCE}

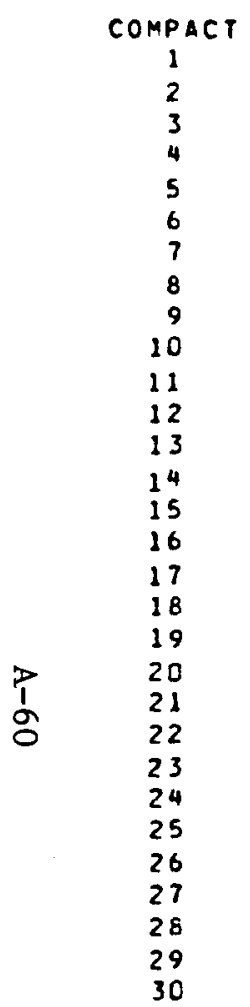

KERNEL MIGRAIION (MICRONS) AVERAGE AMOEBA EO. TEMP.

PEAK

$\begin{array}{ll}563 . & .00 \\ 612 . & .00\end{array}$

$.0 O$
$.0 O$

612 .

675 .

.00
.00

.00
$.0 \mathrm{C}$

736.

784.

828.

866 .

900 .

928 .

952.

970 .

985.

1000

1009.

1009.

1014.

1015

1014.

1008 .

998.

989 .

973.

961.

941.

921.

$\begin{array}{lll}.0 \mathrm{C} & 901 . & .00\end{array}$

856.

.00

.00

.00

.01

.01

.02

.03

.04

.05

.06

.06
.07

.06

.06

.06

.05

.04

.03

.02
.02

.02
.01

.01

$.0 \mathrm{C} \quad 901.00$

.00

.00
.00

866 .

DEFECIIVE COATINGS
.03
.05
.07
.08
.09
.10
.11
.12
.12
.12
.12
.13
.13
.13
.12
.12
.12
.12
.11
.11
.11
.10
.09
.09
.08
.08
.07
.06
.05
.03

FUEL FAILURE ( $z$ )

PRESSURE VESSEL KERNEL MIGRATION TOTAL

$\begin{array}{lll}.09 & .00 & .12 \\ .15 & .00 & .20\end{array}$

$.15 \quad .00 \quad .20$

$\begin{array}{lll}.19 & .00 & .26 \\ .23 & .00 & .32\end{array}$

.26

.0 .39

$.31 \quad .00 \quad .42$

$.32 .00 \quad .44$

$.33 \quad .00 \quad .45$

$\begin{array}{lll}.34 & .00 & .46\end{array}$

$.34 \quad .00 \quad .47$

.00000078

$.34 \quad .00 \quad .47$

$\begin{array}{lll}.34 & .00 & .47\end{array}$

$\begin{array}{lll}.34 & .00 & .46 \\ .33 & .00 & .45\end{array}$

$\begin{array}{lll}.33 & .00 & .45 \\ .32 & .00 & .44\end{array}$

$.31 \quad .00 \quad .43$

$.30 \quad .00 \quad .41$

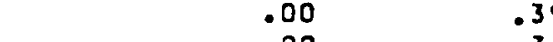

$\begin{array}{lll}.26 & .00 & .35\end{array}$

$\begin{array}{lll}.24 & .00 & .33 \\ 23 & .00 & .31\end{array}$

$\begin{array}{lll}.23 & .00 & .31 \\ .21 & .00 & .28\end{array}$

$.19 \quad .00 \quad .26$

.00
.00

.14
.08

.19

AMOEBA EOUIVALENT TEMPERATURES (C) CORRESPONO TO AVERAGE KERNEL MIGRATIONS

KERNEL MIGRATION OISTANCES CALCULATEO USING $50 \%$ CONFIDENCE LEVEL KMC

FUEL FAILUPE CORRESPONDS TO $50 X$ CONF IOENCE LEVEL 
C11-07 COAPACT 1

TIME INTERVAL
.0- 27.0
$27.0=62.7$
$62.7=68.6$
$68.6=202.1$
$202.1=252.4$
TIME AVERAGED
RMS

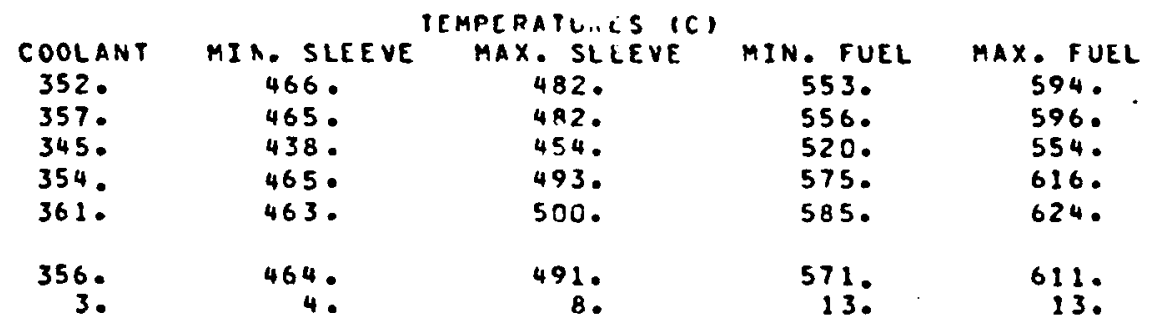

FAST FLUENCE

$120 * 25 N / M * 21$

.00
.03

.03
.08

.09

.26

C11-07 COMPACT 2

$p$
1
$a$
-

TIME INTERVAL

$.0-27.0$
$27.0=62.7$

$62.7-68.6$

$68.6=202.1$

$202.1-252.4$

TIME AVERAGED

FMS

COOLANT TEMPERATURES (C)

361.

361.

353.

363.

369 .

489.

486.

457 .

487.

MAX. SLE

507.

507.

476.

542 .

486.

523.

MIN. FUEL

584.

589.

551.

622 .

642 .

615.

MAX . FUEL

630.

635 .

589

667 .

687.

364.

5 .

C11-07 COMPACT 3

TIME INTERVAL TIAE 027.0 $.0-27.0$
$27.0=62.7$ $62.7=68.6$ $68.6-202.1$ $202.1-252.4$

TIME AVEPAGEO RMS

COOLANT
372.
376
362
374
379
375
3.

MIA. SLEEV
$5322^{\circ}$
$526^{\circ}$
$491^{\circ}$
$520^{\circ}$
$523^{\circ}$
527.
6.

TEMPERATURES (C)

IN. FUEL 647. $555^{\circ}$

517.

580.

653.

609 .

698.

575.

720.

688.

MAX. FUEL

661.
22.

FAST FLUENCE

$(10 * 25 \mathrm{~N} / \mathrm{M} * 2)$

.00

.06
.13

.15

.43

\section{C11-07 COMPACT 4}

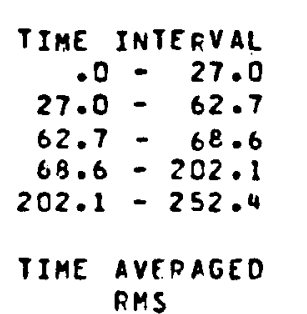

COOLANT
386.
389.
373.
387
391.
387
3.

MIA. SLEEVE
$577^{\circ}$
569.
529.
573.
559
569.
8.

IERPERATURES (C)

$\begin{array}{lc} \\ \text { N. FUEL MAX FUEL } \\ 711 . & 780 . \\ 719 . & 788 . \\ 669 . & 727 . \\ 7750^{\circ} & 8450^{\circ} \\ 791 . & 856 . \\ & \\ 761 . & 829 . \\ 32 . & 32 .\end{array}$

FAST FLUENCE

(10*25 N/M**2)

.00

.09

.21
.23 
C11-07 COMPACT 5

\begin{tabular}{|c|c|c|c|c|c|c|c|}
\hline TIME & IN TERVAL & COOLANT & MIR. SLEEVE & $\begin{array}{l}\text { MPERATL S IC) } \\
\text { MAX. SLEEVE }\end{array}$ & MIN. FUEL & MAX. FUEL & $\begin{array}{l}\text { FAST FLUENCE } \\
(10 * 25 N / M * 2)\end{array}$ \\
\hline $\begin{array}{r}.0 \\
27: 0 \\
62: 7\end{array}$ & $\begin{array}{l}-\quad 27.0 \\
=\quad 62.7 \\
-\quad 68.6\end{array}$ & $\begin{array}{l}401 . \\
403 . \\
385\end{array}$ & $\begin{array}{l}613 . \\
603 . \\
558 .\end{array}$ & $\begin{array}{l}647 . \\
641 . \\
593 .\end{array}$ & $\begin{array}{l}760 . \\
769 . \\
714 .\end{array}$ & $\begin{array}{l}837 . \\
845 . \\
779 .\end{array}$ & $\begin{array}{l}.00 \\
.10 \\
.24\end{array}$ \\
\hline TIME & $\begin{array}{l}\text { AVERAGED } \\
\text { RMS }\end{array}$ & $\begin{array}{r}402 . \\
3\end{array}$ & $\begin{array}{r}603 \\
10\end{array}$ & $\begin{array}{r}666 . \\
18 .\end{array}$ & $\begin{array}{l}817 . \\
36 .\end{array}$ & $\begin{array}{l}893 . \\
36 .\end{array}$ & \\
\hline
\end{tabular}

C11-07 COMPACT 6

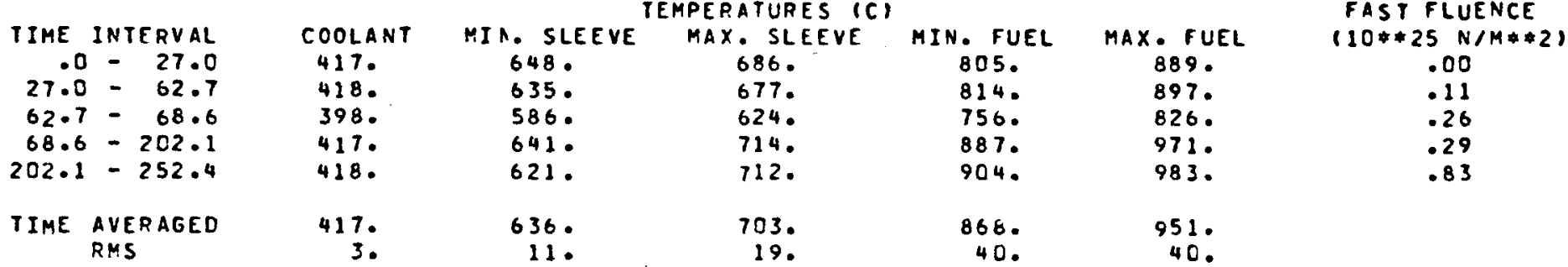

C11-0T COMPACT 7

TIME INTERVAL

$$
.0-27.0
$$$$
27.0=62.7
$$

IIME AVEPAGED

- TEMPERATURES (C)

COOLANT
434.
434.
412.
434.
433
433
3

MIA. SLEEVE

679 .

664.

611

671.

$$
\begin{aligned}
& 720 . \\
& 710 . \\
& 653 .
\end{aligned}
$$

747.

741 .

736.

665

19.

mI

MIN. FUEL

844.

854.

792 .

932.

950 .

912

43.

MAX. FUEL

C11-07 COMPACT 8

TIME INTERVAL

$$
.0-27.0
$$

$.0-27.0$
$27.0-62.7$

$62.7-68.6$

$68.6-202.1$

IIME AVEPAGED

RMS
TEMPERATURES (C)

$\begin{array}{cc}\text { COOLANT } & \text { MIA. SLEEV } \\ 452 . & 707 . \\ 451 . & 690 . \\ 427 . & 634 . \\ 451 . & 698 . \\ 449 . & 674 .\end{array}$

MAX. SLEEVE<smiles>Nc1ccccc1</smiles>

750 .

738 .

677 .

776.

769.

764.
19.

691.
14.

450.
MIN. FUEL

877 .

887.

970 .

992.

949.

46.
933.

942 .

866 .

1022 .

1034 .

1000.

43.

FASI FLUENCE

$(10 * 25 N / H * 2)$

$$
\begin{aligned}
& .00 \\
& .12 \\
& .28 \\
& .31 \\
& .89
\end{aligned}
$$

FAST FLUENCE

$(20 * 25 N / A * 2)$

MAX. FUEL

970 .

979.

899.

1063.

1000 .

.00

.12

.29

.32

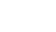




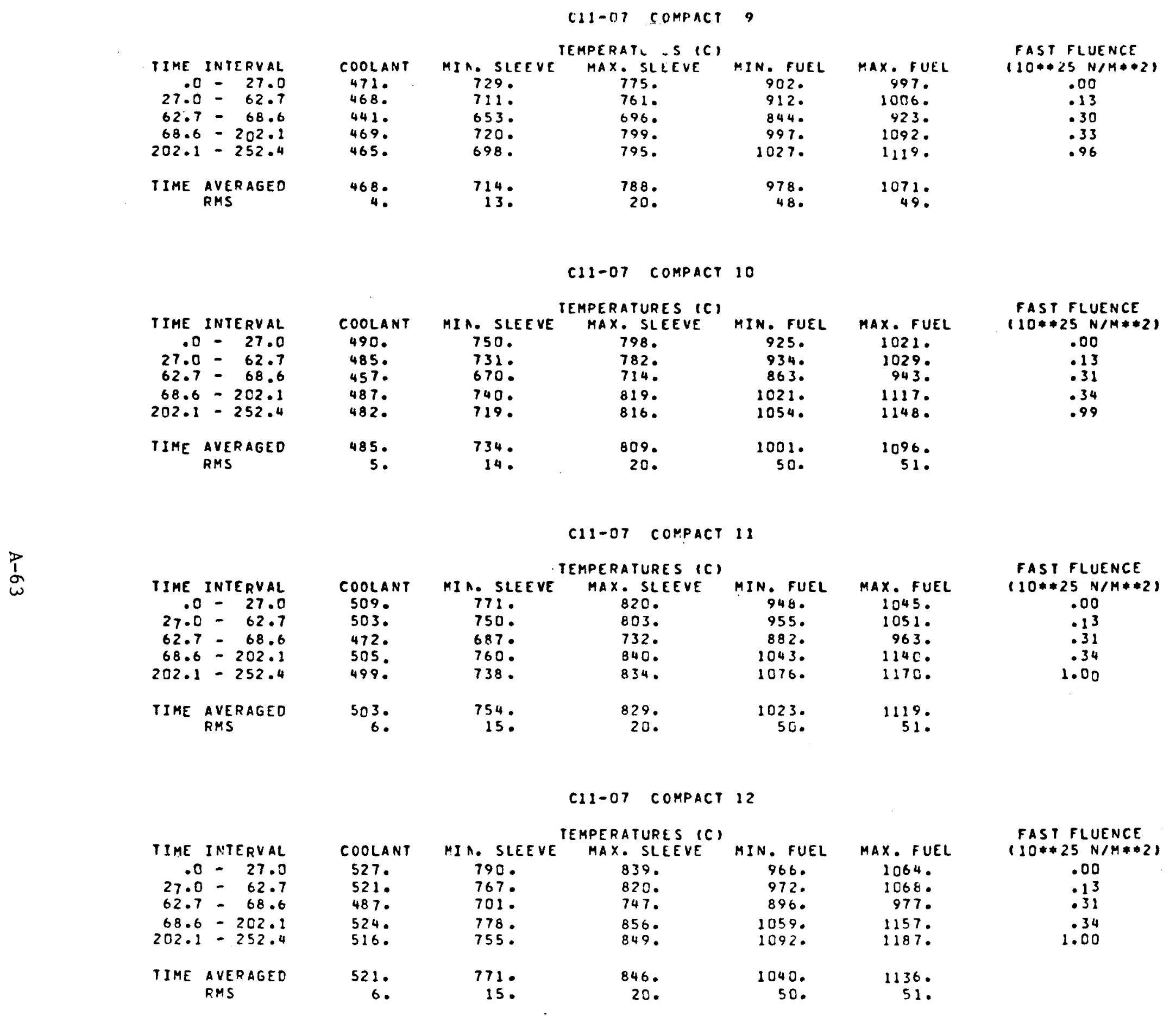


C11-07 ROMPACI 13

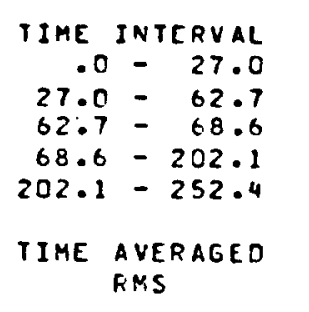

COOLANT
546.
538.
502.
542.
533.
539.
7.

MIA. SLEEV
807.
783.
715.
794.
773.
788.
15.

TEMPERATL.CS (C)

$\begin{array}{cc}\text { EMPERATL.CS }(C) \\ \text { MAX. SLEEVE } \\ 857 . & \text { MIN. FUEL } \\ 836 . & 983 . \\ 760 . & 987 . \\ 872 . & 909 . \\ 865 . & 1073 . \\ & 1108 . \\ 861 . & 1054 . \\ 20 . & 50 .\end{array}$

MAX. FUEL
1080.
1083.
989.
1170.
1204.
1150.
51.
FASI FLUENCE
$110 * 25 N / M * 2$ )

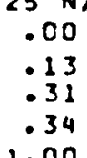

C11-07 COMPACT 14

TIME INTERVAL
$.0-27.0$
$27.0-62.7$
$62.7-68.6$
$68.6=202.1$
$202.1-252.4$
TIME AVERAGED
RMS

COOLANT
565.
555.
518.
560
550
557
8.

MIA. SLEE
822.
798.
728.
810
789
803
15

TEMPERATURES (C)

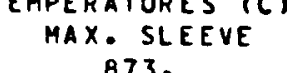

MIN. FUEL

$\begin{array}{ccc}\text { X. SLEEVE } & \text { MIN. FUEL } & \text { MAX.FUEL } \\ 873 . & 998 . & 1094 . \\ 851 . & 1000 . & 1095 . \\ 773 . & 919 . & 990^{\circ} \\ 885 . & 1084 . & 1180 . \\ 880 . & 1120 . & 1210 . \\ 875 . & & \\ 20 . & 1066 . & 1162 .\end{array}$

FAST FLUENCE

(10*25N/M**2)

.00

.31

.34

.99

\section{C11-07 COMPACT 15}

?

TIME INTERVAL
$.0-27.0$
$27.0=62.7$
$62.7=68.6$
$68.6=202.1$
$202.1-252.4$
TIME AVERAGED
RMS

COOLANT
583.
573.
532.
577.
567.
574.
8.

MIN. SLEE
836.
810
739
823.
803.
817.
15.

IEMPERATURES (C)

$\begin{array}{cc}\text { MAX. SLEEVE } & \text { MIN. FUEL } \\ 887 . & 1009 . \\ 863 . & 1009 . \\ 784 . & 927 . \\ 806 . & 1091 . \\ 892 . & 1130 . \\ 887 . & 1075 . \\ 20 . & 48 .\end{array}$

MAX. FUEL
1103.
1102.
1005.
1186.
1224.

FAST FLUENCE

(10*25 N/M*21

.00

.13

.30

.33

1169.

50.

\section{C11-07 COMPACT 16}

TIME INTERVAL
$.0-\quad 27.0$
$27.0-62.7$
$62.7-68.6$
$68.6-202.1$
$202.1-252.4$
TIME AVERAGED
RMS

COOLANT
602.
589.
547.
595.
584.
592.
9.

MIA. SLEEV
849.
823.
750.
836.
817.
830.
16.

IEMPERATURES (C)

$\begin{array}{ccc}\text { MAX. SLEEVE } & \text { MIN. FUEL } & \text { MAX. FUEL } \\ 900^{\circ} & 1020^{\circ} & 1113 . \\ 875^{\circ} & 1018^{\circ} & 1110 . \\ 794 . & 934 . & 1111 . \\ 907 . & 1097 . & 1191 . \\ 903 . & 1135 . & 1229 . \\ 898 . & 1082 . & 1174 . \\ 19 . & 47 . & 48 .\end{array}$

FAST FLUENCE

(10*25 N/H**2)

.00

.13

.30

.33
.97 
C11-07 COMPACT 17

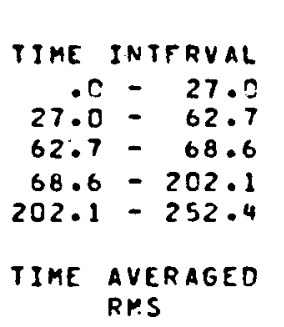

RYS

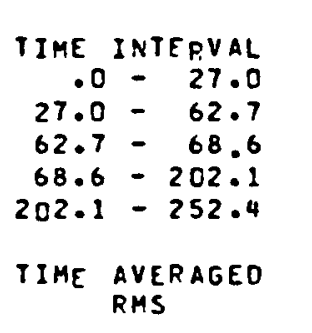

$p$
1
on

$\begin{array}{ll}\text { COOLANT MIN. SLEEVE } \\ 619 . & 859 . \\ 606 . & 832 . \\ 561 . & 758 . \\ 612 . & 845 . \\ 600 . & 828 . \\ 608 . & 839 .\end{array}$

TEMPERATL ES (C)

MAX. SLEEVE
908.
893.
800.
914.
911.

MIN. TUEL 1025 . 1021 . 936.

097.

906.

19.

1083.

\section{C21-07 COMPACT 18}

\begin{tabular}{|c|c|c|c|c|}
\hline \multicolumn{5}{|c|}{ TEMPERATURES $(C)$} \\
\hline COOLANT & MI A. SLEEVE & MAX. SLEEVE & MIN. & MAX: FUEL \\
\hline 636. & 869. & 917. & 1030. & 1118. \\
\hline 622. & 841. & 800 . & 1024. & 1111. \\
\hline 575. & 766. & 807 & 938. & 1011. \\
\hline 628. & 854. & 920. & 1097. & 1185. \\
\hline 616. & 839. & 919. & 1137. & 1227. \\
\hline 625. & 849. & 913. & 1084. & 1172. \\
\hline 10. & 16. & 19. & 44. & - \\
\hline
\end{tabular}

\section{C11-07 COMPACY 19}

\begin{tabular}{ccccc}
\multicolumn{5}{c}{ TEMPERATURES $(C)$} \\
COOLANT & MIA. SLEEVE MAX. SLEEVE & MIN. FUEL & MAX. FUEL \\
653. & 879. & 926. & 1037. & 1122. \\
637. & 850. & 898. & 1028. & 1113. \\
589. & 774. & 814. & 941. & 1012. \\
644. & 864. & 927. & 1098. & 1184. \\
632. & 847. & 924. & 1133. & 1220. \\
641. & 858. & 920. & 1085. & 1170. \\
10. & 16. & 19. & 42. & 43.
\end{tabular}

C11-07 COMPACT 20

TIME INTERVAL $.0-27.0$ $.0=27.0$
$27.0=62.7$
$62.7=68.6$ $68.6-202.1$ $202.1-252.4$

TIME AVERAGED RMS

TEMPERATURES (C)

COOLANT MIR. SLEEVE MAX. SLEEVE

669 .

669 .

652 .

660 .

647.

886 .

857 .

780

871 .

854 .

656.

11 .
MAX. SL

932.

819.

932.

927 .

924.

865.
MIN FUEL

1028.

940

1095 .

1126.

1082. 39.
MAX. FUEL

1116.

1011.

1186

11828.

1173.
FAST FLUENCE

$110 * 25 \mathrm{~N} / \mathrm{M} * 2$

.00
.12

.12

.32

.95

FAST FLUENCE

$(10 * 25 N / M * 2)$

.00

.12

.31

.92

FAST FLUENCE

$110 * 25 N / M * 21$

.00

.12

.30

.89

FAST FLUENCE

(10*25 N/M**2)

AXX. FUEL

.00

.11

.26
.29

.86

$1177^{\circ}$

1210 .

1164. 
C11-07 COMPACT 21

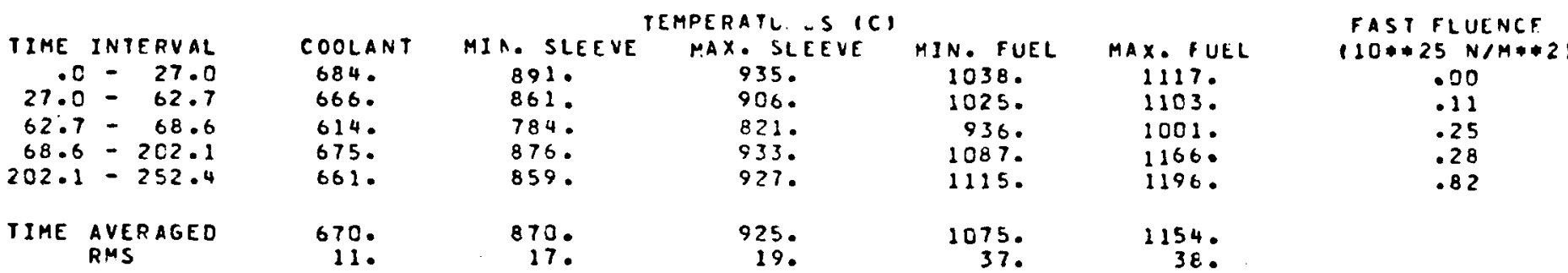

C11-07 COMPACT 22

TIME INTERVAL

$$
.0-27.0
$$

$.0-27.0$
$27.0-62.7$

$62.7-68.6$

$68.6-202.1$

$202.1-252.4$

TIME AVERAGED RHS

$p$
1
$g$
$g$

TIME INTERVAL
$27.0-27.0$
$62.7-62.7$
$68.6=202.1$
$202.1-252.4$
TIME AVERAGED
FMS

COOLANT
699.
680.
626.
689.
675.
684
12.

MIA. SLEEVE

TEMPERATURES (C)

$$
897 .
$$

788 .

881 .

MAX. SLEEVE

X SLEEVE MIN
939.
909.
823.
934.

MIN. FUEL

1038.

1022 .

933.

929.

1107.

38 .

875.

928.

1069.

35.

MAX. FUEL

1113.

1097 .

995.

1156.

1185.

FAST FLUENCE

(10*25 N/M**2)

.00

.10

.24

.26
.78

17.

19.

1145

\section{C11-07 COMPACT 23}

COOLANT
713.
6930
6370
7020
688.
698.
12.

TEMPERATURES (C) $898^{\circ}$

789.

789.
882.

867 .

MAX. SL
938.
907.

938.
907.
822.

Mrn

N. FUEL

1014.

924.

932.

1068.

37.

877.

925.

1058.

MAX. FUEL

1102.

1084.

983.
1138.

1164.

FAST FLUENCE

$(10 * 25 N / M * 2)$

.00

.10

.23

.25
.74

1128

\section{C11-07 COMPACT 24}

TIME INTERVAL

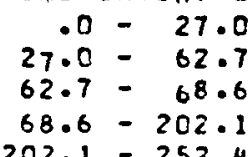

COOLANT
725.
704
647
714
701
710
12.

MIA. SLEEVE
897.
866.
788.
881.
870.
876.
16.

TEMPERATURES (C)

$\begin{array}{ccc}\text { MAX. SLEEVE } & \text { MIN. FUEL } & \text { MAX. FUEL } \\ 933 . & 1021 . & 1086 . \\ 903 . & 1002 . & 1066 . \\ 818 . & 912 . & 967 . \\ 926 . & 1050 . & 1116 . \\ 924 . & 1078 . & 1147 . \\ 921 . & 1043 . & 1109 . \\ 18 . & 31 . & 33 .\end{array}$

FASI FLUENCE

$(10 * 25 N / M * 21$

.00

.09
.21

.23

.69

16.

18.

31.

33. 
CL1-UT CUMPACT 25

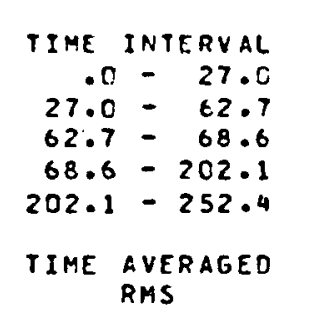

COOLANT
737.
716.
657.
726.
712.
721.
13.

MIN. SLTEV
898.
866.
788.
881.
868.
876.
16.

TEMPERATL S $S$ (C)

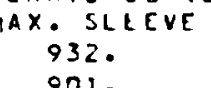

MIN FUEL

FAST FLUENCE.

$10 * 25 N / M * * 2$

1015.

1076.

.00

$$
816 .
$$

993.

1054.

1099.

1057.1121.

.20

.21
.64

1091.

\section{C11-07 COMPACT 26}

TIME INTERVAL $.0-27.0$
$27.0=62.7$ $62.7-68.6$ $68.6-202.1$ $202.1-252.4$

TIME AVERAGED RMS

COOLANT
748.
726.
666.
736.
723.
732.
13.

MIA. SLEEV
895.
864.
786.
879.
865.
874.
16.

IEMPERATURES (C)

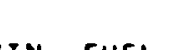

\section{C11-07 COMPACT 27}

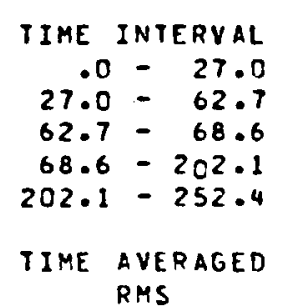

COOLANT
758.
735
674
746.
732
741.
13.

MIA. SLEEV
891.
860.
783.
875.
863.
870
16.

TEMPERATURES (C)

MAX. SLEEVE
920.
889.
806.
909.
902.

MIN. FUEL

990.
906.
879.
1003.

MAX. FUEL

FAST FLUENCE

$110 * 25$ NIM**21 1041. 1017 . 921 . 1054 .

$1070^{\circ}$

FAST FLUENCE $(10 * 25 N / M * 2)$ .00 .08

.18

.20
.59 .00 .01 .16 .18 .54 $\begin{array}{rr}996 . & 1047 . \\ 23 . & 25 .\end{array}$

\section{C11-07 COMPACT 28}

\begin{tabular}{|c|c|c|c|c|c|c|}
\hline & \multicolumn{5}{|c|}{ m } \\
\hline $\begin{array}{l}\text { TIME } \\
27.0 \\
27.0\end{array}$ & $\begin{array}{r}\text { INTERVAL } \\
-\quad 27.0 \\
-\quad 62.7\end{array}$ & $\begin{array}{l}\text { COOLANT } \\
767 \\
743\end{array}$ & $\begin{array}{l}\text { MI A. SLEEVE } \\
888^{\circ} \\
858 .\end{array}$ & $\begin{array}{c}\text { MAX. SLEEVE } \\
914^{\circ} \\
883 .\end{array}$ & $\begin{array}{l}\text { MIN. FUEL } \\
979 . \\
954 .\end{array}$ & $\begin{array}{l}\text { MAX. FUEL } \\
1026 . \\
1000 .\end{array}$ \\
\hline $\begin{array}{r}62.7 \\
68.6 \\
202.1\end{array}$ & $\begin{array}{r}-68.6 \\
=202.1 \\
-252.4\end{array}$ & $\begin{array}{l}681 . \\
755 \\
741 .\end{array}$ & $\begin{array}{l}780 . \\
872 . \\
858 .\end{array}$ & $\begin{array}{l}801 . \\
902 . \\
892 .\end{array}$ & $\begin{array}{l}867 . \\
987 . \\
991 .\end{array}$ & $\begin{array}{l}906 \\
1033 \\
1039\end{array}$ \\
\hline TIME & $\begin{array}{l}\text { AVERAGED } \\
\text { RMS }\end{array}$ & $\begin{array}{l}750 . \\
13 .\end{array}$ & $\begin{array}{r}867 . \\
16 .\end{array}$ & $\begin{array}{c}896 . \\
17 .\end{array}$ & $\begin{array}{r}979 . \\
21 .\end{array}$ & $\begin{array}{r}1026 \\
22 .\end{array}$ \\
\hline
\end{tabular}

FAST FLUENCE

(10*25 N/H*21 .00

.15

.16 
C11-01 COMPACI 29

\begin{tabular}{|c|c|c|c|c|c|c|c|}
\hline TIME & INTERVAL & COOLANT & MIN. SLEEVE & $\begin{array}{l}\text { MPERATL IS (C) } \\
\text { MAX. SLEEVE }\end{array}$ & MIN. FUEL & MAX. FUEL & $\begin{array}{l}\text { FAST FLUENCE } \\
(10 * 25 N / M * 2)\end{array}$ \\
\hline $\begin{array}{r}.0 \\
27.0 \\
62.7\end{array}$ & $\begin{array}{l}-\quad 27.0 \\
=\quad 62.7 \\
-\quad 68.6\end{array}$ & $\begin{array}{l}776 . \\
751 . \\
688 .\end{array}$ & $\begin{array}{l}884 . \\
853 . \\
776 .\end{array}$ & $\begin{array}{l}906 . \\
876 . \\
795 .\end{array}$ & $\begin{array}{l}966 . \\
939 . \\
853 .\end{array}$ & $\begin{array}{l}1007 . \\
980 . \\
886 .\end{array}$ & $\begin{array}{l}.00 \\
.05 \\
.12\end{array}$ \\
\hline $\begin{array}{r}68.6 \\
202.1\end{array}$ & $\begin{array}{l}-202.1 \\
-252.4\end{array}$ & $\begin{array}{l}763 . \\
748 .\end{array}$ & $\begin{array}{l}868 . \\
853\end{array}$ & $\begin{array}{l}893 . \\
882 .\end{array}$ & $\begin{array}{l}967 . \\
969 .\end{array}$ & $\begin{array}{l}1009 \\
1012\end{array}$ & .13 \\
\hline TIME & $\begin{array}{l}\text { AVERAGED } \\
\text { RMS }\end{array}$ & $\begin{array}{l}758 . \\
14 .\end{array}$ & $\begin{array}{l}862 . \\
16 .\end{array}$ & $\begin{array}{l}888 . \\
17 .\end{array}$ & $\begin{array}{l}961 . \\
19 .\end{array}$ & $\begin{array}{r}1002 . \\
20\end{array}$ & \\
\hline
\end{tabular}

C11-07 COMPACT 30

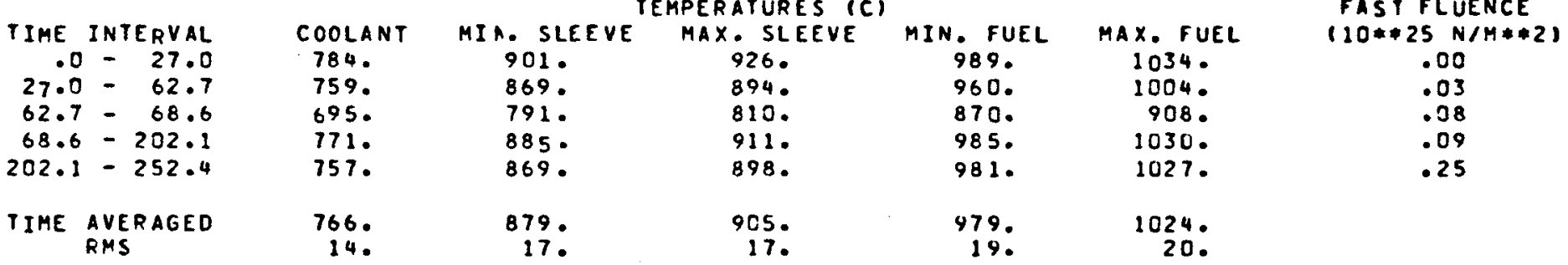


C11-07 FUEL PERFORMANCE

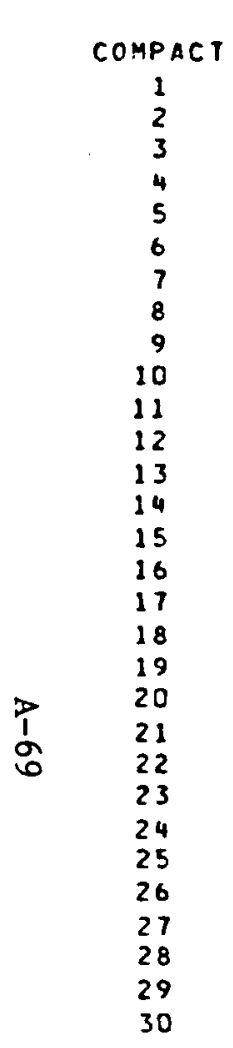

$\begin{array}{ccc}\text { KERNEL MIGRATION (MICRONS) } & \\ \text { AYERAGE } & \text { AMOEBA EO. TEMP. } & \text { PEAK } \\ .00 & 599 . & .00 \\ .00 & 650 . & .00 \\ .00 & 728 . & .00 \\ .00 & 809 . & .00 \\ .00 & 870 . & .00 \\ .01 & 925 . & .01 \\ .02 & 975 . & .03 \\ .05 & 1016 . & .06 \\ .08 & 1047 . & .11 \\ .12 & 1074 . & .16 \\ .17 & 1096 . & .23 \\ .22 & 1113 . & .29 \\ .27 & 1130 . & .36 \\ .32 & 1142 . & .41 \\ .35 & 1149 . & .45 \\ .37 & 1145 . & .48 \\ .36 & 1146 . & .46 \\ .34 & 1146 . & .44 \\ .33 & 1145 . & .42 \\ .29 & 1139 . & .37 \\ .24 & 1132 . & .26 \\ .20 & 1123 . & .20 \\ .15 & 1106 . & .14 \\ .11 & 11086 . & .10 \\ .08 & 1070 . & .07 \\ .05 & 1049 . & .04 \\ .03 & 1027 . & .03 \\ .02 & 1006 . & .02 \\ .01 & 983 . & .03 \\ .02 & 1004 . & \end{array}$

OEFECTIVE COATINGS
.03
.04
.06
.07
.08
.08
.09
.09
.10
.10
.10
.10
.10
.10
.10
.10
.09
.09
.09
.09
.08
.08
.07
.07
.06
.06
.05
.05
.04
.03

FUEL FAILURE ( $z$ ) PRESSURE VESSEL KERNEL MIGRATION

$\begin{array}{lll}.07 & .00 & .00 \\ .12 & .00 & .10 \\ .15 & .00 & .12\end{array}$

OTAL
.10

.00

.00

.00

.01

.19

.21

.23

.00

.00

.00

00

.00

.26

.26
.27

.00

.00
.00

.28
.28

.00

.00

.27

.27
.27

. 00

- 0 ก

.00

.00

.25
.25

.24

AMOEBA EQUIVALENT TEMPERATURES $(C)$ CORRESPONO TO AVERAGE KERNEL MIGRATIONS KERNEL MIGRATION OISTANCES CALCULATED USING SOX CONFIOENCE LEVEL KMC FUEL FAILURE CORRESPONOS TO $50 \%$ CONF IOENCE LEYEL 
E11-07 COMPACT 1

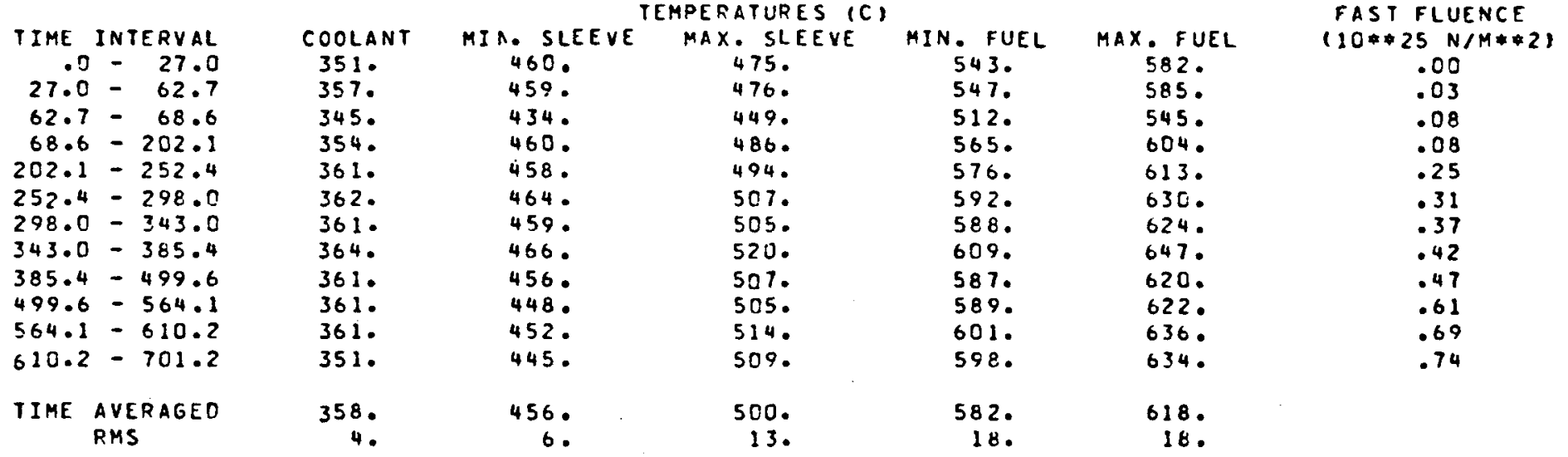

TIME INTERVAL
$.0-27.0$
$27.0=62.7$
$62.7-68.6$
$68.6=202.1$
$202.1-252.4$
$252.4-298.0$
$298.0-343.0$
$343.0-385.4$
$385.4=499.6$
$499.6=564.1$
$564.1-610.2$
$610.2-701.2$
TIME AVERAGED
RMS

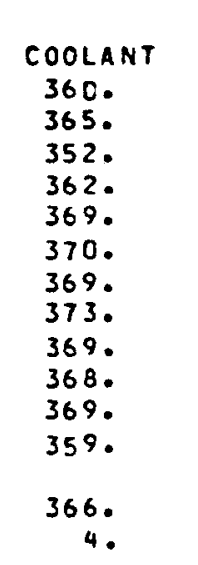

E11-C7 COMPACT

$\begin{array}{ccccc}\text { MEMPERATURES (C) } & & \text { FAST FLUENCE } \\ \text { MIA. SLEEVE MAX. SLEEVE } & \text { MIN. FUEL } & \text { MAX. FUEL } & \text { (10\%25 N/M\#2) } \\ 481 . & 496 . & 573 . & 616 . & .00 \\ 479 . & 499 . & 579 . & 622 . & .05 \\ 451 . & 469 . & 541 . & 578 . & .13 \\ 480 . & 518 . & 610 . & 654 . & .14 \\ 480 . & 535 . & 632 . & 675 . & .41 \\ 487 . & 550 . & 651 . & 695 . & .51 \\ 480 . & 545 . & 644 . & 686 . & .69 \\ 489 . & 561 . & 667 . & 711 . & .78 \\ 477 . & 541 . & 636 . & 674 . & 1.01 \\ 471 . & 541 . & 642 . & 682 . & 1.13 \\ 477 . & 551 . & 655 . & 697 . & 1.22 \\ 471 . & 545 . & 650 . & 692 . & \\ 478 . & 535 . & 632 . & 673 . & \\ 6 . & 18 . & 25 . & 25 . & \end{array}$


E11-07 COMPACT 3

\begin{tabular}{|c|c|c|c|c|c|c|c|}
\hline \multirow{2}{*}{\multicolumn{2}{|c|}{ TIME INIERVAL }} & \multicolumn{5}{|c|}{ TEMPERATURES (C) } & \multirow{2}{*}{$\begin{array}{c}\text { FASI FLUENCE } \\
(10 * 25 N / M * 2) \\
.00\end{array}$} \\
\hline & & $\begin{array}{l}\text { COOLANT } \\
370 .\end{array}$ & $\begin{array}{c}\text { MIR. SLEEVE } \\
522 \text {. }\end{array}$ & $\begin{array}{c}\text { MAX. SLEEVE } \\
544 .\end{array}$ & MIN. FUEL & $\begin{array}{c}\text { MAX FUEL } \\
687\end{array}$ & \\
\hline $\begin{array}{l}27.0 \\
62.7 \\
68.6\end{array}$ & $\begin{array}{l}-62.7 \\
=\quad 68.6 \\
-202.1\end{array}$ & $\begin{array}{l}375 \\
361 \\
373\end{array}$ & $\begin{array}{l}518 \\
484 \\
520\end{array}$ & $\begin{array}{l}543 . \\
508 \\
569\end{array}$ & $\begin{array}{l}640 \\
597 \\
683\end{array}$ & $\begin{array}{l}694 . \\
643 . \\
738\end{array}$ & $\begin{array}{l}.07 \\
.17 \\
.18\end{array}$ \\
\hline $\begin{array}{l}202.1 \\
252.4 \\
298.0\end{array}$ & $\begin{array}{l}=252.4 \\
=298.0 \\
-343.0\end{array}$ & $\begin{array}{l}378 . \\
381 . \\
379 .\end{array}$ & $\begin{array}{l}516 . \\
526 \\
517\end{array}$ & $\begin{array}{l}585 \\
603 \\
595\end{array}$ & $\begin{array}{l}706 \\
729 \\
720\end{array}$ & $\begin{array}{l}759 . \\
784 . \\
772 .\end{array}$ & $\begin{array}{l}.53 \\
.67 \\
.79\end{array}$ \\
\hline $\begin{array}{l}343.0 \\
385.4 \\
499.6\end{array}$ & $\begin{array}{l}-385.4 \\
-499.6 \\
-564.1\end{array}$ & $\begin{array}{l}383 . \\
379 \\
377\end{array}$ & $\begin{array}{l}527 . \\
513 . \\
506 .\end{array}$ & $\begin{array}{l}614 . \\
588 . \\
589 .\end{array}$ & $\begin{array}{l}746 . \\
709 . \\
717\end{array}$ & $\begin{array}{l}601 . \\
756 . \\
767\end{array}$ & $\begin{array}{l}.91 \\
1.02 \\
1.32\end{array}$ \\
\hline $\begin{array}{l}564.1 \\
610.2\end{array}$ & $\begin{array}{l}-610.2 \\
=701.2\end{array}$ & $\begin{array}{l}378 . \\
369 .\end{array}$ & $\begin{array}{l}514 . \\
509 .\end{array}$ & $\begin{array}{l}601 . \\
596 .\end{array}$ & $\begin{array}{l}733 . \\
729 .\end{array}$ & $\begin{array}{l}785 . \\
781 .\end{array}$ & $\begin{array}{l}1.48 \\
1.60\end{array}$ \\
\hline IIME & $\begin{array}{l}\text { AVERAGED } \\
\text { RMS }\end{array}$ & $\begin{array}{r}376 . \\
4 .\end{array}$ & $\begin{array}{r}516 . \\
7 .\end{array}$ & $\begin{array}{l}585 . \\
19 .\end{array}$ & $\begin{array}{r}705 . \\
30\end{array}$ & $\begin{array}{r}758 \\
30 \text { : }\end{array}$ & \\
\hline
\end{tabular}

TIME INTERVAL
$.0=27.0$
$27.0=62.7$
$62.7=68.6$
$68.6=202.1$
$202.1=252.4$
$252.4=298.0$
$298.0=343.0$
$343.0=385.4$
$385.4=499.6$
$499.6=564.1$
$564.1=610.2$
$610.2=701.2$
TIME AVERAGED
RMS

$\begin{array}{cc}\text { COOLANT } & \\ 383^{\circ} & \text { TELEVE } \\ 387 . & 5655^{\circ} \\ 371 . & 558 . \\ 385 . & 519 . \\ 390 . & 562 . \\ 393 . & 551 . \\ 391 . & 563 . \\ 395 . & 553 . \\ 390 . & 564 . \\ 388 . & 547 . \\ 390 . & 536 . \\ 381 . & 545 . \\ 388 . & 541 . \\ 4 . & 552 . \\ & 10 .\end{array}$

E11-07 COMPACT

TEMPERATURES (C)

$\begin{array}{ccc}\text { MAX. SLEEVE } & \text { MIN FUEL } & \text { MAX. FUEL } \\ 592 . & 694 . & 760 . \\ 590^{\circ} & 702 . & 768 . \\ 549 . & 654 . & 709 . \\ 621 . & 757 . & 823 . \\ 629 . & 774 . & 837 . \\ 651 . & 802 . & 867 . \\ 640 . & 791 . & 852 . \\ 662 . & 822 . & 887 . \\ 631 . & 780 . & 635 . \\ 627 . & 783 . & 841 . \\ 641 . & 803 . & 863 . \\ 637 . & 803 . & 863 . \\ & & \\ 630 . & 776 . & 838 . \\ 18 . & 33 . & 32 .\end{array}$

FAST FLUENCE

$(10 * 25 N / H * 2)$ .00

.08

.20

.22

.65

.81

1.24

1.60

1.80 


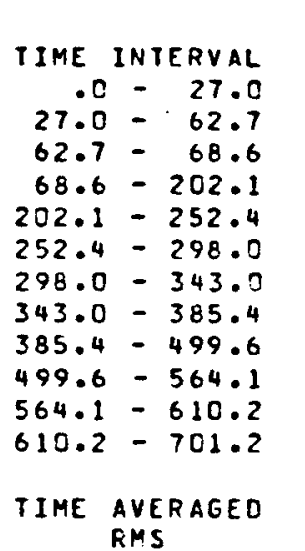
RMS
TIME INTERVAL

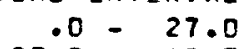

$27.0=27.0$

$62.7-68.6$

$68.6-202.1$

$202.1-252.4$

$252.4-298.0$

$298.0-343.0$

$343.0-385.4$

$385.4-499.6$

$499.6-564.1$

$664.1=610.2$

$610.2=701.2$

TIME AVERAGED RMS
E11-07 COMPACT 5

MIN. SLEE TEMPERATURES (C)

COOLANT

397.

HIN. SLEE VE

MAX. SLEEVE

631.

591.

581.

$548^{\circ}$

596.

399 .

402 .

407.

404.

403.

399.

402 .

393 .

401.

596 .

584.

597 .

578.

560 .

570 .

567 .

661.

666.

678 .

791 .

667.

653.

670.

667.

MIN. FUEL

740 .

750 .

697.

812.

830.

852 .

887.

842 .

838 .

862 .

866.

MAX. FUEL

813.

758 .

886.

901.

935 .

920 .

959.

903.

900 .

927.

932 .

$834 . \quad 902$.

582
13.

665.

37.

E11-07 COMPACT

COOLANT MIA. SLEEVE TEMPERATURES (C)

413.632 .

621 .

$4140^{\circ} \quad 6210^{\circ}$

414.628 .

416.6211.

424.627.

417 . 606 .

412.582 .

415.5594.

591.

667 .

661 .

611.

611.
697.

699.

726.

711.

736.

700 .

678.

696.

415.
5.

610

697.

19.
HIN. FUEL

782.

793.

736.

863.

919.

907.

946.

901.

891.

920.

930 .

889.

43.

MAX. FUEL

862.
873.

873.

943.

958 .

996.

981 .

1025 .

968 .

958 .

$99 \mathrm{C}$.

1000.

963.
FAST FLUENCE

(10*25 N/M**2)

$$
.00
$$

.10

.23

.25

.73
.91

1.08

1.24

1.40

1.80

2.02

2. 18

FAST FLUENCE

(1O**25 N/M*2)

.00
.10
.25
.27
.80
.99
1.18
1.36
1.53
1.96
2.20
2.38

.00

.25

.27
.80

.99

1.18

1.36

1.96

2.38 


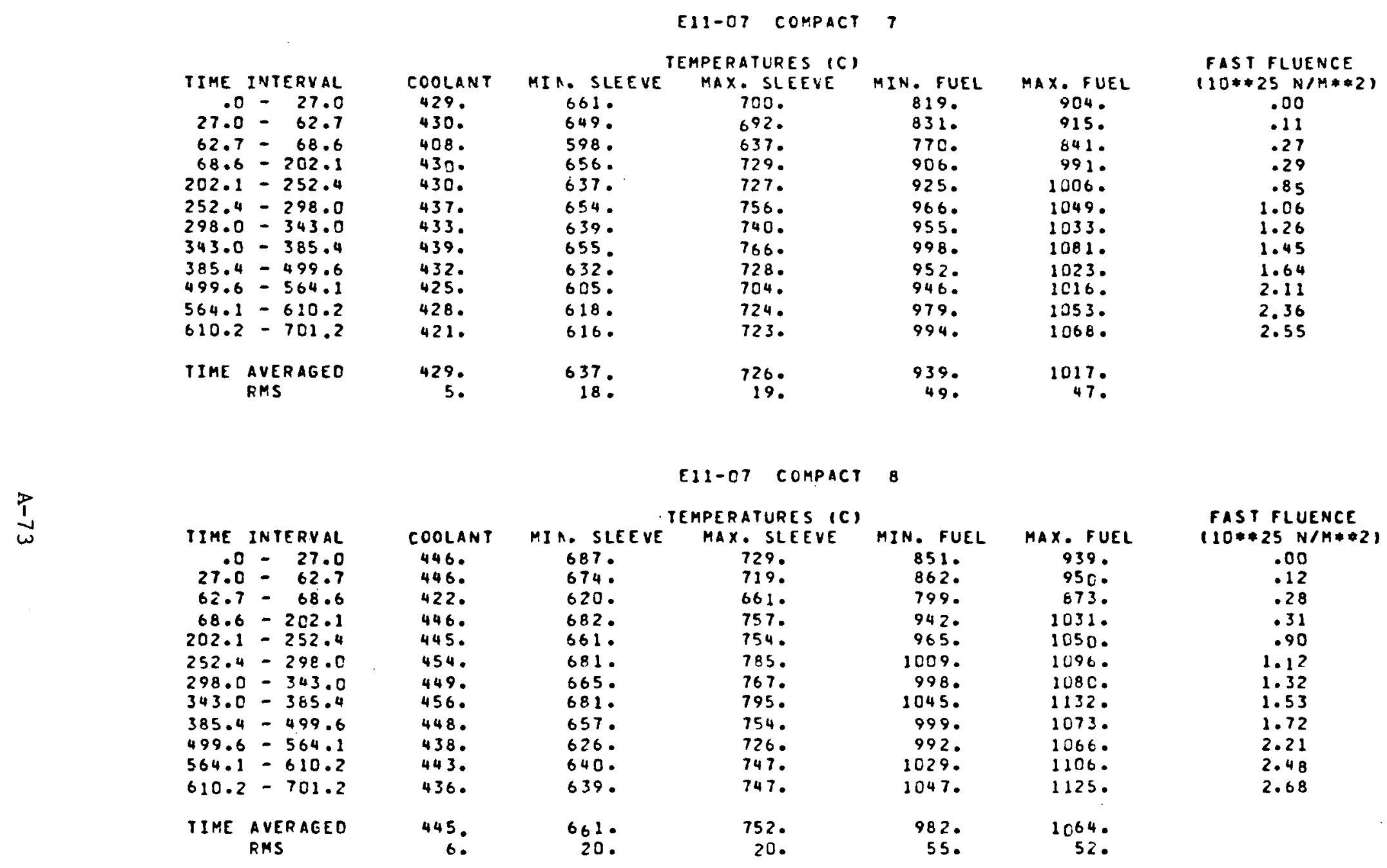


E11-07 COMPACT 9

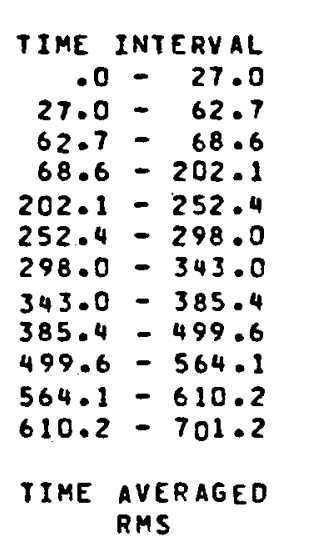

\begin{tabular}{|c|}
\hline $\begin{array}{l}\text { COOLANT } \\
464 .\end{array}$ \\
\hline $\begin{array}{l}462 . \\
436 . \\
463 .\end{array}$ \\
\hline $\begin{array}{l}461 . \\
471 . \\
465 .\end{array}$ \\
\hline $\begin{array}{l}473 . \\
464 .\end{array}$ \\
\hline $457^{\circ}$ \\
\hline 461. \\
\hline
\end{tabular}

MIN. SLEEV
709.
694.
637.
703.
685
706.
689.
706.
680.
646.
661.
661.
683.
20

TEMPERATURES (C)

MAX. SLEEVE M
752.
741.
679.
778.
778.
811.
792.
821.
778.
745.
768.
769.
775.
22.

IN. FUEL

875.

FAST FLUENCE

FAST FLUENCE

965.

820.076.

968.1295.

999.1087.

$1046 . \quad 1236$.

1035.1121.

1084.1175.

1038.1116.

1030.1106.

1069 .

1170 .

.00

.12
.29

.31

.92

1.15

1.37

1.58

1.77

2.28

2.56

$$
\text { RMS }
$$

20 .

22.

1017.

61.

1102.

\section{E11-07 COMPACT 10}

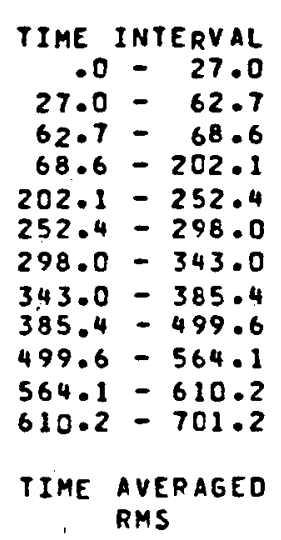

COOLANT
482.
479.
451.
480
477.
489.
482.
490.
480.
467.
473.
467.
478.
7.

MIA. SLEEVE
729.
$712 \circ$
654.
$722 \circ$
705.
728.
709.
728.
701.
667.
683.
683.
704
20

TEMPERATURES (C)

MAX. SLEEVE
773.
760.
696.
798.
799.
833.
812.
842.
798.
768.
792.
793.
796.
22.

MIN FUEL

907 .

838.

990.

1025.

1075.

1114.

1068 .

1067.

1109.

MAX. FUEL

FASI FLUENCE

(10**25 N/H**2)

1131.

988.

997.

915.

1082.

1115.

1166.

1150 .

1207 .

1147 .

1146.

1191.

.00

.12

.29

.95

1.18

1.40

1.62
1.82

2.35

2.63

2.63

$\begin{array}{rr}1047 . & 1133 . \\ 67 . & 65 .\end{array}$ 
E11-07 COMPACT 11

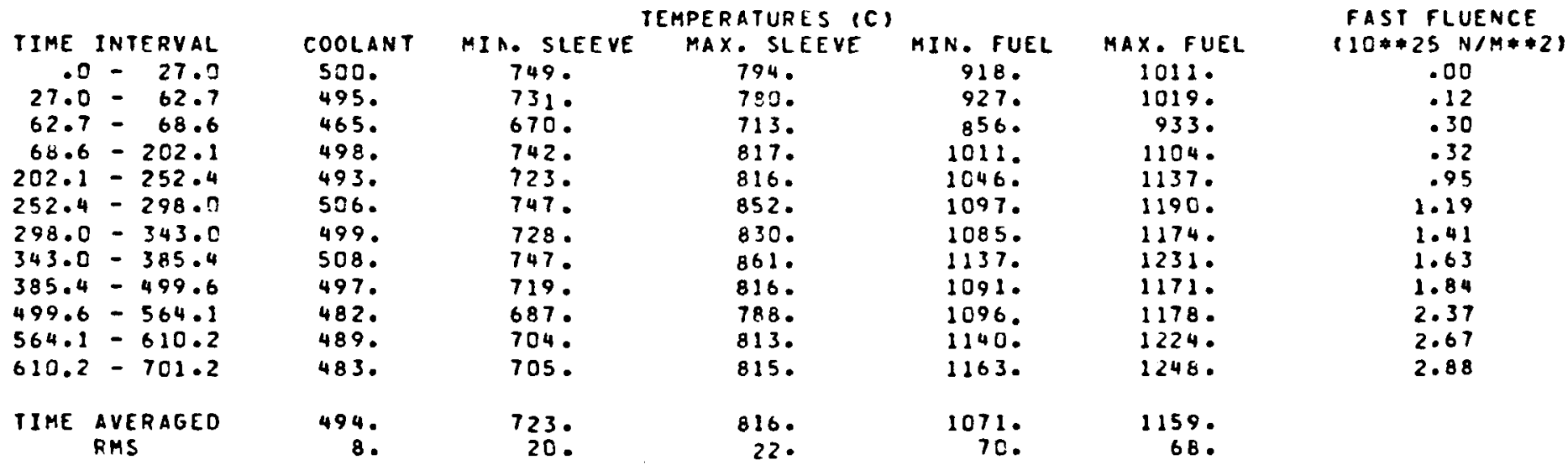

TIME AYERAGED RMS

E11-07 COMPACT 12

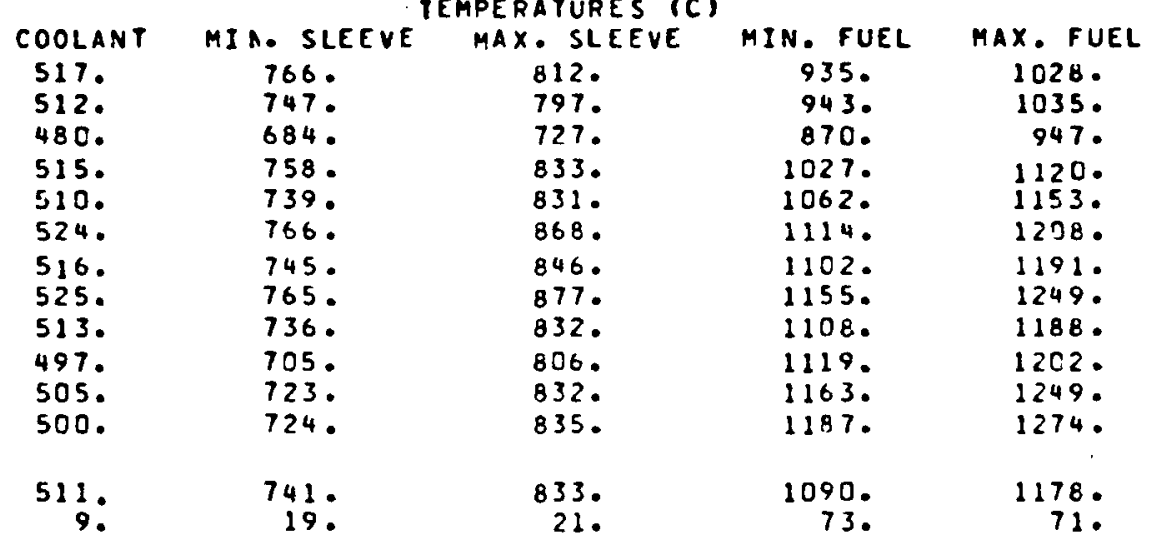

FASI FLUENCE (10*25 N/M**2)

$$
\begin{aligned}
& .00 \\
& .13 \\
& .30 \\
& .33 \\
& .96 \\
& 1.20 \\
& 1.42 \\
& 1.64 \\
& 1.85 \\
& 2.39 \\
& 2.69 \\
& 2.91
\end{aligned}
$$


11-07 COMPACT 13

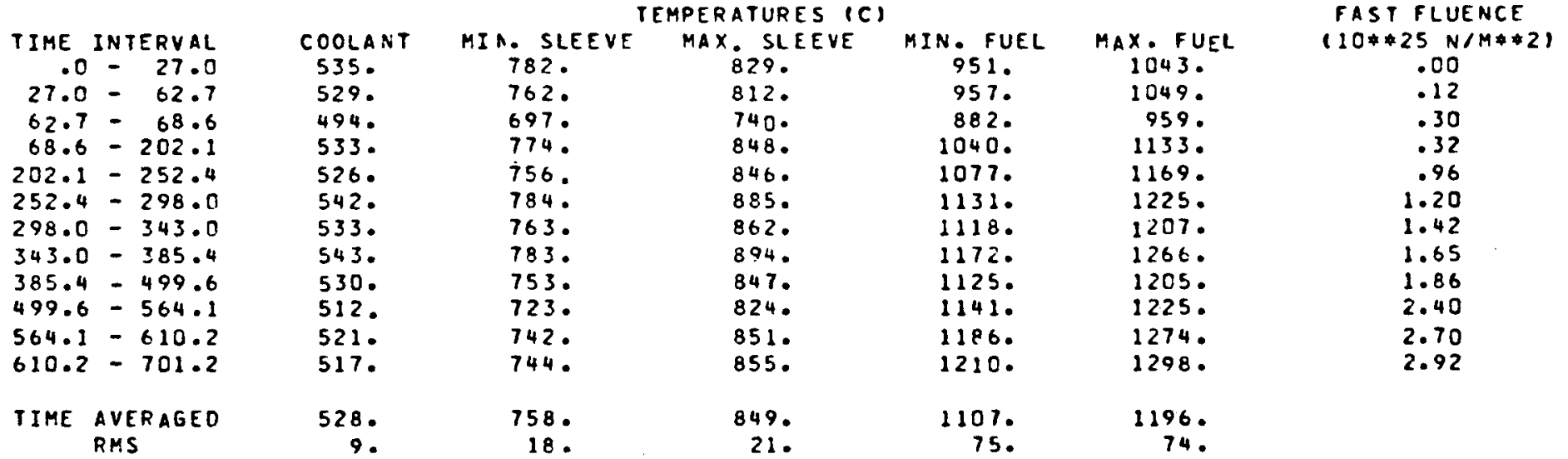

E11-07 COMPACT 14

TIME INTERVAL
$.0-27.0$
$27.0=62.7$
$62.7=68.6$
$68.6=202.1$
$202.1=252.4$
$252.4-298.0$
$298.0-343.0$
$343.0-385.4$
$385.4=499.6$
$499.6=564.1$
$564.1=610.2$
$610.2=701.2$
TIME AVERAGED
RMS

$\begin{array}{cc}\text { COOLANT } & \text { MIR. SLEEVE } \\ 553 . & 797 . \\ 545 . & 776 . \\ 509 . & 710 . \\ 550 . & 789 . \\ 542 . & 771 . \\ 560 . & 800 . \\ 550 . & 779 . \\ 561 . & 800 . \\ 547 . & 769 . \\ 528 . & 739 . \\ 537 . & 759 . \\ 533 . & 762 . \\ 545 . & 774 . \\ 10 . & 18 .\end{array}$

TEMPERATURES (C)

$\begin{array}{ccc}\text { MAX. SLEEVE } & \text { MIN. FUEL } & \text { MAX. FUEL } \\ 845^{\circ} & 965^{\circ} & 1057 . \\ 826 . & 969 . & 1060 . \\ 752 . & 890^{\circ} & 968 . \\ 861 . & 1050^{\circ} & 1143 . \\ 860^{\circ} & 1089 . & 1181 . \\ 890^{\circ} & 1140^{\circ} & 1237 . \\ 876 . & 1131 . & 1219 . \\ 908 . & 1184 . & 1278 . \\ 861 . & 1136 . & 1217 . \\ 839 . & 1155 . & 1239 . \\ 867 . & 1200 . & 1288 . \\ 871 . & 1225 . & 1313 . \\ 864 . & 1120 . & 1208 . \\ 22 . & 76 . & 75 .\end{array}$

FAST FLUENCE

$(10 * 25 N / M * 2)$

.00
.12
.29
.32
.95
1.19
1.41
1.64
1.84
2.39
2.69
2.92


E11-07 COMPACT 15

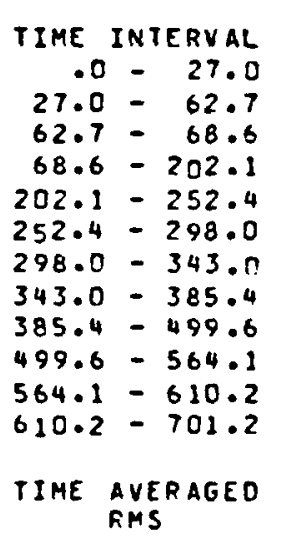

COOLANT
570.
562.
523.
567.
559.
578.
567.
579.
564.
543.
554.
550.
561.
10.

$\begin{array}{cc} & \text { TEMPERATURES }(C) \\ \text { MIA. SLEEVE } & \text { MAX. SLEEVE } \\ 810^{\circ} & 857 . \\ 788 . & 838 . \\ 720 . & 762 . \\ 811 . & 871 . \\ 786 . & 872 . \\ 816 . & 913 . \\ 793 . & 888 . \\ 815 . & 921 . \\ 784 . & 874 . \\ 755 . & 854 . \\ 775 . & 882 . \\ 779 . & 887 . \\ 789 . & 877 . \\ 18 . & 22 .\end{array}$

MIN. FUEL 1066. 978.1068 . $899 . \quad 974$.

1057.1148.

1098.1189.

1154.1247.

$1140.01228^{\circ}$

$11930^{\circ} \quad 1287$.

1168.1253.

1214.1302.

1214.01302

1130
78

1218

\section{E11-07 COMPACT 16}
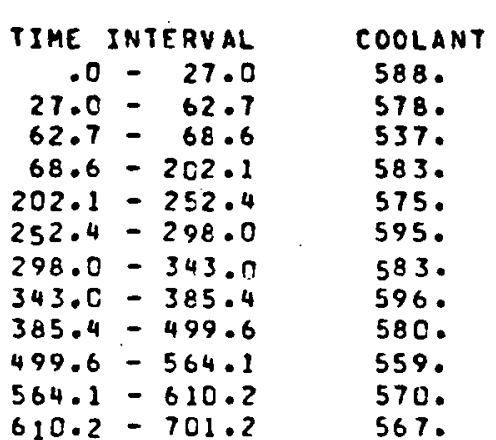
588.

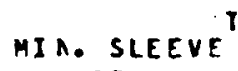

TEMPERATURES (C) MIN. FUEL 823. 870 . 986. 578. 700.

849. 987 .

730

882 .

575.2798.

$595.2830^{\circ}$

$583 . \quad 807$.

$596 . \quad 829$.

$580 . \quad 797$.

559. 770.

570.791.

862 .

$1064^{\circ}$

899.11160.

932.11198 .

885.11150.

868.01177.

897. 1223.

$\begin{array}{ll}897^{\circ} & 1223^{\circ} \\ 902 . & 1247^{\circ}\end{array}$

MAX. FUEL

1075.

FAST FLUENCE

(10*25 N/M*2)

567.

795 .

888.

1137.

1075.

980 .

1153.

1194.

1252 .

.12

.12
.28

.31

.92
1.16

1.38

1.60

1291 .

1229 .

1261.

1311.

1336 .

2.65

2.65
2.87

TIME averagen RMS

11.

17.

22 .

1224. 
E11-0T COMPACT 17

MIA. SLEEVE MAXPERATURES (C)

TIME INTERVAL

$.0-27.0$

$27.0-62.7$

$62.7-68.6$

$68.6-202.1$

$202.1-252.4$

$252.4-298.0$

$298.0-343.0$

$385.4=499.6$

$499.6-564.1$

$564.1-610.2$

$610.2-701.2$

TIME AVERAGED RMS
$343.0-385.4$

COOLANT
604.
593.
550.
599.
590.
612.
600.
613.
596.
575.
586.
584.
594.
11.

$8320^{\circ}$
$8090^{\circ}$
738.
822.
809.
$8420^{\circ}$
$8180^{\circ}$
841.
808.
784.
806.
811.
814.
16.

$$
\begin{aligned}
& 857 . \\
& 778 .
\end{aligned}
$$

888.

$$
890 .
$$

932.

$$
940 .
$$

$$
893 .
$$

$$
881 \text {. }
$$$$
910 .
$$$$
915 .
$$

MIN FUEL

991.

990.

908.

1064 .

1105 .

1161.

1198.

1150.

1182 .

1228 .

1252.

898.

1139.

79.

MAX. FUEL

1077.

1075.

980.

1150

1193.

1231 .

1289.

1227 .

1266.

1316 .

1340 .

1224.

\section{E11-07 COMPACT 18}

IIME INTERVAL$$
.0-27.0
$$

$27.0=62.7$

$62.7=68.6$

$68.6-202.1$

$202.1-252.4$

$252.4-298.0$

$298.0-343.0$

$343.0-385.4$

$385.4-499.6$

$499.6-564.1$

$564.1=610.2$

$610.2-701.2$

IIME AVERAGEO RMS

COOLANT
620.
608
563
615
606.
629
616.
630
612.
590
603
601
610
11.

620 .

MIA. SLEEVE

TEMPERATURES (C)

841.

817 .

745.

831 .

820

854 .

829

853.

819.

797.

820

825 .

825 .

16.

\begin{abstract}
886.
864.

784.

784.
894.

897.

940.

914.

948 .

901 .

892.

922.

928.

906.
23.
\end{abstract}

MIN. FUEL

996.

909.

1063.

1106.

1145

1197.

1149.

1185 .

1231.

1255 .

1080 .

1076.

979.

1148.

1192 .

1250 .

1229 .

1286.

1225 .

1269.

1318.

1343 .

1140.

1224.

81.
FAST FLUENCE

(10*25 N/M**2)

.00

.00

.28

.30
.90

1.14

1.35

1.57

1.77

2.60

2.82

FASI FLUENCE

$110 * 25 \quad N / M * 2)$

$$
.00
$$

.00
.11
.27

.27

.30

.88
1.11

1. 32

1.53

1.72

2.25

2.55

2.77 
E11-07 COMPACT 19

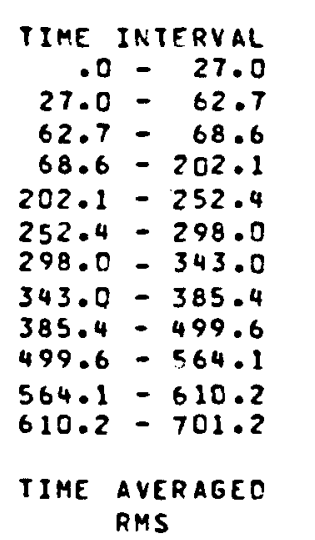

COOLANT
636.
623.
576.
630.
621.
646.
631.
646.
627.
605.
619.
618.
626
12.

MIA. SLEEVE
851.
826.
753.
840.
828.
863.
838.
862.
828.
810.
833.
838
835
16.

TEMPERATURES (C)

$\begin{array}{ccc}\text { MAX. SLEEYE } & \text { MIN. FUEL } & \text { MAX.FUEL } \\ 894^{\circ} & 1002 . & 1 C 83 . \\ 871 . & 997 . & 1077 . \\ 791 . & 912 . & 980 . \\ 901 . & 1064 . & 1146 . \\ 902 . & 1102 . & 1186 . \\ 940^{\circ} & 1158 . & 1244 . \\ 919 . & 1141 . & 1222 . \\ 953 . & 1192 . & 1278 . \\ 907 . & 1144 . & 1217 . \\ 902 . & 1186 . & 1268 . \\ 932 . & 1231 . & 1317 . \\ 939 . & 1255 . & 1342 . \\ & & \\ 913 . & 1138 . & 1220 . \\ 24 . & 78 . & 80 .\end{array}$

FAST FLUENCE

110*25 N/M*:21

.00

.11

.26
.29

.85

1.08

1.28

1.49

1.68

2.17

2.48

\section{E11-07 COMPACT 20}

$\frac{p}{0}$

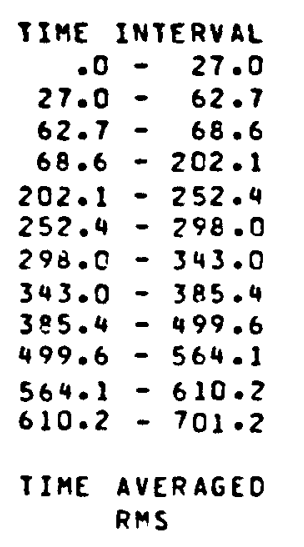

COOLANT
6510
6370
589.
6450
6350
662.
646.
662.
6420
620
634
6340
641.
12.

MIN. SLEEVE
858.
833.
759.
847.
835.
871.
845.
870.
835.
822.
845.
851.
844.
15.

IEMPERATURES (C) MAX. SLEEVE

MIN. FUEL

900.
876.
795.
905.
905.
949.
922.
956.
911.
912.
943.
949.
919.
25.

1004.

MAX. FUEL

FAST FLUENCE

$(10 * 25 N / M * 2)$

1061. 1140.

2096. 1177.

$1152 . \quad 1234$.

1133.1212

$1184^{\circ} \quad 1267$.

1135.1206.

1185.1267 .

$12310^{\circ} \quad 1316$.

$\begin{array}{rr}1135 . & 1214 . \\ 78 . & 80 .\end{array}$

.00
.11
.25
.28
.82
1.04
1.23
1.43
1.62
2.12
2.41
2.62


E11-07 COMPACT 21

\begin{tabular}{|c|c|c|c|c|c|c|}
\hline \multirow{2}{*}{\multicolumn{2}{|c|}{ TIME INTERVAL }} & \multicolumn{5}{|c|}{ APERATURES I } \\
\hline & & COOLANT & MIA. SLEEVE & MAX. SLEEVE & MIN. FUEL & MAX. FUEL \\
\hline $\begin{array}{r}.0 \\
27.0 \\
62.7\end{array}$ & $\begin{array}{l}-\quad 27.0 \\
=\quad 62.7 \\
-\quad 68.6\end{array}$ & $\begin{array}{l}666 . \\
651 . \\
600 .\end{array}$ & $\begin{array}{l}862 . \\
837 . \\
762 .\end{array}$ & $\begin{array}{l}903 . \\
879 . \\
797 .\end{array}$ & $\begin{array}{r}1003 \\
993 \\
908\end{array}$ & $\begin{array}{r}1078 . \\
1068 . \\
970 .\end{array}$ \\
\hline $\begin{array}{r}68.6 \\
202.1 \\
252.4\end{array}$ & $\begin{array}{l}-202.1 \\
-252.4 \\
-298.0\end{array}$ & $\begin{array}{l}659 . \\
649 . \\
677 .\end{array}$ & $\begin{array}{l}852 . \\
839 . \\
876 .\end{array}$ & $\begin{array}{l}906 . \\
905 . \\
949 .\end{array}$ & $\begin{array}{l}1054 . \\
1085 . \\
1140 .\end{array}$ & $\begin{array}{l}1129^{\circ} \\
11620^{\circ} \\
1219^{\circ}\end{array}$ \\
\hline $\begin{array}{l}298.0 \\
343.0\end{array}$ & $\begin{array}{l}-343.0 \\
=385.4\end{array}$ & 661. & 850. & 923. & $\begin{array}{l}11210 \\
1170^{\circ}\end{array}$ & $\begin{array}{l}1196 . \\
1250^{\circ}\end{array}$ \\
\hline $\begin{array}{l}385.4 \\
499.6 \\
564.1 \\
610.2\end{array}$ & $\begin{array}{l}-499.6 \\
-564.1 \\
-610.2 \\
-701.2\end{array}$ & $\begin{array}{l}656 . \\
635 . \\
650 . \\
650 .\end{array}$ & $\begin{array}{l}840^{\circ} \\
8320^{\circ} \\
856 . \\
862 .\end{array}$ & $\begin{array}{l}912 . \\
920 . \\
950 . \\
957 .\end{array}$ & $\begin{array}{l}11220^{\circ} \\
11790^{\circ} \\
1225^{\circ} \\
1248^{\circ}\end{array}$ & $\begin{array}{l}1190^{\circ} \\
1259^{\circ} \\
1300^{\circ} \\
1332^{\circ}\end{array}$ \\
\hline II ME & $\begin{array}{l}\text { AVERAGED } \\
\text { RMS }\end{array}$ & $\begin{array}{r}656 . \\
12 .\end{array}$ & $\begin{array}{r}851 . \\
15 .\end{array}$ & $\begin{array}{r}922 . \\
26 .\end{array}$ & $\begin{array}{r}1126 . \\
77 .\end{array}$ & $\begin{array}{r}1202 . \\
81 .\end{array}$ \\
\hline
\end{tabular}

FAST FLUENCE

(10*25 N/M* 2

.00
.10
.24
.26
.79
.99
1.18
1.37
1.55
2.04
2.32
2.52

TIME INTERVAL

$.0-27.0$

$27.0-62.7$

$27.0=62.7$
$62.7=68.6$

$68.6-202.1$

$202.1-252.4$

$252.4-298.0$

$298.0-343.0$

$343.0-385.4$

$385.4-499.6$

$499.6=564.1$

$564.1-610.2$

$610.2-701.2$

TIME AVERAGEO

RMS

15 .

26.

E11-07 COMPACT 22

COOLANT
680.
663.
612.
673.
662.
691.
674.
691.
670
649
6650
665.
670
12.

HIA. SLEEVE

EMPERATURES (C)

867 .

867.

841.

756.

$856^{\circ}$.

883 :

857 .

881.

847 .

839 .

$833^{\circ}$

870 .

906 .

906.

881.

799.

907.

907.

925.

914.

922.

954.

961 .

857.

925.

25.
MIN. FUEL

1002 .

991.

991.

1047.

1078 .

1132 .

1112 .

1160.

1112.

1167.

1212.

1235 .

1117.

74.

MAX. FUEL

1074 .

1062.

964.

1120 .

1152.

1208 .

$1184^{\circ}$

1278.

1244.

1293 .

1316 .

1191.
FAST FLUENCE

$(10 * 25 N / M * 2)$

.00
.10
.23
.25
.75
.94
1.12
1.30
1.47
1.94
2.21
2.41


E11-07 COMPACT 23

TIME INTERVAL
$.0=27.0$
$27.0=62.7$
$62.7=68.6$
$68.6=202.1$
$202.1=252.4$
$252.4=298.0$
$298.0=343.0$
$343.0=385.4$
$385.4=499.6$
$499.6=564.1$
$564.1=610.2$
$610.2=701.2$
TIME AVERAGEO
RMS

\begin{tabular}{|c|c|}
\hline $\begin{array}{l}\text { COOLANT } \\
\text { O93. }\end{array}$ & $\begin{array}{c}\text { MIA. SLEEVE } \\
869 \text {. }\end{array}$ \\
\hline 676. & 842 . \\
\hline $\begin{array}{l}622 . \\
685\end{array}$ & $\begin{array}{l}767 . \\
858 .\end{array}$ \\
\hline 675. & 847. \\
\hline $\begin{array}{l}705 . \\
687 .\end{array}$ & $\begin{array}{l}885^{\circ} \\
859^{\circ}\end{array}$ \\
\hline 705. & 884. \\
\hline $\begin{array}{l}683 . \\
663 .\end{array}$ & $\begin{array}{l}849 . \\
845^{\circ}\end{array}$ \\
\hline 679. & $870^{\circ}$ \\
\hline 680. & 877. \\
\hline 683 & $\begin{array}{r}860 . \\
16 .\end{array}$ \\
\hline
\end{tabular}

TEMPERATURES (C)

\begin{tabular}{|c|c|c|}
\hline $\begin{array}{l}\text { MAX SLEEVE } \\
905 . \\
880 .\end{array}$ & $\begin{array}{c}\text { MIN FUEL } \\
996 . \\
983 .\end{array}$ & $\begin{array}{c}\text { MAX FUEL } \\
1064 . \\
1050 .\end{array}$ \\
\hline $\begin{array}{l}798 . \\
905 .\end{array}$ & $\begin{array}{r}897 . \\
1035 .\end{array}$ & $\begin{array}{r}953 . \\
1103 .\end{array}$ \\
\hline 903. & 1062. & 1132. \\
\hline $\begin{array}{l}949 . \\
922 .\end{array}$ & $\begin{array}{l}1116 . \\
1095 .\end{array}$ & $\begin{array}{l}1188^{\circ} \\
11630^{\circ}\end{array}$ \\
\hline $\begin{array}{l}954 . \\
912 . \\
925 .\end{array}$ & $\begin{array}{l}1142 . \\
1094 . \\
1155 .\end{array}$ & $\begin{array}{l}12144^{\circ} \\
1156^{\circ} \\
1229^{\circ}\end{array}$ \\
\hline $\begin{array}{l}957 . \\
964 .\end{array}$ & $\begin{array}{l}1199 . \\
1221 .\end{array}$ & $\begin{array}{l}1276 . \\
1290^{\circ}\end{array}$ \\
\hline $\begin{array}{r}924 . \\
27 .\end{array}$ & $\begin{array}{r}1103 . \\
73\end{array}$ & $\begin{array}{r}1173 . \\
77 .\end{array}$ \\
\hline
\end{tabular}

FAST FLUENCE
$\begin{gathered}(10 * 25 N / M * 2) \\ .00 \\ .09 \\ .22 \\ .24 \\ .71 \\ .89 \\ 1.06 \\ 1.24 \\ 1.39 \\ 1.84 \\ 2.10 \\ 2.29\end{gathered}$

E11-07 COMPACT 24

$\stackrel{?}{\substack{1 \\ \hdashline}}$

TIME INTERVAL
$. C=27.0$
$27.0=62.7$
$62.7=68.6$
$68.6=202.1$
$202.1=252.4$
$252.4=298.0$
$298.0=343.0$
$343.0=385.4$
$385.4=499.6$
$499.6=564.1$
$564.1=610.2$
$610.2=701.2$
TIME AVERAGED
RMS

$\begin{array}{ll}\text { COOLANT MIA. SLEEVE } \\ 705 . & 867 . \\ 687 . & 841 . \\ 632 . & 766 . \\ 697^{\circ} & 856 . \\ 687 . & 849 . \\ 718 . & 888 . \\ 700 . & 862 . \\ 717 . & 887 . \\ 695 . & 852 . \\ 676 . & 849 . \\ 693 . & 875 . \\ 695 . & 881 . \\ & \\ 695 . & 862 . \\ 12 . & 17 .\end{array}$

TEMPERATURES (C)

\begin{tabular}{|c|c|c|}
\hline $\begin{array}{l}\text { MAX. SLEEYE } \\
\text { 9C1. } \\
875 .\end{array}$ & $\begin{array}{c}\text { MIN. FUEL } \\
986 . \\
971 .\end{array}$ & $\begin{array}{c}\text { MAX. FUEL } \\
1049 . \\
1033 .\end{array}$ \\
\hline $\begin{array}{l}794 . \\
899 . \\
902 .\end{array}$ & $\begin{array}{l}885 \\
1019 . \\
1050\end{array}$ & $\begin{array}{r}937 \\
1082 \\
1116\end{array}$ \\
\hline $\begin{array}{l}947 . \\
920 .\end{array}$ & $\begin{array}{l}1103 . \\
1081 .\end{array}$ & $\begin{array}{l}1171 . \\
1146 .\end{array}$ \\
\hline $\begin{array}{l}953 . \\
911 . \\
925 .\end{array}$ & $\begin{array}{l}1126 . \\
1079 . \\
1137 .\end{array}$ & $\begin{array}{l}1195 \\
1138 \\
1208\end{array}$ \\
\hline $\begin{array}{l}956 . \\
964 .\end{array}$ & $\begin{array}{l}1181 . \\
1202 .\end{array}$ & $\begin{array}{l}1254^{\circ} \\
1276^{\circ}\end{array}$ \\
\hline $\begin{array}{r}921 . \\
29 .\end{array}$ & $\begin{array}{r}1087 . \\
71 .\end{array}$ & $\begin{array}{r}1153 . \\
75 .\end{array}$ \\
\hline
\end{tabular}

FAST FLUENCE

(10*25 N/M**2)

$$
\begin{aligned}
& .00 \\
& .08 \\
& .20 \\
& .22 \\
& .66 \\
& .84 \\
& 1.00 \\
& 1.16 \\
& 1.31 \\
& 1.73 \\
& 1.98 \\
& 2.16
\end{aligned}
$$


E11-07 COMPACT 25

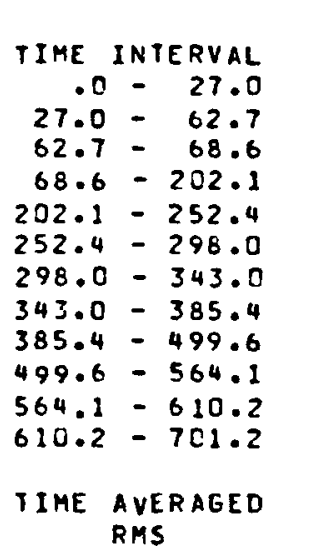

COOLANT
716.
697.
641
708
698
730
711
729
706
688
706
708
707
12

MIA. SLEEVE
868.
841.
767.
857.
848.
887.
86.
886.
851.
851.
876.
884.
862.
17.

TenPE

MAXTURES (C)

$\begin{array}{cc}\text { MAX. SLEEVE } & \text { MIN. FUEL } \\ 900 . & 980 . \\ 874 . & 963 . \\ 793 . & 877 . \\ 896 . & 1007 . \\ 895 . & 1029 . \\ 940 . & 1091 . \\ 913 . & 1059 . \\ 945 . & 1102 . \\ 904 . & 1056 . \\ 921 . & 1115 . \\ 952 . & 1157 . \\ 961 . & 1177 . \\ 917 . & 1068 . \\ 28 . & 66 .\end{array}$

MAX. FUEL

1038.

1021.

926.

1001.

1091.

1144.

1118.

1165 .

$111 \mathrm{C}$.

1182.

1226

1247.

1129.

E11-07 COMPACT 26

$\begin{array}{cc}\text { IIME INTERVAL } & \text { COOLANT } \\ .0-27.0 & 726 . \\ 27.0-62.7 & 707 . \\ 62.7-68.6 & 650 . \\ 68.6=202.1 & 718 . \\ 202.1-252.4 & 708 . \\ 252.4=298.0 & 741 . \\ 298.0-343.0 & 722 . \\ 343.0-385.4 & 740 . \\ 385.4=499.6 & 716 . \\ 499.6-564.1 & 700 . \\ 564.1=610.2 & 718 . \\ 610.2-701.2 & 720 . \\ \text { TIME AVERAGED } & 718 . \\ \text { RMS } & 12 .\end{array}$

TEMPERATURES $(C)$

$\begin{array}{ccc} & \text { TEMPERATURES IC, } \\ \text { KIA. SLEEVE } & \text { MAX. SLEEVE } & \text { MIN. FUEL } \\ 866 . & 895 . & 970 . \\ 839 . & 869 . & 952 . \\ 765 . & 789 . & 860 . \\ 855 . & 890 . & 991 . \\ 845 . & 887 . & 1009 . \\ 885 . & 932 . & 1060 . \\ 859 . & 905 . & 1037 . \\ 883 . & 936 . & 1078 . \\ 850 . & 897 . & 1033 . \\ 852 . & 917 . & 1093 . \\ 878 . & 948 . & 1133 . \\ 885 . & 957 . & 1152 . \\ 861 . & 911 . & 1048 . \\ 18 . & 28 . & 62 .\end{array}$

62.
MAX. FUEL

1023.

1005 .

911.

1045 .

1066 .

1117 .

1091 .

1136.

1082 .

1155 .

1199.

1204.

67.
FAST FLUENCE

$(10 * 25 N / M * 2)$

.00

.00
.08
.19
.20

.20
.61
.77

.92

1.07

1.21

1.60

1.84

2.01

FAST FLUENCE

(10*:25 N/A* 2 )

.00
.07
.17
.19
.57
.71
.85
.99
1.11
1.48
1.70
1.86


E11-07 COMPACT 27

TIME INTERVAL
$.0-27.0$
$27.0-62.7$
$62.7-68.6$
$68.6-202.1$
$202.1-252.4$
$252.4-298.0$
$298.0-343.0$
$343.0-385.4$
$385.4=499.6$
$499.6-564.1$
$564.1-610.2$
$610.2-701.2$
TIME AVERAGED
RMS

COOLANT
736.
716.
657
727
717
751.
731.
750.
726.
710
729
732
728
13.

MIA. SLEEVE MEMPERATURES (C)

862.
836.
761.
851.
843.
883.
857.
881.
848.
851.
876.
883.
859.
18.

MAX. SLEEVE
898.

MIN. FUEL

957.

MAX. FUEL

862.

783 .

882 .

880 .

898.

928.

890 .

909.

940.

937.

1006.

986.

894.

973.11022 .

990.11042 .

1039.1092 .

1016.1066.

1055.1108.

1011.1056.

1066.1124.

1105.1165.

1183 .

FAST FLUENCE

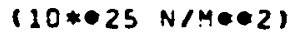

$$
\begin{aligned}
& .00 \\
& .07 \\
& .16 \\
& .17 \\
& .51 \\
& .65 \\
& .77 \\
& .90 \\
& 1.02 \\
& 1.35 \\
& 1.55 \\
& 1.69
\end{aligned}
$$

TIME INTERVAL

$$
\begin{array}{r}
.0-27.0 \\
27.0=62.7
\end{array}
$$

$62.7-68.6$

$68.6-202.1$

$202.1-252.4$

$252.4-298.0$

$298.0-343.0$

$343.0-385.4$

$385.4-499.6$

$499.6-564.1$

$564.1=610.2$

$610.2-701.2$

903.
28.

1026

1078

\section{E11-07 COMPACT 28}

TIME AVERAGED

COOLANT
744.
724.
664.
736
725
760
740
759.
734.
720
739
742.
737
13.

TEMPERATURES (C)

MIA. SLEEVE

859.
833.
759.
848.
838.
878.
852.
876.
843.
846.
872.
879.
855.
18.

$$
883 \text {. }
$$

MIN. FUE

857.
778.

876.

870.

914.

888 .

916.

879.

897 .

927 .

937.

946.

925.

842.

958.

966.
1014.

990.

1027.

984 .

1034 .

1072

1087.

MAX. FUEL

991.

969.

$870^{\circ}$

1002 .

1013.

1061.

1035.

1074 .

1025.

1086.

1126.

893.

1001.

1048

FAST FLUENCE

(10*25 N/M*2)

.00

.06
.14

.14
.15

.45

.57

.68

.79

1.19

1.36

1.49 
E11-07 COMPACT 29

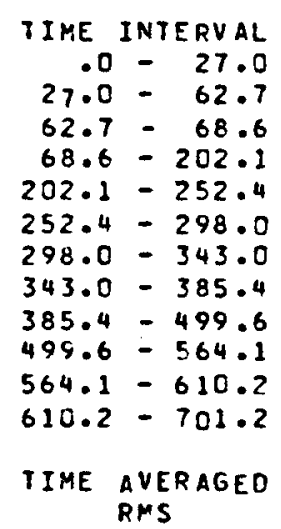

COOLANT
752.
731.
670.
743
733
768
748
767.
742.
728
748.
752
745
13.

MIA. SLEEVE
855
828
755
844
834.
874.
848.
872.
840.
841.
867.
874.
850
18.

IEMPERATURES (C)

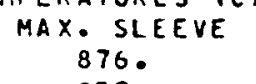
933.

MAX FUE

FAST FLUENCE

876.
850.
772.
868.
933.

861.

904.

878.
906.

906.

883

922 .

$$
928 .
$$

944.

991.

966.
1001.

959.

1000 .

1036.

973.

$(10 * 25 N / M * 2$

883.

1050.
950.
862.

989.

986.
1033.

1006.

1043.

995.

1084 .

1099.

.00
.05
.11
.12
.37
.47
.56
.65
.73
.97
1.12
1.22

$\begin{array}{rr}975 . & 1017 . \\ 44 . & 47 .\end{array}$

E11-07 COMPACT 30

$\begin{array}{rc}\text { TIME INTERVAL } & \text { COOLANT } \\ .0-27.0 & 760 . \\ 27.0=62.7 & 739 . \\ 62.7=68.6 & 677 . \\ 68.6-202.1 & 751 . \\ 202.1=252.4 & 741 . \\ 252.4-298.0 & 777 . \\ 298.0=343.0 & 756 . \\ 343.0-385.4 & 776 . \\ 385.4=499.6 & 750 . \\ 499.6=564.1 & 737 . \\ 564.1=610.2 & 757 . \\ 610.2-701.2 & 761 . \\ \text { TIME AVEPAGED } & 753 . \\ \text { RMS } & 13 .\end{array}$

MIR. SLEEVE
871.
844.
769.
860.
848.
890.
863.
888.
855.
852.
878.
886.
865
17

TEMPERATURES (C)

$\begin{array}{ccc}\text { MAX. SLEEVE } & \text { MIN.FUEL } & \text { MAX.FUEL } \\ 895^{\circ} & 950^{\circ} & 998 . \\ 867 . & 931 . & 974 . \\ 787 . & 845 . & 881 . \\ 885 . & 956 . & 1000 . \\ 870^{\circ} & 957 . & 1001 . \\ 919 . & 1003 . & 1048 . \\ 892 . & 970^{\circ} & 1019 . \\ 920 . & 1010 . & 1056 . \\ 882 . & 967 . & 1006 . \\ 889 . & 995 . & 1043 . \\ 918 . & 1030 . & 1080 . \\ 927 . & 1044 . & 1094 . \\ 895 . & 983 . & 1028 . \\ 21 . & 36 . & 39 .\end{array}$

FASI FLUENCE

(10*25 N/M*2)

$$
\begin{aligned}
& .00 \\
& .03 \\
& .07 \\
& .08 \\
& .24 \\
& .31 \\
& .37 \\
& .42 \\
& .48 \\
& .64 \\
& .73 \\
& .80
\end{aligned}
$$


E11-07 FUEL PERFORMANCE

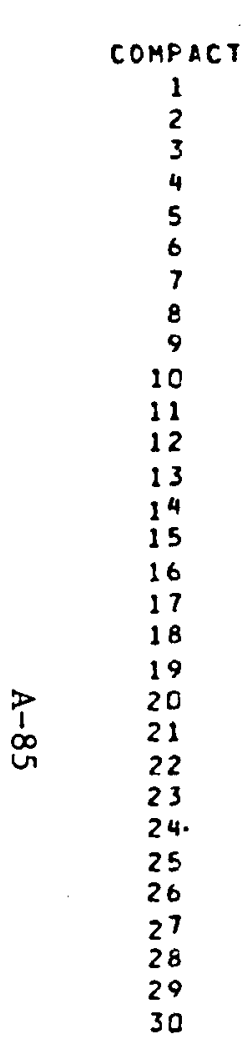

$\begin{array}{ccc}\text { KERNEL MIGRATION (MICRONS) } \\ \text { AVERAGE } & \text { AMOEBAEQ. TEMP. } & \text { PEAK } \\ .00 & 609 . & .00 \\ .00 & 666 . & .00 \\ .00 & 747 . & .00 \\ .00 & 823 . & .00 \\ .01 & 888 . & .01 \\ .03 & 947 . & .04 \\ .08 & 1003 . & .11 \\ .20 & 1052 . & .26 \\ .38 & 1083 . & .49 \\ .65 & 1118 . & .84 \\ .98 & 1145 . & 1.25 \\ 1.32 & 1169 . & 1.68 \\ 1.73 & 1189 . & 2.21 \\ 2.05 & 1201 . & 2.62 \\ 2.35 & 1214 . & 3.01 \\ 2.56 & 1221 . & 3.27 \\ 2.6 C & 1224 . & 3.33 \\ 2.60 & 1226 . & 2.32 \\ 2.48 & 1223 . & 3.17 \\ 2.31 & 1219 . & 2.96 \\ 1.99 & 1210 . & 2.55 \\ 1.64 & 1199 . & 2.10 \\ 1.27 & 1181 . & 1.62 \\ .94 & 1164 . & 1.20 \\ .62 & 1138 . & .80 \\ .40 & 1114 . & .52 \\ .24 & 1085 . & .31 \\ .13 & 1053 . & .17 \\ .07 & 1018 . & .09 \\ .07 & 1018 . & .09\end{array}$

DEFECTIVE COATINGS

.07

.07

.15

.18
.20

.22

.23

.24

.26

.26

.27

.27

.26

.26

.25
.25

.22

.22

.21

.20

.19

.16

.14

.11
FUEL FAILURE (2)

PRESSURE VESSEL KERNEL HIGRATION TOTAL

.19
.31
.40
.49
.55
.60
.64
.67
.69
.71
.72
.73
.74
.74
.74
.73
.72
.72
.70
.69
.66
.63
.60
.57
.52
.48
.43
.38
.31
.20

$\begin{array}{ll}.00 & .25 \\ .00 & .42 \\ .00 & .55\end{array}$

$.00 \quad .67$

.075

.82

$.00 \quad .87$

$.00 \quad .92$

$\begin{array}{ll}.00 & .92 \\ .00 & .95\end{array}$

$.00 \quad .97$

$\begin{array}{ll}.00 & .97 \\ .00 & .99\end{array}$

$\begin{array}{ll}.00 & .99 \\ .00 & 1.00\end{array}$

$.00 \quad 1.01$

$.00 \quad 1.00$

$.00 \quad 1.00$

$.00 \quad .99$

$.00 \quad .98$

$.00 \quad .97$

$.00 \quad .95$

AMOEBA EOUIVALENT TEMPERATURES (C) CORRESPOND TO AVERAGE KERNEL MIGRATIONS KERNEL MIGRATION DISTANCES CALCULATED USING SOZ CONFIDENCE LEVEL KMC

FUEL FAILURE CORRESPONDS TO $50 \%$ CONFIDENCE LEVEL 
E14-01 UUMPACI 1

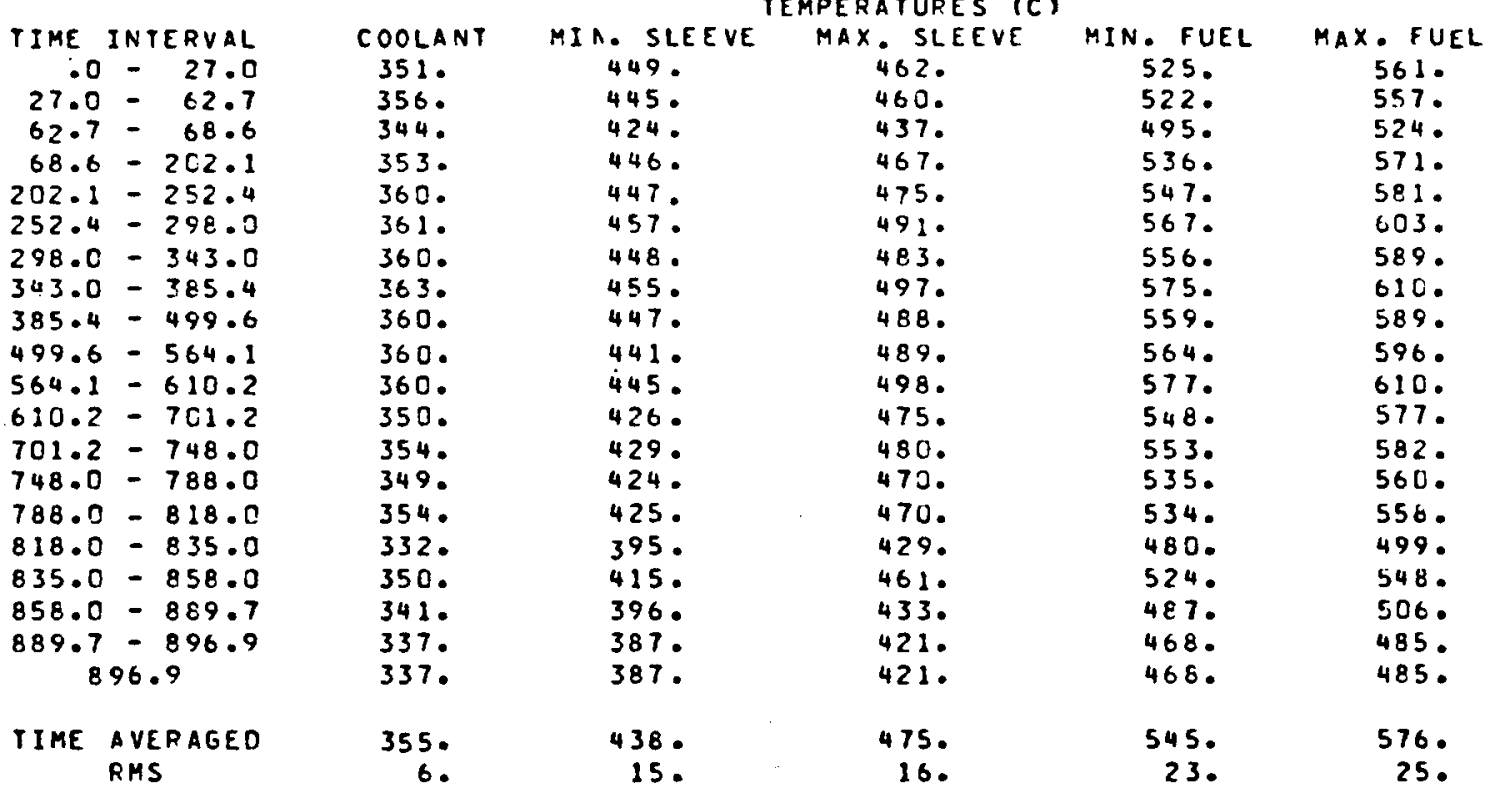

FASI FLUENCE

(10*25 N/M\#2)

.00

06

.06

.06
.18

.23

.27

.31

.35

.45

.50

.54

.61
.64

.68

.70

.71

.73

.76
.76

TIME INTERVAL $.0-27.0$
$27.0-62.7$ $62.7-68.6$ $68.6-202.1$ $202.1-252.4$

$252.4-298.0$

$298.0-343.0$

$343.0-385.4$

$385.4-499.6$

$499.6-564.1$

$564.1-610.2$

$610.2-701.2$

$701.2-748.0$

$748.0-788.0$
$788.0-816.0$

$788.0-816.0$
$818.0-835.0$

$835.0-858.0$

$858.0-889.7$

$889.7-896.9$

896.9

TIME AVERAGED RUS

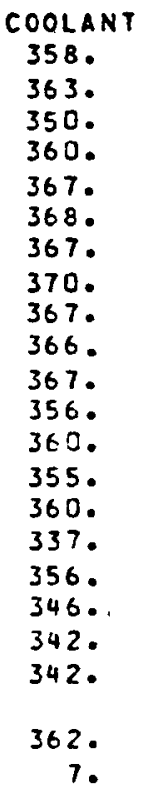

TEMPERATURES (C)

MI ^. SLEEVE

MAX. SLEEVE

MIN . FUEL

MAX - FUEL

FAST FLUENCE

$(10 * 25 \quad N / M * 2)$

468 .

462.

440.

483.

464.

467 .

478 .

467.

475.

466 .

462 .

467.

446.

448.

444.

447.

415 .

435.

413.

403.

479.

455.

493.

509.

530.

536.

536.

527 .

536.

511.

499.

503.

457.

491.

459.

552.

549.

592 .

588.

$52 \mathrm{C}$.
573.

594.

621 .

553.

612.

633.

608.

629.

608 .

619.

645.

670.

632.

600 .

578.

581.

520 .

569.

524.

502.

443 .

502 .

057 .

671 .

634.

608 .

611 .

542.

$598^{\circ}$

548.

522.

523.

.00

.04

.10
.10

.30
.37

.51

458.

507.

591.

627.

.73

. 82

1.06

1.12

1.16

1.18
1.21

1.25

1.26

16.

21.

32 . 
14-01 - JMPACT

\begin{tabular}{|c|c|c|c|c|c|c|c|}
\hline & \multicolumn{5}{|c|}{ TEMPERATURES (C) } & FAST FLUENCE \\
\hline $\begin{array}{l}\text { TIME } \\
27.0 \\
27.0\end{array}$ & $\begin{array}{l}\text { INTERVAL } \\
-\quad 27.0 \\
-\quad 62.7\end{array}$ & $\begin{array}{l}\text { COOLANT } \\
368 \\
371\end{array}$ & $\begin{array}{l}\text { MIA. SLEEVE } \\
505 . \\
496 .\end{array}$ & $\begin{array}{l}\text { MAX. SLEEVE } \\
524 . \\
517 .\end{array}$ & $\begin{array}{c}\text { MIN. FUEL } \\
607 . \\
603 .\end{array}$ & $\begin{array}{c}\text { MAX. FUEL } \\
657 . \\
651 .\end{array}$ & $\begin{array}{c}110 * 25 \quad N / H * 21 \\
.00 \\
.05\end{array}$ \\
\hline $\begin{array}{l}62.7 \\
68.6\end{array}$ & $\begin{array}{r}\quad 68.6 \\
-\quad 202.1\end{array}$ & $\begin{array}{l}358 . \\
369\end{array}$ & $\begin{array}{l}469 . \\
499 .\end{array}$ & $\begin{array}{l}490 . \\
536 .\end{array}$ & $\begin{array}{l}570 . \\
635 .\end{array}$ & $\begin{array}{l}611 . \\
685 .\end{array}$ & $\begin{array}{l}.12 \\
.14\end{array}$ \\
\hline $202 \cdot 1$ & -252.4 & 375. & 499. & 553. & 659. & 708 & .39 \\
\hline $\begin{array}{l}252.4 \\
298.0\end{array}$ & $\begin{array}{l}-298.0 \\
-\quad 343.0\end{array}$ & $\begin{array}{l}378 . \\
375 .\end{array}$ & $\begin{array}{l}513 . \\
500 .\end{array}$ & $\begin{array}{l}579 . \\
565 .\end{array}$ & $\begin{array}{l}693 . \\
675 .\end{array}$ & $\begin{array}{l}744 . \\
722 .\end{array}$ & .49 \\
\hline 343.0 & -285.4 & 379. & 509. & 584. & 701 & 751. & .67 \\
\hline $\begin{array}{l}385.4 \\
499.6\end{array}$ & $\begin{array}{l}-499.6 \\
-564.1\end{array}$ & $\begin{array}{l}376 . \\
374 .\end{array}$ & $\begin{array}{l}498 . \\
494 .\end{array}$ & $\begin{array}{l}566 . \\
571 .\end{array}$ & $\begin{array}{l}674 . \\
688 .\end{array}$ & $\begin{array}{l}717 . \\
735 .\end{array}$ & $\begin{array}{l}.75 \\
.96\end{array}$ \\
\hline $\begin{array}{l}564.1 \\
610.2 \\
701.2\end{array}$ & $\begin{array}{l}-610.2 \\
=701.2 \\
-748.0\end{array}$ & $\begin{array}{l}375 \\
363 \\
367\end{array}$ & $\begin{array}{l}500 . \\
476 . \\
478\end{array}$ & $\begin{array}{l}582 . \\
549 . \\
553 .\end{array}$ & $\begin{array}{l}703 . \\
661 . \\
663 .\end{array}$ & $\begin{array}{l}751 . \\
704 . \\
706 .\end{array}$ & $\begin{array}{l}1.08 \\
1.17 \\
1.32\end{array}$ \\
\hline $\begin{array}{l}748.0 \\
788.0 \\
828.0\end{array}$ & $\begin{array}{l}=788.0 \\
=816.0 \\
=835.0\end{array}$ & $\begin{array}{l}363 . \\
367 \\
344\end{array}$ & $\begin{array}{l}474 . \\
471 . \\
436 .\end{array}$ & $\begin{array}{l}539 . \\
534 \\
484\end{array}$ & $\begin{array}{l}637 \\
628 . \\
560 .\end{array}$ & $\begin{array}{l}675 . \\
664 . \\
587 .\end{array}$ & $\begin{array}{l}1.39 \\
1.46 \\
1.52\end{array}$ \\
\hline $\begin{array}{r}835.0 \\
858.0 \\
889.7 \\
8 .\end{array}$ & $\begin{array}{l}-858.0 \\
-889.7 \\
-896.9 \\
96.9\end{array}$ & $\begin{array}{l}362 . \\
351 . \\
347 \\
347\end{array}$ & $\begin{array}{l}457 . \\
432 . \\
420 . \\
420 .\end{array}$ & $\begin{array}{l}521 . \\
483 . \\
466 . \\
466 .\end{array}$ & $\begin{array}{l}614 . \\
562 . \\
536 . \\
536 .\end{array}$ & $\begin{array}{l}649 . \\
590 . \\
561 . \\
561 .\end{array}$ & $\begin{array}{l}1.55 \\
1.58 \\
1.63 \\
1.64\end{array}$ \\
\hline TIME & $\begin{array}{l}\text { AVERAGED } \\
\text { RMS }\end{array}$ & $\begin{array}{r}370 . \\
7\end{array}$ & $\begin{array}{r}489 . \\
20 .\end{array}$ & $\begin{array}{r}549 . \\
26 .\end{array}$ & $\begin{array}{r}653 \\
37\end{array}$ & $\begin{array}{r}697 . \\
41 .\end{array}$ & \\
\hline
\end{tabular}

E14-01 COMPACT 4

TIME INTERVAL<smiles></smiles>

$2.0-27.0$

$62.7-68.6$

$68.6-202.1$

$202.1-252.4$

$252.4-298.0$

$298.0-343.0$

$343.0-385.4$

$385.4-499.6$

$499.6-564.1$

$564.1-610.2$

$610.2-701.2$

$701.2-748.0$

$748.0-788.0$

$788.0-818.0$

$818.0-835.0$

$835.0-858.0$

$858.0-889.7$

$889.7-896.9$

$$
896.9
$$

TIME AVERAGEO RMS
IEMPERATURES (C)

\begin{tabular}{|c|c|}
\hline COOLANT & MIA. \\
\hline $\begin{array}{l}379^{\circ} \\
382^{\circ} \\
367^{\circ}\end{array}$ & $\begin{array}{l}543 . \\
531 . \\
501 .\end{array}$ \\
\hline $\begin{array}{l}380^{\circ} \\
385^{\circ} \\
388 .\end{array}$ & $\begin{array}{l}536^{\circ} \\
530^{\circ} \\
547^{\circ}\end{array}$ \\
\hline $\begin{array}{l}385 . \\
389 .\end{array}$ & $\begin{array}{l}531 . \\
542 .\end{array}$ \\
\hline $\begin{array}{l}385 . \\
383 . \\
385 .\end{array}$ & $\begin{array}{l}529^{\circ} \\
521^{\circ} \\
529^{\circ}\end{array}$ \\
\hline $\begin{array}{l}372^{\circ} \\
376 . \\
372^{\circ}\end{array}$ & $\begin{array}{l}502 . \\
503 . \\
499 .\end{array}$ \\
\hline $\begin{array}{l}3750^{\circ} \\
352{ }^{\circ} \\
369 .\end{array}$ & $\begin{array}{l}491 . \\
454 . \\
475 .\end{array}$ \\
\hline $\begin{array}{l}358 . \\
353 . \\
353 .\end{array}$ & $\begin{array}{l}447 . \\
434 . \\
434 .\end{array}$ \\
\hline $\begin{array}{r}379 . \\
8 .\end{array}$ & $\begin{array}{r}518 \\
25 .\end{array}$ \\
\hline
\end{tabular}

MIN. FUEL

\section{3. \\ 658 .}

568 .

558.

526 .

581 .

592.

623.

606 .

627.

606 .

607.

619

582.

585 .

571 .

558 .

505

543.

502 .

483.

484.

586.

30 .
620 .

699.

718 .

758 .

737.1

766 .

735.

745.

762 .

715.

717.

689.

594.

652 .

595 .

566.

567.

709.
44.
MAX. FUEL

723.

716 .

670

758.

818 .

792

792

825 .

786.

818 .

765 .

767.

732.

708 .

624

091.

627 .

594.

594.

761 .
FAST FLUENCE

$(10 * 25 N / M * 2)$

.00
.06
.15
.16
.48
.59
.70
.81
.91
1.17
1.31
1.41
1.59
1.68
1.77
1.83
1.86
1.90
1.96
1.97 
E14-01 -UMPACT 5

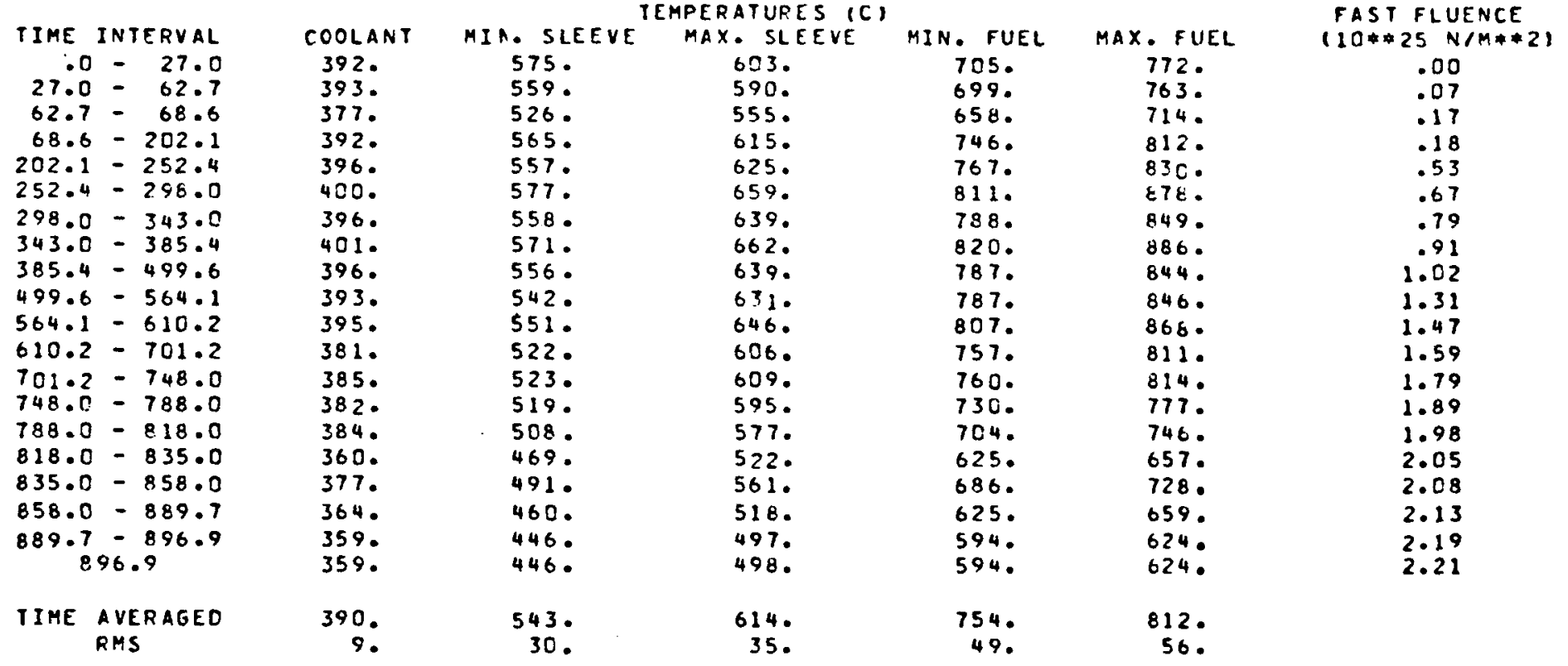

TIHE INTERVAL

$$
.0-27.0
$$

$.0=27.0$
$27.0=62.7$

$62.7=68.6$

$68.6-202.1$

$202.1=252.4$

$252.4=258.0$

$343.0-385.4$

$343.0=385.4$
$385.4=499.6$

$499.6-564.1$

$564.1-610.2$

$610.2-701.2$

$701.2-748.0$

$748.0-788.0$

$818.0=835.0$

$818.0=835.0$
$635.0=858.0$

$835.0=858.0$
$858.0=889.7$

$889.7-896.9$

896.9

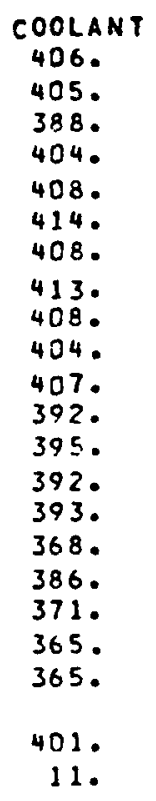

TEMPERATURES (C)

$\begin{array}{ccc}\text { MIA. SLEEVE MAX. SLEEVE } & \text { MIN. FUEL } \\ 604 . & 636 . & 744 . \\ 586 . & 620 . & 736 . \\ 550 . & 581 . & 693 . \\ 593 . & 647 . & 789 . \\ 583 . & 655 . & 810 . \\ 605 . & 692 . & 859 . \\ 584 . & 669 . & 834 . \\ 597 . & 693 . & 869 . \\ 582 . & 669 . & 835 . \\ 563 . & 654 . & 828 . \\ 572 . & 670 . & 851 . \\ 541 . & 628 . & 799 . \\ 542 . & 631 . & 803 . \\ 539 . & 617 . & 772 . \\ 523 . & 594 . & 738 . \\ 483 . & 537 . & 655 . \\ 505 . & 576 . & 720 . \\ 472 . & 531 . & 654 . \\ 457 . & 509 . & 621 . \\ 457 . & 5090^{\circ} & 621 . \\ 565 . & 641 . & 796 . \\ 34 . & 39 . & 54 .\end{array}$

MAX. FUEL

817.

806.

754.

861 .

879.

932.

901.

941.

896.

890 .

$9150^{\circ}$

860.

821.

689.

764.

690.

652 .

653 .

FAST FLUENCE

(10*25 N/M**2)

IIME AVERAGED RMS

858. 
E14-01 -UMPACT 7

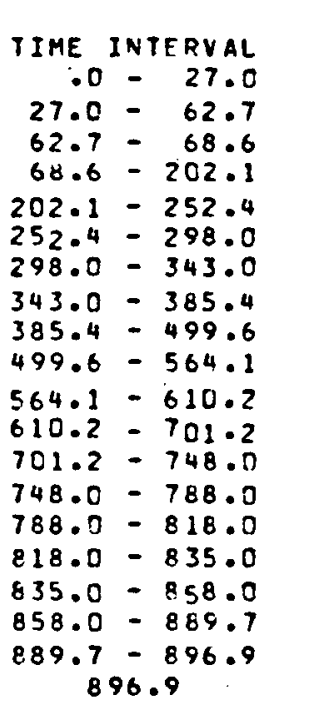

TIME AVERAGED RMS

7
1
$\infty$
0

$\begin{array}{cc} & \\ \text { COOLANT } & \text { MIA. SLEEVE } \\ 421 . & 631 . \\ 419 . & 610 . \\ 400 . & 572 . \\ 418 . & 618 . \\ 420 . & 606 . \\ 427 . & 631 . \\ 421 . & 607 . \\ 426 . & 621 . \\ 421 . & 605 . \\ 415 . & 583 . \\ 418 . & 594 . \\ 402 . & 561 . \\ 406 . & 562 . \\ 403 . & 558 . \\ 403 . & 538 . \\ 377 . & 496 . \\ 394 . & 518 . \\ 379 . & 484 . \\ 372 . & 467 . \\ 372 . & 467 . \\ 413 . & 587 . \\ 12 . & 38 .\end{array}$

rene

MAX OURES (C)

$X$ SLEEVE
665.
646.
605.
675.
680.

777 .

769.

723.

$826^{\circ}$

680.

720 .

694.

720.
694.

694.

695.

650.

653.

639 .

609.

551.

591.

520.

521.

665.

43.

847 .

900.

873.

911.

876.

870 .

896.

842.

847

815.

772 .

685 .

754.

684.

649.

836 .

57.

855.

843.

787.

903.

920 .

978 .

944.

984.

987.

941.

964.

903.

908 .

868 .

819 .

721.
800.

800.

681.

682 .

901.

65.

E14-01 COMPACT 8

TEMPERATURES (C)

\begin{tabular}{|c|c|}
\hline $\begin{array}{l}\text { COOLANT } \\
436 . \\
432 .\end{array}$ & $\begin{array}{c}\text { MIA. SLEEVE } \\
654 . \\
631 .\end{array}$ \\
\hline $\begin{array}{l}413 . \\
433 .\end{array}$ & $\begin{array}{l}591 . \\
640 .\end{array}$ \\
\hline $\begin{array}{l}433 . \\
442 . \\
434 .\end{array}$ & $\begin{array}{l}627 . \\
6550 \\
629 .\end{array}$ \\
\hline $\begin{array}{l}440 . \\
434 . \\
427 .\end{array}$ & $\begin{array}{l}644 \\
627 \\
602 .\end{array}$ \\
\hline $\begin{array}{l}431 . \\
414 \\
417\end{array}$ & $\begin{array}{l}614 . \\
579 . \\
579 .\end{array}$ \\
\hline $\begin{array}{l}414 . \\
412 . \\
386 .\end{array}$ & $\begin{array}{l}576 . \\
551 . \\
509 .\end{array}$ \\
\hline $\begin{array}{l}403 . \\
386 . \\
379 .\end{array}$ & $\begin{array}{l}531 . \\
494 . \\
477^{\circ}\end{array}$ \\
\hline 379. & 477. \\
\hline $\begin{array}{r}425 . \\
14 .\end{array}$ & $\begin{array}{r}607 . \\
42 .\end{array}$ \\
\hline
\end{tabular}

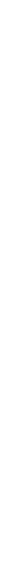

AX. SLEEVE

691 .

670.

626.

699.

704.

746.

718 .

717.

697.

716.

669.

672.

659.

623.

604.

$530^{\circ}$

530.

686.
46.
HIN. FUEL

806.

796.

748.

857 .

881 .

937.

908 .

949.

913.

906.

934.

880.

886 .

853.

803.

713.

785.

674.

675.

870.
14.

42.

$6 \mathrm{~S}$.
MAX. FUEL

887.

874 .

915.

957.

1018.

983.

1029.

982.

975.

1006.

943.

949

908 .

851.

832.

750.
708.

708 .

939.

69.
FAST FLUENCE

$10 * 25 \quad N / 4 * 21$

.00

.09

.22

.63

.93

1.07

1.20

1.53

1.72
1.86

2.09

2.21
2.31

2. 38

2.42

2.47

2.54

IIME AVERAGEO RMS

FAST FLUENCE

$110 * 25 N / M * * 21$ .00

.09

.21

.66

.82
.97

1.12

1.26

1.81

1.95

2.32

2.42

2.50

2.54

2.66

2.67 
E14-02 -UMPACT 9

TIME INTERVAL

.0 027.0 $.0=27.0$
$27.0=62.7$ $62.7-68.6$ $68.6-202.1$ $202.1-252.4$ $252.4=222.4$ $298.0-343.0$ $343.0-385.4$ $385.4-499.6$ $499.6-564.1$ $564.1=610.2$ $610.2-701.2$ $701.2-748.0$ $748.0-788.0$ $723.0-818.0$ $818.0-835.0$

$835.0-858.0$ $858.0-889.7$ $889.7-896.9$ 896.9

TIME AVEFAGED FMS

$p$
1
0
TEMPERATURES (C)

\begin{tabular}{|c|c|}
\hline $\begin{array}{l}\text { COOLANT } \\
452 .\end{array}$ & $\operatorname{MIR.}{ }_{674}$ SLEEV \\
\hline 446. & 649. \\
\hline $\begin{array}{l}425 . \\
447 .\end{array}$ & $\begin{array}{l}607 . \\
659 .\end{array}$ \\
\hline $\begin{array}{l}447 . \\
457{ }^{\circ} \\
448 .\end{array}$ & $\begin{array}{l}648 . \\
677^{\circ} \\
650^{\circ}\end{array}$ \\
\hline 455. & 666. \\
\hline $\begin{array}{l}448 . \\
440 .\end{array}$ & $\begin{array}{l}647 . \\
620 .\end{array}$ \\
\hline $\begin{array}{l}444 . \\
425^{\circ} \\
429^{\circ}\end{array}$ & $\begin{array}{l}632^{\circ} \\
5950^{\circ} \\
596 .\end{array}$ \\
\hline $\begin{array}{l}4260^{\circ} \\
4220^{\circ} \\
3950^{\circ}\end{array}$ & $\begin{array}{l}593 . \\
564 . \\
520 .\end{array}$ \\
\hline $\begin{array}{l}412 . \\
394 .\end{array}$ & $\begin{array}{l}542 . \\
504 .\end{array}$ \\
\hline 386. & 486. \\
\hline 386. & 486. \\
\hline 438. & 625. \\
\hline
\end{tabular}

MAX. SLEEVE

MIN. FUEL

MAX. FUEL

712.

698.

642.

726.

770 .

740.

768.

739.

715

686.

689.

676.

635.

615 .

563.

539.

704.
50.

828 .

816.

767.
879.

879.

910.

969.

939.

982.

946.

937.

967.

920 .

886.

829.

736 .

810.

696.

899.

64.

910.

896.

835 .

960.

985.

1054.

1017.

1065.

1017.

1041.

978 .

985.

943.

878 .

774.

658.

774.

730 .

969.

72.

E14-01 COMPACT 10

TIME INTERVAL$$
\begin{array}{r}
.0=27.0 \\
27.0=62.7
\end{array}
$$

$62.7-68.6$

$68.6-202.1$

$202.1-252.4$

$252.4-298.0$

$298.0-343.0$

$343.0-385.4$

$385.4-499.6$

$499.6-564.1$

$564.1-610.2$

$610.2-701.2$

$701.2-748.0$

$748.0-788.0$

$788.0-818.0$

$818.0-835.0$

$835.0-858.0$

$858.0-889.7$

$889.7-896.9$

896.9

TIME AVERAEED

$$
\text { RNS }
$$

TEMPERATURES (C)

$\begin{array}{cc}\text { COOLANT } & \text { MIA. SLEEV } \\ 468 . & 692 . \\ 461 . & 665 . \\ 438 . & 621 . \\ 462 . & 675 . \\ 461 . & 666 . \\ 473 . & 697 . \\ 462 . & 667 . \\ 469 . & 694 . \\ 463 . & 665 . \\ 452 . & 639 . \\ 457 . & 652 . \\ 437 . & 613 . \\ 440 . & 613 . \\ 438 . & 611 . \\ 433 . & 578 . \\ 404 . & 533 . \\ 422 . & 555 . \\ 402 . & 515 . \\ 393 . & 496 . \\ 393 . & 496 . \\ 451 . & 642 . \\ 18 . & 46 . \\ 4 & \end{array}$

MIN. FUEL

847.

334.

783 .

898 .

932.

994.

963.

1007.

971.

969.

1001.

945.

954.

920 .

858.

838.

838.

719.

720.

925.

66.
AX. FUE

930.

914.

852 .

980 .

1013 .

1080 .

1042 .

1091.

1091.

1044.

1043.

1078 .

1013 .

1021.

979.

908 .

800 .

888 .

800 .

755 .

755 .

997.

74.
FAST FLUENCE

$110 * 25 \mathrm{~N} / \mathrm{M} * 21$

.00

.09
.21

.21
.23

.68

.85

1.16

1.30

1.87

2.01

2.27

2.40

2.50

2.58

2.62

2.67

2.74
2.76

FAST FLUENCE

(10*25 N/M*21

.00

.09

.22
.24

.70

.87

1.03

1.19

1.33

1.71

1.92

2.07
2.33

2.46

2.57

2.65

2.69

2.75
2.82

2.84 
E14-01 -UMPACT \&

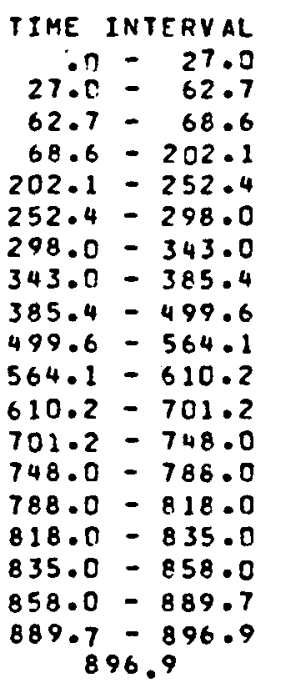

IME AVERAGED RMS

$\begin{array}{ll}\text { COOLANT } & \text { MIA. SLEEVE } \\ 484 . & 710 . \\ 475 . & 681 . \\ 451 . & 636 . \\ 479 . & 692 . \\ 475 . & 682 . \\ 488 . & 714 . \\ 476 . & 684 . \\ 484 . & 701 . \\ 477 . & 681 . \\ 465 . & 656 . \\ 471 . & 670 . \\ 449 . & 630 . \\ 453 . & 629 . \\ 451 . & 628 . \\ 443 . & 590 . \\ 414 . & 545 . \\ 431 . & 566 . \\ 410 . & 525 . \\ 401 . & 505 . \\ 401 . & 505 . \\ 465 . & 658 . \\ 20 . & 48 .\end{array}$

MAX SIEEVE)

MAX - SLEEVE

MIN. FUEL

$$
\begin{aligned}
& 722 . \\
& 673 \text {. }
\end{aligned}
$$

752.

759.

806.

772.

802.

771.

753.

722 .

725.

711.

661.

599.

639.

558 .

558.

738.

53.
E14-01 COMPACT 12

TEMPERATURES (C)

TIME INTERVAL $.0-27.0$
$27.0=62.7$ $62.7-68.6$ $68.6-202.1$ $202.1-252.4$ $252.4-298.0$ $298.0=343.0$ $298.0=343.0$
$343.0=385.4$ $343.0=385.4$
$385.4=499.6$ $385.4=499.6$ $499.6-564.1$ $564.1-610.2$ $610.2-701.2$

$701.2-748.0$ $748.0-788.0$ $788.0-818.0$ $818.0-835.0$ $835.0-858.0$ $858.0-889.7$ $889.7-896.9$ 896.9

TIME AVERAGED

\begin{tabular}{|c|c|}
\hline COOLANT & MIN. SLEEVE \\
\hline $\begin{array}{l}500 . \\
489 . \\
464 . \\
492 .\end{array}$ & $\begin{array}{l}725 . \\
695 . \\
648 . \\
707 .\end{array}$ \\
\hline $\begin{array}{l}489 . \\
504 . \\
490^{\circ}\end{array}$ & $\begin{array}{l}696 . \\
730 . \\
698 .\end{array}$ \\
\hline $\begin{array}{l}499 . \\
491 .\end{array}$ & $\begin{array}{l}717 . \\
696 .\end{array}$ \\
\hline 478 & 672. \\
\hline $\begin{array}{l}484 . \\
462 .\end{array}$ & $\begin{array}{l}687 . \\
645 .\end{array}$ \\
\hline 465. & 644. \\
\hline $\begin{array}{l}464^{\circ} \\
454^{\circ}\end{array}$ & $\begin{array}{l}643 . \\
603 .\end{array}$ \\
\hline $\begin{array}{l}4240^{\circ} \\
4410^{\circ} \\
418 .\end{array}$ & $\begin{array}{l}556 . \\
577^{\circ} \\
534^{\circ}\end{array}$ \\
\hline $\begin{array}{l}408 . \\
408 .\end{array}$ & $\begin{array}{l}513 . \\
514 .\end{array}$ \\
\hline 478. & 673. \\
\hline 22. & 49. \\
\hline
\end{tabular}
RMS

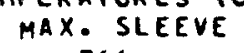

$$
\begin{aligned}
& 760^{\circ} \\
& 7366^{\circ} \\
& 6850^{\circ} \\
& 7660^{\circ} \\
& 772 . \\
& 8211^{\circ} \\
& 7850^{\circ} \\
& 8150^{\circ} \\
& 7850^{\circ} \\
& 769 . \\
& 791 . \\
& 737 . \\
& 739 . \\
& 726 . \\
& 673 . \\
& 610 . \\
& 649 . \\
& 593 . \\
& 566 . \\
& 567 . \\
& 752 . \\
& 54 .
\end{aligned}
$$

866.

852 .

799.

916.

949.

1014.

981.

1027 .

991.

995.

1029 .

972.

981 .

948 .

879.

781.

859.

779.

737.

946.
67.

946.
67.

MAX. FUEL

951.

933.

999.

1032.

101.

1061.

1112 .

1064.

1071 .

1107 .

1042.

1051.

1008 .

930 .

820

909 .

819.

773.

1020
75.

MIN. FUEL

966.

681 .

1013 .

1046 .

1117 .

1076.

1128 .

1080

1093 .

1131.

1065.

1074 .

1074

1031.

949.

838 .

928.

836.

$788^{\circ}$

795 .

752.

753.

1038 .

68.1038
FAST FLUENCE

$10 * 25 N / H * 21$

.00

.09
.22

.24

.70

.88
2.04

1.24

1.20

1.73

1.94

2.10

2.37

2.50

2.61

2.69

2.74

2.79

2.87
2.88

FAST FLUENCE

(10*25 N/M*2)

$$
\begin{aligned}
& .00 \\
& .10 \\
& .22 \\
& .24 \\
& .70 \\
& .88 \\
& 1.05 \\
& 1.21 \\
& 1.35 \\
& 1.74 \\
& 1.96 \\
& 2.12 \\
& 2.39 \\
& 2.53 \\
& 2.64 \\
& 2.72 \\
& 2.77 \\
& 2.83 \\
& 2.90 \\
& 2.92
\end{aligned}
$$


[14-01 UUMPACT 13

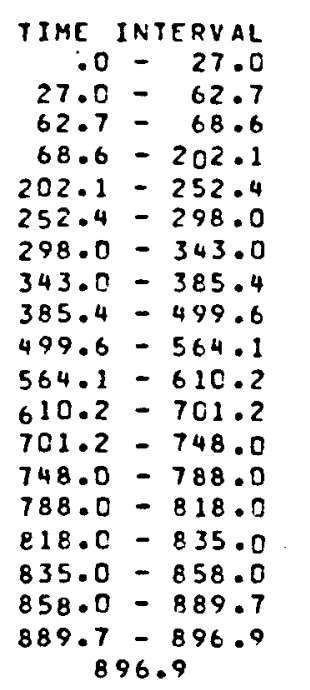

TIRE AVERAGED RMS

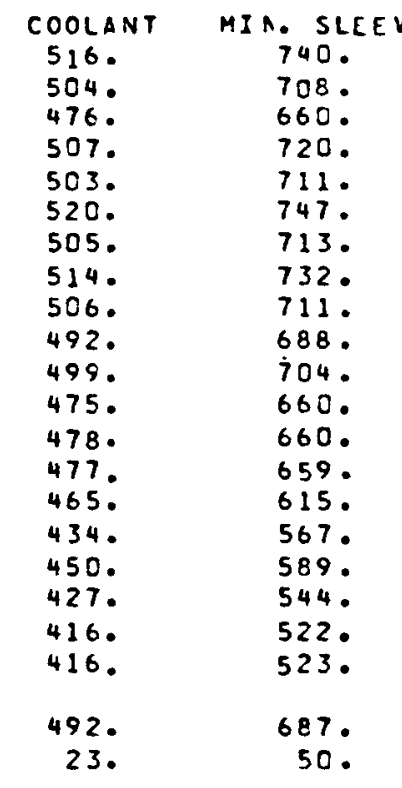

TEMPERATURES (C)

IN.

780.
749.
696.
779.

896.

877.

942 .

977.

1044.

836 .

798 .

829.

798 .

785 .

$8 \cap 8$.

752 .

754.

742 .

685 .

621.

660 .

602.
575 .

575 .

765.

55 .

1010.

1021.

1036 .

1073.

1014.

1024.

991.

915.

815 .

895.

811.

768 .

980

70.

MAX. FUEL

980.

958.

1024.

1024.

1060 .

1091.

1143.

1095 .

1115 .

1154.

1087 .

1096 .

1054 .

968 .

855 .

946.

853.

804 .

805 .

1055.

78 .

E14-01 COMPACT 14

TIME INTERVAL

$$
\begin{aligned}
& \text {. INTERVAL } \\
& .027 .0
\end{aligned}
$$

$.0-27.0$
$27.0=62.7$

$62.7-68.6$

$68.6-202.1$

$202.1-252.4$

$252.4-298.0$

$298.0-343.0$

$343.0-385.4$

$385.4-499.6$

$499.6-564.1$

$564.1-610.2$

$610.2=701.2$

$71.2-748.0$

$748.0-788.0$

$818.0=835.0$

$835.0-858.0$

$858.0-889.7$

$889.7-896.9$

896.9

TIME AVERAGED

$$
\text { RMS }
$$

$\begin{array}{cc}\text { COOLANT } & \text { MIA. SLE } \\ 5322^{\circ} & 7530^{\circ} \\ 518 . & 720^{\circ} \\ 489 . & 671 . \\ 522 . & 733 . \\ 518 . & 724 . \\ 536 . & 761 . \\ 519 . & 726 . \\ 529 . & 746 . \\ 520^{\circ} & 724 . \\ 505 . & 702 . \\ 513 . & 719 . \\ 488 . & 673 . \\ 490 . & 673 . \\ 490 . & 672 . \\ 475 . & 628 . \\ 444 . & 579 . \\ 460 . & 600 . \\ 435 . & 554 . \\ 424 . & 531 . \\ 424 . & 532 . \\ 506 . & 700 . \\ 25 . & 51 .\end{array}$

TEMPERATURES $(C)$

$\begin{array}{ccccc}\text { SLEEVE MAX. SLEEVE } & \text { MIN. FUEL } & \text { MAX.FUEL } & (10 * 25 N / M * * 21 \\ 753 . & 794 . & 908 . & 991 . & .00 \\ 720 . & 761 . & 886 . & 968 . & .09 \\ 671 . & 737 . & 830 . & 900 . & .22 \\ 733 . & 790 . & 951 . & 1033 . & .24 \\ 724 . & 797 . & 988 . & 1071 . & .70 \\ 761 . & 849 . & 1056 . & 1144 . & .87 \\ 726 . & 810 . & 1020 . & 1101 . & 1.04 \\ 746 . & 841 . & 106.8 . & 1154 . & 1.20 \\ 724 . & 810 . & 1032 . & 1106 . & 1.35 \\ 702 . & 797 . & 1049 . & 1128 . & 1.74 \\ 719 . & 821 . & 1086 . & 1168 . & 1.96 \\ 673 . & 764 . & 1027 . & 1100 . & 2.13 \\ 673 . & 766 . & 1038 . & 1110 . & 2.40 \\ 672 . & 754 . & 1005 . & 1068 . & 2.54 \\ 628 . & 698 . & 931 . & 985 . & 2.66 \\ 579 . & 632 . & 830 . & 870 . & 2.74 \\ 600 . & 671 . & 910 . & 962 . & 2.79 \\ 554 . & 612 . & 825 . & 867 . & 2.95 \\ 531 . & 583 . & 780 . & 817 . & 2.95 \\ 532 . & 584 . & 781 . & 818 . & \\ 750 . & 777 . & 992 . & 1067 . & \\ 51 . & 56 . & 70 . & 77 . & \end{array}$


E14-01 COPPACT 15

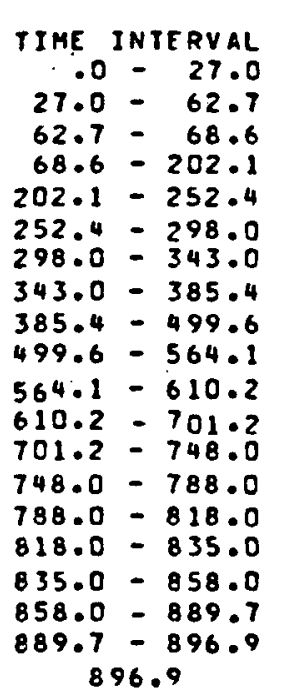

TIME AVERAGED

TIME INTERVAL

$$
.0-27.0
$$

$27.0=62.7$

$62.7-68.6$

$68.6-202.1$

$202.1-252.4$

$252.4-298.0$

$298.0-343.0$

$343.0-385.4$

$385.4-499.6$

$499.6-564.1$

$564.1-610.2$

$610.2-701.2$

$701.2=748.0$

$748.0-788.0$

$788.0-818.0$

$818.0-835.0$

$835.0-858.0$

$858.0-889.7$

$889.7-896.9$

896.9

TIME AVERAGED RMS

\begin{tabular}{|c|c|}
\hline $\begin{array}{l}\text { COOLANT } \\
547 .\end{array}$ & MIA. SLEEV \\
\hline $\begin{array}{l}532 . \\
502 \\
536\end{array}$ & $\begin{array}{l}731 . \\
680 . \\
744\end{array}$ \\
\hline 532. & 736. \\
\hline $\begin{array}{l}551 . \\
533^{\circ}\end{array}$ & $\begin{array}{l}775 \circ \\
739 .\end{array}$ \\
\hline 544. & 759. \\
\hline $\begin{array}{l}534 . \\
519^{\circ}\end{array}$ & $\begin{array}{l}737 \\
716 .\end{array}$ \\
\hline $\begin{array}{l}527 . \\
500 .\end{array}$ & $\begin{array}{l}733 . \\
686 .\end{array}$ \\
\hline $\begin{array}{l}503 . \\
503 .\end{array}$ & $\begin{array}{l}686^{\circ} \\
6850^{\circ}\end{array}$ \\
\hline $\begin{array}{l}486 . \\
454 .\end{array}$ & $\begin{array}{l}641 . \\
591 .\end{array}$ \\
\hline 470 & 612. \\
\hline 444. & 564. \\
\hline $\begin{array}{l}431 . \\
432 .\end{array}$ & $\begin{array}{l}541 . \\
541 .\end{array}$ \\
\hline 519. & 712. \\
\hline 27 & 51. \\
\hline
\end{tabular}

TEMPERATURES (C)

MAX. SLEEVE MIN. FUEL

806.
771.
716.

771.

716.

808 .

860 .

820 .

852.

820.

810.

835.

776.

767.

710.

644.

683.

621.

592.

593.

MIN. FUEL

917.

837 .

958 .

997.

1065.

1076 .

1040 .

1062 .

1100 .

1040.

1050 .

1018.

946.

844.

924.

837.

792.

788.

1002.

70 .

MAX. FUEL

1000 .

974.

905.

1038 .

1079.

1153.

1109.

1162.

1114.

1141.

1182.

1113.

1123.

1081 .

1001.

885.

977 .

880 .

$830^{\circ}$

$83 \mathrm{C}$.

FAST FLUENCE

$(10 * 25 N / M * 2)$

.00
.09
.22
.24
.69
.87
1.03
1.19
1.34
1.74
1.96
2.12
2.40
2.54
2.66
2.75
2.79
2.86
2.94
2.95

$1077 \circ$

E14-01 COMPACT 16

TEMPERATURES (C)

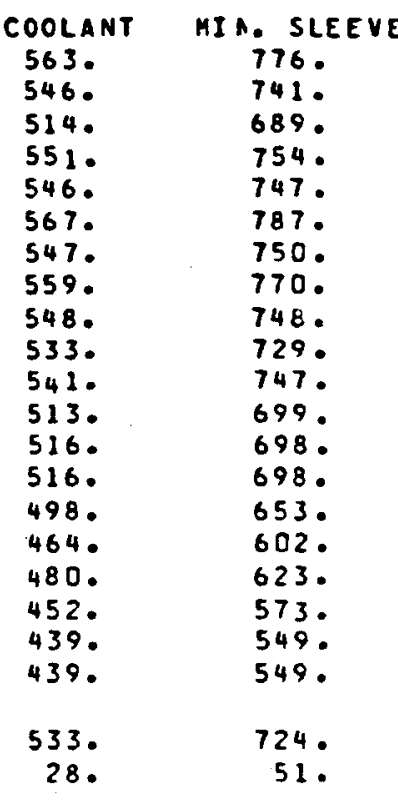

MAX. SLEEVE MIN. FUEL

\begin{abstract}
812.
\end{abstract}
724.

809 .

817.

870 .

829 .

860 .

829 .

822.

647 .

787.

789.

778 .

722 .

654.

693.

630 .

601.

927

MAX. FUEL

FAST FLUENCE

$(10 * 25 N / A * 2)$

1007 .

980.

910.

1043.

1084 .

1258.

1113.

1165 .

1118.

1150 .

1192:

1122 .

1132 .

1090.

1012.

$10122^{\circ}$
896.

988 .

890 .

839.

839.

.00
.09
.21
.23
.68
.85
1.02
1.17
1.32
1.71
1.93
2.09
2.37
2.52
2.63
2.72
2.77
2.83
2.91
2.93

798.

56.

1010

70.

1083. 
E14-01 COMPACT 17

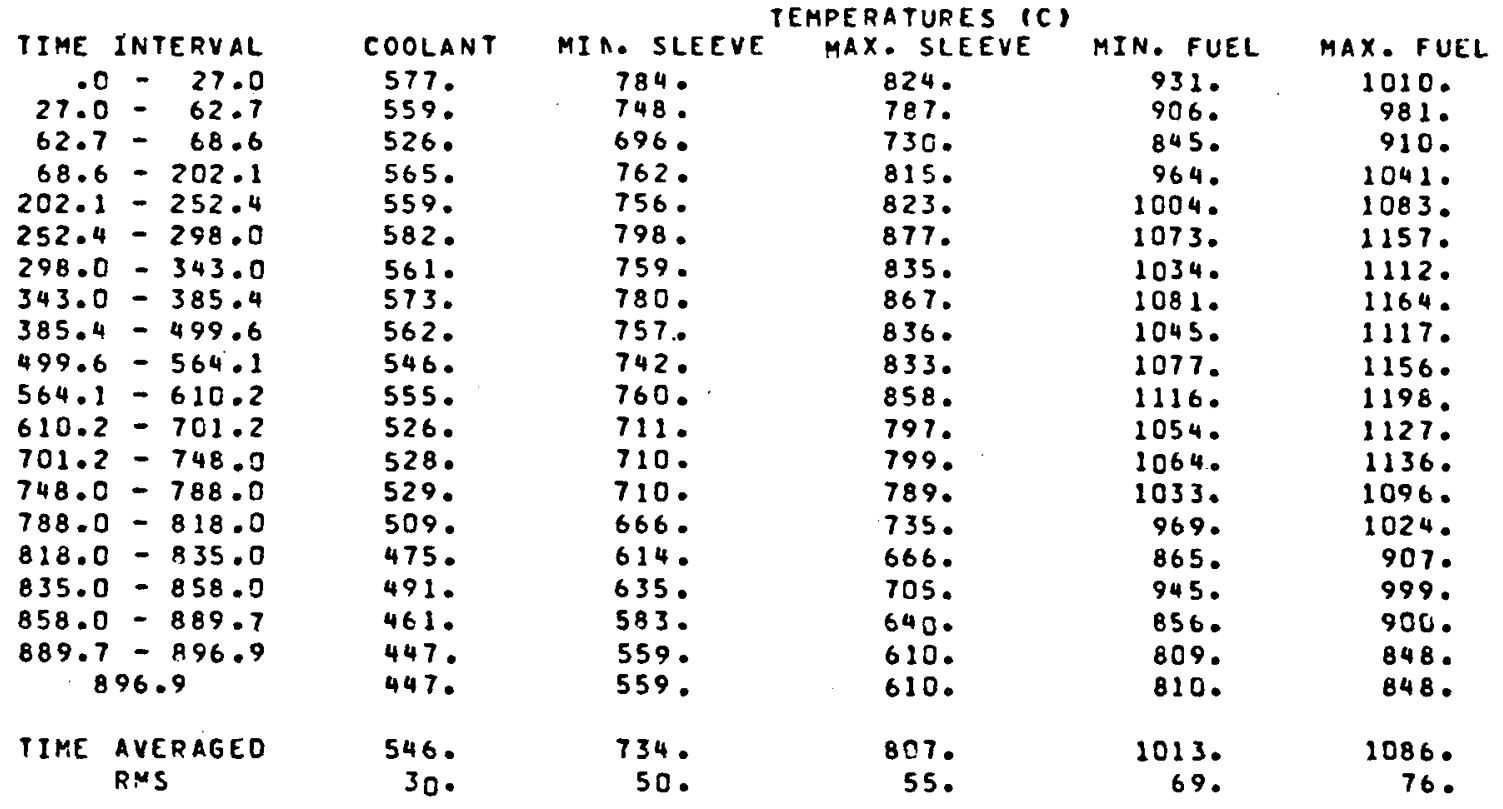

FAST FLUENCE

$(10 * 25 N / M * * 2)$

.00
.09
.21
.23
.66
.83
.99
1.15
1.29
1.68
1.90
2.06
2.33
2.47
2.59
2.68
2.73
2.79
2.87
2.89

E14-01 COMPACT 18

TIME INTERVAL $.0-27.0$ $.0=27.0$
$67.0-62.7$ $62.7-68.6$ $68.6-202.1$ $202.1-252.4$ $252.4-298.0$ $298.0=343.0$ $343.0-385.4$ $385.4-499.6$ $499.6-564.1$ $564.1-610.2$ $610.2-701.2$ $701.2-748.0$ $748.0-788.0$ $788.0-818.0$ $818.0-835.0$ $835.0=858.0$ $858.0-889.7$

$\begin{array}{cc}\text { COOLANT } & \text { MIN. SLEEVE } \\ 592 . & 792 . \\ 572 . & 7550^{\circ} \\ 537 . & 7020^{\circ} \\ 578 . & 769 . \\ 573 . & 765 . \\ 597 . & 807 . \\ 575 . & 768 . \\ 587 . & 790 . \\ 576 . & 767 . \\ 559 . & 753 . \\ 569 . & 772 . \\ 539 . & 721 . \\ 541 . & 720 . \\ 542 . & 721 . \\ 520 . & 679 . \\ 485 . & 626 . \\ 501 . & 646 . \\ 470 . & 593 . \\ 455 . & 568 . \\ 456 . & 568 . \\ & \\ 559 . & 744 . \\ 31 . & 50 .\end{array}$

IEMPERATURES (C)
MAX. SLEEVE

831.
793.
735.
820.
830.
884.
841.
873.

IN. FUEL MAX. FUEL

FAST FLUENCE

$(10 * 25 N / M * 2)$

935. 1012

908.981.

$846.910^{\circ}$

964.1039.

1005. 1083.

1074.1157.

1081.

1081.1162.

$\begin{array}{ll}1045 . & 1115^{\circ} \\ 1081 . & 1159^{\circ}\end{array}$

1120.1201.

1058.1130 .

1067.1139.

1037.1099.

979. 1035 .

875.917.

954.1200.

908.

$\begin{array}{ll}816 . & 855 . \\ 817 . & 856 .\end{array}$

.00

.09

.20
.22

.65

.81

.97

1.12

1.26

1.64

1.86

2.02

2.29

2.43

2.55

2.64

2.69

2.75

2.83

2.85

IIME AVERAGED RMS

50 .

814.

1016.

2087. 
E14-01 COMPACT 19

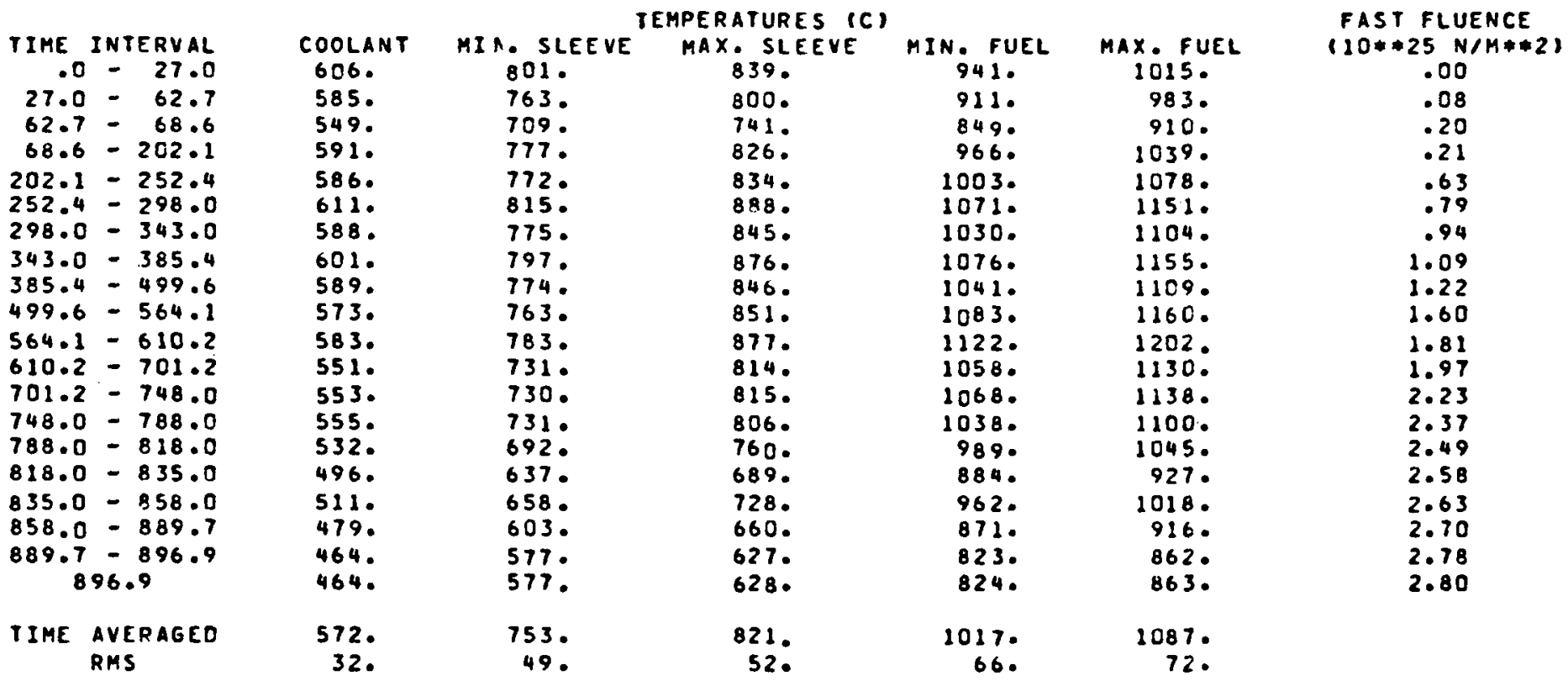

E14-01 COMPACT 20

TIME INTERVAL<smiles>[13CH3][13CH3]</smiles>

$27.0=27.0$

$62.7-68.6$

$68.6-202.1$

$202.1-252.4$

$252.4-298.0$

$298.0-343.0$

$343.0-385.4$

$385.4=499.6$

$499.6-564.1$

$564.1-610.2$

$610.2-701.2$

$701.2-748.0$

$748.0-788.0$

$788.0-818.0$

$818.0-835.0$

$835.0-858.0$

$858.0-889.7$

$889.7-896.9$

896.9

TIME AVERAGED RMS
TEMPERATURES (C)

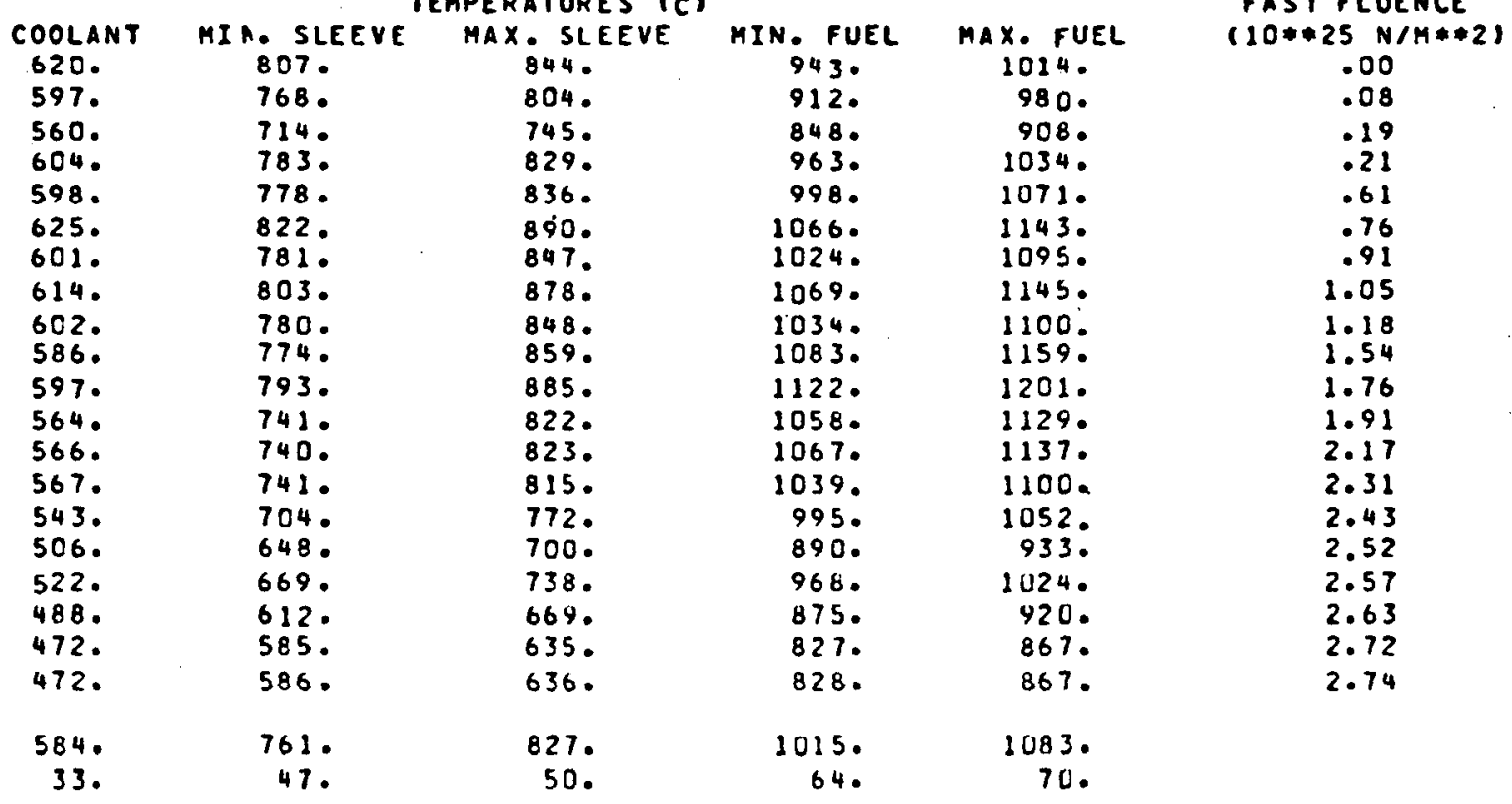


E14-01 COMPACT 21

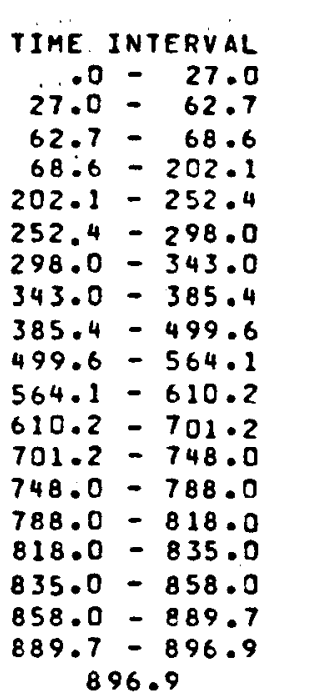

TIME AVERAGED RMS

COOLANT
633.
608.
570
616.
610.
638
613.
627.
614.
598.
610
576.
578
580
555.
517
532.
497.
480.
480.
596.
34.

MIA. SLEEVE
811.
772.
717.
786.
782.
826.
785.
807.
784.
782.
802.
749.
748.
750.
715.
659.
679.
621.
593.
594.
767.
46.

TEKPERATURES ' (C)

\begin{tabular}{|c|c|c|}
\hline $\begin{array}{c}\text { MAX. SLEEVE } \\
847 \text {. } \\
806 .\end{array}$ & $\begin{array}{c}\text { MIN. FUEL } \\
942 . \\
909 .\end{array}$ & $\begin{array}{c}\text { MAX.FUEL } \\
1009 . \\
974 .\end{array}$ \\
\hline $\begin{array}{l}746 \\
830 \\
836\end{array}$ & $\begin{array}{l}845 . \\
958 . \\
989 .\end{array}$ & $\begin{array}{l}902 \\
1025 \\
1059\end{array}$ \\
\hline $\begin{array}{l}890 . \\
846 . \\
877\end{array}$ & $\begin{array}{l}1056 . \\
1014 . \\
1057\end{array}$ & $\begin{array}{l}1130 . \\
1082 \\
1130\end{array}$ \\
\hline $\begin{array}{l}848 . \\
864 .\end{array}$ & $\begin{array}{l}1023 . \\
1079\end{array}$ & $\begin{array}{l}1086 . \\
1154 .\end{array}$ \\
\hline $\begin{array}{l}891^{\circ} \\
827 . \\
828 . \\
821 .\end{array}$ & $\begin{array}{l}1118 . \\
10530^{\circ} \\
10610^{\circ} \\
1035 .\end{array}$ & $\begin{array}{l}1195^{\circ} \\
1122 . \\
1130^{\circ} \\
1094^{\circ}\end{array}$ \\
\hline $\begin{array}{l}783 . \\
710 .\end{array}$ & $\begin{array}{l}998 . \\
893 .\end{array}$ & $\begin{array}{r}1055 . \\
936 .\end{array}$ \\
\hline 747. & 970 & 1025. \\
\hline 677. & 877. & 922. \\
\hline 643. & 828. & $868^{\circ}$ \\
\hline 643. & 829. & 868. \\
\hline $\begin{array}{r}830 \\
48 .\end{array}$ & $\begin{array}{r}1009 \\
62\end{array}$ & $\begin{array}{r}1075 \\
68 \text {. }\end{array}$ \\
\hline
\end{tabular}

FAST FLUENCE

FAST FLUENCE
$(10 * 25 N / M * 2)$

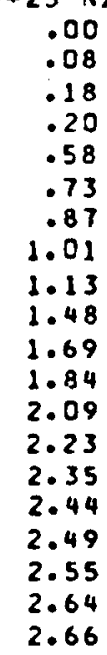

68.

\section{E14-01 COMPACT 22}

TIME INTERVAL $.0-27.0$ $27.0-62.7$ $62.7-68.6$ $68.6-202.1$

$202.1-252.4$

$252.4-298.0$

$298.0-343.0$

$343.0-385.4$

$385.4-499.6$

$499.6-564.1$

$564.1-610.2$

$610.2=701.2$

$701.2=746.0$

$748.0-788.0$

$788.0-818.0$

$818.0-835.0$

$835.0-858.0$

$858.0-889.7$

$889.7-896.9$

896.9

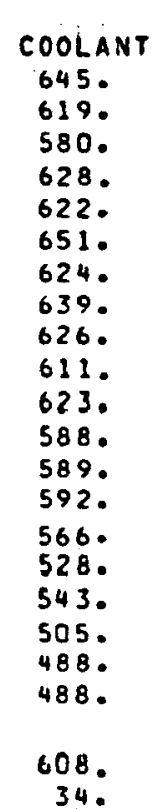

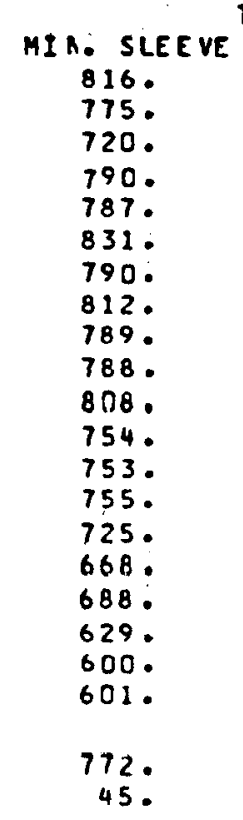

TEMPERATURES (C)

IIME AVERAGEO RMS

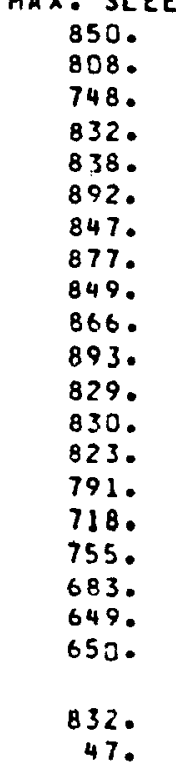

MIN: FUEL

940.

906.

842 .

953.

983.

1049

1006.

1015 .

1069.

1107.

1043 .

1050 .

1025 .

998.

894.

968.

$B 75$.

826.

827 .

1006.

969.

897.

1017.

1051.

1120 .

1072 .

1119.

1075 .

1241.

$1188^{\circ}$

1109 .

1116.

1082.

1055 .

436.

1024.

919.

$8655^{\circ}$

1002.

60.
FAST FLUENCE

(20*25 N/H**2)

.00

.07

.17

.19

.55

.69
.83

.96

1.08

1.41

1.61

1.76

2.00

2.13

2.25

2.34

2.40

2.46

2.55

2.57 
E14-01 COMPACT 23

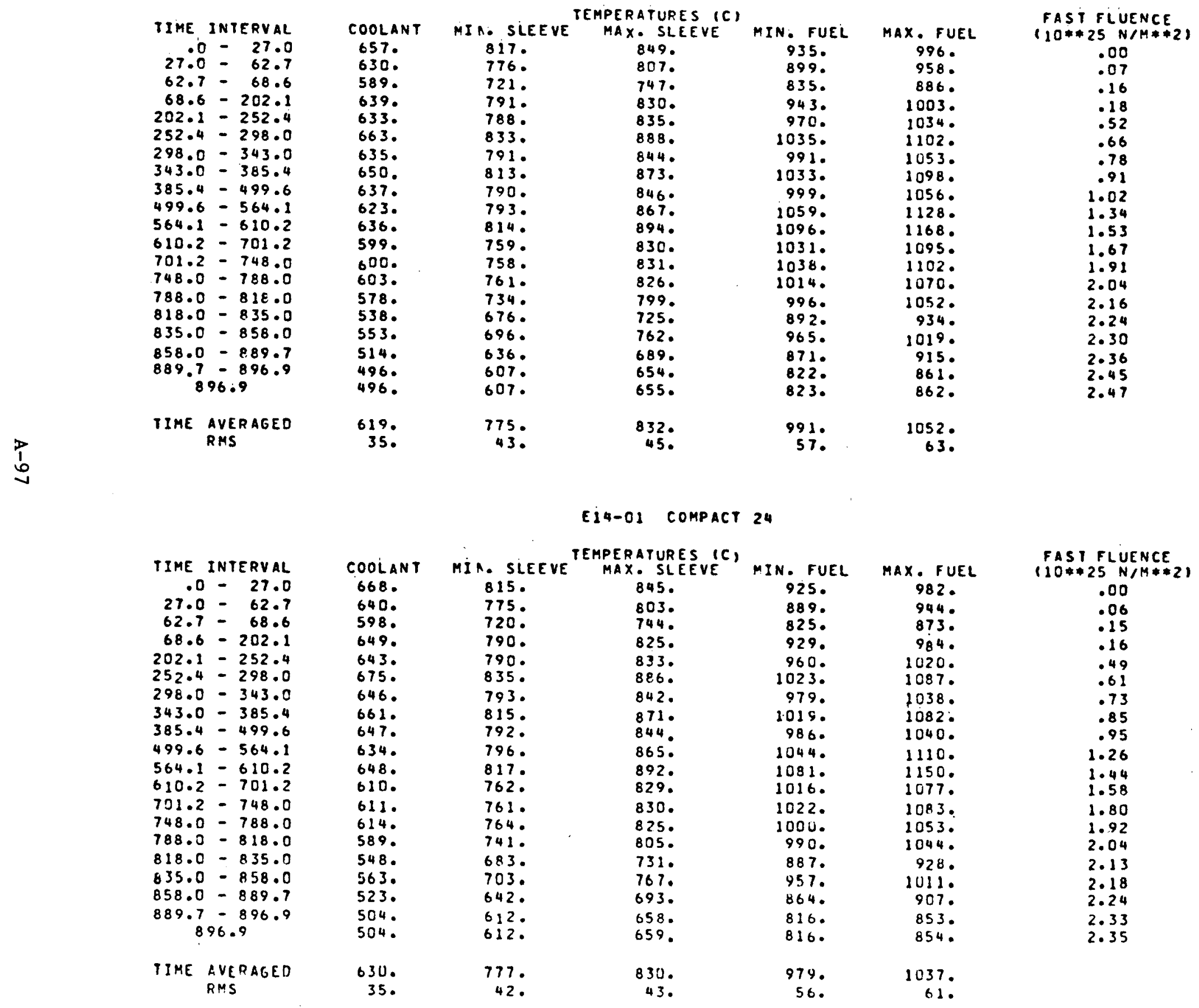


E14-01 COMPACT 25

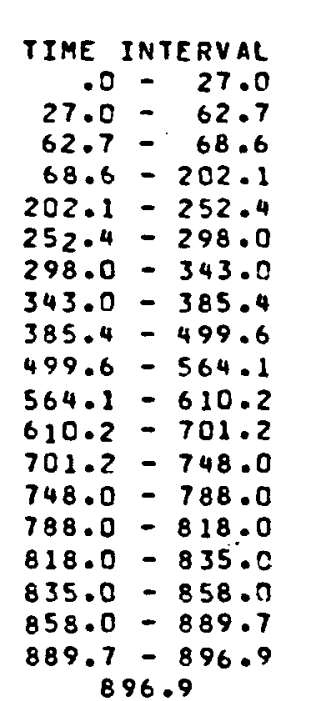

TIME AVERAGEO RMS

$p$
1
0
TEMPERATURES (C)

COOLANT
678.
649.
606.
658.
652.
685.
6550
671.
657.
645.
659.
620.
621.
$625 \circ$
600.
558.
573.
531.
512.
512.
639.
35.

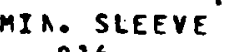

816.

775 .

720 .

790.

788.

701.

791.

813.

797 .

818 .

763.

762 .

765 .

748 .

689.

709 .

647 .

617 .

617 .

778 .

40.
MIN. FUEL

919.

882.

818.

919.

943.

1004.

960.

966.

1025.

1061.

996.

1001 .

981 .

982 .

880 .

948 .

855 .

807.

808 .

963.

51.

827.
41.
MAX. FUEL

973.

933.

863.

971.

998.

1014.

$1055^{\circ}$

1055 .

1087 .

1125.

1053.

1059.

1031.

1035 .

920.

1000.

844.

1017.

56.
FAST FLUENCE

$(10 * 25 \quad N / M * 22)$

.00

.06

.14

.45

.45
.57

.68

.78

.88

1.17

1.34

1.68

1.79

1.99

2.04

2.10

2.19

2.20

E14-01 COMPACT 26

TIME INTERVAL

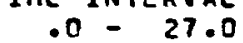

$27.0-62.7$

$62.7-68.6$

$68.6-202.1$

$202.1-252.4$

$252.4-296.0$

$298.0=343.0$

$343.0-385.4$

$385.4-499.6$

$499.6-564.1$

$564.1-610.2$

$610.2-701.2$

$701.2-748.0$

$748.0-788.0$

$788.0=818.0$

$818.0-835.0$

$835.0-858.0$

$858.0-889.7$

$889.7=896.9$

896.9

TEMPERATURES (C)

COOLANT

687 .

657.

614.

667.

661.

695.

664.

680.

666.

659.

669.

629.

634.

610.

568.

582.

$539^{\circ}$

519 .

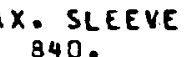

MIN. FUEL

910.

814.

773 .

718.

797 .

739.

785 .

831.

811 .

788 .

797 .

818 .

764.

762 .

766.

752 .

693.

712.

519.

620 .

620 .

817.

820 .

872.

827

855 .

829.

855 .

822 .

820 .

821 .

819.

810.

771.

$697^{\circ}$

662 .

662 .

809.

MAX. FUEL

FAST FLUENCE

$110 * 25 N / M * 22$

959.

.00

.06

.14

.14

953.

925.9976.

985.11039.

977.

945 .

1006 .

1040 .

976.

981.

963.

969.

870.

934.

842.

795.

796.

990.
1029.

.52

.63

.72

.81
1.08

991.

1064.

1101.

1.24

1.35

1034.

1009.

1020.

1.55

2.66

1.77

1.85

1.90

984.

882 .

830 .

831.

1.96
2.04

2.05

IIHC AVERAGED

717.

622.

946.

35 .

39.

39.

48.

496. 


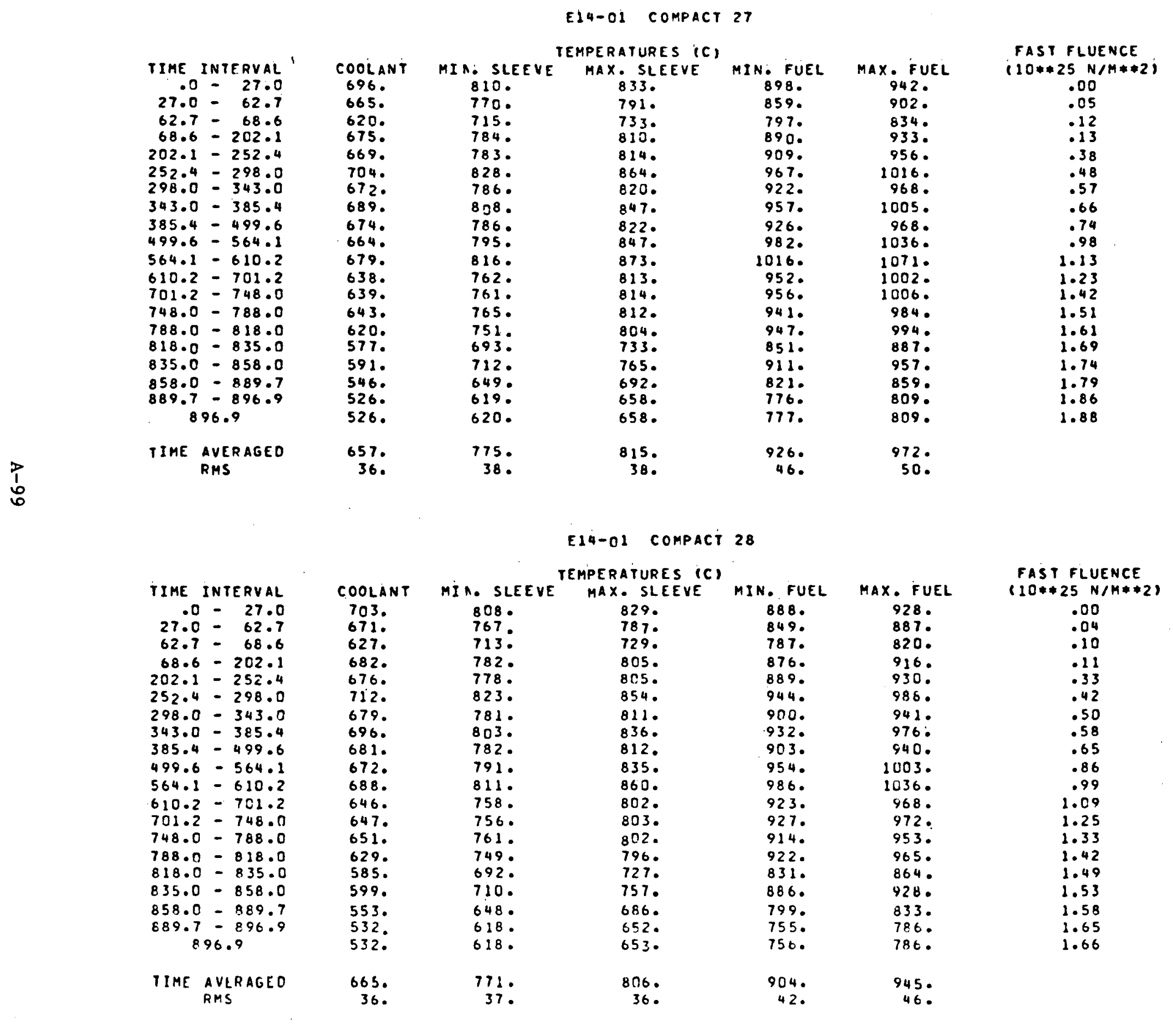


E14-01 COMPACT 29

IIME INTERVAL

$.0-27.0$

$27.0-62.7$

$62.7-68.6$

$68.6-202.1$

$202.1-252.4$

$252.4-298.0$

$298.0-343.0$

$343.0-385.4$

$385.4-499.6$

$499.6-564.1$

$564.1-610.2$

$610.2-701.2$

$701.2=748.0$

$748.0-788.0$

$788.0-818.0$

$818.0-635.0$

$835.0-858.0$

$858.0-889.7$

$889.7-896.9$

896.9

TIME AVERAGED RMS

$\frac{p}{1}$

\begin{tabular}{|c|c|}
\hline COOLANT & $M I A, S L$ \\
\hline $\begin{array}{l}710 . \\
678 . \\
632 .\end{array}$ & $\begin{array}{l}804 . \\
763 . \\
709 .\end{array}$ \\
\hline 688 & 778. \\
\hline $\begin{array}{l}683 . \\
719 .\end{array}$ & $\begin{array}{l}774 . \\
819 .\end{array}$ \\
\hline 686. & 777. \\
\hline 703. & 799. \\
\hline 688. & 778 . \\
\hline 680. & 785. \\
\hline 695 . & 806. \\
\hline $\begin{array}{l}653 . \\
654 .\end{array}$ & $7525^{\circ}$ \\
\hline 658 & 756. \\
\hline 637. & 748. \\
\hline 592. & 691. \\
\hline $\begin{array}{l}606 . \\
560 .\end{array}$ & $\begin{array}{l}708 . \\
646 .\end{array}$ \\
\hline $\begin{array}{l}538 \\
538\end{array}$ & $\begin{array}{l}617 . \\
617 .\end{array}$ \\
\hline 672. & 767. \\
\hline 36. & 37 \\
\hline
\end{tabular}

TEMPERATURES (C)

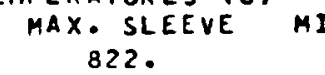

FUEL MAX. FUEL

B76.

912.

836.

775.

724.

798 .

797.

802 .

827.

803.

822 .

$846^{\circ}$

789.

790.

791.

721.

689.

647 .

647.

796.

870.

923.

879 .

910.

881.

924.

954.

893.

896.

885 .

898 .

811.

861.

736.

737 .

881.

38 .

870.

805 .

896.
908.

963.

916.

948.

915.

967.

999.

933.

936.

920 .

938 .

842 .

901.

810.

764.

765

918.

E14-01 COMPACT 30

TIME INTERVAL $.0-27.0$ $27.0-62.7$ $62.7-68.6$ $68.6-202.1$ $202.1-252.4$

$252.4-298.0$

$298.0=343.0$

$343.0-385.4$

$385.4-499.6$

$499.6-564.1$

$564.1=610.2$

$610.2-701.2$

$701.2-748.0$

$748.0-788.0$

$788.0-818.0$

$818.0-835.0$

$835.0-858.0$

$858.0-889.7$

$889.7-896.9$

896.9

IIME AVERAGED RMS
TEMPERATURES (C)

\begin{tabular}{|c|c|c|c|c|}
\hline \multirow[b]{2}{*}{$\begin{array}{l}\text { COOLANT } \\
718 .\end{array}$} & \multicolumn{3}{|c|}{ TEMPERATURES (C) } & \multirow[b]{2}{*}{$\begin{array}{c}\text { MAX } \\
935 \text {. FEL }\end{array}$} \\
\hline & $\begin{array}{c}\text { MIA. SLEEVE } \\
818 \text {. }\end{array}$ & $\begin{array}{c}\text { MAX. SLEEVE } \\
839 .\end{array}$ & $\begin{array}{c}\text { MIN. FUEL } \\
896 .\end{array}$ & \\
\hline $\begin{array}{l}684^{\circ} \\
638 . \\
695\end{array}$ & $\begin{array}{l}777 . \\
721 . \\
792 .\end{array}$ & $\begin{array}{l}796 . \\
737 . \\
813 .\end{array}$ & $\begin{array}{l}854 . \\
790 . \\
876 .\end{array}$ & $\begin{array}{l}891 . \\
823{ }^{\circ} \\
915^{\circ}\end{array}$ \\
\hline $\begin{array}{l}690 . \\
727 \\
693\end{array}$ & $\begin{array}{l}787 . \\
833 . \\
791 .\end{array}$ & $\begin{array}{l}81.0 . \\
859 . \\
814 .\end{array}$ & $\begin{array}{l}882 . \\
936 . \\
889 .\end{array}$ & $\begin{array}{l}922^{\circ} \\
978^{\circ} \\
928^{\circ}\end{array}$ \\
\hline $\begin{array}{l}710 . \\
695 \\
687\end{array}$ & $\begin{array}{l}813 . \\
791 . \\
795 .\end{array}$ & $\begin{array}{l}839 . \\
815 . \\
826 .\end{array}$ & $\begin{array}{l}919 . \\
889 . \\
921 .\end{array}$ & $\begin{array}{l}961 . \\
925^{\circ} \\
965^{\circ}\end{array}$ \\
\hline $\begin{array}{l}703 . \\
660 . \\
661 .\end{array}$ & $\begin{array}{l}816 . \\
762{ }^{\circ} \\
761 .\end{array}$ & $\begin{array}{l}850 . \\
792^{\circ} \\
792^{\circ}\end{array}$ & $\begin{array}{l}949 . \\
880^{\circ} \\
890^{\circ}\end{array}$ & $\begin{array}{l}996 . \\
929 . \\
931 .\end{array}$ \\
\hline $\begin{array}{l}666 . \\
644 . \\
599 .\end{array}$ & $\begin{array}{l}766 . \\
750 . \\
693 .\end{array}$ & $\begin{array}{l}794 . \\
781 . \\
717 .\end{array}$ & $\begin{array}{l}881 . \\
877 . \\
791 .\end{array}$ & $\begin{array}{l}9170^{\circ} \\
915^{\circ} \\
824 .\end{array}$ \\
\hline $\begin{array}{l}613 . \\
566 .\end{array}$ & $\begin{array}{l}710 . \\
648 .\end{array}$ & $\begin{array}{l}742 . \\
674 .\end{array}$ & $\begin{array}{l}840 . \\
760 .\end{array}$ & $\begin{array}{l}878 . \\
790 .\end{array}$ \\
\hline $\begin{array}{l}543 . \\
544 .\end{array}$ & $\begin{array}{l}618 . \\
619 .\end{array}$ & $\begin{array}{l}641 . \\
642 .\end{array}$ & $\begin{array}{l}720 . \\
120 .\end{array}$ & $\begin{array}{l}746 . \\
747 .\end{array}$ \\
\hline 679. & 778 & 804. & 884. & 923. \\
\hline 36. & 40. & 39. & 40. & 43. \\
\hline
\end{tabular}

FAST FLUENCE

$(10 * 25 N / M * * 2)$

.00

.04

.09

.27

.34

.41
.47

.47

.71

.81
.89

1.02

1.09

1.17

1.22

1.26

1.30

1.36
1.37

FAST FLUENCE

(10**25 N/M**2)

.00

.02

.02

.06

.28

.22

.27

. 31

.35

.53

.58

.67

.71
.77

.85
.85

.92

.93 


\section{E14-01 FUEL PERFORMANCE}

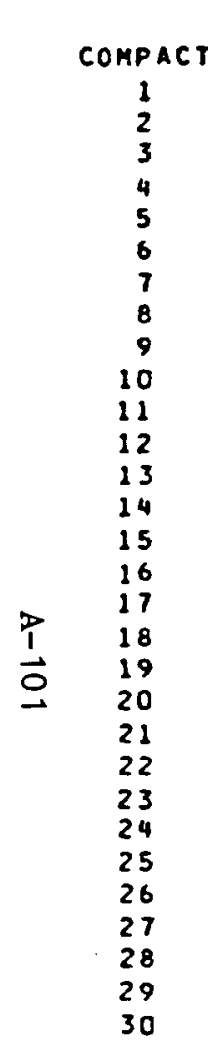

$\begin{array}{ccc}\text { KERNEL MIGRAIION (MICRONSI } & \\ \text { AVERAGE } & \text { AMOEBA EQ. TEMP. } & \text { PEAK } \\ .00 & 574 . & .00 \\ .00 & 629 . & .00 \\ .00 & 700 . & .00 \\ .00 & 762 . & .00 \\ .00 & 813 . & .00 \\ .01 & 861 . & .01 \\ .01 & 904 . & .02 \\ .03 & 941 . & .04 \\ .06 & 973 . & .08 \\ .10 & 1002 . & .13 \\ .15 & 1024 . & .19 \\ .20 & 1036 . & .26 \\ .27 & 1052 . & .35 \\ .33 & 1064 . & .42 \\ .39 & 1077 . & .50 \\ .43 & 1085 . & .55 \\ .44 & 1088 . & .56 \\ .44 & 1091 . & .57 \\ .43 & 1089 . & .55 \\ .40 & 1088 . & .51 \\ .34 & 1080 . & .44 \\ .28 & 1069 . & .36 \\ .22 & 1055 . & .28 \\ .16 & 1040 . & .21 \\ .11 & 1019 . & .14 \\ .07 & 998 . & .09 \\ .04 & 973 . & .05 \\ .02 & 946 . & .03 \\ .01 & 917 . & .02 \\ .01 & 921 . & \end{array}$

DEFECTIVE COATINGS
.06
.10
.13
.16
.18
.19
.20
.21
.22
.23
.23
.23
.24
.24
.24
.23
.23
.23
.22
.22
.21
.21
.20
.19
.18
.16
.15
.13
.11
.07

FUEL FAILURE ( $x$ )

PRESSURE VESSEL KERNEL MIGRATION TOTAL

$\begin{array}{lll}.17 & \text { KERNEL MIGRATION } & \text { TOT } \\ .28 & .00 & .23 \\ .28 & .00 & .38\end{array}$

.23

.36

.43

.49
.53

.50
.59

.61

.62

.63

.64

.65

.65

.64

.64

.63
.62

.62
.60

.58

.56

.54

.48

.45

.41

.30

.21

.00
.00
.00
.00
.00
.00
.00
.00
.00
.00
.00
.00
.00
.00
.00
.00
.00
.00
.00
.00
.00
.00
.00
.00
.00
.00
.00
.00

.49

.59

.66
.72

.77

.83

.85

.88

.88

.88

.88

.87

.86

.84
.82

.82
.80

.77

.74

.70

.66

.62

.56
.50

.41
.28

AMOEBA EQUTVALENT TEMPERATURES (C) CORRE SPONO TO AVERAGE KERNEL MIGRATIONS

KERNEL HIGRATION OISTANCES CALCULATEO USING $50 \%$ CONFIDENCE LEVEL KMC

FUEL FAILURE CORRESPONOS TO 502 CONFIDENCE LEVEL 
